\title{
New Applications of Methyl 2-Chloro-2-cyclopropylideneacetate \\ Towards the Synthesis of Biologically \\ Important Heterocycles
}

\author{
DISSERTATION \\ zur Erlangung des Doktorgrades \\ der Mathematisch-Naturwissenschaftlichen Fakultäten \\ der Georg-August-Universität zu Göttingen \\ vorgelegt von \\ Suryakanta Dalai \\ aus \\ Jagatsingpur, Indien
}

Göttingen 2005 

D7

Referent: $\quad$ Prof. Dr. A. de Meijere

Korreferent: $\quad$ Prof. Dr. Dr. h. c. L.-F. Tietze

Tag der mündlichen Prüfung: 24.06.2005 

Die vorliegende Arbeit wurde unter der Leitung von Herrn Prof. A. de Meijere in der Zeit von September 2001 bis Mai 2005 im Institut für Organische und Biomolekulare Chemie der GeorgAugust-Universität Göttingen angefertigt.

Meinem Lehrer, Herrn Prof. A. de Meijere, möchte ich an dieser Stelle für die Überlassung des interessanten Themas, seine hilfreichen Anregungen, seine Diskussionsbereitschaft und die stete Unterstützung während der Anfertigung dieser Arbeit herzlich danken. 

To My Parents 

Table of Contents

1. Introduction

2. Main Part 11

2.1. An Advanced Synthesis of Methyl 2-Chloro- and 2-Bromo-2-cyclopropylideneacetate 11

2.2. Tetrahydroquinazolinone Derivatives 14

2.2.1. Synthesis of amidines from nitriles 14

2.2.2. Synthesis of cyclobutene-annelated pyrimidinones 15

2.2.3. Cyclobutene ring opening and subsequent Diels-Alder reaction 16

2.2.4. Microwave assisted Diels-Alder reaction 18

2.2.5. "One pot" synthesis of tetrahydroquinazolinones__ 20

2.2.6. Reductive elimination of sulfone group __ 20

2.2.7. Alkylation of tetrahydroquinazolinones __ 23

2.2.8. Nucleophilic substitution of SMe group for thesynthesis of 2-amino tetrahydroquinazolinones 25

2.3. Spirocyclopropane Analogue of Tadalafil __ 27

2.3.1. Considerations___ 27

2.3.2. Synthesis of a spirocyclopropanated tryptophan methyl ester __ 28

2.3.3. The Pictet-Spengler reaction for the synthesis of tetrahydro- $\beta$-carbolines __ 29

2.3.4. Synthesis of spirocyclopropane analogue of hydantoin lead to Tadalafil and Tadalafil

2.4. Synthesis of Spirocyclopropaneoxazolines

2.4.1. Synthesis of spirocyclopropaneoxazolinecarboxylic acid and attempted transformation to oxazole or thiazole derivatives 33

2.4.2. Coupling of oxazolinecarboxylic acids with anilines and subsequent Mitsunobu reaction for the synthesis of benzoxazole derivatives

2.4.3. Pd-Catalyzed $\mathrm{C}-\mathrm{N}$ or $\mathrm{C}-\mathrm{C}$ bond formation reaction for the synthesis of aminoaryl or biaryl-substituted oxazolines

2.5. Reaction of Methylenethiourea with Methyl 2-Chloro2-cyclopropylideneacetate 43 
2.6. Sequential Addition of Grignard Reagents and Aldehydes to Methyl 2-Chloro-2cyclopropylideneacetate _ـ 45

2.7. Synthesis of 6-Amino-3-azabicyclo[3.1.0]hexane ___ 51

3. Experimental Part _ 55

3.1. General Remarks _ـ 55

3.2. Synthesis of Compounds

3.2.1. Advanced synthesis of methyl 2-chloro- and 2-bromo-2-cyclopropylideneacetate

3.2.2. Synthesis of 5,6,7,8-tetrahydroquinazolinone derivatives ___ 60

3.2.3. Synthesis of cyclopropyl analogue of Tadalafil ___ 88

3.2.4. Synthesis of spirocyclopropaneoxazolines ___ 97

3.2.5. Michael addition of dialkythiourea onto methyl 2-chloro-2-cyclopropylideneacetate

3.2.6. Sequential addition of Grignard reagent and aldehyde with methyl 2-chloro-2cylopropylideneacetate 125

3.2.7. Synthesis of 6-amino-3-azabicyclo[3.1.0]hexanes 138

4. Summary 143

5. Spectral Data 151

6. Crystal Data 167

7. Reference 187 


\section{Introduction}

Organic synthesis has a long history that can be traced back to ancient times, although it was not recognized as such, due to lack of scientific knowledge. As a science, arguably the synthesis of urea by Wöhler in the year 1828 is the beginning of organic synthesis. This synthesis was followed by other milestones such as synthesis of acetic acid (Kolbe, 1885), glucose (Fisher, 1890), $\alpha$-terpineols (Perkin, 1904), camphor (Komppa, 1903), torpinone (Robinson, 1917), quinine (Woodward, 1944), and many other natural products. ${ }^{[1]}$ Today, organic synthesis can be broadly divided into two main parts: target oriented (total synthesis) and method oriented. The target oriented molecule can be a natural product or a designed molecule. The organic chemist is free to imagine and design unlimited numbers of new molecules never seen before either in nature or in the laboratory. This molecular design process is often guided by the particular interests of the chemist and can be aided by molecular modeling studies. These designed molecules can be of theoretical, physical, material science or biological interest. Undoubtedly, the most fertile area of molecular design for the organic chemist is that of biologically interesting molecules. Frequently the designed molecule are based on the structures of bioactive natural products (natural product analogue) or a completely imagined molecule targeted towards a specific biological action. Today molecular design, chemical synthesis and the biological evaluation is a powerful multidisciplinary approach to drug discovery and development. For this reason it has been a great challenge for a synthetic chemist to synthesize analogues of natural products.

From the last twenty years there has been a lot of work directed towards the cyclopropyl group as a special substituent in biologically active molecules. Natural and synthetic compounds bearing a cyclopropyl group are endowed with large spectrum of biological properties ranging from enzyme inhibitions to insecticidal, antifungal, herbicidal, antimicrobial, antibiotic, antibacterial, antitumor and antiviral activities. ${ }^{[2]}$ The cyclopropane ring, due to its unusual bonding and inherent ring strain $(27.5 \mathrm{kcal} / \mathrm{mol})$ is unique among carbocycles in both its properties and reactions. ${ }^{[3]}$ Consequently numerous methods have been developed for the construction of cyclopropanes. Among them metal-catalyzed cyclopropanation, in particular that of alkenes with ethyl diazoacetate, is one of the most simple and straightforward approaches for the preparation of cyclopropanes. Initially copper complexes were widely used as catalysts for the cyclopropanation of alkenes with diazo compounds. ${ }^{[4]}$ Subsequently, rhodium carboxylates have been reported to be highly effective catalyst for cyclopropanation. ${ }^{[5]}$ Among cyclopropanes, cyclopropanols have long 
stimulated a spate of synthetic and mechanistic studies due to their unique reactivity. ${ }^{[6]}$ Cyclopropanols are prone to ring-opening reaction and can easily be converted into certain classes of organic compounds in particular, to carbonyl compounds by ring opening followed by reaction with electrophiles. ${ }^{[7]}$ In 1989, Kulinkovich et al. have developed a synthetically useful reaction in which a titanacyclopropane intermediate act as a formal 1,2-dicarbanionic equivalent, and thus leads to the formation of two new carbon-carbon bonds, allowing the conversion of ester to caclopropanols. ${ }^{[8]}$ This Kulinkovich reaction has been extended by de Meijere et al. for the synthesis of cyclopropyl amines from $N, N$-dialkycarboxamide. ${ }^{[9]}$

This is an important milestone in the field of cyclopropane chemistry, because around 200 pharmaceutically relevant compounds contains cyclopropyl amine moiety. Some examples of biologically important molecules containing cyclopropyl group are Ciprofloxacin (1) ${ }^{[10]}$ and Trovaflaxine (2) ${ }^{[11]}$ (Figure 1) which belong to a major class of fluoroquinolones antibacterials with great therapeutic potential and are widely used to treat patients with infections.
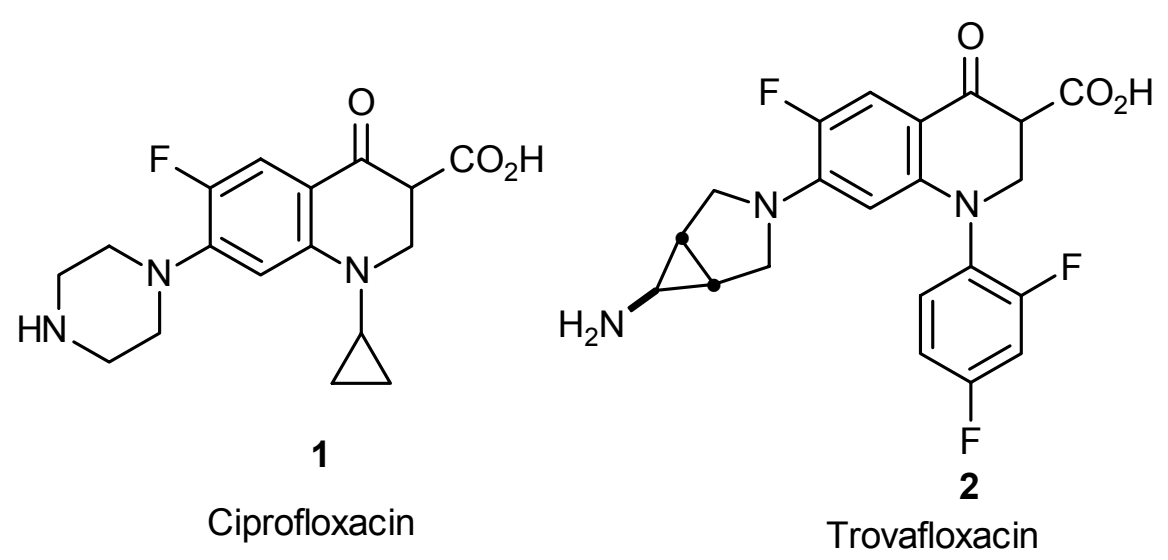

Figure 1. Sructure of quinolone antibacterial agents, Ciprofloxacin (1), Trovafloxacin (2).

Nature also makes use of cyclopropyl groups even in amino acids, ${ }^{[12]}$ and most of the naturally occurring amino acids with a cyclopropyl group, as well as most of the cyclopropyl analogues of natural amino acids, are responsible for the observed biological activities of compounds containing them as constituents. The design and synthesis of unnatural amino acid for incorporation into peptides in order to confer conformational constraint, leading to potential improvements in biological activity, as well as to increase biostability, continues to be of great interest. ${ }^{[13]}$ The studies by Hruby et al. have shown that topographically constrained amino acid analogues can lead to dramatic change in potency and receptor selectivity of the peptide and have 
provided valuable insights about the role which play in amino acid residues in the peptide interaction with the receptor. ${ }^{[14]}$

The synthetic methodology based on the reactions of highly functionalized methylenecyclopropanes, has been growing steadily over the past decade. ${ }^{[15]}$ In particular the acceptoractivated methylenecyclopropanes, like (substituted) alkyl cyclopropylideneacetates (3), ${ }^{[16]}$ alkyl 2-bromo-2-cyclopropylideneacetates $(\mathbf{4})^{[17]}$ and methyl 2-chloro-2-cyclopropylideneacetates $(\mathbf{5})^{[18]}$ (Figure 2) are highly functionalized valuable building blocks for organic synthesis.

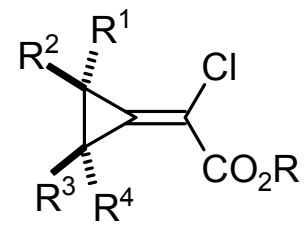

3

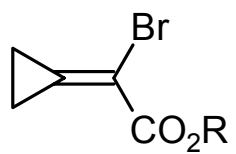

4<smiles>COC(OC)C(Cl)=C1CC1</smiles>

5

Figure 2. Alkyl 2-chloro-2-cyclopropylideneacetates 3, 5 and the bromo analogue 4.

In the year 1982 de Meijere et al. has described a synthetic method for synthesis of variously substituted methyl 2-chloro-2-cyclopropylideneacetates (3-Me) starting from tetrachlorocyclopropene (6). ${ }^{[18]}$ Tetrachlorocyclopropene readily available from tetrachloroethane and sodium trichloro-acetate, ${ }^{[19]}$ undergoes thermal ring opening in an autoclave to perchlorovinylcarbene (7), which can be efficiently trapped with a large number of alkenes to form 1chloro-1-(trichloroethenyl)-cyclopropane 8.

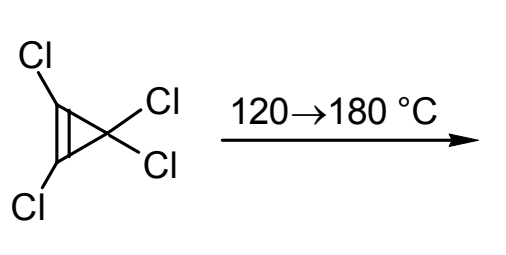

6

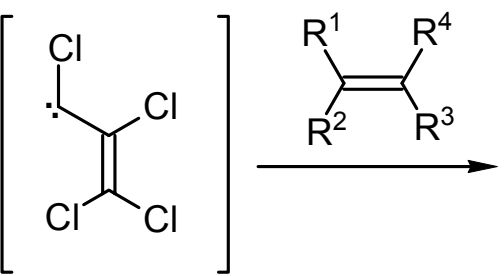

7<smiles>[R]C1([R])C([R])([R])C1(Cl)C(Cl)=C(Cl)Cl</smiles>

8
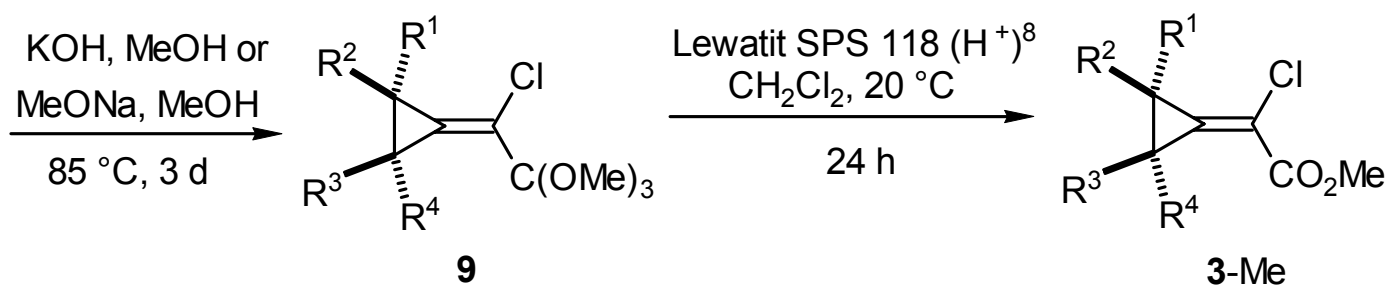

Scheme 1. Synthesis of substituted methyl 2-chloro-2-cyclopropylideneacetates (3-Me). 
From these intermediates, substituted methyl 2-chloro-2-cyclopropylideneacetates (3-Me) can be obtained by treatment with potassium hydroxide or sodium methoxide in methanol followed by acidic hydrolysis of the resulting orthoesters 9 (Scheme 1). The bromo analogues alkyl 2-bromo2-cyclopropylideneacetates 4 can be synthesized by the Wittig olefinations of cyclopropanone ethyl hemiacetal with appropriately substituted triphenyl methylenephosphoranes. ${ }^{[17]}$ These methylene cyclopropanes, in particular methyl 2-chloro-2-cyclopropylideneacetate (5) are very reactive Michael acceptor than any other 3,3-di-substituted acrylates. The addition of most nucleophiles occurs smoothly and this is partly due to the strain release upon conversion of $s p$ - to $s p^{2}$-hybridized carbon ${ }^{[3]}$ and is also due to the presence of the $\alpha$-chloro substituent. ${ }^{[20]}$ Due to its enhanced reactivities and multi-functionalities, $\mathbf{5}$ can be applied in a broad sense towards elegant syntheses of spirocyclopropanated carbo- ${ }^{[21]}$ and heterocycles, ${ }^{[22]}$ various cyclopropyl-group containing amino-acids ${ }^{[23]}$ as well as biologically active conformationally restricted peptide mimics $^{[24]}$ with different ring size and substituent. This can be achieved either by chemical transformations of the primary Michael adducts or by addition of a bidentate nucleophile on to 5 . The 1,4-addition of the nitrogen nucleophile with $\mathbf{5}$ proceeds very smoothly which after nucleophilic substitution of chlorine give the alanine analogue 10. ${ }^{[25]}$ The Michael adducts of diphenymethylene-amine (benzophenone imine) or benzylamine with $\mathbf{5}$ efficiently undergo further transformation to give cyclobutene derivatives (11). ${ }^{[23]}$ Amidines react with $\mathbf{5}$ in the presence of triethyl amine in dioxane to give cyclobutene annelated pyrimidinones (12) by Michael reaction followed by domino transformation. ${ }^{[26]}$ The reaction of $\mathbf{5}$ with aryl amides in presence of $\mathrm{NaH}$ or with aryl thioamide in presence of $\mathrm{NaHCO}_{3}$ in acetonitrile generates spirocyclopropane oxazolines $(\mathbf{1 3})^{[27]}$ and thiazolines (14). ${ }^{[28]}$ The kind of product obtained by Michael addition depends on the nature of reagents used. When $\mathrm{Ti}(\mathrm{iPrO})_{4}$ is used, reaction of aryl thioamides with $\mathbf{5}$ undergoe a sixmembered ring-closure to give the corresponding 5,6-dihydro-1,3-thiazin-4-ones (15). ${ }^{\text {[28] }}$ Pyrrolo[3,2-e]diazepinedione derivatives (16) can also be prepared from $\mathbf{5}^{[24]}$ A broad variety of geometrically defined spirocyclopropane bicyclic peptides $\mathbf{1 7}$ can also be obtained from $\mathbf{5}$ by sequence of reactions ${ }^{[24]}$ (Scheme 2). 
<smiles>[R]C1NC(=O)C(Cl)C2(CC2)S1</smiles>

15<smiles></smiles><smiles>[Y7]C1C(=O)N2C([R])C(=O)N([R])C3(CC3)C2C(=O)N1[R]</smiles><smiles>[R]C1=NC(C(C)=O)C2(CC2)S1</smiles>

14<smiles>COC(=O)C(Cl)=C1CC1</smiles><smiles>[CH]1C=C1</smiles><smiles>NC(C(=O)O)C1(N)CC1</smiles><smiles>[R]C1=NC2(CC2)C(C(C)=O)O1</smiles>

13

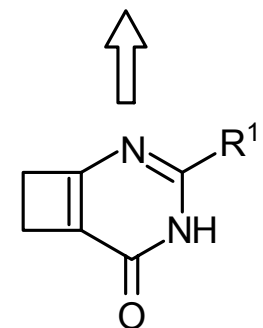

ß

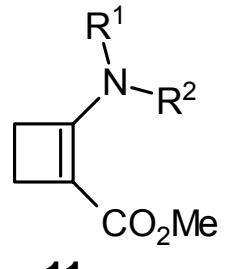

11

Scheme 2. Retrosynthetic analysis of cyclobutene derivatives, spirocyclopropanated carbo- and hetero-cycles from methyl 2-chloro-2-cyclopropylideneacetate (5).

The reaction of aryl amides or thioamide with 5 for the synthesis of oxazolines (13) or thiazolines (14) are particularly very interesting from a biological point of view, since oxazolines and thiazolines are present in many biologically active natural products ${ }^{[29]}$ and are found as a peptide link modification widespread into metabolites from bacterial and merine origin. ${ }^{[30]}$ The conformation constraint introduced by these "peptide mimic" can be used for the design of peptide analogue of pharmacologically interesting molecules. For this reason, these heterocyclic units have been used as scaffolding devices in peptide sequences which lead to the stabilization of the reverse turn secondary structure. ${ }^{[31]}$ Chiral oxazoline derivatives are used for enantiospecific total synthesis of natural products ${ }^{[32]}$ and are also widely applied as ligands in asymmetric synthesis. ${ }^{[33]}$ Oxazolines have been also shown to be suitable precursors for the paclitaxel side chain. ${ }^{[34]}$ Paclitaxel $\left(\right.$ Taxol $^{\mathbb{}}$ ) 18, originally isolated from the pacific yew (Taxus brevifolia), ${ }^{[35]}$ has become an important anticancer drug, especially for the treatment of refractory ovarian cancer, small-cell 
lung cancer, and metastatic breast disease. ${ }^{[36]}$ The first total synthesis of Taxol was achieved by Nicolaou et al., ${ }^{[37]}$ where the side chain was attached by ring-opening condensation with an appropriately substituted $\beta$-lactam. Since cyclopropyl groups have been proved to be highly effective in improving the activity of many biologically active compounds, ${ }^{[2 a, b][38]}$ several cyclopropane-bearing analogues of paclitaxel have been synthesized and shown to have improved or retained anticancer activity. ${ }^{[39]}$ The phenyl derivative of the above spirocyclopropanated oxazoline $(\mathbf{1 3}-\mathrm{Ph})$ has been used for the synthesis of $\mathrm{C}-3^{\prime}$-cyclopropanated Taxol analogue 19 (Scheme 3). ${ }^{[40]}$

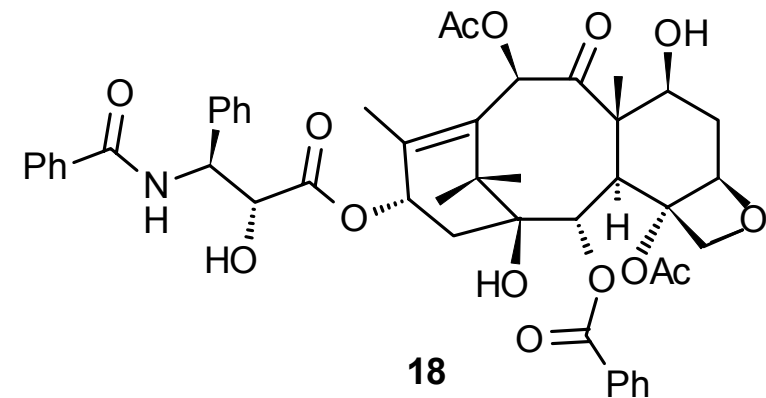

Taxol<smiles>O=C(NC1([C@H](O)C(=O)O)CC1)c1ccccc1</smiles>
$\mathrm{HO}$

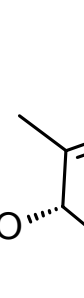

$\mathrm{AcO}$

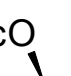

$O=$

19

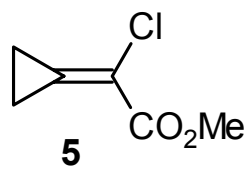

\{<smiles>COC(=O)C1OC(c2ccccc2)=NC12CC2</smiles>

13-Ph

Cyclopropyl analogue of Taxol

Scheme 3. Structure of Taxol and retrosynthetic analysis for the cyclopropane derivatives of it.

The reaction of methyl 2-chloro-2-cyclopropylideneacetate 5 with amidine producing cyclobutene-annelated pyrimidinones (12) resemble hetero analogues of benzocyclobutenes and undergo cyclobutene ring opening followed by Diels-Alder reaction with dienophile to give tetrahydroquinazolinone derivatives. ${ }^{[26]}$ Tetrahydroquinazolinones are important class of heterocycles and have found to be attracted by pharmaceutical chemists. ${ }^{[41]}$ One good example containing tetrahydroquinazolinones is the Folic acid analogue (20). Folic acid (21) (Figure 3) one of the important B vitamins, is a precursor for the biogenetic synthesis of the cofactor, 
tetrahydrofolic acid conjugate. ${ }^{[42]}$ This later in turn, serves both as a formyl and hydroxymethyl transfer agent in a variety of biological system. The analogue 5,8-dideaza-5,6,7,8-tetrahydrofolic acid (20) have attracted considerable attention in chemistry ${ }^{[43]}$ and biology. ${ }^{[4]}$ A wide range of biological activities has been discovered for such compound, like anticancer properties, antimicrobial activity against Streptococcus feacium, inhibition of dihydrofolate reductive and thymidilate synthase, ${ }^{[45]}$ as well as an ability to be a good substrate for mouse liver folylpolyglutamate synthetase. ${ }^{[46]}$

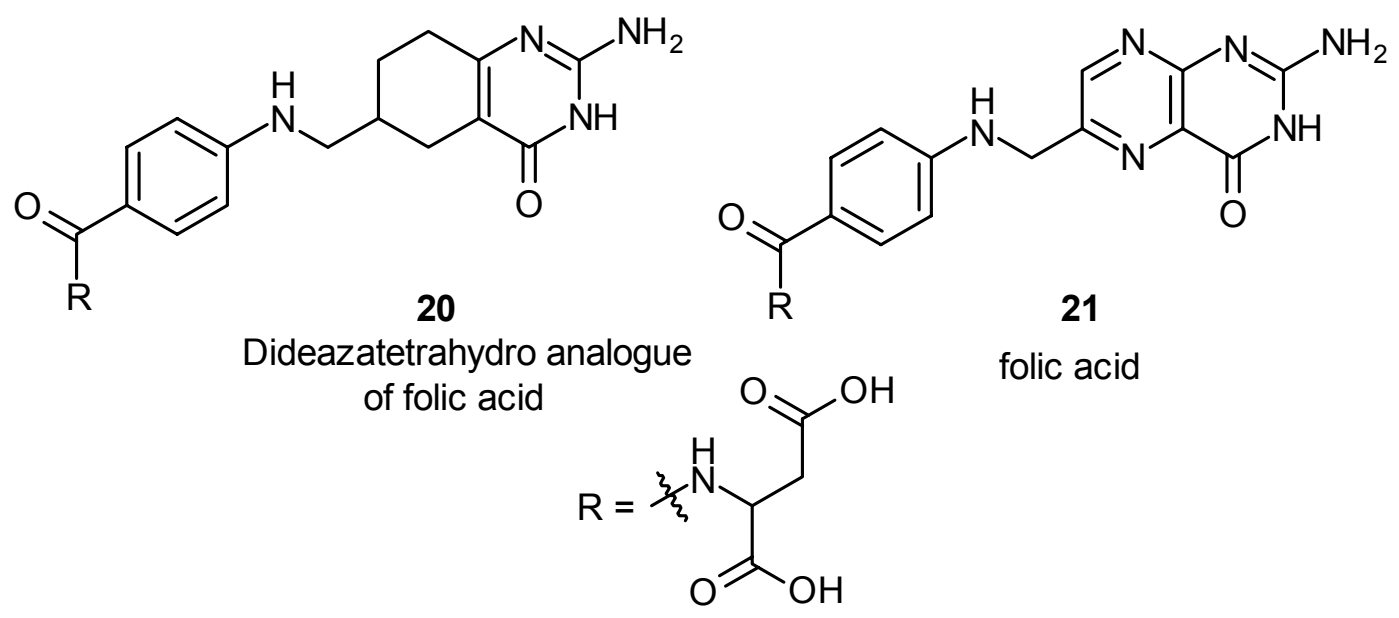

Figure 3. Structure of Folic acid and tetrahydro analogue of Folic acid.

Although methyl 2-chloro-2-cyclopropylideneacetate (5) have shown to be potential Michael acceptor, this building block have not really been easily available yet. The method described by de Meijere et al. requires a halide-resistant autoclave for the thermal ring opening reaction and the overall atom economy is rather poor. ${ }^{[18]}$ The previously developed synthesis of the bromo derivative (4-Me) starts from cyclopropanone ethyl hemiacetal (the preparation of which requires the generation as well as handling of finely dispersed sodium) and the Wittig olefinations with the appropriately substituted triphenyl-methylenephosphoranes do not provide good yields of the product. With the more recently developed conversion of carboxylic acid esters to cyclopropanols, the Kulinkovich reaction ${ }^{[8]}$ in hand, an advanced synthesis of 5 or 4-Me ought to be developed.

It is mentioned before that pyrimidinones and tetrahydroquinazolinones are among the most important class of heterocycles that are present in many biologically active molecules. Literature shows that, only a few different methods for the synthesis of tetrahydroquinazolinone derivatives are described. ${ }^{[47]}$ Since a versatile method for the synthesis of cyclobutene annelated 
pyrimidinones is known, ${ }^{[26]}$ an appropriate method has to be developed for conversion to variously substituted tetrahydro quinazolinones (22). Lewis-acid catalyzed Michael addition of indoles onto Michael acceptors ${ }^{[48]}$ provides 3 -substituted tryptophane analogues ${ }^{[49]}$ and other indole derivatives. ${ }^{[50]}$ In view of the fact that methyl 2-chloro-2-cyclopropylideneacetate (5) is a particularly reactive Michael acceptor a synthetic method to cyclopropane analogue of tryptophan ought to be achieved by Michael addition of $\mathbf{5}$ onto indole, and to use it as a precursor for the synthesis of cyclopropane analogue of the PDE5 inhibitors, the hydrantoin lead structure to Tadalafil (25) ${ }^{[51]}$ and Tadalafil (26) ${ }^{[52]}$ itself.

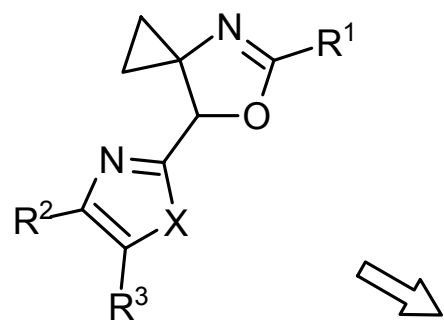

23<smiles>[R]c1nc2c(c(=O)[nH]1)CC([R])C([R])C2[R]</smiles><smiles>[R16]</smiles><smiles>COC(OC)C(Cl)=C1CC1</smiles>

24

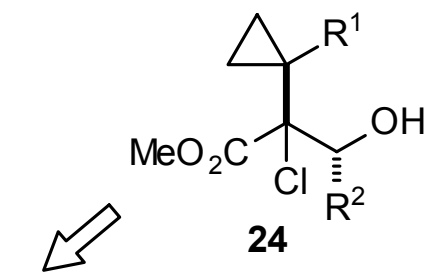<smiles>CCCCN1C(=O)[C@H]2CC3(CC3)c3c([nH]c4ccccc34)[C@H](c3ccc(OC)cc3)N2C1=O</smiles>

5<smiles>C1=CC1</smiles><smiles>C1=CC1</smiles><smiles>C1=CC1</smiles><smiles>COC(=O)C1N2NCN=C2SC12CC2</smiles>

27

25<smiles>CN1CC(=O)N2C(c3ccc4c(c3)OCO4)c3[nH]c4ccccc4c3C3(CC3)[C@@H]2C1=O</smiles>

26

Scheme 4. Retrosynthetic analysis for the synthesis of different molecule from $\mathbf{5}$.

Since a method for the synthesis of spirocyclopropane oxazoline carboxylate is well established the conversion of the carboxylate group to a herterocycle such as oxazole, thiazole or benzoxazole 
(23) has to be found out. In a same way as with thioamides, the Michael addition of alkylated thiourea to produce thiazolines (27) has to be studied.

Domino processes ${ }^{[53]}$ consisting of a Michael addition and an aldol reaction, ${ }^{[54]}$ especially the one known as the Robinson annelation ${ }^{[55]}$ were the first of these now extremely popular sequential reactions which provide a one-pot access to complex skeletons from relatively simple starting materials. ${ }^{[56]}$ After the successful Michael addition, a sequential Michael addition-aldol reaction of Grignard reagents and aldehydes onto 5 for the synthesis of chlorohydrins (24) has to be investigated (Scheme 4).

The aim of this work can be summarized as follows:

1. An advanced, simple and economical method for the synthesis of the acceptor-activated methylenecyclopropanes 5 and 4-Me.

2. Synthesis of dihydro- and tetrahydro-quinazolinones by Michael addition of amidine onto 5 followed by Diels-Alder reaction with suitable dienophiles.

3. Synthesis of variously substituted spirocyclopropane oxazolines containing benzoxazole.

4. Synthesis of spirocyclopropane analogue of tryptophane and its use for the synthesis of Tadalafil (26) and hydrantoin lead structure to Tadalafil (25).

5. Sequential addition of Grignard reagent and aldehydes with $\mathbf{5}$ for synthesis of chlorohydrins (24). 


\section{Main Part}

\subsection{An Advanced Synthesis of Methyl 2-chloro- and 2-bromo-2-cyclopropylideneacetate}

The first aim of this project was to develop a advanced method for the preparation of the methylenecyclopropane 4-Me and 5 by the use of the Kulinkovich reaction. ${ }^{[8]}$ It was assumed that the cyclopropanol obtained form the Kulinkovich reaction of the ester $\mathbf{2 9}$ could be converted to the carboxylic acid $\mathbf{2 8}$ as a key intermediate which in turn could be transformed to the desired methylene cyclopropanes. The retrosynthetic analysis to $\mathbf{4 - M e}$ and $\mathbf{5}$ is presented in Scheme $5 .{ }^{[57]}$
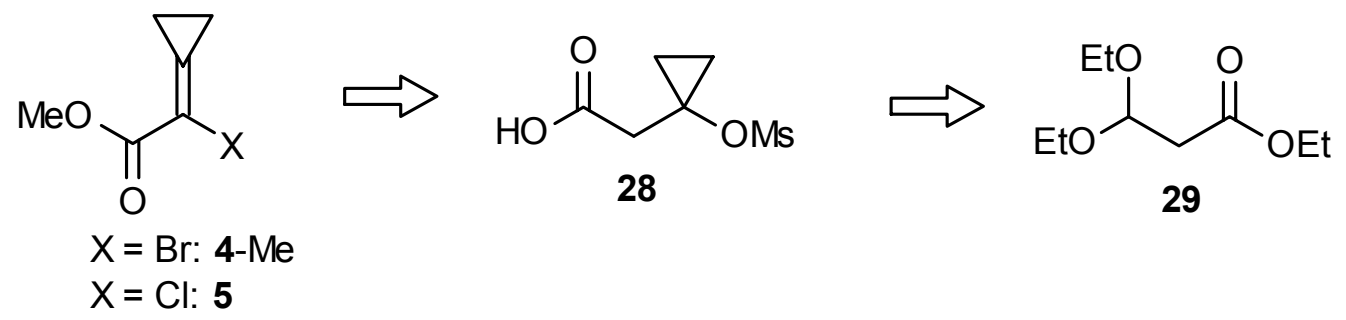

Scheme 5. Retrosynthetic analysis for the synthesis of Methyl 2-chloro- and 2-bromocyclopropylideneacetate.

Having the 2-(1'-mesyloxycyclopropyl)acetic acid (28) in mind as a key intermediate for the synthesis of 4-Me and 5, compound 29 with a masked carboxyl group was considered as a precursor. Although the methyl analogue (methyl 3,3-dimethoxypropionate) is commercially available or easily prepared by Walker oxidation of methyl acylate, ${ }^{[58]}$ the ethyl 3,3dimethoxypropionate (29) could be prepared on a large scale (4 mol) starting from ethyl vinyl ether, tetrachloromethane and ethanol by a little modification of the method described in the literature. ${ }^{[59]}$ When the reaction was carried out at $50{ }^{\circ} \mathrm{C}$, as described in the literature, the product (29) was obtained only in $12 \%$ after 2 days. However, by increasing the reaction temperature to 70 ${ }^{\circ} \mathrm{C}$ the product could be isolated in $62 \%$ yield after $15 \mathrm{~h}$. The Kulinkovich reductive cyclopropanation of 29 under the established conditions yielded the cyclopropanol 31 in $96 \%$ yield even on a reasonably large scale (ca. 1 mol). ${ }^{[60]}$ The cyclopropanol 31 was cleanly transformed under DMAP-catalysis (5 mol\%) in the presence of triethylamine as to the corresponding mesylate. It has been observed that this mesylate is not stable and decomposes when standing for a long time at room temperature. Anyway, this compound could be deprotected 
and oxidized in situ with 1.5 equiv. of Oxone, crystallization from diethyl ether afforded the pure acid 28 as a colorless crystalline compound (Scheme 6).

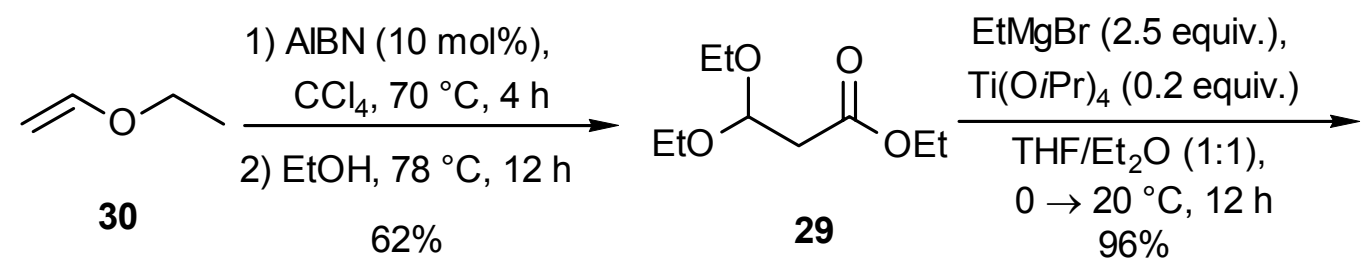

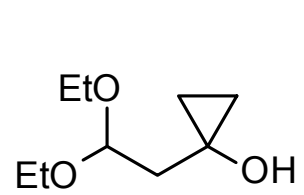

31

1) $\mathrm{MsCl}$, cat. DMAP,

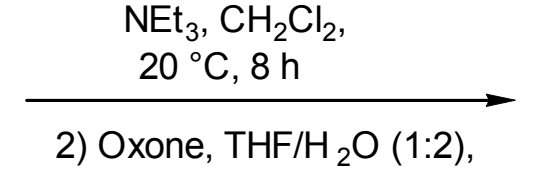

$20{ }^{\circ} \mathrm{C}, 18 \mathrm{~h}$<smiles>COC1(CC(=O)O)CC1</smiles>

28

Scheme 6. Preparation of 2-(1'-mesyloxycyclopropyl)acetic acid from ethyl vinyl ether.

Attempted $\alpha$-bromination of the in situ generated acid chloride of $\mathbf{2 8}$ with a catalytic amount of chlorosulfonic acid and molecular bromine at $85^{\circ} \mathrm{C}$ failed, just like the first attempts to halogenate 28 with NCS in dichloromethane, probably because of the poor solubility of $\mathbf{2 8}$ and the acid chloride of $\mathbf{2 8}$ in tetrachloromethane. However, 1,2-dichloroethane as a better solvent for the starting material upon chlorination with NCS and subsequent treatment with methanol led to the $\alpha$-chloro ester 32a in 87\% yield (Scheme 3).
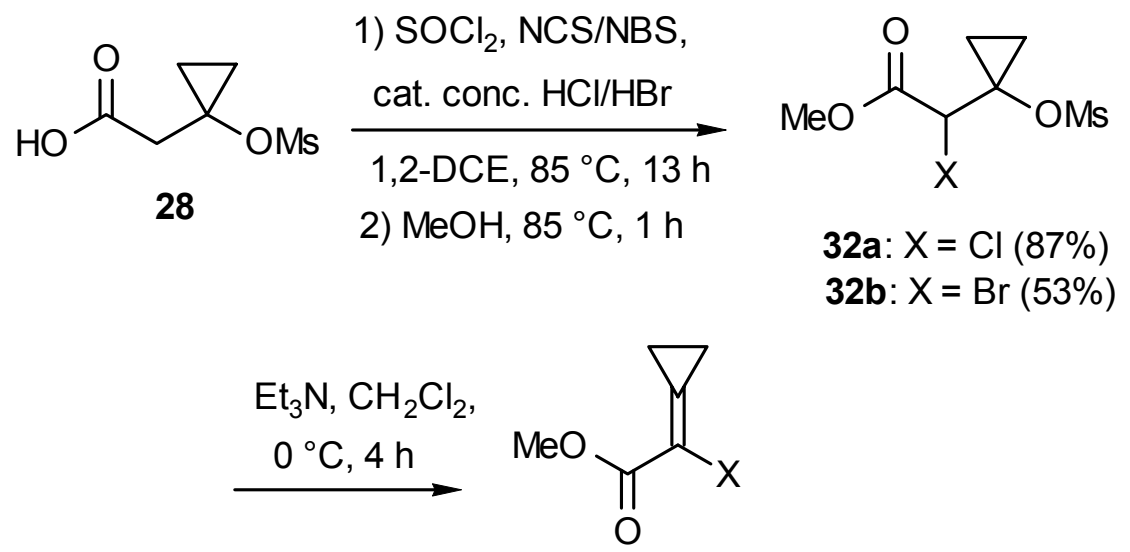

4-Me: $\mathrm{X}=\mathrm{Br}(99 \%)$

5: $\mathrm{X}=\mathrm{Cl}(77 \%)$

Scheme 7. Transformation of $\mathbf{2 8}$ to methyl 2-halo-2-cyclopropylideneacetates 4-Me and $\mathbf{5 .}$ 
Under the same conditions, the acid chloride of $\mathbf{2 8}$ could be transformed to the $\alpha$-bromo ester $\mathbf{3 2 b}$ with NBS and a catalytic amount of concentrated hydrobromic acid in 53\% yield. Although 32a and 29b could be purified by silica gel chromatography, they appeared not to be very stable under these conditions. Therefore, the crude halogenated esters 32a and 32b were directly subjected to dehydrohalogenation by treatment with triethylamine in dichloromethane at $0{ }^{\circ} \mathrm{C}$. The resulting methyl 2-chloro-2-cyclopropylideneacetate (5) could be purified by bulb to bulb distillation and crystallization from pentane $/ \mathrm{Et}_{2} \mathrm{O}$ while bromo analogue 4-Me was purified by chromatography in very good yields (Scheme 7). ${ }^{[61]}$ 


\subsection{Tetrahydroquinazolinone Derivatives}

\subsubsection{Synthesis of amidines from nitriles}

Among various methods for preparation of amidines from nitriles, ${ }^{[62]}$ we found that the method described by Reed et al. ${ }^{[62 b]}$ was the best for our substrates. When a THF solution of pchlorobenzyl nitrile was added to a $1 \mathrm{~N}$ solution of LiHMDS in hexane, followed by quenching with $6 \mathrm{~N}$ isopropanolic $\mathrm{HCl}$, the corresponding amidine 34b was isolated in 93\% yield. Following the same method other amidines $\mathbf{3 4} \mathbf{c}-\mathbf{e}$ were formed and isolated in good to excellent yield. Surprisingly, under the same conditions $o$-phenyl benzonitrile failed to give the corresponding amidine 34f. This compound was prepared by the method descried by Wolfgang von der Sall et al. ${ }^{[62 \mathrm{e}]}$ When a solution of $o$-phenyl benzonitrile in toluene was added to a freshly prepared solution of $\mathrm{MeAl}(\mathrm{Cl}) \mathrm{NH}_{2}$ in toluene, $\mathbf{3 4 f}$ was isolated in $58 \%$ yield. In the same way $\mathbf{3 4 g}$ was prepared with $84 \%$ yield. These results are summarized in Scheme 8 and Table 1.

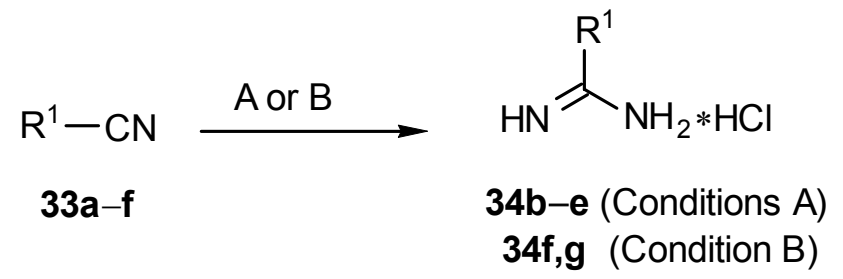

Scheme 8. Synthesis of amidines $(\mathbf{3 4 b}-\mathbf{g})$ from nitriles 33a-f. Conditions A: LiHMDS (1 N , hexane), THF, $25^{\circ} \mathrm{C}, 4 \mathrm{~h}, 6 \mathrm{~N} \mathrm{HCl}(\mathrm{PrOH})$; conditions $\mathrm{B}: \mathrm{Me}_{3} \mathrm{Al}$ (1 M, toluene), $\mathrm{NH}_{4} \mathrm{Cl}$, toluene, $120^{\circ} \mathrm{C}, 15 \mathrm{~h}$.

Table 1. Yield and reaction conditions of preparation of amidines $\mathbf{3 4 b}-\mathbf{g}$ from nitriles.

\begin{tabular}{|c|c|c|c|c|}
\hline Entry & $\mathrm{R}^{1}$ & Reaction Conditions & Product & Yield (\%) \\
\hline & $p-\mathrm{ClC}_{6} \mathrm{H}_{4}$ & $\mathbf{A}$ & 34b & 93 \\
\hline & $o-\mathrm{BrC}_{6} \mathrm{H}_{4}$ & $\mathbf{A}$ & $34 c$ & 91 \\
\hline & $o-\mathrm{FC}_{6} \mathrm{H}_{4}$ & $\mathbf{A}$ & 34d & 80 \\
\hline & $p-\left(\mathrm{C}_{6} \mathrm{H}_{5} \mathrm{CH}_{2} \mathrm{O}\right) \mathrm{C}_{6} \mathrm{H}_{4}$ & $\mathbf{A}$ & $34 \mathrm{e}$ & 92 \\
\hline & $o-\mathrm{PhC}_{6} \mathrm{H}_{4}$ & $\mathbf{B}$ & $34 f$ & 58 \\
\hline & 3-(5-chloro-benzo[b]thiophene) & B & $34 \mathrm{~g}$ & 84 \\
\hline
\end{tabular}




\subsubsection{Synthesis of cyclobutene-annelated pyrimidinones}

When a mixture of methyl 2-chloro-2-cyclopropylidineacetate 5 and 2 equivalents of benzamidine hydrochloride 34a was stirred in dioxane in the presence of 4 equivalents of triethylamine for $48 \mathrm{~h}$ at room temperature, 3-phenyl-2,4-diazabicyclo[4.2.0]octa-1(6),2-diene-5-one (35a) was isolated in $83 \%$ yield. $\left[{ }^{63}\right]$ The product $\mathbf{3 5 a}$ is very less soluble either in $\mathrm{Et}_{2} \mathrm{O}$ or in dichloromethane but could be easily precipitated from $\mathrm{MeOH}$. Similarly, under the same conditions the other pyrimidinones $\mathbf{3 5 b}-\mathbf{j}$ were obtained in good yields (68-82 \%).

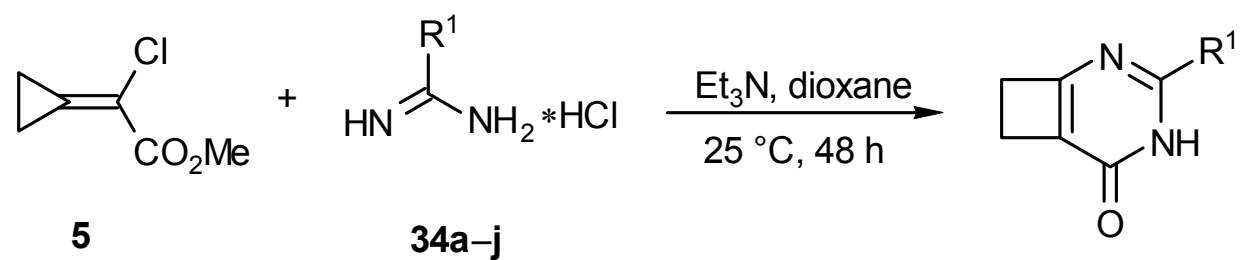

$35 \mathbf{a}-\mathbf{j}$

Scheme 9. Michael addition of amidines $(\mathbf{3 4 a}-\mathbf{j})$ to $\mathbf{5}$ for the synthesis of cyclobutene annelated pyrimidinones 35a-j.

Table 2. Yields of the pyrimidinones from Michael addition of amidines onto 5.

\begin{tabular}{|c|c|c|c|c|}
\hline Entry & Amidines & $\mathrm{R}^{1}$ & Product & Yield (\%) \\
\hline 1 & $34 \mathbf{a}$ & $\mathrm{Ph}$ & $35 \mathbf{a}$ & 83 \\
\hline 2 & $34 b$ & $p-\mathrm{ClC}_{6} \mathrm{H}_{4}$ & $35 b$ & 78 \\
\hline 3 & $34 c$ & $o-\mathrm{BrC}_{6} \mathrm{H}_{4}$ & $35 \mathrm{c}$ & 76 \\
\hline 4 & 34d & $o-\mathrm{FC}_{6} \mathrm{H}_{4}$ & 35d & 82 \\
\hline 5 & $34 \mathrm{e}$ & $p-\left(\mathrm{C}_{6} \mathrm{H}_{5} \mathrm{CH}_{2} \mathrm{O}\right) \mathrm{C}_{6} \mathrm{H}_{4}$ & $35 \mathrm{e}$ & 68 \\
\hline 6 & $34 f$ & $o-\mathrm{PhC}_{6} \mathrm{H}_{4}$ & $35 f$ & 80 \\
\hline 7 & $34 \mathrm{~g}$ & 3-(5-chloro-benzo[b]thiophene) & $35 \mathrm{~g}$ & 74 \\
\hline 8 & $34 \mathrm{~h}$ & $\mathrm{SMe}$ & $35 \mathrm{~h}$ & 74 \\
\hline 9 & $34 \mathbf{i}$ & $\mathrm{N}(\mathrm{Bn})_{2}$ & $35 \mathrm{i}$ & 66 \\
\hline 10 & $34 \mathbf{j}$ & $\mathrm{OMe}$ & $35 j$ & 38 \\
\hline
\end{tabular}


Less reactive $S$-methylisothiourea hemisulpahte and dibenzyl guanidine ${ }^{[64]}$ produced the corresponding pyrimidinones $35 \mathrm{~h}$ and $35 \mathrm{i}$ at $50{ }^{\circ} \mathrm{C}$ in $74 \%$ and $66 \%$ yield respectively. Similarly the $O$-methylurea also furnished the pyrimidinone $\mathbf{3 5 j}$ in $38 \%$ yield(Scheme 9 , Table 2).

\subsubsection{Cyclobutene ring opening and subsequent Diels-Alder reaction}

Diels-Alder reaction $^{[65]}$ is a powerful construction protocol in organic synthesis, an easy method for the synthesis of the carbon-carbon bond formation of cyclic and bicyclic organic compounds. A wide variety of natural products skeleton has been constructed using this method. ${ }^{[66]}$ The above cyclobutene-annelated pyrimidinones 35 resemble hetero analogues of benzocyclobutene and it was interesting to open the cyclobutene ring so that the in situ formed butadiene would react with a suitable ethylene equivalent dienophile to give tetrahydroquinazolinone derivatives. The low reactivity of alkenes and alkynes as dienophilic reagents rank as one of the foremost limitations of Diels-Alder cycloaddition chemistry. It is well known that to achieve the [4+2] cycloaddition of ethylene to butadienes a temperature of $175^{\circ} \mathrm{C}$ and a pressure of 6000 psi or more are require. ${ }^{[67]}$ Therefore, a number of ethylene and acetylene equivalents have been developed to circumvent this problem. Among them, of particular interest and synthetically important are the dienophiles that are activated by sulphur, such as sulphoxide and sulfone. The best known is phenyl vinyl sulfone developed by L. P. Paquette et al. ${ }^{[68]}$ Although this sulfone has been used as a dienophile in an intramolecular Diels-Alder reaction with cyclobutene ring, ${ }^{[69]}$ a regioselective intermolecular addition to cyclobutene ring has never been achieved. In order to optimize the reaction conditions, we chose the compound 35a. The reactions of 35a with excess of phenyl vinyl sulfone (36) in toluene or in 1,2-dichlorobenzene at $175^{\circ} \mathrm{C}$ in a closed Pyrex tube for $12 \mathrm{~h}$ gave the corresponding Diels-Alder adduct 37a in very poor yields (Table 1, entries 1, 2), but interestingly only one regioisomer was found. As the melting point of 36 is $68{ }^{\circ} \mathrm{C}$, the reaction was carried out in liquid of 36. When a mixture of 35a and 10-fold excess of 36 were heated at $175{ }^{\circ} \mathrm{C}$ for $12 \mathrm{~h}$ under solvent free conditions, 2-phenyl-6-benzenesulfonyl-5,6,7,8-tetrahydroquinazolin-4(3H)one 37a was isolated in $84 \%$ yield as one regioisomer (entry 3). Its structure was assigned on the basis of ${ }^{1} \mathrm{H}-\mathrm{NMR},{ }^{13} \mathrm{C}-\mathrm{NMR}, \mathrm{HMBC}, \mathrm{HMQC}$, MS as well as single crystal structure of the 6-ethyl derivative of it (41b). Decreasing the amount of 36 to 4 equivalents did not alter the yield (entry 4), but further going down to 1.5 equiv. or decreasing the temperature substantially reduced the yield (entries 5 and 6). These results are summarized in Table 3. 
Table 3. Optimization of reaction conditions and yield of Diels-Alder reactions of 35a with phenyl vinyl sulfone 36 .

\begin{tabular}{cccc}
\hline Entry & Equiv. of $\mathbf{3 3}$ & Reaction conditions & Yield of $\mathbf{3 7 a}(\%)$ \\
\hline 1 & excess & 1,2-dichlorobenzene, $175^{\circ} \mathrm{C}, 12 \mathrm{~h}$ & 25 \\
2 & excess & toluene, $175^{\circ} \mathrm{C}, 12 \mathrm{~h}$ & 32 \\
3 & 10 & neat, $175^{\circ} \mathrm{C}, 12 \mathrm{~h}$ & 84 \\
4 & 4 & neat, $175^{\circ} \mathrm{C}, 12 \mathrm{~h}$ & 83 \\
5 & 1.5 & neat, $175^{\circ} \mathrm{C}, 12 \mathrm{~h}$ & 49 \\
6 & 4 & neat, $165^{\circ} \mathrm{C}, 12 \mathrm{~h}$ & 39 \\
\hline
\end{tabular}

Once the optimal conditions were found for 35a (entry 4), we performed the ring-opening reactions to $\mathbf{3 5 a}-\mathbf{i}$ and synthesized the corresponding adducts $\mathbf{3 7 a}-\mathrm{h}$ in good yields (Scheme 10, Table 4). Surprisingly 35i decomposed under these reaction conditions.<smiles>[R]c1nc2c(c(=O)[nH]1)CC2</smiles>

$35 a-i$<smiles>C=CS(=O)(=O)c1ccccc1</smiles>

36<smiles>[R]c1nc2c(c(=O)[nH]1)CC(S(=O)(=O)c1ccccc1)CC2</smiles>

$37 a-h$

Scheme 10. Thermal Diels-Alder reaction of 35 with phenyl vinyl sulfone.

Tabale 4: Yeild of Diels-Alder reaction products $37 \mathbf{a}-\mathbf{h}$ from the starting material $\mathbf{3 5 a}-\mathbf{h}$.

\begin{tabular}{ccccc}
\hline Entry & Compound 35 & $\mathrm{R}^{1}$ & Product & Yield (\%) \\
\hline 1 & $\mathbf{3 5 a}$ & $\mathrm{Ph}$ & $\mathbf{3 7 a}$ & 83 \\
2 & $\mathbf{3 5 b}$ & $p-\mathrm{ClC}_{6} \mathrm{H}_{4}$ & $\mathbf{3 7 b}$ & 59 \\
3 & $\mathbf{3 5 c}$ & $o-\mathrm{BrC}_{6} \mathrm{H}_{4}$ & $\mathbf{3 7 c}$ & 43 \\
4 & $\mathbf{3 5 d}$ & $o-\mathrm{FC}_{6} \mathrm{H}_{4}$ & $\mathbf{3 7 d}$ & 70 \\
5 & $\mathbf{3 5}$ & $p-\left(\mathrm{C}_{6} \mathrm{H}_{5} \mathrm{CH}_{2} \mathrm{O}\right) \mathrm{C}_{6} \mathrm{H}_{4}$ & $\mathbf{3 7 e}$ & 66 \\
6 & $\mathbf{3 5 f}$ & $o-\mathrm{PhC}_{6} \mathrm{H}_{4}$ & $\mathbf{3 7 f}$ & 65 \\
7 & $\mathbf{3 5 g}$ & $3-(5-$ chloro-benzo $[\mathrm{b}]$ thiophene $)$ & $\mathbf{3 7 g}$ & 88 \\
8 & $\mathbf{3 5 h}$ & $\mathrm{SMe}$ & $\mathbf{3 7 h}$ & 56 \\
9 & $\mathbf{3 5 i}$ & $\mathrm{N}(\mathrm{Bn})_{2}$ & $\mathbf{3 7 i}$ & 00 \\
\hline
\end{tabular}




\subsubsection{Microwave assisted Diels-Alder reaction}

Microwave-assisted organic reactions have attracted the attention of synthetic chemists ${ }^{[70]}$ as they can decrease the reaction time, can be performed efficiently with less byproducts and most importantly in solvent free conditions. Due to all these factors more focus is been paid now a days to microwave assisted organic reaction. Microwaves have been used to carry out organometallic cross-coupling reactions, heterocycles synthesis, solid-phase synthesis, condensations as well as cycloaddition reactions. ${ }^{[71]}$ Since the Diels-Alder reaction carried out above is a thermal reaction and needed temperature of at least $175^{\circ} \mathrm{C}$, the application of microwaves irradiation in this case is very significant. Although efforts have been paid to promote the Diels-Alder reaction ${ }^{[72]}$ by microwave irradiation, a cyclobutene ring-opening followed by Diels-Alder reactions has never been achieved before. For this reason we were interested in carrying out the ring-opening reaction and Diels-Alder reaction using microwaves. For optimizing the reaction conditions, we tried the reaction between 35a and 36 at $170{ }^{\circ} \mathrm{C}$ for $10 \mathrm{~min}$. Although the reaction was completed (as observed by HPLC) the crude reaction mixture was completely black, which might be due to decomposition of sulfone in these conditions. Despite the difficulty of purifying by column chromatography, $59 \%$ of product $37 \mathbf{a}$ was isolated. It was surprising that the yield increased to $64 \%$ and $70 \%$ when the temperature was decreased to 160 and $150{ }^{\circ} \mathrm{C}$ respectively, the crude reaction mixture was still black in colour.

Table 5. Optimisation of the reaction conditions and yield for microwave assisted reaction.

\begin{tabular}{cccc}
\hline Entry & Temperature & Time & Yield 37a (\%) \\
\hline 1 & $170^{\circ} \mathrm{C}$ & $10 \mathrm{~min}$ & 59 \\
2 & $160^{\circ} \mathrm{C}$ & $10 \mathrm{~min}$ & 64 \\
3 & $150{ }^{\circ} \mathrm{C}$ & $10 \mathrm{~min}$ & 70 \\
4 & $140^{\circ} \mathrm{C}$ & $10 \mathrm{~min}$ & 57 \\
5 & $140^{\circ} \mathrm{C}$ & $20 \mathrm{~min}$ & 69 \\
6 & $130^{\circ} \mathrm{C}$ & $20 \mathrm{~min}$ & 55 \\
7 & $130^{\circ} \mathrm{C}$ & $30 \mathrm{~min}$ & 66 \\
8 & $120^{\circ} \mathrm{C}$ & $20 \mathrm{~min}$ & 20 \\
\hline
\end{tabular}


Further decreasing the temperature to $140{ }^{\circ} \mathrm{C}$ for $10 \mathrm{~min}$, led to decrease in yield to $57 \%$ (entry 4 ), as $21 \%$ of starting material was recovered. The extension of the reaction time to 20 min increased the yield to $69 \%$ (entry 5). Other conditions tried (entries 6,7,8) never gave better yields than entry 5. These results are summarized in Table 5.

Once the optimal conditions (entry 5) were found the other cyclobutene pyrimidinones were also reacted with 36 to give the products $\mathbf{3 7 a}-\mathbf{h}$ in yield ranging from 52 to $69 \%$. Although the yields are lower in comparision with the use of oil bath, the higher reaction temperature and longer time could be avoided by using microwaves. As observed before, the reaction with $\mathbf{3 7 i}$ did not give any product. These results are summarized in Scheme 11 and Table 6.<smiles>[R]c1nc2c(c(=O)[nH]1)CC2</smiles>

$35 a-i$<smiles>CCS(=O)(=O)c1ccccc1</smiles>

36

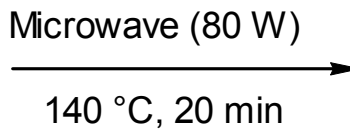

$140^{\circ} \mathrm{C}, 20 \mathrm{~min}$<smiles>[R]c1nc2c(c(=O)[nH]1)CC(S(=O)(=O)c1ccccc1)CC2</smiles>

$37 a-h$

Scheme 11. Optimised conditions for the microwave assisted Diels-Alder reaction.

Table 6. Yields of the microwave assisted Diels-Alder reaction.

\begin{tabular}{cccc}
\hline Entry & $\mathrm{R}^{1}$ & Product & Yield (\%) \\
\hline 1 & $\mathrm{Ph}$ & $\mathbf{3 7 a}$ & 69 \\
2 & $p-\mathrm{Cl}-\mathrm{Ph}$ & $\mathbf{3 7 b}$ & 57 \\
3 & $o-\mathrm{Br}-\mathrm{Ph}$ & $\mathbf{3 7}$ & 62 \\
4 & $o-\mathrm{F}-\mathrm{Ph}$ & $\mathbf{3 7 d}$ & 57 \\
5 & $p-\left(\mathrm{C}_{6} \mathrm{H}_{5} \mathrm{CH}_{2} \mathrm{O}\right) \mathrm{Ph}$ & $\mathbf{3 7 e}$ & 63 \\
6 & $o-$ biphenyl & $\mathbf{3 7 f}$ & 63 \\
7 & $3-(5-$-chloro-benzo[b]thiophene $)$ & $\mathbf{3 7 g}$ & 52 \\
8 & $\mathrm{SMe}$ & $\mathbf{3 7 h}$ & 63 \\
9 & $\mathrm{~N}(\mathrm{Bn})_{2}$ & $\mathbf{3 7 i}$ & Decomposition \\
\hline
\end{tabular}




\subsection{5. "One pot" synthesis of tetrahydroquinazolinones}

After the stepwise Michael addition and Diels-Alder reactions were successfully performed, it was interesting to carry out these two operations in "one pot" fashion. Indeed, when phenyl vinyl sulfone was added to the crude reaction mixture of benzamidine hydrochloride $\mathbf{3 4 a}$ and $\mathbf{5}$ in presence of triethyl amine after $48 \mathrm{~h}$ in a Pyrex bottle and heated to $175^{\circ} \mathrm{C}$ for $12 \mathrm{~h}, \mathbf{3 7 a}$ was isolated in 43\% yield. A little higher yield was obtained in case of 34d (Scheme 12).

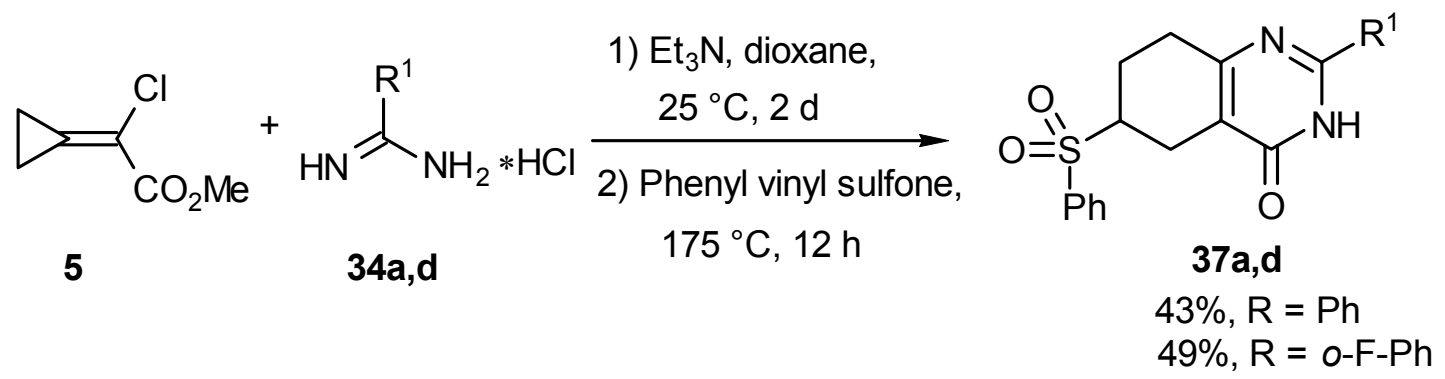

Scheme 12. One pot synthesis of tetrahydroquinazolinones $\mathbf{3 7 a , d}$ from $\mathbf{5}$ and amidines $\mathbf{3 4 a , d .}$

\subsubsection{Reductive elimination of sulfone group}

Once the tetrahydroquinazoline ring has been formed, the next issue was to remove the sulfonyl group. Although several methods are reported for this purpose, the one which involvs $\mathrm{Na} / \mathrm{Hg}$ amalgam in the presence of $\mathrm{Na}_{2} \mathrm{HPO}_{4}{ }^{[73]}$ has been most widely used. However, when 34a (as well as its $O$-TMS protected or $N$-Boc protected equivalent) was reacted with $\mathrm{Na} / \mathrm{Hg}$ and $\mathrm{Na}_{2} \mathrm{HPO}_{4}(4$ equiv. each) in $\mathrm{MeOH}$, only (deprotected) starting material was isolated without any reductive elimination. By changing the reagents to $\mathrm{Na}-\mathrm{sand} / \mathrm{EtOH},{ }^{[74]}$ only substantial amount of benzaldehyde was isolated (Scheme 13). 
<smiles>O=c1[nH]c(-c2ccccc2)nc2c1CC(S(=O)(=O)c1ccccc1)CC2</smiles>

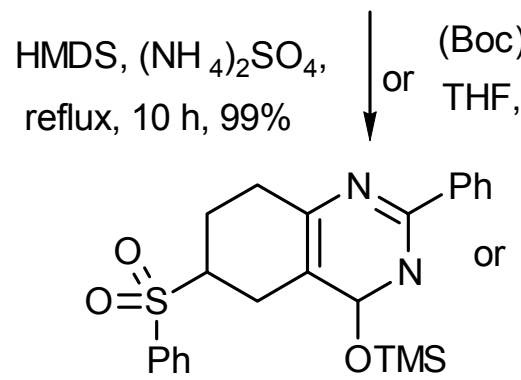

37a-TMS

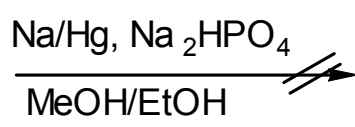

$2 \mathrm{Ot}_{3} \mathrm{~N}, \mathrm{DMAP}$
$25^{\circ} \mathrm{C}, 3 \mathrm{~h}, 82 \%$<smiles>O=c1[nH]c(-c2ccccc2)nc2c1CCCC2</smiles>

$39 a$<smiles>Cc1ccc(-c2nc(-c3ccccc3)[nH]c(=O)c2CCCO)c(O)c1</smiles>

$39 a$

Scheme 13. Removal of sulfone group by using $\mathrm{Na} / \mathrm{Hg}$ amalgam or $\mathrm{Na}-\mathrm{sand} / \mathrm{EtOH}$.

This problem was solved by using a two step procedure: basic elimination of the $\mathrm{PhSO}_{2}$-group followed by Pd-catalysed hydrogenation of the resulting double bond. When 3 equiv. of $\mathrm{KO} t \mathrm{Bu}$ were added to a THF solution of $\mathbf{3 7 a}$, the elimination product $\mathbf{3 8 a}$ was obtained in $96 \%$ yield after $2 \mathrm{~h}$. The subsequent hydrogenation of 38a under $\mathrm{Pd} / \mathrm{C}$, in methanol led to the target substance 2phenyl-5,6,7,8-tetrahydroquinazolinone 39a in 91\% yield. In a similar manner, 39c-f were obtained from $\mathbf{3 7} \mathbf{c}-\mathbf{f}$ in excellent yields over two steps. Due to the low solubility of $\mathbf{3 8 b}$ and $\mathbf{3 8 e}$ in $\mathrm{MeOH}$, the reaction was carried out in $\mathrm{AcOH}$ to give 39b and 39e in 94\% and 93\% yield respectively. In case of $\mathbf{3 8 g}$, the hydrogenation reaction was not successful due to the presence of SMe group which may be due to poisoning of the catalyst. Instead of the desired product, some amount of the material without $S \mathrm{Me}$ group was isolated. These results are summarised in Scheme 14 and Table 7. 
<smiles>[R]c1nc2c(c(=O)[nH]1)CC(S(=O)(=O)c1ccccc1)CC2</smiles><smiles>[R1]c1nc2c(c(=O)[nH]1)C=CCC=C2</smiles>

$\mathrm{Pd} / \mathrm{C}, \mathrm{H}_{2}$,
$\mathrm{MeOH} / \mathrm{AcOH}$,
$25^{\circ} \mathrm{C}, 4 \mathrm{~h}$<smiles>[R]c1nc2c(c(=O)[nH]1)CCCC2</smiles>

39a-f

Scheme 14. Basic elimination of $\mathrm{PhSO}_{2} \mathrm{H}$ group followed by hydrogenation: preparation of 2substituted tetrahydroquinazolinones $\mathbf{3 9 a}-\mathbf{f}$.

Table 7. Yields of elimination and hydrogenation reaction.

\begin{tabular}{cccccc}
\hline Entry & $\mathrm{R}^{1}$ & $\mathbf{3 8}$ & Yield (\%) & $\mathbf{3 9}$ & Yield (\%) \\
\hline 1 & $\mathrm{Ph}$ & $\mathbf{3 8 a}$ & 96 & $\mathbf{3 9 a}$ & 91 \\
2 & $p-\mathrm{Cl}-\mathrm{Ph}$ & $\mathbf{3 8 b}$ & 95 & $\mathbf{3 9 b}$ & 94 \\
3 & $o-\mathrm{Br}-\mathrm{Ph}$ & $\mathbf{3 8 c}$ & 93 & $\mathbf{3 9 c}$ & 91 \\
4 & $o-\mathrm{F}-\mathrm{Ph}$ & $\mathbf{3 8 d}$ & 94 & $\mathbf{3 9 d}$ & 92 \\
5 & $p-\left(\mathrm{C}_{6} \mathrm{H}_{5} \mathrm{CH}_{2} \mathrm{O}\right) \mathrm{Ph}$ & $\mathbf{3 8 e}$ & 92 & $\mathbf{3 9 e}$ & $93^{*}$ \\
6 & $o-$ biphenyl & $\mathbf{3 8 f}$ & 87 & $\mathbf{3 9 f}$ & 93 \\
7 & $\mathrm{SMe}$ & $\mathbf{3 8 g}$ & 98 & $\mathbf{3 9 g}$ & 00 \\
8 & $3-(5-\mathrm{Cl}-1$-benzo-thiophene $)$ & $\mathbf{3 8 h}$ & 00 & &
\end{tabular}

*) With out benzyl group. 


\subsubsection{Alkylation of tetrahydroquinazolinones}

The presence of a strong electron acceptor at C-6 position - phenyl sulfonyl group - makes possible further derivatization at this centre, namely alkylation of the corresponding anion generated from an $\mathrm{O}$ - or $\mathrm{N}$-protected precursor. Surprisingly, when a THF solution of $N$-Boc protected compound (37a-Boc) was subjected to $n \mathrm{BuLi}$ conditions followed by treatment with MeI, no alkylation was observed at C-6 instead, $N$-Boc-2-phenyl-8-methyl-6-phenylsulfonyltetrahydroquinazolin-one $\mathbf{4 0}$ was isolated. Changing the base from $n \mathrm{BuLi}$ to either LDA or NaHMDS gave the same product in lower yield (Scheme 15).<smiles>O=C(O)n1c(-c2ccccc2)nc2c(c1=O)CC(S(=O)(=O)c1ccccc1)CC2</smiles>

$37 a-B o c$

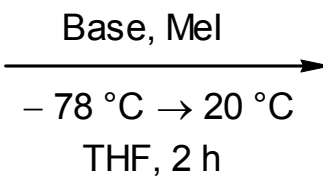

THF, $2 \mathrm{~h}$<smiles>CC1CC(S(=O)(=O)c2ccccc2)Cc2c1nc(-c1ccccc1)n(C(=O)O)c2=O</smiles>

40

\begin{tabular}{ccc}
\hline Entry & Base & Yield 40 (\%) \\
\hline 1 & $n$ BuLi & 28 \\
2 & LDA & 19 \\
3 & NaHMDS & 14 \\
\hline
\end{tabular}

Scheme 15. Alkylation at C-8 of $N$ - 37a-Boc.

However, substitution at C-6 position could be achieved by changing the protecting group. Thus, when $O$-TMS protected compound (37a-TMS) was subjected to $n \mathrm{BuLi}$ conditions followed by treatment with alkyl halides, the desired products 41a-b were obtained in good yields. The structure of 41b could be known from X-ray crystallographic analysis (Figure 4) in which the sulfonyl group is attached to C-6 of the tetrahydro-ring to correspond to the outcome of a rigioselective Diels-Alder reaction. Elimination of sulfone group followed by hydrogenation lead to 2-phenyl-6-alkyltetrahydroquinazolinones $\mathbf{4 2 a - b}$ (Scheme 16). 
<smiles>COc1nc(-c2ccccc2)nc2c1CC(S(=O)(=O)c1ccccc1)CC2</smiles>

37a-TMS
1) $n \mathrm{BuLi}, \mathrm{THF}$, $-78^{\circ} \mathrm{C}, 30 \mathrm{~min}$

2) $\mathrm{R}^{2} \mathrm{X},-78^{\circ} \mathrm{C} \rightarrow \mathrm{RT}$, $2 \mathrm{~h}$<smiles>[R]C1(S(=O)(=O)c2ccccc2)CCc2nc(-c3ccccc3)[nH]c(=O)c2C1</smiles>

41a,b

$\mathrm{KOtBu}, \mathrm{THF}$, $25^{\circ} \mathrm{C}, 2 \mathrm{~h}$<smiles>[R]C1=Cc2c(nc(-c3ccccc3)[nH]c2=O)CC1</smiles>

43a,b

Scheme 16. Alkylation at C-6 position of tetrahydroquinazolinone

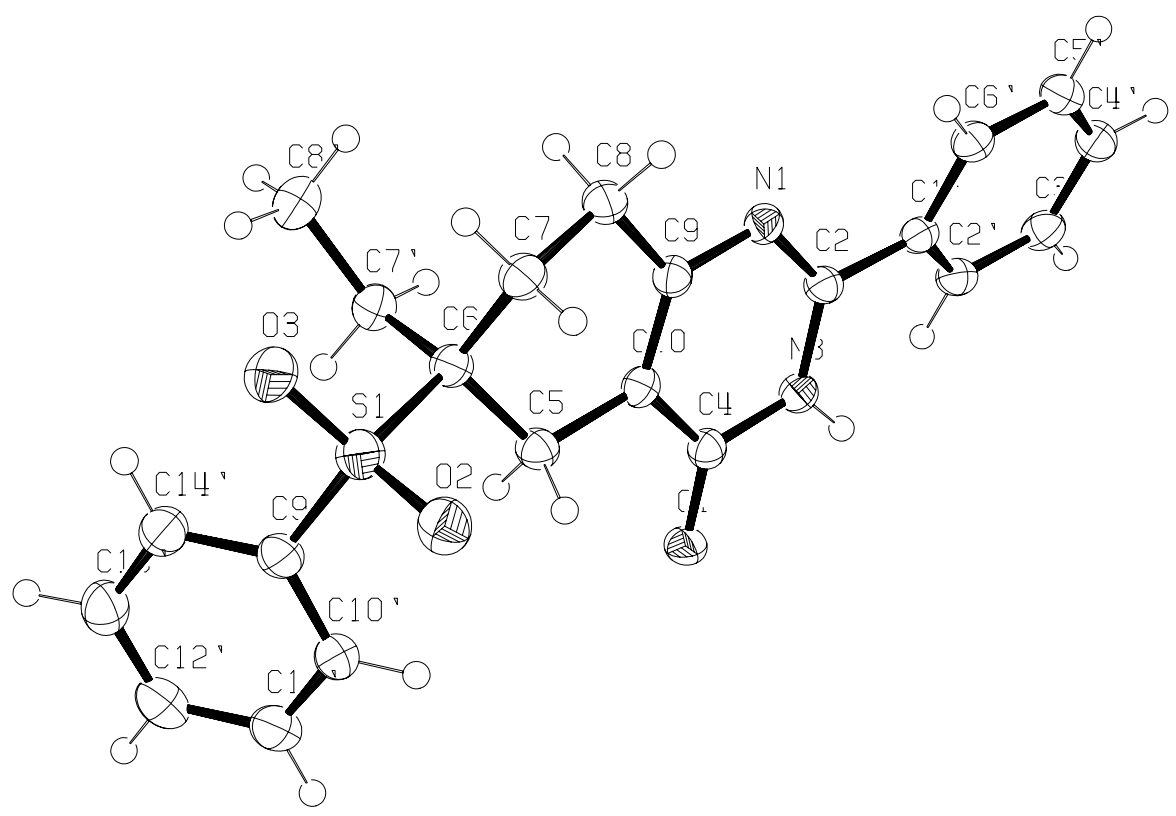

Figure. 4. Molecular structure of $\mathbf{4 1 b}$ in crystal. 
Table 8. Alkylation at sulfone centre (C-6) followed by the removal of $\mathrm{PhSO}_{2}$-group to prepare 2alkyl-tetrahydroquinazolinones $\mathbf{4 2 a , b .}$

\begin{tabular}{ccccccc}
\hline $\mathrm{R}^{2} \mathrm{X}$ & $\mathbf{4 1}$ & Yield (\%) & $\mathbf{4 3}$ & Yield (\%) & $\mathbf{4 2}$ & Yield (\%) \\
\hline $\mathrm{MeI}$ & $\mathbf{4 1 a}$ & 83 & $\mathbf{4 3 a}$ & 95 & $\mathbf{4 2 a}$ & 96 \\
$\mathrm{EtBr}$ & $\mathbf{4 1 b}$ & 86 & $\mathbf{4 3 b}$ & 97 & $\mathbf{4 2 b}$ & 96 \\
\hline
\end{tabular}

After successful substitution at C-6, the intention was to explore the possibility of getting a substituent also at the position C-7. An obvious option was to use a phenyl propenyl sulfone. Indeed, when 35a was reacted with $(E)$-p-tolyl-1-propenyl sulfone (36-Me), the corresponding cycloaddition product (44) was isolated, albeit in $27 \%$ yield, as a mixture of two diasteriomers in a ratio of 1:1. An attempt to introduce an ethyl group failed, because the corresponding sulfone polymerized under these reaction conditions (Scheme 17).

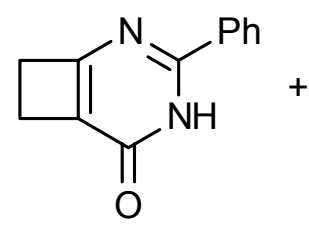

$35 a$

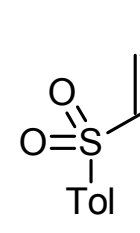

36-Me

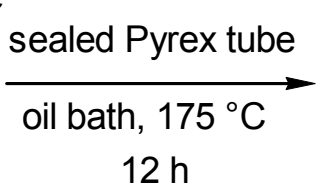

$27 \%$<smiles></smiles>

44

Scheme 17. Reaction of $p$-tolyl-1-propenyl sulfone 36-Me with 35a.

\subsubsection{Nucleophilic substitution of SMe group for the synthesis of 2-amino tetrahydroquinazo- linones}

In order to check the opportunity of further derivatization at C-2, we intended to substitute the $S \mathrm{Me}$ group in $\mathbf{3 7 h}$ by secondary amines. Initial attempt to get 2-morpholinotetrahydroquinazolinone by reacting $37 \mathrm{~h}$ with 2 equivalents of morpholine in DMF at $180{ }^{\circ} \mathrm{C}$ for $12 \mathrm{~h}$ in a sealed Pyrex bottle failed, and this experiment afforded only 2-dimethylamino-6phenylsulfonyltetrahydroquinazolinone (48) in 78\% yield. Obviously, this product has been formed due to the instability of DMF at higher temperature and substitution of SMe by dimethyl amine. This was confirmed by heating $\mathbf{3 7 h}$ in an excess of DMF at $180{ }^{\circ} \mathrm{C}$ without morpholine: the same product (48) was isolated in $86 \%$ yield. When $37 \mathrm{~h}$ was heated at $180{ }^{\circ} \mathrm{C}$ with an excess of morpholine without solvent for $12 \mathrm{~h}, 2$-morpholino-6-phenylsulfonyl tetrahydroquinazolinone 
(45a) was obtained in 93\% yield. In a similar way, $N$-benzyl and $N$-methyl piperazine successfully gave the corresponding substitution products $\mathbf{4 5}$, c in $92 \%$ and $91 \%$ yield, respectively. Unlike before, 5 equiv. of $\mathrm{KO} t \mathrm{Bu}$ and a longer period of time $(15 \mathrm{~h})$ were necessary to complete the elimination reaction in $45 \mathbf{a}-\mathbf{c}$ to prepare $47 \mathbf{a}-\mathbf{c}$. Hydrogenation of $47 \mathbf{a}-\mathbf{c}$ by using standard conditions gave 2-morpholinyl, 2-piperazinyl and 2- $N$-methylpiperazinyl derivatives 46a-c in very good yield (Scheme 18, Table 9).

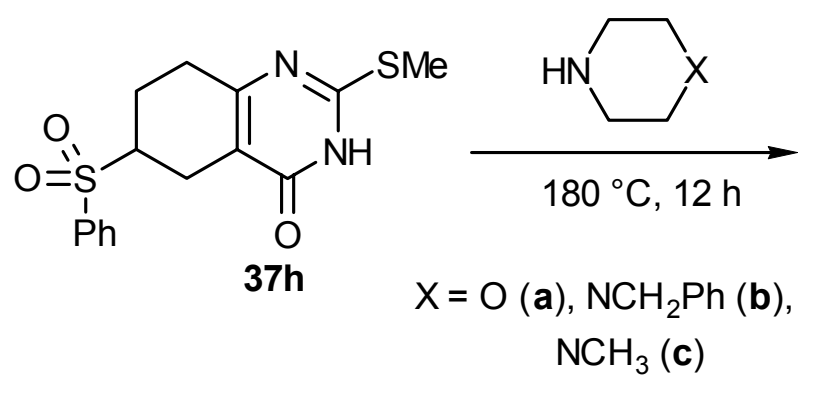<smiles>[X]CCN(CC)c1nc2c(c(=O)[nH]1)CC(S(=O)(=O)c1ccccc1)CC2</smiles><smiles>[X]C1(C)CCN(c2nc3c(c(=O)[nH]2)CCCC3)CC1</smiles>
$\frac{\mathrm{Pd} / \mathrm{C}, \mathrm{H}_{2}}{\mathrm{MeOH}, 12 \mathrm{~h}}$ \begin{tabular}{c|c}
$\mathrm{KOtBu}$ & $\mathrm{THF}$ \\
$25{ }^{\circ} \mathrm{C}$ & $15 \mathrm{~h}$
\end{tabular} 46a-c<smiles>[X]CCN(CC)c1nc2c(c(=O)[nH]1)C=CCC2</smiles>
$47 a-c$

Scheme 18. Nucleophilic substitution of SMe group with secondary amines.

Table 9. Yields of nucleophilic substitution of $S M e$ group in $\mathbf{3 7 h}$ with amines.

\begin{tabular}{ccccccc}
\hline Amines & $\mathbf{4 5}$ & Yield $(\%)$ & $\mathbf{4 7}$ & Yield $(\%)$ & $\mathbf{4 6}$ & Yield (\%) \\
\hline morpholine & $\mathbf{4 5 a}$ & 93 & $\mathbf{4 7 a}$ & 84 & $\mathbf{4 6 a}$ & 94 \\
$N$-benzylpiperazine & $\mathbf{4 5 b}$ & 92 & $\mathbf{4 7 b}$ & 94 & $\mathbf{4 6} \mathbf{b}^{*}$ & 87 \\
$N$-Methypiperazine & $\mathbf{4 5 c}$ & 91 & $\mathbf{4 7 c}$ & 88 & $\mathbf{4 6 c}$ & 83 \\
\hline
\end{tabular}

*) Without benzyl group. 


\subsection{Cyclopropane Analogue of Tadalafil}

\subsubsection{Considerations}

In recent years there has been an increasing interest for the phosphodiesterases (PDEs) inhibitors. PDEs are super-family of enzymes that degrades the intracellular messenger cyclic guanosine monophosphate (cGMP) and are distributed throughout the vascular smooth muscle tissue and to a lesser extends in the lung, kidney, and platelets. ${ }^{[75]}$ Of the 12 PDE gene families discovered till now, cGMP-specific PDE5 carries out the principal cGMP hydrolyzing activities in human corpus cavernosum tissue. PDE5 are drug targets for the treatment of various diseases, particularly for the treatment of cardiovascular disease, such as hypertension, and congestive heart failure. ${ }^{[76]}$ More interestingly it is reported that PDE5 plays an important role in the mechanism of penile erection. The most promising PDE5 inhibitors are Zaprinast (49), Sildenafil (50) (Viagra ${ }^{\circledR}$, Pfizer) and Vardenafil (51) (Levirta ${ }^{\circledR}$, Bayer) and for that reason Sildenafil and Verdanafil were approved for the treatment of erectile disfunction (ED). ${ }^{[77]}$<smiles>CCCOc1ccccc1-c1nc2[nH]nnc2c(=O)[nH]1</smiles>

49

Zaprinast

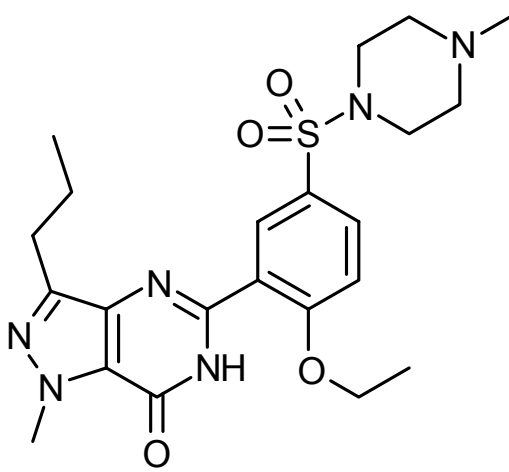

50 Sildenafil (Viagra)

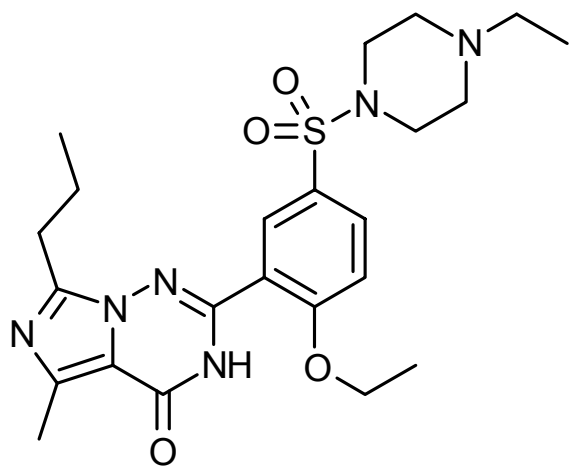

51

Vardenafil (Levitra)

Figure 5. Structures of PDE5 inhibitors: Zaprinast, Vardenafil and Sildenafil.

Despite the efficacy of Sildenafil, clinically significant adverse effect has been noted, ${ }^{[78]}$ and it has been proposed that some of the side effects may be due to lack of selectivity for PDE5 ${ }^{[79]}$ The search for a better PDE5 inhibitor compared to PDE6 led to the discovery of Tadalafil (52) (Cialis ${ }^{\circledR}$, Lilly ICOS) ${ }^{[52]}$ via the hydantoin lead structure (53). It was found that Tadalafil has 1000-fold selectivity for PDE5 versus PDE6 as against 12-fold in case of Sildenafil. ${ }^{[80]}$ These 
hererocycles (52 and 53) have a common heterocyclic skeleton ( $\beta$-carboline ring) which is present in many indole alkaloids. ${ }^{[81]}$

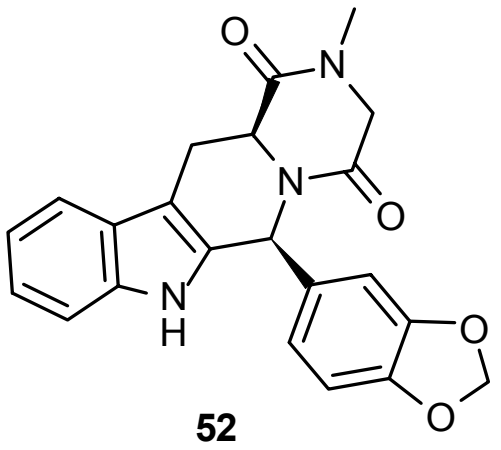

Tadalafil

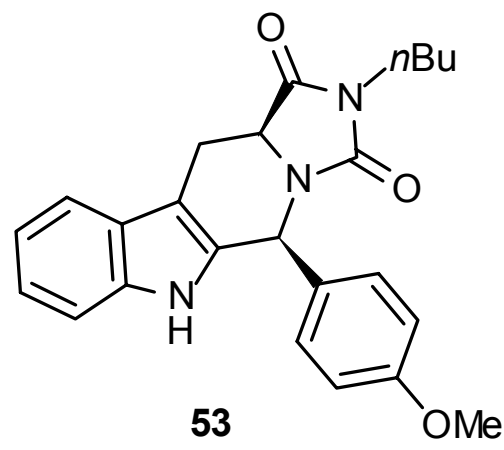

Hydrantoin lead sructure

of Tadalafil

Figure 6. Structure of Tadalafil, hydrantoin lead structure of Tadalafil, and natural product Fumitremorgine $\mathrm{C}$ and Demethoxyfumitremorgine $\mathrm{C}$.

The synthesis of all these tetrahydro- $\beta$-carbolines (52 and 53) have been achieved starting from tryptophan. ${ }^{[80]}$ Tryptophane is an essential residue in numerous peptide hormones and is a part of many naturally occurring indole alkaloids ${ }^{[82]}$ for which its replacement by conformationally restricted analogues e.g. dimethyl or cyclopropyl derivatives has considerable potential. ${ }^{[83]}$ Since cyclopropyl groups have proved to be highly effective in improving the activity of many biologically active compounds, ${ }^{[2 a, b][38]}$ it was interesting to synthesize cyclopropyl analogue of tryptophane and using it as a precursor to synthesize spirocyclopropyl analogue of hydrantoin lead structute to Tadalafil (25) and Tadalafil (26) it self. ${ }^{[57]}$ This idea was executed to demonstrate once again the enhanced tendency with which a suitable 1,1-disubstituted cyclopropane derivative undergoes ring closure due to the enforced favorable conformation just like a correspondingly substituted 2,2-disubstituted propane derivative. ${ }^{[84]}$

\subsubsection{Synthesis of a spirocyclopropanated tryptophan methyl ester}

The potent Michael acceptor methyl 2-chloro-2-cyclopropylideneacetate (5), in presence of ethylaluminum dichloride undergoes addition of indole 54 to furnish $\mathbf{5 5}$ in $85 \%$ yield after 6 h. The cyclopropanated tryptophane methyl ester $\mathbf{5 6}$ could be prepared by nucleophilic substitution 
of the chloride under phase transfer catalysis with sodium azide in water and subsequent reduction of the azido ester 57 with tin(II) chloride with an overall yield of 90\%. These results are summerised in Scheme 19.

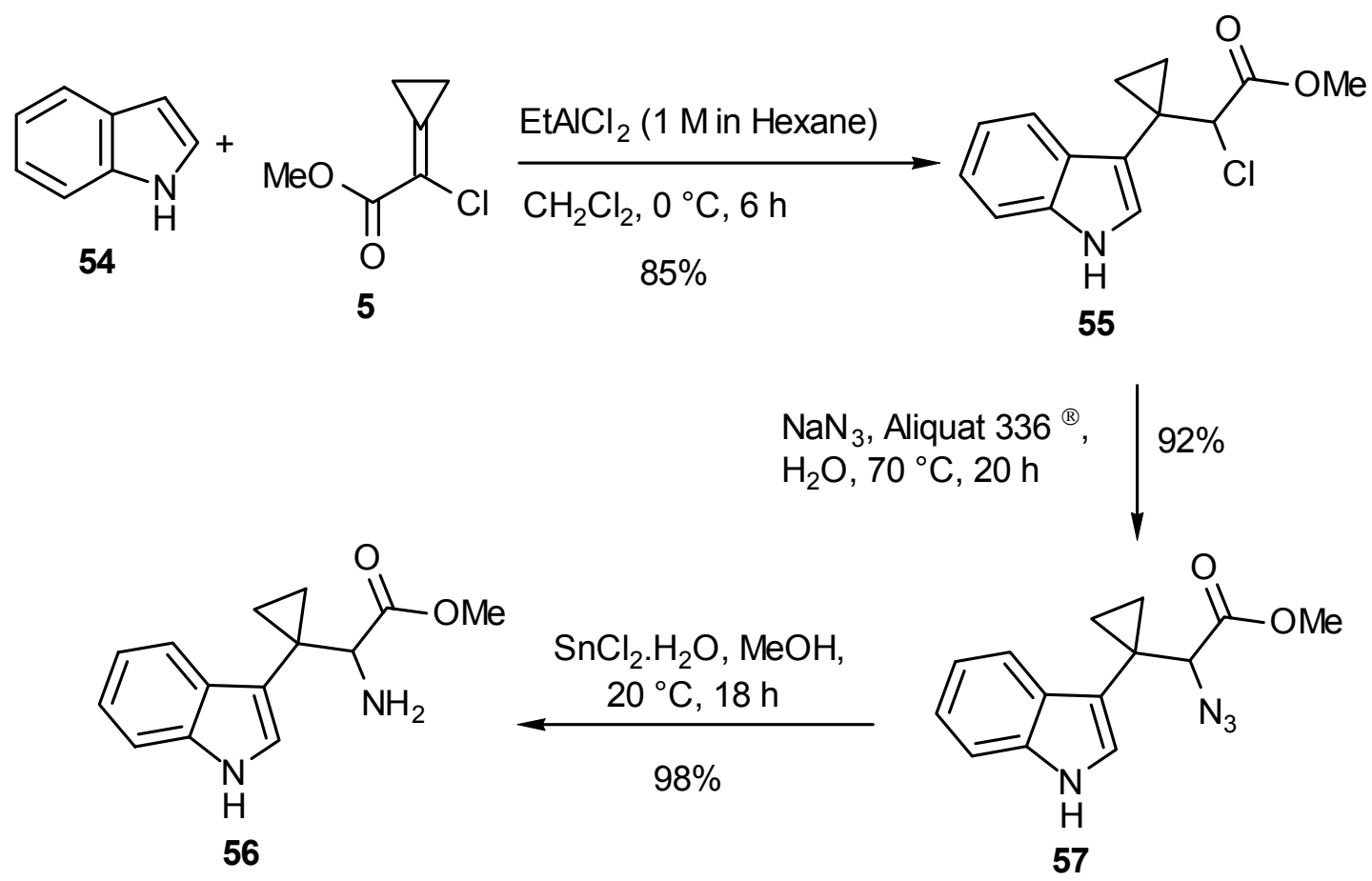

Scheme 19. Michael addition of 5 onto indole (54) and synthesis of cyclopropanated tryptophan methyl ester (56).

\subsubsection{The Pictet-Spengler reaction for synthesis of tetrahydro- $\beta$-carbolines}

The Pictet-Spengler reaction has long been an important reaction for the synthesis of both indole and isoquinoline alkaloids and also has been of vital importance in the synthesis of numerous tetrahydro- $\beta$-Carbolines which is a common skeleton in indole alkaloids and their analogues mostly with interesting biological activities. ${ }^{[82]}$ As expected condensation of 56 with $p$ anisaldehyde in trimethyl orthoformate yielded the corresponding imine 58a after $8 \mathrm{~h}$ at $20{ }^{\circ} \mathrm{C}$ almost quantitatively. Treatment with 4 equiv. of trifluoroacetic acid at $0{ }^{\circ} \mathrm{C}$ and subsequent stirring at $20^{\circ} \mathrm{C}$ for $15 \mathrm{~h}$ gave the tetrahydro- $\beta$-carboline 59a in $87 \%$ yield as a mixture of two diastereomers (cis/trans $=1: 1.7)$ which could not be separated by column chromatography. The pure trans-isomer was separated by crystallization from ethyl acetate and the mother liquer was found to contain a mixture of both isomers (cis/trans $=5: 1)$. In a similar way the reaction of 56 
with piperonal yielded the corresponding tetrahydro- $\beta$-carbolines $\mathbf{5 9 b}$ in $89 \%$ with a ratio of cis/trans $=1: 2.5$ (Scheme 20). In this case the trans-isomer could be separated by crystallization from ethyl acetate/pentane and the mother liquer was found to contain cis/trans $=3: 1$. The assignment of cis/trans-steriochemistry for tetrahydro- $\beta$-carbolines $\mathbf{5 9 a}, \mathbf{b}$ were based on a detailed study of the ${ }^{1} \mathrm{H}$ and ${ }^{13} \mathrm{C}$ spectroscopy and by comparison with the literature data, well established by Cook. ${ }^{[85]}$ Thus, the signals for C-1 and C-3 in trans-isomers appear at higher field in carbon spectrum than the analogous carbons of the corresponding cis-isomer probably due to 1,3 interactions present in trans-isomer. Moreover, the ${ }^{1} \mathrm{H}-\mathrm{NMR}$ signals at $\mathrm{C}-1$ is more shielded in the cis-isomer compared to the trans-isomer.

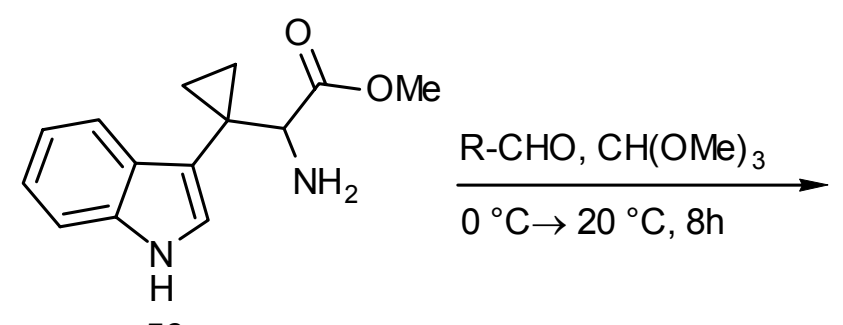

56
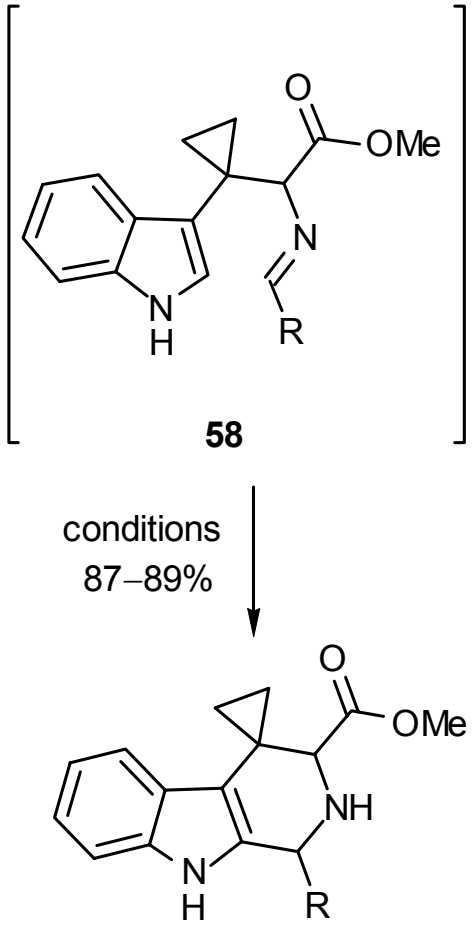

$59 a, b$

\begin{tabular}{ccccc}
\hline Compound & $\mathrm{R}$ & Conditions & Yield (\%) & d.r. (cis/trans) \\
\hline 59a & $p$-anisyl & $\mathrm{TFA}, \mathrm{CH}_{2} \mathrm{Cl}_{2}, 0 \rightarrow 20{ }^{\circ} \mathrm{C}, 15 \mathrm{~h}$ & 87 & $(1: 1.7)$ \\
59b & piperonyl & $\mathrm{TFA}, \mathrm{CH}_{2} \mathrm{Cl}_{2}, 0 \rightarrow 20{ }^{\circ} \mathrm{C}, 12 \mathrm{~h}$ & 89 & $(1: 2.5)$
\end{tabular}

Scheme 20. Pictet-Spengler reactions of 56 with aldehydes. 


\subsubsection{Synthesis of cyclopropyl analogue of hydantoin lead to Tadalafil and Tadalafil}

Treatment of the tetrahydrocarboline trans-59a with $n$-butyl isocyanate in refluxing ethyl methyl ketone for $12 \mathrm{~h}$ led to the hydantoin lead structure trans-25 (91\%) and similarly when a mixture of cis/trans-59a (5:1) was refluxed in ethyl methyl ketone with $n$-butyl isocyanate, the resulted mixture could be purified by column chromatography to give cis-25 in $89 \%$ yield, calculated according to the starting cis-59a.
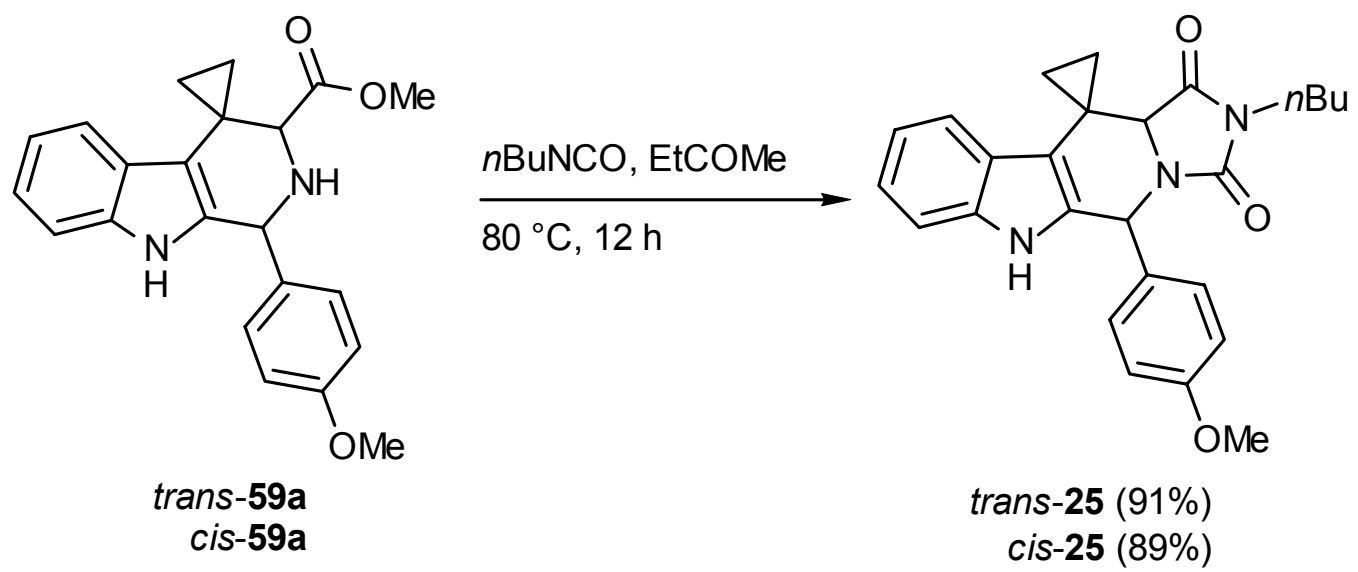

Scheme 21. Synthesis spirocyclopropyl analogue of hydrantion lead to tadalafil 25 from the corresponding $\beta$-carboline 59.

In order to synthesize the tadalafil analogue the tetrahydrocarboline derivative, trans-59b was acylated with chloroacetyl chloride to provide the $N$-(chloroacetyl) derivative trans-60 in 93\% yield, which upon reaction with $40 \%$ aq. methylamine in ethanol underwent nucleophilic substitution and subsequent ring-closing amide formation to lead to the spirocyclopropanated tadalafil analogue trans-26 in $86 \%$ yield. In a similar way cis/trans-59b (3:1) was acylated to provide a chromatograpically separable product to give cis-60 in $81 \%$ yield (calculated from pure cis-59) which upon reaction with $40 \%$ aq. methylamine in ethanol generated the cis-26 in $81 \%$ yield (Scheme 22). ${ }^{[86]}$ 


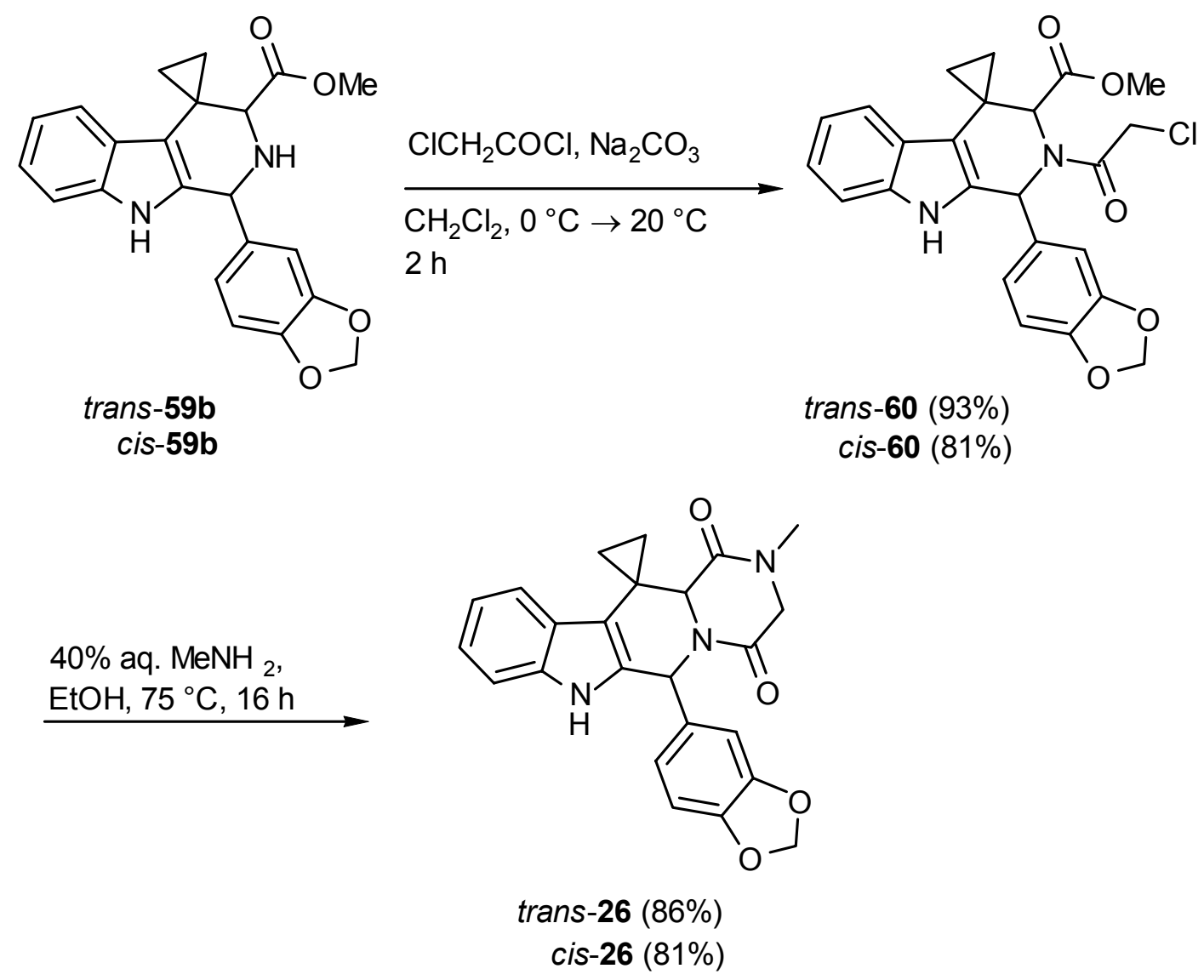

Scheme 22. Synthesis of spirocyclopropane analogue of Tadalafil (26). 


\subsection{Synthesis of Spirocyclopropyl Oxazolines}

2.4.1. Synthesis of spirocyclopropane oxazoline carboxylic acid and attempted transformation to oxazole or thiazole derivatives

Recently, a new and efficient method for the preparation of spirocyclopropane substituted oxazoline carboxylic acid methyl ester by the reaction of carboxamides with methyl 2-chloro-2cyclopropylideneacetate (5) has been developed. ${ }^{[27]}$ On the basis of this work further effords were directed to the transformation of the methyl ester group to heterocycle derivatives such as oxazole and thiazole (61). The conceivable synthesis of the target molecule (61) was considered from the $\alpha$-chloro ketone (62) which could be easily obtained from the corresponding oxazoline methyl ester (13) (Scheme 23).

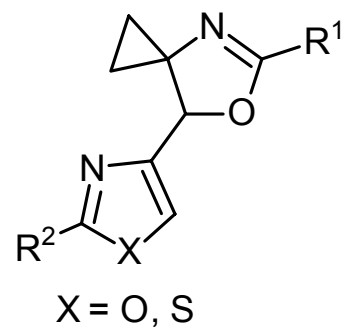

61

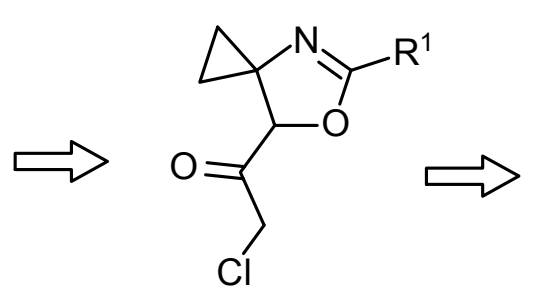

62

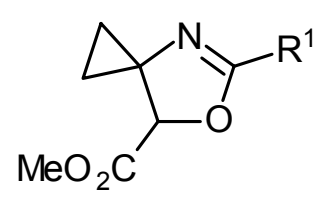

13

Scheme 23. Retrosynthetic analysis for the synthesis of heterocycles from oxazoline methyl ester.

By adopting the method described by de Meijere et al., when methyl 2-chloro-2cyclopropylideneacetate (5) in acetonitrile solution was treated with 4-bromo benzamide (63a) in the presence of sodium hydride at $0{ }^{\circ} \mathrm{C}$ and the mixture was allowed to warm up to room temperature after $2 \mathrm{~h}$, the 5-(4-bromophenyl)-6-oxa-4-aza-spiro[2.4]hept-4-ene-7-carboxylic acid methyl ester (13a) was isolated in 51\% yield. Under the same conditions, other aryl amides gave the corresponding oxazolines $\mathbf{1 3 b}-\mathbf{e}$ in yields ranging from 54 to $81 \%$. Under basic conditions ( $1 \mathrm{~N}$ aq. $\mathrm{NaOH}$ in $\mathrm{H}_{2} \mathrm{O} / \mathrm{THF}$ ), the hydrolysis of 13a gave the oxazoline carboxylic acid $64 \mathbf{a}$ in excellent yield (91\%) after $2 \mathrm{~h}$. The product could be purified by column chromatography using $\mathrm{Et}_{2} \mathrm{O} / \mathrm{AcOH}$ as eluent. In the similar procedure other oxazoline methyl esters were converted to the corresponding carboxylic acid $(\mathbf{6 4 b}-\mathbf{e})$ in very good yield. These results are summarized in Scheme 24 and Table 10. 


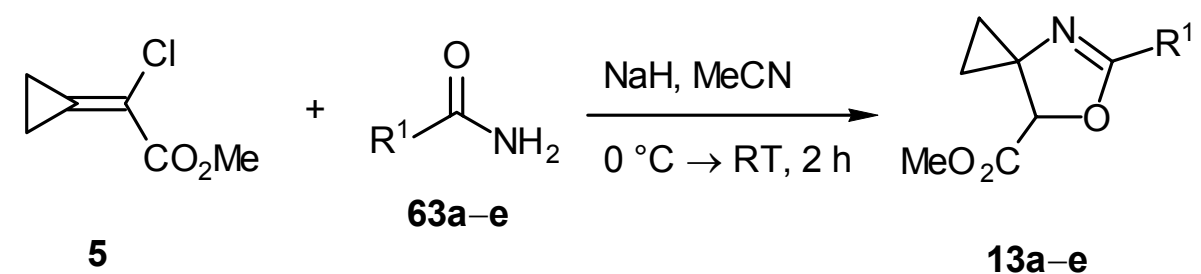

1) $1 \mathrm{~N} \mathrm{NaOH}, \mathrm{H}_{2} \mathrm{O} / \mathrm{THF}(4: 1)$

2) $\mathrm{AcOH}, 25^{\circ} \mathrm{C}, 2 \mathrm{~h}$

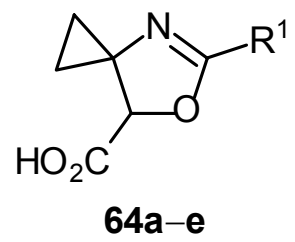

Scheme 24. Michael addition of aryl amides onto methyl 2-chloro-2-cyclopropylideneacetate 5 with ensuing intramolecular nucleophilic substitution to yield spirocyclopropaneoxazolinecarboxylic acids $64 \mathbf{a}-\mathbf{e}$.

Table 10. Yields of methyl spirocyclopropaneoxazoline carboxylates 13a-e from $\mathbf{5}$ and arenecarboxamides and hydrolysis of $13 \mathbf{a}-\mathbf{e}$ to oxazolinecarboxylic acids $64 \mathbf{a}-\mathbf{e}$.

\begin{tabular}{cccccc}
\hline Entry & $\mathrm{R}^{1}$ & $\mathbf{1 3}$ & Yield (\%) & $\mathbf{6 4}$ & Yield (\%) \\
\hline 1 & $4-\mathrm{Br}-\mathrm{C}_{6} \mathrm{H}_{4}$ & $\mathbf{1 3 a}$ & 51 & $\mathbf{6 4 a}$ & 91 \\
2 & $3-\mathrm{Br}_{-} \mathrm{C}_{6} \mathrm{H}_{4}$ & $\mathbf{1 3 b}$ & 64 & $\mathbf{6 4 b}$ & 90 \\
3 & $3-\mathrm{CF}_{3}-\mathrm{C}_{6} \mathrm{H}_{4}$ & $\mathbf{1 3 c}$ & 47 & $\mathbf{6 4 c}$ & 89 \\
4 & $4-\mathrm{CF}_{3}-\mathrm{C}_{6} \mathrm{H}_{4}$ & $\mathbf{1 3 d}$ & 81 & $\mathbf{6 4 d}$ & 93 \\
5 & $\mathrm{Ph}$ & $\mathbf{1 3 e}$ & 54 & $\mathbf{6 4 e}$ & 89 \\
\hline
\end{tabular}

With the oxazolinecarboxylic acids in hand further effords were made to convert them into $\alpha$ chloro ketone. The classical method for the synthesis of $\alpha$-chloroketones involves the conversion of the carboxylic acid to an $\alpha$-diazoketone and the subsequent acidolysis with $\mathrm{HCl}^{[87]}$ When the half ester formed from 64e and isobutylchloroformate was treated with diazomethane followed by reaction with $\mathrm{HCl}\left(3 \mathrm{~N}_{\text {in }} \mathrm{Et}_{2} \mathrm{O}\right)$ the corresponding $\alpha$-chloroketone $(62-\mathrm{Ph})$ was obtained in $52 \%$ yield. The same product $(62-\mathrm{Ph})$ could also be obtained in $51 \%$ yield by using the method described by Polniaszek and coworkers by using LDA (5 equiv.) and $\mathrm{CH}_{2} \mathrm{ICl}$ (4 equiv. $)^{[88]}$ directly from the ester 13e in one step without the use of diazomethane. However, the reaction of $\alpha$ chloroketone $(62-\mathrm{Ph})$ with benzamide or benzothioamide either in $\mathrm{NaH} / \mathrm{DMF}$ at $45^{\circ} \mathrm{C}$ or in 
$\mathrm{Et}_{3} \mathrm{~N} / \mathrm{EtOH}$ at $70{ }^{\circ} \mathrm{C}$ for $15 \mathrm{~h}$ did not lead to any product containing oxazole or thiazole ring, only starting material was recovered. Only in the case when the reaction was carried out with 4(trifluoromethyl)-benzothioamide in the presence of $\mathrm{NaOAc}$ in $\mathrm{CH}_{3} \mathrm{CN}$ at $50{ }^{\circ} \mathrm{C}$ for $24 \mathrm{~h}$, the nucleophilic substitution product (65) was isolated in 59\% yield. Several trials for the ring closure reaction by using molecularsieve or by using para-toluenesulfonic acid in a Dean-Stark apparatus did not lead to the thiazole ring. These resuts are presented in the Scheme 25.

For 64e: $\mathrm{R}=\mathrm{H}$

1) $\mathrm{Et}_{3} \mathrm{~N}$, isobutylchloroformate,<smiles>[R]OC(=O)C1OC(c2ccccc2)=NC12CC2</smiles>

$64 e$ or $13 e$ $-20^{\circ} \mathrm{C}, 30 \mathrm{~min}$

2) Diazomethane, $0^{\circ} \mathrm{C}, 30 \mathrm{~min}$

3) $\mathrm{HCl}\left(3 \mathrm{~N}\right.$ in $\left.\mathrm{Et}_{2} \mathrm{O}\right)$, $25^{\circ} \mathrm{C}, 2 \mathrm{~h}, 52 \%$

or for 13e: $\mathrm{R}=\mathrm{Me}$

LDA, $\mathrm{CH}_{2} \mathrm{ICl}$, THF, $-78^{\circ} \mathrm{C} \rightarrow 20^{\circ} \mathrm{C}, 2 \mathrm{~h}$, $51 \%$<smiles>O=C(CCl)C1OC(c2ccccc2)=NC12CC2</smiles>

62-Ph<smiles>FC(F)(F)c1ccc(-c2nc(C3OC(c4ccccc4)=NC34CC4)cs2)cc1</smiles>

66
Molecularsieb, $\mathrm{CH}_{3} \mathrm{CN}$, $80^{\circ} \mathrm{C}, 12 \mathrm{~h}$

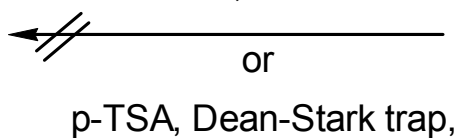

Toluene, $80^{\circ} \mathrm{C}, 12 \mathrm{~h}$

$50{ }^{\circ} \mathrm{C}, 1 \mathrm{~d}$ $59 \%$<smiles>CC(C)(C)C(=S)c1ccc(C(F)(F)F)cc1</smiles>

$63 f$

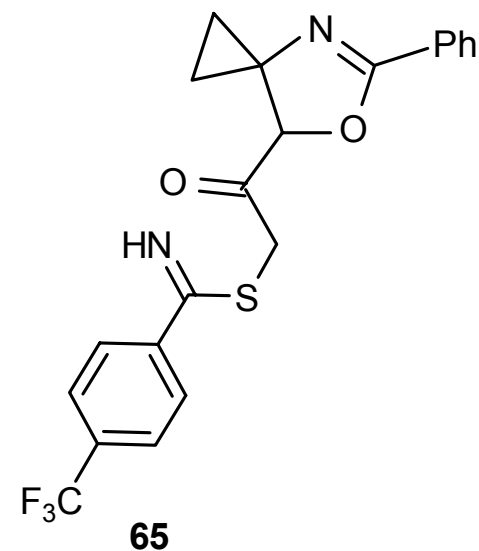

Scheme 25. Synthesis of $\alpha$-chloroketone derivative (62-Ph) from oxazoline carboxylic acid (64e) or methyl ester (13e) and conceivable further transformation to thiazoline ring (66). 
2.4.2. Coupling of oxazoline carboxylic acids with anilines and subsequent Mitsunobu reaction for the synthesis of benzoxazole derivatives

After failing to produce the oxazole or thiazole ring it was interesting to synthesize a benzoxazole derivative of spirocyclopropane oxazolines. The obvious choice was to consider the orthohydroxy aniline so that the coupling reaction with oxazoline carboxylic acid followed by ring closing reaction would afford the desired benzoxazole. In order to optimize the condition for coupling reaction, 5-(4-bromophenyl)-6-oxa-4-aza-spiro[2.4]hept-4-ene-7-carboxylic acid (64a) and 3trifluoromethyl aniline was chosen. When the reaction was carried out in the presence of DCC and DMAP in DCM as solvent at $20^{\circ} \mathrm{C}$ for $18 \mathrm{~h}$, no product was obtained. The reaction also failed in a condition of DCC, HOBT in DCM for $18 \mathrm{~h} .^{[89]}$

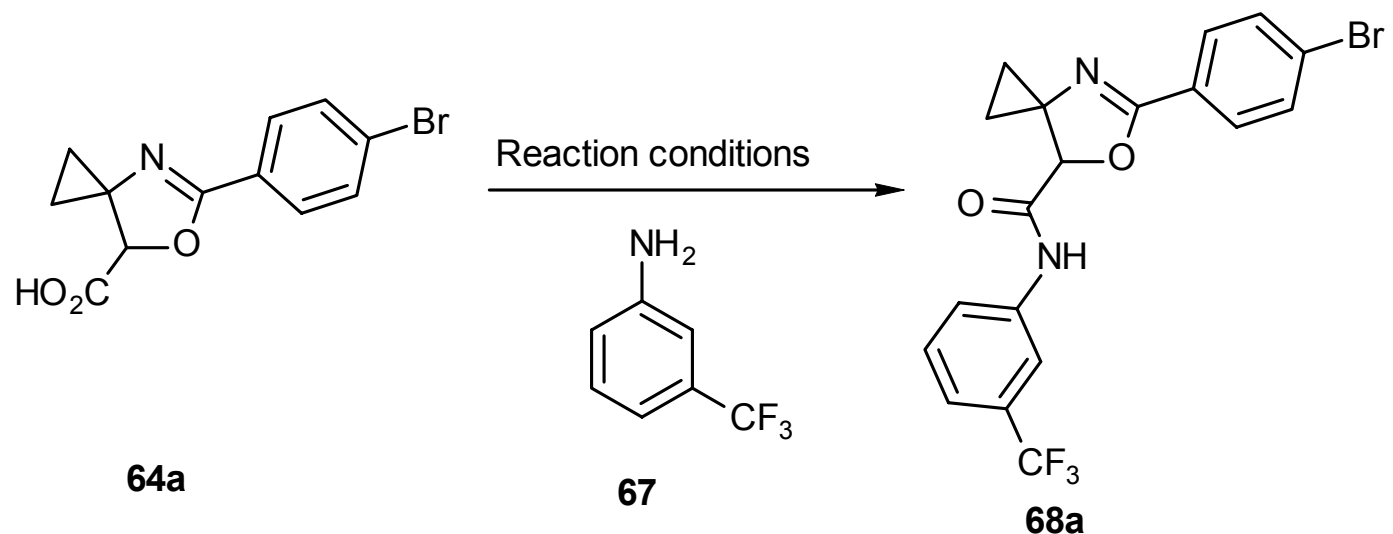

Scheme 26. Optimization of reaction conditions for the coupling reaction of 64 a with $4-\mathrm{CF}_{3^{-}}$ aniline.

Table 11. Reaction conditions and yield for the coupling reaction of $\mathbf{4 6 a}$ with paratrifluoromethylaniline.

\begin{tabular}{lll}
\hline Entry & Reaction conditions & 68a Yield (\%) \\
\hline 1 & DCC, DMAP, DCM, 18 h & 00 \\
2 & DCC, HOBT, colidine, DCM, $18 \mathrm{~h}$ & 00 \\
3 & $\mathrm{SOCl}_{2}, \mathrm{DCM}, 60{ }^{\circ} \mathrm{C}, 12 \mathrm{~h}$ & decomposition \\
4 & $\mathrm{HOAt}, \mathrm{EDC} \cdot \mathrm{HCl}$, colidine, $20{ }^{\circ} \mathrm{C}, 12 \mathrm{~h}$ & $92 \%$ \\
\hline
\end{tabular}


Another idea to convert the carboxylic group to acid chloride by $\mathrm{SOCl}_{2}$ and then reacting with the aniline also failed. Finally when the reaction was carried out in the presence of $\mathrm{HOAt}, \mathrm{EDC} \cdot \mathrm{HCl}$ and colidine at $20{ }^{\circ} \mathrm{C}$ for $12 \mathrm{~h}$ in DCM, the coupling product $68 \mathrm{a}$ was obtained in $92 \%$ yield (Scheme 27, Table 12).

With the optimal conditions the oxazolines $64 \mathbf{b}-\mathbf{d}$ were coupled with 3-trifluoromethyl aniline to give the corresponding amide $\mathbf{6 8 b}-\mathbf{d}$ in good yield. The reaction of $\mathbf{4 6 d}$ with 3-chloro-6-hydroxyaniline gave the product $\mathbf{6 8 e}$ in $85 \%$ yield. The other substituted 6-hydroxy anilines also coupled with $\mathbf{6 4 d}$ to give the product $\mathbf{6 8 f}-\mathbf{h}$ in yield ranging from 55 to $78 \%$. The reaction of $64 \mathbf{e}$ with 3chloro-6-hydroxy-aniline under the same conditions gave the product $\mathbf{6 8 i}$ in $92 \%$ yield. These results are summerized in Scheme 27 and Table 12.

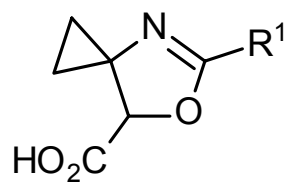

$64 a-e$

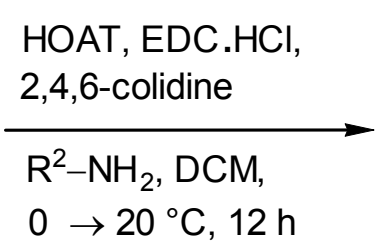

$0 \rightarrow 20^{\circ} \mathrm{C}, 12 \mathrm{~h}$

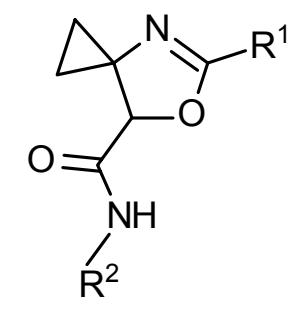

$68 \mathrm{a}-\mathrm{j}$

Scheme 27. Coupling reaction of oxazoline carboxylic acids $(64 a-e)$ with anilines for the synthesis of amide $\mathbf{6 8 a}-\mathbf{j}$.

Table 12. Yields of the coupling of oxazoline carboxylic acids with anilines.

\begin{tabular}{cccccc}
\hline Entry & $\mathbf{6 4}$ & $\mathrm{R}^{1}$ & $\mathrm{R}^{2}$ & $\mathbf{6 8}$ & Yield (\%) \\
\hline 1 & $\mathbf{6 4 a}$ & $4-\mathrm{Br}_{-}-\mathrm{C}_{6} \mathrm{H}_{4}$ & $3-\mathrm{CF}_{3}-\mathrm{C}_{6} \mathrm{H}_{4}$ & $\mathbf{6 8 a}$ & 92 \\
2 & $\mathbf{6 4 b}$ & $3-\mathrm{Br}_{-}-\mathrm{C}_{6} \mathrm{H}_{4}$ & $3-\mathrm{CF}_{3}-\mathrm{C}_{6} \mathrm{H}_{4}$ & $\mathbf{6 8 b}$ & 82 \\
3 & $\mathbf{6 4 c}$ & $3-\mathrm{CF}_{3}-\mathrm{C}_{6} \mathrm{H}_{4}$ & $3-\mathrm{CF}_{3}-\mathrm{C}_{6} \mathrm{H}_{4}$ & $\mathbf{6 8 c}$ & 81 \\
4 & $\mathbf{6 4 d}$ & $4-\mathrm{CF}_{3}-\mathrm{C}_{6} \mathrm{H}_{4}$ & $3-\mathrm{CF}_{3}-\mathrm{C}_{6} \mathrm{H}_{4}$ & $\mathbf{6 8 d}$ & 90 \\
5 & $\mathbf{6 4 d}$ & $4-\mathrm{CF}_{3}-\mathrm{C}_{6} \mathrm{H}_{4}$ & $3-\mathrm{Cl}_{-}-6-\mathrm{OH}-\mathrm{C}_{6} \mathrm{H}_{3}$ & $\mathbf{6 8 e}$ & 85 \\
6 & $\mathbf{6 4 d}$ & $4-\mathrm{CF}_{3}-\mathrm{C}_{6} \mathrm{H}_{4}$ & $3-\mathrm{OCF}-6-\mathrm{OH}-\mathrm{C}_{6} \mathrm{H}_{3}$ & $\mathbf{6 8 f}$ & 55 \\
7 & $\mathbf{6 4 d}$ & $4-\mathrm{CF}_{3}-\mathrm{C}_{6} \mathrm{H}_{4}$ & $3,5-\mathrm{di}-\mathrm{Cl}-6-\mathrm{OH}-\mathrm{C}_{6} \mathrm{H}_{2}$ & $\mathbf{6 8 g}$ & 79 \\
8 & $\mathbf{6 4 d}$ & $4-\mathrm{CF}_{3}-\mathrm{C}_{6} \mathrm{H}_{4}$ & $3-\mathrm{CF}-6-\mathrm{OH}-\mathrm{C}_{6} \mathrm{H}_{3}$ & $\mathbf{6 8 h}$ & 78 \\
9 & $\mathbf{6 4 e}$ & $\mathrm{C}_{6} \mathrm{H}_{5}$ & $3-\mathrm{Cl}-6-\mathrm{OH}-\mathrm{C}_{6} \mathrm{H}_{3}$ & $\mathbf{6 8 i}$ & 92 \\
10 & $\mathbf{6 4 d}$ & $\mathrm{C}_{6} \mathrm{H}_{5}$ & $6-\mathrm{SH}-\mathrm{C}_{6} \mathrm{H}_{4}$ & $\mathbf{6 8 j}$ & 0 \\
\hline
\end{tabular}


When 68i was reacted with $\mathrm{P}_{2} \mathrm{O}_{5}$ in $\mathrm{CCl}_{4}$, no product was found, only the starting material was recovered. A very small amount of the product $69 \mathrm{e}(12 \%)$ was isolated when the reaction was carried out in PPTS in DCE at $85{ }^{\circ} \mathrm{C}$ for $12 \mathrm{~h}$. The desired product was not observed when the same reaction was done in toluene in a Dean-Stark trap at $85^{\circ} \mathrm{C}$. Finnaly under the Mitsunobu condition $^{[90]}\left(\mathrm{Ph}_{3} \mathrm{P}, \mathrm{DEAD}\right)$ the product was isolated in $85 \%$ yield. These results are given in Scheme 28, Table 13.

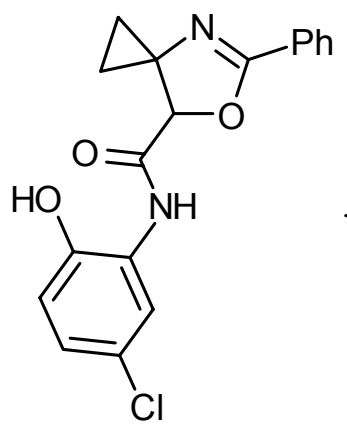

$68 \mathrm{i}$
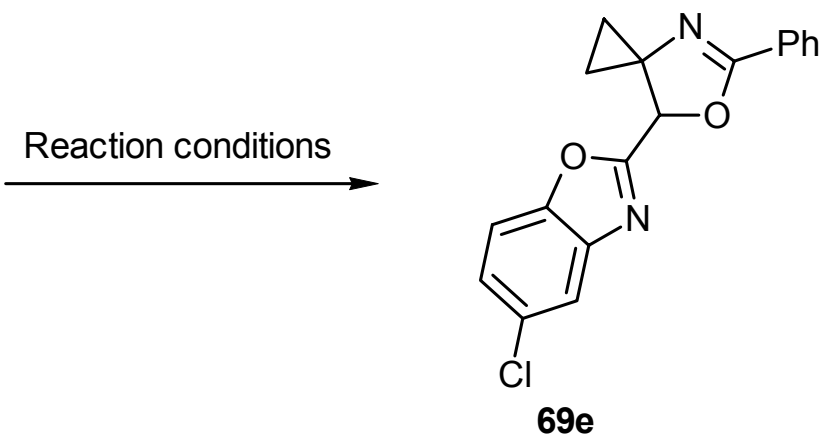

Scheme 28. Optimisation of reaction conditions for synthesis of bezoxazole derivatives.

Table 13. Reaction conditions and yield of dehydro cyclization reaction for synthesis of benzoxazole derivative.

\begin{tabular}{ccc}
\hline Entry & Raction conditions & Yield 69e (\%) \\
\hline 1 & $\mathrm{P}_{2} \mathrm{O}_{5}, \mathrm{CCl}_{4}, 80^{\circ} \mathrm{C}, 10 \mathrm{~h}$ & 00 \\
2 & $\mathrm{PPTS}, \mathrm{DCE}, 85^{\circ} \mathrm{C}, 12 \mathrm{~h}$ & 12 \\
3 & $\mathrm{PPTS}$, Dean-Stark trap, toluene, $80{ }^{\circ} \mathrm{C}, 12 \mathrm{~h}$ & 00 \\
4 & $\mathrm{Ph}_{3} \mathrm{P}, \mathrm{DEAD}, \mathrm{THF}, 0 \rightarrow 20{ }^{\circ} \mathrm{C}, 10 \mathrm{~h}$ & 83 \\
\hline
\end{tabular}

After finding the optimized condition for the dehydro cyclisation reaction, other $o$-hydroxy amides 68f-i were also transformed to the corresponding benzoxazoles in very good yield. These resuts are given in Scheme 29. 

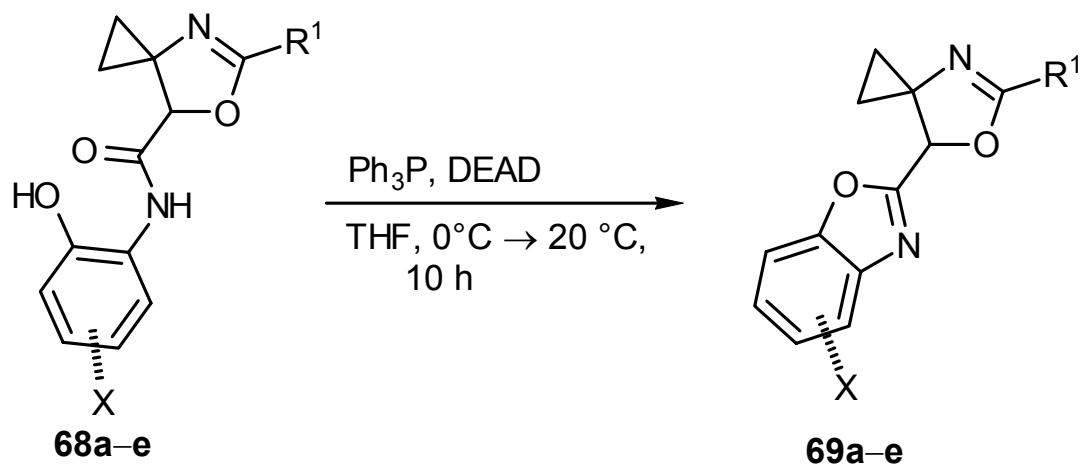

Scheme 29. Synthesis of benzoxazole derivatives of oxazolines by Mitsunobu reaction conditions.

Table 13. Yields of benzoxazole derivatives of oxazolines by Mitsunobu reaction.

\begin{tabular}{cccccc}
\hline Entry & $\mathbf{6 8}$ & $\mathrm{R}^{1}$ & $\mathrm{X}$ & $\mathbf{6 9}$ & Yield (\%) \\
\hline 1 & $\mathbf{6 8 e}$ & $4-\mathrm{CF}_{3}-\mathrm{C}_{6} \mathrm{H}_{4}$ & $3-\mathrm{Cl}$ & $\mathbf{6 9 a}$ & 85 \\
2 & $\mathbf{6 8 f}$ & $4-\mathrm{CF}_{3}-\mathrm{C}_{6} \mathrm{H}_{4}$ & $3-\mathrm{OCF}_{3}$ & $\mathbf{6 9 b}$ & 81 \\
3 & $\mathbf{6 8 g}$ & $4-\mathrm{CF}_{3}-\mathrm{C}_{6} \mathrm{H}_{4}$ & $3,5-\mathrm{di}-\mathrm{Cl}$ & $\mathbf{6 9 c}$ & 88 \\
4 & $\mathbf{6 8 h}$ & $4-\mathrm{CF}_{3}-\mathrm{C}_{6} \mathrm{H}_{4}$ & $3-\mathrm{CF}_{3}$ & $\mathbf{6 9 d}$ & 81 \\
5 & $\mathbf{6 8 i}$ & $\mathrm{C}_{6} \mathrm{H}_{5}$ & $3-\mathrm{Cl}$ & $\mathbf{6 9 e}$ & 83 \\
\hline
\end{tabular}

2.4.3. Pd-Catalyzed $C-N$ or $C-C$ bond formation reaction for the synthesis of amino-aryl or biaryl substituted oxazolines.

The palladium-catalyzed coupling of amines with aryl halides, commonly known as BuchwaldHartwig reaction ${ }^{[91]}$ has matured from a synthetic loboratory procedure to a technique that is widely used in natural product synthesis as well as in other field of academic interest. This reaction also opened a new chapter in the field of transition metal-catalyzed cross coupling chemistry with a new approach for the synthesis of aniline derivatives. ${ }^{[92]}$ After successful synthesis of benzoxazole derivatives, it was interesting to try Buchwald-Hartwig reaction of the aryl bromide group present in 5-(4-bromo-phenyl)-6-oxa-4-aza-spiro[2.4]hept-4-ene-7-carboxylic acid (3-trifluoromethyl-phenyl)-amide (68a). The initial attempts for the reaction of 68a with the well established condition $\left[\mathrm{Pd}_{2}(\mathrm{dba})_{3}, \mathrm{BINAP}, \mathrm{Na} t \mathrm{OBu}\right]$ in toluene for $18 \mathrm{~h}$ failed to give any desired products with complete decomposition of 68a. It was concluded that the free amide group might not be stable in the reaction conditions. So by methylating the amide group of $68 \mathbf{a}$ with 
$\mathrm{Me}_{2} \mathrm{SO}_{4}, \mathrm{~K}_{2} \mathrm{CO}_{3}$ in acetone followed by the palladium-catalyzed reaction with morpholine, the coupling product 71a was isolated in $71 \%$ yield after $16 \mathrm{~h}$. In order to optimize the amount of catalyst we found that the best yield was obtained when $5 \mathrm{~mol} \%$ of $\operatorname{Pd}_{2}(\mathrm{dba})_{3}$ and $7.5 \mathrm{~mol} \%$ of \pm BINAP were used. Other secondary amines such as pyrolidine under the same reaction condition gave the product $\mathbf{7 1 b}$ in $90 \%$ yield, but in the case of $N$-methyl and $N$-benzyl piperazine the decreased yield (59 and 62\% respectively, entries 3 and 4) was observed. Another interesting amine, $N, N$-dibenzyl-3-azabicyclo[3.1.0]hexane also proceeded smoothly to afford the product 71e in 67\% yield. These five different amines were also reacted with 5-(3-bromo-phenyl)-6-oxa4-aza-spiro[2.4]hept-4-ene-7-carboxylic acid methyl-(3-trifluoromethyl-phenyl)-amide (70b) to give the corresponding aminated products. In the case of $N$-methyl and $N$-benzyl piperazine very poor yield (13 and 12\% respectively, entry 8 and 9 ) was obtained and the reaction was not finished even if the reaction time was increased to $40 \mathrm{~h}$. These results are given in Scheme 30 and Table 14.

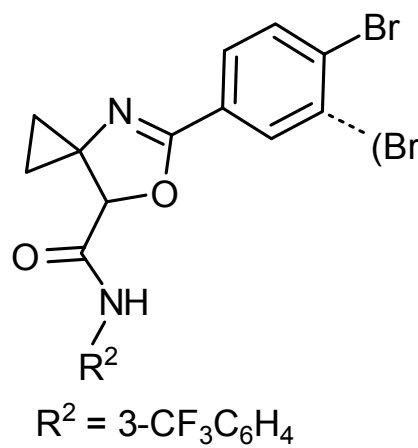

$68 \mathbf{a}-\mathbf{b}$

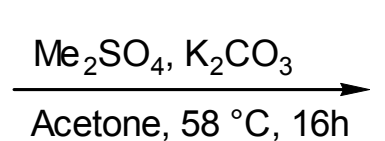

70a: $92 \%$

70b: $89 \%$

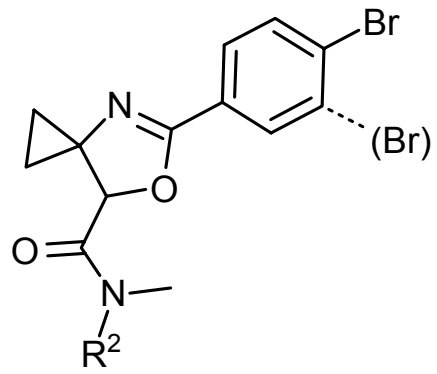

$70 a-b$

$$
\begin{aligned}
& \mathrm{Pd}_{2}(\mathrm{dba})_{3}(5 \mathrm{~mol} \%), \\
& \pm \mathrm{BINAP}(7.5 \mathrm{~mol} \%) \\
& \underset{\mathrm{NaOtBu}, \mathrm{Nu}-\mathrm{H}, \text { Toluene, }}{\longrightarrow} \\
& \quad 80^{\circ} \mathrm{C}, 16 \mathrm{~h}
\end{aligned}
$$

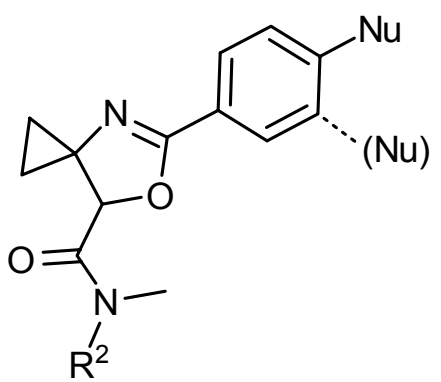

$71 \mathrm{a}-\mathrm{j}$

Scheme 30. Methylation of amide group followed by Buchwald amination of aryl-bromides (70a,b) for the synthesis of aminoaryl substituted oxazolines (71a-j). 
Table 14. Yield of Buchwald-Hartwig amination of aryl-bromides for the synthesis of aminoaryl substituted oxazolines

\begin{tabular}{ccccc}
\hline Entry & $\mathbf{7 0}$ & Nu-H & $\mathbf{7 1}$ & Yield (\%) \\
\hline 1 & $\mathbf{7 0 a}$ & morpholine & $\mathbf{7 1 a}$ & 71 \\
2 & $\mathbf{7 0 a}$ & pyrolidine & $\mathbf{7 1 b}$ & 90 \\
3 & $\mathbf{7 0 a}$ & $N$-methylpiperazine & $\mathbf{7 1 c}$ & 59 \\
4 & $\mathbf{7 0 a}$ & $N$-Benzylpiperazine & $\mathbf{7 1 d}$ & 62 \\
5 & $\mathbf{7 0 a}$ & $N, N$-dibenzyl-3-azabicyclo[3.1.0]hexane & $\mathbf{7 1 e}$ & 67 \\
6 & $\mathbf{7 0 b}$ & morpholine & $\mathbf{7 1 f}$ & 82 \\
7 & $\mathbf{7 0 b}$ & pyrolidine & $\mathbf{7 1 g}$ & 73 \\
8 & $\mathbf{7 0 b}$ & $N$-methylpiperazine & $\mathbf{7 1 h}$ & 13 \\
9 & $\mathbf{7 0 b}$ & $N$-Benzylpiperazine & $\mathbf{7 1 i}$ & 12 \\
10 & $\mathbf{7 0 b}$ & $N, N$-dibenzyl-3-azabicyclo[3.1.0]hexane & $\mathbf{7 1 j}$ & 81 \\
\hline
\end{tabular}

Another interesting Pd-catalyzed reaction of aryl halides with boronic acid the Suzuki reaction represents a powerful means for the preparation of numerous products important in pharmaceutical and material science. ${ }^{[93]}$ After successful Buchwald-Hartwig reaction the scope of the Suzuki reaction of the aryl bromide group present in 5-(4-bromo-phenyl)-6-oxa-4-aza-spiro[2.4]hept-4ene-7-carboxylic acid methyl-(3-trifluoromethylphenyl)-amide (70a) was also tested. After trying several reaction of 70a with phenyl boronic acid it was found that $10 \mathrm{~mol} \%$ of $\mathrm{Pd}(\mathrm{OAc})_{2}$ and 40 $\mathrm{mol} \%$ of $\mathrm{Ph}_{3} \mathrm{P}$ was necessary to complete the reaction and the product 72a was isolated in $90 \%$ yield after 16 h. $p$-Trifluoromethoxy phenyl boronic acid under the same reaction condition gave the product 72b in $84 \%$ yield. 3-Thiophene- and 2-naphthaleneboronic acid also yielded the product 72c,d in 65 and 85\% respectively. These results are given in Scheme 31 and Table 15. 


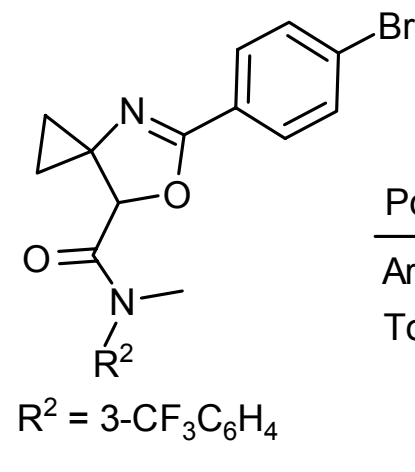

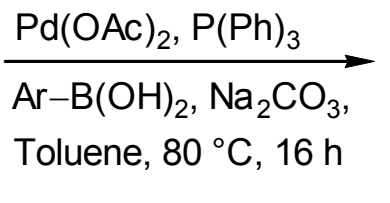

$70 a$<smiles>[R]N(C)C(=O)C1OC(c2ccc([Al])cc2)=NC12CC2</smiles>

$72 a-d$

Scheme 31. Palladium catalyzed cross-coupling reaction of $\mathbf{7 0 a}$ with aryl boronic acids.

Table 15.Yield of the Palladium catalyzed cross-coupling reaction of 72a with aryl boronic acids.

\begin{tabular}{cccc}
\hline Entry & $\mathrm{Ar}$ & $\mathbf{7 2}$ & Yield \\
\hline 1 & $\mathrm{C}_{6} \mathrm{H}_{5}$ & $\mathbf{7 2 a}$ & 90 \\
2 & $4-\mathrm{OCF}_{3}-\mathrm{C}_{6} \mathrm{H}_{4}$ & $\mathbf{7 2 b}$ & 80 \\
3 & $\mathrm{C}_{4} \mathrm{H}_{3} \mathrm{~S}$ & $\mathbf{7 2 c}$ & 65 \\
4 & $\mathrm{C}_{10} \mathrm{H}_{6}$ & $\mathbf{7 2 d}$ & 85 \\
\hline
\end{tabular}




\subsection{Reaction of Tetra- and trimethylenethiourea with Methyl 2-Chloro-2- cyclopropylideneacetate}

After the successful synthesis of spirocyclopropanated oxazolines it was interesting to study the Michael addition of urea or thiourea derivatives. Dimethyl urea or dimethyl thiourea did not react with the Michael acceptor 5 either in the presence of $\mathrm{Et}_{3} \mathrm{~N}$ in dioxane or $\mathrm{NaH}$ in acetonitrile. Under the same reaction conditions, tetramethylene urea also did not give any product corresponding to a Michael addition. But in the case of tetramethylene thiourea, when the reaction was carried out in the presence of $\mathrm{Et}_{3} \mathrm{~N}$ in a solution of dioxane, the tricyclic product 74a corresponding to the Michael addition and ring closure reaction was isolated in $82 \%$ yield as a hydrochloride salt. The same product 74a was also be obtained, when the reaction was carried out in the presence of $\mathrm{NaH}$ but in lower yield (49\%). The reaction also occurs with trimethylenethioura in presence of either $\mathrm{Et}_{3} \mathrm{~N}$ or $\mathrm{NaH}$ to give the product $\mathbf{7 4 b}$ in 41 and $29 \%$ yield respectively. The hydrochloride moiety could not be detected by mass spectrometry.
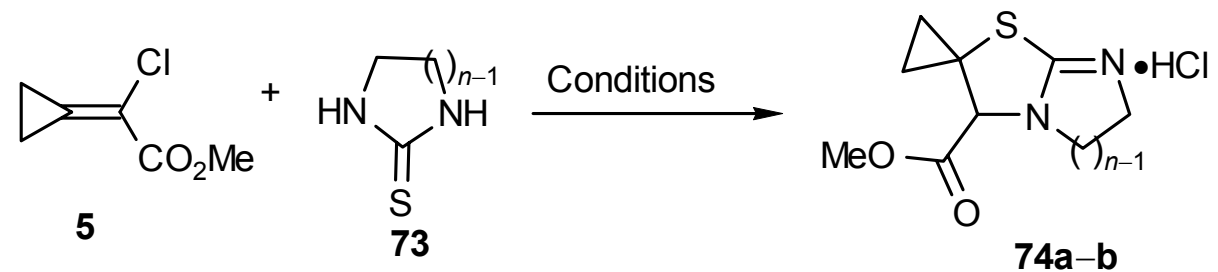

Scheme 32. Michael reaction of oligomethylenethioureas with 5.

Table 16. Yields of the reaction of oligomethylenethioureas with 5.

\begin{tabular}{ccccc}
\hline Entry & Conditions & $n$ & $\mathbf{7 4}$ & Yield (\%) \\
\hline 1 & $\mathrm{Et}_{3} \mathrm{~N}$, dioxane, $20{ }^{\circ} \mathrm{C}, 15 \mathrm{~h}$ & 4 & $\mathbf{7 4 a}$ & 82 \\
2 & Same as above & 3 & $\mathbf{7 4 b}$ & 41 \\
3 & same & 2 & $\mathbf{7 4 c}$ & 00 \\
4 & $\mathrm{NaH}, \mathrm{MeCN}, 0 \rightarrow 20 \mathrm{C}, 12 \mathrm{~h}$ & 4 & $\mathbf{7 4 a}$ & 49 \\
5 & same & 3 & $\mathbf{7 4 b}$ & 29 \\
6 & same & 2 & $\mathbf{7 4 c}$ & 00 \\
\hline
\end{tabular}

Since the product $\mathbf{7 4 b}$ could be readily crystallized from $\mathrm{Et}_{2} \mathrm{O}$, the structure was rigorously proved by an X-ray crystal structure alalysis (Figure 7). The reaction did not occur with 
diethylenethiourea (Scheme 32, Table 16). The compound 74b decomposed when washed with 1 $\mathrm{N} \mathrm{NaOH}$ in order to get the free base.

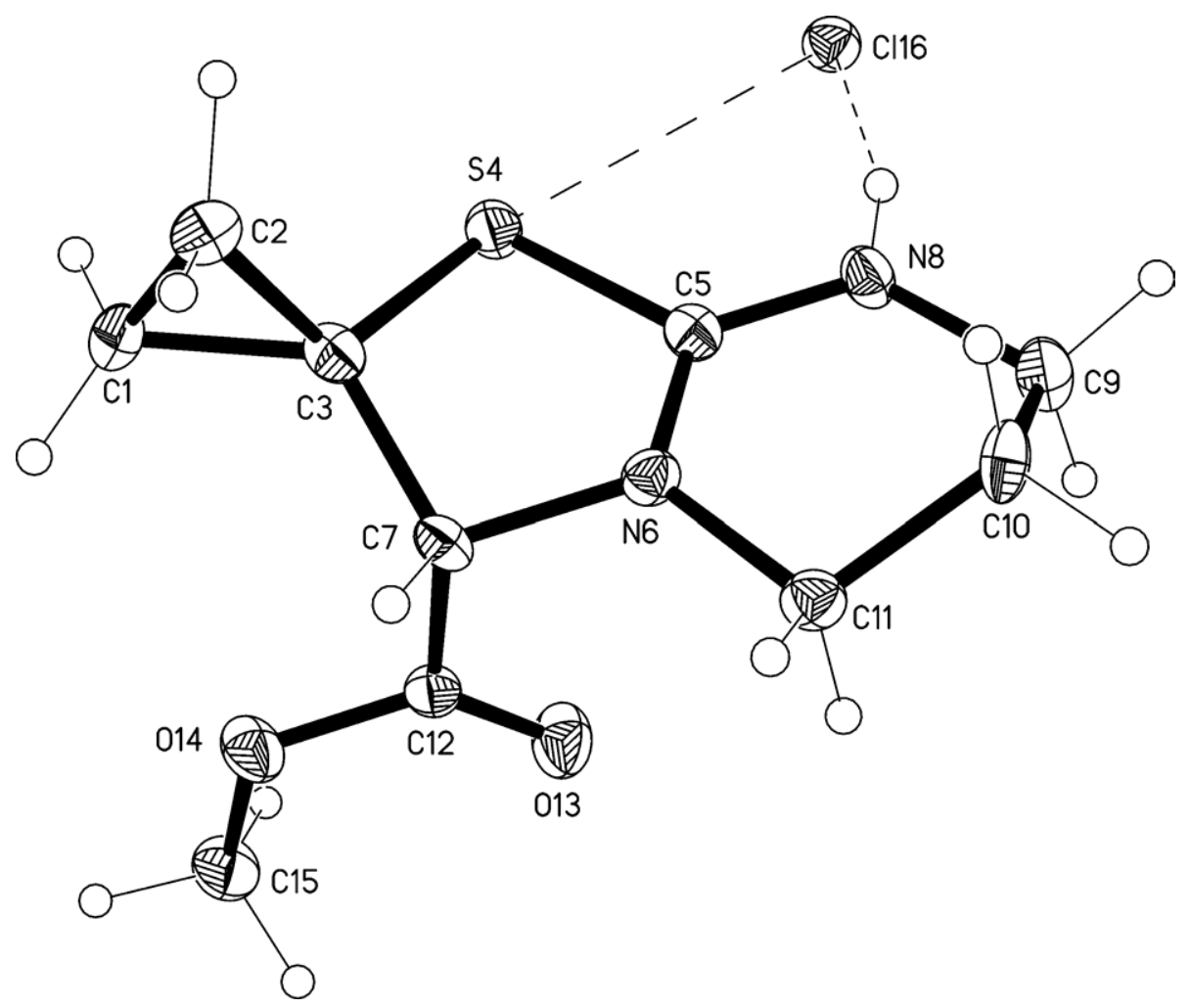

Figure. 7. Molecular structure of $\mathbf{7 4 b}$ in crystal. 


\subsection{Sequential Addition of Grignard Reagents and Aldehydes to Methyl 2-chloro-2- cyclopropylideneacetate}

The smooth bromine-magnesium exchange on Michael-acceptors like tert-butyl bromocrotonate and even $\beta, \beta$-disubstituted $\alpha$-bromoacrylonitriles with isopropylmagnesium halides as reported by Knochel et al., ${ }^{[94]}$ led to functionalised alkenylmagnesium halides which cleanly reacted with various electrophilies. In an attempt to adopt these conditions for the conversion of the very reactive Michael acceptor methyl 2-chloro-2-cyclopropylideneacetate (5) to the corresponding methyl 2-cyclopropylidene-2-(chloro-magnesuim)acetate, treatment of 5 with one equivalent of iPrMgCl at $0{ }^{\circ} \mathrm{C}$ apparently did not lead to the insertion of the metal into the carbon-chlorine bond, but to 1,4-addition of the Grignard reagent on to the $\alpha, \beta$-unsaturated ester. Upon trapping of the magnesium enolate with benzaldehyde, the Michael-aldol product 75a-iPr was isolated in very good yield (92\%, Table 17) and as a single diastereomer ( $>97 \%$ anti $\left.2 S^{*}, 3 R^{*}\right)$ as indicated by the ${ }^{1} \mathrm{H}-\mathrm{NMR}$ spectrum of the crude product. The scope of this reaction sequence of $\mathbf{5}$ was screened varying the Grignard reagents and the aldehydes to lead to the corresponding $\beta$-chloroalcohols $75 \mathbf{a}-\mathbf{l}-\mathrm{R}^{1}$ in moderate to excellent yield (27-92\%, Scheme 33, Table 17), all of which could be purified by column chromatography. The same diastereoselectivity was observed for the reactions with cyclohexyl- and ethylmagnesium bromide, albeit the yields were poor (27 and 32\%, respectively).

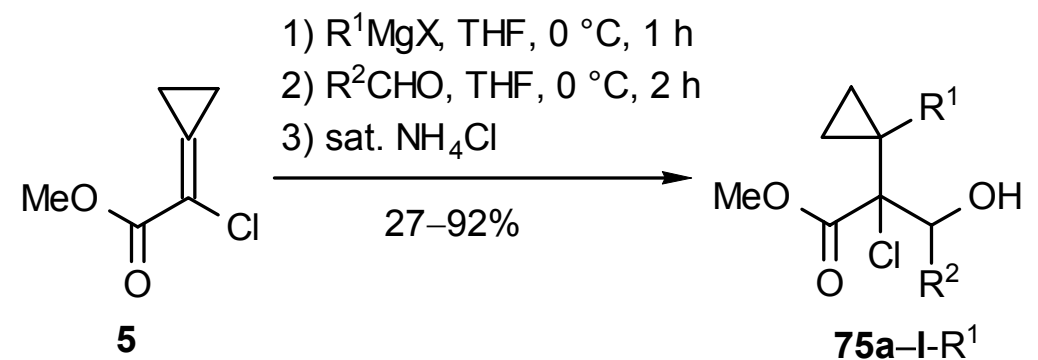

Scheme 33. Addition of Grignard reagents to methyl 2-chloro-cyclopropylideneacetate (5) and trapping of the formed magnesium enolate with aldehydes.

However, the reaction with phenyl- and $p$-fluorophenyl-magnesium chloride did not proceed as cleanly as those with isopropylmagnesium chloride, albeit the product could be purified by crystallization followed by column chromatography (Table 1, entries 4, 6). The reactions of 
vinylmagnesium bromide and phenylethynylmagnesium chloride led to an unidentified mixture of products and a $60 \%$ yield of 1,3-diphenylprop-2-ynol, respectively, the latter resulting from a direct attack of the Grignard reagent on benzyldehyde. Mesitylmagnesium bromide did not react cleanly with 5 to yield the Michael adduct, most probably due to its steric bulk. Upon addition of 1 equiv. of cuprous cyanide to the reaction mixture from $\mathbf{5}$ and vinylmagnesium bromide prior to the addition of benzaldehyde, the Michael-aldol adduct 75-Vin was formed, but as a mixture of antiand syn-diastereomers (69:31). The same decreased diastereoselectivity was observed in the reaction of 5 with $\mathrm{PhMgCl}$ and $\mathrm{PhCHO}$ in the presence of $\mathrm{CuCN}$ (entries 8 and 5). Since compound $\mathbf{7 5 b} \mathbf{b}$ - $\operatorname{Pr}$ readily crystallized, the relative configurations of the quaternary and the tertiary stereogenic center could be deduced from a single crystal X-ray structure analysis (Figure 7) to correspond to the outcome of an anti-diastereoselective aldol reaction in the second step.

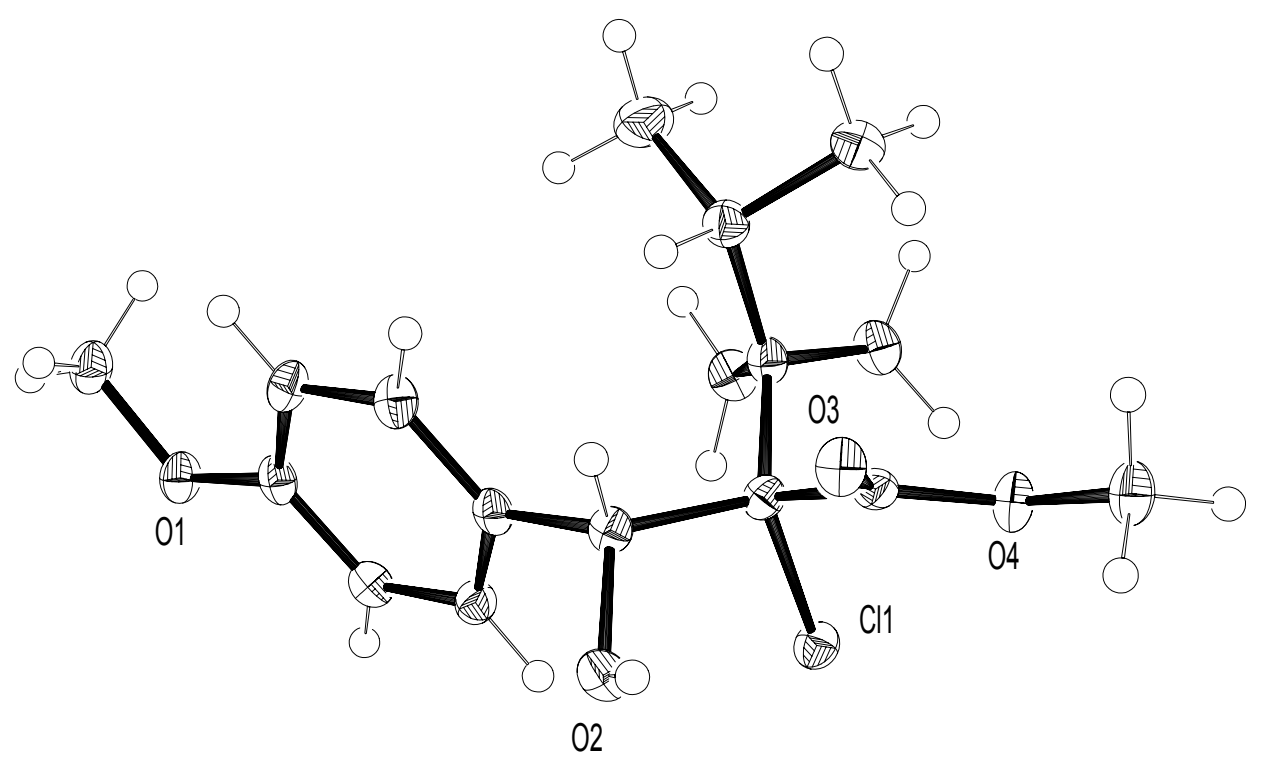

Figure 7. Structure of compound $\mathbf{7 5 b} \mathbf{b}$-iPr in the crystal indicating a $\left(2 S^{*}, 3 R^{*}\right)$-configuration corresponding to an anti-aldol. 
Table 17. Addition of organomagnesium halides to methyl 2-chloro-cyclopropylideneacetate (17) and trapping of the formed magnesium enolate with aldehydes

\begin{tabular}{|c|c|c|c|c|c|}
\hline Entry & $\mathrm{R}^{1}$ & $X$ & $\mathrm{R}^{2}$ & Product & $d\left((\%)^{\mathrm{a}}[\right.$ d.r. $\left.]\right)$ \\
\hline 1 & iPr & $\mathrm{Cl}$ & $\mathrm{Ph}$ & 75a-iPr & $92[>97: 3]$ \\
\hline 2 & cHex & $\mathrm{Br}$ & $\mathrm{Ph}$ & 75a-cHex & $27[>97: 3]$ \\
\hline 3 & $\mathrm{Et}$ & $\mathrm{Br}$ & $\mathrm{Ph}$ & 75a-Et & $32[>97: 3]$ \\
\hline 4 & $\mathrm{Ph}$ & $\mathrm{Cl}$ & $\mathrm{Ph}$ & 75a-Ph & $42[>97: 3]$ \\
\hline 5 & $\mathrm{Ph}$ & $\mathrm{Cl}$ & $\mathrm{Ph}$ & 75a-Ph & $35^{\mathrm{b}}[66: 34]$ \\
\hline 6 & $4-\mathrm{F}-\mathrm{C}_{6} \mathrm{H}_{4}$ & $\mathrm{Cl}$ & $\mathrm{Ph}$ & 75a-4- $\mathrm{FC}_{6} \mathrm{H}_{4}$ & $39[>97: 3]$ \\
\hline 7 & Vin & $\mathrm{Br}$ & $\mathrm{Ph}$ & 75a-Vin & $-^{\mathrm{c}}$ \\
\hline 8 & Vin & $\mathrm{Br}$ & $\mathrm{Ph}$ & 75a-Vin & $64^{\mathrm{b}}[69: 31]$ \\
\hline 9 & $\mathrm{PhC} \equiv \mathrm{C}$ & $\mathrm{Cl}$ & $\mathrm{Ph}$ & 75a-PhC $\equiv \mathrm{C}$ & $-^{\mathrm{d}}$ \\
\hline 10 & $2,4,6-\mathrm{Me}_{3}-\mathrm{C}_{6} \mathrm{H}_{2}$ & $\mathrm{Br}$ & $\mathrm{Ph}$ & 75a-2,4,6- $\mathrm{Me}_{3} \mathrm{C}_{6} \mathrm{H}_{2}$ & $0^{\mathrm{c}}$ \\
\hline 11 & $\mathrm{iPr}$ & $\mathrm{Cl}$ & $4-\mathrm{OMeC}_{6} \mathrm{H}_{4}$ & 75b-iPr & $72[>97: 3]$ \\
\hline 12 & $\mathrm{iPr}$ & $\mathrm{Cl}$ & $3,4-\left(\mathrm{OCH}_{2} \mathrm{O}\right) \mathrm{C}_{6} \mathrm{H}_{3}$ & 75c-iPr & $78[>97: 3]$ \\
\hline 13 & $\mathrm{iPr}$ & $\mathrm{Cl}$ & $4-\mathrm{NMe}_{2} \mathrm{C}_{6} \mathrm{H}_{4}$ & 75d-iPr & $80[>97: 3]$ \\
\hline 14 & $\mathrm{iPr}$ & $\mathrm{Cl}$ & $3,4,5-(\mathrm{OMe}){ }_{3} \mathrm{C}_{6} \mathrm{H}_{2}$ & 75e-iPr & $72[>97: 3]$ \\
\hline 15 & iPr & $\mathrm{Cl}$ & $\mathrm{C}_{6} \mathrm{~F}_{5}$ & 75f-iPr & $78[>97: 3]$ \\
\hline 16 & iPr & $\mathrm{Cl}$ & $2-\mathrm{F}-4-\mathrm{NO}_{2} \mathrm{C}_{6} \mathrm{H}_{3}$ & 75g-iPr & $83[>97: 3]$ \\
\hline 17 & iPr & $\mathrm{Cl}$ & $4-\mathrm{FC}_{6} \mathrm{H}_{4}$ & 75h-iPr & $80[>97: 3]$ \\
\hline 18 & iPr & $\mathrm{Cl}$ & $4-\mathrm{NO}_{2} \mathrm{C}_{6} \mathrm{H}_{4}$ & 75i-iPr & $73[>97: 3]$ \\
\hline 19 & $\mathrm{iPr}$ & $\mathrm{Cl}$ & $\mathrm{H}$ & 75j-iPr & $61^{\mathrm{e}}[>97: 3]$ \\
\hline 20 & iPr & $\mathrm{Cl}$ & $\mathrm{CO}_{2} \mathrm{Et}$ & 75k-iPr & $72[>97: 3]$ \\
\hline 21 & iPr & $\mathrm{Cl}$ & $\mathrm{C}_{3} \mathrm{H}_{7}$ & 75l-iPr & $32^{\mathrm{e}}[>97: 3]$ \\
\hline
\end{tabular}

${ }^{\mathrm{a}}$ Stoichiometry used normally: $\mathbf{5}: \mathrm{R}^{1} \mathrm{MgX}: \mathrm{R}^{2} \mathrm{CHO}=1: 1: 1.05$; isolated yields.

${ }^{\mathrm{b}} 1$ Equivalent of $\mathrm{CuCN}$ was added prior to the aldehyde.

${ }^{\mathrm{c}}$ Complicated mixture of unidentified products.

${ }^{\mathrm{d}}$ Only 1,3-diphenylprop-2-ynol was isolated in 60\% yield.

e 5 Equivalents each of paraformaldehyde and butyraldehyde were used. 
As far as the aldehydes were concerned, various substituted benzaldehydes were screened and found to give consistently good yields (72-83\%) independent of their substituents and substitution pattern (Table 17, entries 11-18). In case of aliphatic aldehyde five equiv. each of paraformaldehyde and butyraldehyde was used to complete the reaction although the yields were only 61 and 32\% respectively (Table 17, entries 19,21). Another interesting aldehyde, ethylglyoxylate reacted in the general way to give the corresponding product in $72 \%$ yield.

Comparable processes with trialkylboranes ${ }^{[95]}$ yielded mixtures of syn- and anti-diastereomers (20:80 to $70: 30$, in the best cases) in good chemical yields but with the waste of two organyl residues from the borane. The extraordinarily high diastereoselectivity upon the addition of the enolate formed upon reaction of isopropylmagnesium chloride with $\mathbf{5}$ and benzaldehyde may be rationalized with the six-membered transition structure, typical for aldol additions, in which the bulky alkyl residue containing the 1,1-disubstituted cyclopropane ring, prefers an equatorial as in 76 rather than an axial position as in 77 (Scheme 34).

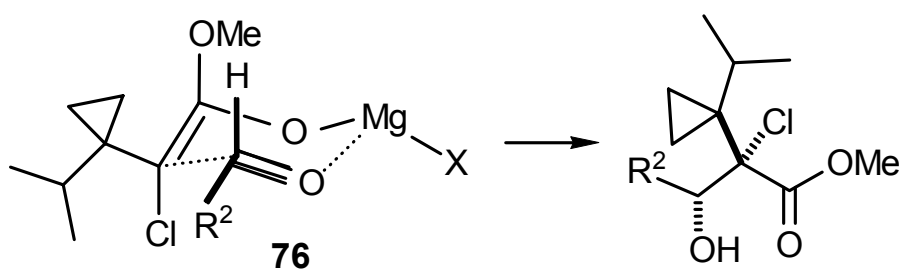

anti-75-iPr
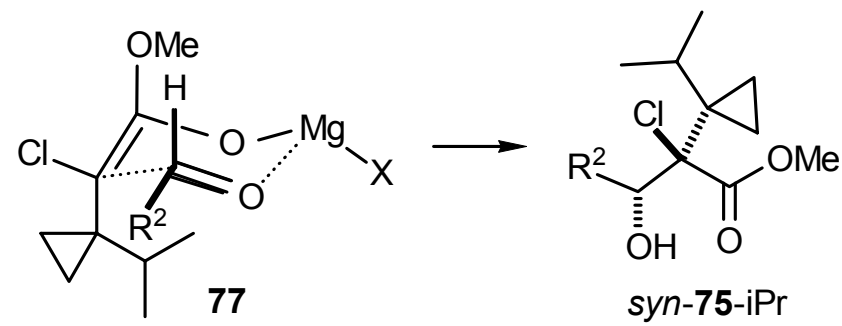

syn-75-iPr

Scheme 34. Rationalization of the selective formation of the anti-aldols in the sequential addition of iPrMgCl and aldehydes to $\mathbf{5}$.

The Michael adduct 78-iPr of isopropylmagnesium chloride to $\mathbf{5}$ was obtained in virtually quantitative yield (98\%), upon quenching the reaction before adding an aldehyde, after $30 \mathrm{~min}$ with aqueous ammonium chloride solution. The corresponding adduct 78 -Vin of vinylmagnesium bromide could also be obtained in $80 \%$ yield by adding 5 to the Grignard reagent in the presence of one equiv. of cuprous cyanide, and 0.5 equiv. of $\mathrm{BF}_{3}{ }^{\circ} \mathrm{OEt}_{2}$ at $-78{ }^{\circ} \mathrm{C}$, followed by aqueous 
work-up. The adduct 78-iPr was also obtained as the sole product when benzonitrile was added to the enolate formed from isopropylmagnesium bromide and $\mathbf{5}$.

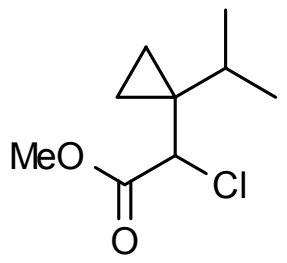

78-iPr

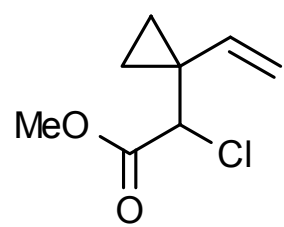

78-Vin

Figure 10. Structures of the Michael adduct of 5 with Grignard reagents.

The $\alpha$-chloro- $\beta$-hydroxy esters 2 which are - in most cases - diastereoselectively formed in this one-pot sequential Michael addition-aldol reaction from 5, isopropylmagnesium chloride and an aldehyde, offer themselves as precursors to $\alpha, \beta$-epoxy esters of type 79 which can also be made by a Darzens reaction. In fact, when 75-iPr in tetrahydrofuran was treated at ambient temperature with potassium tert-butoxide, it underwent clean cyclization to the diastereomerically pure $\alpha, \beta$ epoxyester 79-iPr with the aryl and the 1-isopropylcyclopropyl group on the same side of the oxirane ring.<smiles>COc1ccc([C@@H](O)[C@@](Cl)(C(C)C)C2(C(C)C)CC2)cc1</smiles>

75b-iPr

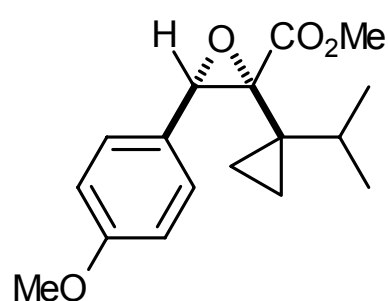

$79 b-\mathrm{iPr}$

Scheme 35. Diastereoselective formation of the Darzen-type $\alpha, \beta$-epoxy ester 79b-iPr.

The analogous $\alpha, \beta$-epoxy esters 79a-iPr was obtained directly from methyl 2-bromo-2cyclopropylideneacetate (4-Me) upen treatment with isopropylmagnesium chloride and benzaldehyde in 96\% yield (Scheme 36). Apparently the bromohydrine analogous to 75a-iPr is not stable under the work-up condition and undergoes cyclisation by intramolecular nucleophilic displacement even in the ammonium chloride-buffered medium. 


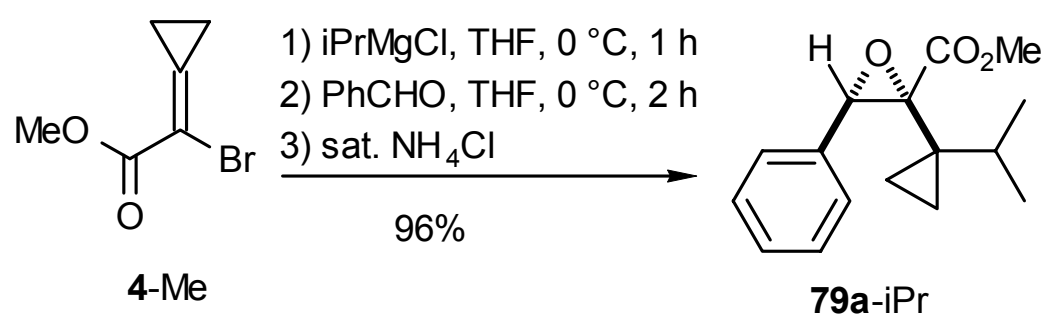

Scheme 36. Diastereoselective formation of the Darzen-type $\alpha, \beta$-epoxy ester 79a-iPr directly from 5. 


\subsection{Synthesis of 6-amino-3-azabicyclo[3.1.0]hexane}

It has already noted before that Trovafloxacin $(\mathbf{1 1})^{[11]}$ which has a potent activity against Gramnegative, Gram-positive and anaerobic bacteria contains a 3-azabicyclo[3.1.0]hex-6-yl amine substituent on C-7 of the naphthyridinon moiety. Very recently 3,6-disubstituted azabicyclo[3.1.0] hexyl amine derivatives has reported to be muscarinic receptor antagonist. ${ }^{[96]}$ In view of the biological activity of the compound containing 3-azabicyclo[3.1.0]hex-6-yl amine it was interesting to synthesize this amine as well as other orthogonally protected derivative of it and find out a condition for the nucleophilic substitution with aryl halides. Recently Kulinkovich has developed a method in which a titanacyclopropane intermediate has been used for the synthesis of cyclopropanol from ester. ${ }^{[8]} \mathrm{A}$ analogous method has been developed by de Meijere for the synthesis of cyclopropyl amine from ester. ${ }^{[9]}$ In the mean time Helmchen et al. ${ }^{[97]}$ has described an efficient synthesis of 1-Boc-2,5-dihydro-1H-pyrrole (81) by protection reaction of dially amine followed by ring closing metathesis using Grubbs first generation catalyst. ${ }^{[98]}$ Initial attempts to prepare (81) from Boc-diallyl amine as described by Helmchen was not successful. The reaction did not come to end and only $30 \%$ of starting material was converted to product even if after stirring for $24 \mathrm{~h}$ and increasing the catalyst loading to $2 \mathrm{~mol} \%$. This problem was solved by distilling the starting material and storing it in the presence small amount of molecular sieb prior to the metathesis reaction and by using glove box for transferring the catalyst. In this way the reaction was successful and gave the product in $99 \%$ yield even in large scale $(1.1 \mathrm{~mol})$ and with the use of only $0.1 \mathrm{~mol} \%$ of catalyst. The orthogonally protected diamine exo-6-(N,Ndibenzylamino)-3-aza-bicyclo[3,1,0]hexane-3-carboxylic acid tert-butyl ester (82) was prepared from $(\mathbf{8 1})$ in $71 \%$ yield (270 g, from $1 \mathrm{~mol}$ of starting material). Debenzylation of 82 by using $\mathrm{Pd} / \mathrm{C}$ in methanol gave the mono-Boc protected diamine (84). It must be noted that for the debenzylation reaction $\mathrm{Pd} / \mathrm{C}$ purchased from the Merck was the best. The same reaction carried out by using Pd/C from Aldrich, Fluka or Lancaster did not go to the end even after 4 days of stirring. The reaction of 82 with $2 \mathrm{~N} \mathrm{HCl}$ in EtOAc resulted in deprotection of Boc group and gave the hydrochloride salt of $\mathbf{8 3}$ from which the free amine $\mathbf{8 3}$ was liberated in $98 \%$ by treatment with $10 \mathrm{~N}$ aq. $\mathrm{NaOH}$ solution. These results are given in Scheme 37. 


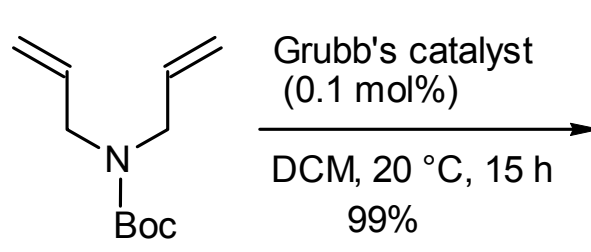

80

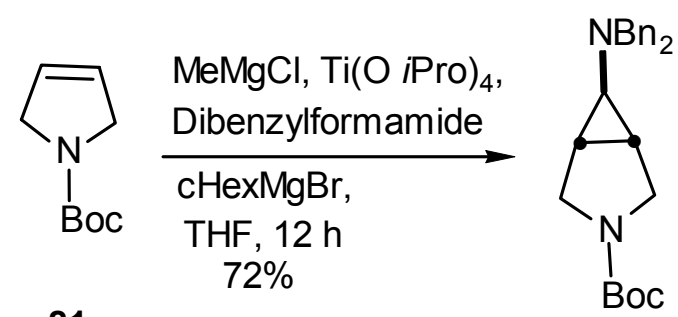

81

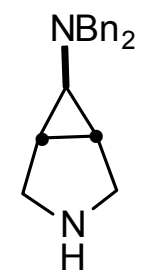

83
82

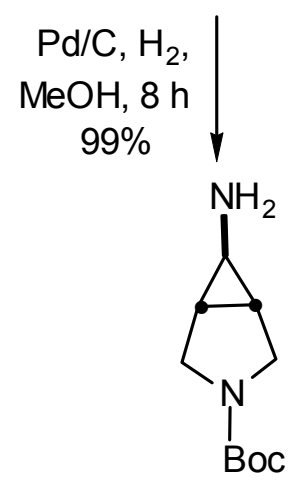

84

Scheme 37. Synthesis of exo-6-( $N, N$-dibenzylamino)-3-aza-bicyclo[3,1,0]hexane-3-carboxylic acid tert-butyl ester (82) and mono-deprotected products 83 and 84.

Since the protecting group play and important role in organic synthesis ${ }^{[99]}$ it was interesting to change the protection of the primary amine group from dibenzyl group to benzyloxy carbonyl group ( $\mathrm{Z}$ group). When a solution of $\mathbf{8 4}$ in acetone was treated with 1.1 equiv. of $\mathrm{ZOSu}$ in presence of $1 \mathrm{~N}$ aq. $\mathrm{NaHCO}_{3}$ the orthogonally protected product 85 was obtained in $85 \%$ of yield. Deprotection of the Boc group was achieved by using the same method described for compound 83. These results are summarized in Scheme 38.<smiles>NC1C2CN(C(=O)OCc3ccccc3)CC12</smiles>

84<smiles>[Z]NC1[C@H]2CN(C(=O)OCc3ccccc3)C[C@H]12</smiles>

85
1) $2 \mathrm{~N} \mathrm{HCl}$ in EtOAc $20^{\circ} \mathrm{C}, 15 \mathrm{~h}$

2) $10 \mathrm{~N} \mathrm{NaOH}$ $98 \%$<smiles>[Z]NC1C2CNCC21</smiles>

86

Scheme 38. Protection of primary and deprotection of secondary anine group. 
Since aromatic amines plays an important role in many areas including pharmaceutical, agrochemicals, photography and electronic materials ${ }^{[100]}$ it was interesting to study the reactivity towards the nucleophilic substitution of benzyl iodide with the free amine 83. By adopting the palladium-catalyzed amination protocol developed by Buchwald ${ }^{[91 \mathrm{a}]}$ by using $10 \mathrm{~mol} \% \mathrm{Pd}_{2}(\mathrm{dba})_{3}$, $30 \mathrm{~mol} \% \pm \mathrm{BINAP}$ in the presence of $\mathrm{Na} t \mathrm{OBu}$ in a solution of toluene the product $(\mathbf{8})$ was isolated only in 35\% yield. Recently Buchwald et al. has reported a Copper-catalyzed amination of alkyl amine with aryl iodide in the presence of $\mathrm{K}_{3} \mathrm{PO}_{4}$ and ethylene glycol. ${ }^{[101]} \mathrm{By}$ using this method when 2.1 equiv. of benzyl iodide was reacted with $\mathbf{8 3}$ in the presence of $10 \mathrm{~mol} \%$ of $\mathrm{CuI}$ in a solvent isopropanol at $80{ }^{\circ} \mathrm{C}$ for $24 \mathrm{~h}$ the compound 87 was isolated in $73 \%$ yield. It is noted that the product 87 is very sensitive towards silica gel but could be purified by crystallized from methanol. The attempted debenzylation of 87 with $\mathrm{Pd} / \mathrm{C}, \mathrm{H}_{2}$ in methanol did not give any product even after $48 \mathrm{~h}$, instead, only starting material and 30\% of product corresponding to the monodebenzylation reaction was identified by NMR spectra. By changing the solvent to $\mathrm{MeOH} / \mathrm{AcOH}$ $(20: 1)$ or $\mathrm{MeOH} / 6 \mathrm{~N} \mathrm{HCl}$ in isopropanol did not give any product. The reaction also failed by changing the catalyst to $\mathrm{Pd}(\mathrm{OH})_{2} / \mathrm{C}$ in ethyl acetate as solvent. Finally by changing the solvent to dimethyl acetamide the product $\mathbf{8 8}$ was isolated in $90 \%$ yield after $48 \mathrm{~h}$ by using $\mathrm{Pd} / \mathrm{C}$ as catalyst. These results are presented in Scheme 39.

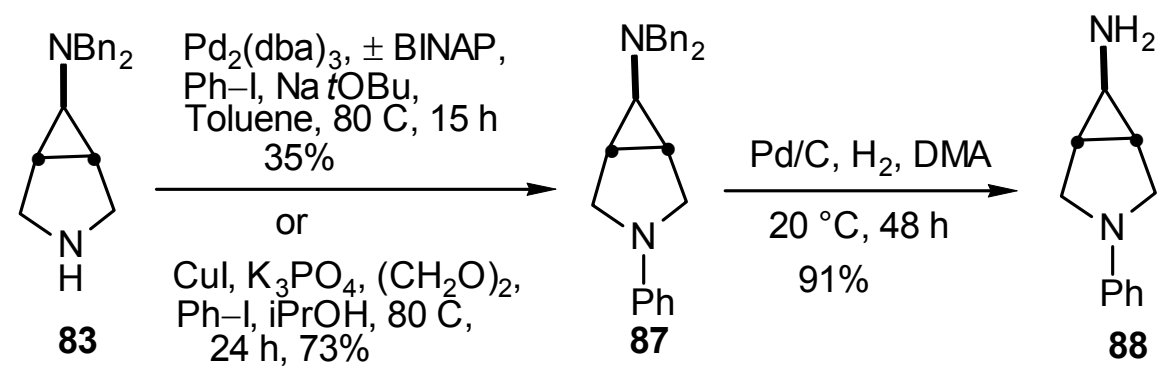

Scheme 39. Metel-catalyzed amination of aryl iodide with $\mathbf{8 3}$. 


\section{Experimental Part}

\subsection{General Remarks}

All reagents were used as purchased from commercial suppliers without further purification. All reactions in non-aq. solvents were carried out using standard Schlenk techniques under an atmosphere of dry nitrogen. Solvents were purified and dried according to conventional methods prior to use; diethyl ether $\left(\mathrm{Et}_{2} \mathrm{O}\right)$ and tetrahydrofuran (THF) were freshly distilled from sodium/benzophenone. - Solvents are abbreviated as follows: EtOAc $=$ ethyl acetate, MEK $=$ ethyl methyl ketone, $\mathrm{MeOH}=$ methanol, $\mathrm{EtOH}=$ ethanol, $\mathrm{DCM}=$ dichloromethane, $\mathrm{AcOH}=$ acetic acid, DMF $=$ dimethyl formamide. $-{ }^{1} \mathrm{H}$ - and ${ }^{13} \mathrm{C}$-NMR spectra were recorded at ambient temperature with a Bruker AM $250\left(250 \mathrm{MHz}\right.$ for ${ }^{1} \mathrm{H}$ and $62.9 \mathrm{MHz}$ for $\left.{ }^{13} \mathrm{C}\right)$, a Varian UNITY-200 $\left(200 \mathrm{MHz}\right.$ for ${ }^{1} \mathrm{H}$ and $50.3 \mathrm{MHz}$ for ${ }^{13} \mathrm{C}$ ) or a Varian UNITY-300 $\left(300 \mathrm{MHz}\right.$ for ${ }^{1} \mathrm{H}$ and $75.5 \mathrm{MHz}$ for ${ }^{13} \mathrm{C}$ ) instrument. Chemical shifts $\delta$ are given in ppm relative to resonances of solvent $\left({ }^{1} \mathrm{H}: 7.26\right.$ ppm for $\mathrm{CHCl}_{3} ;{ }^{13} \mathrm{C}: 77.0 \mathrm{ppm}$ for $\mathrm{CDCl}_{3}$ ), coupling constants $J$ are given in Hertz. Characterization of the multiplicity of signals: $\mathrm{s}=$ singlet, $\mathrm{br} \mathrm{s}=$ broad singlet, $\mathrm{d}=$ doublet, $\mathrm{t}=$ triplet, $\mathrm{m}=$ multiplet. - The multiplicities of signals were determined by the DEPT or the APT technique: DEPT: $+=$ primary or tertiary (positive DEPT-signal),$-=$ secondary (negative DEPTsignal), $\mathrm{C}_{\text {quat }}=$ quaternary $\mathrm{C}$-atom; $\mathrm{APT}:+=$ primary or tertiary (positive APT-signal),$-=$ secondary or quaternary C-atom (negative APT-signal). - IR: Bruker IFS 66. - MS: Finnigan MAT 95, $70 \mathrm{eV}$. - HRMS: Finnigan MAT 95 using preselected ion peak matching at $\mathrm{R}=10000$ to be within \pm 2 ppm. - Chromatography: Separations were carried out on Merck Silica 60 (0.063-0.200 $\mathrm{mm}, 70-230$ mesh ASTM). The dimensions of the columns are given as "diameter $\times$ height of the silica column”. - TLC: Macherey-Nagel, TLC plates Alugram ${ }^{\circledR}$ Sil G/UV 254 . Detection under UVlight at $254 \mathrm{~nm}$, development with MOPS reagent (10\% molybdophosphoric acid, solution in ethanol). - Melting points: apparatus according to Dr. Tottoli (Büchi); values are uncorrected. Elemental analyses: Mikroanalytisches Laboratorium des Instituts für Organische und Biomolekulare Chemie der Universität Göttingen. 


\subsection{Synthesis of Compounds}

\subsubsection{Advanced synthesis of methyl 2-chloro- and 2-bromo-2-cyclopropylideneacetate}

Ethyl 3,3-diethoxypropionate (29): Into a two litre three necked round-bottomed flask equipped with magnetic stirring bar, reflux condenser, addition funnel and a thermometer, $400 \mathrm{~mL}$ of $\mathrm{CCl}_{4}$ was taken with $3.3 \mathrm{~g}(20 \mathrm{mmol}, 10 \mathrm{Mol} \%)$ of azobisisobutyronitrile. This solution was heated to $70{ }^{\circ} \mathrm{C}$ and Etyl vinyl ether (144 g, 2 Mol) was added over a period of $1 \mathrm{~h}$. After stirring for 3 hour at this temperature, at which<smiles>CCOC(=O)CC(OCC)OCC</smiles>
point the vapour temperature increased from $50{ }^{\circ} \mathrm{C}$ to $70{ }^{\circ} \mathrm{C}$, the reaction mixture was cooled down to $\mathrm{RT}$ and $400 \mathrm{~mL}$ of $\mathrm{EtOH}$ was added to it and the reaction mixture was refluxed for $12 \mathrm{~h}$, cooled down to RT, diluted with $500 \mathrm{~mL}$ of ether and washed with $\mathrm{H}_{2} \mathrm{O}(2 \times 300 \mathrm{~mL})$, sat. aq. $\mathrm{NaHCO}_{3}(2 \times 300 \mathrm{~mL})$, dried over $\mathrm{MgSO}_{4}$ and solvent removed in vacuo. The crude product was distilled at reduced pressure (b.p. $86-89{ }^{\circ} \mathrm{C}, 30 \mathrm{mbar}$ ) to yield $236 \mathrm{~g}(62 \%)$ of pure product as a colourless oil. Other physical properties are same with the literature. ${ }^{[99]}$

1-(2,2-Diethoxyethyl)cyclopropanol (31): To a solution of $190 \mathrm{~g}(1.0 \mathrm{~mol})$ of 29 in $1.5 \mathrm{~L}$ of $\mathrm{THF} / \mathrm{Et}_{2} \mathrm{O}(1: 1)$ was added $59.5 \mathrm{~mL}(200 \mathrm{mmol})$ of titanium(IV) isopropoxide at $0{ }^{\circ} \mathrm{C}$ and $1.25 \mathrm{~L}$ of $\mathrm{EtMgBr}\left(2.0 \mathrm{M}\right.$ solution in $\left.\mathrm{Et}_{2} \mathrm{O}, 2.50 \mathrm{~mol}\right)$ over $2 \mathrm{~h}$. The deep black solution was stirred at $20^{\circ} \mathrm{C}$ for $12 \mathrm{~h}$ then $1 \mathrm{~L}$ of $\mathrm{Et}_{2} \mathrm{O}$ was added and the reaction was quenched by slow addition of $200 \mathrm{~mL}$ of water at $0{ }^{\circ} \mathrm{C}$, and the

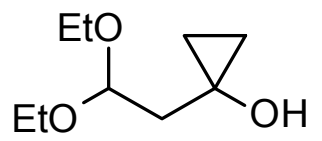
mixture stirred until the precipitation was complete. After filtration of the precipitate, the filtrate was dried over $\mathrm{MgSO}_{4}$ and then filtered over Celite ${ }^{\circledR}$. Removal of the solvent in vacuo (water bath $<40{ }^{\circ} \mathrm{C}$ ) yielded $171 \mathrm{~g}(96 \%)$ of $\mathbf{1 7}$ as a slightly yellow liquid. - IR (film): $v=3420 \mathrm{~cm}^{-1}, 2938$, 2835, 1717, 1700, 1457, 1387, 1192, 1122, 1053. $-{ }^{1} \mathrm{H}-\mathrm{NMR}\left(\mathrm{CDCl}_{3}, 250 \mathrm{MHz}\right): \delta=0.41-0.44$ (m, $\left.2 \mathrm{H}, \mathrm{cPr}-\mathrm{CH}_{2}\right), 0.73-80\left(\mathrm{~m}, 2 \mathrm{H}, \mathrm{cPr}-\mathrm{CH}_{2}\right), 1.21$ (t, $\left.{ }^{3} J=7.3 \mathrm{~Hz}, 6 \mathrm{H}, 2 \mathrm{CH}_{3}\right), 1.85$ (d, $\left.{ }^{3} J=7.2 \mathrm{~Hz}, 2 \mathrm{H}, \mathrm{CH}_{2}\right), 3.42-3.78\left(\mathrm{~m}, 5 \mathrm{H}, 2 \mathrm{CH}_{2}+\mathrm{OH}\right), 4.77\left(\mathrm{t},{ }^{3} \mathrm{~J}=7.2 \mathrm{~Hz}, 1 \mathrm{H}, \mathrm{CH}\right) .-{ }^{13} \mathrm{C}-$ NMR $\left(\mathrm{CDCl}_{3}, 62.9 \mathrm{MHz}, \mathrm{DEPT}\right): \delta=12.4$ (-, $\left.2 \mathrm{C}, \mathrm{cPr}-\mathrm{C}\right), 15.3\left(+, 2 \mathrm{C}, \mathrm{CH}_{3}\right), 40.8\left(-, \mathrm{CH}_{2}\right), 53.1$ $\left(\mathrm{C}_{\text {quat }}, \mathrm{cPr}-\mathrm{C}\right), 61.7\left(-, 2 \mathrm{C}, \mathrm{OCH}_{2}\right), 102.9(+, \mathrm{CH}) .-\mathrm{MS}(\mathrm{EI}, 70 \mathrm{eV}), \mathrm{m} / \mathrm{z}(\%): 129$ (6), 128 (10), 103 (49), 83 (92), 75 (48), 55 (63), 47 (100). $-\mathrm{C}_{9} \mathrm{H}_{18} \mathrm{O}_{3}$ (174.2): calcd. C 62.04, $\mathrm{H}$ 10.41, found: $\mathrm{C}$ $61.79, \mathrm{H} 10.38$. 
2-(1'-Mesyloxycyclopropyl)acetic acid (28). To a solution of $171 \mathrm{~g}(955 \mathrm{mmol})$ of 31 in $1.5 \mathrm{~L}$ of DCM were added $121 \mathrm{~g}(1.2 \mathrm{~mol})$ of triethyl-amine, $6.10 \mathrm{~g}(50 \mathrm{mmol})$ of DMAP and slowly within $30 \mathrm{~min} 120 \mathrm{~g}(1.05 \mathrm{mmol})$ of mesyl chloride. The solution was stirred for $8 \mathrm{~h}$ at $20^{\circ} \mathrm{C}$, extracted with $1 \mathrm{~N} \mathrm{HCl}(1 \times 500 \mathrm{~mL})$ and water $(1 \times 500 \mathrm{~mL})$. Drying over $\mathrm{Na}_{2} \mathrm{SO}_{4}$ yielded the raw mesylate as brown oil,

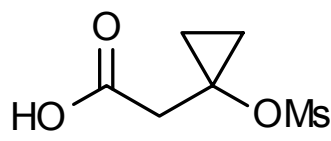
which was dissolved in $1.20 \mathrm{~L}$ of $\mathrm{THF} / \mathrm{H}_{2} \mathrm{O}(1: 2)$. To this solution was added $922 \mathrm{~g}$ (1.50 mol) of Oxone ${ }^{\circledR}$ and the suspension was stirred at $20^{\circ} \mathrm{C}$ for $18 \mathrm{~h}$. Water $(1 \mathrm{~L})$ was added and the aq. phase was extracted with ethyl acetate $(3 \times 1 \mathrm{~L})$. Drying over $\mathrm{MgSO}_{4}$, evaporation of the solvent and recrystallization of the residue from $\mathrm{Et}_{2} \mathrm{O}$ yielded $108 \mathrm{~g}(58 \%)$ of 28 as colorless crystals, m. p. $=96-98^{\circ} \mathrm{C}$. - IR (film): $v=3032 \mathrm{~cm}^{-1}, 1710,1424,1408,1331,1265,1230,1184,1155$, 942, 907. $-{ }^{1} \mathrm{H}-\mathrm{NMR}\left(\mathrm{CDCl}_{3}, 250 \mathrm{MHz}\right): \delta=0.90-0.96\left(\mathrm{~m}, 2 \mathrm{H}, \mathrm{cPr}^{-\mathrm{CH}_{2}}\right), 1.39-1.47(\mathrm{~m}, 2 \mathrm{H}$, cPr- $\mathrm{CH}_{2}$ ), 2.91 (s, $2 \mathrm{H}, \mathrm{CH}_{2}$ ), 3.05 (s, $3 \mathrm{H}, \mathrm{CH}_{3}$ ), 10.85-11.23 (br. s., $1 \mathrm{H}, \mathrm{CO}_{2} \mathrm{H}$ ). $-{ }^{13} \mathrm{C}-\mathrm{NMR}$ $\left(\mathrm{CDCl}_{3}, 50.3 \mathrm{MHz}, \mathrm{APT}\right): \delta=12.5(-, 2 \mathrm{C}, \mathrm{cPr}-\mathrm{C}), 39.9\left(+, \mathrm{CH}_{3}\right), 41.7\left(-, \mathrm{CH}_{2}\right), 63.6\left(\mathrm{C}_{\text {quat }}, \mathrm{cPr}-\right.$ C), $173.6\left(\mathrm{C}_{\text {quat }}, \mathrm{C}=\mathrm{O}\right) .-\mathrm{MS}(\mathrm{DCI}, 70 \mathrm{eV}), \mathrm{m} / z(\%): 406$ (3) $\left[2 \mathrm{M}^{+}+\mathrm{NH}_{4}{ }^{+}\right], 212$ (100) $\left[\mathrm{M}^{+}+\mathrm{NH}_{4}^{+}\right] .-\mathrm{C}_{6} \mathrm{H}_{10} \mathrm{O}_{5} \mathrm{~S}$ (194.2): calcd. C 37.11, H 5.19, found: C 37.39, H 4.96.

Methyl 2-chloro(1-methanesulfonyloxycyclopropyl)acetate (32a): To a solution of $108 \mathrm{~g}$ $(557 \mathrm{mmol})$ of 28 in $1.5 \mathrm{~L}$ of 1,2-dichloroethane was added $51.3 \mathrm{~mL}(696 \mathrm{mmol})$ of thionyl chloride, and the solution was heated under reflux for $60 \mathrm{~min}$. At room temperature were added $111 \mathrm{~g}(836 \mathrm{mmol})$ of NCS and $1 \mathrm{~mL}$ of conc. aq. $\mathrm{HCl}$, and the solution was heated at $90{ }^{\circ} \mathrm{C}$ for $12 \mathrm{~h}$. At $20^{\circ} \mathrm{C} \mathrm{MeOH}$ $(100 \mathrm{~mL})$ was added, and the solution stirred at this temperature for an

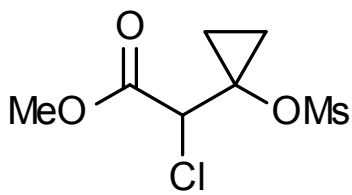
additional $1 \mathrm{~h}$. The solvent was removed in vacuo, the residue suspended in cold $\left(0^{\circ} \mathrm{C}\right)$ tetrachloromethane, and the mixture was filtered to remove succinamide. Removal of the solvent afforded $130.1 \mathrm{~g}(97 \%)$ of 32a as yellow oil, which was 90\% pure by NMR and used in the next step without further purification. An analytical sample was purified on $100 \mathrm{~g}$ of silica gel (column, $4 \times 20 \mathrm{~cm}, \mathrm{PE} / \mathrm{Et}_{2} \mathrm{O}=3: 1 ; R_{\mathrm{f}}=0.16$ ). - IR (film): $v=3045 \mathrm{~cm}^{-1}, 2830,1717,1456,1424,1408$, 1329, 1175, 987, 935, 882. ${ }^{1} \mathrm{H}-\mathrm{NMR}\left(\mathrm{CDCl}_{3}, 250 \mathrm{MHz}\right): \delta=0.84\left(\mathrm{~m}, 2 \mathrm{H}, \mathrm{cPr}-\mathrm{CH}_{2}\right), 1.33(\mathrm{~m}$, $\left.2 \mathrm{H}, \mathrm{cPr}-\mathrm{CH}_{2}\right), 3.01$ (s, $\left.3 \mathrm{H}, \mathrm{CH}_{3}\right), 3.77$ (s, $\left.3 \mathrm{H}, \mathrm{OCH}_{3}\right), 4.58$ (s, $\left.1 \mathrm{H}, \mathrm{Cl}-\mathrm{CH}\right) .-{ }^{13} \mathrm{C}-\mathrm{NMR}\left(\mathrm{CDCl}_{3}\right.$, $62.9 \mathrm{MHz}): \delta=10.0(-, 2 \mathrm{C}, \mathrm{cPr}-\mathrm{C}), 39.3\left(+, \mathrm{CH}_{3}\right), 52.0\left(+, \mathrm{OCH}_{3}\right), 62.0\left(\mathrm{C}_{\text {quat }}, \mathrm{cPr}-\mathrm{C}\right), 63.2(+$, 
Cl-CH), $170.8\left(\mathrm{C}_{\text {quat }}, \mathrm{C}=\mathrm{O}\right) .-\mathrm{MS}(\mathrm{DCI}, 70 \mathrm{eV}), \mathrm{m} / z(\%): 244 / 242(2 / 7)\left[\mathrm{M}^{+}\right], 207$ (63) $\left[\mathrm{M}^{+}-\mathrm{Cl}\right]$, 129 (100), 99 (35), 97 (28). - $\mathrm{C}_{7} \mathrm{H}_{11} \mathrm{ClO}_{5} \mathrm{~S}$ (242.7): calcd. C 34.65, H 4.57, found: C 34.60, $\mathrm{H}$ 4.56 .

Methyl 2-chloro-2-cyclopropylideneacetate (5). To a solution of $130.1 \mathrm{~g}$ of the above crude 32a in $1 \mathrm{~L}$ of DCM kept at $0{ }^{\circ} \mathrm{C}$ was added $81 \mathrm{~g}(55.2 \mathrm{mmol})$ of triethylamine, and the solution was stirred at this temperature for $8 \mathrm{~h}$. DCM $(500 \mathrm{~mL})$ and aq. $1 \mathrm{~N} \mathrm{HCl}(3 \times 100 \mathrm{~mL})$ were added and the phases were separated. Drying of the organic phase over $\mathrm{MgSO}_{4}$ and removal of the solvent in vacuo yielded the crude product which

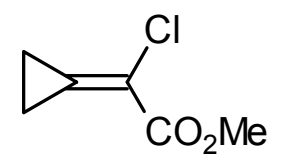
was bulb to bulb distilled at 1 torr with a dry-ice cooling bath, crystallized with $\mathrm{PE} / \mathrm{Et}_{2} \mathrm{O}(100 \mathrm{~mL})$ at $-20{ }^{\circ} \mathrm{C}$ to yield $58 \mathrm{~g}$ (77\% from 28$)$ of 5 as colorless crystals. $-{ }^{1} \mathrm{H}-\mathrm{NMR}\left(\mathrm{CDCl}_{3}, 250 \mathrm{MHz}\right)$ : $\delta=1.41-1.50\left(\mathrm{~m}, 2 \mathrm{H}, \mathrm{cPr}-\mathrm{CH}_{2}\right), 1.66-1.75\left(\mathrm{~m}, 2 \mathrm{H}, \mathrm{cPr}-\mathrm{CH}_{2}\right), 3.83\left(\mathrm{~s}, 3 \mathrm{H}, \mathrm{CH}_{3}\right) .-{ }^{13} \mathrm{C}-\mathrm{NMR}$ $\left(\mathrm{CDCl}_{3}, 62.9 \mathrm{MHz}\right): \delta=5.5$ (-, cPr-C), 9.7 (-, cPr-C), $52.9\left(+, \mathrm{CH}_{3}\right), 114.7\left(\mathrm{C}_{\text {quat }}, \mathrm{cPr}-\mathrm{C}\right), 139.1$ $\left(\mathrm{C}_{\text {quat }}, \mathrm{Cl}-\mathrm{C}\right), 162.7\left(\mathrm{C}_{\text {quat }}, \mathrm{C}=\mathrm{O}\right)$. The additional experimental data are identical to those reported in literature. ${ }^{[18]}$

Methyl 2-bromo(1-methanesulfonyloxycyclopropyl)acetate (32b). To a solution of $4.00 \mathrm{~g}$ (20.6 mmol) of 28 in $50 \mathrm{~mL}$ of DCE was added $1.82 \mathrm{~mL}(25.0 \mathrm{mmol})$ of thionyl chloride, and the solution was heated under reflux for $30 \mathrm{~min}$. At ambient temperature were added $4.44 \mathrm{~g}(25.0 \mathrm{mmol})$ of NBS and 4 drops of conc. aq. $\mathrm{HBr}$, and the solution was heated under reflux at $90^{\circ} \mathrm{C}$ for $4 \mathrm{~h} . \mathrm{MeOH}$ $(50 \mathrm{~mL})$ was added at $20{ }^{\circ} \mathrm{C}$, and the solution was stirred for an

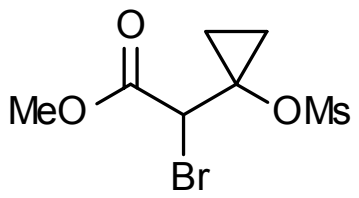
additional $1 \mathrm{~h}$. The solvents were removed in vacuo, the residue suspended in tetrachloromethane, and the mixture filtered. Removal of the solvent and chromatographic purification of the residue on $40 \mathrm{~g}$ of silica gel (column, $\left.3 \times 20 \mathrm{~cm}, \mathrm{PE} / \mathrm{Et}_{2} \mathrm{O}=3: 1 ; R_{\mathrm{f}}=0.19\right)$ afforded $2.98 \mathrm{~g}(53 \%)$ of $\mathbf{3 2 b}$ as colourless oil. - IR (film): $v=3031 \mathrm{~cm}^{-1}, 2944,1423,1366,1168 .-{ }^{1} \mathrm{H}-\mathrm{NMR}\left(\mathrm{CDCl}_{3}, 250 \mathrm{MHz}\right)$ : $\delta=0.82\left(\mathrm{~m}, 2 \mathrm{H}, \mathrm{cPr}-\mathrm{CH}_{2}\right), 1.21\left(\mathrm{~m}, 2 \mathrm{H}, \mathrm{cPr}-\mathrm{CH}_{2}\right), 3.08\left(\mathrm{~s}, 3 \mathrm{H}, \mathrm{CH}_{3}\right), 3.82\left(\mathrm{~s}, 3 \mathrm{H}, \mathrm{OCH}_{3}\right), 4.85$ (s, $1 \mathrm{H}, \mathrm{Br}-\mathrm{CH}) .-{ }^{13} \mathrm{C}-\mathrm{NMR}\left(\mathrm{CDCl}_{3}, 62.9 \mathrm{MHz}\right): \delta=11.2(-, 2 \mathrm{C}, \mathrm{cPr}-\mathrm{C}), 40.4\left(+, \mathrm{CH}_{3}\right), 52.5(+$, $\left.\mathrm{OCH}_{3}\right), 62.3\left(\mathrm{C}_{\text {quat }}, \mathrm{cPr}-\mathrm{C}\right), 63.2(+, \mathrm{Br}-\mathrm{CH}), 171.2\left(\mathrm{C}_{\text {quat }}, \mathrm{C}=\mathrm{O}\right) .-\mathrm{MS}(70 \mathrm{eV}), \mathrm{m} / z(\%): 288 / 286$ (3/4) $\left[\mathrm{M}^{+}\right], 209$ (63) $\left[\mathrm{M}^{+}-\mathrm{Br}\right], 207$ (19), 129 (100), 79 (28), 56 (75). - $\mathrm{C}_{7} \mathrm{H}_{11} \mathrm{BrO}_{5} \mathrm{~S}(287.1)$ : calcd. C 29.28, H 3.86, found: C 29.17, H 3.83. 
Methyl 2-bromo-2-cyclopropylideneacetate (4-Me). To a solution of $2.18 \mathrm{~g}$ (15.6 mmol) of 32b in $25 \mathrm{~mL}$ of DCM kept at $0{ }^{\circ} \mathrm{C}$ were added $1.21 \mathrm{~g}(12.4 \mathrm{mmol})$ of triethylamine, and the solution was stirred at this temperature for $4 \mathrm{~h}$. DCM $(100 \mathrm{~mL})$ and $1 \mathrm{~N}$ aq. $\mathrm{HCl}(10 \mathrm{~mL})$ were added. Separation of the phases, drying of the

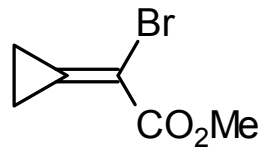
organic phase over $\mathrm{MgSO}_{4}$ and removal of the solvent in vacuo yielded $1.52 \mathrm{~g}$ $(99 \%)$ of $\mathbf{3 2 b}$ as a colourless crystalline solid. $-{ }^{1} \mathrm{H}-\mathrm{NMR}\left(\mathrm{CDCl}_{3}, 250 \mathrm{MHz}\right): \delta=1.39(\mathrm{~m}, 2 \mathrm{H}$, cPr-CH 2$), 1.76\left(\mathrm{~m}, 2 \mathrm{H}, \mathrm{cPr}-\mathrm{CH}_{2}\right), 3.82\left(\mathrm{~s}, 3 \mathrm{H}, \mathrm{CH}_{3}\right) .-{ }^{13} \mathrm{C} \mathrm{NMR}\left(\mathrm{CDCl}_{3}, 62.9 \mathrm{MHz}\right): \delta=7.0(-$, cPr-C), 12.1 (-, cPr-C), $53.2\left(+, \mathrm{CH}_{3}\right), 103.7\left(\mathrm{C}_{\text {quat }}, \mathrm{cPr}-\mathrm{C}\right), 143.6\left(\mathrm{C}_{\text {quat }}, \mathrm{Br}-\mathrm{C}\right), 162.6\left(\mathrm{C}_{\text {quat }}, \mathrm{C}=\mathrm{O}\right)$. 


\subsubsection{Synthesis of 5,6,7,8-tetrahydroquinazolinone derivatives}

General procedure for synthesis of amidine from nitriles (GP 1): Into a dry reaction flask charged with $1 \mathrm{M}$ LiHMDS in THF, the corresponding solution of nitriles (33a-d) in THF was added, and the reaction mixture was kept stirring at $20^{\circ} \mathrm{C}$ for $4 \mathrm{~h}$, at which point $6 \mathrm{~N}$ isopropanolic $\mathrm{HCl}$ (4 equiv) was added. The crude reaction mixture was kept at $0{ }^{\circ} \mathrm{C}$ overnight. The precipitated product was filtered, washed with diethyl ether, dried to yield the amidine hydrochlorides as colourless solids.

p-Chlorobenzamidine hydrochloride (34b): Using the GP 1, $3.5 \mathrm{~g}(91 \%)$ of $\mathbf{3 4 b}$ were obtained from $p$-chlorobenzonitrile $(2.76 \mathrm{~g}, 20.0 \mathrm{mmol})$ and $22.0 \mathrm{~mL}$ of $1 \mathrm{M}$ LiHMDS as a white solid, m.p. $238^{\circ} \mathrm{C}$ (lit. m.p. $243-245{ }^{\circ} \mathrm{C}$ ) ${ }^{[102]}$. - IR $(\mathrm{KBr}): v=3239 \mathrm{~cm}^{-1}, 3054,1678,1460,1401,1036,715 .-{ }^{1} \mathrm{H}-\mathrm{NMR}(250$ MHz, [D $\left.\mathrm{D}_{6}\right] \mathrm{DMSO}$ ): $\delta=7.60-7.77$ (m, $2 \mathrm{H}$ ), 7.85-7.97 (m, $2 \mathrm{H}$ ), 8.4 (br. s, $\mathrm{NH}) .-{ }^{13} \mathrm{C}-\mathrm{NMR}\left(62.9 \mathrm{MHz},\left[\mathrm{D}_{6}\right] \mathrm{DMSO}\right), \delta=126.8\left(\mathrm{C}_{\text {quat }}\right.$, aryl-C), 129.4

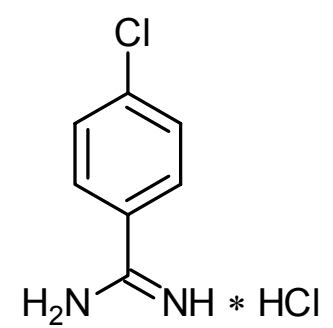
(+, 2 C, aryl-C), $130.6\left(+, 2\right.$ C, aryl-C), $139.1\left(\mathrm{C}_{\text {quata }}\right.$, aryl-C), $165.1\left(\mathrm{C}_{\text {quat }}, \mathrm{NCN}\right)$.

o-Bromobenzamidine hydrochloride (34c): Using the GP 1, $4.3 \mathrm{~g}(91 \%)$ of $\mathbf{3 4 c}$ were obtained from $o$-bromobenzonitrile (3.68 g, $20.0 \mathrm{mmol})$ and $22 \mathrm{~mL}$ of $1 \mathrm{M} \mathrm{LiHMDS}$ as a white solid, m.p. $>250{ }^{\circ} \mathrm{C}$. $-\mathrm{IR}(\mathrm{KBr}): v=3228 \mathrm{~cm}^{-1}, 3059,1669$, 1458, 1401, 1030, 728. $-{ }^{1} \mathrm{H}-\mathrm{NMR}\left(250 \mathrm{MHz}, \mathrm{CD}_{3} \mathrm{OD}\right): \delta=4.92$ (br. s, $\mathrm{NH}), 7.54-7.68(\mathrm{~m}, 3 \mathrm{H}), 7.74-7.86(\mathrm{~m}, 1 \mathrm{H}) .-{ }^{13} \mathrm{C}-\mathrm{NMR}(62.9 \mathrm{MHz}$,

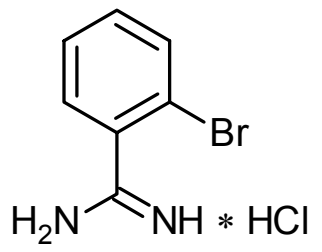
$\left.\mathrm{CD}_{3} \mathrm{OD}\right): \delta=121.1\left(\mathrm{C}_{\text {quat }}\right), 129.5(+), 131.0(+), 133.3\left(\mathrm{C}_{\text {quat }}\right), 134.8(+), 135.0(+), 168.4(\mathrm{NCN})$. $-\mathrm{MS}(\mathrm{DCI}, 70 \mathrm{eV}) \mathrm{m} / z(\%): 399(6)\left[2 \mathrm{M}-2 \mathrm{Na}+\mathrm{H}^{+}\right], 216\left[\mathrm{M}-\mathrm{HCl}+\mathrm{NH}_{4}{ }^{+}\right], 199(100)[\mathrm{M}-$ $\left.\mathrm{HCl}+\mathrm{H}^{+}\right]$.

o-Fluorobenzamidine hydrochloride (34d): Using the GP 1, $2.8 \mathrm{~g}(80 \%)$ of $\mathbf{3 4 d}$ were obtained from $o$-fluorobenzonitrile $(2.40 \mathrm{~g}, 20.0 \mathrm{mmol})$ and $22.0 \mathrm{~mL}$ of $1 \mathrm{M}$ LiHMDS as a white solid, m.p. $98-100{ }^{\circ} \mathrm{C} .-\mathrm{IR}(\mathrm{KBr}): v=3477 \mathrm{~cm}^{-1}$, 3144, 1701, 1674, 1476, 1401, 1228, 774, 684. - ${ }^{1} \mathrm{H}-\mathrm{NMR}(250 \mathrm{MHz}$, $\mathrm{CD}_{3} \mathrm{OD}$ ): $\delta=4.98$ (br. s, NH), 7.38-7.49 (m, $\left.2 \mathrm{H}\right), 7.64-7.80$ (m, $\left.1 \mathrm{H}\right)$.<smiles></smiles>
${ }^{13} \mathrm{C}-\mathrm{NMR}\left(62.9 \mathrm{MHz}, \mathrm{CD}_{3} \mathrm{OD}, \mathrm{DEPT}\right): \delta=118.2\left(+, \mathrm{d},{ }^{2} J_{\mathrm{C}-\mathrm{F}}=9.2 \mathrm{~Hz}\right.$, aryl-C), $118.8\left(\mathrm{C}_{\text {quat }}, \mathrm{d},{ }^{2} J_{\mathrm{C}-}\right.$ 
${ }_{\mathrm{F}}=3.2 \mathrm{~Hz}$, aryl-C), $126.6\left(+\right.$, aryl-C), $131.4(+$, aryl-C $), 136.8(+), 161.2\left(\mathrm{C}_{\text {quat }}, \mathrm{d},{ }^{1} J_{\mathrm{C}-\mathrm{F}}=253.7 \mathrm{~Hz}\right.$, aryl-C), 164.9 ( $\left.\mathrm{C}_{\text {quat }}, \mathrm{NCN}\right)$.

p-Benzyloxybenzamidine hydrochloride (34e): Using the GP 1, $2.42 \mathrm{~g}(92 \%)$ of 34e were obtained from $o$-benzyloxybenzonitrile $(2.09 \mathrm{~g}, 10 \mathrm{mmol})$ and $11 \mathrm{~mL}$ of $1 \mathrm{~m} \mathrm{LiHMDS}$ as a white solid, m.p. 181-182 ${ }^{\circ} \mathrm{C}$ (lit. m.p. $\left.179-180{ }^{\circ} \mathrm{C}\right) .{ }^{[103]}-\mathrm{IR}(\mathrm{KBr}): v=3317 \mathrm{~cm}^{-1}, 3125$, 1677, 1609, 1486, 1267, 1190, 1010, 837, 763. - ${ }^{1} \mathrm{H}-\mathrm{NMR}(250 \mathrm{MHz}$, $\left.\mathrm{CD}_{3} \mathrm{OD}\right): \delta=4.92$ (br. s, NH), 5.24 (s, $\left.2 \mathrm{H}\right), 7.19-7.28(\mathrm{~m}, 2 \mathrm{H}), 7.32-7.52$ (m, $5 \mathrm{H}) 7.79-7.84(\mathrm{~m}, 2 \mathrm{H}) .-{ }^{13} \mathrm{C}-\mathrm{NMR}\left(75.5 \mathrm{MHz}, \mathrm{CD}_{3} \mathrm{OD}\right): \delta=71.4(-$ , $\left.\mathrm{OCH}_{2}\right), 116.7$ (+, aryl-C), $121.14\left(\mathrm{C}_{\text {quat, }}\right.$ aryl-C), 128.7 (+, aryl-C), 129.2

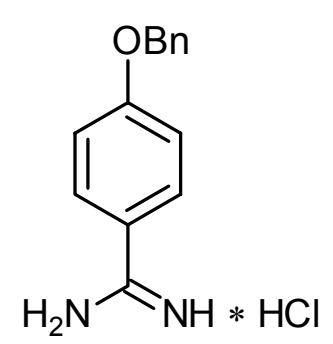
(+, aryl-C), $129.6\left(+\right.$, aryl-C), 131.1 (+, aryl-C), $137.6\left(\mathrm{C}_{\mathrm{ipso}}\right), 164.9\left(\mathrm{C}_{\mathrm{ipso}}\right), 168.4(\mathrm{NCN})$.

o-Phenylbenzamidine (34f): To a suspension of $\mathrm{NH}_{4} \mathrm{Cl}(2.14 \mathrm{~g}, 40.0 \mathrm{mmol})$ in $40 \mathrm{~mL}$ of toluene was added $\mathrm{Me}_{3} \mathrm{Al}(2 \mathrm{M}$ in toluene, $40.0 \mathrm{mmol})$ over a period of $30 \mathrm{~min}$ at $5{ }^{\circ} \mathrm{C}$. The temperature was then allowed to reach $25^{\circ} \mathrm{C}$, and stirring was continued until evolution of methane ceased $(\sim 2 \mathrm{~h})$. To this solution of $\mathrm{MeAl}(\mathrm{Cl}) \mathrm{NH}_{2}, o$-biphenyl nitrile (2.86 g, $16.0 \mathrm{mmol})$ was added in $5 \mathrm{~mL}$ of toluene during a period of $5 \mathrm{~min}$, and the resulting solution was refluxed for $20 \mathrm{~h}$. After cooling, the crude reaction mixture was poured onto a suspension of $20 \mathrm{~g}$ of $\mathrm{SiO}_{2}$ in $100 \mathrm{~mL}$ of<smiles>N=C(N)c1ccccc1-c1ccccc1</smiles>
dichloromethane, filtered and the solid residue was washed with $2 \times 50 \mathrm{~mL}$ of $\mathrm{MeOH}$. The solvent was removed in vacuo from the combined solutions. The residue was suspended in $100 \mathrm{~mL}$ of water, $30 \mathrm{~mL}$ of $2 \mathrm{~N} \mathrm{HCl}$ were added and the suspension was extracted with ethylacetate $(2 \times 50$ $\mathrm{mL}$ ). To the aq. layer $60 \mathrm{~mL}$ of $2 \mathrm{~N} \mathrm{NaOH}$ were added and the solution was extracted with DCM $(3 \times 50 \mathrm{~mL})$. This DCM layer was dried over $\mathrm{MgSO}_{4}$, filtered, and the solvent was removed in vacuo to yield $1.83 \mathrm{~g}(58 \%)$ of $\mathbf{3 4 f}$ as a white solid, m.p. $148-150{ }^{\circ} \mathrm{C}$ (lit. m.p. $\left.149-151{ }^{\circ} \mathrm{C}\right) .{ }^{[62 \mathrm{e}]}$ IR (KBr): $v=3408 \mathrm{~cm}^{-1}, 3059,1674,1639,1600,1427,1199,744,701 .-{ }^{1} \mathrm{H}-\mathrm{NMR}(250 \mathrm{MHz}$, $\left.\left[\mathrm{D}_{6}\right] \mathrm{DMSO}\right): \delta=5.4$ (br. s, NH), 7.31-7.55 (m, $\left.9 \mathrm{H}\right) .-{ }^{13} \mathrm{C}-\mathrm{NMR}\left(62.9 \mathrm{MHz},\left[\mathrm{D}_{6}\right] \mathrm{DMSO}\right): \delta=$ 127.3 (aryl-C), 128.1 (aryl-C), 128.3 (aryl-C), 128.6 (aryl-C), 129.2 (aryl-C), 136.2 (aryl-C), 137.5 (aryl-C), 139.0 (aryl-C), 140.5 (aryl-C), 165.9 (NCN). - MS (70 eV), m/z (\%): 196 (10) [M $\left.{ }^{+}\right], 195$ (100) $\left[\mathrm{M}^{+}-1\right], 178(31), 77(8)$. 
3-(5-chloro-benzo[b]thiophene) amidine (34g): Following the method described for $\mathbf{3 4 f} 420 \mathrm{mg}$ ( $84 \%)$ of $34 \mathrm{~g}$ were obtained from the corresponding nitrile $(460 \mathrm{mg}, 2.38 \mathrm{mmol})$, as a white solid, m.p. $>250{ }^{\circ} \mathrm{C}$. - IR (KBr): $v=3042 \mathrm{~cm}^{-1}, 1656,1551,1639,1408$, 1082, 866, 829. $-{ }^{1} \mathrm{H}-\mathrm{NMR}\left(250 \mathrm{MHz}, \mathrm{CDCl}_{3}\right): \delta=7.46\left(\mathrm{dd},{ }^{3} J=8.4\right.$ $\left.\mathrm{Hz},{ }^{4} J=1.8 \mathrm{~Hz}, 1 \mathrm{H}, \mathrm{Ar}-\mathrm{H}\right), 7.83\left(\mathrm{~d},{ }^{3} J=8.3 \mathrm{~Hz}, 1 \mathrm{H}, \mathrm{Ar}-\mathrm{H}\right), 7.98\left(\mathrm{~d},{ }^{4} J\right.$ $=1.3 \mathrm{~Hz}, 1 \mathrm{H}, \mathrm{Ar}-\mathrm{H}), 8.17(\mathrm{~s}, 1 \mathrm{H}, \mathrm{Ar}-\mathrm{H}) .-{ }^{13} \mathrm{C} \mathrm{NMR}(62.9 \mathrm{MHz}$,

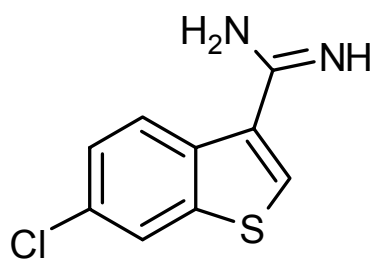
$\left.\mathrm{CDCl}_{3}, \mathrm{DEPT}\right): \delta=106.5\left(\mathrm{C}_{\text {quat }}\right.$, aryl-C $), 113.7\left(\mathrm{C}_{\text {quat }}\right.$, aryl-C $), 122.1(+$, aryl-C), 123.8 (+, aryl-C), 126.9 (+, aryl-C), $132.7\left(\mathrm{C}_{\text {quat, }}\right.$ aryl-C), $136.5\left(\mathrm{C}_{\text {quat }}\right.$, aryl-C), 138.3 (C $\mathrm{C}_{\text {quat, }}$ aryl-C), 139.1 (+, aryl-C), $163.3(\mathrm{NCN}) .-\mathrm{MS}(70 \mathrm{eV}), \mathrm{m} / z(\%): 210(100)\left[\mathrm{M}^{+}\right], 194$ (75), 159 (40),

\section{General Procedure for Michael addition of amidines 34a-j onto 5 (GP 2):}

A solution of methyl 2-chloro-2-cyclopropylidineacetate 5, amidine 34 (2 eqiv.) and of $\mathrm{Et}_{3} \mathrm{~N}$ (4 equiv.) was stirred in anhydrous dioxane at room temp. for $48 \mathrm{~h}$. After filtration the solid residue was suspended in DCM and washed with water. The aq. layer was washed three times with DCM. The organic layers were combined with the filtrate, dried over $\mathrm{MgSO}_{4}$, evaporated in vacuo and the crude product was purified by column chromatography.

3-Phenyl-2,4-diazabicyclo[4.2.0]octa-1(6),2-dien-5-one (35a): The crude product obtained from 5 (365 mg, $2.50 \mathrm{mmol}$ ) and benzamidine hydrochloride (34a, $793 \mathrm{mg}, 5.00 \mathrm{mmol}$ ) in $25 \mathrm{~mL}$ of dioxane according to GP 2 was purified by column chromatography $\left(R_{\mathrm{f}}=\right.$ $\left.0.3, \mathrm{Et}_{2} \mathrm{O}, 1.5 \times 30 \mathrm{~cm}, 30 \mathrm{~g} \mathrm{SiO}_{2}\right)$ to yield $411 \mathrm{mg}(83 \%)$ of $35 \mathrm{a}$ as a white solid, m.p. $191^{\circ} \mathrm{C}$. - IR (KBr): $v=3008 \mathrm{~cm}^{-1}, 2937,1670,1557$, 1498, 1321, 1082, 838, 766. ${ }^{1} \mathrm{H}-\mathrm{NMR}\left(250 \mathrm{MHz}, \mathrm{CDCl}_{3}\right): \delta=3.01$ (t, $J$ $=3.2 \mathrm{~Hz}, 2 \mathrm{H}), 3.22(\mathrm{t}, J=3.2 \mathrm{~Hz}, 2 \mathrm{H}), 7.47-7.53(\mathrm{~m}, 3 \mathrm{H}$, aryl-H), $8.05-$

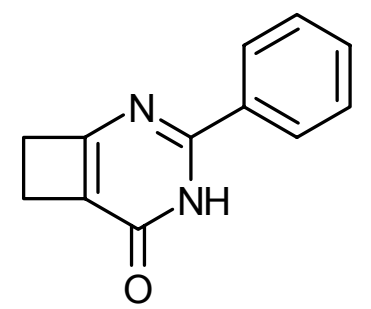
$8.2(\mathrm{~m}, 2 \mathrm{H}$, aryl-H), 12.54 (br. s, $1 \mathrm{H}, \mathrm{NH}) .-{ }^{13} \mathrm{C}-\mathrm{NMR}\left(62.9 \mathrm{MHz}, \mathrm{CDCl}_{3}, \mathrm{DEPT}\right): \delta=25.7(-$, $\mathrm{CH}_{2}$ ), 33.7 (-, $\left.\mathrm{CH}_{2}\right), 124.1$ ( $\left.\mathrm{C}_{\text {quat }}, \mathrm{C}-6\right), 127.6$ (+, aryl-C), 128.9 (+, aryl-C), 131.7 (+, aryl-C), 132.5 ( $\mathrm{C}_{\text {ipso }}$, aryl-C), 158.6 ( $\left.\mathrm{C}_{\text {quat }}, \mathrm{C}-1 / \mathrm{C}-3\right), 160.52$ ( $\left.\mathrm{C}_{\text {quat, }}, \mathrm{C}-3 / \mathrm{C}-1\right), 171.9$ ( $\left.\mathrm{C}_{\text {quat, }}, \mathrm{C}-5\right) .-\mathrm{MS}$ (70 $\mathrm{eV}), m / z(\%): 198(100)\left[\mathrm{M}^{+}\right], 170(7)\left[\mathrm{M}^{+}-\mathrm{C}_{2} \mathrm{H}_{4}\right], 104$ (91) $\left[\mathrm{HN}=\mathrm{CPh}^{+}\right], 77$ (44) $\left[\mathrm{C}_{5} \mathrm{H}_{5}^{+}\right] .-$ $\mathrm{C}_{12} \mathrm{H}_{10} \mathrm{~N}_{2} \mathrm{O}$ (198.2): calcd. C 72.71, H 5.08, N 14.13; found C 72.44, H 5.38, N 14.03. 
3-(p-Chlorophenyl)-2,4-diazabicyclo[4.2.0]octa-1(6),2-dien-5-one (35b): The crude product obtained from 5 (365 mg, $2.50 \mathrm{mmol}$ ), p-chlorobenzamidine hydrochloride (34b, $955 \mathrm{mg}, 5.00$ $\mathrm{mmol})$ and triethylamine $(1.01 \mathrm{~g}, 10.0 \mathrm{mmol})$ in $25 \mathrm{~mL}$ of dioxane according to GP 2 was purified by column chromatography $\left(R_{\mathrm{f}}=0.31\right.$, $\left.\mathrm{DCM} / \mathrm{MeOH}=25: 1,1.5 \times 20 \mathrm{~cm}, 25 \mathrm{~g} \mathrm{SiO}_{2}\right)$ to yield $455 \mathrm{mg}(78 \%)$ of 35b as a white solid, m.p. $218-219{ }^{\circ} \mathrm{C}$. $-\mathrm{IR}(\mathrm{KBr}): v=3078 \mathrm{~cm}^{-1}$, 2938, 1668, 1520, 1491, 1322, 1092, 1076, 837, 760. - ${ }^{1} \mathrm{H}-\mathrm{NMR}(250$

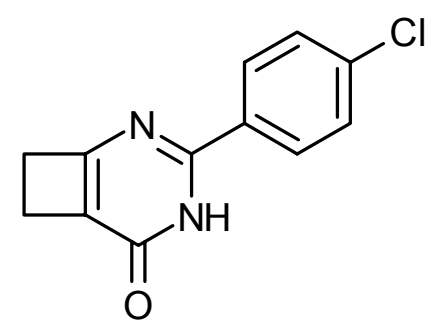
MHz, [D $\left.\left.\mathrm{D}_{6}\right] \mathrm{DMSO}\right): \delta=2.9(\mathrm{t}, J=3.1 \mathrm{~Hz}, 2 \mathrm{H}), 3.15(\mathrm{t}, J=3.1 \mathrm{~Hz}, 2 \mathrm{H}), 7.6(\mathrm{~d}, J=8.5 \mathrm{~Hz}, 2 \mathrm{H}$, aryl-H), $8.05\left(\mathrm{~d}, J=8.5 \mathrm{~Hz}, 2 \mathrm{H}\right.$, aryl-H). $-{ }^{13} \mathrm{C}-\mathrm{NMR}\left(62.9 \mathrm{MHz},\left[\mathrm{D}_{6}\right] \mathrm{DMSO}\right), \delta=25.1\left(-, \mathrm{CH}_{2}\right)$, $33.6\left(-, \mathrm{CH}_{2}\right), 126.1\left(\mathrm{C}_{\text {quat }}, \mathrm{C}-6\right), 128.9$ (+, aryl-C), 129.8 (+, aryl-C), $136.6\left(\mathrm{C}_{\text {quat }}, \mathrm{C}_{\text {ipso }}\right), 152.1$ $\left(\mathrm{C}_{\text {quat }}, \mathrm{C}-\mathrm{Cl}\right), 158.2\left(\mathrm{C}_{\text {quat }}, \mathrm{C}-1 / \mathrm{C}-3\right), 159.6\left(\mathrm{C}_{\text {quat, }}, \mathrm{C}-3 / \mathrm{C}-1\right), 171.5\left(\mathrm{C}_{\text {quat }}, \mathrm{C}-5\right) .-\mathrm{MS}(70 \mathrm{eV}), \mathrm{m} / \mathrm{z}$ (\%): 234/232 (33/100) $\left[\mathrm{M}^{+}\right], 204$ (6) $\left[\mathrm{M}^{+}-\mathrm{C}_{2} \mathrm{H}_{4}\right], 138 / 140(57 / 18)\left[\mathrm{HN}=\mathrm{CC}_{6} \mathrm{H}_{4} \mathrm{Cl}^{+}\right], 111$ (15) $\left[\mathrm{C}_{5} \mathrm{H}_{4} \mathrm{Cl}^{+}\right] .-\mathrm{C}_{12} \mathrm{H}_{9} \mathrm{ClN}_{2} \mathrm{O}$ (232.7): calcd. C 61.95, H 3.90, N 12.04; found $\mathrm{C} 61.63, \mathrm{H} \mathrm{3.78,} \mathrm{N}$ 11.83 .

3-(o-Bromophenyl)-2,4-diazabicyclo[4.2.0]octa-1(6),2-dien-5-one (35c): The crude product obtained from 5 (1.10 g, $7.50 \mathrm{mmol}) o$-bromobenzamidine hydrochloride (34c, $3.50 \mathrm{~g}, 15.0 \mathrm{mmol})$ and triethylamine $(3.03 \mathrm{~g}, 30.0 \mathrm{mmol})$ in $60 \mathrm{~mL}$ of dioxane according to GP 2 was purified by column chromatography $\left(R_{\mathrm{f}}=0.35, \mathrm{Et}_{2} \mathrm{O} / \mathrm{MeOH}=\right.$ $50: 1,3 \times 30 \mathrm{~cm}$., $\left.50 \mathrm{~g} \mathrm{SiO}_{2}\right)$ to yield $1.59 \mathrm{~g}(76 \%)$ of $\mathbf{3 5} \mathbf{c}$ as a white solid, m.p. $178-179{ }^{\circ} \mathrm{C} .-\mathrm{IR}(\mathrm{KBr}): v=2925 \mathrm{~cm}^{-1}, 2847,1662,1540,1472$, 1326, 1089, 778, 762. $-{ }^{1} \mathrm{H}-\mathrm{NMR}\left(200 \mathrm{MHz}, \mathrm{CDCl}_{3}\right): \delta=3.0(\mathrm{t}, J=4.2$

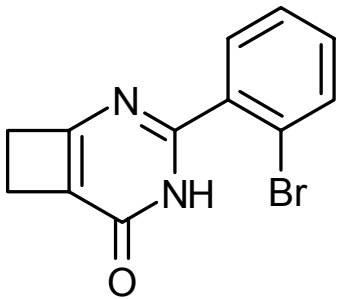
Hz, 2 H), $3.2(\mathrm{t}, J=4.2 \mathrm{~Hz}, 2 \mathrm{H}), 7.3-7.5$ (m, $2 \mathrm{H}$, aryl-H), 7.52-7.7 (m, $2 \mathrm{H}$, aryl-H), 11.0 (br. s, $1 \mathrm{H}, \mathrm{NH}) .-{ }^{13} \mathrm{C}-\mathrm{NMR}\left(50.3 \mathrm{MHz}, \mathrm{CDCl}_{3}\right): \delta=25.4\left(-, \mathrm{CH}_{2}\right), 33.7\left(-, \mathrm{CH}_{2}\right), 120.9\left(\mathrm{C}_{\text {quat }}, \mathrm{C}-6\right)$, 125.1 ( $\left.\mathrm{C}_{\text {quat, }} \mathrm{C}-\mathrm{Br}\right), 127.7$ (+, aryl-C), 130.9 (+, aryl-C), 132.0 (+, aryl-C), 133.7 (+, aryl-C), 134.6 $\left(\mathrm{C}_{\text {quat }}, \mathrm{C}_{\text {ipso }}\right), 157.6$ ( $\left.\mathrm{C}_{\text {quat }}, \mathrm{C}-1 / \mathrm{C}-3\right), 160.3$ ( $\left.\mathrm{C}_{\text {quat }}, \mathrm{C}-3 / \mathrm{C}-1\right), 171.2\left(\mathrm{C}_{\text {quat }}, \mathrm{C}-5\right) .-\mathrm{MS}(70 \mathrm{eV}), \mathrm{m} / \mathrm{z}$ (\%): 279/277 (21/22) $\left[\mathrm{M}^{+}+1\right], 278 / 276(97 / 100)\left[\mathrm{M}^{+}\right], 184 / 182(41 / 42)\left[\mathrm{HNCC}_{6} \mathrm{H}_{4} \mathrm{Br}^{+}\right], 102(58)$, 95 (79). $-\mathrm{C}_{12} \mathrm{H}_{10} \mathrm{BrN}_{2} \mathrm{O}$ (277.1): calcd. C 52.01, H 3.27, N 10.11; found C 51.92, H 3.37, N 9.94. 
3-(o-Fluorophenyl)-2,4-diazabicyclo[4.2.0]octa-1(6),2-dien-5-one (35d): The crude product obtained from 5 (730 mg, $5.00 \mathrm{mmol}$ ), o-fluorobenzamidine hydrochloride (34d, $1.75 \mathrm{~g}, 10.0$ mmol $)$ and triethylamine $(2.02 \mathrm{~g}, 20.0 \mathrm{mmol})$ in $50 \mathrm{~mL}$ of dioxane according to GP 2 was purified by column chromatography $\left(R_{\mathrm{f}}=0.5\right.$, $\left.\mathrm{Et}_{2} \mathrm{O} / \mathrm{MeOH}=25: 1,3 \times 30 \mathrm{~cm}, 50 \mathrm{~g} \mathrm{SiO}_{2}\right)$ to yield $884 \mathrm{mg}(82 \%)$ of $\mathbf{3 5 d}$ as a white solid, m.p. $184-185^{\circ} \mathrm{C} .-\mathrm{IR}(\mathrm{KBr}): v=3095 \mathrm{~cm}^{-1}, 2976,2947$, 1668, 1617, 1532, 1319, 1223, 1084, 753. - ${ }^{1} \mathrm{H}-\mathrm{NMR}\left(200 \mathrm{MHz}, \mathrm{CDCl}_{3}\right)$ : $\delta=3.04(\mathrm{t}, J=4.2 \mathrm{~Hz}, 2 \mathrm{H}), 3.25(\mathrm{t}, J=4.2 \mathrm{~Hz}, 2 \mathrm{H}), 7.16-7.42(\mathrm{~m}, 2 \mathrm{H}$,

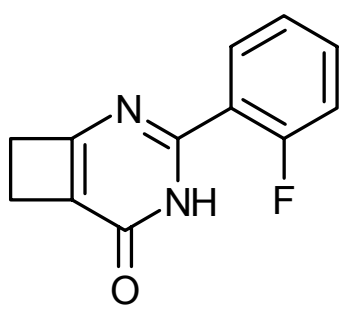
aryl-H), 7.48-7.62 (m, $1 \mathrm{H}$, aryl-H), 7.91-8.20 (m, $1 \mathrm{H}$, aryl-H), 10.22 (br. s, $1 \mathrm{H}, \mathrm{NH}) \cdot-{ }^{13} \mathrm{C}-$ $\operatorname{NMR}\left(50.3 \mathrm{MHz}, \mathrm{CDCl}_{3}\right): \delta=25.4\left(-, \mathrm{CH}_{2}\right), 33.8\left(-, \mathrm{CH}_{2}\right), 116.6\left(+, \mathrm{d},{ }^{2} J_{\mathrm{C}-\mathrm{F}}=22.9 \mathrm{~Hz}\right) 120.1(\mathrm{~d}$, $\left.{ }^{2} J_{\mathrm{C}-\mathrm{F}}=22.9 \mathrm{~Hz}, \mathrm{C}_{\mathrm{ipso}}\right), 125.0\left(+, \mathrm{d},{ }^{3} J_{\mathrm{C}-\mathrm{F}}=3.5 \mathrm{~Hz}\right), 130.9\left(+, \mathrm{d},{ }^{4} J_{\mathrm{C}-\mathrm{F}}=1.5 \mathrm{~Hz}\right), 133.6\left(+, \mathrm{d},{ }^{3} J_{\mathrm{C}-\mathrm{F}}=\right.$ $9.2 \mathrm{~Hz}), 156.7\left(\mathrm{C}_{\text {quat }}, \mathrm{C}-1 / \mathrm{C}-3\right), 156.8\left(\mathrm{C}_{\text {quat, }}, \mathrm{C}-3 / \mathrm{C}-1\right), 160.2\left(\mathrm{C}_{\text {quat }}, \mathrm{d},{ }^{1} J_{\mathrm{C}-\mathrm{F}}=251 \mathrm{~Hz}\right), 171.0$ $\left(\mathrm{C}_{\text {quat }}, \mathrm{C}-5\right) .-\mathrm{MS}(70 \mathrm{eV}), \mathrm{m} / z(\%): 217(17)\left[\mathrm{M}^{+}+1\right], 216(100)\left[\mathrm{M}^{+}\right], 122(42)\left[\mathrm{HN}=\mathrm{CC}_{6} \mathrm{H}_{4} \mathrm{~F}^{+}\right]$, 102 (20), 95 (24) $\left[\mathrm{C}_{6} \mathrm{H}_{4} \mathrm{~F}^{+}\right] .-\mathrm{C}_{12} \mathrm{H}_{10} \mathrm{FN}_{2} \mathrm{O}$ (116.2): calcd. C 66.66, H 4.20, N 12.96; found $\mathrm{C}$ 66.50, H 4.28, N 13.03 .

3-(p-Benzyloxyphenyl)-2,4-diazabicyclo[4.2.0]octa-1(6),2-dien-5-one (35e): The crude product obtained from 5 (660 $\mathrm{mg}, 4.50 \mathrm{mmol})$ p-benzyloxybenzamidine hydrochloride (34e, $2.4 \mathrm{~g}, 9.00$ mol) and triethylamine $(1.82 \mathrm{~g}, 18.0 \mathrm{~mol})$ in $50 \mathrm{~mL}$ of dioxane according to GP 2 was purified by column chromatography $\left(R_{\mathrm{f}}=\right.$ $0.34, \mathrm{DCM} / \mathrm{MeOH}=25: 1,3 \times 30 \mathrm{~cm}, 50 \mathrm{~g}, \mathrm{SiO}_{2}$ ) to yield $930 \mathrm{mg}$ $(68 \%)$ of 35e as a white solid, m.p. $212-213{ }^{\circ} \mathrm{C}$. $-\mathrm{IR}(\mathrm{KBr})$ : $v=3088 \mathrm{~cm}^{-1}, 2936,1664,1608,1504,1303,1252,1189,843$, 762. $-{ }^{1} \mathrm{H}-\mathrm{NMR}\left(300 \mathrm{MHz}, \mathrm{CDCl}_{3}\right): \delta=3.01(\mathrm{t}, J=3.8 \mathrm{~Hz}, 2 \mathrm{H})$,

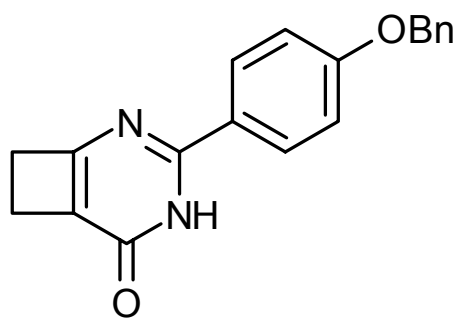
$3.20(\mathrm{t}, J=3.8 \mathrm{~Hz}, 2 \mathrm{H}), 5.15$ (s, $\left.2 \mathrm{H}, \mathrm{OCH}_{2}\right), 7.04-7.12$ (m, $2 \mathrm{H}$, aryl-H), 7.30-7.47 (m, $5 \mathrm{H}$, arylH), 7.95-8.22 (m, $2 \mathrm{H}$, aryl-H), 11.48 (br. s, $1 \mathrm{H}, \mathrm{NH}) .-{ }^{13} \mathrm{C}-\mathrm{NMR}\left(50.3 \mathrm{MHz},\left[\mathrm{D}_{6}\right] \mathrm{DMSO}\right)$ : $\delta=24.6\left(-, \mathrm{CH}_{2}\right), 32.9\left(-, \mathrm{CH}_{2}\right), 69.4\left(-, \mathrm{OCH}_{2}\right), 114.7\left(+\right.$, aryl-C), $122.5\left(\mathrm{C}_{\text {quat }}, \mathrm{C}-6\right), 124.9$ $\left(\mathrm{C}_{\mathrm{ipso}}\right), 127.6\left(+\right.$, aryl-C), 127.8 (+, aryl-C), 128.4 (+, aryl-C), 129.4 (+, aryl-C), $136.5\left(\mathrm{C}_{\mathrm{ipso}}\right.$, arylC), 156.7 ( $\mathrm{C}_{\text {ipso }}$, aryl-C), 159.9 ( $\left.\mathrm{C}_{\text {quat, }}, \mathrm{C}-1 / \mathrm{C}-3\right), 160.9$ (C $\left.\mathrm{C}_{\text {quat }}, \mathrm{C}-3 / \mathrm{C}-1\right), 169.9$ ( $\left.\mathrm{C}_{\text {quat }}, \mathrm{C}-5\right)$. - MS (70 eV), $m / z$ (\%): $304(8)\left[\mathrm{M}^{+}\right], 91(100)\left[\mathrm{C}_{7} \mathrm{H}_{7}^{+}\right] .-\mathrm{C}_{19} \mathrm{H}_{16} \mathrm{~N}_{2} \mathrm{O}_{2}$ (304.6): calcd. C 74.98, H 5.30, N 9.20; found C 74.78, H 5.01, N 8.98. 
3-(Biphenyl-2-yl)-2,4-diazabicyclo[4.2.0]octa-1(6),2-dien-5-one (35f): The crude product obtained from $5(527 \mathrm{mg}, 3.6 \mathrm{mmol})$ o-biphenylbenzamidine (34f, $1.40 \mathrm{~g}, 7.20 \mathrm{~mole})$ and triethylamine (1.44 g, $14.2 \mathrm{~mol})$ in $25 \mathrm{~mL}$ of dioxane according to GP 2 was purified by column chromatography $\left(R_{\mathrm{f}}=0.41, \mathrm{Et}_{2} \mathrm{O}, 1.5 \times 20 \mathrm{~cm}, 20\right.$ $\left.\mathrm{g} \mathrm{SiO}_{2}\right)$ to yield $425 \mathrm{mg}(80 \%)$ of $\mathbf{3 5 f}$ as a white solid, m.p. $176-177{ }^{\circ} \mathrm{C} .-$ IR (KBr): $v=2928 \mathrm{~cm}^{-1}, 1675,1540,1478,1323,1088,976,745,698 .-$ ${ }^{1} \mathrm{H}-\mathrm{NMR}\left(250 \mathrm{MHz}, \mathrm{CDCl}_{3}\right): \delta=2.92(\mathrm{t}, J=4.2 \mathrm{~Hz}, 2 \mathrm{H}), 3.18(\mathrm{t}, J=4.2$

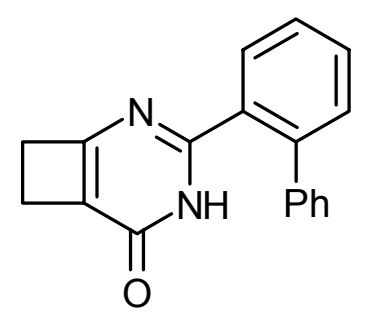
$\mathrm{Hz}, 2 \mathrm{H}), 7.22-7.41$ (m, $5 \mathrm{H}$, aryl-H), 7.44-7.78 (m, $4 \mathrm{H}$, aryl-H), 9.22 (br. s, $1 \mathrm{H}, \mathrm{NH}) .-{ }^{13} \mathrm{C}-$ NMR (50.3 MHz, $\left.\mathrm{CDCl}_{3}\right): \delta=25.0\left(-, \mathrm{CH}_{2}\right) 33.5\left(-, \mathrm{CH}_{2}\right), 123.8\left(\mathrm{C}_{\text {quat }}, \mathrm{C}-6\right), 127.7$ (+, aryl-C), 128.1 (+, aryl-C), 128.8 (+, aryl-C), 129.8 (+, aryl-C), 130.7 (+, aryl-C), $132.3\left(\mathrm{C}_{\mathrm{ipso}}\right.$, aryl-C), $139.2\left(\mathrm{C}_{\text {ipso }}\right.$, aryl-C), $140.6\left(\mathrm{C}_{\mathrm{ipso}}\right.$, aryl-C), 157.3 ( $\left.\mathrm{C}_{\text {quat }}, \mathrm{C}-1 / \mathrm{C}-3\right), 161.9\left(\mathrm{C}_{\text {quat }}, \mathrm{C}-3 / \mathrm{C}-1\right), 170.8$ $\left(\mathrm{C}_{\text {quat }}, \mathrm{C}-5\right)$. - MS (70 eV), m/z (\%): $274(42)\left[\mathrm{M}^{+}\right], 273(100)\left[\mathrm{M}^{+}-1\right], 245$ (8), $178(11)$.

3-(5-chloro-benzo[b]thiophene)-2,4-diazabicyclo[4.2.0]octa-1(6),2-dien-5-one (35g): The crude product obtained from $5(219 \mathrm{mg}, 1.50 \mathrm{mmol})$ 5-chloro-benzo[b]thiophen-3-yl-amidine (34g, 420 $\mathrm{mg}, 2.0 \mathrm{~mol})$ and triethylamine $(404 \mathrm{~g}, 4.0 \mathrm{~mol})$ in $25 \mathrm{~mL}$ of dioxane according to GP 2 was purified by column chromatography $\left(R_{\mathrm{f}}=0.37\right.$, $\left.\mathrm{DCM} / \mathrm{MeOH}=25: 1,1.5 \times 20 \mathrm{~cm}, 20 \mathrm{~g} \mathrm{SiO}_{2}\right)$ to yield $330 \mathrm{mg}(74 \%)$ of 35g as a white solid, m.p. $218^{\circ} \mathrm{C}$. $-\mathrm{IR}(\mathrm{KBr}): v=3109, \mathrm{~cm}^{-1}, 2985,1934$, 1664, 1552, 1538, 1245, 1075, 858, 757. - ${ }^{1} \mathrm{H}-\mathrm{NMR}(200 \mathrm{MHz}$, [D $\mathrm{D}_{6}$ DMSO): $\delta=2.90(\mathrm{t}, J=3.2 \mathrm{~Hz}, 2 \mathrm{H}), 3.20(\mathrm{t}, J=3.2 \mathrm{~Hz}, 2 \mathrm{H}), 7.46$ (dd, ${ }^{3} J=8.2 \mathrm{~Hz},{ }^{4} J=1.83 \mathrm{~Hz}, 1 \mathrm{H}$, aryl-H), $8.05\left(\mathrm{~d},{ }^{3} J=8.9 \mathrm{~Hz}, 1 \mathrm{H}\right.$,

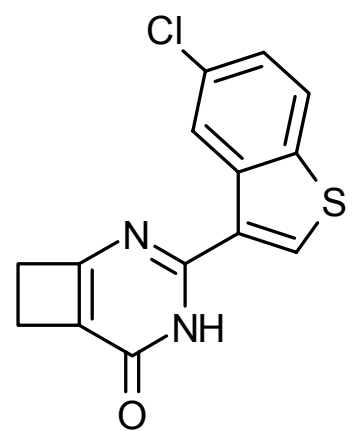
aryl-H), 8.72 (s, $2 \mathrm{H}$, aryl-H), 11.9 (br. s, $1 \mathrm{H}, \mathrm{NH}) .-{ }^{13} \mathrm{C}-\mathrm{NMR}\left(50.3 \mathrm{MHz},\left[\mathrm{D}_{6}\right] \mathrm{DMSO}\right): \delta=24.8$ (-, $\left.\mathrm{CH}_{2}\right), 33.1$ (-, $\left.\mathrm{CH}_{2}\right), 124.0$ ( $\left.\mathrm{C}_{\text {quat }}, \mathrm{C}-6\right), 124.2$ (+, aryl-C), 124.5 (+, aryl-C), 125.1 (+, aryl-C), 127.4 (-, aryl-C), 130.3 (-, aryl-C), 134.7 (+, aryl-C), 137.4 (-, aryl-C), 138.2 (-, aryl-C), 155.9

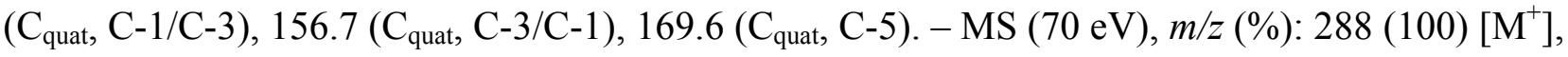
193 (45), 159 (40), 95 (89). $-\mathrm{C}_{14} \mathrm{H}_{9} \mathrm{ClN}_{2} \mathrm{OS}$ (288.8): calcd. C 58.23, H 3.14, N 9.70; found $\mathrm{C}$ 58.53, H 3.07, N 9.67. 
3-Methylthio-2,4-diazabicyclo[4.2.0]octa-1(6),2-dien-5-one (35h): In $50 \mathrm{~mL}$ of Schlank flask $\mathbf{5}$ (365 mg, $2.50 \mathrm{mmol}$ ), $S$-methylisothiourea hemisulphate 34h (1.39 g, $5.00 \mathrm{mmol})$ and triethylamine $(1.52 \mathrm{~g}, 15.0 \mathrm{mmol})$ in $25 \mathrm{~mL}$ of anhydrous dioxane were stirred at $50^{\circ} \mathrm{C}$ for $48 \mathrm{~h}$. After filtration, the solid residue was dissolved in water and extracted with DCM $(2 \times 25 \mathrm{~mL})$. After removing the solvent in vacuum, the crude product was purified by column chromatography $\left(R_{\mathrm{f}}=\right.$

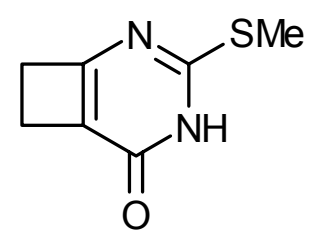
$\left.0.3, \mathrm{DCM} / \mathrm{MeOH}=25: 1,1.5 \times 30 \mathrm{~cm}, 30 \mathrm{~g} \mathrm{SiO}_{2}\right)$ to yield $310 \mathrm{mg}(74 \%)$ of $\mathbf{3 5 h}$ as a white solid, m.p. $215^{\circ} \mathrm{C}$. - IR (KBr): $v=2996 \mathrm{~cm}^{-1}, 2928,1654,1541,1456,1396,1297,1189,922,751$. ${ }^{1} \mathrm{H}-\mathrm{NMR}\left(250 \mathrm{MHz}, \mathrm{CDCl}_{3}\right): \delta=2.58(\mathrm{~s}, 3 \mathrm{H}, \mathrm{SMe}), 2.95\left(\mathrm{t}, J=3.1 \mathrm{~Hz}, 2 \mathrm{H}, \mathrm{CH}_{2}\right), 3.15(\mathrm{t}, J=$ $3.1 \mathrm{~Hz}, 2 \mathrm{H}, \mathrm{CH}_{2}$ ), 11.5 (br. s, $\left.1 \mathrm{H}, \mathrm{NH}\right) .-{ }^{13} \mathrm{C}-\mathrm{NMR}\left(62.9 \mathrm{MHz},\left[\mathrm{D}_{6}\right.\right.$ ]DMSO, DEPT): 13.4 (-, $\left.\mathrm{CH}_{2}\right), 24.9\left(-, \mathrm{CH}_{2}\right), 33.2\left(+, \mathrm{CH}_{3}\right), 120.4\left(\mathrm{C}_{\text {quat }}, \mathrm{C}-6\right), 152.3\left(\mathrm{C}_{\text {quat }}, \mathrm{C}-1 / \mathrm{C}-3\right), 156.6\left(\mathrm{C}_{\text {quat }}, \mathrm{C}-3 / \mathrm{C}-\right.$ 1), $164.98\left(\mathrm{C}_{\text {quat }}, \mathrm{C}-5\right)$. - MS (70 eV), $m / z(\%): 169(12)\left[\mathrm{M}^{+}+1\right], 168(100)\left[\mathrm{M}^{+}\right], 121(14)\left[\mathrm{M}^{+}-\right.$ $\mathrm{SMe}$, 93 (22), 74 (16). $-\mathrm{C}_{7} \mathrm{H}_{8} \mathrm{~N}_{2} \mathrm{OS}$ (168.2): calcd. C 49.98, H 4.79, N 16.65; found C 49.66, $\mathrm{H}$ 5.14, N 16.50.

3-Dibenzylamino-2,4-diazabicyclo[4.2.0]octa-1(6),2-dien-5-one (35i): The crude product obtained from 5 (366 mg, $2.5 \mathrm{mmol}$ ), dibenzylguanidine (1.38 g, $5 \mathrm{mmol}$ ) was subjected to chromatography to get $520 \mathrm{mg}(66 \%)$ of $\mathbf{3 5 i}$ as a white solid, m.p. $204{ }^{\circ} \mathrm{C}, R_{\mathrm{f}}=0.5$ (DCM:MeOH = 25:1). $-{ }^{1} \mathrm{H}-\mathrm{NMR}\left(250 \mathrm{MHz}, \mathrm{CDCl}_{3}\right): \delta=2.77\left(\mathrm{t},{ }^{3} \mathrm{~J}=\right.$ $\left.3.3 \mathrm{~Hz}, 2 \mathrm{H}, \mathrm{CH}_{2}\right), 3.03\left(\mathrm{t},{ }^{3} \mathrm{~J}=3.3 \mathrm{~Hz}, 2 \mathrm{H}, \mathrm{CH}_{2}\right), 4.75$ (s, $\left.4 \mathrm{H}, \mathrm{Bn}-\mathrm{H}\right)$, 7.17-7.20 (m, 4 H, aryl-H), 7.23-7.33 (m, 6 H, aryl-H), 9.60 (br. s, 1 $\mathrm{H}, \mathrm{NH}) .-{ }^{13} \mathrm{C}-\mathrm{NMR}\left(62.9 \mathrm{MHz}, \mathrm{CD}_{3} \mathrm{OD}\right): \delta=23.9\left(-, \mathrm{CH}_{2}\right), 33.5(-$, $\left.\mathrm{CH}_{2}\right), 50.4\left(-, \mathrm{Bn}-\mathrm{CH}_{2}\right), 112.2\left(\mathrm{C}_{\text {quat }}, \mathrm{C}-6\right), 127.1$ (+, aryl-C), 127.7 (+,

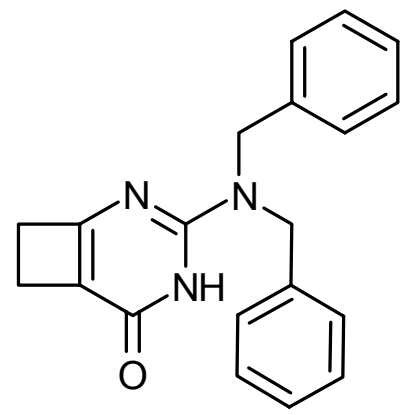
aryl-C), 128.8 (+, aryl-C), 135.8 ( $\mathrm{C}_{\text {quat }}$, aryl-C), 157.5 ( $\left.\mathrm{C}_{\text {quat }}, \mathrm{C}-1 / \mathrm{C}-3\right)$, 157.9 ( $\left.\mathrm{C}_{\text {quat }}, \mathrm{C}-3 / \mathrm{C}-1\right), 173.0$ ( $\left.\mathrm{C}_{\text {quat }}, \mathrm{C}-5\right) .-\mathrm{MS}$ (70 eV), m/z (\%): 317 (10) $\left[\mathrm{M}^{+}\right], 227$ (15), 226 (100) $\left[\mathrm{M}^{+}\right], 91$ (35). HRMS 317.1528 (correct mass).

3-Methoxy-2,4-diazabicyclo[4.2.0]octa-1(6),2-dien-5-one (35j): The crude product obtained from 5 (366 mg, $2.50 \mathrm{mmol})$, Omethyl-urea $(1.23 \mathrm{~g}, 10 \mathrm{mmol})$ and $\mathrm{Et}_{3} \mathrm{~N}(1.52 \mathrm{~g}, 15 \mathrm{mmol})$ in $25 \mathrm{~mL}$ of dioxane at $50{ }^{\circ} \mathrm{C}$ according to GP 2 was subjected to column chromatography $\left(R_{\mathrm{f}}=0.20, \mathrm{Et}_{2} \mathrm{O}\right)$ to yield $144 \mathrm{mg}(38 \%)$ of $\mathbf{3 5 j}$ as a white solid, m.p. $198^{\circ} \mathrm{C} .-{ }^{1} \mathrm{H}$ NMR $\left(250 \mathrm{MHz}, \mathrm{CDCl}_{3}\right): \delta=2.92$ 
(t, $\left.J=3.2 \mathrm{~Hz}, 2 \mathrm{H}, \mathrm{CH}_{2}\right), 3.08$ (t, $\left.J=3.1 \mathrm{~Hz}, 2 \mathrm{H}, \mathrm{CH}_{2}\right), 3.98$ (s, $3 \mathrm{H}, \mathrm{OMe}$ ), 11.5 (br. s, $\left.1 \mathrm{H}, \mathrm{NH}\right)$. $-{ }^{13} \mathrm{C}$ NMR (62.9 MHz, [D 6 DMSO, DEPT): $24.2\left(-, \mathrm{CH}_{2}\right), 33.1\left(-, \mathrm{CH}_{2}\right)$, $55.5\left(+, \mathrm{CH}_{3}\right), 117.9\left(\mathrm{C}_{\text {quat }}, \mathrm{C}-6\right), 159.7\left(\mathrm{C}_{\text {quat }}, \mathrm{C}-1 / \mathrm{C}-3\right), 160.6\left(\mathrm{C}_{\text {quat }}, \mathrm{C}-3 / \mathrm{C}-\right.$ 1), $171.5\left(\mathrm{C}_{\text {quat }}, \mathrm{C}-5\right)$. -MS (70 eV), $m / z(\%): 152(100)\left[\mathrm{M}^{+}\right], 122(21), 94$ (15), 58 (33). $-\mathrm{C}_{7} \mathrm{H}_{8} \mathrm{~N}_{2} \mathrm{O} 2$ (152.2): calcd. C 55.26, H 5.30, N 18.41; found $\mathrm{C}$

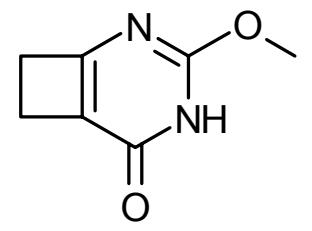
55.50, H 5.30, N 18.64.

\section{General Procedure for Diels-Alder reaction of 35 with phenyl vinyl sulfone (GP 3):}

6-Benzenesulfonyl-5,6,7,8-tetrahydroquinazolin-4(3H)-one (37): In a sealed Pyrex tube, 1 equiv. of 2,4-diazabicyclo[4.2.0]octa-1(6),2-dien-5-ones (35) were stirred with 4 equiv. of phenyl vinyl sulfone (36) at $175{ }^{\circ} \mathrm{C}$ for $12 \mathrm{~h}$. The mixture was allowed to cool down to room temperature, dissolved in $\mathrm{DCM} / \mathrm{MeOH}$ (10:1) and was purified by column chromatography.

\section{General Procedure for microwave assisted Diels.Alder reaction (GP 4):}

6-Benzenesulfonyl-5,6,7,8-tetrahydroquinazolin-4(3H)-one (37): In a $10 \mathrm{~mL}$ glass tube, 2,4diazabicyclo[4.2.0]octa-1(6),2-dien-5-ones (35) (0.5 mmol) and phenyl-vinyl sulphone (336 mg, 2 mmol) were taken. The vessel was sealed with a septum and placed in to a microwave cavity. Microwave irradiation of $80 \mathrm{~W}$ was used, the temperature being ramped from RT to $140{ }^{\circ} \mathrm{C}$. Once $140{ }^{\circ} \mathrm{C}$ was reached, the reaction mixture was held at this temperature for $20 \mathrm{~min}$. After allowing the mixture to cool to RT, the reaction vessel was opened. This crude product was purified by column chromatography.

6-Benzenesulfonyl-2-phenyl-5,6,7,8-tetrahydroquinazolin-4(3H)-one (37a): The crude product obtained from 3-phenyl-2,4-diazabicyclo[4.2.0]octa-1(6),2-diene-5-one (35a, $198 \mathrm{mg}, 1.00 \mathrm{mmol}$ ) and phenyl vinyl sulfone $(670 \mathrm{mg}, 4.00 \mathrm{mmol})$ according to GP 3 was purified by column chromatagraphy $\left(R_{\mathrm{f}}=0.41\right.$, $\mathrm{Et}_{2} \mathrm{O} / \mathrm{MeOH}=25: 1,1 \times 30 \mathrm{~cm}, 25 \mathrm{~g} \mathrm{SiO}_{2}$ ) to yield $303 \mathrm{mg}$ $(81 \%)$ of $37 \mathbf{a}$ as a white solid, m.p. $>250{ }^{\circ} \mathrm{C}$. $-\mathrm{IR}(\mathrm{KBr})$ : $v=2931 \mathrm{~cm}^{-1}, 1637,1551,1316,1146,1085,699 .-{ }^{1} \mathrm{H}-\mathrm{NMR}$

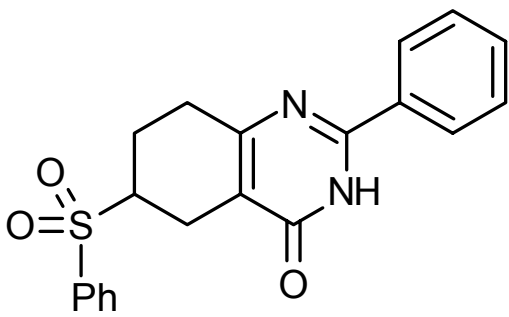
(200 MHz, [D $\mathrm{D}_{6}$ DMSO): $\delta=1.60-1.83$ (m, $\left.1 \mathrm{H}\right), 2.10-2.30(\mathrm{~m}, 1 \mathrm{H}), 2.30-2.45(\mathrm{~m}, 1 \mathrm{H}), 2.65-$ $2.82(\mathrm{~m}, 3 \mathrm{H}), 3.6-3.8[\mathrm{~m}, 1 \mathrm{H}, \mathrm{C}(6)-\mathrm{H}]$, 7.4-7.6 (m, $3 \mathrm{H}$, aryl-H), 7.60-7.85 (m, $3 \mathrm{H}$, aryl-H), 7.85-8.10 (m, 4 H, aryl-H), 12.6 (br. s, $1 \mathrm{H}, \mathrm{NH}) .-{ }^{13} \mathrm{C}-\mathrm{NMR}\left(50.3 \mathrm{MHz},\left[\mathrm{D}_{6}\right] \mathrm{DMSO}\right): \delta=21.1$ 
(-, $\left.\mathrm{CH}_{2}\right), 21.7\left(-, \mathrm{CH}_{2}\right), 29.8\left(-, \mathrm{CH}_{2}\right), 57.4(+, \mathrm{C}-6), 115.9(\mathrm{C}-4 \mathrm{a}), 127.5(+), 128.5(+), 128.5(+)$, $129.5(+), 131.3(+), 132.2\left(\mathrm{C}_{\mathrm{ipso}}\right), 134.1(+), 136.9\left(\mathrm{C}_{\mathrm{ipso}}\right), 153.9(\mathrm{C}-8 \mathrm{a}), 159.0(\mathrm{C}-2 / \mathrm{C}-4), 162.5$ (C-4/C-2). - MS (70eV), m/z (\%): 366 (2) $\left[\mathrm{M}^{+}\right], 225$ (24) $\left[\mathrm{M}^{+}-\mathrm{SO}_{2} \mathrm{Ph}\right], 224$ (100) $\left[\mathrm{M}^{+}-\mathrm{SO}_{2} \mathrm{Ph}-\right.$ $\mathrm{H}], 180$ (8), 104 (12), 77 (18) [ $\left.\mathrm{Ph}^{+}\right] .-\mathrm{C}_{20} \mathrm{H}_{18} \mathrm{~N}_{2} \mathrm{O}_{3} \mathrm{~S}$ (366.5): calcd. C 65.56, H 4.95, N 7.64; found C 65.89, H 4.86, N 7.49.

Using microwaves $126 \mathrm{mg}(69 \%)$ of $\mathbf{3 7 a}$ was obtained according to GP 4 which has same physical datas as given above.

6-Benzenesulfonyl-2-(p-chlorophenyl)-5,6,7,8-tetrahydroquinazolin-4(3H)-one (37b): The crude product obtained from 3-(p-chlorophenyl)-2,4-diazabicyclo[4.2.0]octa-1(6),2-diene-5-one (35b, $400 \mathrm{mg}, 1.70 \mathrm{mmol})$ and phenyl vinyl sulfone $(1.15 \mathrm{~g}, 6.80$ mmol) according to GP 3 was purified by column chromatagraphy $\left(R_{\mathrm{f}}=0.41, \mathrm{DCM} / \mathrm{MeOH}=25: 1,3 \times 30\right.$ $\left.\mathrm{cm}, 50 \mathrm{~g} \mathrm{SiO}_{2}\right)$ to yield $410 \mathrm{mg}(59 \%)$ of $\mathbf{3 7 b}$ as a white solid, m.p. $>250{ }^{\circ} \mathrm{C}$. - IR $(\mathrm{KBr}): v=3067 \mathrm{~cm}^{-1}, 2946$, $1655,1548,1506,1321,1146,1087,842,749,689 .-{ }^{1} \mathrm{H}-$

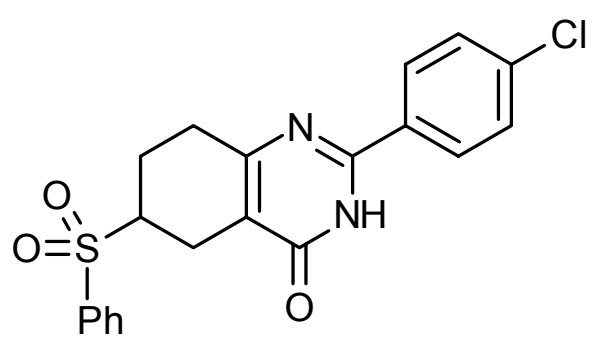
NMR (250 MHz , [D $\mathrm{D}_{6}$ DMSO): $\delta=1.68-1.80$ (m, $\left.1 \mathrm{H}\right), 2.20-2.75(\mathrm{~m}, 5 \mathrm{H}), 3.60-3.71$ [m, $1 \mathrm{H}$, C(6)-H], 7.52-7.62 (m, $2 \mathrm{H}$, aryl-H), 7.71-7.80 (m, $3 \mathrm{H}$, aryl-H), 7.85-7.97 (m, $2 \mathrm{H}$, aryl-H), 8.02-8.11 (m, $2 \mathrm{H}$, aryl-H). ${ }^{13} \mathrm{C}-\mathrm{NMR}\left(63.9 \mathrm{MHz},\left[\mathrm{D}_{6}\right] \mathrm{DMSO}\right): \delta=21.5\left(-, \mathrm{CH}_{2}\right), 22.0\left(-, \mathrm{CH}_{2}\right)$, $30.0\left(-, \mathrm{CH}_{2}\right), 57.5$ (+, C-6), $117.0\left(\mathrm{C}_{\text {qaut }}, \mathrm{C}-4 \mathrm{a}\right), 128.8$ (+, aryl-C), 128.9 (+, aryl-C), $129.5(+$, aryl-C), 129.8 (+, aryl-C), $131.0\left(\mathrm{C}_{\text {qaut }}, \mathrm{C}-\mathrm{Cl}\right), 134.4$ (+, aryl-C), $136.6\left(\mathrm{C}_{\mathrm{ipso}}\right), 137.1\left(\mathrm{C}_{\mathrm{ipso}}\right), 153.1$ (C-8a), $159.6\left(\mathrm{C}_{\mathrm{qaut}}, \mathrm{C}-2 / \mathrm{C}-4\right), 162.5\left(\mathrm{C}_{\mathrm{qaut}}, \mathrm{C}-4 / \mathrm{C}-2\right) .-\mathrm{MS}(70 \mathrm{eV}), m / z(\%): 400(1)\left[\mathrm{M}^{+}\right]$, $261 / 259 \quad(10 / 36) \quad\left[\mathrm{M}^{+}-\mathrm{SO}_{2} \mathrm{Ph}\right], \quad 260 / 258 \quad(32 / 100) \quad\left[\begin{array}{llll}\mathrm{M}^{+}-\mathrm{HSO}_{2} \mathrm{Ph}\end{array}\right], \quad 140 / 138 \quad(5 / 13)$ $\left[\mathrm{HN}=\mathrm{CC}_{6} \mathrm{H}_{4} \mathrm{Cl}^{+}\right] .-\mathrm{C}_{20} \mathrm{H}_{17} \mathrm{ClN}_{2} \mathrm{O}_{3} \mathrm{~S}$ (400.9): calcd. C 59.92, H 4.27, N 6.99; found C 59.70, 4.04, 6.67 .

Using microwaves $114 \mathrm{mg}(57 \%)$ of $\mathbf{3 7 b}$ was obtained according to GP 4 which has same physical datas as given above.

6-Benzenesulfonyl-2-(o-bromophenyl)-5,6,7,8-tetrahydroquinazolin-4(3H)-one (37c): The crude product obtained from 3-(o-bromophenyl)-2,4-diazabicyclo-[4.2.0]octa-1(6),2-dien-5-one (35c, 1.10 $\mathrm{g}, 3.60 \mathrm{mmol})$ and phenyl vinyl sulfone $(2.32 \mathrm{~g}, 12.0 \mathrm{mmol})$, according to GP 3 was purified by column chromatagraphy $\left(R_{\mathrm{f}}=0.43, \mathrm{Et}_{2} \mathrm{O} / \mathrm{MeOH}=25: 1,3 \times 30 \mathrm{~cm}, 50 \mathrm{~g} \mathrm{SiO}_{2}\right)$ to yield $683 \mathrm{mg}$ 
(43\%) of 37c as a white solid, m.p. $221-22{ }^{\circ} \mathrm{C} .-\mathrm{IR}(\mathrm{KBr}): v=3064 \mathrm{~cm}^{-1}, 2932,1653,1604$, 1544, 1447, 1301, 1147, 1084, 764, 690. ${ }^{1} \mathrm{H}-\mathrm{NMR}\left(300 \mathrm{MHz}, \mathrm{CDCl}_{3}\right): \delta=1.80-2.01(\mathrm{~m}, 1 \mathrm{H})$, 2.42-3.01 [m, $1 \mathrm{H}, \mathrm{C}(6)-\mathrm{H}], 7.35-7.45$ (m, $2 \mathrm{H}$, aryl-H), 7.537.75 (m, 5 H, aryl-H), 7.95-8.01 (m, 2 H, aryl-H), 11.04 (br. s, $1 \mathrm{H}, \mathrm{NH}) .-{ }^{13} \mathrm{C}-\mathrm{NMR}\left(50.3 \mathrm{MHz}, \mathrm{CDCl}_{3}\right): \delta=21.5\left(-, \mathrm{CH}_{2}\right)$, $22.1\left(-, \mathrm{CH}_{2}\right), 30.6\left(-, \mathrm{CH}_{2}\right), 58.6(+, \mathrm{C}-6), 117.6\left(\mathrm{C}_{\text {qaut }}, \mathrm{C}-4 \mathrm{a}\right)$, $120.9\left(\mathrm{C}_{\text {qaut }}, \mathrm{C}-\mathrm{Br}\right), 127.8$ (+, aryl-C), $129.0(+$, aryl-C), 129.3

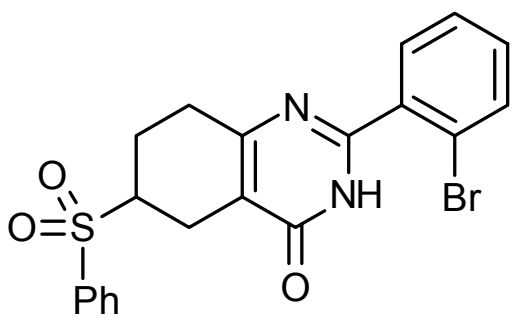
(+, aryl-C), 130.9 (+, aryl-C), 132.1 (+, aryl-C), $133.6\left(+\right.$, aryl-C), 134.0 (+, aryl-C), $134.6\left(\mathrm{C}_{\mathrm{ipso}}\right)$, $136.8\left(\mathrm{C}_{\text {ipso }}\right), 154.3\left(\mathrm{C}_{\text {qaut }}, \mathrm{C}-8 \mathrm{a}\right), 160.3\left(\mathrm{C}_{\text {qaut }}, \mathrm{C}-2 / \mathrm{C}-4\right), 162.4\left(\mathrm{C}_{\mathrm{qaut}}, \mathrm{C}-4 / \mathrm{C}-2\right) .-\mathrm{MS}(70 \mathrm{eV}), \mathrm{m} / z$ (\%): 446/444 (1/1) $\left[\mathrm{M}^{+}\right], 318 / 316$ (9/10), 304/302 (95/100) [ $\left.\mathrm{M}^{+}-\mathrm{HSO}_{2} \mathrm{Ph}\right], 260 / 258$ (11/11) 141 (18) $\left[\mathrm{PhSO}_{2}^{+}\right], 77$ (52) $\left[\mathrm{Ph}^{+}\right] .-\mathrm{C}_{20} \mathrm{H}_{17} \mathrm{BrN}_{2} \mathrm{O}_{3} \mathrm{~S}$ (445.3): calcd. C 53.93, H 3.85, N6.29; found C 54.22, H 3.71, N 6.34 .

Using microwaves $138 \mathrm{mg}$ (62\%) of $37 \mathrm{c}$ was obtained according to GP 4 which has same physical datas as given above.

6-Benzenesulfonyl-2-(o-fluorophenyl)-5,6,7,8-tetrahydroquinazolin-4(3H)-one (37d): The crude product obtained from 3-(o-fluorophenyl)-2,4-diazabicyclo-[4.2.0]octa-1(6),2- dien-5-one (35d, 648 $\mathrm{mg}, 3 \mathrm{mmol}$ ) and phenyl vinyl sulfone ( $2.01 \mathrm{~g}, 12.0 \mathrm{mmol}$ ), according to GP 3 was purified by column chromatagraphy $\left(R_{\mathrm{f}}\right.$ $=0.45, \mathrm{Et}_{2} \mathrm{O} / \mathrm{MeOH}=25: 1,3 \times 30 \mathrm{~cm}, 50 \mathrm{~g} \mathrm{SiO}_{2}$ ) to yield 881 $\mathrm{mg}(70 \%)$ of $\mathbf{3 7 d}$ as a white solid, m.p. $201-202^{\circ} \mathrm{C}$. - IR ( $\left.\mathrm{KBr}\right)$ : $v=3073 \mathrm{~cm}^{-1}, 2941,1672,1603,1558,1449,1308,1146$,

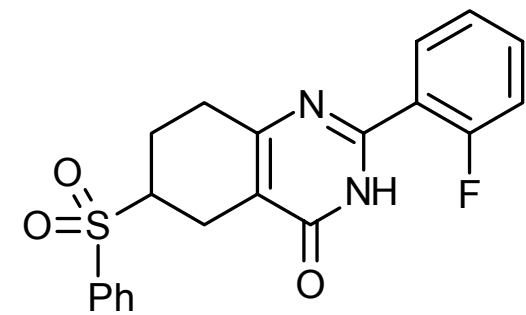
1084, 779, 689. $-{ }^{1} \mathrm{H}-\mathrm{NMR}\left(200 \mathrm{MHz}, \mathrm{CDCl}_{3}\right): \delta=1.81-2.12(\mathrm{~m}, 1 \mathrm{H}), 2.48-3.11(\mathrm{~m}, 5 \mathrm{H}), 3.22-$ 3.40 [m, 1 H, C(6)-H], 7.10-7.38 (m, 3 H, aryl-H), 7.50-7.81 (m, 4 H, aryl-H), 7.95-8.20 (m, 2 H, aryl-H), 10.67 (br. s, $1 \mathrm{H}, \mathrm{NH}) .-{ }^{13} \mathrm{C}-\mathrm{NMR}\left(50.3 \mathrm{MHz}, \mathrm{CDCl}_{3}\right): \delta=21.4\left(-, \mathrm{CH}_{2}\right), 22.3\left(-, \mathrm{CH}_{2}\right)$, $30.7\left(-, \mathrm{CH}_{2}\right), 59.0(+), 116.6\left(+, \mathrm{d},{ }^{2} J_{\mathrm{C}-\mathrm{F}}=22.9 \mathrm{~Hz}\right), 117.7(\mathrm{C}-4 \mathrm{a}), 119.4\left(\mathrm{~d},{ }^{2} J_{\mathrm{C}-\mathrm{F}}=9.2 \mathrm{~Hz}, \mathrm{C}_{\mathrm{ipso}}\right)$, $125.1\left(+, \mathrm{d},{ }^{3} J_{\mathrm{C}-\mathrm{F}}=3.1 \mathrm{~Hz}\right), 129.0(+), 129.3\left(+\right.$, aryl-C), $131.0\left(+, \mathrm{d},{ }^{4} J_{\mathrm{C}-\mathrm{F}}=1.1 \mathrm{~Hz}\right.$, aryl-C), 133.7 $\left(+, \mathrm{d},{ }^{3} J_{\mathrm{C}-\mathrm{F}}=9.2 \mathrm{~Hz}\right.$, aryl-C), $133.9\left(+\right.$, aryl-C), $136.8\left(\mathrm{C}_{\mathrm{ipso}}\right.$, aryl-C), $150.5\left(\mathrm{~d},{ }^{3} J_{\mathrm{C}-\mathrm{F}}=1.5 \mathrm{~Hz}\right.$, arylC), $160.1\left(\mathrm{C}_{\text {qaut }}, \mathrm{C}-2\right), 160.5\left(\mathrm{~d},{ }^{1} J_{\mathrm{C}-\mathrm{F}}=250.6 \mathrm{~Hz}, \mathrm{C}-\mathrm{F}\right), 163.0\left(\mathrm{C}_{\text {qaut }}, \mathrm{C}-4\right) .-\mathrm{MS}(70 \mathrm{eV}), \mathrm{m} / z(\%)$ : 384 (1) $\left[\mathrm{M}^{+}\right], 243(23)\left[\mathrm{M}^{+}-\mathrm{SO}_{2} \mathrm{Ph}\right], 242$ (100) $\left[\mathrm{M}^{+}-\mathrm{HSO}_{2} \mathrm{Ph}\right], 122$ (16), 77 (22) $\left[\mathrm{Ph}^{+}\right]$. $\mathrm{C}_{20} \mathrm{H}_{10} \mathrm{FN}_{2} \mathrm{O}_{3} \mathrm{~S}$ (384.4): calcd. C 62.49, H 4.46, N 7.29; found C 62.30, H 4.30, N 7.11. 
Using microwaves $110 \mathrm{mg}(57 \%)$ of $\mathbf{3 7 d}$ was obtained according to GP 4 which has same physical datas as given above.

6-Benzenesulfonyl-2-(p-benzyloxyphenyl)-5,6,7,8-tetrahydroquinazolin-4(3H)one (37e): The crude product obtained from 3-(p-benzyloxyphenyl)-2,4-diazabicyclo[4.2.0]octa-1(6),2-dien-5-one (35e, $652 \mathrm{mg}, 2.1 \mathrm{mmol})$ and phenyl vinyl sulfone $(2.43 \mathrm{~g}$, $8.50 \mathrm{mmol}$ ), according to GP 3 was purified by column chromatagraphy $\left(R_{\mathrm{f}}=0.40, \mathrm{DCM} / \mathrm{MeOH}=25: 1,3 \times 30\right.$ $\left.\mathrm{cm}, 50 \mathrm{~g} \mathrm{SiO}_{2}\right)$ to yield $670 \mathrm{mg}(66 \%)$ of $37 \mathrm{e}$ as a white solid, m.p. $>250{ }^{\circ} \mathrm{C}$. - IR $(\mathrm{KBr}): v=3072 \mathrm{~cm}^{-1}, 2939$, $1649,1607,1547,1516,1304,1259,1144,1085,837$,

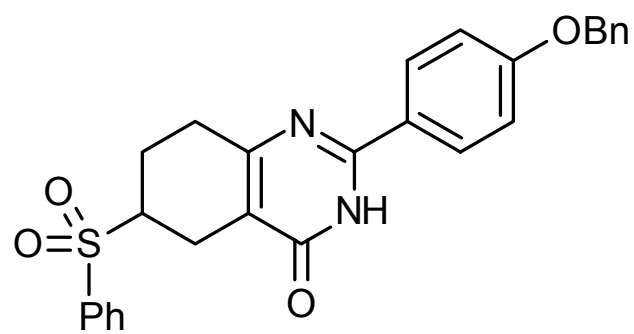
742, 687. $-{ }^{1} \mathrm{H}-\mathrm{NMR}\left(300 \mathrm{MHz},\left[\mathrm{D}_{6}\right] \mathrm{DMSO}\right): \delta=1.71-1.84(\mathrm{~m}, 1 \mathrm{H}), 2.18-2.31(\mathrm{~m}, 1 \mathrm{H}), 2.40$ $2.58(\mathrm{~m}, 1 \mathrm{H}), 2.64-2.78(\mathrm{~m}, 3 \mathrm{H}), 3.55-3.73$ [m, $1 \mathrm{H}, \mathrm{C}(6)-\mathrm{H}], 5.21$ (s, $2 \mathrm{H}), 7.05-7.12$ (m, $2 \mathrm{H}$, aryl-H), 7.31-7.48 (m, 5 H, aryl-H), 7.64-7.8 (m, 3 H, aryl-H), 7.90-7.98 (m, 2 H, aryl-H), 8.048.11 (m, $2 \mathrm{H}$, aryl-H), 12.15 (br. s, $1 \mathrm{H}, \mathrm{NH}) .-{ }^{13} \mathrm{C}-\mathrm{NMR}$ (50.3 MHz, [D $]$ DMSO): $\delta=21.1(-$,

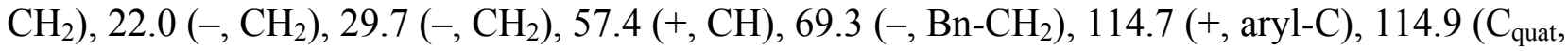
C-4a), $124.3\left(\mathrm{C}_{\text {quat, }}, \mathrm{C}_{\text {ipso }}\right), 127.6$ (+, aryl-C), 127.8 (+, aryl-C), 128.4 (+, aryl-C), 128.4 (+, aryl-C), $129.2\left(+\right.$, aryl-C), 129.5 (+, aryl-C), 134.0 (+, aryl-C), $136.5\left(\mathrm{C}_{\mathrm{ipso}}\right), 136.9\left(\mathrm{C}_{\mathrm{ipso}}\right), 153.8\left(\mathrm{C}_{\text {quat }}, \mathrm{C}-\right.$

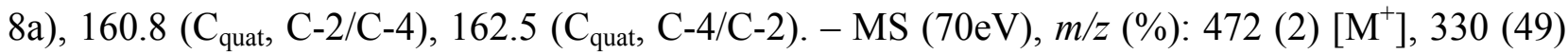
$\left[\mathrm{M}^{+}-\mathrm{SO}_{2} \mathrm{Ph}\right], 91(100)\left[\mathrm{C}_{7} \mathrm{H}_{7}^{+}\right] .-\mathrm{C}_{27} \mathrm{H}_{24} \mathrm{~N}_{2} \mathrm{O}_{4} \mathrm{~S}$ (472.6): calcd. C 68.63, H 5.12, N 5.93; found $\mathrm{C}$ 68.80, H 5.04, N 6.08 .

Using microwaves $149 \mathrm{mg}(63 \%)$ of $\mathbf{3 7 e}$ was obtained according to GP 4 which has same physical datas as given above.

6-Benzenesulfonyl-2-(o-biphenyl)-5,6,7,8-tetrahydroquinazolin-4(3H)-one (37f): The crude product obtained from 3-(o-biphenyl)-2,4-diazabicyclo[4.2.0]-octa-1(6),2-dien-5-one (35f, $501 \mathrm{mg}, 1.83$ $\mathrm{mmol}$ ) and phenyl vinyl sulfone (1.23 $\mathrm{g}, 7.32 \mathrm{mmol})$, according to GP 3 was purified by column chromatagraphy $\left(R_{\mathrm{f}}=0.31\right.$, $\mathrm{Et}_{2} \mathrm{O} / \mathrm{MeOH}=25: 1,3 \times 30 \mathrm{~cm}, 50 \mathrm{~g} \mathrm{SiO}_{2}$ ) to yield $522 \mathrm{mg}$ (65\%) of 37f as a white solid, m.p. $194-195{ }^{\circ} \mathrm{C}$. - IR (KBr): $v=3059 \mathrm{~cm}^{-1}, 3059,2933,1647,1546,1447,1320,1302$,

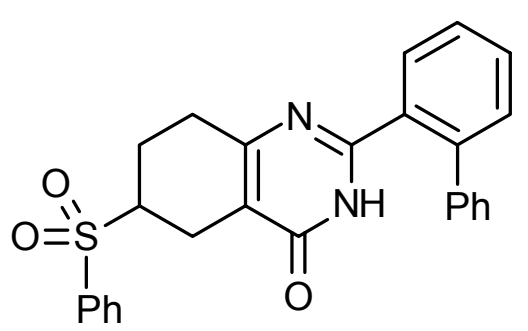
1145, 1085, 742, 688. $-{ }^{1} \mathrm{H}-\mathrm{NMR}\left(250 \mathrm{MHz}, \mathrm{CDCl}_{3}\right): \delta=1.82-1.95$ (m, $\left.1 \mathrm{H}\right), 2.42-2.95(\mathrm{~m}, 5 \mathrm{H})$, 
3.21-3.31 [m, $1 \mathrm{H}, \mathrm{C}(6)-\mathrm{H}], 7.20-7.39$ (m, $5 \mathrm{H}$, aryl-H), 7.42-7.78 (m, $7 \mathrm{H}$, aryl-H), 7.90-8.02 (m, $2 \mathrm{H}$, aryl-H), 9.1 (br. s, $1 \mathrm{H}, \mathrm{NH}) .-{ }^{13} \mathrm{C}-\mathrm{NMR}\left(62.9 \mathrm{MHz}, \mathrm{CDCl}_{3}\right): \delta=21.3\left(-, \mathrm{CH}_{2}\right), 22.1(-$, $\left.\mathrm{CH}_{2}\right), 30.5\left(-, \mathrm{CH}_{2}\right), 59.1(+, \mathrm{CH}), 116.5\left(\mathrm{C}_{\text {quat }}, \mathrm{C}-4 \mathrm{a}\right), 127.7$ (+, aryl-C), 128.1 (+, aryl-C), 128.4 (+, aryl-C), 128.9 (+, aryl-C), 129.0 (+, aryl-C), 130.0 (+, aryl-C), 130.9 (+, aryl-C), 131.0 (+, aryl-C), 131.7 (+, aryl-C), $133.9\left(\mathrm{C}_{\mathrm{ipso}}\right), 136.9\left(\mathrm{C}_{\mathrm{ipso}}\right), 139.2\left(\mathrm{C}_{\mathrm{ipso}}\right), 140.7\left(\mathrm{C}_{\mathrm{ipso}}\right), 155.7\left(\mathrm{C}_{\text {quat }}, \mathrm{C}-\right.$ 8a), 160.1 ( $\left.\mathrm{C}_{\text {quat }}, \mathrm{C}-2 / \mathrm{C}-4\right), 162.0\left(\mathrm{C}_{\text {quat }}, \mathrm{C}-4 / \mathrm{C}-2\right) .-\mathrm{MS}(70 \mathrm{eV}), \mathrm{m} / z(\%)$ : $442(4)\left[\mathrm{M}^{+}\right], 300(100)$ $\left[\mathrm{M}^{+}-\mathrm{SO}_{2} \mathrm{Ph}\right], 180$ (16), 122 (17). $-\mathrm{C}_{26} \mathrm{H}_{22} \mathrm{~N}_{2} \mathrm{O}_{3} \mathrm{~S}$ (442.5): calcd. C 70.57, H 5.01, N 6.33; found C 70.53, H 4.98, N 6.04.

Using microwaves $139 \mathrm{mg}(63 \%)$ of $\mathbf{3 7 f}$ was obtained according to GP 4 which has same physica datas as given above.

\section{6-Benzenesulfonyl-2-(5-chloro-benzo[b] thiophene-3-yl)-5,6,7,8-tetrahydroquinazolin-4(3H)-one}

(37g): The crude product obtained from 3-(5-chloro-benzo[b]thiophene)-2,4-diazabicyclo[4.2.0]octa-1(6),2-dien-5-one (35g, $180 \mathrm{mg}, 0.63 \mathrm{mmol})$ and phenyl vinyl sulfone (420 mg, $4.00 \mathrm{mmol}$ ), according to GP 3 was purified by column chromatagraphy $\left(R_{\mathrm{f}}=0.45, \mathrm{DCM} / \mathrm{MeOH}=\right.$ $\left.25: 1,1.5 \times 15 \mathrm{~cm}, 20 \mathrm{~g} \mathrm{SiO}_{2}\right)$ to yield $256 \mathrm{mg}(88 \%)$ of $\mathbf{3 7} \mathrm{g}$ as a pale yellow solid, m.p. $>250{ }^{\circ} \mathrm{C}$. - IR $(\mathrm{KBr}): v=3084 \mathrm{~cm}^{-1}$, 1640, 1563, 1417, 1312, 1147, 1078, 806. - ${ }^{1} \mathrm{H}-\mathrm{NMR}(200$ MHz, [D $\left.\left.\mathrm{D}_{6}\right] \mathrm{DMSO}\right): \delta=1.60-1.89(\mathrm{~m}, 2 \mathrm{H}), 2.08-2.56(\mathrm{~m}, 2 \mathrm{H})$,

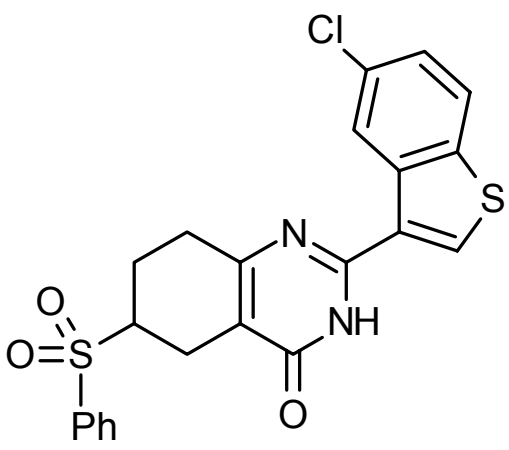
2.59-3.02 (m, 2 H), 3.48-3.67 [m, 1 H, C(6)-H], 7.41-7.54 (m, 1 H, aryl-H), 7.68-7.80 (m, 3 H, aryl-H), 7.85-8.00 (m, $2 \mathrm{H}$, aryl-H), 8.04-8.19 (m, $2 \mathrm{H}$, aryl-H), 8.52-8.73 (m, $1 \mathrm{H}$, aryl-H). ${ }^{13} \mathrm{C}-\mathrm{NMR}\left(62.9 \mathrm{MHz}, \mathrm{CDCl}_{3}\right): \delta=21.0\left(-, \mathrm{CH}_{2}\right), 21.8\left(-, \mathrm{CH}_{2}\right), 29.8\left(-, \mathrm{CH}_{2}\right), 57.4(+$, aryl-C), 116.3 (C-4a), 124.4 (+, 2 C, aryl-C), 125.1 (+, aryl-C), 126.8 (-, aryl-C), 128.5 (+, 2 C, aryl-C), 129.5 (+, 2 C, aryl-C), 130.2 (+, aryl-C), 134.1 (+, aryl-C), 134.4 (+, aryl-C), $136.9\left(\mathrm{C}_{\mathrm{ipso}}\right), 137.4$ $\left(\mathrm{C}_{\text {ipso }}\right), 138.2\left(\mathrm{C}_{\text {ipso }}\right), 150.0\left(\mathrm{C}_{\text {quat }}, \mathrm{C}-8 \mathrm{a}\right), 158.9$ ( $\left.\mathrm{C}_{\text {quat }}, \mathrm{C}-2 / \mathrm{C}-4\right), 162.0\left(\mathrm{C}_{\text {quat }}, \mathrm{C}-4 / \mathrm{C}-2\right) .-\mathrm{MS}(70$ eV), $m / z$ (\%): 456 (1) $\left[\mathrm{M}^{+}\right], 314$ (100) $\left[\mathrm{M}^{+}-\mathrm{SO}_{2} \mathrm{Ph}\right], 125$ (20), 77 (57). - $\mathrm{C}_{22} \mathrm{H}_{17} \mathrm{ClN}_{2} \mathrm{O}_{3} \mathrm{~S}_{2}$ (457.0): calcd. C 57.83, H 3.75, N 6.13; found C 57.52, H 3.55, N 6.02.

Using microwaves $118 \mathrm{mg}(52 \%)$ of $\mathbf{3 7} \mathbf{g}$ was obtained according to GP 4 which has same physical datas as given above. 
6-Benzenesulfonyl-2-(methylthio)-5,6,7,8-tetrahydroquinazolin-4(3H)-one (37h): The crude product obtained from $35 \mathrm{~h}(168 \mathrm{mg}, 1.00 \mathrm{mmol})$ and phenyl vinyl sulfone (672 $\mathrm{g}, 7.32 \mathrm{mmol})$, according to GP 3 was purified by column chromatagraphy $\left(R_{\mathrm{f}}=\right.$ $\left.0.41, \mathrm{DCM} / \mathrm{MeOH}=25: 1,1.5 \times 20 \mathrm{~cm}, 25 \mathrm{~g} \mathrm{SiO}_{2}\right)$ to yield 189 $\mathrm{mg}(56 \%)$ of $\mathbf{3 7 h}$ as a white solid, m.p. $>250{ }^{\circ} \mathrm{C}$. $-\mathrm{IR}(\mathrm{KBr})$ : $v=3059 \mathrm{~cm}^{-1}, 2918,2847,1644,1576,1448,1315,1150,1088$,

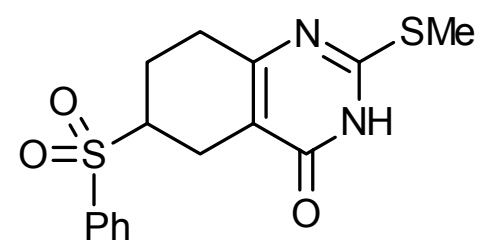
723, 689. - ${ }^{1} \mathrm{H}-\mathrm{NMR}\left(200 \mathrm{MHz},\left[\mathrm{D}_{6}\right] \mathrm{DMSO}\right): \delta=1.57-1.79$ (m, $\left.1 \mathrm{H}\right), 2.11-2.38$ (m, $\left.3 \mathrm{H}\right), 2.43$ (s, $3 \mathrm{H}), 2.58-2.70(\mathrm{~m}, 2 \mathrm{H}), 3.52-3.73[\mathrm{~m}, 1 \mathrm{H}, \mathrm{C}(6)-\mathrm{H}]$, 7.60-7.81 (m, 3 H, aryl-H), 7.85-7.95 (m, $2 \mathrm{H}$, aryl-H), 12.56 (br. s, $1 \mathrm{H}, \mathrm{NH}) .-{ }^{13} \mathrm{C}-\mathrm{NMR}\left(50.3 \mathrm{MHz},\left[\mathrm{D}_{6}\right] \mathrm{DMSO}\right): \delta=12.5\left(+, \mathrm{CH}_{3}\right)$, $21.0\left(-, \mathrm{CH}_{2}\right), 21.4\left(-, \mathrm{CH}_{2}\right), 29.8\left(-, \mathrm{CH}_{2}\right), 57.3(+, \mathrm{CH}), 117.2\left(\mathrm{C}_{\text {quat }}, \mathrm{C}-4 \mathrm{a}\right), 128.4$ (+, aryl-C), 129.5 (+, aryl-C), 134.0 (+, aryl-C), $136.9\left(\mathrm{C}_{\text {ipso }}\right) 154.3$ ( $\left.\mathrm{C}_{\text {quat }}, \mathrm{C}-8 \mathrm{a}\right), 159.8\left(\mathrm{C}_{\text {quat }}, \mathrm{C}-2 / \mathrm{C}-4\right), 162.7$ $\left(\mathrm{C}_{\text {quat }}, \mathrm{C}-4 / \mathrm{C}-2\right)$. - MS (70 eV), m/z (\%): 336 (2) $\left[\mathrm{M}^{+}\right], 195$ (18), 194 (100) $\left[\mathrm{M}^{+}-\mathrm{SO}_{2} \mathrm{Ph}\right] .-$ $\mathrm{C}_{15} \mathrm{H}_{16} \mathrm{~N}_{2} \mathrm{O}_{3} \mathrm{~S}_{2}$ (336.4): calcd. C 53.55, H 4.79, N 8.33; found C 53.56, H 4.52, N 8.20.

Using microwaves $106 \mathrm{mg}$ (63\%) of $\mathbf{3 7 h}$ was obtained according to GP 4 which has same physical datas as given above.

General Procedure for elimination of solfonyl group (GP 5): 2-Aryl-7,8-dihydroquinazolin4(3H)-one (38): To a suspension of above sulfone 37 in THF, 3 equiv. of $\mathrm{KO} t \mathrm{Bu}$ were added and the resulting solution was stirred for $2 \mathrm{~h}$ at room temperature, poured into a separating funnel with sat. aq. $\mathrm{NH}_{4} \mathrm{Cl}$ solution $(10 \mathrm{~mL})$ and extracted with $\mathrm{DCM}(3 \times 20 \mathrm{~mL})$. The organic layer was dried over $\mathrm{MgSO}_{4}$, after filtration, the solvent was evaporated in vacuum to yield $\mathbf{3 8}$ which was used for the next reaction without further purification.

2-Phenyl-7,8-dihydroquinazolin-4(3H)-one (38a): From the sulfone 37a (366 $\mathrm{mg}, 1.00 \mathrm{mmol}$ ) and $\mathrm{KO} t \mathrm{Bu}(336 \mathrm{mg}, 3.00 \mathrm{mmol})$ were obtained according to GP 5, $210 \mathrm{mg}(96 \%)$ of 38a as a pale yellow solid $\left(R_{\mathrm{f}}=0.5\right.$, hexane/ethylacetate $\left.=1: 1\right)$, m.p. $241{ }^{\circ} \mathrm{C} .-$ IR $(\mathrm{KBr}): v=3032 \mathrm{~cm}^{-1}, 2932,1653,1505,1317,930,718 .-{ }^{1} \mathrm{H}-\mathrm{NMR}$ $\left(250 \mathrm{MHz}, \mathrm{CDCl}_{3}\right): \delta=2.42-2.58[\mathrm{~m}, 2 \mathrm{H}, \mathrm{C}(7)-\mathrm{H}], 2.89[\mathrm{t}, J=9.7 \mathrm{~Hz}$, $2 \mathrm{H}, \mathrm{C}(8)-\mathrm{H}], 6.04[\mathrm{dt}, J=9.7,4.3 \mathrm{~Hz}, 1 \mathrm{H}, \mathrm{C}(6)-\mathrm{H}], 6.73$ [dt, $J=9.7$, $1.8 \mathrm{~Hz}, 1 \mathrm{H}, \mathrm{C}(5)-\mathrm{H}]$, 7.48-7.56 (m, $3 \mathrm{H}$, aryl-H), 8.14-8.22 (m, $2 \mathrm{H}$,

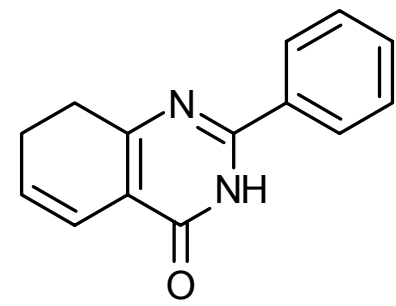
aryl-H), 12.58 (br. s, $1 \mathrm{H}, \mathrm{NH}) .-{ }^{13} \mathrm{C}-\mathrm{NMR}\left(62.9 \mathrm{MHz}, \mathrm{CDCl}_{3}\right): \delta=22.7\left(-, \mathrm{CH}_{2}\right), 29.6\left(-, \mathrm{CH}_{2}\right)$, 117.2 (C-4a), 119.5 (+, C-5), 127.4 (+, aryl-C), 127.6 (+, aryl-C), 128.9 (+, aryl-C), 131.7 (+, C- 
6), $132.1\left(\mathrm{C}_{\text {ipso }}\right), 154.6\left(\mathrm{C}_{\text {quat, }}, \mathrm{C}-8 \mathrm{a}\right), 161.4\left(\mathrm{C}_{\text {quat }}, \mathrm{C}-2 / \mathrm{C}-4\right), 161.9$ ( $\left.\mathrm{C}_{\text {quat }}, \mathrm{C}-4 / \mathrm{C}-2\right) .-\mathrm{MS}(70 \mathrm{eV})$, $m / z(\%): 224(100)\left[\mathrm{M}^{+}\right], 223$ (98), 180 (19) $\left[\mathrm{M}^{+}-\mathrm{CONH}_{2}\right], 104$ (14) $\left[\mathrm{PhCNH}^{+}\right], 77$ (20) $\left[\mathrm{Ph}^{+}\right] .-$ $\mathrm{C}_{14} \mathrm{H}_{12} \mathrm{~N}_{2} \mathrm{O}$ (224.3): calcd. C 74.98, H 5.39, N 12.55; found C 74.71, H 5.31, N 12.31.

2-(p-Chlorophenyl)-7,8-dihydroquinazolin-4(3H)-one (38b): From the sulfone 37b (200 mg, 0.50 $\mathrm{mmol})$ and $\mathrm{KO} t \mathrm{Bu}(168 \mathrm{mg}, 1.50 \mathrm{mmol})$ were obtained according to GP 5, $123 \mathrm{mg}(95 \%)$ of $\mathbf{3 8 b}$ as a pale yellow solid $\left(R_{\mathrm{f}}=0.48\right.$, hexane/ethylacetate $\left.=1: 1\right)$, m.p. $>$ $250{ }^{\circ} \mathrm{C}$. - IR (KBr): $v=3029 \mathrm{~cm}^{-1}, 2934,1652,1504,1389,1176$, 1091, 738. $-{ }^{1} \mathrm{H}-\mathrm{NMR}\left(250 \mathrm{MHz}, \mathrm{CDCl}_{3}\right): \delta=2.42-2.56[\mathrm{~m}, 2 \mathrm{H}$, $\mathrm{C}(7)-\mathrm{H}], 2.87$ [t, $J=9.6 \mathrm{~Hz}, 2 \mathrm{H}, \mathrm{C}(8)-\mathrm{H}], 6.06$ [dt, $J=9.5,4.3 \mathrm{~Hz}$, $1 \mathrm{H}, \mathrm{C}(6)-\mathrm{H}], 6.71$ [dt, $J=9.5,1.8 \mathrm{~Hz}, 1 \mathrm{H}, \mathrm{C}(5)-\mathrm{H}], 7.48-7.56$ (m,

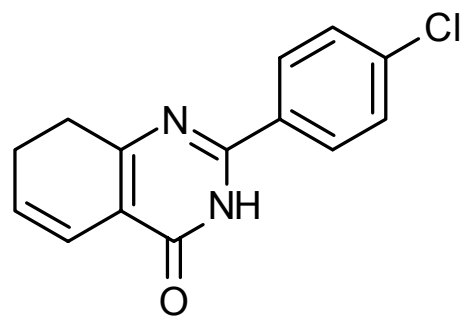
$2 \mathrm{H}), 8.16-8.27$ (m, $2 \mathrm{H}), 13.1$ (br. s, $1 \mathrm{H}, \mathrm{NH}) .-{ }^{13} \mathrm{C}-\mathrm{NMR}\left(62.9 \mathrm{MHz}, \mathrm{CDCl}_{3}\right): \delta=22.3(-$, $\left.\mathrm{CH}_{2}\right), 28.7$ (-, $\left.\mathrm{CH}_{2}\right), 116.8$ (C-4a), 119.6 (+, C-5), 127.7 (+), 128.9 (+, aryl-C), 129.5 (+, aryl-C), 131.7 (+, C-6), 136.5 ( $\left.\mathrm{C}_{\text {ipso }}\right), 153.3$ ( $\left.\mathrm{C}_{\text {quat }}, \mathrm{C}-8 \mathrm{a}\right), 159.6$ (C $\left.\mathrm{C}_{\text {quat }}, \mathrm{C}-2 / \mathrm{C}-4\right), 162.5$ ( $\left.\mathrm{C}_{\text {quat, }}, \mathrm{C}-4 / \mathrm{C}-2\right)$.

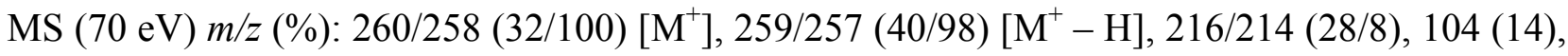
77 (20) $\left[\mathrm{Ph}^{+}\right] .-\mathrm{C}_{14} \mathrm{H}_{11} \mathrm{ClN}_{2} \mathrm{O}$ (258.7): calcd. C 65.00, H 4.29, N 10.83; found C 64.93, H 4.08, N 10.99 .

2-(o-Bromophenyl)-7,8-dihydroquinazolin-4(3H)-one (38c): From the sulfone 37c (400 mg, 0.90 $\mathrm{mmol}$ ) and $\mathrm{KO} t \mathrm{Bu}(302 \mathrm{mg}, 2.70 \mathrm{mmol}$ ) was obtained according to GP 5, $285 \mathrm{mg}(94 \%)$ of $\mathbf{3 8 c}$ as a pale yellow solid $\left(R_{\mathrm{f}}=0.55, \mathrm{Et}_{2} \mathrm{O}\right)$, m.p. $202{ }^{\circ} \mathrm{C} .-\mathrm{IR}(\mathrm{KBr}): v=3035$ $\mathrm{cm}^{-1}, 2836,1665,1491,1324,1183,928,767,735 .-{ }^{1} \mathrm{H}-\mathrm{NMR}(200$ $\left.\mathrm{MHz}, \mathrm{CDCl}_{3}\right): \delta=2.15-2.30[\mathrm{~m}, 2 \mathrm{H}, \mathrm{C}(7)-\mathrm{H}], 2.85[\mathrm{t}, J=9.1 \mathrm{~Hz}, 2 \mathrm{H}$, C(8)-H], 6.04 [dt, $J=9.6,4.4 \mathrm{~Hz}, 1 \mathrm{H}, \mathrm{C}(6)-\mathrm{H}], 6.59$ [dt, $J=9.6,1.7$ $\mathrm{Hz}, 1 \mathrm{H}, \mathrm{C}(5)-\mathrm{H}], 7.28-7.81$ (m, $4 \mathrm{H}$ ), 11.55 (br. s, $1 \mathrm{H}, \mathrm{N}-\mathrm{H}) .-{ }^{13} \mathrm{C}-$

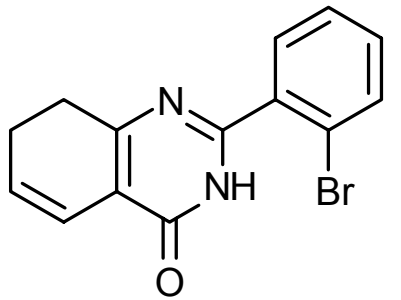
$\operatorname{NMR}\left(50.3 \mathrm{MHz}, \mathrm{CDCl}_{3}\right): \delta=22.5\left(-, \mathrm{CH}_{2}\right), 29.0\left(-, \mathrm{CH}_{2}\right), 118.0(\mathrm{C}-$ 8a), 119.3 (+, C-5), 121.1 ( $\left.\mathrm{C}_{\text {quat }}, \mathrm{C}-\mathrm{Br}\right), 127.7$ (+, C-6), 127.9 (+, aryl-C), 131.1 (+, aryl-C), 131.8 (+, aryl-C), 133.7 (+, aryl-C), 134.3 ( $\left.\mathrm{C}_{\text {ipso }}\right), 154.4$ (C $\left.\mathrm{C}_{\text {quat }}, \mathrm{C}-8 \mathrm{a}\right), 160.6\left(\mathrm{C}_{\text {quat }}, \mathrm{C}-2 / \mathrm{C}-4\right), 160.6\left(\mathrm{C}_{\text {quat }}\right.$, C-4/C-2). - MS (70 eV), m/z (\%): 304/302 (96/100) [M+], 203/301 (98/80), 259/257 (24/26), 102 (28). $-\mathrm{C}_{14} \mathrm{H}_{11} \mathrm{BrN}_{2} \mathrm{O}$ (303.2): calcd. C 55.47, H 3.66, N 9.24; found C 55.22, H 3.70, N 9.03. 
2-(o-Fluorophenyl)-7,8-dihydroquinazolin-4(3H)-one (38d): From the sulfone 37d (384 mg, 1.00 $\mathrm{mmol})$ and $\mathrm{KO} t \mathrm{Bu}(336 \mathrm{mg}, 3.00 \mathrm{mmol})$ were obtained according to GP 5, $223 \mathrm{mg}(92 \%)$ of $\mathbf{3 8 d}$ as a pale yellow solid $\left(R_{\mathrm{f}}=0.51, \mathrm{Et}_{2} \mathrm{O}\right)$, m.p. $191{ }^{\circ} \mathrm{C}$. $-\mathrm{IR}(\mathrm{KBr})$ : $v=3043 \mathrm{~cm}^{-1}, 2934,2886,1653,1559,1327,1220,1181,1122,774$. ${ }^{1} \mathrm{H}-\mathrm{NMR}\left(250 \mathrm{MHz}, \mathrm{CDCl}_{3}\right): \delta=2.38-2.52[\mathrm{~m}, 2 \mathrm{H}, \mathrm{C}(7)-\mathrm{H}], 2.85[\mathrm{t}, J$ $=9.4 \mathrm{~Hz}, 2 \mathrm{H}, \mathrm{C}(8)-\mathrm{H}], 5.98\left[\mathrm{dt},{ }^{3} J=9.4,4.4 \mathrm{~Hz}, 1 \mathrm{H}, \mathrm{C}(6)-\mathrm{H}\right], 6.61$ [dt, $J=9.5,1.7 \mathrm{~Hz}, 1 \mathrm{H}, \mathrm{C}(5)-\mathrm{H}], 7.12-7.35(\mathrm{~m}, 2 \mathrm{H}), 7.41-7.54(\mathrm{~m}, 1$ H), 7.98-8.12 (m, $1 \mathrm{H}), 11.51$ (br. s, $1 \mathrm{H}, \mathrm{NH}) .-{ }^{13} \mathrm{C}-\mathrm{NMR}(62.9 \mathrm{MHz}$,

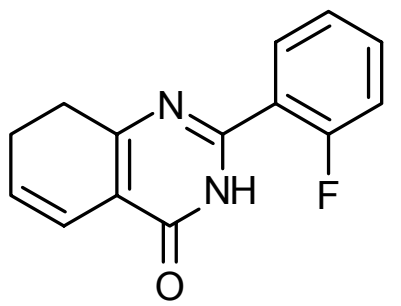
$\left.\mathrm{CDCl}_{3}\right): \delta=22.5\left(-, \mathrm{CH}_{2}\right), 29.0\left(-, \mathrm{CH}_{2}\right), 116.5\left(+, \mathrm{d},{ }^{2} J_{\mathrm{C}-\mathrm{F}}=22.7 \mathrm{~Hz}\right), 118.0(\mathrm{C}-4 \mathrm{a}), 119.4(+, \mathrm{C}-$ 5), $120.1\left(\mathrm{C}_{\text {quat }}, \mathrm{d},{ }^{2} J_{\mathrm{C}-\mathrm{F}}=9.0 \mathrm{~Hz}\right.$, aryl-C), $124.8\left(+, \mathrm{d},{ }^{3} J_{\mathrm{C}-\mathrm{F}}=3.1 \mathrm{~Hz}\right.$, aryl-C), $127.8(+, \mathrm{C}-6), 133.3$ $\left(+, \mathrm{d},{ }^{3} J_{\mathrm{C}-\mathrm{F}}=9.2 \mathrm{~Hz}\right.$, aryl-C), $134.5\left(\mathrm{C}_{\mathrm{ipso}}\right), 155.3\left(\mathrm{C}_{\text {quat }}, \mathrm{d},{ }^{1} J_{\mathrm{C}-\mathrm{F}}=250.6 \mathrm{~Hz}\right), 157.5\left(\mathrm{C}_{\text {quat }}, \mathrm{C}-8 \mathrm{a}\right)$, 158.4 (C quat $\left._{1}, \mathrm{C}-2 / \mathrm{C}-4\right), 162.4$ (C $\left.\mathrm{C}_{\text {quat }}, \mathrm{C}-4 / \mathrm{C}-2\right) .-\mathrm{MS}$ (70 eV), m/z (\%): $242(100)\left[\mathrm{M}^{+}\right], 241$ (85), 198 (24), 102 (12). $-\mathrm{C}_{14} \mathrm{H}_{11} \mathrm{FN}_{2} \mathrm{O}$ (242.3): calcd. C 69.41, H 4.58, N 11.56; found $\mathrm{C} 69.22, \mathrm{H}$ $4.83, \mathrm{~N} 11.45$.

2-(p-Benzoyloxyphenyl)-7,8-dihydroquinazolin-4(3H)-one (38e): From the sulfone 37d (400 mg, $0.85 \mathrm{mmol})$ and $\mathrm{KO} t \mathrm{Bu}(285 \mathrm{mg}, 2.50 \mathrm{mmol})$ were obtained according to GP 5, $258 \mathrm{mg}(92 \%)$ of 38e as a pale yellow solid $\left(R_{\mathrm{f}}=0.62, \mathrm{DCM} / \mathrm{MeOH}=25: 1\right)$, m.p. $247{ }^{\circ} \mathrm{C}$. - IR (KBr): $v=3032 \mathrm{~cm}^{-1}, 2943,1646,1606,1512$, 1305, 999, 753, 677. $-{ }^{1} \mathrm{H}-\mathrm{NMR}\left(250 \mathrm{MHz}, \mathrm{CDCl}_{3}\right): \delta=2.40$ $2.58(\mathrm{~m}, 2 \mathrm{H}), 2.85[\mathrm{t}, J=9.1 \mathrm{~Hz}, 2 \mathrm{H}, \mathrm{C}(8)-\mathrm{H}], 5.15(\mathrm{~s}, 2 \mathrm{H})$, $6.01[\mathrm{dt}, J=9.6,4.4 \mathrm{~Hz}, 1 \mathrm{H}, \mathrm{C}(6)-\mathrm{H}], 6.7[\mathrm{dt}, J=9.6,1.7 \mathrm{~Hz}, 1$

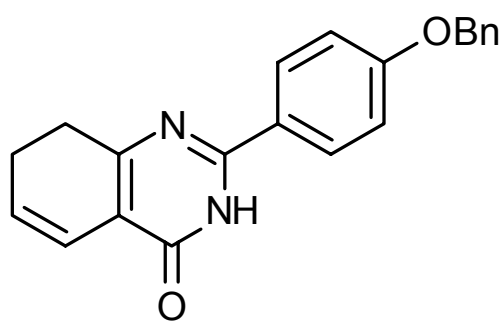
H, C(5)-H], 7.02-7.18 (m, 2 H, aryl-H), 7.30-7.62 (m, 5 H, aryl-H), 8.06-8.22 (m, 2 H, aryl-H), 12.23 (br. s, $1 \mathrm{H}, \mathrm{NH}) .-{ }^{13} \mathrm{C}-\mathrm{NMR}\left(75.5 \mathrm{MHz}, \mathrm{CDCl}_{3}\right): \delta=22.7\left(-, \mathrm{CH}_{2}\right), 28.6\left(-, \mathrm{CH}_{2}\right), 69.4(-$, Bn- $\left.\mathrm{CH}_{2}\right), 114.8$ (C-4a), 119.5 (+, C-5), 126.34 (+, C-6), 127.7 (+, aryl-C), 127.9 (+, aryl-C), 128.4 (+, aryl-C), 129.2 (+, aryl-C), $131.5\left(\mathrm{C}_{\mathrm{ipso}}\right), 136.5\left(\mathrm{C}_{\mathrm{ipso}}\right), 140.8\left(\mathrm{C}_{\mathrm{ipso}}\right), 155.9\left(\mathrm{C}_{\text {quat }}, \mathrm{C}-8 \mathrm{a}\right), 159.9$

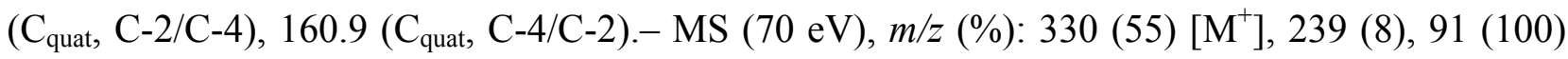
$\left[\mathrm{C}_{7} \mathrm{H}_{7}^{+}\right]$.

2-(o-Biphenyl)-7,8-dihydroquinazolin-4(3H)-one (38f): From the sulfone 37f (300 $\mathrm{mg}, 0.68$ $\mathrm{mmol}$ ) and $\mathrm{KO} t \mathrm{Bu}(224 \mathrm{mg}, 2.00 \mathrm{mmol})$ was obtained according to GP 5, $177 \mathrm{mg}(87 \%)$ of $\mathbf{3 8 f}$ as a pale yellow solid $\left(R_{\mathrm{f}}=0.55, \mathrm{Et}_{2} \mathrm{O}\right)$, m.p. $193{ }^{\circ} \mathrm{C} .-\mathrm{IR}(\mathrm{KBr}): v=3070 \mathrm{~cm}^{-1}, 2936,1634,1549$, 
1507, 1321, 1165, 979, 699. $-{ }^{1} \mathrm{H}-\mathrm{NMR}\left(250 \mathrm{MHz}, \mathrm{CDCl}_{3}\right): \delta=2.38-2.51[\mathrm{~m}, 2 \mathrm{H}, \mathrm{C}(7)-\mathrm{H}], 2.88$ [t, $J=9.2 \mathrm{~Hz}, 2 \mathrm{H}, \mathrm{C}(8)-\mathrm{H}$ ], 5.98 [dt, $J=9.5$ and $4.4 \mathrm{~Hz}, 1 \mathrm{H}, \mathrm{C}(6)-\mathrm{H}$ ], 6.57 [dt, $J=9.5,1.7 \mathrm{~Hz}, 1$ H, C(5)-H], 7.22-7.36 (m, 5 H), 7.44-7.62 (m, 3 H), 7.76-7.82 (m, 1 H) 9.51 (br. s, $1 \mathrm{H}, \mathrm{NH}) .-{ }^{13} \mathrm{C}-\mathrm{NMR}\left(62.9 \mathrm{MHz}, \mathrm{CDCl}_{3}\right): \delta=22.5(-$, $\left.\mathrm{CH}_{2}\right), 28.7$ (-, $\left.\mathrm{CH}_{2}\right), 117.2$ (C-4a), 119.3 (+, C-5), 127.8 (+, C-6), 127.9 (+, 2 C, aryl-C), $128.6(+, 2$ C, aryl-C), 129.1 (+, 2 C, aryl-C), 130.2 (+, aryl-C), $130.9\left(+\right.$, aryl-C), $131.1\left(+\right.$, aryl-C), $131.5\left(\mathrm{C}_{\mathrm{ipso}}\right), 139.1\left(\mathrm{C}_{\mathrm{ipso}}\right)$,

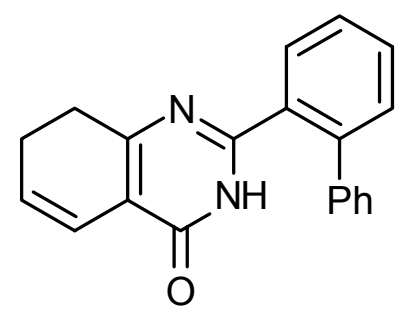
$140.8\left(\mathrm{C}_{\text {ipso }}\right), 155.8$ ( $\left.\mathrm{C}_{\text {quat, }}, \mathrm{C}-8 \mathrm{a}\right), 159.8$ ( $\left.\mathrm{C}_{\text {quat }}, \mathrm{C}-2 / \mathrm{C}-4\right), 160.0\left(\mathrm{C}_{\text {quat, }}, \mathrm{C}-4 / \mathrm{C}-2\right) .-\mathrm{MS}(70 \mathrm{eV}), \mathrm{m} / \mathrm{z}$ (\%): $301(28)\left[\mathrm{M}^{+}+1\right], 300(100)\left[\mathrm{M}^{+}\right], 299$ (40), 180 (38), 122 (43), 77 (78). - $\mathrm{C}_{20} \mathrm{H}_{16} \mathrm{~N}_{2} \mathrm{O}$ (300.4): calcd. C 79.98, H 5.37, N 9.33; found C 79.64, H 5.24, N 9.57.

2-Methylthio-7,8-dihydroquinazolin-4(3H)-one (38h): From the sulfone 37h (336 mg, $1.00 \mathrm{mmol})$ and $\mathrm{KO} t \mathrm{Bu}(336 \mathrm{mg}, 3.00 \mathrm{mmol}$ ) was obtained according to GP 5, $181 \mathrm{mg}(98 \%)$ of $\mathbf{3 8 h}$ as a pale yellow solid $\left(R_{\mathrm{f}}=0.45 \mathrm{Et}_{2} \mathrm{O}\right)$, m.p. $214{ }^{\circ} \mathrm{C}$. $-\mathrm{IR}(\mathrm{KBr}): v=2922 \mathrm{~cm}^{-1}$, 2836, 1641, 1623, 1540, 1271, 1138, 1203, 943. - ${ }^{1} \mathrm{H}-\mathrm{NMR}(250 \mathrm{MHz}$, $\left.\mathrm{CDCl}_{3}\right): \delta=2.38-2.49[\mathrm{~m}, 2 \mathrm{H}, \mathrm{C}(7)-\mathrm{H}], 2.60(\mathrm{~s}, 3 \mathrm{H}), 2.76[\mathrm{t}, J=9.3 \mathrm{~Hz}$, $2 \mathrm{H}, \mathrm{C}(8)-\mathrm{H}], 5.98$ [dt, $J=9.7$ and $4.3 \mathrm{~Hz}, 1 \mathrm{H}, \mathrm{C}(6)-\mathrm{H}], 6.61$ [dt, $J=9$.<smiles>CSc1nc2c(c(=O)[nH]1)C=CCC2</smiles>
and $1.8 \mathrm{~Hz}, 1 \mathrm{H}, \mathrm{C}(5)-\mathrm{H}], 12.9$ (br. s, $1 \mathrm{H}, \mathrm{NH}) .-{ }^{13} \mathrm{C}-\mathrm{NMR}(62.9 \mathrm{MHz}$, $\left.\mathrm{CDCl}_{3}\right): \delta=13.3\left(+, \mathrm{CH}_{3}\right), 22.4\left(-, \mathrm{CH}_{2}\right), 29.4\left(-, \mathrm{CH}_{2}\right), 114.6(\mathrm{C}-4 \mathrm{a}), 119.3(+, \mathrm{C}-5), 126.0(+, \mathrm{C}-$ 6), 157.0 ( $\left.\mathrm{C}_{\text {quata }}, \mathrm{C}-8 \mathrm{a}\right), 161.2\left(\mathrm{C}_{\text {quat }}, \mathrm{C}-2 / \mathrm{C}-4\right), 162.2$ (C $\left.\mathrm{C}_{\text {quat }}, \mathrm{C}-4 / \mathrm{C}-2\right)$. - MS (70 eV), m/z (\%): 194 (100) $\left[\mathrm{M}^{+}\right], 147$ (12), 121 (16), 92 (14). $-\mathrm{C}_{9} \mathrm{H}_{10} \mathrm{~N}_{2} \mathrm{OS}$ (194.3): calcd. C 55.65, H 5.19, N 14.42; found C 55.39, H 5.32, N 14.16.

General Procedure for hydrogenation reaction (GP 6):

2-Aryl-5, 6, 7,8-tetrahydroquinazolin-4(3H)-one (39): Into a $50 \mathrm{~mL}$ flame-dried flask flushed with nitrogen, $\mathrm{Pd} / \mathrm{C}(10 \% \mathrm{Pd} \mathrm{w} / \mathrm{w})$ was added, followed by $10 \mathrm{~mL}$ of $\mathrm{MeOH}$. This mixture was stirred under hydrogen atmosphere and after 30 min a solution of $\mathbf{3 8}$ in $\mathrm{MeOH}$ was added with a syringe and stirring was continued at room temperature until the reaction completed. Reaction mixture was filtered through a pad of Celite ${ }^{\circledR}$, and the solvent was removed in vacuo to yield $\mathbf{3 9}$ as a white solid. 
2-Phenyl-5,6,7,8-tetrahydroquinazolin-4(3H)-one (39a): The crude reaction mixture obtained from 38a (224 mg, $1.00 \mathrm{mmol}$ ), $10 \mathrm{mg}$ of $\mathrm{Pd} / \mathrm{C}$ in $20 \mathrm{~mL}$ of MeOH after $4 \mathrm{~h}$, according to GP 6 , afforded $206 \mathrm{mg}(91 \%)$ of 39a as a solid, m.p. $224{ }^{\circ} \mathrm{C}$. - IR (KBr): $v=2934 \mathrm{~cm}^{-1}, 2848,1634,1550,1319,1165,979,698 .-{ }^{1} \mathrm{H}-\mathrm{NMR}$ $\left(250 \mathrm{MHz}, \mathrm{CDCl}_{3}\right): \delta=1.75-1.91(\mathrm{~m}, 4 \mathrm{H}), 2.50-2.65(\mathrm{~m}, 2 \mathrm{H}), 2.71-$ 2.78 (m, $2 \mathrm{H}), 7.40-7.58$ (m, $3 \mathrm{H}$, aryl-H), 8.11-8.22 (m, $2 \mathrm{H}$, aryl-H), 12.4 (br. s, $1 \mathrm{H}, \mathrm{NH}) .-{ }^{13} \mathrm{C}-\mathrm{NMR}\left(62.9 \mathrm{MHz}, \mathrm{CDCl}_{3}\right): \delta=21.8(-$,

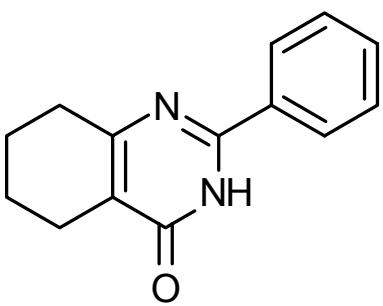
$\left.\mathrm{CH}_{2}\right), 21.9\left(-, \mathrm{CH}_{2}\right), 22.3\left(-, \mathrm{CH}_{2}\right), 31.9\left(-, \mathrm{CH}_{2}\right), 120.2\left(\mathrm{C}_{\text {quat }}, \mathrm{C}-4 \mathrm{a}\right), 127.5$ (+, aryl-C), $128.8(+$, aryl-C), 131.4 (+, aryl-C), $132.5\left(\mathrm{C}_{\text {ipso }}\right), 153.2$ ( $\left.\mathrm{C}_{\text {quat }}, \mathrm{C}-8 \mathrm{a}\right), 162.5$ (C $\left.\mathrm{C}_{\text {quat }}, \mathrm{C}-2 / \mathrm{C}-4\right), 164.5\left(\mathrm{C}_{\text {quat, }}, \mathrm{C}-\right.$

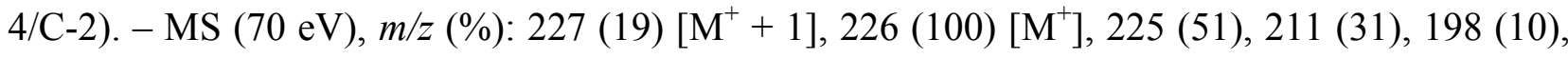
104 (21). $-\mathrm{C}_{14} \mathrm{H}_{14} \mathrm{~N}_{2} \mathrm{O}$ (226.3): calcd. C 74.31, H 6.24, N 12.38; found C 74.34, H 6.55, N 12.29.

2-(p-Chlorophenyl)-5,6,7,8-tetrahydroquinazolin-4(3H)-one (39b): The crude reaction mixture obtained from 38b $(51.6 \mathrm{mg}, 0.20 \mathrm{mmol}), 20 \mathrm{mg}$ of $\mathrm{Pd} / \mathrm{C}$ in $10 \mathrm{~mL}$ of AcOH after $8 \mathrm{~h}$ according to GP 6, afforded $49 \mathrm{mg}$ (94\%) of $\mathbf{3 9 b}$ as a solid, m.p. $=255^{\circ} \mathrm{C}$. - IR $(\mathrm{KBr}): v=3070 \mathrm{~cm}^{-1}, 2936,1634,1549,1507,1321,1014,929$, 699. $-{ }^{1} \mathrm{H}-\mathrm{NMR}\left(250 \mathrm{MHz}, \mathrm{CDCl}_{3}\right): \delta=1.74-1.93(\mathrm{~m}, 4 \mathrm{H}), 2.50$ $2.62(\mathrm{~m}, 2 \mathrm{H}), 2.66-2.79(\mathrm{~m}, 2 \mathrm{H}), 7.42-7.58(\mathrm{~m}, 2 \mathrm{H}$, aryl-H), 8.13-8.24 (m, $2 \mathrm{H}$, aryl-H), 12.92 (br. s, $1 \mathrm{H}, \mathrm{NH}) .-{ }^{13} \mathrm{C}-\mathrm{NMR}$

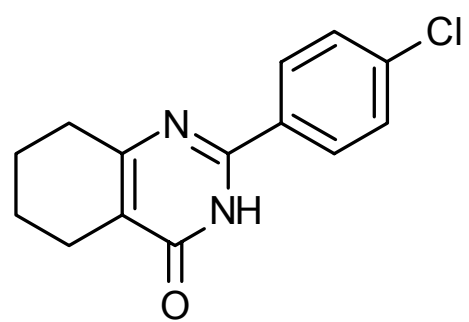
$\left(62.9 \mathrm{MHz}, \mathrm{CDCl}_{3}\right): \delta=21.8,\left(-, \mathrm{CH}_{2}\right), 21.9\left(-, \mathrm{CH}_{2}\right), 22.4\left(-, \mathrm{CH}_{2}\right), 32.0\left(-, \mathrm{CH}_{2}\right), 120.4\left(\mathrm{C}_{\text {quat }}\right.$, C-4a), 127.4 (+, aryl-C), 128.9 (+, aryl-C), 129.0 ( $\left.\mathrm{C}_{\text {ipso }}\right), 131.4\left(\mathrm{C}_{\text {quat, }}, \mathrm{C}-\mathrm{Cl}\right), 157.5$ ( $\mathrm{C}_{\text {quat, }}, \mathrm{C}-2 / \mathrm{C}-$ 8a), 157.6 ( $\left.\mathrm{C}_{\text {quat }}, \mathrm{C}-8 \mathrm{a} / \mathrm{C}-2\right), 162.5$ (C $\left.\mathrm{C}_{\text {quat }}, \mathrm{C}-4\right)$. - MS (70 eV), $m / z(\%): 262 / 260(16 / 51)\left[\mathrm{M}^{+}\right], 226$ (100) $\left[\mathrm{M}^{+}-\mathrm{C} 1+1\right], 225$ (52) $\left[\mathrm{M}^{+}-\mathrm{Cl}\right], 211$ (37), 104 (32), 77 (26).

2-(o-Bromophenyl)-5,6,7,8-tetrahydroquinazolin-4(3H)-one (39c): The crude reaction mixture obtained from 38c $(100 \mathrm{mg}, 0.33 \mathrm{mmol}) 20 \mathrm{mg}$ of $\mathrm{Pd} / \mathrm{C}(10 \%$ by weight) in $20 \mathrm{~mL} \mathrm{MeOH}$ after $4 \mathrm{~h}$, according to GP 6, yielded $93 \mathrm{mg}$ (92\%) of 39c as a white solid, m.p. $193{ }^{\circ} \mathrm{C} .-\mathrm{IR}(\mathrm{KBr}): v=3035 \mathrm{~cm}^{-}$ ${ }^{1}$, 2944, 1648, 1559, 1319, 1227, 1031, 977, 927, 760, 728. - ${ }^{1} \mathrm{H}-\mathrm{NMR}$ $\left(250 \mathrm{MHz}, \mathrm{CDCl}_{3}\right): \delta=1.72-1.91(\mathrm{~m}, 4 \mathrm{H}), 2.38-2.51(\mathrm{~m}, 2 \mathrm{H}), 2.60$ 2.78 (m, 2 H), 7.28-7.42 (m, 2 H, aryl-H), 7.51-7.68 (m, 2 H, aryl-H),

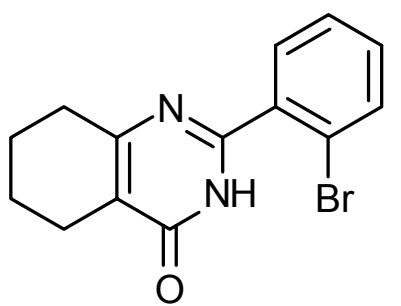
12.12 (br. s, $1 \mathrm{H}, \mathrm{NH}) .-{ }^{13} \mathrm{C}-\mathrm{NMR}\left(62.9 \mathrm{MHz}, \mathrm{CDCl}_{3}\right): \delta=21.5\left(-, \mathrm{CH}_{2}\right), 21.8\left(-, \mathrm{CH}_{2}\right), 22.1(-$, 
$\left.\mathrm{CH}_{2}\right), 31.6\left(-, \mathrm{CH}_{2}\right), 121.1\left(\mathrm{C}_{\text {quat }}, \mathrm{C}-4 \mathrm{a}\right), 127.6$ (+, aryl-C), 131.0 (+, aryl-C), 131.7 (+, aryl-C), 133.4 (+, aryl-C), $134.6\left(\mathrm{C}_{\text {ipso }}\right), 156.2$ ( $\left.\mathrm{C}_{\text {quat, }}, \mathrm{C}-8 \mathrm{a}\right), 161.8$ ( $\left.\mathrm{C}_{\text {quat }}, \mathrm{C}-2\right), 163.6\left(\mathrm{C}_{\text {quat }}, \mathrm{C}-4\right)$. - MS (70 $\mathrm{eV}), m / z$ (\%): 304/306 (100/95) $\left[\mathrm{M}^{+}\right], 289 / 291$ (28/27), 225 (11) $\left[\mathrm{M}^{+}-\mathrm{Br}\right] .-\mathrm{C}_{14} \mathrm{H}_{13} \mathrm{BrN}_{2} \mathrm{O}$ (305.2): calcd. C 55.10, H 4.29, N 9.18; found C 55.32, H 4.14, N 8.97.

2-(o-Fluorophenyl)-5,6,7,8-tetrahydroquinazolin-4(3H)-one (39d): The crude reaction mixture obtained from 38d (100 mg, $0.41 \mathrm{mmol}), 20 \mathrm{mg}$ of $\mathrm{Pd} / \mathrm{C}$ in $20 \mathrm{~mL}$ of $\mathrm{MeOH}$ after $4 \mathrm{~h}$ according to GP 6, afforded $93 \mathrm{mg}(92 \%)$ of $39 \mathrm{~d}$ as a solid, m.p. $170^{\circ} \mathrm{C}$. - IR (KBr): $v=3026 \mathrm{~cm}^{-1}, 2952,1647,1564,1327,1233,1163,979,928,761 .-$ ${ }^{1} \mathrm{H}-\mathrm{NMR}\left(250 \mathrm{MHz}, \mathrm{CDCl}_{3}\right): \delta=1.66-1.92(\mathrm{~m}, 4 \mathrm{H}), 2.49-2.61(\mathrm{~m}, 2$ H), 2.63-2.74 (m, 2 H), 7.16-7.34 (m, $2 \mathrm{H}$, aryl-H), 7.22-7.58 (m, $1 \mathrm{H}$, aryl-H), 8.14-8.25 (m, $1 \mathrm{H}$, aryl-H), 10.2 (br. s, $1 \mathrm{H}, \mathrm{NH}) .-{ }^{13} \mathrm{C}-\mathrm{NMR}$

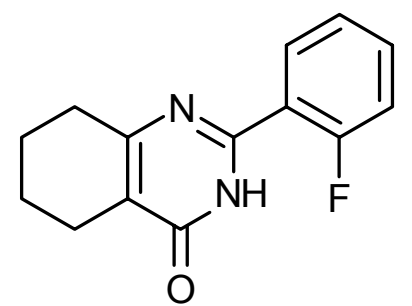
$\left(62.9 \mathrm{MHz}, \mathrm{CDCl}_{3}\right): \delta=21.6,\left(-, \mathrm{CH}_{2}\right), 21.9\left(-, \mathrm{CH}_{2}\right), 22.2\left(-, \mathrm{CH}_{2}\right)$, $31.7\left(-, \mathrm{CH}_{2}\right), 116.4\left(+, \mathrm{d},{ }^{2} J_{\mathrm{C}-\mathrm{F}}=22.9 \mathrm{~Hz}\right), 120.3\left(\mathrm{C}_{\text {quat }}, \mathrm{d},{ }^{2} J_{\mathrm{C}-\mathrm{F}}=9.2 \mathrm{~Hz}\right), 121.2\left(\mathrm{C}_{\text {quat }}, \mathrm{C}-4 \mathrm{a}\right)$, $124.8\left(+, \mathrm{d},{ }^{3} J_{\mathrm{C}-\mathrm{F}}=3.1 \mathrm{~Hz}\right), 130.9\left(+\right.$, aryl-C), $132.5\left(+, \mathrm{d},{ }^{3} J_{\mathrm{C}-\mathrm{F}}=9.2 \mathrm{~Hz}\right.$, aryl-C), $154.0\left(\mathrm{C}_{\text {quat }}, \mathrm{d}\right.$, ${ }^{1} J_{\mathrm{C}-\mathrm{F}}=250.6 \mathrm{~Hz}$, aryl-C), $161.5\left(\mathrm{C}_{\text {quat }}, \mathrm{C}-8 \mathrm{a}\right), 162.4\left(\mathrm{C}_{\text {quat }}, \mathrm{C}-2 / \mathrm{C}-4\right), 162.7$ (C $\left.\mathrm{C}_{\text {quat }}, \mathrm{C}-4 / \mathrm{C}-2\right) .-\mathrm{MS}$ (70eV), $m / z(\%): 244(100)\left[\mathrm{M}^{+}\right], 243$ (48), 229 (28), 122 (16). - $\mathrm{C}_{14} \mathrm{H}_{13} \mathrm{FN}_{2} \mathrm{O}$ (244.3): calcd. $\mathrm{C}$ 68.84, H 5.56, N 11.47; found C 69.09, H 5.21, N 11.61.

2-[(p-Benzoyloxy)phenyl]-5,6,7,8-tetrahydroquinazoline-4(3H)-one (39e): The crude reaction mixture obtained from 38e (165 mg, $0.50 \mathrm{mmol}), 25 \mathrm{mg}$ of $\mathrm{Pd} / \mathrm{C}$ in $10 \mathrm{~mL}$ of AcOH after $8 \mathrm{~h}$ according to GP 6 , afforded $113 \mathrm{mg}(93 \%)$ of 39e as a solid, m.p. $>250{ }^{\circ} \mathrm{C} .-\mathrm{IR}(\mathrm{KBr}): v=3430 \mathrm{~cm}^{-1}, 2940,1641,1515,1324$, 1289, 1182, 1113, 932, 847, 768. - ${ }^{1} \mathrm{H}-\mathrm{NMR}(300 \mathrm{MHz}$, [D $\mathrm{D}_{6}$ DMSO): $\delta=1.61-1.80(\mathrm{~m}, 4 \mathrm{H}), 2.31-2.41(\mathrm{~m}, 2 \mathrm{H}), 2.54$ 2.61 (m, $2 \mathrm{H}), 6.78-6.89$ (m, $2 \mathrm{H}$, aryl-H), 7.88-7.99 (m, $2 \mathrm{H}$, aryl-H), 10.6 (br. s, $1 \mathrm{H}, \mathrm{NH}) . \quad-{ }^{13} \mathrm{C}-\mathrm{NMR}(75.5 \mathrm{MHz}$,

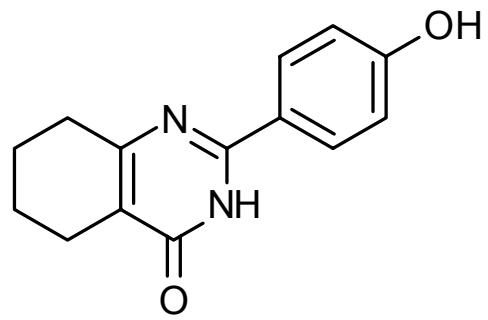
[D $\left.\left.\mathrm{D}_{6}\right] \mathrm{DMSO}\right): \delta=21.5,\left(-, \mathrm{CH}_{2}\right), 21.6\left(-, \mathrm{CH}_{2}\right), 21.9\left(-, \mathrm{CH}_{2}\right), 31.1\left(-, \mathrm{CH}_{2}\right), 115.2(+, 2 \mathrm{C}$, arylC), $118.0\left(\mathrm{C}_{\text {ipso }}\right), 122.8$ ( $\left.\mathrm{C}_{\text {ipso }}\right), 129.1$ (+, 2 C, aryl-C), 153.0 ( $\left.\mathrm{C}_{\text {quat }}, \mathrm{C}-8 \mathrm{a}\right), 160.5\left(\mathrm{C}_{\text {quat }}, \mathrm{C}-2 / \mathrm{C}-4\right)$,

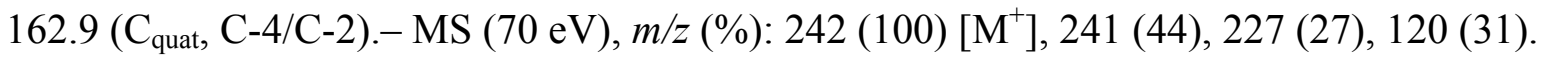


2-(o-Biphenyl)-5,6, 7,8-tetrahydroquinazolin-4(3H)-one (39f): The crude reaction mixture obtained from $38 \mathrm{f}(120 \mathrm{mg}, 0.40 \mathrm{mmol}), 10 \mathrm{mg}$ of $\mathrm{Pd} / \mathrm{C}$ in $20 \mathrm{~mL}$ of $\mathrm{MeOH}$ after $4 \mathrm{~h}$ according to GP 6 afforded $112 \mathrm{mg}(93 \%)$ of 39f as a solid, m.p. $190{ }^{\circ} \mathrm{C}$. - IR (KBr): $v=3027 \mathrm{~cm}^{-1}, 2936,1643,1566,1324,1225,1170,978,764 .-{ }^{1} \mathrm{H}-$ NMR (250 MHz, $\left.\mathrm{CDCl}_{3}\right): \delta=1.66-1.84(\mathrm{~m}, 4 \mathrm{H}), 2.38-2.48(\mathrm{~m}, 2 \mathrm{H})$, 2.57-2.66 (m, $2 \mathrm{H}), 7.21-7.42(\mathrm{~m}, 5 \mathrm{H}$, aryl-H), 7.48-7.61 (m, $2 \mathrm{H}$, aryl-H), 7.72-7.79 (m, $2 \mathrm{H}$, aryl-H), 11.1 (br. s, $1 \mathrm{H}, \mathrm{NH}) .-{ }^{13} \mathrm{C}-\mathrm{NMR}$

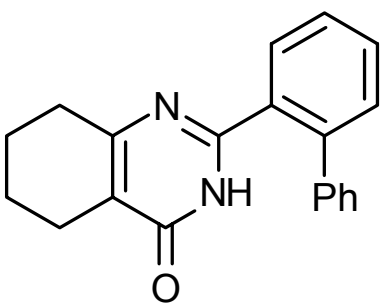
(62.9 MHz, $\left.\mathrm{CDCl}_{3}\right): \delta=21.6\left(-, \mathrm{CH}_{2}\right), 21.7\left(-, \mathrm{CH}_{2}\right), 22.1\left(-, \mathrm{CH}_{2}\right), 31.6\left(-, \mathrm{CH}_{2}\right), 119.9\left(\mathrm{C}_{\text {quat }}\right.$, C-4a), 127.3 (+, aryl-C), 127.4 (+, aryl-C), 128.1 (+, aryl-C), 128.3 (+, aryl-C), 129.0 (+, aryl-C), 130.0 (+, aryl-C), 130.7 (+, aryl-C), $132.1\left(\mathrm{C}_{\mathrm{ipso}}\right), 139.5$ (+, aryl-C), $140.7\left(\mathrm{C}_{\mathrm{ipso}}\right), 156.1\left(\mathrm{C}_{\text {quat }}, \mathrm{C}-\right.$

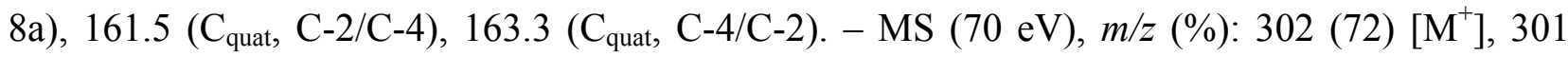
(35), 180 (58), 124 (100). $-\mathrm{C}_{22} \mathrm{H}_{18} \mathrm{~N}_{2} \mathrm{O}$ (302.4): calcd. C 79.44, $\mathrm{H}$ 6.00, N 9.26; found $\mathrm{C} 79.89, \mathrm{H}$ $5.68, \mathrm{~N} 9.49$.

6-Benzenesulfonyl-4-oxo-2-phenyl-5,6,7,8-tetrahydroquinazoline-4(H)-3-carboxylic acid tertbutyl ester (37a-Boc): To a suspension of $\mathbf{3 7 a}$ (183 $\mathrm{mg}, 0.50 \mathrm{mmol})$ in $10 \mathrm{~mL}$ of THF was added $(\mathrm{Boc})_{2} \mathrm{O}(218 \mathrm{mg}, 1.00 \mathrm{mmol}), \mathrm{Et}_{3} \mathrm{~N}(50.6 \mathrm{mg}, 0.50 \mathrm{mmol})$ and DMAP (122 $\mathrm{mg}, 0.5 \mathrm{mmol})$ at room temperature, and the resulting solution was stirred for $2 \mathrm{~h}$. The reaction mixture was diluted with $25 \mathrm{~mL}$ of DCM and washed with $10 \mathrm{~mL} 1 \mathrm{~N} \mathrm{HCl}$. The oganic layer was separated, aqueous layer was extracted

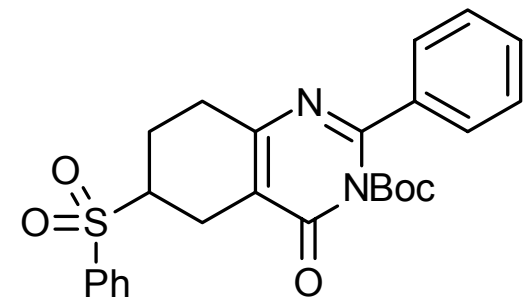
with $\mathrm{DCM}(2 \times 20 \mathrm{~mL})$. The combined organic layers were dried over $\mathrm{MgSO}_{4}$, the solvent was removed and the crude product was purified by column chromatography $\left(R_{\mathrm{f}}=0.5\right.$, hexane/ethylacetate $=2: 1,1 \times 20 \mathrm{~cm}$, "flash" $\left.\mathrm{SiO}_{2}\right)$ to yield $191 \mathrm{mg}(82 \%)$ of the product as a white solid, m.p. $153{ }^{\circ} \mathrm{C}$. - IR (KBr): $v=3066 \mathrm{~cm}^{-1}, 2985,1752,1595,1421,1249,1146 .-{ }^{1} \mathrm{H}-$ NMR $\left(250 \mathrm{MHz}, \mathrm{CDCl}_{3}\right): \delta=1.62(\mathrm{~s}, 9 \mathrm{H}), 1.84-2.04(\mathrm{~m}, 1 \mathrm{H}), 2.40-2.56(\mathrm{~m}, 1 \mathrm{H}), 2.79-3.25$ (m, 4 H), 3.31-3.44 [m, 1 H, C(6)-H], 7.42-7.51 (m, 3 H, aryl-H), 7.58-7.78 (m, $3 \mathrm{H}$, aryl-H), 7.95-8.02 (m, $2 \mathrm{H}$, aryl-H), 8.28-8.39 (m, $2 \mathrm{H}$, aryl-H). $-{ }^{13} \mathrm{C}-\mathrm{NMR}\left(62.9 \mathrm{MHz}, \mathrm{CDCl}_{3}\right): \delta=21.8$ $\left(-, \mathrm{CH}_{2}\right), 22.0\left(-, \mathrm{CH}_{2}\right), 27.5(+), 31.0\left(-, \mathrm{CH}_{2}\right), 58.9(+, \mathrm{CH}), 85.2\left(\mathrm{C}_{\text {quat }}\right), 114.5\left(\mathrm{C}_{\text {quat, }}, \mathrm{C}-4 \mathrm{a}\right)$, 128.2 (+, aryl-C), 128.4 (+, aryl-C), 129.0 (+, aryl-C), 129.4 (+, aryl-C), 130.6 (+, aryl-C), 134.2 $\left(\mathrm{C}_{\text {ipso }}\right), 136.4$ (+, aryl-C), 136.5 ( $\left.\mathrm{C}_{\text {ipso }}\right), 148.9$ (C $\left.\mathrm{C}_{\text {quat }}, \mathrm{C}-8 \mathrm{a}\right), 162.6$ ( $\left.\mathrm{C}_{\text {quat, }}, \mathrm{C}-2 / \mathrm{C}-4\right), 163.4\left(\mathrm{C}_{\text {quat }}, \mathrm{C}-\right.$ 4/C-2), $167.0\left(\mathrm{C}_{\text {quat }}, \mathrm{C}=\mathrm{O}\right) .-\mathrm{MS}(70 \mathrm{eV}), \mathrm{m} / z(\%): 466(1)\left[\mathrm{M}^{+}\right], 225(20), 224(100)\left[\mathrm{M}^{+}-\right.$ 
$\left.\mathrm{SO}_{2} \mathrm{Ph}-\mathrm{Boc}-\mathrm{H}\right], 57$ (16). $-\mathrm{C}_{25} \mathrm{H}_{26} \mathrm{~N}_{2} \mathrm{O}_{5} \mathrm{~S}$ (466.6): calcd. C 64.36, H 5.62, N 6.00; found $\mathrm{C}$ 64.22, H 5.51, N 5.86.

6-Benzenesulfonyl-8-methyl-4-oxo-2-phenyl-5,6,7,8-tetrahydroquinazoline-4(H)-3-carboxylic acid tert-butyl ester (40): To a solution of 37a-Boc (233 mg, $0.50 \mathrm{mmol})$ in $10 \mathrm{~mL}$ of THF was added $n \operatorname{BuLi}\left(0.24 \mathrm{~mL}, 2.45 \mathrm{M}\right.$ in hexane) at $-78^{\circ} \mathrm{C}$ over a period of $15 \mathrm{~min}$. The dark red solution was stirred at this temperature and after $15 \mathrm{~min}$ MeI (92 mg, $0.65 \mathrm{mmol})$ in $1 \mathrm{~mL}$ THF was added, cooling bath was removed, and stirring was continued at room temperature for $2 \mathrm{~h}$. The reaction mixture was poured into a separating funnel containing $10 \mathrm{~mL}$ of sat. aq. $\mathrm{NH}_{4} \mathrm{Cl}$ solution

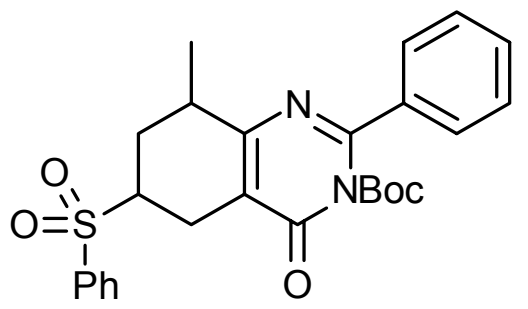
and extracted with $\mathrm{Et}_{2} \mathrm{O}(3 \times 15 \mathrm{~mL})$. Combined organic solutions were dried over $\mathrm{MgSO}_{4}$. Removal of the solvent, followed by column chromatography $\left(R_{\mathrm{f}}=0.55\right.$, hexane/ethylacetate $=$ 2:1, $1 \times 20 \mathrm{~cm}$, "flash" $\left.\mathrm{SiO}_{2}\right)$ yielded $66 \mathrm{mg}(28 \%)$ of the title product as a white solid, m.p. $98^{\circ} \mathrm{C}$. - IR (KBr): $v=3029 \mathrm{~cm}^{-1}, 2981,1762,1540,1410,1243,1148,856 . \quad{ }^{1} \mathrm{H}-\mathrm{NMR}$ (300 $\left.\mathrm{MHz} \mathrm{CDCl}_{3}\right): \delta=1.35(\mathrm{~d}, J=7.3 \mathrm{~Hz}, 3 \mathrm{H}), 1.58(\mathrm{~s}, 9 \mathrm{H}) 2.04-2.32(\mathrm{~m}, 2 \mathrm{H}), 2.75-3.12(\mathrm{~m}, 2 \mathrm{H})$, 3.25-3.39 (m, $1 \mathrm{H}), 3.40-3.52$ [m, $1 \mathrm{H}, \mathrm{C}(6)-\mathrm{H}]$, 7.38-7.46 (m, 3 H, aryl-H), 7.58-7.77 (m, $3 \mathrm{H}$, aryl-H), 7.92-7.98 (m, $2 \mathrm{H}$, aryl-H), 8.30-8.39 (m, $2 \mathrm{H}$, aryl-H). $-{ }^{13} \mathrm{C}-\mathrm{NMR}\left(50.3 \mathrm{MHz}, \mathrm{CDCl}_{3}\right)$ : $\delta=21.6(+), 22.2\left(-, \mathrm{CH}_{2}\right), 27.9(+), 28.4\left(-, \mathrm{CH}_{2}\right), 34.8(+), 55.8(+), 85.4\left(\mathrm{C}_{\text {quat }}\right), 114.0(\mathrm{C}-4 \mathrm{a})$, 128.1 (+, aryl-C), 128.3 (+, aryl-C), 129.1 (+, aryl-C), $129.5(+), 131.2(+$, aryl-C), 134.2 (+, arylC), $136.4\left(\mathrm{C}_{\text {ipso }}\right), 149.2\left(\mathrm{C}_{\text {quat, }}, \mathrm{C}-8 \mathrm{a}\right), 162.5$ (C $\left.\mathrm{C}_{\text {quat }}, \mathrm{C}-2 / \mathrm{C}-4\right), 163.4\left(\mathrm{C}_{\text {quat, }}, \mathrm{C}-4 / \mathrm{C}-2\right)$ - - MS (DCI, 70 eV), $m / z(\%): 498$ (5) $\left[\mathrm{M}^{+}+\mathrm{NH}_{4}\right], 481(100)\left[\mathrm{M}^{+}+\mathrm{H}\right], 381$ (79), 341 (82). - $\mathrm{C}_{26} \mathrm{H}_{28} \mathrm{~N}_{2} \mathrm{O}_{5} \mathrm{~S}$ (480.6): calcd. C 64.98, H 5.87, N 5.83; found C 64.62, H 5.57, N 5.85.

6-Benzenesulfonyl-2-phenyl-4-trimethylsilyloxy-5,6,7,8-tetrahydroquinazolin (37a-TMS): Into a $25 \mathrm{~mL}$ dry reaction flask $37 \mathrm{a}(1.60 \mathrm{~g}, 4.40 \mathrm{mmol})$ and $20 \mathrm{mg}$ of $\left(\mathrm{NH}_{4}\right)_{2} \mathrm{SO}_{4}$ was added in $15 \mathrm{ml}$ of HMDS. This reaction mixture was refluxed for $15 \mathrm{~h}$. After cooling to $20^{\circ} \mathrm{C}$ HMDS was removed in vacuo, the reaction mixture was taken in $20 \mathrm{~mL}$ of DCM, washed with $5 \mathrm{~mL}$ of water and dried over $\mathrm{MgSO}_{4}$. After

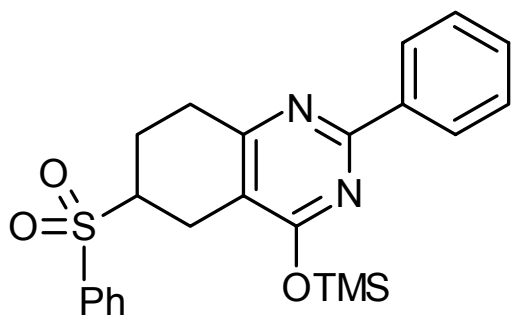
filtration, removal of the solvent yielded $1.91 \mathrm{~g}(99 \%)$ of 37a-TMS as a white solid, m.p. > $250{ }^{\circ} \mathrm{C} .-{ }^{1} \mathrm{H}-\mathrm{NMR}\left(250 \mathrm{MHz}, \mathrm{CDCl}_{3}\right): \delta=0.45(\mathrm{~s}, 9 \mathrm{H}), 1.80-1.98(\mathrm{~m}, 1 \mathrm{H}), 2.35-2.51(\mathrm{~m}, 1 \mathrm{H})$, 
2.75-2.87 (m, 2 H), 3.02-3.21 (m, 2 H), 3.25-3.41 (m, $1 \mathrm{H}), 7.18-7.32$ (m, 3 H, aryl-H), 7.587.75 (m, $3 \mathrm{H}$, aryl-H), 7.95-8.03 (m, $2 \mathrm{H}$, aryl-H), 8.25-8.36 (m, $2 \mathrm{H}$, aryl-H). ${ }^{13} \mathrm{C}-\mathrm{MNR}(62.9$ $\left.\mathrm{MHz}_{\mathrm{CDCl}}\right): \delta=0.31\left(+, \mathrm{TMS}-\mathrm{CH}_{3}\right), 21.9\left(-, \mathrm{CH}_{2}\right), 22.2\left(-, \mathrm{CH}_{2}\right), 30.81\left(-, \mathrm{CH}_{2}\right), 59.5(+)$, $112.3(\mathrm{C}-4 \mathrm{a}), 127.3(+), 127.8(+), 128.4(+$, aryl-C), $128.9(+$, aryl-C), $129.0(+$, aryl-C), $130.2(+$, aryl-C), 134.0 (+, aryl-C), 136.8 ( $\left.\mathrm{C}_{\mathrm{ipso}}\right), 137.5\left(\mathrm{C}_{\mathrm{ipso}}\right), 161.4\left(\mathrm{C}_{\text {quat }}, \mathrm{C}-8 \mathrm{a}\right), 164.1\left(\mathrm{C}_{\text {quat }}, \mathrm{C}-2 / \mathrm{C}-4\right)$, $166.4\left(\mathrm{C}_{\text {quat }}, \mathrm{C}-4 / \mathrm{C}-2\right) .-\mathrm{MS}(70 \mathrm{eV}), \mathrm{m} / z(\%): 438$ (2) $\left[\mathrm{M}^{+}\right], 296$ (100) $\left[\mathrm{M}^{+}-\mathrm{SO}_{2} \mathrm{Ph}\right], 281$ (23) $\left[\mathrm{M}^{+}-\mathrm{SO}_{2} \mathrm{Ph}-\mathrm{Me}\right], 247$ (63), 175 (25).

6-Benzenesulfonyl-6-methyl-2-phenyl-5,6,7,8-tetrahydroquinazolin-4(3H)-one (41a): To a cooled solution of sulfone 37a-TMS (439 mg, $1.00 \mathrm{mmol})$ in $15 \mathrm{~mL}$ THF, $n$ BuLi (1.77 M in hexane, 0.62 $\mathrm{mL}$ ) was added at $-78^{\circ} \mathrm{C}$ over a period of $15 \mathrm{~min}$. The resulting mixture was stirred for additional $15 \mathrm{~min}$. and MeI (156 mg, $1.10 \mathrm{mmol}$ ) in $1 \mathrm{ml}$ THF was added. The cooling bath was removed, and stirring was continued at RT for $2 \mathrm{~h}$. The reaction mixture was poured into a separating funnel containing $10 \mathrm{~mL}$ of sat. aq. $\mathrm{NH}_{4} \mathrm{Cl}$ solution and extracted with $\mathrm{DCM}(3 \times 15$

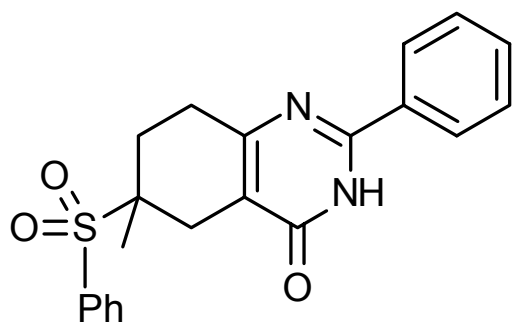
$\mathrm{mL})$. Removal of solvent followed by column chromatography $\left(R_{\mathrm{f}}=0.32, \mathrm{DCM} / \mathrm{MeOH}=25: 1\right.$, $\left.1.5 \times 30 \mathrm{~cm}, 25 \mathrm{~g} \mathrm{SiO}_{2}\right)$ yielded $315 \mathrm{mg}(83 \%)$ of $41 \mathrm{a}$ as a white solid, m.p. $>250{ }^{\circ} \mathrm{C} .-\mathrm{IR}(\mathrm{KBr})$ : $v=3057 \mathrm{~cm}^{-1}, 2943,1644,1553,1447,1300,1153,1088,701 .-{ }^{1} \mathrm{H}-\mathrm{NMR}(250 \mathrm{MHz}$, [D ${ }_{6}$ DMSO): $\delta=1.22(\mathrm{~s}, 3 \mathrm{H}), 1.84-2.51(\mathrm{~m}, 3 \mathrm{H}), 2.62-2.83(\mathrm{~m}, 3 \mathrm{H}), 7.38-7.60$ (m, $3 \mathrm{H}$, aryl$\mathrm{H}), 7.63-8.10\left(\mathrm{~m}, 7 \mathrm{H}\right.$, aryl-H). $-{ }^{13} \mathrm{C}-\mathrm{NMR}\left(62.9 \mathrm{MHz},\left[\mathrm{D}_{6}\right] \mathrm{DMSO}\right): \delta=18.1(+), 26.2\left(-, \mathrm{CH}_{2}\right)$, $27.8\left(-, \mathrm{CH}_{2}\right), 28.1\left(-, \mathrm{CH}_{2}\right), 60.4\left(\mathrm{C}_{\text {quat }}\right), 115.7\left(\mathrm{C}_{\text {quat }}, \mathrm{C}-4 \mathrm{a}\right), 127.8(+$, aryl-C), 128.8 (+, aryl-C), $129.6(+), 130.5$ (+, aryl-C), $132.4\left(+\right.$, aryl-C), $134.6\left(\mathrm{C}_{\mathrm{ipso}}\right), 154.1\left(\mathrm{C}_{\text {quat }}, \mathrm{C}-8 \mathrm{a}\right), 158.4\left(\mathrm{C}_{\text {quat }}, \mathrm{C}-2\right)$, $164.2\left(\mathrm{C}_{\text {quat }}, \mathrm{C}-4\right) .-\mathrm{MS}(70 \mathrm{eV}), \mathrm{m} / z(\%): 380(1)\left[\mathrm{M}^{+}\right], 238$ (100) $\left[\mathrm{M}^{+}-\mathrm{SO}_{2} \mathrm{Ph}\right], 77$ (30). $\mathrm{C}_{21} \mathrm{H}_{20} \mathrm{~N}_{2} \mathrm{O}_{3} \mathrm{~S}$ (380.5): calcd. C 66.30, H 5.30, N 7.36; found C 66.13, H 5.60, N 7.61.

6-Benzenesulfonyl-6-ethyl-2-phenyl-5,6,7,8-tetrahydroquinazolin-4(3H)-one (41b): The crude mixture obtained from 37a-TMS (439 mg, $1.00 \mathrm{mmol}), n \mathrm{BuLi}(1.77 \mathrm{M}$ in hexane, $0.62 \mathrm{~mL}$ ) and $\operatorname{EtBr}(119 \mathrm{mg}, 1.1 \mathrm{mmol})$ according to the method given above for 41a was purified by column chromatography $\left(R_{\mathrm{f}}=0.38, \mathrm{DCM} / \mathrm{MeOH}=25: 1,1.5 \times 30 \mathrm{~cm}, 25 \mathrm{~g} \mathrm{SiO}_{2}\right)$ to afford $339 \mathrm{mg}(86 \%)$ of 41b as a white solid, m.p. $242{ }^{\circ} \mathrm{C}$. - IR (KBr): $v=3065 \mathrm{~cm}^{-1}, 2941,1644,1554,1447,1301$, 
1151, 1079, 763, 692. - ${ }^{1} \mathrm{H}-\mathrm{NMR}\left(250 \mathrm{MHz},\left[\mathrm{D}_{6}\right] \mathrm{DMSO}\right): \delta=1.11(\mathrm{t}, J=7.3 \mathrm{~Hz}, 3 \mathrm{H}), 1.62-1.81$ (m, $2 \mathrm{H}), 2.12-2.44(\mathrm{~m}, 3 \mathrm{H}), 2.71-3.02(\mathrm{~m}, 3 \mathrm{H}), 7.41-7.74$ (m, $6 \mathrm{H}$, aryl-H), 7.91-8.21 (m, $4 \mathrm{H}$, aryl-H), 12.7 (br. s, $1 \mathrm{H}$, $\mathrm{NH}) .-{ }^{13} \mathrm{C}-\mathrm{NMR}\left(62.9 \mathrm{MHz},\left[\mathrm{D}_{6}\right] \mathrm{DMSO}\right): \delta=8.6(+), 23.3(-$, $\left.\mathrm{CH}_{2}\right), 24.4\left(-, \mathrm{CH}_{2}\right), 28.4\left(-, \mathrm{CH}_{2}\right), 28.8\left(-, \mathrm{CH}_{2}\right), 67.9\left(\mathrm{C}_{\text {quat }}\right)$, $116.2\left(\mathrm{C}_{\text {quat }}, \mathrm{C}-4 \mathrm{a}\right), 127.5(+$, aryl-C), $128.8(+$, aryl-C), 129.0 $(+), 130.1\left(+\right.$, aryl-C), $131.8\left(+\right.$, aryl-C), $133.8\left(\mathrm{C}_{\mathrm{ipso}}\right), 135.7(+$,

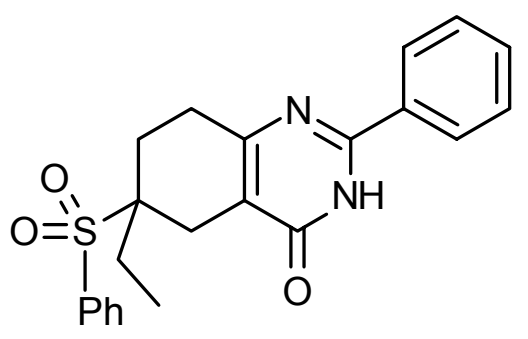
aryl-C), 153.9 ( $\left.\mathrm{C}_{\text {quat }}, \mathrm{C}-8 \mathrm{a}\right), 160.7$ (C $\left.\mathrm{C}_{\text {quat }}, \mathrm{C}-2 / \mathrm{C}-4\right), 164.4\left(\mathrm{C}_{\text {quat }}, \mathrm{C}-4 / \mathrm{C}-2\right)-\mathrm{MS}(70 \mathrm{eV}), \mathrm{m} / z(\%)$ : 394 (1) $\left[\mathrm{M}^{+}\right], 253$ (52) $\left[\mathrm{M}^{+}-\mathrm{SO}_{2} \mathrm{Ph}\right], 252$ (100) $\left[\mathrm{M}^{+}-\mathrm{SO}_{2} \mathrm{Ph}-\mathrm{H}\right], 237$ (14), 211 (15), 104 (11).

6-Methyl-2-phenyl-7,8-dihydroquinazolin-4(3H)-one (43a): From the sulfone 41a (265 mg, 0.70 $\mathrm{mmol})$ and $\mathrm{KO} t \mathrm{Bu}(235 \mathrm{mg}, 2.10 \mathrm{mmol})$ according to the GP 5 were obtained $160 \mathrm{mg}(96 \%)$ of 43a as a pale yellow solid, m.p. $218{ }^{\circ} \mathrm{C}, R_{\mathrm{f}}=0.6$ (hexane/ethyl acetate $=1: 2) .-\operatorname{IR}(\mathrm{KBr}): v=3031 \mathrm{~cm}^{-1}, 2924,1636,1506,1436$, 1314, 1182, 932, 772, 699. $-{ }^{1} \mathrm{H}-\mathrm{NMR}\left(250 \mathrm{MHz}, \mathrm{CDCl}_{3}\right): \delta=1.96$ (s, $3 \mathrm{H}), 2.39[\mathrm{t}, J=9.1 \mathrm{~Hz}, 2 \mathrm{H}, \mathrm{C}(7)-\mathrm{H}], 2.89[\mathrm{t}, J=9.1 \mathrm{~Hz}, 2 \mathrm{H}$, $\mathrm{C}(8)-\mathrm{H}], 6.46$ [s, $1 \mathrm{H}, \mathrm{C}(5)-\mathrm{H}], 7.42-7.61$ (m, $3 \mathrm{H}$, aryl-H), 8.18-

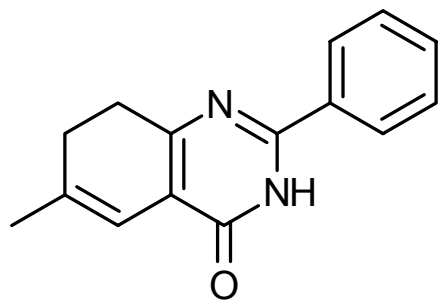
$8.36(\mathrm{~m}, 2 \mathrm{H}$, aryl-H), 13.3 (br. s, $1 \mathrm{H}, \mathrm{NH}) .-{ }^{13} \mathrm{C}-\mathrm{NMR}\left(62.9 \mathrm{MHz}, \mathrm{CDCl}_{3}\right): \delta=23.4\left(+, \mathrm{CH}_{3}\right)$, $28.3\left(-, \mathrm{CH}_{2}\right), 29.9\left(-, \mathrm{CH}_{2}\right), 114.3$ (+, C-5), 117.8 (C $\left.\mathrm{C}_{\text {quat }}, \mathrm{C}-4 \mathrm{a}\right), 127.5$ (+, aryl-C), 128.8 (+, arylC), 131.3 (+, aryl-C), 132.3 ( $\left.\mathrm{C}_{\text {ipso }}\right), 137.7$ ( $\left.\mathrm{C}_{\text {quat }}, \mathrm{C}-6\right), 153.4$ ( $\left.\mathrm{C}_{\text {quat }}, \mathrm{C}-8 \mathrm{a}\right), 159.4\left(\mathrm{C}_{\text {quat }}, \mathrm{C}-2 / \mathrm{C}-4\right)$,

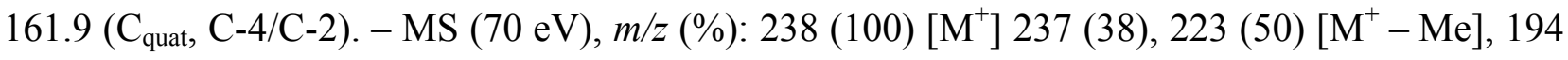
(10) $\left[\mathrm{M}^{+}-\mathrm{CONH}_{2}\right], 104$ (14), $77(10)\left[\mathrm{Ph}^{+}\right]$.

6-Ethyl-2-phenyl-7,8-dihydroquinazolin-4(3H)-one (43b): From the sulfone 41b (197 mg, 0.50 $\mathrm{mmol})$ and $\mathrm{KO} t \mathrm{Bu}(168 \mathrm{mg}, 1.50 \mathrm{mmol})$ according to the GP 5 were obtained $118 \mathrm{mg}(98 \%)$ of 43b as a pale yellow solid m.p. $198{ }^{\circ} \mathrm{C}, R_{\mathrm{f}}=0.6$ (hexane/ethylacetate $=1: 2) .-$ IR $(\mathrm{KBr}): v=3020 \mathrm{~cm}^{-1}, 2955,2922,1630$, 1532, 1321, 1098, 922, 699. - ${ }^{1} \mathrm{H}-\mathrm{NMR}\left(250 \mathrm{MHz}, \mathrm{CDCl}_{3}\right): \delta=$ $1.16(\mathrm{t}, J=7.3 \mathrm{~Hz}, 3 \mathrm{H}), 2.26(\mathrm{q}, J=7.3 \mathrm{~Hz}, 2 \mathrm{H}), 2.40[\mathrm{t}, J=8.9$ $\mathrm{Hz}, 2 \mathrm{H}, \mathrm{C}(7)-\mathrm{H}], 2.89[\mathrm{t}, J=8.9 \mathrm{~Hz}, 2 \mathrm{H}, \mathrm{C}(8)-\mathrm{H}], 6.47$ [s, $2 \mathrm{H}$,

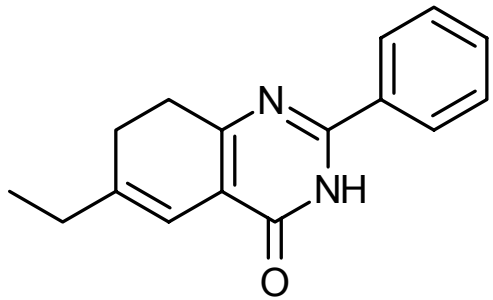
$\mathrm{C}(5)-\mathrm{H}], 7.42-7.61(\mathrm{~m}, 3 \mathrm{H}$, aryl-H), 8.22-8.38 (m, $2 \mathrm{H}$, aryl-H), 13.45 (br. s, $1 \mathrm{H}, \mathrm{NH}) .-{ }^{13} \mathrm{C}-$ NMR (62.9 MHz, $\left.\mathrm{CDCl}_{3}\right): \delta=11.8(+), 26.9\left(-, \mathrm{CH}_{2}\right), 23.0\left(-, \mathrm{CH}_{2}\right), 30.1\left(-, \mathrm{CH}_{2}\right), 112.4(+, \mathrm{C}-$ 
5), $117.8\left(\mathrm{C}_{\text {quat }}, \mathrm{C}-4 \mathrm{a}\right), 127.5$ (+, aryl-C), 128.7 (+, aryl-C), 131.3 (+, aryl-C), $132.3\left(\mathrm{C}_{\text {quat }}, \mathrm{C}-6\right)$, $143.2\left(\mathrm{C}_{\text {ipso }}\right), 153.4$ ( $\left.\mathrm{C}_{\text {quat }}, \mathrm{C}-8 \mathrm{a}\right), 159.7$ ( $\left.\mathrm{C}_{\text {quat }}, \mathrm{C}-2 / \mathrm{C}-4\right), 162.0$ ( $\left.\mathrm{C}_{\text {quat }}, \mathrm{C}-4 / \mathrm{C}-2\right) .-\mathrm{MS}(70 \mathrm{eV}), \mathrm{m} / \mathrm{z}$

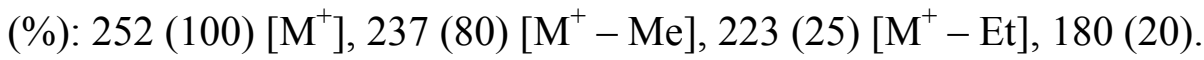

6-Methyl-2-phenyl-5,6,7,8-tetrahydroquinazolin-4(3H)-one (42a): The crude reaction mixture obtained from 43a (100 mg, $0.42 \mathrm{mmol}$ ), $22 \mathrm{mg}$ of $\mathrm{Pd} / \mathrm{C}$ in $25 \mathrm{~mL}$ of MeOH after $4 \mathrm{~h}$ according to the GP 6 afforded $97 \mathrm{mg}(96 \%)$ of $\mathbf{4 2 a}$ as a solid, m.p. $237^{\circ} \mathrm{C}$. - IR (KBr): $v=3072 \mathrm{~cm}^{-1}, 2948,1641,1507,1316,1073,697 .-{ }^{1} \mathrm{H}-$ $\operatorname{NMR}\left(250 \mathrm{MHz}, \mathrm{CDCl}_{3}\right): \delta=1.12\left(\mathrm{~d}, J=6.5 \mathrm{~Hz}, 3 \mathrm{H}, \mathrm{CH}_{3}\right), 1.38$ $1.56(\mathrm{~m}, 1 \mathrm{H}), 1.76-2.15(\mathrm{~m}, 3 \mathrm{H}), 2.68-2.86(\mathrm{~m}, 3 \mathrm{H}), 7.20-7.38$ (m, 3 H, aryl-H), 8.02-8.14 (m, 2 H, aryl-H), 11.68 (br. s, 1 H, NH).

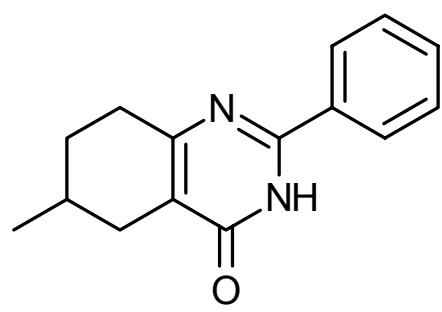
${ }^{13}{ }^{13}$-NMR (62.9 MHz, $\left.\mathrm{CDCl}_{3}\right): \delta=21.5(+), 28.0(+), 30.1\left(-, \mathrm{CH}_{2}\right), 30.4\left(-, \mathrm{CH}_{2}\right), 31.8\left(-, \mathrm{CH}_{2}\right)$, $119.6\left(\mathrm{C}_{\text {quat }}, \mathrm{C}-4 \mathrm{a}\right), 127.5$ (+, aryl-C), 128.8 (+, aryl-C), 131.3 (+, aryl-C), $132.4\left(\mathrm{C}_{\mathrm{ipso}}\right), 153.3$ ( $\left.\mathrm{C}_{\text {quat }}, \mathrm{C}-8 \mathrm{a}\right), 162.2$ (C $\left.\mathrm{C}_{\text {quat }}, \mathrm{C}-2 / \mathrm{C}-4\right), 164.8$ (C $\left.\mathrm{C}_{\text {quat }}, \mathrm{C}-4 / \mathrm{C}-2\right) .-\mathrm{MS}(70 \mathrm{eV}), \mathrm{m} / z(\%): 240(100)$ $\left[\mathrm{M}^{+}\right], 225$ (90) $\left[\mathrm{M}^{+}-\mathrm{Me}\right], 198$ (49), 104 (31), 77 (16). $-\mathrm{C}_{15} \mathrm{H}_{16} \mathrm{~N}_{2} \mathrm{O}$ (240.3): calcd. C 74.97, $\mathrm{H}$ 6.71, N 11.66; found C 74.77, H 6.99, N 11.53.

6-Ethyl-2-phenyl-5,6,7,8-tetrahydroquinazolin-4(3H)-one (42b): The crude reaction mixture obtained from $43 \mathbf{b}$ ( $76 \mathrm{mg}, 0.30 \mathrm{mmol}$ ), $15 \mathrm{mg}$ of $\mathrm{Pd} / \mathrm{C}$ in $25 \mathrm{~mL} \mathrm{MeOH}$ after $4 \mathrm{~h}$, according to the GP 6, afforded $74 \mathrm{mg}(96 \%)$ of $\mathbf{4 2 b}$ as a solid, m.p. $221^{\circ} \mathrm{C}$. - IR (KBr): $v=2922 \mathrm{~cm}^{-1}, 1642,1549,1315,919,697 .-{ }^{1} \mathrm{H}-\mathrm{NMR}$ $\left(250 \mathrm{MHz}, \mathrm{CDCl}_{3}\right): \delta=1.02(\mathrm{t}, J=7.3 \mathrm{~Hz}, 3 \mathrm{H}), 1.24-1.68(\mathrm{~m}, 4$ H), 1.80-2.15 (m, 2 H), 2.55-3.00 (m, $3 \mathrm{H}), 7.36-7.60$ (m, $3 \mathrm{H}$, aryl-H), 8.10-8.32 (m, $2 \mathrm{H}$, aryl-H), 13.12 (br. s, $1 \mathrm{H}, \mathrm{NH}) .-{ }^{13} \mathrm{C}-$

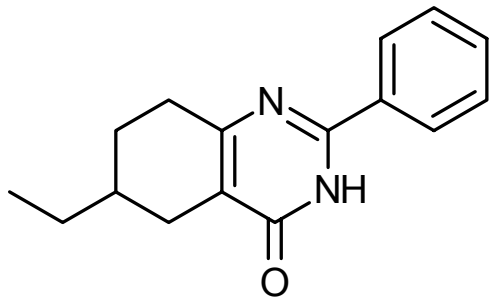
NMR (62.9 MHz, $\left.\mathrm{CDCl}_{3}\right): \delta=11.5(+), 27.9\left(-, \mathrm{CH}_{2}\right), 28.1\left(-, \mathrm{CH}_{2}\right), 28.8\left(-, \mathrm{CH}_{2}\right), 31.8\left(-, \mathrm{CH}_{2}\right)$, $34.7(+), 119.6\left(\mathrm{C}_{\text {quat }}, \mathrm{C}-4 \mathrm{a}\right), 127.5$ (+, aryl-C), 128.7 (+, aryl-C), $131.3\left(+\right.$, aryl-C), $132.4\left(\mathrm{C}_{\mathrm{ipso}}\right)$, 153.2 ( $\left.\mathrm{C}_{\text {quat }}, \mathrm{C}-8 \mathrm{a}\right), 162.4$ (C $\left.\mathrm{C}_{\text {quat }}, \mathrm{C}-2 / \mathrm{C}-4\right), 164.9$ (C $\left.\mathrm{C}_{\text {quat }}, \mathrm{C}-4 / \mathrm{C}-2\right) .-\mathrm{MS}(70 \mathrm{eV}), \mathrm{m} / z(\%): 254(80)$ $\left[\mathrm{M}^{+}\right], 225$ (100) [ $\left.\mathrm{M}^{+}-\mathrm{Et}\right], 198$ (36), 104 (22). $-\mathrm{C}_{16} \mathrm{H}_{18} \mathrm{~N}_{2} \mathrm{O}$ (254.3): calcd. C 75.56, H 7.15, N 11.01; found. C 75.36, H 7.45, N 10.88.

6-p-Toluenesulfonyl-7-methyl-2-phenyl-5,6,7,8-tetrahydroquinazolin-4(3H)one (44): 35a (99 mg, $0.50 \mathrm{mmol}$ ) and (E)-p-tolyl-1-propenyl sulfone 36-Me (392 $\mathrm{mg}, 2.00 \mathrm{mmol})$ were heated at 175 
${ }^{\circ} \mathrm{C}$ for $12 \mathrm{~h}$ in $10 \mathrm{~mL}$ Pyrex tube. After cooling down to room temperature, the reaction mixture was dissolved in $\mathrm{DCM} / \mathrm{MeOH}$ and purified by column chromatography $\left(R_{\mathrm{f}}=0.40\right.$, hexane/ethyl acetate $=1: 2)$ to yield $53 \mathrm{mg}(27 \%)$ of the title compound as a white solid, m.p. $=247^{\circ} \mathrm{C} .-\mathrm{IR}(\mathrm{KBr}): v=3034 \mathrm{~cm}^{-1}, 2927$, 1653, 1507, 1302, 1142, 1018. - ${ }^{1} \mathrm{H}-\mathrm{NMR}\left(250 \mathrm{MHz}, \mathrm{CDCl}_{3}\right): \delta$ $=1.26(\mathrm{~d}, J=7.8 \mathrm{~Hz}, 3 \mathrm{H}), 2.41-2.76(\mathrm{~m}, 4 \mathrm{H}), 2.88-3.04(\mathrm{~m}, 1$ H), 3.13-3.36 (m, $1 \mathrm{H}), 7.32-7.55$ (m, $6 \mathrm{H}), 7.72-7.82(\mathrm{~m}, 2 \mathrm{H}$, aryl-H), 8.06-8.16 (m, $2 \mathrm{H}$, aryl-H), 12.8 (br. s, NH). $-{ }^{13} \mathrm{C}-$

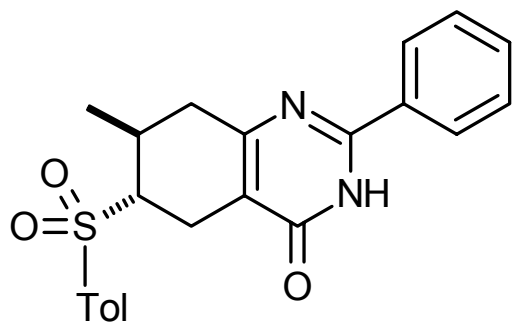
$\operatorname{NMR}\left(62.9 \mathrm{MHz}, \mathrm{CDCl}_{3}\right): \delta=20.4\left(+, \mathrm{CH}_{3}\right), 21.7\left(+, \mathrm{CH}_{3}\right), 27.2\left(-, \mathrm{CH}_{2}\right), 37.3\left(-, \mathrm{CH}_{2}\right), 63.2(+$, $\mathrm{CH}), 115.6$ (C-4a), 127.5 (+, aryl-C), 128.7 (+, aryl-C), 128.9 (+, aryl-C), 130.0 (+, aryl-C), 131.7 (+, aryl-C), 132.0 (+, aryl-C), $135.2\left(\mathrm{C}_{\text {ipso }}\right), 144.8$ (C $\left.\mathrm{C}_{\text {quat, }}, \mathrm{C}-8 \mathrm{a}\right), 154.1$ ( $\left.\mathrm{C}_{\text {quat, }}, \mathrm{C}-2\right), 163.7$ ( $\mathrm{C}_{\text {quat }}, \mathrm{C}-$

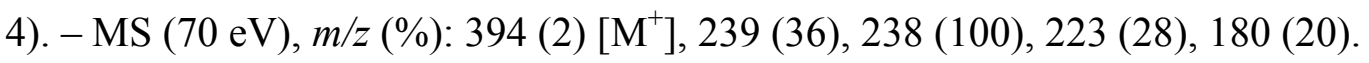

Nucleophilic substitution of SMe group by amines 6-Benzenesulfonyl-2-(morpholine-4-yl)-5,6,7,8-tetrahydroquinazolin-4(3H)-one (45a): 37h (168 $\mathrm{mg}, 0.5 \mathrm{mmol})$ and morpholine $(2 \mathrm{~mL})$ were tightly sealed in a $10 \mathrm{~mL}$ of Pyrex bottle and heated at $180{ }^{\circ} \mathrm{C}$ for $15 \mathrm{~h}$. The reaction mixture was cooled to RT, excess of morpholine was removed in vacuo, and crude product was filtered through a pad of $\mathrm{SiO}_{2}(2 \times 3 \mathrm{~cm}, 10 \mathrm{~g}$, $\mathrm{DCM} / \mathrm{MeOH}=10: 1)$ to yield $175 \mathrm{mg}(93 \%)$ of $\mathbf{3 1 a}$ as a white solid, m.p. $>250^{\circ} \mathrm{C} .-\mathrm{IR}(\mathrm{KBr}): v=2902 \mathrm{~cm}^{-1}, 2848,1656$,

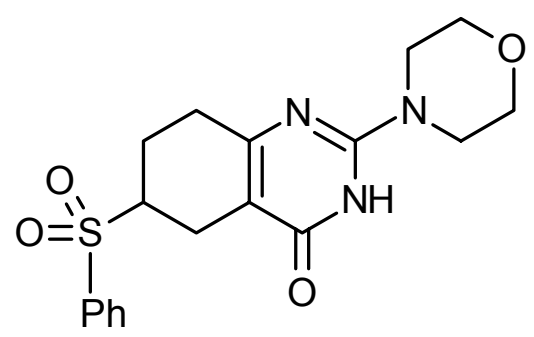
1590, 1395, 1300, 1267, 1146, 1114, 979, 742, 722. - ${ }^{1} \mathrm{H}-\mathrm{NMR}\left(250 \mathrm{MHz},\left[\mathrm{D}_{6}\right] \mathrm{DMSO}\right): \delta=1.55-$ $1.72(\mathrm{~m}, 1 \mathrm{H}), 2.05-2.32(\mathrm{~m}, 2 \mathrm{H}), 3.21-3.72(\mathrm{~m}, 12 \mathrm{H}), 7.60-7.81$ (m, $3 \mathrm{H}$, aryl-H), 7.82-7.92 (m, $2 \mathrm{H}$, aryl-H), 8.86 (br. s, $1 \mathrm{H}, \mathrm{NH}) .-{ }^{13} \mathrm{C}-\mathrm{NMR}\left(62.9 \mathrm{MHz},\left[\mathrm{D}_{6}\right] \mathrm{DMSO}\right): \delta=21.5\left(-, \mathrm{CH}_{2}\right)$, $21.7\left(-, \mathrm{CH}_{2}\right), 44.8\left(-, 2 \mathrm{C}, \mathrm{NCH}_{2}\right), 58.1(+), 65.8\left(-, 2 \mathrm{C}, \mathrm{OCH}_{2}\right), 128.7$ (+, aryl-C), $129.8(+$, arylC), 134.3 (+, aryl-C), 137.2 ( $\left.\mathrm{C}_{\text {ipso }}\right) 151.9$ ( $\left.\mathrm{C}_{\text {quat, }}, \mathrm{C}-8 \mathrm{a}\right), 159.1$ ( $\left.\mathrm{C}_{\text {quat, }}, \mathrm{C}-2\right), 163.4\left(\mathrm{C}_{\text {quat }}, \mathrm{C}-4\right)$. - MS (70 eV), m/z (\%): $375(8)\left[\mathrm{M}^{+}\right], 233(100)\left[\mathrm{M}^{+}-\mathrm{SO}_{2} \mathrm{Ph}\right], 202(23), 176(10) .-\mathrm{C}_{18} \mathrm{H}_{21} \mathrm{~N}_{3} \mathrm{O}_{4} \mathrm{~S}$ (375.5): calcd. C 57.58, H 5.64, N 11.19; found C 57.26, H 5.65, N 11.53.

\section{2-(4-benzylpiperazin-1-yl)-6-Benzenesulfonyl-5,6,7,8-tetrahydroquinazolin-4(3H)-one}

(45b):

Following the method given above for $45 \mathbf{a}, 45 \mathrm{~b}$ was obtained from $\mathbf{3 7 h}(750 \mathrm{mg}, 2.23 \mathrm{mmol})$ and $\mathrm{N}$-benzylpiperazine $(1.57 \mathrm{~g}, 8.92 \mathrm{mmol})$ in $92 \%(953 \mathrm{mg})$ yield as a white solid, m.p. $>250^{\circ} \mathrm{C}$. - 
IR (KBr): $v=2937 \mathrm{~cm}^{-1}, 2816,1653,1576,1304,1262,1144,745 .-{ }^{1} \mathrm{H}-\mathrm{NMR}(250 \mathrm{MHz}$, [D $]$ DMSO): 1.52-1.71 (m, $1 \mathrm{H}), 2.08-2.42(\mathrm{~m}, 7 \mathrm{H}), 3.41-3.63(\mathrm{~m}, 9 \mathrm{H}), 7.17-7.38(\mathrm{~m}, 5 \mathrm{H}$, aryl-H), 7.62-7.94 (m, $5 \mathrm{H}$, aryl-H). ${ }^{-13} \mathrm{C}-\mathrm{NMR}(50.3$ $\left.\mathrm{MHz},\left[\mathrm{D}_{6}\right] \mathrm{DMSO}\right): \delta=20.9\left(-, \mathrm{CH}_{2}\right), 21.2\left(-, \mathrm{CH}_{2}\right), 29.6$ $\left(-, \mathrm{CH}_{2}\right), 43.9\left(-, \mathrm{NCH}_{2}\right), 51.6\left(-, \mathrm{NCH}_{2}\right), 58.1(+), 61.4$ (-, Bn-CH$), 104.4$ (C-4a), 126.4 (+, aryl-C), $127.6(+$, aryl-C), $128.0(+$, aryl-C), 128.3 (+, aryl-C), $128.9(+$, aryl-C), 133.4 (+, aryl-C), $137.1\left(\mathrm{C}_{\mathrm{ipso}}\right), 137.5\left(\mathrm{C}_{\mathrm{ipso}}\right)$,

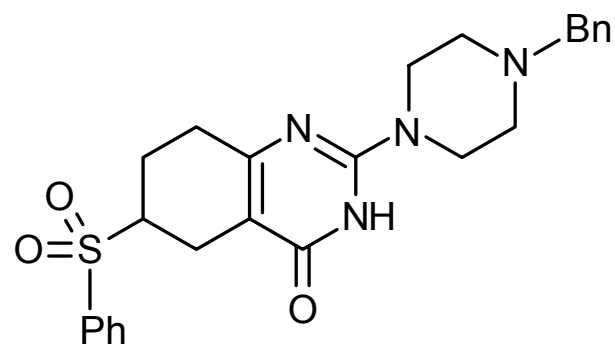
$152.5\left(\mathrm{C}_{\text {quat }}, \mathrm{C}-8 \mathrm{a}\right), 159.4\left(\mathrm{C}_{\text {quat }}, \mathrm{C}-2\right), 163.1$ (C $\left.\mathrm{C}_{\text {quat }}, \mathrm{C}-4\right) .-\mathrm{MS}(70 \mathrm{eV}), m / z(\%): 464(14)\left[\mathrm{M}^{+}\right]$, 429 (12), 412 (16), 318 (68), 159 (96), 91 (100). - $\mathrm{C}_{25} \mathrm{H}_{28} \mathrm{~N}_{4} \mathrm{O}_{3} \mathrm{~S}$ (464.6): calcd. C 64.63, H 6.07, N 12.06; found C 64.48, H 6.17, N 11.97.

6-Benzenesulfonyl-2-(4-methylpiperazin-1-yl)-5,6,7,8-tetrahydroquinazolin-4(3H)-one (45c):

Following the method given above for 45a, 45c was obtained from $37 \mathbf{h}$ (336 $\mathrm{mg}, 1.00 \mathrm{mmol})$ and $N$-methyl piperazine $(2 \mathrm{~mL})$ in $91 \%$ yield $(352 \mathrm{mg})$ as a white solid, m.p. $>250{ }^{\circ} \mathrm{C}$. $-\mathrm{IR}(\mathrm{KBr}): v=3232 \mathrm{~cm}^{-1}, 2930$, $2797,1631,1585,1301,1266,1147,1083,1003,721 .-{ }^{1} \mathrm{H}-$ NMR (250 MHz, $\left.\mathrm{CDCl}_{3}\right): \delta=1.78-1.92\left(\mathrm{~m}, 1 \mathrm{H}, \mathrm{CH}_{2}\right), 2.38$ $\left(\mathrm{s}, 3 \mathrm{H}, \mathrm{CH}_{3}\right), 2.41\left(\mathrm{~m}, 4 \mathrm{H}, \mathrm{CH}_{2}\right), 2.43-2.80(\mathrm{~m}, 5 \mathrm{H}$, $\left.\mathrm{NCH}_{2}\right), 3.13-3.26(\mathrm{~m}, 1 \mathrm{H}, \mathrm{CH}), 3.68-3.81\left(\mathrm{~m}, 4 \mathrm{H}, \mathrm{NCH}_{2}\right)$,

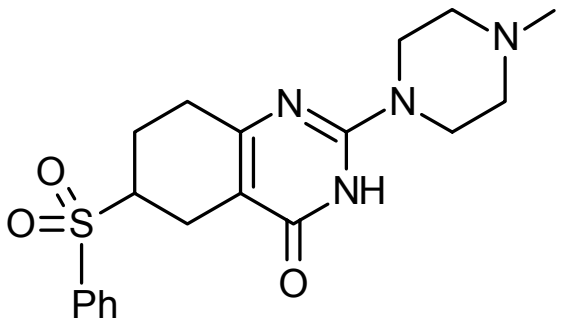
7.52-7.71 (m, 3 H, aryl-H), 8.89-8.98 (m, 2 H, aryl-H) 11.42 (br. s, $1 \mathrm{H}, \mathrm{NH}) .-{ }^{13} \mathrm{C}-\mathrm{NMR}(50.3$ MHz, [D 6 DMSO): $\delta=21.0\left(-, \mathrm{CH}_{2}\right), 21.2\left(-, \mathrm{CH}_{2}\right), 29.6\left(-, \mathrm{CH}_{2}\right), 43.8\left(-, \mathrm{NCH}_{2}\right), 45.1(+), 53.7$ (-, $\left.\mathrm{NCH}_{2}\right), 58.0(+), 104.4(\mathrm{C}-4 \mathrm{a}), 128.0$ (+, aryl-C), 129.0 (+, aryl-C), 133.4 (+, aryl-C), 137.1 $\left(\mathrm{C}_{\text {ipso }}\right), 152.6$ ( $\left.\mathrm{C}_{\text {quat }}, \mathrm{C}-8 \mathrm{a}\right), 159.3$ ( $\left.\mathrm{C}_{\text {quat }}, \mathrm{C}-2\right), 163.2$ (C $\left.\mathrm{C}_{\text {quat }}, \mathrm{C}-4\right) .-\mathrm{MS}(70 \mathrm{eV}), \mathrm{m} / z(\%): 388(16)$ $\left[\mathrm{M}^{+}\right], 318$ (100), 306 (24), 176 (74), 83 (55), 71 (26). - $\mathrm{C}_{19} \mathrm{H}_{22} \mathrm{~N}_{4} \mathrm{O}_{3} \mathrm{~S}$ (388.5): calcd. C 58.74, $\mathrm{H}$ 6.23, N 14.42; found C 58.64, H 6.14, N 14.29.

2-(Morpholin-4-yl)-7,8-dihydroquinazolin-4(3H)-one (47a): The crude product obtained from 45a (175 mg, $0.47 \mathrm{mmol}), \mathrm{KO} t \mathrm{Bu}(264 \mathrm{mg}, 2.35 \mathrm{mmol})$ in $10 \mathrm{~mL}$ THF, according to GP 5, after $15 \mathrm{~h}$ was purified by column chromatography $\left(R_{\mathrm{f}}=0.45, \mathrm{Et}_{2} \mathrm{O} / \mathrm{MeOH}=25: 1,1.5 \times 30 \mathrm{~cm}, 25 \mathrm{~g} \mathrm{SiO}_{2}\right)$ to yield $60 \mathrm{mg}(84 \%)$ of $47 \mathrm{a}$ as a white solid, m.p. $228-230^{\circ} \mathrm{C} .-\mathrm{IR}(\mathrm{KBr}): \mathrm{nu}(\mathrm{tillde})=2924 \mathrm{~cm}^{-1}$, $2849,1637,1585,1382,1263,1171,1115,987,862,729 .-{ }^{1} \mathrm{H}-\mathrm{NMR}\left(250 \mathrm{MHz}, \mathrm{CDCl}_{3}\right): \delta=$ 
2.28- $2.40\left(\mathrm{~m}, 2 \mathrm{H}, \mathrm{CH}_{2}\right), 2.62[\mathrm{t}, J=9.6 \mathrm{~Hz}, 2 \mathrm{H}, \mathrm{C}(8)-\mathrm{H}], 3.77-3.84\left(\mathrm{~m}, 8 \mathrm{H}, \mathrm{NCH}_{2}\right), 6.45$ [dt, $J$ $=9.7$ and $4.3 \mathrm{~Hz}, 1 \mathrm{H}, \mathrm{C}(6)-\mathrm{H}], 6.73$ [dt, $J=9.7$ and $1.8 \mathrm{~Hz}, 1 \mathrm{H}, \mathrm{C}(5)-$ $\mathrm{H}], 12.12$ (br. s, $1 \mathrm{H}, \mathrm{NH}) .-{ }^{13} \mathrm{C}-\mathrm{NMR}\left(62.9 \mathrm{MHz} \mathrm{CDCl}_{3}\right): \delta=22.6$ (-, $\left.\mathrm{CH}_{2}\right), 30.2\left(-, \mathrm{CH}_{2}\right), 44.9\left(-, \mathrm{NCH}_{2}\right), 66.4\left(-, \mathrm{NCH}_{2}\right), 107.5\left(\mathrm{C}_{\text {quat }}\right.$, C-4a), 119.6 (+, C-5), 122.1 (+, C-6), $152.4\left(\mathrm{C}_{\text {quat }}, \mathrm{C}-8 \mathrm{a}\right), 162.5$ ( $\mathrm{C}_{\text {quat }}$, $\mathrm{C}-2 / \mathrm{C}-4), 164.2\left(\mathrm{C}_{\text {quat }}, \mathrm{C}-4 / \mathrm{C}-2\right)$. - MS (70 eV), m/z (\%): 234 (11) $\left[\mathrm{M}^{+}+1\right], 233(100)\left[\mathrm{M}^{+}\right], 202(62)\left[\mathrm{M}^{+}-\mathrm{CH}_{2} \mathrm{OH}\right], 188(16), 176(30)$.

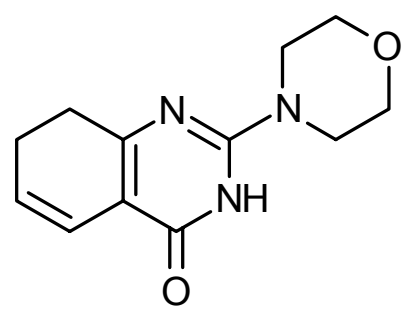
$-\mathrm{C}_{12} \mathrm{H}_{15} \mathrm{~N}_{3} \mathrm{O}_{2}$ (233.3): calcd. C 61.79, H 6.48, N 18.01; found C 61.54, H 6.72, N 17.91.

2-(4-Benzylpiperazin-1-yl)-7,8-dihydroquinazolin-4(3H)-one (47b): The crude product obtained from $45 \mathbf{b}(200 \mathrm{mg}, 0.43 \mathrm{mmol})$ and $\mathrm{KO} t \mathrm{Bu}(480 \mathrm{mg}, 4.30 \mathrm{mmol})$ according to GP 5, was purified by column chromatography $\left(R_{\mathrm{f}}=0.41, \mathrm{DCM} / \mathrm{MeOH}=25: 1\right.$, $\left.1.5 \times 30 \mathrm{~cm}, 25 \mathrm{~g} \mathrm{SiO}_{2}\right)$ to yield $131 \mathrm{mg}(94 \%)$ of $\mathbf{4 7} \mathrm{b}$ as a white solid, m.p. $=196-197^{\circ} \mathrm{C} .-\mathrm{IR}(\mathrm{KBr}): v=3040 \mathrm{~cm}^{-1}, 2953,1636$, 1576, 1388, 1311, 1277, 1170, 1005, 848, 726. - ${ }^{1}$ H-NMR (250 $\left.\mathrm{MHz}, \mathrm{CDCl}_{3}\right): \delta=2.22-2.40(\mathrm{~m}, 2 \mathrm{H}), 2.49-2.63(\mathrm{~m}, 6 \mathrm{H}), 3.54$ (s, $2 \mathrm{H}), 3.72-3.82(\mathrm{~m}, 4 \mathrm{H}), 5.68[\mathrm{dt}, J=9.5$ and $4.3 \mathrm{~Hz}, 1 \mathrm{H}$,

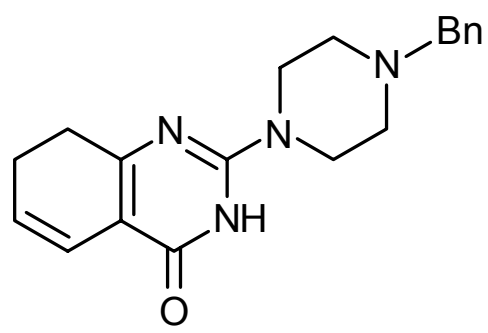
$\mathrm{C}(6)-\mathrm{H}], 6.42[\mathrm{dt}, J=9.5,1.7 \mathrm{~Hz}, 1 \mathrm{H}, \mathrm{C}(5)-\mathrm{H}], 7.26-7.38\left(\mathrm{~m}, 5 \mathrm{H}\right.$, aryl-H). $-{ }^{13} \mathrm{C}-\mathrm{NMR}(62.9$ $\left.\mathrm{MHz}, \mathrm{CDCl}_{3}\right): \delta=22.7\left(-, \mathrm{CH}_{2}\right), 29.7\left(-, \mathrm{CH}_{2}\right), 44.5\left(-, \mathrm{NCH}_{2}\right), 46.0\left(-, \mathrm{NCH}_{2}\right), 54.5(-, \mathrm{Bn}-$ $\mathrm{CH}_{2}$ ), 107.1 (C-4a), 119.8 (+, C-5), 121.6 (+, C-6), 127.2 (+, aryl-C), 128.3 (+, aryl-C), 137.6 $\left(\mathrm{C}_{\text {ipso }}\right), 152.2\left(\mathrm{C}_{\text {quat }}, \mathrm{C}-8 \mathrm{a}\right), 162.5\left(\mathrm{C}_{\text {quat, }}, \mathrm{C}-2 / \mathrm{C}-4\right), 164.2\left(\mathrm{C}_{\text {quat, }}, \mathrm{C}-4 / \mathrm{C}-2\right) .-\mathrm{MS}(70 \mathrm{eV}), \mathrm{m} / \mathrm{z}(\%)$ : $322(71)\left[\mathrm{M}^{+}\right], 189(30), 176(100), 146(38), 91$ (53). $-\mathrm{C}_{19} \mathrm{H}_{22} \mathrm{~N}_{4} \mathrm{O}$ (322.4): calcd. C 70.78, H 6.88, N 17.38; found C 70.45, H 6.47, N 17.50 .

2-(4-Methylpiperazin-1-yl)-7,8-dihydroquinazolin-4(3H)-one (47c): The crude product obtained from $45 \mathrm{c}(220 \mathrm{mg}, 0.57 \mathrm{mmol})$ and $\mathrm{KO} t \mathrm{Bu}(638 \mathrm{mg}, 5.70 \mathrm{mmol})$ according to the GP 5 was purified by column chromatography $\left(R_{\mathrm{f}}=\right.$ $0.40, \mathrm{DCM} / \mathrm{MeOH}=25: 1,1.5 \times 20 \mathrm{~cm}, 25 \mathrm{~g} \mathrm{Al}_{2} \mathrm{O}_{3}$ ) to yield $121 \mathrm{mg}$ (94\%) of $47 \mathbf{c}$ as a white solid, m.p. $=188{ }^{\circ} \mathrm{C} .-\mathrm{IR}(\mathrm{KBr}): v=3101$ $\mathrm{cm}^{-1}, 2935,2792,1654,1582,1387,1267,1140,1005,727 .{ }^{1}{ }^{1} \mathrm{H}-$ NMR $\left(250 \mathrm{MHz}, \mathrm{CDCl}_{3}\right): \delta=2.21-2.38(\mathrm{~m}, 5 \mathrm{H}), 2.41-2.54$ (m, 4<smiles>CN1CCN(c2nc3c(c(=O)[nH]2)C=CCC3)CC1</smiles> 
H), 2.55-2.66 (m, 2 H), 3.64-3.81(m, $4 \mathrm{H}), 5.72$ [dt, $J=9.7$ and 4.3 Hz, $1 \mathrm{H}, \mathrm{C}(6)-\mathrm{H}$ ], 6.48 [dt, $J$ $=9.7$ and $1.8 \mathrm{~Hz}, 1 \mathrm{H}, \mathrm{C}(5)-\mathrm{H}] .{ }^{13} \mathrm{C}-\mathrm{NMR}\left(62.9 \mathrm{MHz}, \mathrm{CDCl}_{3}\right): \delta=22.6\left(-, \mathrm{CH}_{2}\right), 25.5\left(-, \mathrm{CH}_{2}\right)$, $44.4\left(-, \mathrm{NCH}_{2}\right), 46.0(+), 54.5\left(-, \mathrm{NCH}_{2}\right), 107.1$ (C-4a), 119.8 (+, C-5), 121.6 (+, C-6), 156.9 $\left(\mathrm{C}_{\text {quat }}, \mathrm{C}-8 \mathrm{a}\right), 162.5$ (C $\left.\mathrm{C}_{\text {quat }}, \mathrm{C}-2 / \mathrm{C}-4\right), 164.2$ (C $\left.\mathrm{C}_{\text {quat }}, \mathrm{C}-4 / \mathrm{C}-2\right) .-\mathrm{MS}(70 \mathrm{eV}), \mathrm{m} / z(\%): 246(49)\left[\mathrm{M}^{+}\right]$, 189 (12), 176 (100). $-\mathrm{C}_{13} \mathrm{H}_{18} \mathrm{~N}_{4} \mathrm{O}$ (246.3): calcd. C 62.39, H 7.37, N 22.75; found C 62.37, $\mathrm{H}$ 7.23, N 22.59 .

2-(Morpholin-4-yl)-5,6,7,8-tetrahydroquinazolin-4(3H)-one (46a): The crude reaction mixture obtained from $47 \mathrm{a}(100 \mathrm{mg}, 0.43 \mathrm{mmol}), 15 \mathrm{mg}$ of $\mathrm{Pd} / \mathrm{C}$ in $25 \mathrm{~mL} \mathrm{MeOH}$ after $15 \mathrm{~h}$ according to the GP 6 afforded $97 \mathrm{mg}(96 \%)$ of 46a as a white solid, m.p. 204$205^{\circ} \mathrm{C}$. - IR $(\mathrm{KBr}): v=2925 \mathrm{~cm}^{-1}, 2856,1640,1576,1386,1270$, $1165,1121,1001,877,767 .-{ }^{1} \mathrm{H}-\mathrm{NMR}\left(200 \mathrm{MHz}, \mathrm{CDCl}_{3}\right): \delta=1.63-$ 1.90 (m, 4 H), 2.30-2.42 (m, 2 H), 2.43-2.58 (m, 2 H), 3.56-3.92 (m, 8 H), 11.6 (br. s, $1 \mathrm{H}, \mathrm{NH}) .-{ }^{13} \mathrm{C}-\mathrm{NMR}\left(50.3 \mathrm{MHz}, \mathrm{CDCl}_{3}\right): \delta=21.3(-$

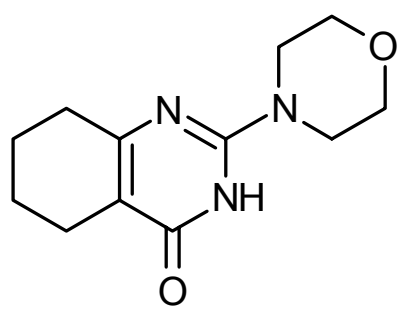
), $23.2\left(-, \mathrm{CH}_{2}\right), 22.5\left(-, \mathrm{CH}_{2}\right), 32.2\left(-, \mathrm{CH}_{2}\right), 44.9$ (-, $\left.\mathrm{NCH}_{2}\right), 66.5\left(-, \mathrm{OCH}_{2}\right), 109.7\left(\mathrm{C}_{\text {quat }}, \mathrm{C}-4 \mathrm{a}\right)$, $156.6\left(\mathrm{C}_{\text {quat }}, \mathrm{C}-8 \mathrm{a}\right), 163.6\left(\mathrm{C}_{\text {quat }}, \mathrm{C}-2 / \mathrm{C}-4\right), 165.7\left(\mathrm{C}_{\text {quat }}, \mathrm{C}-4 / \mathrm{C}-2\right) .-\mathrm{MS}(70 \mathrm{eV}), \mathrm{m} / z(\%): 235(84)$ $\left[\mathrm{M}^{+}\right], 204$ (100) $\left[\mathrm{M}^{+}-\mathrm{CH}_{2} \mathrm{OH}\right], 190$ (40), 178 (90), 150 (47). - $\mathrm{C}_{12} \mathrm{H}_{17} \mathrm{~N}_{3} \mathrm{O}_{2}$ (235.3): calcd. C 61.26, H 7.28, N 17.86; found C 61.41, H 7.40, N 17.65.

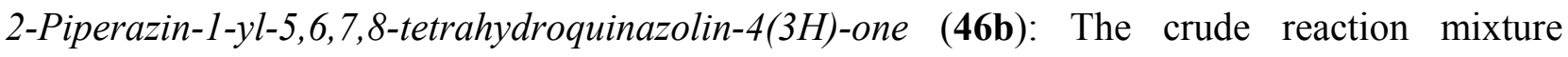
obtained from $\mathbf{4 7 b}(150 \mathrm{mg}, 0.47 \mathrm{mmol}), 15 \mathrm{mg}$ of $\mathrm{Pd} / \mathrm{C}$ in $25 \mathrm{~mL}$ of $\mathrm{MeOH}$ after $15 \mathrm{~h}$ according to the GP 6 afforded $99 \mathrm{mg}(90 \%)$ of 46b as a white solid, m.p. $=121^{\circ} \mathrm{C}$. $-\mathrm{IR}(\mathrm{KBr}): v=2930 \mathrm{~cm}^{-1}$, 1700, 1635, 1576, 1437, 1398, 1267, 998. - ${ }^{1} \mathrm{H}-\mathrm{NMR}(250 \mathrm{MHz}$, $\left.\mathrm{CDCl}_{3}\right): \delta=1.60-1.82(\mathrm{~m}, 4 \mathrm{H}), 2.28-2.54(\mathrm{~m}, 4 \mathrm{H}), 2.84-3.02(\mathrm{~m}, 4$

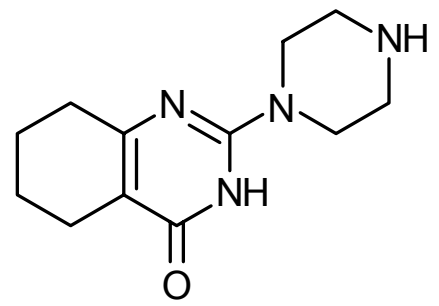
$\mathrm{H}), 3.62-3.71(\mathrm{~m}, 4 \mathrm{H})$, aryl H 11.81 (br. s, $1 \mathrm{H}, \mathrm{NH}) .{ }^{13} \mathrm{C}-\mathrm{NMR}\left(75.5 \mathrm{MHz}, \mathrm{CDCl}_{3}\right): \delta=21.4(-$, $\left.\mathrm{CH}_{2}\right), 22.3\left(-, \mathrm{CH}_{2}\right), 22.6\left(-, \mathrm{CH}_{2}\right), 32.3\left(-, \mathrm{CH}_{2}\right), 45.6\left(-, \mathrm{CH}_{2}\right), 109.2\left(\mathrm{C}_{\text {quat }}, \mathrm{C}-4 \mathrm{a}\right), 151.7\left(\mathrm{C}_{\text {quat }}\right.$,

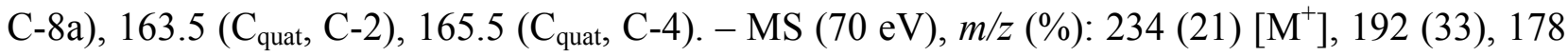
(55), $166(74), 72(100)$. 
2-(4-Methylpiperazin-1-yl)-5,6,7,8-tetrahydroquinazolin-4(3H)-one (46c): The crude reaction mixture obtained from $47 \mathrm{c}$ (100 mg, $0.41 \mathrm{mmol}), 15 \mathrm{mg}$ of $\mathrm{Pd} / \mathrm{C}$ in $25 \mathrm{~mL}$ of $\mathrm{MeOH}$ after $15 \mathrm{~h}$ according to the GP 6 afforded $74 \mathrm{mg}(96 \%)$ of $46 \mathbf{c}$ as a white solid, m.p. $210{ }^{\circ} \mathrm{C}$. - IR (KBr): $v=3091 \mathrm{~cm}^{-1}, 2934,2785,1642,1576$, 1387, 1308, 1267, 1150, 1000, 845. - ${ }^{1} \mathrm{H}-\mathrm{NMR}\left(250 \mathrm{MHz}, \mathrm{CDCl}_{3}\right)$ : $\delta=1.61-1.80(\mathrm{~m}, 4 \mathrm{H}), 2.24-2.40(\mathrm{~m}, 5 \mathrm{H}), 2.42-2.58(\mathrm{~m}, 6 \mathrm{H})$, 3.64-3.74 (m, $4 \mathrm{H}), 11.81$ (br. s, $1 \mathrm{H}, \mathrm{NH}) .-{ }^{13} \mathrm{C}-\mathrm{NMR}(62.9 \mathrm{MHz}$,

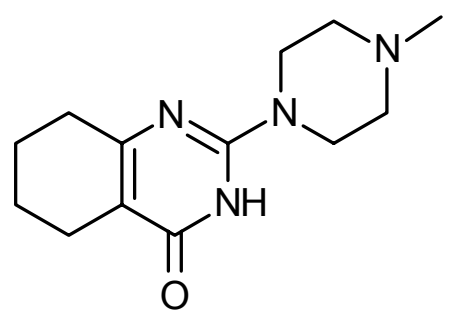
$\left.\mathrm{CDCl}_{3}\right): \delta=21.3\left(-, \mathrm{CH}_{2}\right), 22.3\left(-, \mathrm{CH}_{2}\right), 22.6\left(-, \mathrm{CH}_{2}\right), 32.3\left(-, \mathrm{CH}_{2}\right), 44.5\left(-, \mathrm{NCH}_{2}\right), 46.0(+$, $\left.\mathrm{CH}_{3}\right), 54.6\left(-, \mathrm{NCH}_{2}\right), 109.7\left(\mathrm{C}_{\text {quat, }}, \mathrm{C}-4 \mathrm{a}\right), 151.5\left(\mathrm{C}_{\text {quat, }}, \mathrm{C}-8 \mathrm{a}\right), 163.6\left(\mathrm{C}_{\text {quat, }}, \mathrm{C}-2 / \mathrm{C}-4\right), 165.6\left(\mathrm{C}_{\text {quat }}\right.$, C-4/C-2). - MS (70 eV), m/z (\%): 248 (17) $\left[\mathrm{M}^{+}\right], 178$ (100), 166 (12), 83 (28), 71 (19). $\mathrm{C}_{13} \mathrm{H}_{20} \mathrm{~N}_{4} \mathrm{O}$ (248.3): calcd. C 62.88, H 8.12, N 22.56; found C 62.59, H 8.12, N 22.40.

2-Dimethylamino-6-benzenesulfonyl-5,6,7,8-tetrahydroquinazolin-4(3H)-one (48): 37h (168 mg, $0.5 \mathrm{mmol}$ ) was dissolved in $2 \mathrm{~mL}$ of DMF in a $10 \mathrm{ml}$ pyrex bottle. This bottle was tightly sealed and heated at $180{ }^{\circ} \mathrm{C}$ for $12 \mathrm{~h}$. The reaction mixture was cooled to room temperature, excess of DMF was removed at reduced pressure, and the crude product was filtered through a pad of $\mathrm{SiO}_{2}$ $(2 \times 3 \mathrm{~cm}, 10 \mathrm{~g}, \mathrm{DCM} / \mathrm{MeOH}=10: 1)$ to yield $143 \mathrm{mg}(86 \%)$ of 48 as a white solid, m.p. $=249^{\circ} \mathrm{C}$. $-\mathrm{IR}(\mathrm{KBr}): v=2929 \mathrm{~cm}^{-1}, 1635$,

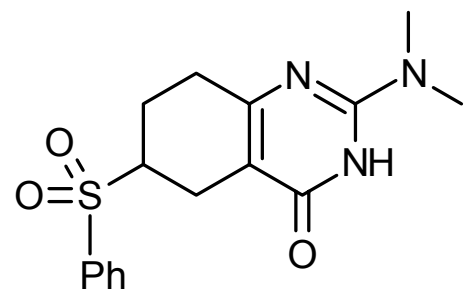
1586, 1302, 1138, 1084. $-{ }^{1} \mathrm{H}-\mathrm{NMR}\left(250 \mathrm{MHz}, \mathrm{CDCl}_{3}\right): \delta=1.71-1.92$ (m, $\left.1 \mathrm{H}\right), 2.31-2.87$ (m, 4 H), $3.11(\mathrm{~s}, 3 \mathrm{H}), 3.13-3.26$ (m, $1 \mathrm{H}), 3.58-3.72(\mathrm{~m}, 1 \mathrm{H})$, 7.53-7.74 (m, 3 H, aryl-H), 7.90-7.99 (m, $2 \mathrm{H}$, aryl-H), 11.59 (br s, $1 \mathrm{H}, \mathrm{NH}) .-{ }^{13} \mathrm{C}-\mathrm{NMR}\left(62.9 \mathrm{MHz}, \mathrm{CDCl}_{3}\right): \delta=21.5\left(-, \mathrm{CH}_{2}\right), 22.0$ $\left(-, \mathrm{CH}_{2}\right), 31.1\left(-, \mathrm{CH}_{2}\right), 37.4\left(+, \mathrm{CH}_{3}\right), 59.9\left(+, \mathrm{CH}_{3}\right), 104.1\left(\mathrm{C}_{\text {quat }}, \mathrm{C}-4 \mathrm{a}\right), 128.9$ (+, aryl-C), 129.1 (+, aryl-C), $133.8\left(+\right.$, aryl-C), $137.1\left(\mathrm{C}_{\text {ipso }}\right), 152.5\left(\mathrm{C}_{\text {quat }}, \mathrm{C}-8 \mathrm{a}\right), 161.9\left(\mathrm{C}_{\text {quat }}, \mathrm{C}-2 / \mathrm{C}-4\right), 164.8\left(\mathrm{C}_{\text {quat }}\right.$, C-4/C-2). - MS (70 eV), m/z (\%): 333 (6) $\left[\mathrm{M}^{+}\right], 192$ (20), 191 (100), 162 (10), 77 (15). $\mathrm{C}_{16} \mathrm{H}_{19} \mathrm{~N}_{3} \mathrm{O}_{3} \mathrm{~S}$ (333.4): calcd. C 57.64, H 5.74, N 12.60; found C 57.58, H 5.45, N 12.48. 


\subsubsection{Synthesis of cyclopropyl analogue of Tadalafil}

Methyl 2-chloro-2-[1-(1H-indol-3-yl)cyclopropyl]acetate (55). To a solution of methyl 2-chloro2-cyclopropylidineacetate $(4.40 \mathrm{~g}, 30.0 \mathrm{mmol})$ and indole $(5.97 \mathrm{~g}, 51.0 \mathrm{mmol})$ in $\mathrm{CH}_{2} \mathrm{Cl}_{2}$ $(150 \mathrm{~mL})$ was added ethylaluminum dichloride $(51.0 \mathrm{~mL}$, $51.0 \mathrm{mmol}, 1 \mathrm{M}$ solution in hexane) at $0{ }^{\circ} \mathrm{C}$, and the mixture was stirred at this temperature for $6 \mathrm{~h}$. The mixture was carefully poured into an ice-cold saturated $\mathrm{Na}_{2} \mathrm{CO}_{3}$ solution $(150 \mathrm{~mL})$. Extraction of the aq. phase with $\mathrm{CH}_{2} \mathrm{Cl}_{2}(3 \times 200 \mathrm{~mL})$, drying of

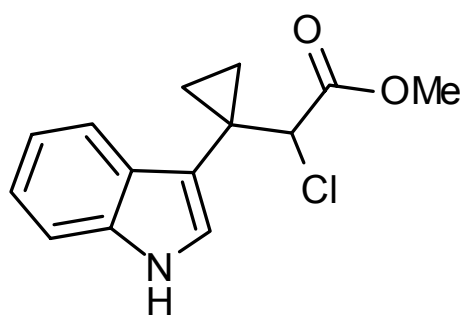
the combined organic phases over $\mathrm{MgSO}_{4}$, evaporation of the solvents and chromatographic purification of the residue on $80 \mathrm{~g}$ of silica gel $(4.5 \times 20 \mathrm{~cm}$, $R_{\mathrm{f}}=0.45$, pentane/ $\left.\mathrm{Et}_{2} \mathrm{O}=5: 1\right)$ yielded $6.70 \mathrm{~g}(85 \%)$ of $\mathbf{5 5}$ as a pale yellow oil. - IR (film): $v=$ $3306 \mathrm{~cm}^{-1}, 3008,2949,1747,1456,1420,1313,1195,1170,1010 .-{ }^{1} \mathrm{H}-\mathrm{NMR}(250 \mathrm{MHz}$, $\left.\mathrm{CDCl}_{3}\right): \delta=1.02-1.24(\mathrm{~m}, 3 \mathrm{H}, \mathrm{cPr}-\mathrm{H}), 1.35-1.46(\mathrm{~m}, 1 \mathrm{H}, \mathrm{cPr}-\mathrm{H}), 3.60\left(\mathrm{~s}, 3 \mathrm{H}, \mathrm{OCH}_{3}\right), 4.31$ (s, $1 \mathrm{H}, 2-\mathrm{H}), 7.06-7.38$ (m, $4 \mathrm{H}$, aryl -H), 7.65-7.72 (m, $1 \mathrm{H}$, aryl-H), 8.00-8.14 (br s, $1 \mathrm{H}, \mathrm{NH})$. ${ }^{13} \mathrm{C}-\mathrm{NMR}\left(62.9 \mathrm{MHz}, \mathrm{CDCl}_{3}, \mathrm{DEPT}\right): \delta=13.6(-, \mathrm{cPr}-\mathrm{C}), 21.6\left(\mathrm{C}_{\text {quat }}, \mathrm{cPr}-\mathrm{C}\right), 52.8\left(+, \mathrm{OCH}_{3}\right)$, $69.2(+, 2-\mathrm{H}), 111.4\left(+\right.$, aryl-C), $113.9\left(\mathrm{C}_{\text {quat }}\right.$, aryl-C), 118.9 (+, aryl-C), 119.4 (+, aryl-C), 121.8 (+, aryl-C), 125.8 (+, aryl-C), $127.2\left(\mathrm{C}_{\text {quat }}\right.$, aryl-C), $135.7\left(\mathrm{C}_{\text {quat }}\right.$, aryl-C), 169.2 $\left(\mathrm{C}_{\text {quat }}, \mathrm{C}=\mathrm{O}\right) .-\mathrm{MS}$ (70 eV); $m / z(\%):$ 265/263 (14/52) $\left[\mathrm{M}^{+}\right], 228(100)\left[\mathrm{M}^{+}-\mathrm{Cl}\right], 196(73), 168(35),\left[\mathrm{M}^{+}-\mathrm{Cl}-\right.$ $\left.\mathrm{CO}_{2} \mathrm{Me}-\mathrm{H}\right], 130$ (29), 83 (28). $-\mathrm{C}_{14} \mathrm{H}_{14} \mathrm{ClNO}_{2}$ (263.7): calcd. 263.0713 (correct HRMS).

Methyl 2-azido-2-[1-(1H-indol-3-yl)cyclopropyl]acetate (57). A suspension of 55 (6.50 g, $24.7 \mathrm{mmol}), \mathrm{NaN}_{3}(4.80 \mathrm{~g}, 73.9 \mathrm{mmol})$ and Aliquat $336^{\circledR}(12.4 \mathrm{~g}, 12.4 \mathrm{mmol})$ in water $(60 \mathrm{~mL})$ was heated at $70{ }^{\circ} \mathrm{C}$ for $20 \mathrm{~h}$. After cooling to $20^{\circ} \mathrm{C}$ the reaction mixture was extracted with $\mathrm{Et}_{2} \mathrm{O}(3 \times 100 \mathrm{~mL})$, and the combined organic phases were dried over $\mathrm{Na}_{2} \mathrm{SO}_{4}$. Removal of the solvent in vacuo and column chromatography of the residue on $80 \mathrm{~g}$ of silica gel $\left(4.5 \times 20 \mathrm{~cm}, R_{\mathrm{f}}=0.36, \mathrm{CH}_{2} \mathrm{Cl}_{2} / \mathrm{MeOH}=10: 1\right)$ yielded $6.10 \mathrm{~g}$

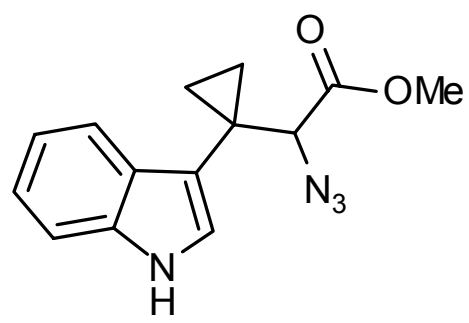
(91\%) of 57 as a slightly yellow oil. - IR (KBr): $v=3329 \mathrm{~cm}^{-1}, 2923,2104,1743,1442,1338$, 1270, 1233, 1033, 746. - ${ }^{1} \mathrm{H}-\mathrm{NMR}\left(250 \mathrm{MHz}, \mathrm{CDCl}_{3}\right): \delta=1.00-1.18$ (m, $\left.3 \mathrm{H}, \mathrm{cPr}-\mathrm{H}\right), 1.24-1.42$ (m, $1 \mathrm{H}, \mathrm{cPr}-\mathrm{H}), 3.68\left(\mathrm{~s}, 3 \mathrm{H}, \mathrm{OCH}_{3}\right), 3.75(\mathrm{~s}, 1 \mathrm{H}, 2-\mathrm{H}), 7.10-7.21(\mathrm{~m}, 3 \mathrm{H}$, aryl-H), 7.33 (d, 
${ }^{3} J=7.5 \mathrm{~Hz}, 1 \mathrm{H}$, aryl-H), $7.70\left(\mathrm{~d},{ }^{3} J=7.5 \mathrm{~Hz}, 1 \mathrm{H}\right.$, aryl-H), $8.00-8.14$ (br s, $\left.1 \mathrm{H}, \mathrm{NH}\right) .-{ }^{13} \mathrm{C}-$ $\operatorname{NMR}\left(62.9 \mathrm{MHz}, \mathrm{CDCl}_{3}, \mathrm{DEPT}\right): \delta=10.9$ (-, cPr-C), 11.9 (-, cPr-C), $20.1\left(\mathrm{C}_{\text {quat }}, \mathrm{cPr}-\mathrm{C}\right), 52.4(+$, $\left.\mathrm{OCH}_{3}\right), 69.2(+, \mathrm{C}-2), 111.4$ (+, aryl-C), 114.6 (C $\mathrm{C}_{\text {quat }}$, aryl-C), 119.0 (+, aryl-C), 119.6 (+, aryl-C), 122.0 (+, aryl-C), 125.3 (+, aryl-C), $127.3\left(\mathrm{C}_{\text {quat }}\right.$, aryl-C), $135.8\left(\mathrm{C}_{\text {quat }}\right.$, aryl-C), $169.6\left(\mathrm{C}_{\text {quat }}, \mathrm{C}=\mathrm{O}\right)$. - MS (70 eV); m/z (\%): 270 (71) $\left[\mathrm{M}^{+}\right], 228(100)\left[\mathrm{M}^{+}-\mathrm{N}_{3}\right], 196(56), 183(58)\left[\mathrm{M}^{+}-\mathrm{N}_{2}-\right.$ $\left.\mathrm{CO}_{2} \mathrm{Me}\right], 168(20)\left[\mathrm{M}^{+}-\mathrm{N}_{3}-\mathrm{CO}_{2} \mathrm{Me}-\mathrm{H}\right], 156$ (62), 129 (47), 117 (12). $-\mathrm{C}_{14} \mathrm{H}_{14} \mathrm{~N}_{4} \mathrm{O}_{2}$ (270.3): calcd. C 62.21, H 5.22; found: C 62.12, H 5.33.

Methyl 2-amino-2-[1-(1H-indol-3-yl)cyclopropyl]acetate (56). To a solution of 57 (6.00 g, $22.2 \mathrm{mmol})$ in $\mathrm{MeOH}(80 \mathrm{~mL})$ was added tin(II) chloride dihydrate $(9.90 \mathrm{~g}, 43.9 \mathrm{mmol})$, and the solution was stirred at $20^{\circ} \mathrm{C}$ for $18 \mathrm{~h}$. After removal of the solvent in vacuo, the residue was taken up in $\mathrm{CH}_{2} \mathrm{Cl}_{2}(150 \mathrm{~mL})$, the solution extracted with sat. $\mathrm{Na}_{2} \mathrm{CO}_{3}$ solution $(100 \mathrm{~mL})$ and the aq. layer reextracted with $\mathrm{CH}_{2} \mathrm{Cl}_{2}(3 \times 150 \mathrm{~mL})$. The combined organic phases were dried over $\mathrm{MgSO}_{4}$, the solvents removed, and the residue purified by column chromatography on $100 \mathrm{~g}$ of silica

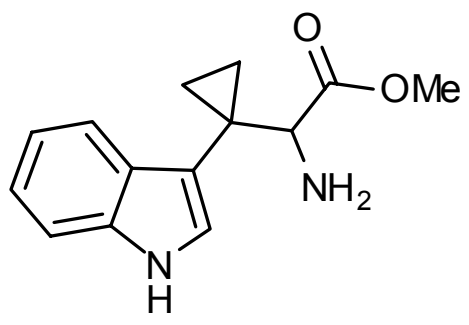
gel $\left(3 \times 30 \mathrm{~cm}, R_{\mathrm{f}}=0.30, \mathrm{CH}_{2} \mathrm{Cl}_{2} / \mathrm{MeOH}=10: 1\right)$ to yield $5.22 \mathrm{~g}(98 \%)$ of $\mathbf{5 6}$ as a pale yellow solid (m. p. $127^{\circ} \mathrm{C}$ ). - IR (film): $v=3348 \mathrm{~cm}^{-1}$ (NH), 3136, 2986, 2945, 2919, $1736(\mathrm{C}=\mathrm{O}), 1584$, 1435, 1199, 1015, 919, 741. - ${ }^{1} \mathrm{H}-\mathrm{NMR}$ (250 MHz, $\left.\mathrm{CDCl}_{3}\right): \delta=0.85-1.09$ (m, $\left.3 \mathrm{H}, \mathrm{cPr}-\mathrm{H}\right), 1.21-$ 1.29 (m, $1 \mathrm{H}, \mathrm{cPr}-\mathrm{H}), 1.65$ (s, $\left.2 \mathrm{H}, \mathrm{NH}_{2}\right), 3.08$ (s, $\left.1 \mathrm{H}, 2-\mathrm{H}\right), 3.65$ (s, $\left.3 \mathrm{H}, \mathrm{OCH}_{3}\right), 7.07-7.20$ (m, $3 \mathrm{H}$, aryl-H), 7.31 (d, ${ }^{3} J=7.7 \mathrm{~Hz}, 1 \mathrm{H}$, aryl-H), $7.70\left(\mathrm{~d},{ }^{3} J=7.7 \mathrm{~Hz}, 1 \mathrm{H}\right.$, aryl-H), 8.21-8.33 (br s, $1 \mathrm{H}, \mathrm{NH}) .-{ }^{13} \mathrm{C}-\mathrm{NMR}\left(62.9 \mathrm{MHz}, \mathrm{CDCl}_{3}, \mathrm{DEPT}\right): \delta=11.6(-, \mathrm{cPr}-\mathrm{C}), 12.2(-, \mathrm{cPr}-\mathrm{C}), 22.2\left(\mathrm{C}_{\text {quat }}\right.$, cPr-C), $51.8\left(+, \mathrm{OCH}_{3}\right), 62.6(+, \mathrm{C}-2), 111.3\left(+\right.$, aryl-C), $115.3\left(\mathrm{C}_{\text {quat }}\right.$, aryl-C), 119.3 (+, aryl-C), 119.5 (+, aryl-C), 122.0 (+, aryl-C), 124.9 (+, aryl-C), 127.8 ( $\mathrm{C}_{\text {quat }}$, aryl-C), 135.9 ( $\mathrm{C}_{\text {quat }}$, aryl-C), $174.7\left(\mathrm{C}_{\text {quat }}, \mathrm{C}=\mathrm{O}\right) .-\mathrm{MS}(70 \mathrm{eV}) ; \mathrm{m} / z(\%): 244(28)\left[\mathrm{M}^{+}\right], 215(12), 185(38)\left[\mathrm{M}^{+}-\mathrm{CO}_{2} \mathrm{Me}\right], 168$ (25), 156 (100), 129 (42), 117 (9). $-\mathrm{C}_{14} \mathrm{H}_{16} \mathrm{~N}_{2} \mathrm{O}_{2}$ (244.3): calcd. C 68.83, H 6.60; found: C 68.65, H 6.84 .

Methyl I'-(4-methoxyphenyl)-2',3',4',9'-tetrahydrospiro-(cyclopropane-1,4'-1H- $\beta$-carboline)-3'carboxylate (59a). To a solution of 56 (1.22 g, $5.00 \mathrm{mmol})$ in trimethyl orthoformate $(25 \mathrm{~mL})$ was added $p$-anisaldehyde $(694 \mathrm{mg}, 5.10 \mathrm{mmol})$ and the solution was stirred at $25^{\circ} \mathrm{C}$ for $8 \mathrm{~h}$. After 
removal of the volatiles in vacuo and dissolving of the residue in $\mathrm{CH}_{2} \mathrm{Cl}_{2}(25 \mathrm{~mL})$, at $0{ }^{\circ} \mathrm{C}$ trifluoroacetic acid $(2.24 \mathrm{~g}, 19.6 \mathrm{mmol})$ was added over a period of $5 \mathrm{~min}$, and the resulting red solution was stirred at $25{ }^{\circ} \mathrm{C}$ for $15 \mathrm{~h}$. Dilution with sat. aq. $\mathrm{NaHCO}_{3}(20 \mathrm{~mL})$, extraction of the aq. phase with $\mathrm{CH}_{2} \mathrm{Cl}_{2}$ $(2 \times 25 \mathrm{~mL})$, washing of the combined organic phases with brine (50 $\mathrm{mL}$ ), drying over $\mathrm{MgSO}_{4}$ and column chromatography of the residue on $50 \mathrm{~g}$ of silica gel $\left(3 \times 15 \mathrm{~cm}, R_{\mathrm{f}}=0.54, \mathrm{Et}_{2} \mathrm{O}\right)$ yielded $1.57 \mathrm{~g}(87 \%)$ of $59 \mathrm{a}$ as a mixture of cis- and trans-diastereomers $(1: 1.7)$. Recrystallization from EtOAc yielded the transdiastereomer ( $830 \mathrm{mg}, 46 \%$ ), as a colorless solid, m. p. $190^{\circ} \mathrm{C}$.

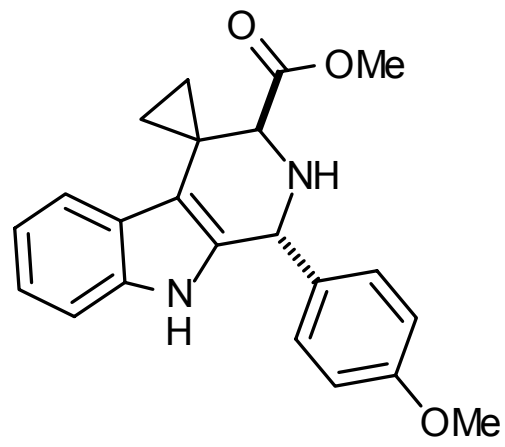
The residue from the mother liquor contained the cis- and trans-diastereomers in a ratio of 5:1, $\mathrm{m}$. p. $91-98{ }^{\circ} \mathrm{C}$. - trans-59a: - IR (KBr): $v=3393 \mathrm{~cm}^{-1}, 3171,2956,1725,1514,1246,1178,1027$. ${ }^{1} \mathrm{H}-\mathrm{NMR}\left(250 \mathrm{MHz}, \mathrm{CDCl}_{3}\right): \delta=1.06-1.12(\mathrm{~m}, 2 \mathrm{H}, \mathrm{cPr}-\mathrm{H}), 1.40-1.48$ (m, $\left.1 \mathrm{H}, \mathrm{cPr}-\mathrm{H}\right), 1.80$ 1.88 (m, $1 \mathrm{H}, \mathrm{cPr}-\mathrm{H}), 2.20-2.81$ (br s, $1 \mathrm{H}, \mathrm{NH}), 3.44$ (s, $1 \mathrm{H}, \mathrm{CH}), 3.70\left(\mathrm{~s}, 3 \mathrm{H}, \mathrm{OCH}_{3}\right), 3.82$ (s, $\left.3 \mathrm{H}, \mathrm{OCH}_{3}\right), 5.52(\mathrm{~s}, 1 \mathrm{H}, \mathrm{CH}), 6.84-6.90(\mathrm{~m}, 2 \mathrm{H}$, aryl-H), 6.94-7.12 (m, $2 \mathrm{H}$, aryl-H), 7.16-7.42 (m, $4 \mathrm{H}$, aryl-H), 7.55 (br s, $1 \mathrm{H}, \mathrm{NH}) .-{ }^{13} \mathrm{C}-\mathrm{NMR}\left(62.9 \mathrm{MHz}, \mathrm{CDCl}_{3}, \mathrm{DEPT}\right): \delta=10.7(-, \mathrm{cPr}-$ C), $13.6(-, \mathrm{cPr}-\mathrm{C}), 20.0\left(\mathrm{C}_{\text {quat }}, \mathrm{cPr}-\mathrm{C}\right), 51.8\left(+, \mathrm{OCH}_{3}\right), 54.2\left(+, \mathrm{OCH}_{3}\right), 55.3(+, \mathrm{CH}), 62.2(+$, $\mathrm{CH}), 111.2\left(+\right.$, aryl-C), $111.5\left(\mathrm{C}_{\text {quat }}\right.$, aryl-C), 114.1 (+, 2 C, aryl-C), 118.8 (+, aryl-C), 119.3 (+, aryl-C), 121.4 (+, aryl-C), 124.5 ( $\mathrm{C}_{\text {quat, }}$ aryl-C), 130.0 (+, $2 \mathrm{C}$, aryl-C), 133.3 ( $\mathrm{C}_{\text {quat }}$, aryl-C), 134.8 ( $\mathrm{C}_{\text {quat, }}$, aryl-C), 136.0 ( $\mathrm{C}_{\text {quat, }}$ aryl-C), 159.5 ( $\mathrm{C}_{\text {quat }}$, aryl-C), $172.7\left(\mathrm{C}_{\text {quat }}, \mathrm{C}=\mathrm{O}\right) .-\mathrm{MS}(70 \mathrm{eV}), \mathrm{m} / \mathrm{z}$ (\%): $362(100)\left[\mathrm{M}^{+}\right], 303$ (75) $\left[\mathrm{M}^{+}-\mathrm{CO}_{2} \mathrm{Me}\right], 195$ (50), 168 (18), $121(22) .-\mathrm{C}_{22} \mathrm{H}_{22} \mathrm{~N}_{2} \mathrm{O}_{3}$ (362.4): C 72.91, H 6.12, N 7.73; found: C 72.83, H 6.05, N 7.58. - cis-59a: - IR (KBr): $v=3394 \mathrm{~cm}^{-1}$, 3004, 2950, 1734, 1512, 1457, 1251, 1174, 1030. ${ }^{1} \mathrm{H}-\mathrm{NMR}\left(250 \mathrm{MHz}, \mathrm{CDCl}_{3}\right): \delta=0.86-0.91$ (m, $1 \mathrm{H}, \mathrm{cPr}-\mathrm{H}), 1.04-1.40$ (m, $2 \mathrm{H}, \mathrm{cPr}-\mathrm{H}), 1.68-1.80$ (m, $1 \mathrm{H}, \mathrm{cPr}-\mathrm{H}), 2.21-2.80$ (br s, $1 \mathrm{H}, \mathrm{NH}$ ), $3.46(\mathrm{~s}, 1 \mathrm{H}, \mathrm{CH}), 3.74\left(\mathrm{~s}, 3 \mathrm{H}, \mathrm{OCH}_{3}\right), 3.84\left(\mathrm{~s}, 3 \mathrm{H}, \mathrm{OCH}_{3}\right), 5.23(\mathrm{~s}, 1 \mathrm{H}, \mathrm{CH}), 6.84-6.92(\mathrm{~m}, 2 \mathrm{H}$, aryl-H), 6.94-7.12 (m, $2 \mathrm{H}$, aryl-H), 7.16-7.42 (m, $4 \mathrm{H}$, aryl-H), 7.52 (br s, $1 \mathrm{H}, \mathrm{NH}) .-{ }^{13} \mathrm{C}-\mathrm{NMR}$ (62.9 MHz, $\left.\mathrm{CDCl}_{3}, \mathrm{DEPT}\right): \delta=9.0$ (-, cPr-C), 11.8 (-, cPr-C), $20.2\left(\mathrm{C}_{\text {quat }}, \mathrm{cPr}-\mathrm{C}\right), 51.8(+$, $\left.\mathrm{OCH}_{3}\right), 55.3(+, \mathrm{CH}), 57.1\left(+, \mathrm{OCH}_{3}\right), 62.5(+, \mathrm{CH}), 111.3\left(+\right.$, aryl-C), $113.4\left(\mathrm{C}_{\text {quat }}\right.$, aryl-C), 114.3 (+, 2 C, aryl-C), 118.8 (+, aryl-C), 119.5 (+, aryl-C), 121.6 (+, aryl-C), 124.5 ( $\mathrm{C}_{\text {quat, }}$, aryl-C), 129.8 (+, $2 \mathrm{C}$, aryl-C), 132.1 ( $\mathrm{C}_{\text {quat }}$, aryl-C), 135.9 ( $\mathrm{C}_{\text {quat }}$, aryl-C), 159.7 ( $\mathrm{C}_{\text {quat }}$, aryl-C), $170.9\left(\mathrm{C}_{\text {quat }}\right.$, $\mathrm{C}=\mathrm{O}) .-\mathrm{MS}(70 \mathrm{eV}), \mathrm{m} / z(\%): 363 / 362(20 / 100)\left[\mathrm{M}^{+}\right], 303(75)\left[\mathrm{M}^{+}-\mathrm{CO}_{2} \mathrm{Me}\right], 302(35), 195$ 
(50), 168 (18), 121 (22). - $\mathrm{C}_{22} \mathrm{H}_{22} \mathrm{~N}_{2} \mathrm{O}_{3}$ (362.4): calcd. C 72.91, H 6.12, N 7.73; found: C 72.49, H 5.98, N 7.61.

Methyl 1'-(1,3-benzodioxole-5-yl)-2',3',4',9'-tetrahydrospiro[cyclopropane-1,4'-(1H- $\beta$ carboline)]-3'-carboxylate (59b). To a solution of $56(1.22 \mathrm{~g}, 5.00 \mathrm{mmol})$ in trimethyl orthoformate $(25 \mathrm{~mL})$ was added piperonal $(750 \mathrm{mg}, 5.00 \mathrm{mmol})$, and the solution was stirred at $25{ }^{\circ} \mathrm{C}$ for $8 \mathrm{~h}$. After removal of the volatiles in vacuo the residue was taken up in $\mathrm{CH}_{2} \mathrm{Cl}_{2}(30 \mathrm{~mL})$, at $0{ }^{\circ} \mathrm{C}$ trifluoroacetic acid $(2.28 \mathrm{~g}, 20.0 \mathrm{mmol})$ was added over a period of $5 \mathrm{~min}$, and the solution was stirred at $25^{\circ} \mathrm{C}$ for $12 \mathrm{~h}$. Dilution with $\mathrm{CH}_{2} \mathrm{Cl}_{2}(50 \mathrm{~mL})$, adding of sat. aq. $\mathrm{NaHCO}_{3}$ $(25 \mathrm{~mL})$, extraction of the aq. phase with $\mathrm{CH}_{2} \mathrm{Cl}_{2}(2 \times 30 \mathrm{~mL})$,

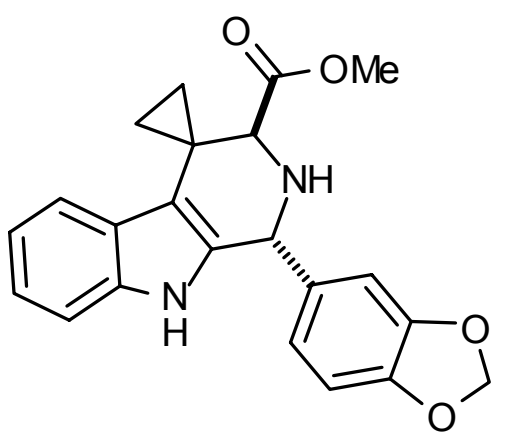
drying of the combined organic phases over $\mathrm{MgSO}_{4}$, evaporation of the solvents and column chromatography of the residue on $50 \mathrm{~g}$ of silica gel $\left(3 \times 15 \mathrm{~cm}, R_{\mathrm{f}}=0.40\right.$, pentane $\left./ \mathrm{Et}_{2} \mathrm{O}=2: 1\right)$ yielded $1.67 \mathrm{~g}(89 \%)$ of $59 \mathrm{~b}$ as a mixture of the cis- and trans-diastereomer $(1: 2.5)$. recrystallization from EtOAc/pentane yielded the trans-diastereomer (980 mg, 52\%) as a colorless solid, m. p. $190^{\circ} \mathrm{C}$. The residue from the mother liquor showed a ratio of $3: 1$ for the cis- and trans-diastereomer, m. p. 79-85 ${ }^{\circ} \mathrm{C}$. - trans-59b: IR (KBr): $v=3271 \mathrm{~cm}^{-1}, 2972,2892,1740$, 1487, 1354, 1238, 1111, 1038. - ${ }^{1} \mathrm{H}-\mathrm{NMR}\left(250 \mathrm{MHz}, \mathrm{CDCl}_{3}\right): \delta=1.06-1.12$ (m, $\left.2 \mathrm{H}, \mathrm{cPr}-\mathrm{H}\right)$, 1.38-1.42 (m, $1 \mathrm{H}, \mathrm{cPr}-\mathrm{H}), 1.80-1.82$ (m, $1 \mathrm{H}, \mathrm{cPr}-\mathrm{H}), 2.20$ (br s, $1 \mathrm{H}, \mathrm{NH}), 3.42$ (s, $1 \mathrm{H}, \mathrm{CH})$, $3.71\left(\mathrm{~s}, 3 \mathrm{H}, \mathrm{OCH}_{3}\right), 5.61(\mathrm{~s}, 1 \mathrm{H}, \mathrm{CH}), 5.94\left(\mathrm{~s}, 2 \mathrm{H}, \mathrm{OCH}_{2} \mathrm{O}\right), 6.72-6.92(\mathrm{~m}, 3 \mathrm{H}$, aryl-H), 6.967.14 (m, 2 H, aryl-H), 7.18-7.38 (m, 2 H, aryl-H), 7.60 (br s, $1 \mathrm{H}, \mathrm{NH}) .-{ }^{13} \mathrm{C}-\mathrm{NMR}(62.9 \mathrm{MHz}$, $\left.\mathrm{CDCl}_{3}, \mathrm{DEPT}\right): \delta=10.7$ (-, cPr-C), $13.6(-, \mathrm{cPr}-\mathrm{C}), 15.2\left(\mathrm{C}_{\text {quat }}, \mathrm{cPr}-\mathrm{C}\right), 51.7\left(+, \mathrm{OCH}_{3}\right), 54.6(+$, $\mathrm{CH}), 62.3(+, \mathrm{CH}), 101.1\left(-, \mathrm{OCH}_{2} \mathrm{O}\right), 108.1$ (+, aryl-C), 108.9 (+, aryl-C), 111.2 (+, aryl-C), $111.5\left(\mathrm{C}_{\text {quat }}\right.$, aryl-C), 118.8 (+, aryl-C), 119.4 (+, aryl-C), $121.4(+$, aryl-C), 122.0 (+, aryl-C), 124.5 ( $\mathrm{C}_{\text {quat }}$, aryl-C), 134.3 ( $\mathrm{C}_{\text {quat }}$, aryl-C), 135.5 ( $\mathrm{C}_{\text {quat }}$, aryl-C), 136.1 ( $\mathrm{C}_{\text {quat }}$, aryl-C), 147.5 ( $\mathrm{C}_{\text {quat }}$, aryl-C), $148.0\left(\mathrm{C}_{\text {quat }}\right.$, aryl-C), $172.9\left(\mathrm{C}_{\text {quat }}, \mathrm{C}=\mathrm{O}\right) .-\mathrm{MS}(70 \mathrm{eV}), m / z(\%): 376(100)\left[\mathrm{M}^{+}\right], 359$ (20), 317 (64) $\left[\mathrm{M}^{+}-\mathrm{CO}_{2} \mathrm{Me}\right], 195$ (10), 168 (18). $-\mathrm{C}_{22} \mathrm{H}_{20} \mathrm{~N}_{2} \mathrm{O}_{4}$ (376.4): calcd. C 70.20, H 5.36, N 7.44; found: C 69.90, H 5.60, N 7.55. - cis-59b: IR (KBr): $v=3302 \mathrm{~cm}^{-1}, 3013,2890,2773$, 1735, 1697, 1487, 1443, 1248, 1039. - ${ }^{1} \mathrm{H}-\mathrm{NMR}\left(250 \mathrm{MHz}, \mathrm{CDCl}_{3}\right): \delta=0.85-0.92(\mathrm{~m}, 1 \mathrm{H}, \mathrm{cPr}-$ H), 1.38-1.42 (m, $2 \mathrm{H}, \mathrm{cPr}-\mathrm{H}), 1.72-1.80$ (m, $1 \mathrm{H}, \mathrm{cPr}-\mathrm{H}), 3.10$ (br s, $1 \mathrm{H}, \mathrm{NH}), 3.75$ (s, $3 \mathrm{H}$, 
$\left.\mathrm{OCH}_{3}\right), 4.28(\mathrm{~s}, 1 \mathrm{H}, \mathrm{CH}), 5.20(\mathrm{~s}, 1 \mathrm{H}, \mathrm{CH}), 5.96\left(\mathrm{~s}, 2 \mathrm{H}, \mathrm{OCH}_{2} \mathrm{O}\right), 6.72-6.94(\mathrm{~m}, 3 \mathrm{H}, \mathrm{Ar}-\mathrm{H})$, 6.98-7.14 (m, 2 H, aryl-H), 7.18-7.38 (m, 2 H, aryl-H), 7.55 (br s, $1 \mathrm{H}, \mathrm{NH}) .-{ }^{13} \mathrm{C}-\mathrm{NMR}(62.9$ $\left.\mathrm{MHz}, \mathrm{CDCl}_{3}, \mathrm{DEPT}\right): \delta=10.7$ (-, cPr-C), 13.6 (-, cPr-C), $20.0\left(\mathrm{C}_{\text {quat }}, \mathrm{cPr}-\mathrm{C}\right), 51.8\left(+, \mathrm{OCH}_{3}\right)$, $54.2(+, \mathrm{CH}), 62.2(+, \mathrm{CH}), 101.4\left(-, \mathrm{OCH}_{2} \mathrm{O}\right), 108.2\left(+\right.$, aryl-C), $111.2\left(+\right.$, aryl-C), $111.5\left(\mathrm{C}_{\text {quat }}\right.$,

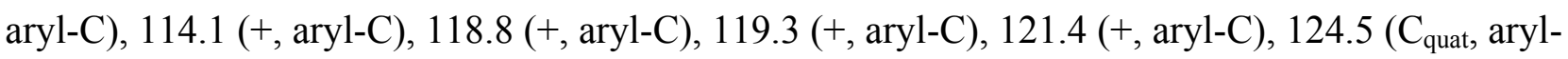
C), 130.0 (+, aryl-C), 133.3 ( $\mathrm{C}_{\text {quat }}$, aryl-C), 134.2 ( $\mathrm{C}_{\text {quat }}$, aryl-C), $134.8\left(\mathrm{C}_{\text {quat }}\right.$, aryl-C), $136.0\left(\mathrm{C}_{\text {quat }}\right.$, aryl-C), 159.5 ( $\mathrm{C}_{\text {quat }}$, aryl-C), $172.7\left(\mathrm{C}_{\text {quat }}, \mathrm{C}=\mathrm{O}\right) .-\mathrm{MS}(70 \mathrm{eV}), \mathrm{m} / z(\%): 376(100)\left[\mathrm{M}^{+}\right], 359$ (20), 317 (64) $\left[\mathrm{M}^{+}-\mathrm{CO}_{2} \mathrm{Me}\right], 315$ (20), 289 (12), 195 (10), 168 (18). - $\mathrm{C}_{22} \mathrm{H}_{20} \mathrm{~N}_{2} \mathrm{O}_{4}$ (376.4): calcd. C 70.20, H 5.36, N 7.44; found: C 70.48, H 5.56, N 7.13.

trans-2'-Butyl-10'-(4-methoxyphenyl)-3a',4',9',10'-tetrahydrospiro[cyclopropane-1,4'-(2', 9',10a'triazacyclopenta[b]fluorene)]-1',3'-dione (trans-25). To a solution of trans-59a (100 mg, $276 \mu \mathrm{mol})$ in methyl ethyl ketone $(5 \mathrm{~mL})$ was added nbutyl isocyanate $(31 \mathrm{mg}, 313 \mu \mathrm{mol})$, and the mixture was stirred at $100{ }^{\circ} \mathrm{C}$ for $18 \mathrm{~h}$. The solvent was removed in vacuo, and the residue purified by chromatography on $15 \mathrm{~g}$ of silica $(3 \times 15 \mathrm{~cm}$, $R_{\mathrm{f}}=0.35$, pentane $\left./ \mathrm{Et}_{2} \mathrm{O}=1: 1\right)$ to yield $108 \mathrm{mg}(91 \%)$ of trans25 as a colorless solid, m. p. $178{ }^{\circ} \mathrm{C}$. - IR $(\mathrm{KBr}): v=3309 \mathrm{~cm}^{-1}$, 2959, 2933, 2866, 1761, 1708, 1512, 1457, 1249, 1174, 1078. ${ }^{1} \mathrm{H}-\mathrm{NMR}\left(250 \mathrm{MHz}, \mathrm{CDCl}_{3}\right): \delta=0.92\left(\mathrm{t},{ }^{3} \mathrm{~J}=8.0 \mathrm{~Hz}, 3 \mathrm{H}, \mathrm{CH}_{3}\right)$,

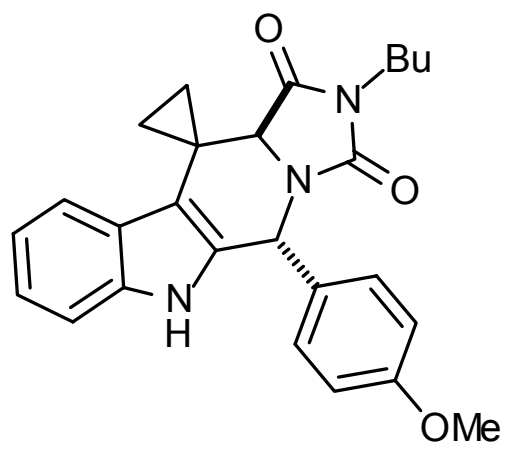
1.08-1.42 (m, 4 H, cPr-H), 1.55-1.62 (m, 3 H, Bu-H), 1.92-2.04 (m, 1 H, Bu-H), 3.40-3.52 (m, $\left.2 \mathrm{H}, \mathrm{NCH}_{2}\right), 3.77$ (s, $\left.3 \mathrm{H}, \mathrm{OCH}_{3}\right), 4.48(\mathrm{~s}, 1 \mathrm{H}, \mathrm{CH}), 6.3$ (s, $\left.1 \mathrm{H}, \mathrm{CH}\right), 6.80-6.91(\mathrm{~m}, 2 \mathrm{H}$, aryl-H), 7.04-7.5 (m, $6 \mathrm{H}$, aryl-H), 8.04 (br s, $1 \mathrm{H}, \mathrm{NH}) .-{ }^{13} \mathrm{C}-\mathrm{NMR}\left(50.3 \mathrm{MHz}, \mathrm{CDCl}_{3}, \mathrm{APT}\right): \delta=7.8(-$, cPr-C), 11.7 (-, cPr-C), 13.6 (+, $\left.\mathrm{CH}_{3}\right), 18.9$ (-, $\left.\mathrm{CH}_{2}\right), 19.9$ (-, cPr-C), 30.1 (-, $\left.\mathrm{CH}_{2}\right), 38.5$ (-, $\left.\mathrm{NCH}_{2}\right), 51.1\left(+, \mathrm{OCH}_{3}\right), 55.3(+, \mathrm{CH}), 56.4(+, \mathrm{CH}), 111.5(+$, aryl-C), $113.3(-$, aryl-C), $114.4(+$, 2 C, aryl-C), 119.0 (+, aryl-C), 120.0 (+, aryl-C), 122.4 (+, aryl-C), 123.9 (-, aryl-C), $129.6(+, 2$ C, aryl-C), 131.1 (-, aryl-C), 131.4 (-, aryl-C), 136.8 (-, aryl-C), 154.7 (-, aryl-C), 159.9 (-,

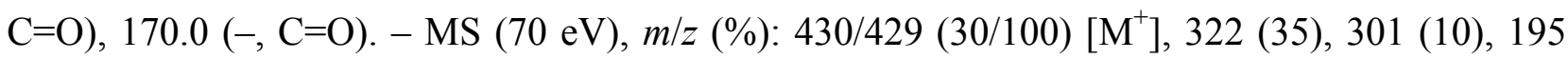
(10). $-\mathrm{C}_{26} \mathrm{H}_{27} \mathrm{~N}_{3} \mathrm{O}_{3}$ (429.5): calcd. C 72.71, H 6.34, N 9.78; found: C 72.57, H 6.32, N 9.57. 
cis-2'-Butyl-10'-(4-methoxyphenyl)-3a',4',9',10'-tetrahydrospiro[cyclopropane-1,4'-(2',9',10a'triazacyclopenta[b]fluorene)]-1',3'-dione (cis-25). To a solution of 59a (480 mg, $1.33 \mathrm{mmol}$, cis/trans $=5: 1)$ in MEK $(20 \mathrm{~mL})$ was added $n$ butyl isocyanate (158 mg, $1.60 \mathrm{mmol}$ ), and the mixture was stirred at $100{ }^{\circ} \mathrm{C}$ for $18 \mathrm{~h}$. Evaporation of the solvents and chromatographic purification of the residue on $20 \mathrm{~g}$ of silica $(3 \times 15 \mathrm{~cm}$, pentane $\left./ \mathrm{Et}_{2} \mathrm{O}=1: 2\right)$ yielded $84 \mathrm{mg}(89 \%$ from starting transdiastereomer) of trans-25 $\left(R_{\mathrm{f}}=0.71\right)$, and $426 \mathrm{mg}(90 \%$ from starting cis-diastereomer) of cis-25 $\left(R_{\mathrm{f}}=0.30\right)$ the latter as a colorless solid, m. p. $104-105^{\circ} \mathrm{C}$. $-\mathrm{IR}(\mathrm{KBr}): v=3290 \mathrm{~cm}^{-1}$,

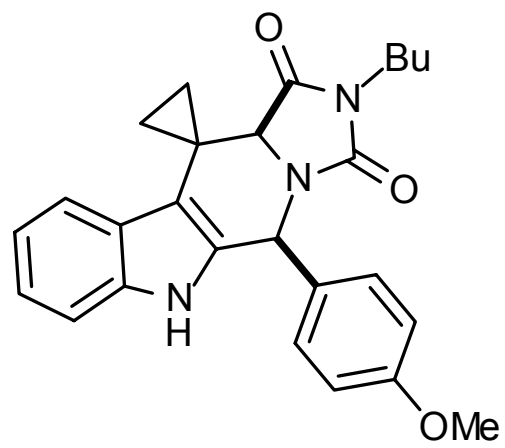
2961 2933, 2871, 1766, 1702, 1512, 1447, 1331, 1244, 1173, 1063. - ${ }^{1} \mathrm{H}-\mathrm{NMR}\left(250 \mathrm{MHz}, \mathrm{CDCl}_{3}\right)$ : $\delta=0.90\left(\mathrm{t},{ }^{3} \mathrm{~J}=8.0 \mathrm{~Hz}, 3 \mathrm{H}, \mathrm{CH}_{3}\right), 1.12-1.38(\mathrm{~m}, 4 \mathrm{H}, \mathrm{cPr}-\mathrm{H}), 1.55-1.62\left(\mathrm{~m}, 2 \mathrm{H}, \mathrm{CH}_{2}\right), 1.85-$ $2.04\left(\mathrm{~m}, 2 \mathrm{H}, \mathrm{CH}_{2}\right), 3.40-3.52\left(\mathrm{~m}, 2 \mathrm{H}, \mathrm{NCH}_{2}\right), 3.80\left(\mathrm{~s}, 3 \mathrm{H}, \mathrm{OCH}_{3}\right), 4.65$ (s, $\left.1 \mathrm{H}, \mathrm{CH}\right), 5.84$ (s, $1 \mathrm{H}, \mathrm{CH}), 6.85-6.91\left(\mathrm{~m}, 2 \mathrm{H}\right.$, aryl-H), 7.04-7.36 (m, $6 \mathrm{H}$, aryl-H), 7.52 (br s, $2 \mathrm{H}$, aryl-H). $-{ }^{13} \mathrm{C}-$ NMR (62.9 MHz, $\left.\mathrm{CDCl}_{3}, \mathrm{DEPT}\right): \delta=7.1(-, \mathrm{cPr}-\mathrm{C}), 11.0(-, \mathrm{cPr}-\mathrm{C}), 13.6\left(+, \mathrm{CH}_{3}\right), 18.9\left(-, \mathrm{CH}_{2}\right)$, $19.9\left(\mathrm{C}_{\text {quat }}, \mathrm{cPr}-\mathrm{C}\right), 30.0\left(-, \mathrm{CH}_{2}\right), 38.3\left(-, \mathrm{NCH}_{2}\right), 55.1\left(+, \mathrm{OCH}_{3}\right), 56.3(+, \mathrm{CH}), 60.5(+, \mathrm{CH})$, 111.4 (+, aryl-C), $112.0\left(\mathrm{C}_{\text {quat, }}\right.$ aryl-C), 114.1 (+, 2 C, aryl-C), 119.0 (+, aryl-C), 119.9 (+, aryl-C), 122.2 (+, aryl-C), $124.0\left(\mathrm{C}_{\text {quat }}\right.$, aryl-C), 129.1 (+, $2 \mathrm{C}$, aryl-C) 130.7 ( $\mathrm{C}_{\text {quat }}$, aryl-C), $134.3\left(\mathrm{C}_{\text {quat }}\right.$, aryl-C), 136.9 ( $\mathrm{C}_{\text {quat, }}$ aryl-C), 154.7 ( $\mathrm{C}_{\text {quat, }}$, aryl-C), $159.5\left(\mathrm{C}_{\text {quat, }}, \mathrm{C}=\mathrm{O}\right), 169.1\left(\mathrm{C}_{\text {quat, }}, \mathrm{C}=\mathrm{O}\right) .-\mathrm{MS}$ (70 eV), m/z (\%): 430/429 (30/100) [ $\left.\mathrm{M}^{+}\right], 322$ (38), 301 (10), 223 (10) 195 (10). - $\mathrm{C}_{26} \mathrm{H}_{27} \mathrm{~N}_{3} \mathrm{O}_{3}$ (429.5): calcd. C 72.71, H 6.34, N 9.78; found: C 72.45, H 6.29, N 9.81.

Methyl trans-1'-(1,3-benzodioxole-5-yl)-2'-(2-chloroacetyl)-2',3',4',9'-tetrahydrospiro-[cyclopropane-1,4'-(1H- $\beta$-carboline)]-3'-carboxylate (trans-60). To a mixture of trans-59b (619 mg, $1.65 \mathrm{mmol})$ and $1 \mathrm{~N} \mathrm{Na}_{2} \mathrm{CO}_{3}(8.25 \mathrm{~mL}, 8.25 \mathrm{mmol})$ in $\mathrm{CH}_{2} \mathrm{Cl}_{2}$ $(30 \mathrm{~mL})$ was added chloroacetyl chloride $(372 \mathrm{mg}, 3.30 \mathrm{mmol})$ at $0{ }^{\circ} \mathrm{C}$, the mixture was stirred at $20^{\circ} \mathrm{C}$ for $2 \mathrm{~h}$, diluted with $\mathrm{CH}_{2} \mathrm{Cl}_{2}(25 \mathrm{~mL})$ and washed with a sat. $\mathrm{NaHCO}_{3}$ solution $(20 \mathrm{~mL})$. Drying over $\mathrm{MgSO}_{4}$, evaporation of the solvents and chromatographic purification of the residue on $25 \mathrm{~g}$ of silica $\left(1.5 \times 20 \mathrm{~cm}, R_{\mathrm{f}}=0.40\right.$, pentane $\left./ \mathrm{Et}_{2} \mathrm{O}=1: 2\right)$ yielded $697 \mathrm{mg}$

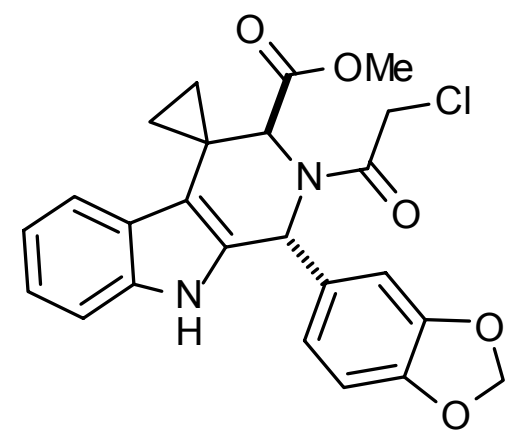


(93\%) of trans-60 as a colorless solid, m. p. $118^{\circ} \mathrm{C} .-\mathrm{IR}(\mathrm{KBr}): v=3302 \mathrm{~cm}^{-1}, 2890,1739,1662$, 1487, 1444, 1394, 1240, 1039. - ${ }^{1} \mathrm{H}-\mathrm{NMR}\left(250 \mathrm{MHz}, \mathrm{CDCl}_{3}\right): \delta=1.06-1.12(\mathrm{~m}, 1 \mathrm{H}, \mathrm{cPr}-\mathrm{H})$, 1.24-1.30 (m, $1 \mathrm{H}, \mathrm{cPr}-\mathrm{H}), 1.52-1.64$ (m, $1 \mathrm{H}, \mathrm{cPr}-\mathrm{H}), 2.18-2.26$ (m, $1 \mathrm{H}, \mathrm{cPr}-\mathrm{H}), 3.65$ (s, $3 \mathrm{H}$, $\left.\mathrm{OCH}_{3}\right), 3.74-4.15\left(\mathrm{~m}, 3 \mathrm{H}, \mathrm{CH}_{2}, \mathrm{CH}\right), 5.81-5.93\left(\mathrm{~m}, 2 \mathrm{H}, \mathrm{CH}_{2}\right), 6.12(\mathrm{~s}, 1 \mathrm{H}, \mathrm{CH}), 6.72-6.80(\mathrm{~m}$, $1 \mathrm{H}$, aryl-H), 6.86-7.00 (m, $2 \mathrm{H}$, aryl-H), 7.02-7.28 (m, $3 \mathrm{H}$, aryl-H), 7.40-7.51 (m, $1 \mathrm{H}$, aryl-H), 8.60 (br s, $1 \mathrm{H}, \mathrm{NH}) .-{ }^{13} \mathrm{C}-\mathrm{NMR}\left(62.9 \mathrm{MHz}, \mathrm{CDCl}_{3}, \mathrm{DEPT}\right): \delta=10.3(-, \mathrm{cPr}-\mathrm{C}), 15.3(-, \mathrm{cPr}-\mathrm{C})$, $19.5\left(\mathrm{C}_{\text {quat }}, \mathrm{cPr}-\mathrm{C}\right), 43.1\left(-, \mathrm{CH}_{2}\right), 52.8\left(+, \mathrm{OCH}_{3}\right), 57.6(+, \mathrm{CH}), 62.2(+, \mathrm{CH}), 101.2\left(-, \mathrm{OCH}_{2} \mathrm{O}\right)$, $106.4\left(\mathrm{C}_{\text {quat }}\right.$, aryl-C), $107.3(+$, aryl-H), $108.6(+$, aryl-H), $111.5(+$, aryl-H), 118.4 (+, aryl-H), $118.8\left(\mathrm{C}_{\text {quat }}\right.$, aryl-H), $119.3\left(+\right.$, aryl-H), $121.4\left(+\right.$, aryl-H), $124.5\left(+\right.$, aryl-H), $135.0\left(\mathrm{C}_{\text {quat }}, 2 \mathrm{C}\right.$, aryl$\mathrm{H}), 136.2\left(\mathrm{C}_{\text {quat }}, 2 \mathrm{C}\right.$, aryl-H), $149.2\left(\mathrm{C}_{\text {quart }}\right.$, aryl-H), $169.4\left(\mathrm{C}_{\text {quart }}, \mathrm{C}=\mathrm{O}\right), 171.8\left(\mathrm{C}_{\text {quat }}, \mathrm{C}=\mathrm{O}\right) .-\mathrm{MS}$ $(70 \mathrm{eV}), m / z$ (\%): 454/452 (22/63) $\left[\mathrm{M}^{+}\right], 417(36)\left[\mathrm{M}^{+}-\mathrm{Cl}\right], 375$ (100) $\left[\mathrm{M}^{+}-\mathrm{COCH}_{2} \mathrm{Cl}\right], 315$ (40), 195 (20). $-\mathrm{C}_{24} \mathrm{H}_{21} \mathrm{ClN}_{2} \mathrm{O}_{5}$ (452.9): calcd. C 63.65, $\mathrm{H}$ 4.67, $\mathrm{N}$ 6.19; found: $\mathrm{C}$ 63.82, $\mathrm{H}$ 4.92, $\mathrm{N}$ 5.96 .

Methyl cis-1'-(1,3-benzodioxole-5-yl)-2'-(2-chloroacetyl)-2',3',4',9'-tetrahydrospiro-[cyclopropane-1,4'-(1H- $\beta$-carboline)]-3'-carboxylate (cis-60). To a mixture of cis-59b (752 mg, $2.00 \mathrm{mmol}$, mixture of cis/trans $=3: 1)$ and $1 \mathrm{~N} \mathrm{Na} \mathrm{CO}_{3} \quad(10.0 \mathrm{~mL}$, $10.0 \mathrm{mmol})$ in $\mathrm{CH}_{2} \mathrm{Cl}_{2}(25 \mathrm{~mL})$ was added chloroacetyl chloride (452 mg, $4.00 \mathrm{mmol}$ ) at $0{ }^{\circ} \mathrm{C}$, the mixture was stirred at $20^{\circ} \mathrm{C}$ for $2 \mathrm{~h}$, diluted with $\mathrm{CH}_{2} \mathrm{Cl}_{2}(25 \mathrm{~mL})$ and washed with sat. $\mathrm{NaHCO}_{3}$ solution $(20 \mathrm{~mL})$. Drying over $\mathrm{MgSO}_{4}$, evaporation of the solvents and chromatographic purification on $25 \mathrm{~g}$ of silica $\left(1.5 \times 20 \mathrm{~cm}, R_{\mathrm{f}}=0.51\right.$, pentane $\left./ \mathrm{Et}_{2} \mathrm{O}=1: 1\right)$ yielded $551 \mathrm{mg}$

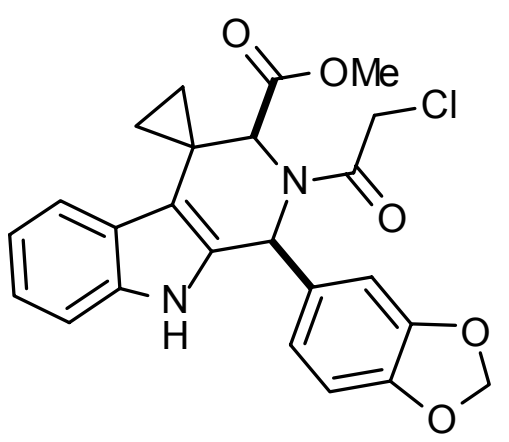
(81\% from pure cis-59) of cis-60 as a colorless solid, m. p. 125-132 C. - IR (KBr): $v=3295 \mathrm{~cm}^{-}$ ${ }^{1}, 2891,1738,1670,1486,1395,1040 .-{ }^{1} \mathrm{H}-\mathrm{NMR}\left(200 \mathrm{MHz}, \mathrm{CDCl}_{3}\right): \delta=0.86-0.92(\mathrm{~m}, 1 \mathrm{H}$, cPr-H), 0.96-1.15 (m, $1 \mathrm{H}, \mathrm{cPr}-\mathrm{H}), 142-1.58$ (m, $1 \mathrm{H}, \mathrm{cPr}-\mathrm{H}), 2.05-2.21$ (m, $1 \mathrm{H}, \mathrm{cPr}-\mathrm{H}), 3.58$ (s, $\left.3 \mathrm{H}, \mathrm{OCH}_{3}\right), 3.92-4.10\left(\mathrm{~m}, 3 \mathrm{H}, \mathrm{CH}_{2}, \mathrm{CH}\right), 5.78-5.95\left(\mathrm{~m}, 2 \mathrm{H}, \mathrm{OCH}_{2} \mathrm{O}\right), 6.08(\mathrm{~s}, 1 \mathrm{H}, \mathrm{CH}), 6.63-$ $6.76(\mathrm{~m}, 1 \mathrm{H}$, aryl-H), 6.82-7.13 (m, $4 \mathrm{H}$, aryl-H), 7.21-7.40 (m, $2 \mathrm{H}$, aryl-H), 7.82 (br s, $1 \mathrm{H}$, $\mathrm{NH}) .-{ }^{13} \mathrm{C}-\mathrm{NMR}\left(50.3 \mathrm{MHz}, \mathrm{CDCl}_{3}, \mathrm{APT}\right): \delta=10.2$ (-, cPr-C), 15.1 (-, cPr-C), 19.6 (-, cPr-C), $43.3\left(-, \mathrm{CH}_{2}\right), 52.7\left(+, \mathrm{OCH}_{3}\right), 57.7(+, \mathrm{CH}), 64.1(+, \mathrm{CH}), 101.3\left(-, \mathrm{OCH}_{2} \mathrm{O}\right), 106.4$, (-, aryl-C), 108.7 (+, aryl-C), 107.4 (+, aryl-C), 111.4 (+, aryl-C), 118.8 (-, aryl-C), 119.2 (-, aryl-C), 119.8 
(+, aryl-C), 122.1 (+, aryl-C), 124.2 (+, aryl-C), 136.3 (-, 2 C, aryl-C), 136.8 (-, 2 C, aryl-C), 147.5 (-, aryl-C), $166.2(-, \mathrm{C}=\mathrm{O}), 171.5(-, \mathrm{C}=\mathrm{O}) .-\mathrm{MS}(70 \mathrm{eV}), m / z(\%): 454 / 452$ (30/79) $\left[\mathrm{M}^{+}\right]$, 417 (48) $\left[\mathrm{M}^{+}-\mathrm{Cl}\right], 375$ (100) $\left[\mathrm{M}^{+}-\mathrm{COCH}_{2} \mathrm{Cl}\right], 315$ (46), 195 (20). $-\mathrm{C}_{24} \mathrm{H}_{21} \mathrm{ClN}_{2} \mathrm{O}_{5}(452.9)$ : calcd. C 63.65, H 4.67, N 6.19; found: C 63.90, H 5.31, N 6.16.

trans-6'-(1,3-Benzodioxole-5-yl)-2'-methyl-12'-spirocyclopropyl-2', 3', 6', 7',12',12a'-hexa-hydropyrazino[1',2':1,6]pyrido[3,4-b]indole-1',4'-dione (trans-26). A solution of trans-60 (596 mg, $1.32 \mathrm{mmol})$ and methylamine $\left(1 \mathrm{~mL}, 40 \%\right.$ in $\left.\mathrm{H}_{2} \mathrm{O}\right)$ in $\mathrm{EtOH}$ $(25 \mathrm{~mL})$ was heated at $75{ }^{\circ} \mathrm{C}$ for $16 \mathrm{~h}$. All volatiles were removed in vacuo, the residue was dissolved in $\mathrm{CH}_{2} \mathrm{Cl}_{2}(30 \mathrm{~mL})$, the solution washed with water $(20 \mathrm{~mL})$ and dried over $\mathrm{MgSO}_{4}$. Evaporation of the solvent and chromatographic purification of the residue on $25 \mathrm{~g}$ of silica $\left(1.5 \times 20 \mathrm{~cm}, R_{\mathrm{f}}=0.23\right.$, pentane/ $\left.\mathrm{Et}_{2} \mathrm{O}=1: 2\right)$ yielded $470 \mathrm{mg}(86 \%)$ of trans-26 as a

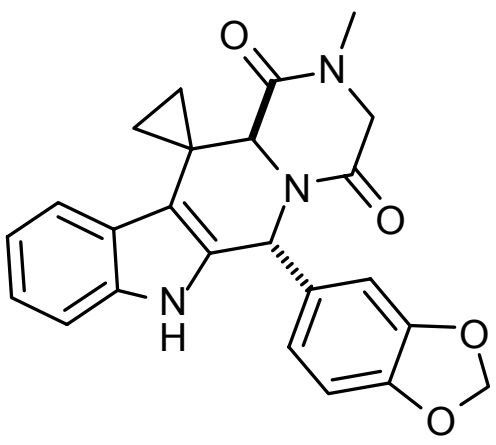
colorless solid, m. p. $>250{ }^{\circ} \mathrm{C}$. - IR (KBr): $v=3299 \mathrm{~cm}^{-1}, 2888,1664,1488,1330,1236,1036$, 935. $-{ }^{1} \mathrm{H}-\mathrm{NMR}\left(250 \mathrm{MHz}, \mathrm{CDCl}_{3}\right): \delta=0.70-0.78(\mathrm{~m}, 1 \mathrm{H}, \mathrm{cPr}-\mathrm{H}), 1.24-1.32(\mathrm{~m}, 1 \mathrm{H}, \mathrm{cPr}-\mathrm{H})$, 1.54-1.82 (m, $2 \mathrm{H}, \mathrm{cPr}-\mathrm{H}), 2.98\left(\mathrm{~s}, 3 \mathrm{H}, \mathrm{CH}_{3}\right), 3.86-4.20\left(\mathrm{~m}, 2 \mathrm{H}, \mathrm{CH}_{2}\right), 4.56(\mathrm{~s}, 1 \mathrm{H}, \mathrm{CH}), 5.32(\mathrm{~s}$, $1 \mathrm{H}, \mathrm{CH}), 5.95$ (s, $\left.2 \mathrm{H}, \mathrm{CH}_{2}\right), 6.68-6.82$ (m, $3 \mathrm{H}$, aryl-H), 6.96-7.20 (m, $2 \mathrm{H}$, aryl-H), 7.28-7.39 (m, $2 \mathrm{H}$, aryl-H), 8.12 (br s, $1 \mathrm{H}, \mathrm{NH}) .-{ }^{13} \mathrm{C}-\mathrm{NMR}$ (62.9 MHz, $\left.\mathrm{CDCl}_{3}, \mathrm{DEPT}\right): \delta=8.2(-, \mathrm{cPr}-\mathrm{C})$, 11.2 (-, cPr-C), $21.0\left(\mathrm{C}_{\text {quat }}, \mathrm{cPr}-\mathrm{C}\right), 33.5\left(+, \mathrm{CH}_{3}\right), 51.5\left(-, \mathrm{CH}_{2}\right), 53.4(+, \mathrm{CH}), 56.8(+, \mathrm{CH})$, $101.3\left(-, \mathrm{CH}_{2}\right), 108.3\left(\mathrm{C}_{\text {quat }}\right.$, aryl-C), 109.1 (+, aryl-C), 111.5 (+, aryl-C), 113.6 (+, aryl-C), 118.9 $\left(\mathrm{C}_{\text {quat }}\right.$, aryl-C), 120.0 (+, aryl-C), 122.4 (+, aryl-C), 122.5 (+, aryl-C), 123.9 (+, aryl-C), 130.4 ( $\mathrm{C}_{\text {quat }}$, aryl-C), 131.4 ( $\mathrm{C}_{\text {quat }}$, aryl-C), 136.5 ( $\mathrm{C}_{\text {quat }}$, aryl-C), $148.0\left(\mathrm{C}_{\text {quat }}\right.$, aryl-C), 148.2 ( $\mathrm{C}_{\text {quat, }}$, arylC), $162.9\left(\mathrm{C}_{\text {quat }}, \mathrm{C}=\mathrm{O}\right), 163.0\left(\mathrm{C}_{\text {quat }}, \mathrm{C}=\mathrm{O}\right) .-\mathrm{MS}(70 \mathrm{eV}), \mathrm{m} / z(\%): 415(100)\left[\mathrm{M}^{+}\right], 315(15), 289$ (48). $-\mathrm{C}_{24} \mathrm{H}_{21} \mathrm{~N}_{3} \mathrm{O}_{4}$ (415.5): calcd. 415.1532 (correct HRMS).

cis-6'-(1,3-Benzodioxole-5-yl)-2'-methyl-12'-spirocyclopropyl-2', 3', 6', 7', 12',12a'-hexahydropyrazino $\left[1^{\prime}, 2^{\prime}: 1,6\right]$ pyrido [3,4-b]indole-1',4'-dione (cis-26). A solution of cis-60 (226 mg, $\left.500 \mu \mathrm{mol}\right)$ and methylamine $\left(0.4 \mathrm{~mL}, 40 \%\right.$ in $\left.\mathrm{H}_{2} \mathrm{O}\right)$ in $\mathrm{EtOH}(10 \mathrm{~mL})$ was heated at $75^{\circ} \mathrm{C}$ for $16 \mathrm{~h}$. The volatiles were removed in vacuo, the residue was dissolved in $\mathrm{CH}_{2} \mathrm{Cl}_{2}(20 \mathrm{~mL})$, washed with water $(10 \mathrm{~mL})$ and dried over $\mathrm{MgSO}_{4}$. Evaporation of the solvents and chromatographic 
purification of the residue on $20 \mathrm{~g}$ of silica $\left(1.5 \times 20 \mathrm{~cm}, R_{\mathrm{f}}=0.40\right.$, pentane $\left./ \mathrm{Et}_{2} \mathrm{O}=1: 1\right)$ yielded $168 \mathrm{mg}(81 \%)$ of cis-26 as a colorless solid, m. p. $>250{ }^{\circ} \mathrm{C}$. $-\mathrm{IR}(\mathrm{KBr}): v=3245 \mathrm{~cm}^{-1}, 2890$, 1668, 1489, 1465, 1332, 1040. - ${ }^{1} \mathrm{H}-\mathrm{NMR}\left(250 \mathrm{MHz}, \mathrm{CDCl}_{3}\right): \delta$ $=1.10-1.16(\mathrm{~m}, 2 \mathrm{H}, \mathrm{cPr}-\mathrm{H}), 1.36-1.40(\mathrm{~m}, 1 \mathrm{H}, \mathrm{cPr}-\mathrm{H}), 1.72-$ $1.78(\mathrm{~m}, 1 \mathrm{H}, \mathrm{cPr}-\mathrm{H}), 3.01\left(\mathrm{~s}, 3 \mathrm{H}, \mathrm{CH}_{3}\right), 3.85-4.20(\mathrm{~m}, 2 \mathrm{H}$, $\left.\mathrm{CH}_{2}\right), 4.52(\mathrm{~s}, 1 \mathrm{H}, \mathrm{CH}), 5.22(\mathrm{~s}, 1 \mathrm{H}, \mathrm{CH}), 5.98\left(\mathrm{~s}, 2 \mathrm{H}, \mathrm{CH}_{2}\right)$, 6.71-6.84 (m, $3 \mathrm{H}$, aryl-H), 6.96-7.20 (m, $2 \mathrm{H}$, aryl-H), 7.267.38 (m, $2 \mathrm{H}$, aryl-H), 8.12 (br s, $1 \mathrm{H}, \mathrm{NH}) .-{ }^{13} \mathrm{C}-\mathrm{NMR}(62.9$ $\left.\mathrm{MHz}, \mathrm{CDCl}_{3}, \mathrm{DEPT}\right): \delta=8.2(-, \mathrm{cPr}-\mathrm{C}), 11.2$ (-, cPr-C), 21.0

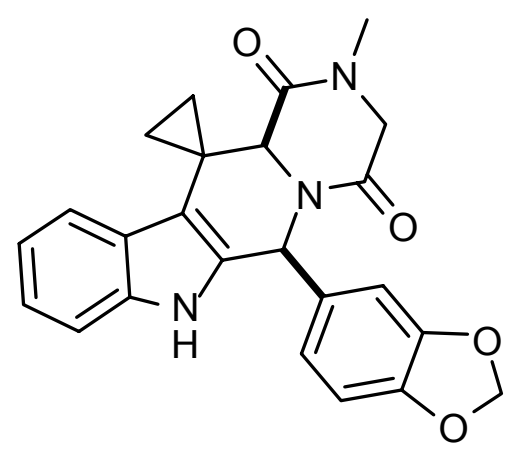
( $\left.\mathrm{C}_{\text {quat }}, \mathrm{cPr}-\mathrm{C}\right), 33.5\left(+, \mathrm{CH}_{3}\right), 51.5\left(-, \mathrm{CH}_{2}\right), 53.4(+, \mathrm{CH}), 56.8(+, \mathrm{CH}), 101.3\left(-, \mathrm{CH}_{2}\right), 108.3$ $\left(\mathrm{C}_{\text {quat }}\right.$, aryl-C), 109.1 (+, aryl-C), 111.5 (+, aryl-C), $113.6\left(+\right.$, aryl-C), $118.9\left(\mathrm{C}_{\text {quat }}\right.$, aryl-C), 120.0 (+, aryl-C), 122.4 (+, aryl-C), 122.5 (+, aryl-C), 123.9 (+, aryl-C), $130.4\left(\mathrm{C}_{\text {quat, }}\right.$ aryl-C), 131.4 ( $\mathrm{C}_{\text {quat, }}$, aryl-C), 136.5 ( $\mathrm{C}_{\text {quat, }}$ aryl-C), $148.0\left(\mathrm{C}_{\text {quat }}\right.$, aryl-C), $148.2\left(\mathrm{C}_{\text {quat, }}\right.$, aryl-C), $162.9\left(\mathrm{C}_{\text {quat, }} \mathrm{C}=\mathrm{O}\right)$,

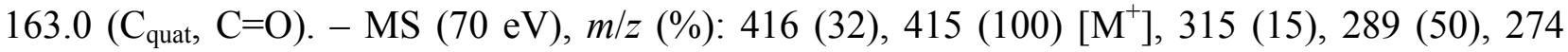
(10). $-\mathrm{C}_{24} \mathrm{H}_{21} \mathrm{~N}_{3} \mathrm{O}_{4}$ (415.5): calcd. C 69.39, H 5.10, N 10.11; found: C 69.12, H 5.09, N 9.91. 


\subsubsection{Synthesis of spirocyclopropyl oxazolines}

General procedure for Michael reaction of carboxamides with 5 (GP 7): A solution of Methyl2-chloro-2-cyclopropylideneacetate (5) and the corresponding amide in anhydrous acetonitrile was treated with $\mathrm{NaH}\left(60 \%\right.$ dispersion in mineral oil) at $0{ }^{\circ} \mathrm{C}$. The resulting suspension was subsequently stirred for $1 \mathrm{~h}$ at this temperature and $2 \mathrm{~h}$ at $20^{\circ} \mathrm{C}$. After removing solvent the pale yellow residue was taken with $300 \mathrm{~mL}$ of diethyl ether, poured into a separating funnel and washed with $100 \mathrm{~mL}$ of water. The aq. layer was extracted with $2 \times 100 \mathrm{~mL}$ of diethyl ether and combined organic layer was dried over $\mathrm{MgSO}_{4}$. After removing the solvent in vacuo the crude product was purified by column chromatography.

5-(4-Bromophenyl)-6-oxa-4-aza-spiro[2.4] hept-4-ene-7-carboxylic acid methyl ester (13a): The crude product obtained from 5 (5.86 g, $40.0 \mathrm{mmol}$ ), 4-bromobenzamide (63a, $8.0 \mathrm{~g}, 40.0 \mathrm{mmol}$ ) and $\mathrm{NaH}(1.60 \mathrm{~g}, 40.0 \mathrm{mmol})$ in $150 \mathrm{~mL}$ of anhydrous acetonitrile according to GP 7 was purified by column chromatography $\left(R_{\mathrm{f}}=\right.$ 0.35 , Pentane $/ \mathrm{Et}_{2} \mathrm{O}=5: 1,3 \times 20 \mathrm{~cm}, 100 \mathrm{~g} \mathrm{SiO}_{2}$ ) to yield $6.20 \mathrm{~g}$ $(51 \%)$ of 13a as a white solid, m.p. $=102{ }^{\circ} \mathrm{C} .-\mathrm{IR}(\mathrm{KBr}): v=$ $3087 \mathrm{~cm}^{-1}, 2954,1767,1651,1403,1214,1066,1011,725 .-{ }^{1} \mathrm{H}-$

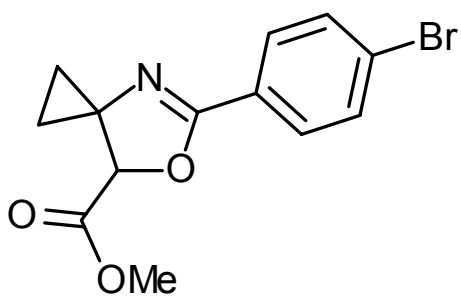
NMR (300 MHz, $\left.\mathrm{CDCl}_{3}\right): \delta=0.85-1.01(\mathrm{~m}, 2 \mathrm{H}, \mathrm{cPr}-\mathrm{H}), 1.14-1.22(\mathrm{~m}, 1 \mathrm{H}, \mathrm{cPr}-\mathrm{H}), 1.28-1.35$ (m, $1 \mathrm{H}, \mathrm{cPr}-\mathrm{H}), 3.78\left(\mathrm{~s}, 3 \mathrm{H}, \mathrm{CH}_{3}\right), 4.91(\mathrm{~s}, 1 \mathrm{H}, \mathrm{CH}), 7.52-7.58(\mathrm{~m}, 2 \mathrm{H}$, aryl-H), 7.78-7.82 (m, $2 \mathrm{H}$, aryl-H). $-{ }^{13} \mathrm{C}$ NMR $\left(75.5 \mathrm{MHz}, \mathrm{CDCl}_{3}, \mathrm{APT}\right): \delta=10.5$ (-, cPr-C), 14.8 (-, cPr-C), $52.3(+$, $\mathrm{CH}_{3}$ ), 53.4 (-, cPr-C), 79.8 (+, CH-C), 125.9 (-, aryl-C), 126.1 (-, aryl-C), 129.4 (+, $2 \mathrm{C}$, aryl-C), 131.7 (+, 2 C, aryl-C), 162.7 (-, CN-C), 169.3 (-, CO-C). - MS (70 eV), m/z (\%): 311/309 (32/36) $\left[\mathrm{M}^{+}\right], 252 / 250$ (100/96) [ $\left.\mathrm{M}^{+}-\mathrm{CO}_{2} \mathrm{Me}\right], 224 / 222$ (22/20), 143 (33). $-\mathrm{C}_{13} \mathrm{H}_{12} \mathrm{BrNO}_{3}$ (310.2): calcd. C 50.35, H 3.90, N 4.52; found C 50.36, H 3.64, N 4.41.

5-(3-Bromophenyl)-6-oxa-4-aza-spiro[2.4]hept-4-ene-7-carboxylic acid methyl ester (13b): The crude product obtained from 5 (6.15 g, $42.0 \mathrm{mmol})$, 3-bromobenzamide $(\mathbf{6 3 b}, 8.40 \mathrm{~g}, 42.0 \mathrm{mmol})$ and $\mathrm{NaH}(1.68 \mathrm{~g}, 42.0 \mathrm{mmol})$ in $150 \mathrm{~mL}$ of anhydrous acetonitrile according to GP 7 was purified by column chromatography $\left(R_{\mathrm{f}}=0.42\right.$, Pentane/ $\left.\mathrm{Et}_{2} \mathrm{O}=5: 1,3 \times 20 \mathrm{~cm}, 100 \mathrm{~g} \mathrm{SiO}_{2}\right)$ to yield $9.36 \mathrm{~g}$ (64\%) of 13b as a solid, m.p. $=79^{\circ} \mathrm{C} .-\mathrm{IR}(\mathrm{KBr}): v=3066 \mathrm{~cm}^{-1}, 2958,1738,1653,1423,1074$, 1025, 1012, 888, 708. $-{ }^{1} \mathrm{H}-\mathrm{NMR}\left(250 \mathrm{MHz}, \mathrm{CDCl}_{3}\right): \delta=0.95-1.05$ (m, $\left.2 \mathrm{H}, \mathrm{cPr}-\mathrm{H}\right), 1.20-1.42$ 
(m, $2 \mathrm{H}, \mathrm{cPr}-\mathrm{H}), 3.80\left(\mathrm{~s}, 3 \mathrm{H}, \mathrm{CH}_{3}\right), 4.90(\mathrm{~s}, 1 \mathrm{H}), 7.22-7.34(\mathrm{~m}, 1 \mathrm{H}$, aryl-H), 7.58-7.64 (m, $1 \mathrm{H}$,

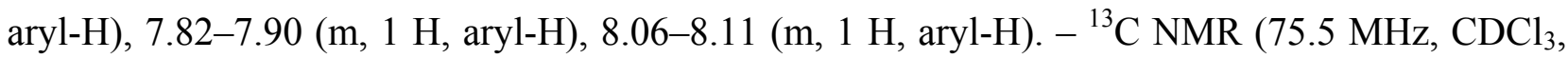
APT): $\delta=10.5$ (-, cPr-C), 14.8 (-, cPr-C), $52.3\left(+, \mathrm{CH}_{3}\right), 53.4(-$, cPr-C), 79.8 (+, CH-C), 122.5 (-, aryl-C), 126.5 (+, aryl-C), 128.9 (, aryl-C), 129.9 (+, aryl-C), 130.9 (+, aryl-C), 134.4 (+, aryl-C), 162.1 (-, CN-C), 169.3 (-, CO-C). - MS (70 eV), $m / z(\%): 311 / 309$ $(24 / 24)\left[\mathrm{M}^{+}\right], 252 / 250(92 / 100)\left[\mathrm{M}^{+}-\mathrm{CO}_{2} \mathrm{Me}\right], 224$ (27), 143 (72).

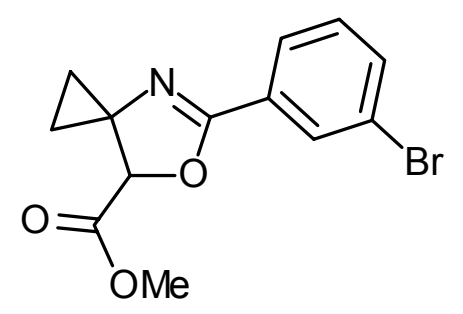
$-\mathrm{C}_{13} \mathrm{H}_{12} \mathrm{BrNO}_{3}$ (310.2): calcd. C 50.35, H 3.90, N 4.52; found C 50.16, H 3.71, N 4.38.

5-(3-Trifluoromethyl-phenyl)-6-oxa-4-aza-spiro[2.4]hept-4-ene-7-carboxylic acid methyl ester (13c): The crude product obtained from 5 (7.32 g, $50.0 \mathrm{mmol})$, 3-trifluoromethylbenzamide (63c, $8.65 \mathrm{~g}, 50.0 \mathrm{mmol})$ and $\mathrm{NaH}(2 \mathrm{~g}, 50.0 \mathrm{mmol})$ in $180 \mathrm{~mL}$ of anhydrous acetonitrile according to GP 7 was purified by column chromatography $\left(R_{\mathrm{f}}=0.35\right.$, Pentane $/ \mathrm{Et}_{2} \mathrm{O}=5: 1,3 \times 20 \mathrm{~cm}, 100 \mathrm{~g}$ $\left.\mathrm{SiO}_{2}\right)$ to yield $7.03 \mathrm{~g}(47 \%)$ of $\mathbf{1 3 c}$ as a viscous oil. $-{ }^{1} \mathrm{H}-\mathrm{NMR}$ $\left(250 \mathrm{MHz}, \mathrm{CDCl}_{3}\right): \delta=0.90-1.05(\mathrm{~m}, 2 \mathrm{H}, \mathrm{cPr}-\mathrm{H}), 1.20-1.42(\mathrm{~m}$,

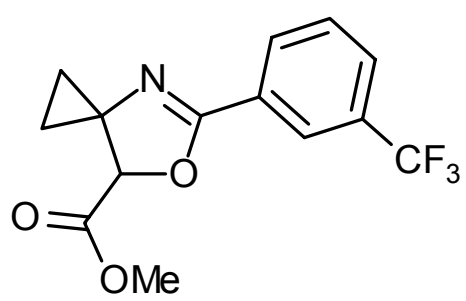
$2 \mathrm{H}, \mathrm{cPr}-\mathrm{H}), 3.82\left(\mathrm{~s}, 3 \mathrm{H}, \mathrm{CH}_{3}\right), 4.95(\mathrm{~s}, 1 \mathrm{H}, \mathrm{CH}), 7.50-7.61(\mathrm{~m}, 1$ $\mathrm{H}$, aryl-H), 7.68-7.75 (m, $1 \mathrm{H}$, aryl-H), 8.10-8.21 (m, $2 \mathrm{H}$, aryl-H). - ${ }^{13} \mathrm{C}-\mathrm{NMR}(62.9 \mathrm{MHz}$, $\left.\mathrm{CDCl}_{3}, \mathrm{DEPT}\right): \delta=10.4$ (-, cPr-C), 14.7 (-, cPr-C), $52.2\left(+, \mathrm{CH}_{3}\right), 53.4(-, \mathrm{cPr}-\mathrm{C}), 79.7(+, \mathrm{CH}-$ C), $123.6\left(\mathrm{q},{ }^{1} J_{\mathrm{C}-\mathrm{F}}=272 \mathrm{~Hz}, \mathrm{CF}_{3}\right), 124.8\left(+, \mathrm{q}\right.$, aryl-C), $127.8\left(+\right.$, aryl-C), $127.9\left(+, \mathrm{q},{ }^{3} J_{\mathrm{C}-\mathrm{F}}=3.9\right.$ $\mathrm{Hz}$, aryl-C) 128.9 (+, aryl-C), $130.9\left(\mathrm{C}_{\text {quat }}, \mathrm{q},{ }^{2} J_{\mathrm{C}-\mathrm{F}}=32.8 \mathrm{~Hz}\right.$, aryl-C), 131.0 (C $\mathrm{C}_{\text {quat }}$, aryl-C), 162.1 (-, CN-C), 169.1 (-, CO-C). - MS (70 eV), m/z (\%): 299 (11) $\left[\mathrm{M}^{+}\right], 240$ (100) $\left[\mathrm{M}^{+}-\mathrm{CO}_{2} \mathrm{Me}\right], 212$ (49), 145 (30). $-\mathrm{C}_{14} \mathrm{H}_{12} \mathrm{~F}_{3} \mathrm{NO}_{3}$ (299.3): calcd. C 56.19, H 4.04, N 4.68; found;

5-(4-Trifluoromethyl-phenyl)-6-oxa-4-aza-spiro[2.4]hept-4-ene-7-carboxylic acid methyl ester (13d). The crude product obtained from 5 (4.03 g, $27.5 \mathrm{mmol})$, 4-trifluoromethylbenzamide 63d $(5.20 \mathrm{~g}, 27.5 \mathrm{mmol})$ and $\mathrm{NaH}(1.1 \mathrm{~g}, 27.5 \mathrm{mmol})$ in $120 \mathrm{~mL}$ of anhydrous acetonitrile according to GP 7 was purified by column chromatography $\left(R_{\mathrm{f}}=0.4\right.$, Pentane $\left./ \mathrm{Et}_{2} \mathrm{O}=5: 1,3 \times 20 \mathrm{~cm}\right)$ to yield $6.60 \mathrm{~g}(81 \%)$ of title compound as a oil which solidified at 0 ${ }^{\circ} \mathrm{C}$ m.p. $=75-77^{\circ} \mathrm{C}$. - IR $(\mathrm{KBr}): v=3021 \mathrm{~cm}^{-1}, 2967,1768$,

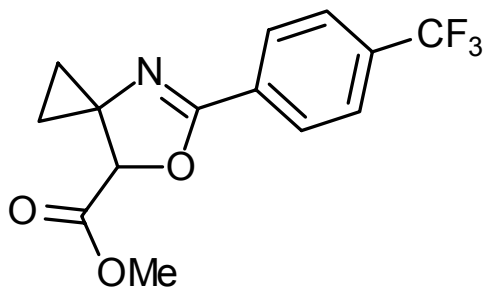
$1648,1415,1317,1170,1080,859 .-{ }^{1} \mathrm{H}-\mathrm{NMR}\left(250 \mathrm{MHz}, \mathrm{CDCl}_{3}\right): \delta=0.92-1.08(\mathrm{~m}, 2 \mathrm{H}, \mathrm{cPr}-$ 
H), 1.20-1.28 (m, 1 H, cPr-H), 1.33-1.41 (m, $1 \mathrm{H}, \mathrm{cPr}-\mathrm{H}), 3.78$ (s, $\left.3 \mathrm{H}, \mathrm{CH}_{3}\right), 4.92$ (s, $\left.1 \mathrm{H}, \mathrm{CH}\right)$, $7.67\left(\mathrm{~d},{ }^{3} \mathrm{~J}=8.3 \mathrm{~Hz}, 2 \mathrm{H}\right.$, aryl-H), $8.05\left(\mathrm{~d},{ }^{3} \mathrm{~J}=8.3 \mathrm{~Hz}, 2 \mathrm{H}\right.$, aryl-H). $-{ }^{13} \mathrm{C}-\mathrm{NMR}(62.9 \mathrm{MHz}$, $\left.\mathrm{CDCl}_{3}, \mathrm{DEPT}\right): \delta=10.6$ (-, cPr-C), 14.9 (-, cPr-C), $52.3\left(+, \mathrm{CH}_{3}\right), 53.4(-, \mathrm{cPr}-\mathrm{C}), 79.8(+, \mathrm{CH}-$ C), $123.4\left(\mathrm{C}_{\text {quat }}, \mathrm{q},{ }^{1} J_{\mathrm{C}-\mathrm{F}}=242 \mathrm{~Hz}, \mathrm{CF}_{3}\right), 124.8\left(+, \mathrm{q},{ }^{3} J_{\mathrm{C}-\mathrm{F}}=3.4 \mathrm{~Hz}, 2 \mathrm{C}\right.$, aryl-C), $128.3(+, 2 \mathrm{C}$, $\operatorname{aryl-C}), 130.2$ (C $\mathrm{C}_{\text {quat }}$, aryl-C), $132.3\left(\mathrm{C}_{\text {quat }}, \mathrm{q},{ }^{2} J_{\mathrm{C}-\mathrm{F}}=32.8 \mathrm{~Hz}\right.$, aryl-C), $162.2\left(\mathrm{C}_{\text {quat }}, \mathrm{CN}-\mathrm{C}\right), 169.1$ $\left(\mathrm{C}_{\text {quat }}, \mathrm{CO}-\mathrm{C}\right) .-\mathrm{MS}(70 \mathrm{eV}), \mathrm{m} / z(\%): 299(20)\left[\mathrm{M}^{+}\right], 240(100)\left[\mathrm{M}^{+}-\mathrm{CO}_{2} \mathrm{Me}\right], 212(60), 172$ (23), 145 (25). $-\mathrm{C}_{14} \mathrm{H}_{12} \mathrm{~F}_{3} \mathrm{NO}_{3}$ (299.3): calcd. C 56.19, H 4.04, N 4.68; found C 56.09, H 3.80, N 4.77 .

5-Phenyl-6-oxa-4-aza-spiro[2.4]hept-4-ene-7-carboxylic acid methyl ester (13e): The crude product obtained from 5 (2.50 g, $17.0 \mathrm{mmol})$, benzamide (63e, $5.20 \mathrm{~g}, 17.0 \mathrm{mmol})$ and $\mathrm{NaH}(595$ $\mathrm{mg}, 17.0 \mathrm{mmol}$ ) in $50 \mathrm{~mL}$ of anhydrous acetonitrile according to GP 7 was purified by column chromatography $\left(R_{\mathrm{f}}=0.38\right.$, Pentane $/ \mathrm{Et}_{2} \mathrm{O}=4: 1$, $3 \times 10 \mathrm{~cm})$ to yield $2.2 \mathrm{~g}(54 \%)$ of title compound as a solid, m.p. $=$ $48{ }^{\circ} \mathrm{C} .-\mathrm{IR}(\mathrm{KBr}): v=2948 \mathrm{~cm}^{-1}, 1732,1449,1284,1057,694 .-{ }^{1} \mathrm{H}-$ NMR (250 MHz, $\left.\mathrm{CDCl}_{3}\right): \delta=0.91-1.08$ (m, $\left.2 \mathrm{H}, \mathrm{cPr}-\mathrm{H}\right), 1.18-1.27$ (m,

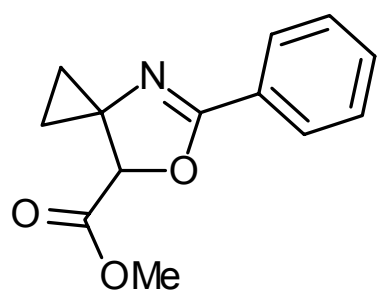
$1 \mathrm{H}, \mathrm{cPr}-\mathrm{H}), 1.32-1.42(\mathrm{~m}, 1 \mathrm{H}, \mathrm{cPr}-\mathrm{H}), 3.80$ (s, $\left.3 \mathrm{H}, \mathrm{CH}_{3}\right), 4.93$ (s, $\left.1 \mathrm{H}, \mathrm{CH}\right), 7.39-7.54(\mathrm{~m}, 3 \mathrm{H}$, aryl-H), 7.91-7.99 (m, $2 \mathrm{H}$, aryl-H). $\left.-{ }^{13} \mathrm{C} \mathrm{NMR} \mathrm{(50.3} \mathrm{MHz,} \mathrm{CDCl}_{3}, \mathrm{APT}\right): \delta=10.4$ (-, cPr-C), 14.7 (-, cPr-C), 52.3 (+, $\left.\mathrm{CH}_{3}\right), 53.3$ (-, cPr-C), 79.7 (+, CH-C), 127.0 (-, aryl-C), 128.0 (+, 2 C, aryl-C), 128.4 (+, 2 C, aryl-C), 131.5 (+, aryl-C), 163.5 (-, CN-C), 169.5 (-, CO-C). - MS (70 $\mathrm{eV}), m / z(\%): 231(35)\left[\mathrm{M}^{+}\right], 172$ (100) $\left[\mathrm{M}^{+}-\mathrm{CO}_{2} \mathrm{Me}\right], 144$ (60), 105 (26).

\section{General procedure for synthesis of oxazoline carboxylic Acid 64a-e from the ester 13a-e (GP} 8): To a solution of oxazoline carboxylic acid methyl ester (13a-e) in MeOH/THF (4:1) at room temperature aq. $\mathrm{NaOH}(1 \mathrm{~N}, 5$ equiv.) was added. The resulting solution was stirred for $30 \mathrm{~min}$, then glacial $\mathrm{AcOH}$ (10 equiv.) was added, stirred for additional $15 \mathrm{~min}$. and the solvent was evaporated in vacuo. The residue was filtered over $\mathrm{SiO}_{2}$ gel $\left(\mathrm{Et}_{2} \mathrm{O} / \mathrm{AcOH}=50: 1\right)$, and the product crystallized from ether/hexane. 
5-(4-Bromophenyl)-6-oxa-4-aza-spiro[2.4] hept-4-ene-7-carboxylic acid (64a).The crude product obtained from 13a (3.70 g, $12 \mathrm{mmol}), \mathrm{NaOH}(2.40 \mathrm{~g})$ and $\mathrm{AcOH}(7.20 \mathrm{~g}, 120 \mathrm{mmol})$ according to GP 8 yielded $3.21 \mathrm{~g}(91 \%)$ of $\mathbf{6 4 a}$ as white solid, m.p. $199^{\circ} \mathrm{C}$. - IR (KBr): $v=3093 \mathrm{~cm}^{-1}, 1745,1633,1591,1491,1199,1092,838 .-$ ${ }^{1} \mathrm{H}-\mathrm{NMR}\left(300 \mathrm{MHz}, \mathrm{CD}_{3} \mathrm{OD}\right): \delta=0.96-1.02(\mathrm{~m}, 1 \mathrm{H}, \mathrm{cPr}-\mathrm{H})$, 1.09-1.20 (m, 2 H, cPr-H), 1.22-1.32 (m, $1 \mathrm{H}, \mathrm{cPr}-\mathrm{H}), 4.95$ (s, $1 \mathrm{H}$, $\mathrm{CH}), 7.61-7.68(\mathrm{~m}, 2 \mathrm{H}$, aryl-H), 7.80-7.85 (m, $2 \mathrm{H}$, aryl-H). -

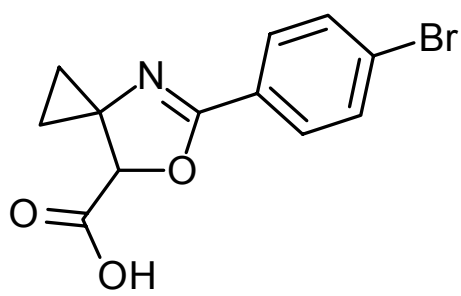
${ }^{13} \mathrm{C}-\mathrm{NMR}\left(75.5 \mathrm{MHz}, \mathrm{CD}_{3} \mathrm{OD}, \mathrm{APT}\right): \delta=10.7$ (-, cPr-C), 15.0 (-, cPr-C), 53.9 (-, cPr-C), $81.0(+$, CH-C), 127.2 (-, aryl-C), 127.4 (-, aryl-C), 130.7 (+, 2 C, aryl-C), 133.0 (+, 2 C, aryl-C), 164.9 (, CN-C), 171.9 (-, CO-C). - MS (70 eV), m/z (\%): $297 / 255$ (46/44) $\left[\mathrm{M}^{+}\right], 252 / 250$ (100/96) $\left[\mathrm{M}^{+}-\right.$ $\mathrm{CO}_{2} \mathrm{H}$ ], 224/222 (36/40), 143 (71). $-\mathrm{C}_{12} \mathrm{H}_{10} \mathrm{BrNO}_{3}$ (296.1): calcd. C 48.67, H 3.40, N 4.73; found C 48.39, H 3.15, N 4.65.

5-(3-Bromophenyl)-6-oxa-4-aza-spiro[2.4] hept-4-ene-7-carboxylic acid (64b). The crude product obtained from 13b (8.5 g, $27.5 \mathrm{mmol}), \mathrm{NaOH}(5.5 \mathrm{~g}, 138 \mathrm{mmol})$ and $\mathrm{AcOH}(16.5 \mathrm{~g}, 275 \mathrm{mmol})$ according to GP 8 yielded $7.31 \mathrm{~g}(90 \%)$ of $\mathbf{6 4 b}$ as a white solid, m.p. $=196{ }^{\circ} \mathrm{C} .-\mathrm{IR}(\mathrm{KBr}): v=3108 \mathrm{~cm}^{-1}, 1728,1637,1431,1201$, 1096, 1032, 791. $-{ }^{1} \mathrm{H}-\mathrm{NMR}\left(300 \mathrm{MHz}, \mathrm{CD}_{3} \mathrm{OD}\right): \delta=0.96-1.04$ (m, $1 \mathrm{H}, \mathrm{cPr}-\mathrm{H}), 1.11-1.22$ (m, $2 \mathrm{H}, \mathrm{cPr}-\mathrm{H}), 1.23-1.37$ (m, $1 \mathrm{H}, \mathrm{cPr}-\mathrm{H})$, 5.01 (s, $1 \mathrm{H}, \mathrm{CH}), 7.39$ (m, $1 \mathrm{H}$, aryl-H), 7.68-7.75 (m, $1 \mathrm{H}$, aryl-H),

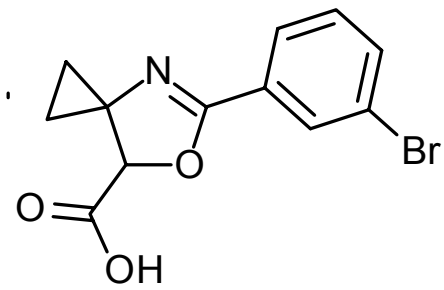
7.86-7.94 (m, $1 \mathrm{H}$, aryl-H), 8.05-8.10 (m, $1 \mathrm{H}$, aryl-H). $-{ }^{13} \mathrm{C}-\mathrm{NMR}\left(75.5 \mathrm{MHz}, \mathrm{CD}_{3} \mathrm{OD}, \mathrm{APT}\right): \delta$ = 10.8 (-, cPr-C), 15.1 (-, cPr-C), 53.9 (-, cPr-C), 81.0 (+, CH-C), 123.4 (-, aryl-C), 127.7 (+, aryl-C), 130.2 (-, aryl-C), 131.6 (+, aryl-C), 131.8 (+, aryl-C), 135.8 (+, aryl-C), 164.3 (-, CN-C), 171.8 (-, CO-C). - MS (70 eV), m/z (\%): 297/295 (42/37) [M+1, 252/250 (98/100) $\left[\mathrm{M}^{+}-\mathrm{CO}_{2} \mathrm{H}\right]$, 143 (68), 116 (20). $-\mathrm{C}_{12} \mathrm{H}_{10} \mathrm{BrNO}_{3}$ (296.1): calcd. C 48.67, H 3.40, N 4.73; found $\mathrm{C} 48.76, \mathrm{H}$ $3.21, \mathrm{~N} 4.80$.

5-(3-Trifluoromethyl-phenyl)-6-oxa-4-aza-spiro[2.4]hept-4-ene-7-carboxylic acid (64c): The crude product obtained from 13c $(6.70 \mathrm{~g}, 22.0 \mathrm{mmol}), \mathrm{NaOH}(4.40 \mathrm{~g}, 110 \mathrm{mmol})$ and $\mathrm{AcOH}(1.32$ g, $220 \mathrm{mmol}$ ) according to GP 8 yielded $5.60 \mathrm{~g}(89 \%)$ of $\mathbf{6 4 c}$ as a white solid, m.p. $=171{ }^{\circ} \mathrm{C}$. - IR $(\mathrm{KBr}): v=3015 \mathrm{~cm}^{-1}, 1734,1641,1447,1329,1303,1135,1027,919 .-{ }^{1} \mathrm{H}-\mathrm{NMR}(250 \mathrm{MHz}$, DMSO-D $): \delta=0.98-1.07$ (m, $1 \mathrm{H}, \mathrm{cPr}-\mathrm{H}), 1.13-1.23$ (m, $2 \mathrm{H}, \mathrm{cPr}-\mathrm{H}), 1.26-1.37$ (m, $1 \mathrm{H}, \mathrm{cPr}-\mathrm{H})$, 
$5.05(\mathrm{~s}, 1 \mathrm{H}, \mathrm{CH}), 7.71\left(\mathrm{~m}, 1 \mathrm{H}\right.$, aryl-H), 7.87 (m, $2 \mathrm{H}$, aryl-H), 8.14-8.23 (m, $2 \mathrm{H}$, aryl-H). - ${ }^{13} \mathrm{C}-$ NMR (62.9 MHz, [D $\mathrm{D}_{6}$ DMSO, DEPT): $\delta=10.0$ (-, cPr-C), 14.5 (, cPr-C), 53.3 (-, cPr-C), 79.1 (+, CH-C), 123.9 ( $\mathrm{C}_{\text {quat }}, \mathrm{q},{ }^{1} J_{\mathrm{C}-\mathrm{F}}=$ $\left.272 \mathrm{~Hz}, \mathrm{CF}_{3}\right), 123.8\left(+, \mathrm{q},{ }^{3} J_{\mathrm{C}-\mathrm{F}}=3.2 \mathrm{~Hz}\right.$, aryl-C), $127.8(+$, arylC), $128.5\left(+, \mathrm{q}^{3} J_{\mathrm{C}-\mathrm{F}}=3.2 \mathrm{~Hz}\right.$, aryl-C), $129.8\left(\mathrm{C}_{\text {quat }}, \mathrm{q},{ }^{2} J_{\mathrm{C}-\mathrm{F}}=33.2\right.$ $\mathrm{Hz}$, aryl-C), 130.5 (+, aryl-C), 131.5 (C $\mathrm{C}_{\text {quat }}$, aryl-C), 161.4 (-, CN-

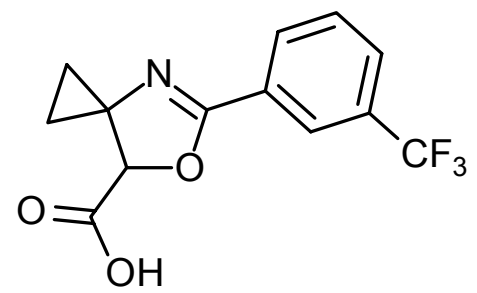
C), 170.3 (-, CO-C). - MS (70 eV), m/z (\%): 285 (24) $\left[\mathrm{M}^{+}\right], 240(100)\left[\mathrm{M}^{+}-\mathrm{CO}_{2} \mathrm{H}\right], 212(81)$, $172(36), 145(44)$.

5-(4-Trifluoromethyl-phenyl)-6-oxa-4-aza-spiro[2.4]hept-4-ene-7-carboxylic acid (64d): The crude product obtained from $13 d$ (5.90 g, $19.2 \mathrm{mmol}), \mathrm{NaOH}(3.84 \mathrm{~g}, 94 \mathrm{mmol})$ and AcOH (11.5 $\mathrm{g}, 192 \mathrm{mmol})$ in $\mathrm{MeOH} / \mathrm{THF}(500 \mathrm{~mL})$ according to GP $8\left(R_{\mathrm{f}}=\right.$ $\left.0.40, \mathrm{Et}_{2} \mathrm{O} / \mathrm{AcOH}=50: 1\right)$ to yield $5.11 \mathrm{~g}(93 \%)$ of $\mathbf{6 4 d}$ as white solid, m.p. $=190-191{ }^{\circ} \mathrm{C} .-\mathrm{IR}(\mathrm{KBr}): v=2755 \mathrm{~cm}^{-1}, 1740$, 1646, 1417, 1332, 1168, 1093, 852. - ${ }^{1} \mathrm{H}-\mathrm{NMR}(300 \mathrm{MHz}$, $\left.\mathrm{CD}_{3} \mathrm{OD}\right): \delta=0.95-1.06(\mathrm{~m}, 1 \mathrm{H}, \mathrm{cPr}-\mathrm{H}), 1.11-1.23(\mathrm{~m}, 2 \mathrm{H}, \mathrm{cPr}-$

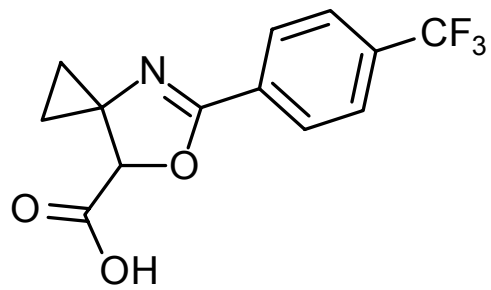
H), 1.06-1.15 (m, 1 H, cPr-H), 5.03 (s, $1 \mathrm{H}, \mathrm{CH}), 7.78$ (d, ${ }^{3} \mathrm{~J}=8.2 \mathrm{~Hz}, 2 \mathrm{H}$, aryl-H), 8.10 (d, ${ }^{3} J=$ $8.2 \mathrm{~Hz}, 2 \mathrm{H}$, aryl-H). $-{ }^{13} \mathrm{C}-\mathrm{NMR}\left(75.5 \mathrm{MHz}, \mathrm{CDCl}_{3}, \mathrm{APT}\right): \delta=10.9$ (-, cPr-C), 15.2 (-, cPr-C), $54.1(-, \mathrm{cPr}-\mathrm{C}), 81.1(+, \mathrm{CH}-\mathrm{C}), 125.3\left(-, \mathrm{q},{ }^{1} J_{\mathrm{C}-\mathrm{F}}=272 \mathrm{~Hz}, \mathrm{CF}_{3}\right), 126.6\left(+, \mathrm{q},{ }^{3} J_{\mathrm{C}-\mathrm{F}}=3.8 \mathrm{~Hz}, 2 \mathrm{C}\right.$, aryl-C), $129.6\left(+, 2 \mathrm{C}\right.$, aryl-C), 131.8 (-, aryl-C), 134.2 (-, q, ${ }^{2} J_{\mathrm{C}-\mathrm{F}}=32.8 \mathrm{~Hz}$, aryl-C), $164.3(-$, CN-C), 171.8 (-, CO-C). - MS (70 eV), m/z (\%): 285 (38) $\left[\mathrm{M}^{+}\right], 240$ (100) $\left[\mathrm{M}^{+}-\mathrm{CO}_{2} \mathrm{H}\right], 212$ (75), 172 (32). $-\mathrm{C}_{13} \mathrm{H}_{10} \mathrm{~F}_{3} \mathrm{NO}_{3}$ (285.2): calcd. C 54.74, $\mathrm{H} 3.53, \mathrm{~N} 4.91$; found $\mathrm{C} 54.35, \mathrm{H} \mathrm{3.25,} \mathrm{N}$ 5.11 .

5-Phenyl-6-oxa-4-aza-spiro[2.4]hept-4-ene-7-carboxylic acid (64e): The crude product obtained from 13e (3.40 g, $14.7 \mathrm{mmol}), \mathrm{NaOH}(2.96 \mathrm{~g}, 74 \mathrm{mmol})$ and $\mathrm{AcOH}(8.80 \mathrm{~g}, 147 \mathrm{mmol})$ in $\mathrm{MeOH} / \mathrm{THF}(200 \mathrm{~mL})$ according to $\mathrm{GP} 8\left(R_{\mathrm{f}}=0.40, \mathrm{Et}_{2} \mathrm{O} / \mathrm{AcOH}=\right.$ 50:1), yielded $5.10 \mathrm{~g}(93 \%)$ of $64 \mathrm{e}$ as a white solid, m.p $182^{\circ} \mathrm{C}$. - IR $(\mathrm{KBr}): v=3061 \mathrm{~cm}^{-1}, 3009,1717,1638,1457,1364,1211,1069,735$. $-{ }^{1} \mathrm{H}-\mathrm{NMR}\left(250 \mathrm{MHz}, \mathrm{CD}_{3} \mathrm{OD}\right): \delta=0.95-1.03$ (m, $\left.1 \mathrm{H}, \mathrm{cPr}-\mathrm{H}\right), 1.05-$ 1.34 (m, 3 H, cPr-H), 4.99 (s, 1 H, CH), 7.40-7.59 (m, 3 H, aryl-H),

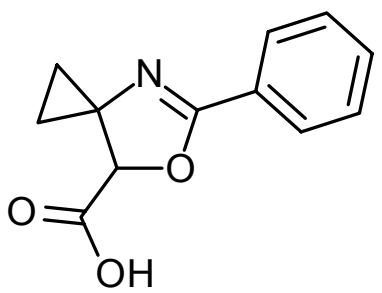
7.86-7.98 (m, $2 \mathrm{H}$, aryl-H). $-{ }^{13} \mathrm{C}-\mathrm{NMR}\left(62.9 \mathrm{MHz}, \mathrm{CD}_{3} \mathrm{OD}, \mathrm{DEPT}\right): \delta=11.0$ (-, cPr-C), $15.3(-$, 
cPr-C), 54.0 ( $\mathrm{C}_{\text {quat }}$, cPr-C), 81.3 (+, CH-C), 128.3 (C $\mathrm{C}_{\text {quat }}$, aryl-C), 129.3 (+, $2 \mathrm{C}$, aryl-C), $129.9(+$, $2 \mathrm{C}$, aryl-C), 133.3 (+, aryl-C), 166.2 (-, CN-C), 172.4 (-, CO-C). - MS (70 eV), m/z (\%): 217 (48) $\left[\mathrm{M}^{+}\right], 172$ (100) $\left[\mathrm{M}^{+}-\mathrm{CO}_{2} \mathrm{H}\right], 144$ (57), 104 (28).

2-Chloro-1-(5-phenyl-6-oxa-4-aza-spiro[2.4]hept-4-en-7-yl)-ethanone (62-Ph): A solution of lithium diisopropyl amine $(35.7 \mathrm{mmol})$ in $40 \mathrm{~mL}$ THF was added dropwise over a period of $30 \mathrm{~min}$ to a solution 5-phenyl-6-oxa-4-aza-spiro[2.4]hept-4-ene-7-carboxylic acid methyl ester 13e $(1.71 \mathrm{~g}, 7.14 \mathrm{mmol})$ and $\mathrm{CH}_{2} \mathrm{ICl}$ in $30 \mathrm{~mL}$ of $\mathrm{THF}$ at $78^{\circ} \mathrm{C}$ and the reaction mixture was stirred for $10 \mathrm{~min}$ at this temperature. At $25^{\circ} \mathrm{C}$ a solution of acetic acid $(10 \mathrm{~mL})$ in $20 \mathrm{~mL}$ of THF was added slowly over a period of $10 \mathrm{~min}$. After stirring for an additional $10 \mathrm{~min}$ the

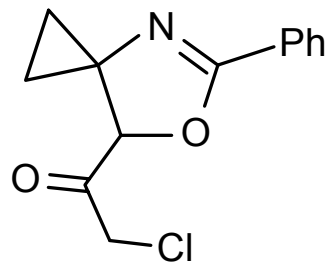
reaction mixture was partitioned between $\mathrm{Et}_{2} \mathrm{O}$ and brine. Organic layer was washed with sat. $\mathrm{NaHCO} 3,5 \% \mathrm{NaHSO}_{3}$ and brine, dried over $\mathrm{MgSO}_{4}$. After removing the solvent the crude product was purified by column chromatography $\left(R_{\mathrm{f}}=0.31\right.$, pentane/ $\left.\mathrm{Et}_{2} \mathrm{O}=5: 1\right)$ to yield $900 \mathrm{mg}$ (51\%) of 62-Ph as a white solid, m.p. $168{ }^{\circ} \mathrm{C} .-{ }^{1} \mathrm{H}-\mathrm{NMR}\left(250 \mathrm{MHz}, \mathrm{CDCl}_{3}\right): \delta=0.89-1.00(\mathrm{~m}, 2$ $\mathrm{H}, \mathrm{cPr}-\mathrm{H}), 1.15-1.24$ (m, $1 \mathrm{H}, \mathrm{cPr}-\mathrm{H}), 1.36-1.42$ (m, $1 \mathrm{H}, \mathrm{cPr}-\mathrm{H}), 4.37$ (d, ${ }^{2} \mathrm{~J}=14.2 \mathrm{~Hz}, 1 \mathrm{H}$, $\left.\mathrm{CH}_{2}\right), 4.57\left(\mathrm{~d},{ }^{2} \mathrm{~J}=14.2 \mathrm{~Hz}, 1 \mathrm{H}, \mathrm{CH}_{2}\right), 4.98$ (s, $\left.1 \mathrm{H}, \mathrm{CH}\right), 7.38-7.59$ (m, 3 H, aryl-H), 7.88-8.00 (m, $2 \mathrm{H}$, aryl-H). $-{ }^{13} \mathrm{C}-\mathrm{NMR}\left(62.9 \mathrm{MHz}, \mathrm{CDCl}_{3}, \mathrm{DEPT}\right): \delta=\delta=10.4$ (-, cPr-C), 14.4 (-, cPr-C), $46.5\left(-, \mathrm{CH}_{2}\right), 53.3\left(\mathrm{C}_{\text {quat }}, \mathrm{cPr}-\mathrm{C}\right), 84.6(+, \mathrm{CH}-\mathrm{C}), 126.3\left(\mathrm{C}_{\text {quat }}\right.$, aryl-C), 127.9 (+, $2 \mathrm{C}$, aryl-C), 128.6 (+, 2 C, aryl-C), 131.9 (+, aryl-C), 162.8 ( $\left.\mathrm{C}_{\text {quat }}, \mathrm{CN}-\mathrm{C}\right), 198.9$ (C $\left.\mathrm{C}_{\text {quat }}, \mathrm{CO}-\mathrm{C}\right) .-\mathrm{MS}(70 \mathrm{eV})$, $m / z(\%): 451 / 449(32 / 30)\left[\mathrm{M}^{+}\right], 184 / 182(95 / 100), 156 / 154(32 / 31)$.

4-Trifluoromethyl-thiobenzimidic acid 2-oxo-2-(5-phenyl-6-oxa-4-aza-spiro[2.4]hept-4-en-7-yl)ethyl ester (65): To a mixture of 62-Ph (100 mg, $0.40 \mathrm{mmol})$ and $\mathrm{NaOAc}(41 \mathrm{mg}, 0.50 \mathrm{mmol})$ in 5 $\mathrm{mL}$ of $\mathrm{CH}_{3} \mathrm{CN}$ was added 4-trifluoromethy benzthioamide $(102 \mathrm{mg}, 0.50 \mathrm{mmol})$ and the reaction mixtute was stirrred at $50^{\circ} \mathrm{C}$ 
for $48 \mathrm{~h}$. After removing solvent the reaction mixture was taken in $25 \mathrm{~mL}^{\text {of }} \mathrm{Et}_{2} \mathrm{O}$ and wased with water, dried over $\mathrm{MgSO} 4$. After removing solvent the crude reaction mixture was purified in column chromatography $\left(R_{\mathrm{f}}=0.28\right.$, pentane/Et ${ }_{2} \mathrm{O}$ $=5: 1)$ to yield $99 \mathrm{mg}(59 \%)$ of 65 as a white solid, m.p. $191{ }^{\circ} \mathrm{C} .-{ }^{1} \mathrm{H}-$ NMR (250 MHz, $\left.\mathrm{CDCl}_{3}\right): \delta=0.78-0.83(\mathrm{~m}, 1 \mathrm{H}, \mathrm{cPr}-\mathrm{H}), 1.30-1.41$ (m, 2 $\mathrm{H}, \mathrm{cPr}-\mathrm{H}), 3.58\left(\mathrm{~d},{ }^{2} \mathrm{~J}=12.5 \mathrm{~Hz}, 1 \mathrm{H}, \mathrm{CH}_{2}\right), 3.79\left(\mathrm{~d},{ }^{2} \mathrm{~J}=14.2 \mathrm{~Hz}, 1 \mathrm{H}\right.$, $\left.\mathrm{CH}_{2}\right), 4.92(\mathrm{~s}, 1 \mathrm{H}, \mathrm{CH}), 7.36-7.55$ (m, $3 \mathrm{H}$, aryl-H), 7.63-7.75 (m, $2 \mathrm{H}$, aryl-H), 7.83-8.01 (m, $4 \mathrm{H}$, aryl-H). $-{ }^{13} \mathrm{C}-\mathrm{NMR}\left(62.9 \mathrm{MHz}, \mathrm{CDCl}_{3}\right.$, DEPT $): \delta=\delta=10.6(-, \mathrm{cPr}-\mathrm{C}), 14.7(-, \mathrm{cPr}-\mathrm{C}), 39.8\left(-, \mathrm{CH}_{2}\right), 50.4\left(\mathrm{C}_{\text {quat }}\right.$, cPr-C), 85.0 (+, CH-C), 109.2 ( $\left.\mathrm{C}_{\text {quat }}, \mathrm{CNH}-\mathrm{C}\right), 125.6$ (C $\mathrm{C}_{\text {quat }}$, aryl-C), 127.1 (+, aryl-C), $127.8(+, 2 \mathrm{C}$, aryl-C), $128.3(+, 2 \mathrm{C}$, aryl-C), $128.8(+, 2 \mathrm{C}$, aryl-C), $131.3\left(\mathrm{C}_{\text {quat }}\right.$, aryl-C). - MS (70 eV), m/z (\%): $418(<1)\left[\mathrm{M}^{+}\right], 246$

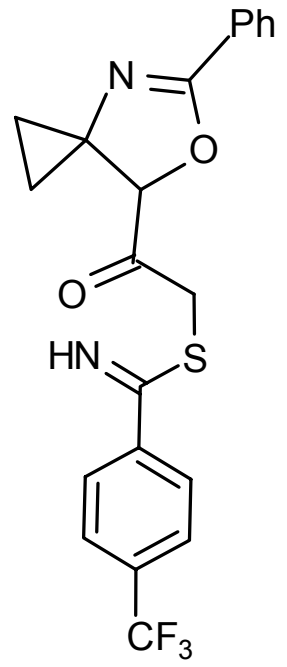
(40), 173 (100), 172 (78).

General procedure for coupling of oxazoline carboxylic acid with anilines (GP 9): To an icecold solution of oxazoline carboxylic acid (64) and HOAt in dry DCM was added EDC. $\mathrm{HCl}$ in one portion, stirred for $15 \mathrm{~min}$. followed by addition of 2,4,6-colidine and respective anilines. The cooling bath was removed and the resulting pale yellow reaction solution was stirred for $12 \mathrm{~h}$ at 20 ${ }^{\circ} \mathrm{C}$. The reaction mixture was filtered through a pad of $\mathrm{SiO}_{2}$ and the crude product was purified by column chromatography.

5-(4-Bromophenyl)-6-oxa-4-aza-spiro[2.4]hept-4-ene-7-carboxylic acid (3-trifluoromethylphenyl)-amide (68a): The crude product obtained from 64a (1.20 g, $4.05 \mathrm{mmol})$, HOAt (606 mg, $4.45 \mathrm{mmol}), \mathrm{EDC} \cdot \mathrm{HCl}(1.16 \mathrm{~g}, 6.07 \mathrm{mmol}), 2,4,6$-colidine (980 $\mathrm{mg}, 8.1 \mathrm{mmol}$ ) and 3-trifluoro-methyl aniline (977 $\mathrm{mg}, 6.07$ mmol) following GP 9 was purified by column chromatography $\left(R_{\mathrm{f}}=0.52\right.$, Pentane $\left./ \mathrm{Et}_{2} \mathrm{O}=1: 1\right)$ to yield $1.64 \mathrm{~g}(92 \%)$ of $68 \mathbf{a}$ as white solid, m.p. $194{ }^{\circ} \mathrm{C}$. - IR (KBr): $v=3327 \mathrm{~cm}^{-1}, 1670,1557$, 1453, 1336, 1125, 1069. - ${ }^{1} \mathrm{H}-\mathrm{NMR}\left(300 \mathrm{MHz}, \mathrm{CDCl}_{3}\right): \delta=$ 0.97-1.16 (m, 2 H, cPr-H), 1.34-1.48 (m, 2 H, cPr-H), 4.98 (s, 1 $\mathrm{H}, \mathrm{CH}), 7.38-7.50(\mathrm{~m}, 2 \mathrm{H}$, aryl-H), 7.59-7.63 (m, $2 \mathrm{H}$, aryl-H)

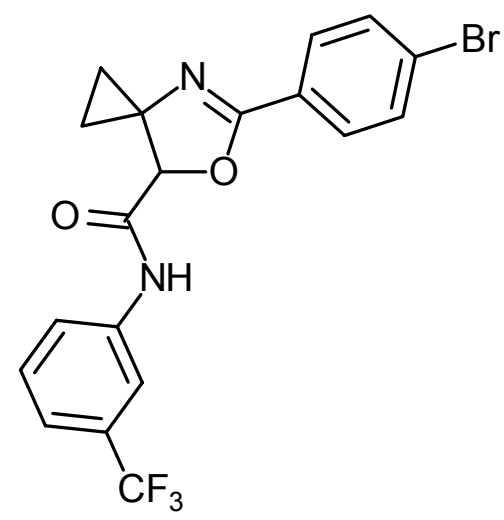
7.72-7.87 (m, $4 \mathrm{H}$, aryl-H), 8.02 (br s, $1 \mathrm{H}, \mathrm{NH}) .-{ }^{13} \mathrm{C}-\mathrm{NMR}\left(75.5 \mathrm{MHz}, \mathrm{CDCl}_{3}, \mathrm{APT}\right): \delta=10.6$ 
(-, cPr-C), 14.4 (-, cPr-C), 53.5 (-, cPr-C), 80.3 (+, CH-C), $116.8\left(+\right.$, q, ${ }^{3} J_{\mathrm{C}-\mathrm{F}}=3.8 \mathrm{~Hz}$, aryl-C), $121.7\left(+, \mathrm{q},{ }^{3} J_{\mathrm{C}-\mathrm{F}}=3.8 \mathrm{~Hz}\right.$, aryl-C), 123.2 (+, aryl-C), 125.5 (-, aryl-C), 126.6 (-, aryl-C), 129.2 $\left(+\right.$, aryl-C), $129.7\left(+, 2 \mathrm{C}\right.$, aryl-C), $131.8\left(-, \mathrm{q},{ }^{2} J_{\mathrm{C}-\mathrm{F}}=32.5 \mathrm{~Hz}\right.$, aryl-C), $132.0(+, 2 \mathrm{C}$, aryl-C), 136.9 (-, aryl-C), 160.7 (-, CN-C), 166.5 (-, CO-C). - MS (70 eV), m/z (\%): 440/438 (21/20) $\left[\mathrm{M}^{+}\right], 280 / 278$ (46/44) $\left[\mathrm{M}^{+}-\mathrm{Br}\right], 253 / 251$ (58/62), 252/250 (68/62), 161 (100). $-\mathrm{C}_{19} \mathrm{H}_{14} \mathrm{BrF}_{3} \mathrm{~N}_{2} \mathrm{O}_{2}$ (439.2): calcd. C 51.96, H 3.21, N 6.38; found C 52.13, H 3.07, N 6.16.

5-(3-Bromophenyl)-6-oxa-4-aza-spiro[2.4] hept-4-ene-7-carboxylic acid (3-trifluoromethylphenyl)-amide (68b): The crude product obtained from 64b (250 mg, $0.85 \mathrm{mmol})$, HOAt (139 $\mathrm{mg}, 1.02 \mathrm{mmol}), \mathrm{EDC} \cdot \mathrm{HCl}(244 \mathrm{mg}$, $1.27 \mathrm{mmol}$ ), 2,4,6-colidine (206 $\mathrm{mg}, 1.70 \mathrm{mmol}$ ) and 3trifluoromethyl aniline $(178 \mathrm{mg}, 1.1 \mathrm{mmol})$ in $20 \mathrm{~mL}$ of DCM following GP 9 was purified by column chromatography $\left(R_{\mathrm{f}}=\right.$ 0.40, Pentane $\left./ \mathrm{Et}_{2} \mathrm{O}=2: 1\right)$ to yield $307 \mathrm{mg}(82 \%)$ of $\mathbf{6 8 b}$ as a white solid, m.p. $=171-172{ }^{\circ} \mathrm{C} .-\mathrm{IR}(\mathrm{KBr}): v=3269 \mathrm{~cm}^{-1}, 3077,1668$, 1552, 1338, 1168, 1063, 810. $-{ }^{1} \mathrm{H}-\mathrm{NMR}\left(300 \mathrm{MHz}, \mathrm{CDCl}_{3}\right): \delta=$ 0.98-1.11 (m, 2 H, cPr-H), 1.36-1.47 (m, 2 H, cPr-H), 5.00 (s, 1

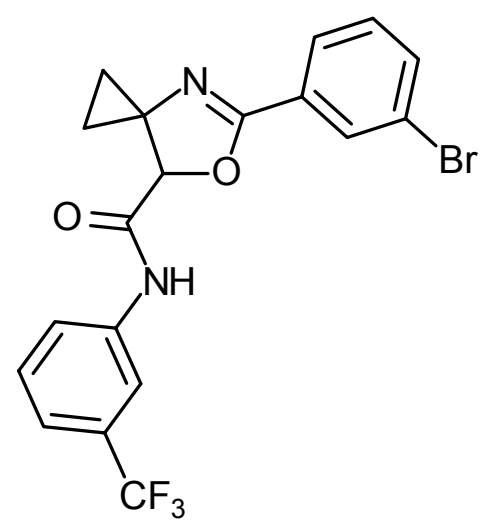
H, CH), 6.76-6.99 (m, 1 H, aryl-H), 7.30-7.47 (m, 2 H, aryl-H), 7.63-7.78 (m, 1 H, aryl-H), 7.82 (s, $1 \mathrm{H}$, aryl-H), 7.84-7.93 (m, $1 \mathrm{H}$, aryl-H), 8.06 (br. s, $1 \mathrm{H}, \mathrm{NH}), 8.11-8.15$ (m, $1 \mathrm{H}$, aryl-H). ${ }^{13} \mathrm{C}-\mathrm{NMR}\left(75.5 \mathrm{MHz}, \mathrm{CDCl}_{3}, \mathrm{APT}\right): \delta=10.7$ (-, cPr-C), 14.4 (-, cPr-C), 53.5 (-, cPr-C), $80.3(+$, $\mathrm{CH}-\mathrm{C}), 116.9\left(+, \mathrm{q},{ }^{3} J_{\mathrm{C}-\mathrm{F}}=3.4 \mathrm{~Hz}\right.$, aryl-C), $121.7\left(+, \mathrm{q},{ }^{3} J_{\mathrm{C}-\mathrm{F}}=3.4 \mathrm{~Hz}\right.$, aryl-C), 122.8 (-, aryl-C), 123.2 (+, aryl-C), 126.4 (+, aryl-C), 128.5 (-, aryl-C), 129.7 (+, aryl-C), 130.3 (+, aryl-C), 130.8 $\left(+\right.$, aryl-C), 131.3 (-, aryl-C), $131.5\left(+, \mathrm{q},{ }^{2} J_{\mathrm{C}-\mathrm{F}}=32.5 \mathrm{~Hz}\right.$, aryl-C) 134.8 (+, aryl-C), 136.9 (-, aryl-

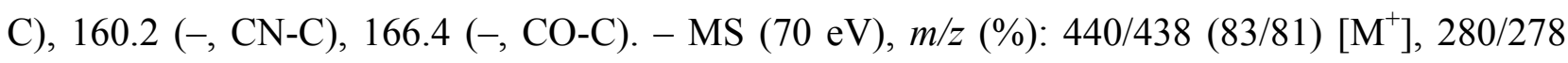
(67/69), 253/251 (98/100), 252/250 (92/84), 161 (69). - $\mathrm{C}_{19} \mathrm{H}_{14} \mathrm{BrF}_{3} \mathrm{~N}_{2} \mathrm{O}_{2}$ (439.2): calcd. C 51.96, H 3.21, N 6.38; found C 52.25, H 2.96, N 6.24.

5-(3-Trifluromethylphenyl)-6-oxa-4-aza-spiro[2.4]hept-4-ene-7-carboxylic acid (3-trifluoromethylphenyl)-amide (68c): The crude product obtained from 64c (100 mg, $0.35 \mathrm{mmol})$, HOAt (57 $\mathrm{mg}, 0.42 \mathrm{mmol}), \mathrm{EDC} \cdot \mathrm{HCl}(101 \mathrm{mg}, 0.53 \mathrm{mmol}), 2,4,6$-colidine (85 $\mathrm{mg}, 0.7 \mathrm{mmol}$ ) and 3trifluoro-methyl aniline ( $86 \mathrm{mg}, 0.53 \mathrm{mmol})$ in $10 \mathrm{~mL}$ of DCM following the GP 9 was purified by column chromatography $\left(R_{\mathrm{f}}=0.35\right.$, Pentane/Diethylether $\left.2: 1\right)$ to yield $121 \mathrm{mg}(81 \%)$ of the title 
compound as a white solid m.p. $=135^{\circ} \mathrm{C} .-\mathrm{IR}(\mathrm{KBr}): v=3259 \mathrm{~cm}^{-1}, 3074,1672,1549,1324$, 1110, 1075, 696. - ${ }^{1} \mathrm{H}-\mathrm{NMR}\left(300 \mathrm{MHz}, \mathrm{CDCl}_{3}\right): \delta=1.00-1.16$ (m, $\left.2 \mathrm{H}, \mathrm{cPr}-\mathrm{H}\right), 1.34-1.52$ (m, 2 $\mathrm{H}, \mathrm{cPr}-\mathrm{H}), 5.04$ (s, $1 \mathrm{H}, \mathrm{CH}), 7.37-7.50(\mathrm{~m}, 2 \mathrm{H}$, aryl-H), 7.62 (t, ${ }^{3} J=8.2 \mathrm{~Hz}, 1 \mathrm{H}$, aryl-H), 7.71-7.84 (m, $3 \mathrm{H}$, aryl-H), 8.03 (br. s, $1 \mathrm{H}, \mathrm{NH}), 8.17\left(\mathrm{~d},{ }^{3} \mathrm{~J}=8.2 \mathrm{~Hz}, 1 \mathrm{H}\right.$, aryl-H), 8.23 (s, $1 \mathrm{H}$, aryl$\mathrm{H}),-{ }^{13} \mathrm{C}-\mathrm{NMR}\left(75.5 \mathrm{MHz}, \mathrm{CDCl}_{3}, \mathrm{APT}\right): \delta=10.8$ (-, cPr-C), 14.4 (-, cPr-C), 53.5 (-, cPr-C), 80.4 (+, CH-C), $116.9\left(+, \mathrm{q},{ }^{3} J_{\mathrm{C}-}\right.$ $\mathrm{F}=3.8 \mathrm{~Hz}$, aryl-C), $121.7\left(+, \mathrm{q},{ }^{3} J_{\mathrm{C}-\mathrm{F}}=3.8 \mathrm{~Hz}\right.$, aryl-C), $123.3(+$, $\mathrm{q},{ }^{3} J_{\mathrm{C}-\mathrm{F}}=3.8 \mathrm{~Hz}$, aryl-C), $124.8\left(+, \mathrm{q},{ }^{3} J_{\mathrm{C}-\mathrm{F}}=3.8 \mathrm{~Hz}\right.$, aryl-C), 127.4 (-, aryl-C), 128.4 (+, aryl-C), 129.4 (+, aryl-C), 129.7 (+,

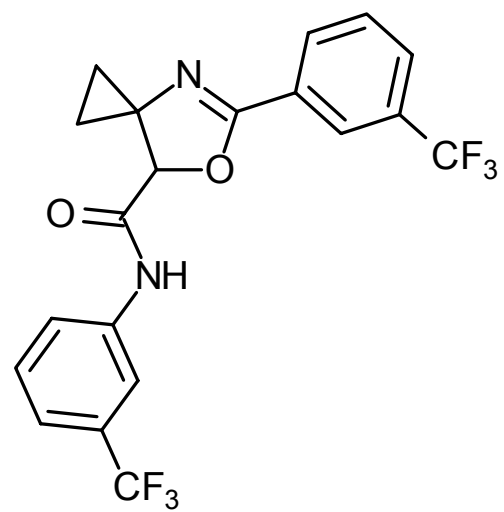
aryl-C), 130.9 (+, aryl-C), $131.2\left(-\right.$, q, ${ }^{2} J_{\mathrm{C}-\mathrm{F}}=33.5 \mathrm{~Hz}$, aryl-C), 131.5 (-, q, ${ }^{2} J_{\mathrm{C}-\mathrm{F}}=33.5 \mathrm{~Hz}$, arylC), 136.9 (-, Ar-C), 160.3 (-, CN-C), 166.3 (-, CO-C). - MS (70 eV), m/z (\%): 428 (32) [M $\left.{ }^{+}\right]$, 268 (48), 241 (100), 240 (86), 173 (56), 161 (52). - $\mathrm{C}_{20} \mathrm{H}_{14} \mathrm{~F}_{6} \mathrm{~N}_{2} \mathrm{O}_{2}$ (428.3): calcd. C 56.08, H 3.29, N 6.54; found C 56.29, H 3.20, N 6.42.

5-(4-Trifluoromethyl-phenyl)-6-oxa-4-aza-spiro[2.4]hept-4-ene-7-carboxylic acid (3-trifluromethyl-phenyl)-amide (68d): The crude product obtained from 64d (285 mg, $1.00 \mathrm{mmol})$, HOAt (163 mg, $1.20 \mathrm{mmol}), \mathrm{EDC} \cdot \mathrm{HCl}(286 \mathrm{mg}, 1.50 \mathrm{mmol}), 2,4,6-$ colidine (242 $\mathrm{mg}, 2.00 \mathrm{mmol})$ and 3-trifluoro-methyl aniline (209 $\mathrm{mg}, 1.30 \mathrm{mmol}$ ) in $15 \mathrm{~mL}$ of DCM following the GP 9 was purified by column chromatography $\left(R_{\mathrm{f}}=0.43\right.$, Pentane: $\left.\mathrm{Et}_{2} \mathrm{O}=2: 1\right)$ to yield $385 \mathrm{mg}(90 \%)$ of $\mathbf{6 8 d}$ as a white solid, m.p. $=162{ }^{\circ} \mathrm{C} .-\mathrm{IR}(\mathrm{KBr}): v=3335 \mathrm{~cm}^{-1}, 2927,1671$, 1558, 1447, 1338, 1128, 1054, 851. - ${ }^{1} \mathrm{H}-\mathrm{NMR}(300 \mathrm{MHz}$, $\left.\mathrm{CDCl}_{3}\right): \delta=0.98-1.17(\mathrm{~m}, 2 \mathrm{H}, \mathrm{cPr}-\mathrm{H}), 1.38-1.50$ (m, $2 \mathrm{H}, \mathrm{cPr}-$ H), 5.02 (s, $1 \mathrm{H}, \mathrm{CH}), 7.38-749$ (m, $2 \mathrm{H}$, aryl-H), 7.70-7.78 (m,

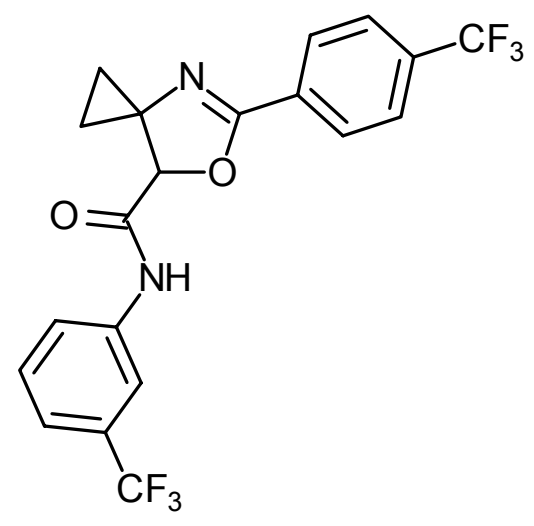
$3 \mathrm{H}$, aryl-H), 7.82 (s, $1 \mathrm{H}$, aryl-H), 8.04 (br. s, $1 \mathrm{H}, \mathrm{NH}), 8.08-8.14$ (m, $2 \mathrm{H}$, aryl-H). - ${ }^{13} \mathrm{C}-\mathrm{NMR}$ (75.5 MHz, $\left.\mathrm{CDCl}_{3}, \mathrm{APT}\right): \delta=10.8$ (-, cPr-C), 14.5 (-, cPr-C), 53.6 (-, cPr-C), 80.4 (+, CH-C), $116.9\left(+, \mathrm{q},{ }^{3} J_{\mathrm{C}-\mathrm{F}}=3.8 \mathrm{~Hz}\right.$, aryl-C), $121.7\left(+, \mathrm{q},{ }^{3} J_{\mathrm{C}-\mathrm{F}}=3.8 \mathrm{~Hz}, \operatorname{aryl}-\mathrm{C}\right), 123.2(+$, aryl-C), $123.2(+$, aryl-C), $125.7\left(+\right.$, q, ${ }^{3} J_{\mathrm{C}-\mathrm{F}}=3.8 \mathrm{~Hz}$, aryl-C), $126.4(+$, aryl-C), $128.2(+, 2 \mathrm{C}$, aryl-C), $129.7(+$, aryl-C), 129.9 (-, aryl-C), 131.3 (-, q, ${ }^{2} J_{\mathrm{C}-\mathrm{F}}=33.5 \mathrm{~Hz}$, aryl-C), 133.5 (-, q, ${ }^{2} J_{\mathrm{C}-\mathrm{F}}=33.5 \mathrm{~Hz}$, arylC), 136.9 (-, aryl-C), 160.3 (-, CN-C), 166.4 (-, CO-C). - MS (70 eV), m/z (\%): $428(44)\left[\mathrm{M}^{+}\right]$, 
268 (51), 241 (100), 240 (86), 161 (31). $-\mathrm{C}_{20} \mathrm{H}_{14} \mathrm{~F}_{6} \mathrm{~N}_{2} \mathrm{O}_{2}$ (428.3): calcd. C 56.08, H 3.29, N 6.54; found C 56.37, H 3.50, N 6.61.

5-(4-Trifluoromethyl-phenyl)-6-oxa-4-aza-spiro[2.4]hept-4-ene-7-carboxylic acid (5-chloro-2hydroxy-phenyl)-amide (68e): The crude product obtained from 64d (285 mg, $1.0 \mathrm{mmol})$, HOAt (163 mg, $1.20 \mathrm{mmol})$, $\mathrm{EDC} \cdot \mathrm{HCl}(286 \mathrm{mg}, 1.5 \mathrm{mmol})$, 2,4,6-colidine (242 $\mathrm{mg}, 2.0$ $\mathrm{mmol})$ and 5-chloro-2-hydroxyaniline $(187 \mathrm{mg}, 1.3 \mathrm{mmol})$ in $15 \mathrm{~mL}$ of DCM following GP 9 was purified by column chromatography $\left(R_{\mathrm{f}}=0.50\right.$, Pentane/Et $\left.{ }_{2} \mathrm{O} 1: 1\right)$ to yield 349 mg $(85 \%)$ of the $68 \mathrm{e}$ as white solid, m.p. $238^{\circ} \mathrm{C}$. - IR $(\mathrm{KBr})$ : $v=3380 \mathrm{~cm}^{-1}, 3102,1653,1552,1428,1327,1136,1176$,

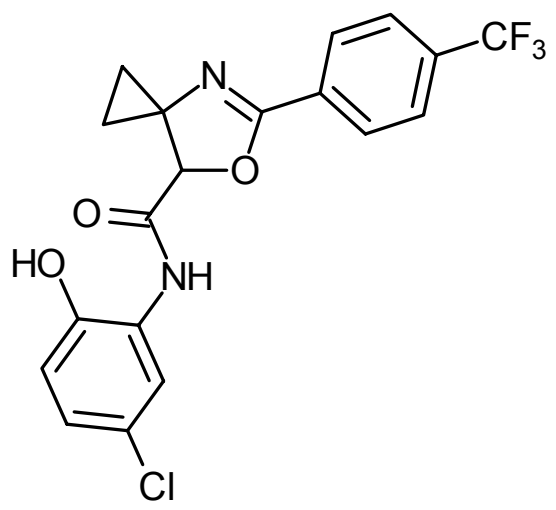
854. - ${ }^{1} \mathrm{H}-\mathrm{NMR}\left(300 \mathrm{MHz}, \mathrm{CDCl}_{3}\right): \delta=0.76-0.92(\mathrm{~m}, 1 \mathrm{H}, c \mathrm{Pr}-\mathrm{H}), 0.97-1.17$ (m, $\left.2 \mathrm{H}, \mathrm{cPr}-\mathrm{H}\right)$, 0.76-0.92 (m, $1 \mathrm{H}, \mathrm{cPr}-\mathrm{H}), 5.01$ (s, 1 H, CH), 6.87-6.92 (m, 1 H, aryl-H), 7.05 (m, 1 H, aryl-H), 7.42-7.58 (m, 3 H, aryl-H), 7.77 (s, 1 H, aryl-H), 7.96-8.00 (m, 2 H, aryl-H), 8.27 (br s, $1 \mathrm{H}, \mathrm{NH})$. $-{ }^{13} \mathrm{C}-\mathrm{NMR}\left(75.5 \mathrm{MHz}, \mathrm{CDCl}_{3}, \mathrm{APT}\right): \delta=10.6$ (-, cPr-C), 14.7 (-, cPr-C), 53.8 (-, cPr-C), 80.6 $(+, \mathrm{CH}-\mathrm{C}), 117.3$ (+, aryl-C), $120.8\left(+\right.$, aryl-C), $125.4\left(+, \mathrm{q},{ }^{3} J_{\mathrm{C}-\mathrm{F}}=3.8 \mathrm{~Hz}\right.$, aryl-C), $126.6(+, \mathrm{q}$, ${ }^{3} J_{\mathrm{C}-\mathrm{F}}=3.8 \mathrm{~Hz}$, aryl-C), $128.2\left(+, 2 \mathrm{C}\right.$, aryl-C), 130.1 (-, aryl-C), $131.3\left(-, \mathrm{q},{ }^{2} J_{\mathrm{C}-\mathrm{F}}=33.5 \mathrm{~Hz}\right.$, arylC), 146.8, 160.6 (-, CN-C), 166.7 (-, CO-C). - MS (DCI), m/z (\%): 411 (100) [M + $\left.\mathrm{H}^{+}\right]$. $\mathrm{C}_{19} \mathrm{H}_{14} \mathrm{ClF}_{3} \mathrm{~N}_{2} \mathrm{O}_{3}$ (410.8): calcd. C 55.56, H 3.44, N 6.82; found C 55.69, H 3.18, N 7.06.

5-(4-Trifluoromethyl-phenyl)-6-oxa-4-aza-spiro[2.4]hept-4-ene-7-carboxylic acid (5-trifluoromethoxy-2-hydroxy-phenyl)-amide (68f) The crude product obtained from 64d (143 mg, $0.50 \mathrm{mmol})$, HOAt (82 mg, 0.60 $\mathrm{mmol}), \mathrm{EDC} \cdot \mathrm{HCl}(143 \mathrm{mg}, 0.75 \mathrm{mmol}), 2,4,6$-colidine (121 $\mathrm{mg}, 1.0 \mathrm{mmol}$ ) and 5-trifluoromethoxy-2-hydroxy aniline (125 mg, $0.65 \mathrm{mmol}$ ) in $10 \mathrm{~mL}$ of DCM following the general procedure 1 (GP 1) was purified by column chromatography $\left(R_{\mathrm{f}}=0.45\right.$, Pentane $\left./ \mathrm{Et}_{2} \mathrm{O}=1: 1\right)$ to yield 127 $\mathrm{mg}(55 \%)$ of $68 \mathrm{f}$ as colourless solid, m.p. $=135^{\circ} \mathrm{C}$. $-\mathrm{IR}$ $(\mathrm{KBr}): v=3378 \mathrm{~cm}^{-1}, 3122,1654,1559,1447,1251,1136$,

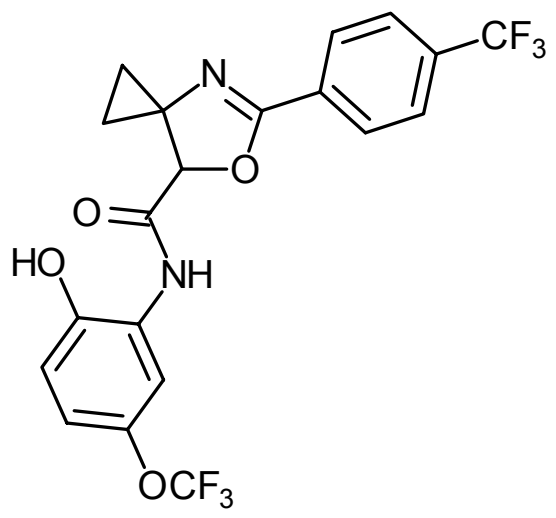
1069, 854. - ${ }^{1} \mathrm{H}-\mathrm{NMR}$ (300 MHz, $\left.\mathrm{CD}_{3} \mathrm{OD}\right): \delta=1.04-1.20$ (m, $\left.2 \mathrm{H}, \mathrm{cPr}-\mathrm{H}\right), 1.30-1.41$ (m, $2 \mathrm{H}$, 
cPr-H), 5.18 (s, 1 H, CH), 6.82-6.93 (m, 2 H, aryl-H), 7.83 (d, ${ }^{3} J=8.2 \mathrm{~Hz}, 2 \mathrm{H}$, aryl-H), 8.02$8.08\left(\mathrm{~m}, 1 \mathrm{H}\right.$, aryl-H), $8.18\left(\mathrm{~d},{ }^{3} J=8.2 \mathrm{~Hz}, 2 \mathrm{H}\right.$, aryl-H). $-{ }^{13} \mathrm{C}-\mathrm{NMR}\left(75.5 \mathrm{MHz}, \mathrm{CD}_{3} \mathrm{OD}, \mathrm{APT}\right): \delta$ $=10.7$ (-, cPr-C), 15.0 (-, cPr-C), 54.7 (-, cPr-C), 81.8 (+, CH-C), 115.2 (+, aryl-C), $116.0(+$, aryl-C), $118.8\left(+\right.$, aryl-C), $121.7\left(-\right.$, q, $\left.{ }^{1} J_{\mathrm{C}-\mathrm{F}}=273 \mathrm{~Hz}, \mathrm{CF}_{3}\right), 126.7\left(+, \mathrm{q},{ }^{3} J_{\mathrm{C}-\mathrm{F}}=3.8 \mathrm{~Hz}\right.$, aryl-C), 127.1 (-, aryl-C), 129.6 (+, 2 C, aryl-C), $131.6\left(-\right.$, aryl-C), 134.4 (-, q, ${ }^{2} J_{\mathrm{C}-\mathrm{F}}=33.5 \mathrm{~Hz}$, aryl-C), 142.3 (-, aryl-C), 147.7 (-, aryl-C), 163.1 (-, CN-C), 168.4 (-, CO-C). - MS (70 eV), m/z (\%): 460 (35) $\left[\mathrm{M}^{+}\right], 296$ (15), 268 (54), 241 (100), 240 (61), 173 (57).

5-(4-Trifluoromethyl-phenyl)-6-oxa-4-aza-spiro[2.4]hept-4-ene-7-carboxylic acid (3,5-dichloro2-hydroxy-phenyl)-amide (68g) The crude product obtained from 64d (143 mg, $0.50 \mathrm{mmol}$ ), HOAt (82 mg, $0.60 \mathrm{mmol}), \mathrm{EDC} \cdot \mathrm{HCl}(143 \mathrm{mg}, 0.75 \mathrm{mmol})$, 2,4,6-colidine (121 mg, $1.0 \mathrm{mmol})$ and 3,5-dichloro-2hydroxy aniline $(126 \mathrm{mg}, 0.65 \mathrm{mmol})$ in $10 \mathrm{~mL}$ of $\mathrm{DCM}$ following GP 9 was purified by column chromatography $\left(R_{\mathrm{f}}=0.41\right.$, Pentane: $\left.\mathrm{Et}_{2} \mathrm{O}=1: 1\right)$ to yield $175 \mathrm{mg}$ of $\mathbf{6 8 g}$ $(79 \%)$ as colourless solid, m.p. $=185^{\circ} \mathrm{C} .-\mathrm{IR}(\mathrm{KBr}): v=$ $3392 \mathrm{~cm}^{-1}, 3132,1676,1596,1412,1326,1140,1056$, 852. $-{ }^{1} \mathrm{H}-\mathrm{NMR}\left(300 \mathrm{MHz}, \mathrm{CDCl}_{3}\right): \delta=0.99-1.20(\mathrm{~m}, 2$

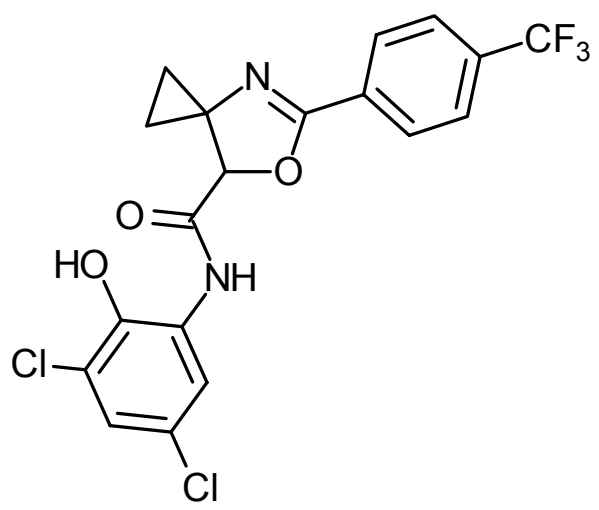
$\mathrm{H}, \mathrm{cPr}-\mathrm{H}), 1.37-1.51$ (m, $2 \mathrm{H}, \mathrm{cPr}-\mathrm{H}), 5.00$ (s, $1 \mathrm{H}, \mathrm{CH}), 6.21$ (br s, $1 \mathrm{H}, \mathrm{OH}), 7.12\left(\mathrm{~d},{ }^{4} \mathrm{~J}=2.5 \mathrm{~Hz}\right.$, $1 \mathrm{H}$, aryl-H), $7.71\left(\mathrm{~d},{ }^{3} \mathrm{~J}=8.0 \mathrm{~Hz}, 2 \mathrm{H}\right.$, aryl-H), $8.07\left(\mathrm{~d},{ }^{3} \mathrm{~J}=8.0 \mathrm{~Hz}, 2 \mathrm{H}\right.$, aryl-H), $8.17\left(\mathrm{~d},{ }^{4} J=2.5\right.$ $\mathrm{Hz}, 1 \mathrm{H}$, aryl-H), 8.78 (br s, $1 \mathrm{H}, \mathrm{NH}) .-{ }^{13} \mathrm{C}-\mathrm{NMR}\left(75.5 \mathrm{MHz}, \mathrm{CDCl}_{3}, \mathrm{APT}\right): \delta=10.5$ (-, cPr-C), 14.9 (-, cPr-C), 53.8 (-, cPr-C), 80.3 (+, CH-C), 119.1 (+, aryl-C), 120.5 (-, aryl-C), 123.6 (-, q, $\left.{ }^{1} J_{\mathrm{C}-\mathrm{F}}=273 \mathrm{~Hz}, \mathrm{CF}_{3}\right), 124.1\left(+\right.$, aryl-C) $125.7\left(+, \mathrm{q},{ }^{3} J_{\mathrm{C}-\mathrm{F}}=3.8 \mathrm{~Hz}\right.$, aryl-C), $125.8(-$, aryl-C), 126.0 (-, aryl-C), 128.1 (+, 2 C, aryl-C), 129.8 (-, aryl-C), 133.7 (-, q, ${ }^{2} J_{\mathrm{C}-\mathrm{F}}=33.2 \mathrm{~Hz}$, aryl-C), $140.0(-$, aryl-C), 160.3 (-, CN-C), 166.7 (-, CO-C). - MS (70 eV), m/z (\%): 446/444 (15/21) [M $\left.{ }^{+}\right], 268$ (59), 241 (100), 240 (60), 173 (51). $-\mathrm{C}_{19} \mathrm{H}_{13} \mathrm{Cl}_{2} \mathrm{~F}_{3} \mathrm{~N}_{2} \mathrm{O}_{3}$ (445.2) calcd. C 51.26, H 2.94, N 6.29; found C 51.51, H 2.95, N 6.53. 
5-(4-Trifluoromethyl-phenyl)-6-oxa-4-aza-spiro[2.4]hept-4-ene-7-carboxylic acid (5-trifluoromethyl-2-hydroxy-phenyl)-amide (68h): The crude product obtained from $\mathbf{6 4 d}$ (143 $\mathrm{mg}, 0.50$ mmol), HOAt ( $82 \mathrm{mg}, 0.60 \mathrm{mmol}), \mathrm{EDC} \cdot \mathrm{HCl}(143 \mathrm{mg}, 0.75$ mmol), 2,4,6-colidine (121 $\mathrm{mg}, \quad 1.0 \mathrm{mmol})$ and 5trifluoromethyl-2-hydroxy aniline $(115 \mathrm{mg}, 0.65 \mathrm{mmol})$ in 10 $\mathrm{mL}$ of DCM following GP 9 was purified by column chromatography $\left(R_{\mathrm{f}}=0.45\right.$, Pentane $\left./ \mathrm{Et}_{2} \mathrm{O}=1: 1\right)$ to yield 167 $\mathrm{mg}(78 \%)$ of $\mathbf{6 8 h}$ as colourless solid, m.p. $134-136{ }^{\circ} \mathrm{C}$. $-\mathrm{IR}$ $(\mathrm{KBr}): v=3383 \mathrm{~cm}^{-1}, 3122,1664,1559,1414,1336,1160$, 1069, 856. $-{ }^{1} \mathrm{H}-\mathrm{NMR}\left(300 \mathrm{MHz}, \mathrm{CD}_{3} \mathrm{OD}\right): \delta=1.04-1.20$

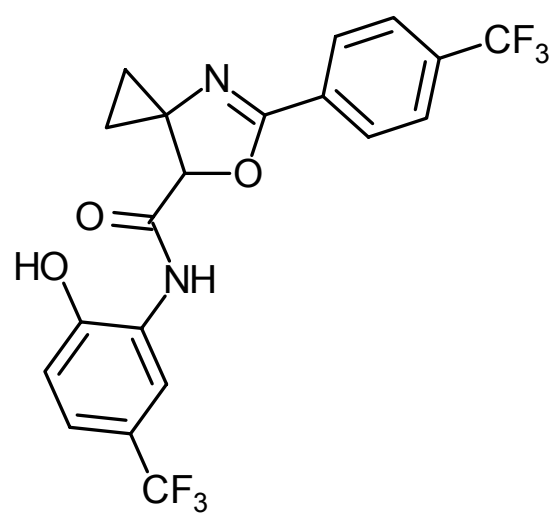
(m, 2 H, cPr-H), 1.24-1.40 (m, 2 H, cPr-H), 5.09 (s, 1 H, CH), 6.97 (d, ${ }^{3} J=7.5$ Hz, 1 H, aryl-H), 7.25-7.32 (m, $1 \mathrm{H}$, aryl-H), $7.82\left(\mathrm{~d},{ }^{3} J=8.1 \mathrm{~Hz}, 2 \mathrm{H}\right.$, aryl-H), $8.18\left(\mathrm{~d},{ }^{3} J=8.1 \mathrm{~Hz}, 2 \mathrm{H}\right.$, aryl-H), 8.39 (br. s, $1 \mathrm{H}, \mathrm{NH}), 8.64-8.70$ (m, $1 \mathrm{H}$, aryl-H). $-{ }^{13} \mathrm{C}-\mathrm{NMR}\left(75.5 \mathrm{MHz}, \mathrm{CDCl}_{3}\right.$, APT): $\delta=10.8$ (-, cPr-C), 14.4 (-, cPr-C), 54.7 (-, cPr-C), 81.8 (+, CH-C), 115.8 (+, aryl-C), $119.2\left(+\right.$, q, ${ }^{3} J_{\mathrm{C}-\mathrm{F}}=$ $3.8 \mathrm{~Hz}$, aryl-C), $122.5\left(+, \mathrm{q},{ }^{3} J_{\mathrm{C}-\mathrm{F}}=32.5 \mathrm{~Hz}\right.$, aryl-C), $123.5\left(+, \mathrm{q},{ }^{3} J_{\mathrm{C}-\mathrm{F}}=3.8 \mathrm{~Hz}\right.$, aryl-C), 124.8 (q, $\left.{ }^{1} J_{\mathrm{C}-\mathrm{F}}=271 \mathrm{~Hz}, \mathrm{CF}_{3}\right), 125.3\left(\mathrm{q},{ }^{1} J_{\mathrm{C}-\mathrm{F}}=262 \mathrm{~Hz}, \mathrm{CF}_{3}\right), 126.7$ (+, aryl-C), $129.6(+, 2 \mathrm{C}$, aryl-C), 131.6 (-, aryl-C), 134.3 (q, ${ }^{2} J_{\mathrm{C}-\mathrm{F}}=33.2 \mathrm{~Hz}$, aryl-C), 151.8 (-, aryl-C), 163.1 (-, CN-C), 168.6 (-, CO-C). - MS (70 eV), m/z (\%): 444 (20) $\left[\mathrm{M}^{+}\right], 268$ (52), 241 (100), 140 (57), 173 (53). $\mathrm{C}_{20} \mathrm{H}_{14} \mathrm{~F}_{6} \mathrm{~N}_{2} \mathrm{O}_{3}$ (444.3). - calcd. C 54.06, H 3.14, N 6.30; found C 54.16, H 3.40, N 6.45.

5-phenyl-6-oxa-4-aza-spiro[2.4] hept-4-ene-7-carboxylic acid (5-chloro-2-hydroxy-phenyl)-amide (68i): The crude product obtained from 64e (185 mg, $0.85 \mathrm{mmol})$, HOAt (139 mg, $1.02 \mathrm{mmol})$, $\mathrm{EDC} \cdot \mathrm{HCl}$ (244 mg, $1.27 \mathrm{mmol}), 2,4,6$-colidine (206 mg, $1.70 \mathrm{mmol}$ ) and 5-chloro-2-hydroxyaniline $(146 \mathrm{mg}, 1.1 \mathrm{mmol})$ in $15 \mathrm{~mL}$ of DCM following GP 9 was purified by column chromatography $\left(R_{\mathrm{f}}=\right.$ 0.25 , Pentane: $\left.\mathrm{Et}_{2} \mathrm{O}=1: 1\right)$ to yield $268 \mathrm{mg}(92 \%)$ of $\mathbf{6 8 i}$ as a white solid, m.p. $211{ }^{\circ} \mathrm{C}$. - IR (KBr): $v=3381 \mathrm{~cm}^{-1}, 3052,1668,1558$, 1431, 1339, 1195, 1033. - ${ }^{1} \mathrm{H}-\mathrm{NMR}\left(300 \mathrm{MHz}, \mathrm{CDCl}_{3}\right): \delta=0.96-$ 1.18 (m, $2 \mathrm{H}, \mathrm{cPr}-\mathrm{H}), 1.34-1.50$ (m, $2 \mathrm{H}, \mathrm{cPr}-\mathrm{H}), 5.01$ (s, $1 \mathrm{H}, \mathrm{CH})$, 6.87-6.92 (m, $1 \mathrm{H}, \mathrm{Ar}-\mathrm{H}), 7.05\left(\mathrm{dd},{ }^{3} J=8.2 \mathrm{~Hz},{ }^{4} J=2.5 \mathrm{~Hz}, 1 \mathrm{H}\right.$,

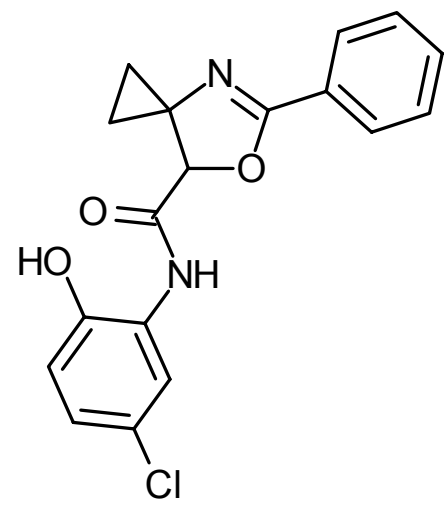
aryl-H) 7.42-7.58 (m, $3 \mathrm{H}$, aryl-H), 7.77 (s, $1 \mathrm{H}$, aryl-H), 7.96-8.00 (m, $2 \mathrm{H}$, aryl-H), 8.27 (br s, $1 \mathrm{H}, \mathrm{NH}) .-{ }^{13} \mathrm{C}-\mathrm{NMR}\left(75.5 \mathrm{MHz}, \mathrm{CDCl}_{3}, \mathrm{APT}\right): \delta=10.6$ (-, cPr-C), 
14.5 (-, cPr-C), 53.7 (-, cPr-C), 80.2 (+, CH-C), 120.1 (+, aryl-C), 121.9 (+, aryl-C), 125.5 (-, aryl-C), 126.6 (-, aryl-C), 127.0 (+, aryl-C), 127.9 (+, 2 C, aryl-C), 128.7 (+, 2 C, aryl-C), 131.9 (+, aryl-C), 146.8 (-, aryl-C), 161.5 (-, CN-C), 168.2 (-, CO-C). - MS (70 eV), m/z (\%): 344/342 (6/20) $\left[\mathrm{M}^{+}\right], 200$ (55), 173 (100), 172 (58), 105 (54). - $\mathrm{C}_{18} \mathrm{H}_{15} \mathrm{ClN}_{2} \mathrm{O}_{3}$ (342.8): calcd. C 63.07, $\mathrm{H}$ 4.41, N 8.17; found C 63.32, H 4.18, N 8.02.

\section{General procedure for synthesis of Oxazoline benzoxazole derivatives (Mitsunabu reaction)}

(GP 10): To a solution of the the above amides (68e-h) and $\mathrm{Ph}_{3} \mathrm{P}$ (2.2 equiv.) in $10 \mathrm{~mL}$ of dry THF was added drop wise a solution of DEAD (2.2 equiv.) in an ice bath. The resulting solution was stirred at $20^{\circ} \mathrm{C}$ for $10 \mathrm{~h}$. This reaction mixture was taken in $\mathrm{Et}_{2} \mathrm{O}(25 \mathrm{~mL}), \mathrm{H}_{2} \mathrm{O}$ was added, the organic layer was separated. The aq. layer was extracted with $\mathrm{Et}_{2} \mathrm{O}(2 \times 25 \mathrm{~mL})$, the combined etheral layer was dried over $\mathrm{MgSO}_{4}$, concentrated and purified by column chromatography to yield the corresponding benzooxazoles.

\section{5-Chloro-2-[5-(4-trifluoromethyl-phenyl)-6-oxa-4-aza-spiro[2.4]hept-4-en-7-yl]-benzooxazole}

(69a): The crude product obtained from $(68 \mathrm{e}, 205 \mathrm{mg}, 0.50 \mathrm{mmol}), \mathrm{Ph}_{3} \mathrm{P}(288 \mathrm{mg}, 1.10 \mathrm{mmol})$ and DEAD (174 mg, $1.10 \mathrm{mmol})$ according to GP 10, was purified by column chromatography $\left(R_{\mathrm{f}}=0.40\right.$, Pentane $\left./ \mathrm{Et}_{2} \mathrm{O}=5: 1\right)$ to yield $162 \mathrm{mg}(83 \%)$ of $\mathbf{6 9 a}$ as a white solid, m.p. $=129^{\circ} \mathrm{C}$. - IR $(\mathrm{KBr}): v=3105 \mathrm{~cm}^{-1}$, 1652, 1453, 1324, 1173, 1130, 1122, 857. - ${ }^{1} \mathrm{H}-\mathrm{NMR}(300$ $\left.\mathrm{MHz}, \mathrm{CDCl}_{3}\right): \delta=0.59-0.66(\mathrm{~m}, 1 \mathrm{H}, \mathrm{cPr}-\mathrm{H}), 1.00-1.12(\mathrm{~m}$, $1 \mathrm{H}, \mathrm{cPr}-\mathrm{H}), 1.21-1.32$ (m, $1 \mathrm{H}, \mathrm{cPr}-\mathrm{H}), 1.38-1.47$ (m, $1 \mathrm{H}$, cPr-H), $5.74(\mathrm{~s}, 1 \mathrm{H}, \mathrm{CH}), 7.33\left(\mathrm{dd},{ }^{3} \mathrm{~J}=8.7 \mathrm{~Hz},{ }^{4} \mathrm{~J}=1.87\right.$

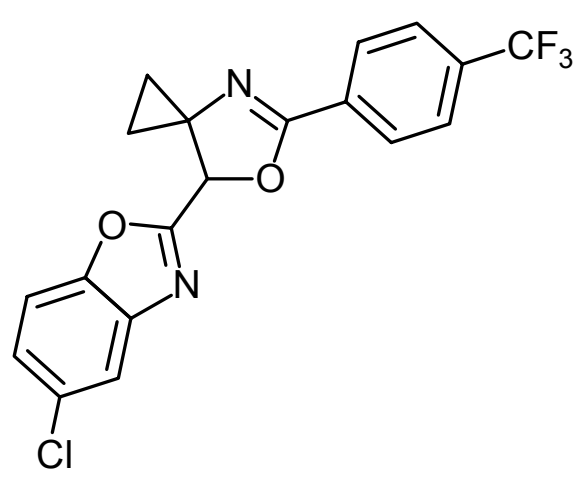
$\mathrm{Hz}, 1 \mathrm{H}$, aryl-H), 7.44 (d, ${ }^{3} J=8.7 \mathrm{~Hz}, 1 \mathrm{H}$, aryl-H), $7.65\left(\mathrm{~d},{ }^{3} \mathrm{~J}=8.1 \mathrm{~Hz}, 2 \mathrm{H}\right.$, aryl-H), 7.71 (s, $1 \mathrm{H}$, aryl-H), $8.08\left(\mathrm{~d},{ }^{3} J=8.1 \mathrm{~Hz}, 2 \mathrm{H}\right.$, aryl-H). $-{ }^{13} \mathrm{C}-\mathrm{NMR}\left(75.5 \mathrm{MHz}, \mathrm{CDCl}_{3}, \mathrm{APT}\right): \delta=11.3(-, \mathrm{cPr}-$ C), 14.9 (-, cPr-C), 54.4 (-, cPr-C), 78.0 (+, CH-C), 111.9 (+, aryl-C), 120.5 (+, aryl-C), $123.2(-$, q, $\left.{ }^{1} J_{\mathrm{C}-\mathrm{F}}=273 \mathrm{~Hz}, \mathrm{CF}_{3}\right), 125.5\left(+, \mathrm{q},{ }^{3} J_{\mathrm{C}-\mathrm{F}}=3.8 \mathrm{~Hz}\right.$, aryl-C), $125.6(+$, aryl-C), $128.4(+, 2 \mathrm{C}$, arylC), 130.1 (-, aryl-C), 130.4 (-, aryl-C), 132.7 (-, q, ${ }^{2} J_{\mathrm{C}-\mathrm{F}}=32.1 \mathrm{~Hz}$, aryl-C), 141.5 (-, aryl-C), 149.5 (-, aryl-C), 162.0 (-, CN-C), 163.3 (-, CN-C). - MS (DCI), m/z (\%): 393 (100) [M+ + H]. $\mathrm{C}_{19} \mathrm{H}_{12} \mathrm{ClF}_{3} \mathrm{~N}_{2} \mathrm{O}_{2}$ (392.8): calcd. C 58.10, H 3.08, N 7.13; found C 58.31, H 2.94, N 6.99. 
5-(Trifluoromethoxy)-2-[5-(4-trifluoromethyl-phenyl)-6-oxa-4-aza-spiro[2.4]hept-4-en-7-yl]-

benzooxazole (69b): The crude product obtained from (68f, 91mg, $0.20 \mathrm{mmol}), \mathrm{Ph}_{3} \mathrm{P}(115 \mathrm{mg}$, $0.44 \mathrm{mmol}$ ) and DEAD (77 $\mathrm{mg}, 0.44 \mathrm{mmol}$ ) according to GP 10 was purified by column chromatography $\left(R_{\mathrm{f}}=0.42\right.$, Pentane/ $\left.\mathrm{Et}_{2} \mathrm{O}=5: 1\right)$ to yield $72 \mathrm{mg}(81 \%)$ of $\mathbf{6 9 b}$ as white solid, m.p. $=105^{\circ} \mathrm{C} .-\mathrm{IR}(\mathrm{KBr}): v=3093 \mathrm{~cm}^{-1}, 1651,1569$, 1475, 1348, 1255, 1160, 810. - ${ }^{1} \mathrm{H}-\mathrm{NMR}$ (300 MHz, $\left.\mathrm{CDCl}_{3}\right): \delta=0.20-0.30(\mathrm{~m}, 1 \mathrm{H}, \mathrm{cPr}-\mathrm{H}), 0.98-1.12(\mathrm{~m}, 1 \mathrm{H}$, cPr-H), 1.23-1.34 (m, $1 \mathrm{H}, \mathrm{cPr}-\mathrm{H}), 1.39-1.50$ (m, $1 \mathrm{H}, \mathrm{cPr}-$ $\mathrm{H}), 5.74(\mathrm{~s}, 1 \mathrm{H}, \mathrm{CH}), 7.20-7.29(\mathrm{~m}, 1 \mathrm{H}$, aryl-H), $7.43-$

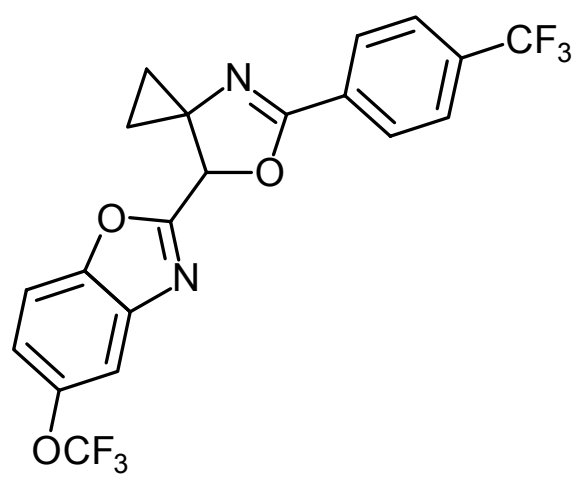
7.58 (m, $1 \mathrm{H}$, aryl-H), 7.61 (s, $1 \mathrm{H}$, aryl-H), $7.68\left(\mathrm{~d},{ }^{3} J=8.0 \mathrm{~Hz}, 2 \mathrm{H}\right.$, aryl-H), 8.09 (d, ${ }^{3} J=8.0 \mathrm{~Hz}$, $2 \mathrm{H}$, aryl-H). $-{ }^{13} \mathrm{C} \mathrm{NMR}\left(75.5 \mathrm{MHz}, \mathrm{CDCl}_{3}, \mathrm{APT}\right): \delta=11.3$ (-, cPr-C), 14.9 (-, cPr-C), 54.4 (-, cPr-C), 78.0 (+, CH-C), 111.7 (+, aryl-C), 113.7 (+, aryl-C), 115.4 (-, q, $\left.{ }^{1} J_{\mathrm{C}-\mathrm{F}}=234 \mathrm{~Hz}, \mathrm{CF}_{3}\right)$, 119.7 (+, aryl-C), 122.1 (-, aryl-C), 125.5 (+, q, ${ }^{3} J_{\mathrm{C}-\mathrm{F}}=3.8 \mathrm{~Hz}$, aryl-C), 128.4 (+, 2 C, aryl-C), 130.1 (-, aryl-C), 133.2 (+, q, ${ }^{2} J_{\mathrm{C}-\mathrm{F}}=32.5 \mathrm{~Hz}$, aryl-C), 141.2 (-, aryl-C), 149.2 (-, aryl-C), 162.0

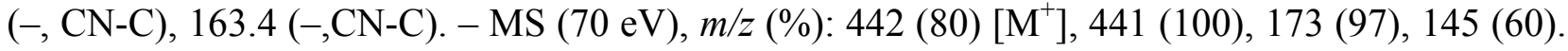
$-\mathrm{C}_{20} \mathrm{H}_{12} \mathrm{~F}_{6} \mathrm{~N}_{2} \mathrm{O}_{3}$ (442.3): calcd. C 54.31, H 2.73, N 6.33; found C 54.37, H 2.92, N 6.18.

\section{5,7-(Dichloro)-2-[5-(4-trifluoromethyl-phenyl)-6-oxa-4-aza-spiro[2.4]hept-4-en-7-yl]-benzo-}

oxazole (69c): The crude product obtained from (68g, 125mg, $0.28 \mathrm{mmol}), \mathrm{Ph}_{3} \mathrm{P}$ (161 $\mathrm{mg}, 0.62$ mmol) and DEAD (108 $\mathrm{mg}, 0.62 \mathrm{mmol})$ according to GP 10 was purified by column chromatography $\left(R_{\mathrm{f}}=0.45\right.$, Pentane/ $\left.\mathrm{Et}_{2} \mathrm{O} 5: 1\right)$, to yield $105 \mathrm{mg}(88 \%)$ of $69 \mathrm{c}$ as colourless solid, m.p. $=120^{\circ} \mathrm{C} .-\mathrm{IR}(\mathrm{KBr}): v=3074 \mathrm{~cm}^{-}$ ${ }^{1}, 1657,1562,1328,1348,1167,1119,848 .-{ }^{1} \mathrm{H}-\mathrm{NMR}$ $\left(300 \mathrm{MHz}, \mathrm{CDCl}_{3}\right): \delta=0.63-0.75(\mathrm{~m}, 1 \mathrm{H}, \mathrm{cPr}-\mathrm{H}), 1.02-$ 1.13 (m, $1 \mathrm{H}, \mathrm{cPr}-\mathrm{H}), 1.26-1.37$ (m, $1 \mathrm{H}, \mathrm{cPr}-\mathrm{H}), 1.01-$ $1.50(\mathrm{~m}, 1 \mathrm{H}, \mathrm{cPr}-\mathrm{H}), 5.77$ (s, $1 \mathrm{H}, \mathrm{CH}), 7.39\left(\mathrm{~d},{ }^{4} \mathrm{~J}=2.4\right.$

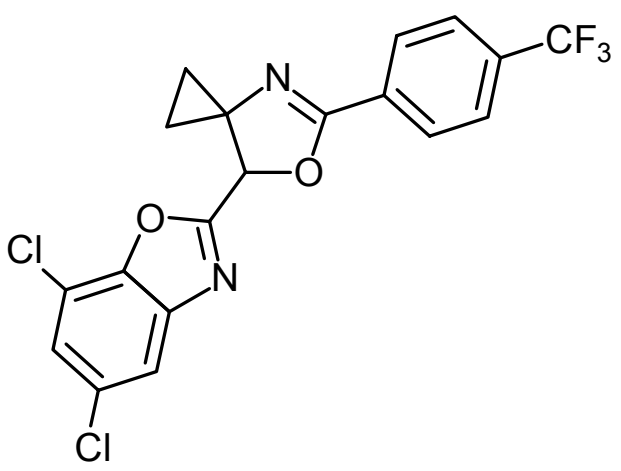
$\mathrm{Hz}, 1 \mathrm{H}$, aryl-H), 7.62 (d, ${ }^{4} J=2.4 \mathrm{~Hz}, 1 \mathrm{H}$, aryl-H), $7.69\left(\mathrm{~d},{ }^{3} J=8.0 \mathrm{~Hz}, 1 \mathrm{H}\right.$, aryl-H), $8.09\left(\mathrm{~d},{ }^{3} J=\right.$ $8.0 \mathrm{~Hz}, 1 \mathrm{H}$, aryl-H). $-{ }^{13} \mathrm{C}-\mathrm{NMR}\left(75.5 \mathrm{MHz}, \mathrm{CDCl}_{3}, \mathrm{APT}\right): \delta=11.5$ (-, cPr-C), 14.9 (-, cPr-C), 54.5 (-, cPr-C), 77.7 (+, CH-C), 117.0 (-, aryl-C), 119.2 (+, aryl-C), 123.8 (-, q, ${ }^{1} J_{\mathrm{C}-\mathrm{F}}=262 \mathrm{~Hz}$, $\left.\mathrm{CF}_{3}\right), 125.5\left(+, \mathrm{q},{ }^{3} J_{\mathrm{C}-\mathrm{F}}=3.8 \mathrm{~Hz}\right.$, aryl-C), 126.4 (+, aryl-C), 128.5 (+, $2 \mathrm{C}$, aryl-C), 130.1 (-, aryl- 
C), 130.7 (-, aryl-C), 133.7 (-, q, ${ }^{2} J_{\mathrm{C}-\mathrm{F}}=33.2 \mathrm{~Hz}$, aryl-C), 142.3 (-, aryl-C), 146.4 (-, aryl-C),

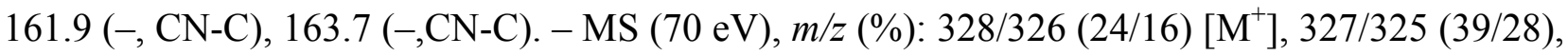
$173(100), 145(65)$.

5-(Trifluoromethyl)-2-[5-(4-trifluoromethyl-phenyl)-6-oxa-4-aza-spiro[2.4]hept-4-en-7-yl]benzooxazole (69d): The crude product obtained from $(\mathbf{6 8 h}, 105 \mathrm{mg}, 0.24 \mathrm{mmol}), \mathrm{Ph}_{3} \mathrm{P}(136 \mathrm{mg}$, $0.52 \mathrm{mmol})$ and DEAD (90 $\mathrm{mg}, 0.52 \mathrm{mmol})$ according to GP 10 was purified by column chromatography $\left(R_{\mathrm{f}}=0.40\right.$ Pentane $\left./ \mathrm{Et}_{2} \mathrm{O}=5: 1\right)$ to yield $85 \mathrm{mg}(81 \%)$ of $69 \mathbf{d}$ as solid m.p. $=145^{\circ} \mathrm{C}$. $-\mathrm{IR}(\mathrm{KBr}): v=3075 \mathrm{~cm}^{-1}, 3046,1650$, 1628, 1579, 1439, 1336, 1187, 1072, 815. - ${ }^{1} \mathrm{H}-\mathrm{NMR}(300$ $\left.\mathrm{MHz} \mathrm{CDCl}_{3}\right): \delta=0.60-0.72(\mathrm{~m}, 1 \mathrm{H}, \mathrm{cPr}-\mathrm{H}), 1.02-1.14(\mathrm{~m}$, $1 \mathrm{H}, \mathrm{cPr}-\mathrm{H}), 1.23-1.34$ (m, $1 \mathrm{H}, \mathrm{cPr}-\mathrm{H}), 1.40-1.52$ (m, $1 \mathrm{H}$, cPr-H), 5.78 (s, 1 H, CH), 7.58-7.66 (m, 2 H, aryl-H), 7.73

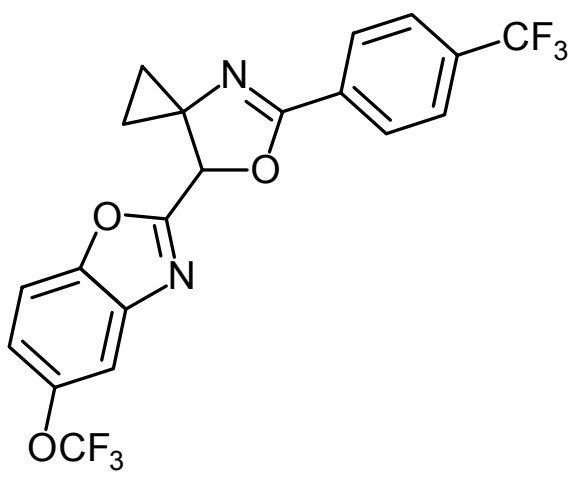
(d, ${ }^{3} J=8.1 \mathrm{~Hz}, 2 \mathrm{H}$, aryl-H), 8.02 (s, $1 \mathrm{H}$, aryl-H), $8.11\left(\mathrm{~d},{ }^{3} \mathrm{~J}=8.1 \mathrm{~Hz}, 2 \mathrm{H}\right.$, aryl-H). $-{ }^{13} \mathrm{C}$ NMR (75.5 MHz, $\left.\mathrm{CDCl}_{3}, \mathrm{APT}\right): \delta=11.3$ (-, cPr-C), 15.0 (-, cPr-C), 54.4 (-, cPr-C), 77.9 (+, CH-C), $111.7\left(+\right.$, aryl-C), $118.4\left(+, \mathrm{q},{ }^{3} J_{\mathrm{C}-\mathrm{F}}=4.5 \mathrm{~Hz}\right.$, aryl-C), $123.2\left(+, \mathrm{q},{ }^{3} J_{\mathrm{C}-\mathrm{F}}=4.5 \mathrm{~Hz}\right.$, aryl-C), $124.2(-$, q, $\left.{ }^{1} J_{\mathrm{C}-\mathrm{F}}=268 \mathrm{~Hz}, \mathrm{CF}_{3}\right), 124.8\left(-, \mathrm{q},{ }^{1} J_{\mathrm{C}-\mathrm{F}}=264 \mathrm{~Hz}, \mathrm{CF}_{3}\right), 125.5\left(+, \mathrm{q},{ }^{3} J_{\mathrm{C}-\mathrm{F}}=3.8 \mathrm{~Hz}\right.$, aryl-C), $127.8\left(-\right.$, q, ${ }^{2} J_{\mathrm{C}-\mathrm{F}}=32.5 \mathrm{~Hz}$, aryl-C), $128.5\left(+, 2 \mathrm{C}\right.$, aryl-C), $130.1\left(-\right.$, aryl-C), $133.2\left(-, \mathrm{q},{ }^{2} J_{\mathrm{C}-\mathrm{F}}=\right.$ $32.5 \mathrm{~Hz}$, aryl-C), 140.6 (-, aryl-C), 152.6 (-, aryl-C), 162.0 (-, CN-C), 163.7 (-, CN-C). - MS (DCI), $m / z(\%): 870$ (1) $\left[2 \mathrm{M}+\mathrm{NH}_{4}^{+}\right], 853(18)\left[2 \mathrm{M}+\mathrm{H}^{+}\right], 444$ (12) $\left[\mathrm{M}+\mathrm{NH}_{4}^{+}\right], 427(100)[\mathrm{M}+$ $\mathrm{H}^{+}$]. $-\mathrm{C}_{20} \mathrm{H}_{12} \mathrm{~F}_{6} \mathrm{~N}_{2} \mathrm{O}_{2}$ (426.3): calcd. C 56.35, H 2.84, N 6.57; found C 56.21, H 2.79, N 6.62.

5-Chloro-2-(5-phenyl-6-oxa-4-aza-spiro[2.4]hept-4-en-7-yl)-benzooxazole (69e): the crude product obtained from 5-phenyl-6-oxa-4-aza-spiro[2.4]hept-4-ene7-carboxylic acid (5-chloro-2-hydroxy-phenyl)-amide (68e, 103mg, $0.3 \mathrm{mmol}), \mathrm{Ph}_{3} \mathrm{P}$ (173 $\left.\mathrm{mg}, 0.66 \mathrm{mmol}\right)$ and DEAD (115 $\mathrm{mg}, 0.66 \mathrm{mmol}$ ) according to GP 10 was purified by column chromatography $\left(R_{\mathrm{f}}=0.35\right.$, Pentane $\left./ \mathrm{Et}_{2} \mathrm{O}=5: 1\right)$ to yield $83 \mathrm{mg}$ $(85 \%)$ of product $69 \mathrm{e}$ as white solid, m.p. $=129{ }^{\circ} \mathrm{C}$. $-\mathrm{IR}(\mathrm{KBr})$ : $v=3077 \mathrm{~cm}^{-1}, 2971,1653,1569,1427,1335,1293,1078,698$.

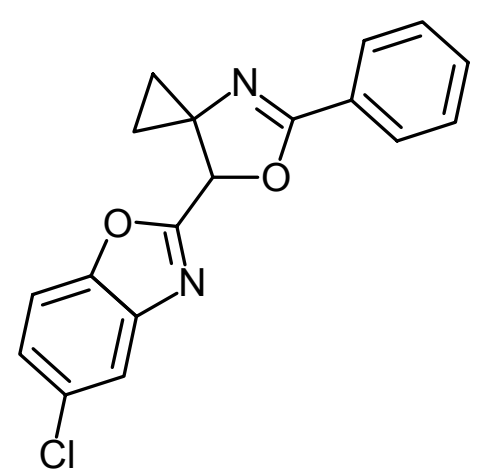
${ }^{1} \mathrm{H}-\mathrm{NMR}\left(300 \mathrm{MHz}, \mathrm{CDCl}_{3}\right): \delta=0.54-0.63(\mathrm{~m}, 1 \mathrm{H}, \mathrm{cPr}-\mathrm{H}), 0.98-1.06(\mathrm{~m}, 1 \mathrm{H}, \mathrm{cPr}-\mathrm{H}), 1.18-$ 
1.28 (m, 1 H, cPr-H), 1.36-1.45 (m, 1 H, cPr-H), 5.70 (s, 1 H, CH), 7.28-7.39 (m, 1 H, aryl-H), $7.40-7.57$ (m, 4 H, aryl-H), 7.69 (s, $1 \mathrm{H}$, aryl-H), 7.95-8.02 (m, $2 \mathrm{H}$, aryl-H). $-{ }^{13} \mathrm{C}-\mathrm{NMR}(75.5$ $\left.\mathrm{MHz}, \mathrm{CDCl}_{3}, \mathrm{APT}\right): \delta=11.1$ (-, cPr-C), 14.7 (-, cPr-C), 54.2 (-, cPr-C), 77.8 (+, CH-C), 111.9 (+, aryl-C), 120.4 (+, aryl-C), 126.1 (+, aryl-C), 126.8 (-, aryl-C), 128.1 (+, 2 C, aryl-C), $128.5(+$, 2 C, aryl-C), 130.2 (-, aryl-C), 131.6 (+, aryl-C), 141.5 (-, aryl-C), 149.5 (-, aryl-C), 163.2 (-,

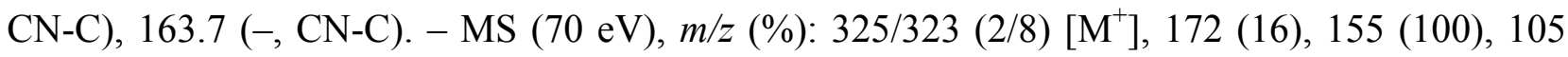
(18), 91 (82). $-\mathrm{C}_{18} \mathrm{H}_{13} \mathrm{ClN}_{2} \mathrm{O}_{2}$ (324.8): calcd. C 66.57, $\mathrm{H} 4.03, \mathrm{~N} 8.63$; found $\mathrm{C} 66.58, \mathrm{H} \mathrm{3.84,} \mathrm{N}$ 8.55 .

5-(4-Bromo-phenyl)-6-oxa-4-aza-spiro[2.4]hept-4-ene-7-carboxylic acid methyl-(3-trifluoromethyl-phenyl)-amide (70a): To a solution of 5-(4-bromophenyl)-6-oxa-4-aza-spiro[2.4]hept-4ene-7-carboxylic acid (3-trifluoromethyl-phenyl)-amides (68a) $(1.50 \mathrm{~g}, 3.42 \mathrm{~mol})$ in $50 \mathrm{~mL}$ acetone was added $\mathrm{Me}_{2} \mathrm{SO}_{4}(657 \mathrm{mg}$, $5.13 \mathrm{mmol})$ and $\mathrm{K}_{2} \mathrm{CO}_{3}(1.42 \mathrm{~g}, 10.3 \mathrm{mmol})$ and the resulting reaction mixture was refluxed for $16 \mathrm{~h}$. After cooling the crude product was filtered through $\mathrm{SiO}_{2}$ (eluent $\mathrm{Et}_{2} \mathrm{O}, 3 \times 4 \mathrm{~cm}, 20 \mathrm{~g}$ $\left.\mathrm{Si}_{2} \mathrm{O}\right)$ and the product purified by column chromatography $\left(R_{\mathrm{f}}=\right.$ 0.35 , Pentane $\left./ \mathrm{Et}_{2} \mathrm{O}=1: 1\right)$ to yield $1.43 \mathrm{~g}(92 \%)$ of $70 \mathrm{a}$ as a white solid, m.p. $=89-90{ }^{\circ} \mathrm{C} .-\mathrm{IR}(\mathrm{KBr}): v=3073 \mathrm{~cm}^{-1}, 2924,1655$,

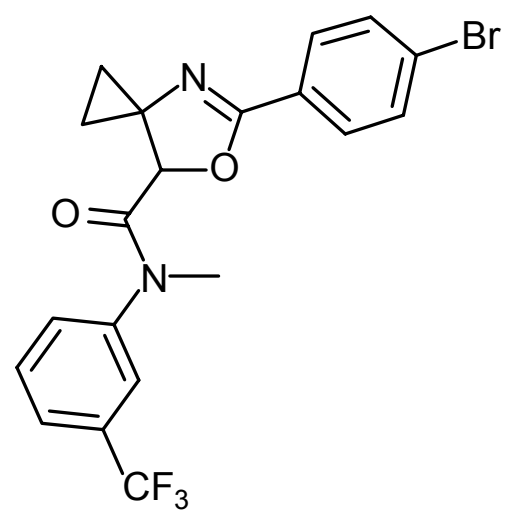
1507, 1333, 1180, 1128, 936, 833, 794. - ${ }^{1} \mathrm{H}-\mathrm{NMR}\left(200 \mathrm{MHz}, \mathrm{CDCl}_{3}\right): \delta=0.72-0.84(\mathrm{~m}, 1 \mathrm{H}$, cPr-H), 1.06-1.39 (m, 3 H, cPr-H), 3.29 (s, 3 H, $\mathrm{CH}_{3}$ ), 5.07 (s, 1 H, CH), 7.21-7.48 (m, 8 H, aryl$\mathrm{H}) .-{ }^{13} \mathrm{C}-\mathrm{NMR}\left(75.5 \mathrm{MHz}, \mathrm{CDCl}_{3}, \mathrm{APT}\right): \delta=12.0(-, \mathrm{cPr}-\mathrm{C}), 14.7(-, \mathrm{cPr}-\mathrm{C}), 39.0\left(+, \mathrm{CH}_{3}\right), 53.7$ (-, cPr-C), $80.9(+, \mathrm{CH}-\mathrm{C}), 119.8\left(\mathrm{q},{ }^{1} J_{\mathrm{C}-\mathrm{F}}=273 \mathrm{~Hz}, \mathrm{CF}_{3}\right), 124.3\left(+, \mathrm{q},{ }^{3} J_{\mathrm{C}-\mathrm{F}}=3.8 \mathrm{~Hz}\right.$, aryl-C), $124.5\left(+, \mathrm{q},{ }^{3} J_{\mathrm{C}-\mathrm{F}}=3.8 \mathrm{~Hz}\right.$, aryl-C), 124.9 (-, aryl-C), 129.1 (+, 2 C, aryl-C), 130.3 (+, aryl-C), $131.2\left(+, 2 \mathrm{C}\right.$, aryl-C), 132.0 (-, q, ${ }^{2} J_{\mathrm{C}-\mathrm{F}}=33.0 \mathrm{~Hz}$, aryl-C), 143.1 (-, aryl-C), 161.6 (-, CN-C), 167.8 (-, CO-C). - MS (70 eV), m/z (\%): 454/452 (7/9) $\left[\mathrm{M}^{+}\right]$, 280/278 (34/36), 202 (100), 174 (86).

5-(3-Bromo-phenyl)-6-oxa-4-aza-spiro[2.4]hept-4-ene-7-carboxylic acid methyl-(3-trifluoromethyl-phenyl)-amide (70b): The crude product obtained from $68 \mathrm{~b}$ (220 mg, $0.5 \mathrm{~mol}), \mathrm{Me}_{2} \mathrm{SO}_{4}$ (96 mg, $0.75 \mathrm{mmol})$ and $\mathrm{K}_{2} \mathrm{CO}_{3}(207 \mathrm{mg}, 1.5 \mathrm{mmol})$ in $10 \mathrm{~mL}$ of acetone according to the method described for 70a was purified by column chromatography $\left(R_{\mathrm{f}}=0.2\right.$, Pentane/Et $\left.2 \mathrm{O}=2: 1\right)$ to yield 
$203 \mathrm{~g}(89 \%)$ of $\mathbf{7 0 b}$ as white solid, m.p. $=110^{\circ} \mathrm{C} .-\mathrm{IR}(\mathrm{KBr}): v=2956 \mathrm{~cm}^{-1}, 1663,1340,1255$, 1124, 1061, 799. - ${ }^{1} \mathrm{H}-\mathrm{NMR}\left(300 \mathrm{MHz}, \mathrm{CDCl}_{3}\right): \delta=0.75-0.87$ (m, $\left.1 \mathrm{H}, \mathrm{cPr}-\mathrm{H}\right), 1.10-1.38$ (m, 3 $\mathrm{H}, \mathrm{cPr}-\mathrm{H}), 3.31$ (s, $\left.3 \mathrm{H}, \mathrm{CH}_{3}\right), 5.07$ (s, $\left.1 \mathrm{H}, \mathrm{CH}\right), 7.08-7.17$ (m, 1 $\mathrm{H}$, aryl-H), 7.21-7.54 (m, $7 \mathrm{H}$, aryl-H). $-{ }^{13} \mathrm{C}-\mathrm{NMR}(75.5 \mathrm{MHz}$, $\left.\mathrm{CDCl}_{3}, \mathrm{APT}\right): \delta=12.1$ (-, cPr-C), $14.8(-, \mathrm{cPr}-\mathrm{C}), 39.1\left(+, \mathrm{CH}_{3}\right)$, 53.7 (-, cPr-C), $80.9(+, \mathrm{CH}-\mathrm{C}), 122.0\left(-\right.$, aryl-C), $\left(+, \mathrm{q},{ }^{3} J_{\mathrm{C}-\mathrm{F}}=\right.$ $3.8 \mathrm{~Hz}$, aryl-C), $124.2\left(+, \mathrm{q},{ }^{3} J_{\mathrm{C}-\mathrm{F}}=3.8 \mathrm{~Hz}\right.$, aryl-C), $126.2(+$, arylC), $128.2(+$, aryl-C), $129.6(+$, aryl-C), 130.3 (+, aryl-C), 131.5 (+, aryl-C), 131.9 (-, aryl-C), 134.2 (-, aryl-C), 143.1 (-, aryl-C), 161.1 (-, CN-C), 167.8 (-, CO-C). - MS (70 eV), m/z (\%):

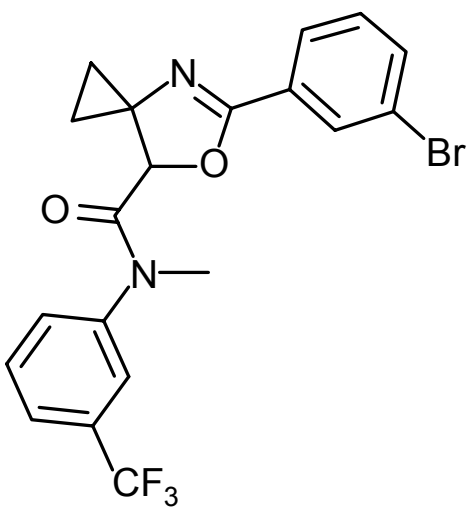
454/452 (21/19) $\left[\mathrm{M}^{+}\right], 280 / 278$ (43/42), 202 (100), 175 (80), 174 (60). - $\mathrm{C}_{20} \mathrm{H}_{16} \mathrm{BrF}_{3} \mathrm{~N}_{2} \mathrm{O}_{2}(453.3)$ : calcd. C 53.00, H 3.56, N 6.18; found C 53.28, H 3.41, N 6.27.

\section{General procedure for amination of aryl bromide in 70 (GP 11) (Buchwald-Hartwig}

Reaction): To an oven-dried Schlenk flask purged with nitrogen, was taken $\operatorname{Pd}_{2}(\mathrm{dba})_{3}(2.0 \mathrm{~mol} \%)$ and $\left( \pm\right.$-BINAP $(3.0 \mathrm{~mol} \%)$ in $5 \mathrm{~mL}$ of toluene. The mixture was heated to $80{ }^{\circ} \mathrm{C}$ with stirring for 5 min to dissolve the BINAP. After cooling, the aryl-bromide (70a or 70b), the corresponding amine and $\mathrm{NaO} t \mathrm{Bu}$ was added and the mixture was heated to $80^{\circ} \mathrm{C}$ for $16 \mathrm{~h}$. the mixture was cooled to room temperature, diluted with $\mathrm{Et}_{2} \mathrm{O}(25 \mathrm{~mL})$, filtered, and concentrated in vacuo. The crude product was then purified by column chromatography.

5-(4-Morpholin-4-yl-phenyl)-6-oxa-4-aza-spiro[2.4]hept-4-ene-7-carboxylic acid methyl-(3trifluoromethyl-phenyl)-amide (71a): The crude product obtained from 70a $(90.6 \mathrm{mg}, 0.20 \mathrm{mmol}), \mathrm{Pd}_{2}(\mathrm{dba})_{3}(3.65$ $\mathrm{mg}),( \pm)$-BINAP $(3.74 \mathrm{mg})$, morpholine $(26.0 \mathrm{mg}, 0.30$ $\mathrm{mmol})$ and $\mathrm{NaO} t \mathrm{Bu}(28.8 \mathrm{mg}, 0.30 \mathrm{mmol})$ according to GP 12 was purified by column chromatography $\left(R_{\mathrm{f}}=0.25\right.$, $\left.\mathrm{Et}_{2} \mathrm{O}\right)$ to yield $65 \mathrm{mg}(71 \%)$ of product which crystallized from $\mathrm{Et}_{2} \mathrm{O}$ at $0{ }^{\circ} \mathrm{C}$ to give the product 71a as a white solid, m.p. $=159-160{ }^{\circ} \mathrm{C} .-\mathrm{IR}(\mathrm{KBr}): v=3084 \mathrm{~cm}^{-1}, 2969$, 2864, 1655, 1607, 1521, 1335, 1228, 1119. - ${ }^{1} \mathrm{H}-\mathrm{NMR}$

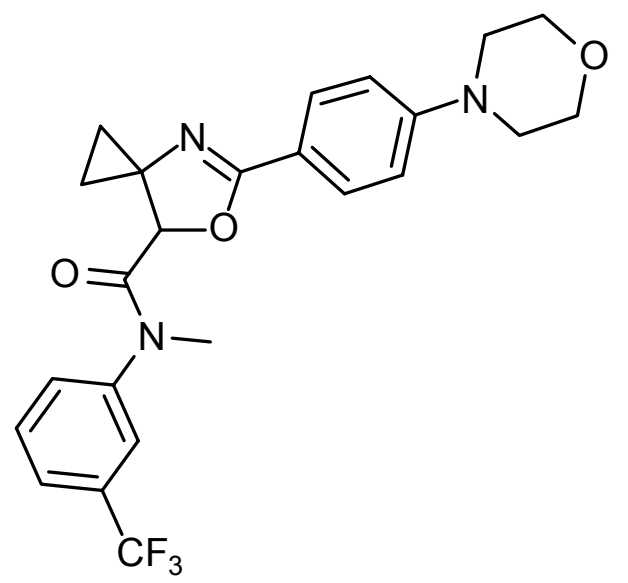
$\left(200 \mathrm{MHz}, \mathrm{CDCl}_{3}\right): \delta=0.62-0.83(\mathrm{~m}, 1 \mathrm{H}, \mathrm{cPr}-\mathrm{H}), 0.98-1.33(\mathrm{~m}, 3 \mathrm{H}, \mathrm{cPr}-\mathrm{H}), 3.02-3.20(\mathrm{~m}, 2$ 
H), $3.28\left(\mathrm{~s}, 3 \mathrm{H}, \mathrm{CH}_{3}\right), 3.66-3.91(\mathrm{~m}, 2 \mathrm{H}), 4.97(\mathrm{~s}, 1 \mathrm{H}, \mathrm{CH}), 6.62\left(\mathrm{~d},{ }^{3} J=8.2 \mathrm{~Hz}, 2 \mathrm{H}\right.$, aryl-H) 7.03-7.50 (m, $6 \mathrm{H}$, aryl-H). $-{ }^{13} \mathrm{C}-\mathrm{NMR}\left(75.5 \mathrm{MHz}, \mathrm{CDCl}_{3}, \mathrm{APT}\right): \delta=11.8(-, \mathrm{cPr}-\mathrm{C}), 14.4(-$, cPr-C), 39.0 (,$\left.+ \mathrm{CH}_{3}\right), 48.1$ (-, $\left.2 \mathrm{C}, \mathrm{CH}_{2}-\mathrm{C}\right), 53.4$ (-, cPr-C), 66.6 (-, $\left.2 \mathrm{C}, \mathrm{CH}_{2}-\mathrm{C}\right), 80.8$ (+, CH-C), $113.6\left(+, 2\right.$ C, aryl-C), $117.0\left(-\right.$, aryl-C), $123.3\left(-\right.$, q, $\left.{ }^{1} J_{\mathrm{C}-\mathrm{F}}=264 \mathrm{~Hz}, \mathrm{CF}_{3}\right), 124.2\left(+, \mathrm{q},{ }^{3} J_{\mathrm{C}-\mathrm{F}}=4.3\right.$ $\mathrm{Hz}$, aryl-C), $124.3\left(+, \mathrm{q},{ }^{3} J_{\mathrm{C}-\mathrm{F}}=3.2 \mathrm{~Hz}\right.$, aryl-C), 129.0 (+, 2 C, aryl-C), 130.1 (+, aryl-C), $131.8(-$, q, ${ }^{2} J_{\mathrm{C}-\mathrm{F}}=32.5 \mathrm{~Hz}$, aryl-C), 143.2 (-, aryl-C), 153.1 (-, aryl-C), 162.4 (-, CN-C), 168.2 (-, CO-C). - MS (70 eV), m/z (\%): 459 (22) $\left[\mathrm{M}^{+}\right], 285$ (16), 257 (20), 190 (100).

5-(4-Pyrrolidin-1-yl-phenyl)-6-oxa-4-aza-spiro[2.4]hept-4-ene-7-carboxylic acid methyl-(3trifluoromethyl-phenyl)-amide (71b): The crude product obtained from 70a (90.6 $\mathrm{mg}, 0.20 \mathrm{mmol})$, $\mathrm{Pd}_{2}(\mathrm{dba})_{3} \quad(3.65 \mathrm{mg}), \quad( \pm)$-BINAP $\quad(3.74 \quad \mathrm{mg})$, pyrrolidine $(28 \mathrm{mg}, 0.40 \mathrm{mmol})$ and $\mathrm{NaO} t \mathrm{Bu}(28.8$ $\mathrm{mg}, \quad 0.30 \mathrm{mmol}$ ) was purified by column chromatography $\left(R_{\mathrm{f}}=0.42, \mathrm{Et}_{2} \mathrm{O}\right)$ to yield $80 \mathrm{mg}$ $(90 \%)$ of $71 \mathbf{b}$ as white solid, m.p. $=152-153{ }^{\circ} \mathrm{C} .-\mathrm{IR}$ $(\mathrm{KBr}): v=3083 \mathrm{~cm}^{-1}, 2961,2852,1637,1607,1527$, 1387, 1334, 1130, 1061. - ${ }^{1} \mathrm{H}-\mathrm{NMR}$ (200 MHz,

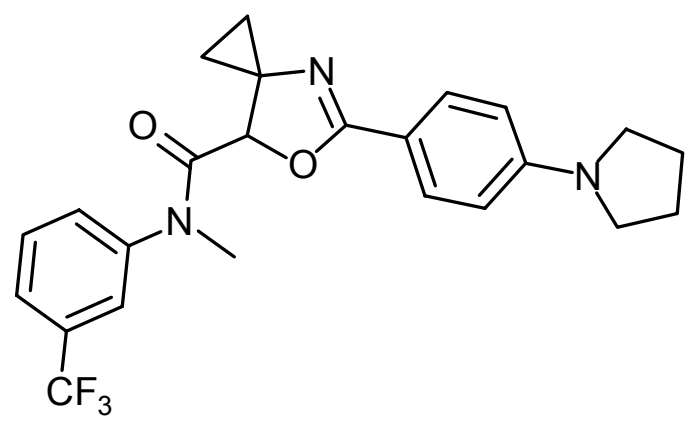
$\left.\mathrm{CDCl}_{3}\right): \delta=0.72-0.80(\mathrm{~m}, 1 \mathrm{H}, \mathrm{cPr}-\mathrm{H}), 1.03-1.14(\mathrm{~m}, 1 \mathrm{H}, \mathrm{cPr}-\mathrm{H}), 1.18-1.34(\mathrm{~m}, 2 \mathrm{H}, \mathrm{cPr}-\mathrm{H})$, 1.94-2.02 (m, $4 \mathrm{H}), 3.11-3.35(\mathrm{~m}, 7 \mathrm{H}), 4.98(\mathrm{~s}, 1 \mathrm{H}, \mathrm{CH}), 6.32\left(\mathrm{~d}, 2 \mathrm{H},{ }^{3} J=8.1 \mathrm{~Hz}\right.$, aryl-H), 7.18-7.38 (m, $5 \mathrm{H}$, aryl-H), 7.47 (s, $1 \mathrm{H}$, aryl-H). $-{ }^{13} \mathrm{C} \mathrm{NMR}\left(75.5 \mathrm{MHz}, \mathrm{CDCl}_{3}, \mathrm{APT}\right): \delta=11.7$ (-, cPr-C), 14.3 (-, cPr-C), 25.4 (-,2 C, $\left.\mathrm{CH}_{2}-\mathrm{C}\right), 39.0\left(+, \mathrm{CH}_{3}\right), 47.4$ (-, $\left.2 \mathrm{C}, \mathrm{CH}_{2}-\mathrm{C}\right), 53.3$ (-, cPrC), $80.8(+, \mathrm{CH}-\mathrm{C}), 110.5\left(+, 2 \mathrm{C}\right.$, aryl-C), $112.8\left(-\right.$, aryl-C), $123.2\left(-, \mathrm{q},{ }^{1} J_{\mathrm{C}-\mathrm{F}}=272 \mathrm{~Hz}, \mathrm{CF}_{3}\right)$, $123.9\left(+, \mathrm{q},{ }^{3} J_{\mathrm{C}-\mathrm{F}}=3.2 \mathrm{~Hz}\right.$, aryl-C), $124.3\left(+, \mathrm{q},{ }^{3} J_{\mathrm{C}-\mathrm{F}}=3.4 \mathrm{~Hz}\right.$, aryl-C), $129.2(+, 2 \mathrm{C}$, aryl-C), 130.1 (+, aryl-C), 131.8 (-, q, ${ }^{2} J_{\mathrm{C}-\mathrm{F}}=32.1 \mathrm{~Hz}$, aryl-C), 143.2 (-, aryl-C), 149.7 (-, aryl-C), 163.1

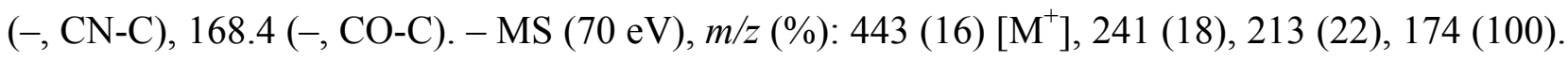
$-\mathrm{C}_{24} \mathrm{H}_{24} \mathrm{~F}_{3} \mathrm{~N}_{3} \mathrm{O}_{2}$ (443.5): calcd. C 65.00, H 5.45, N 9.48; found C 64.69, H 5.16, N 9.22. 
5-[4-(4-Methyl-piperazin-1-yl)-phenyl]-6-oxa-4-aza-spiro[2.4]hept-4-ene-7-carboxylic

acid methyl-(3-trifluoromethyl-phenyl)-amide (71c): The crude product obtained from 70a (90.6 mg, $0.20 \mathrm{mmol}), \mathrm{Pd}_{2}(\mathrm{dba})_{3}(3.65 \mathrm{mg}),( \pm)-\mathrm{BINAP}$ $(3.74 \mathrm{mg}), \quad N$-methylpiperazine $(40 \mathrm{mg}, 0.40$ $\mathrm{mmol})$ and $\mathrm{NaO} t \mathrm{Bu}(28.8 \mathrm{mg}, 0.30 \mathrm{mmol})$ was purified by column chromatography $\left(R_{\mathrm{f}}=0.20\right.$, $\left.\mathrm{CH}_{2} \mathrm{Cl}_{2} / \mathrm{MeOH}=10: 1\right)$ to yield $55 \mathrm{mg}(59 \%)$ of 71c as white solid, m.p. $=147-148{ }^{\circ} \mathrm{C}$. - IR (KBr): $v=2948 \mathrm{~cm}^{-1}, 2843,2794,1653,1644$,

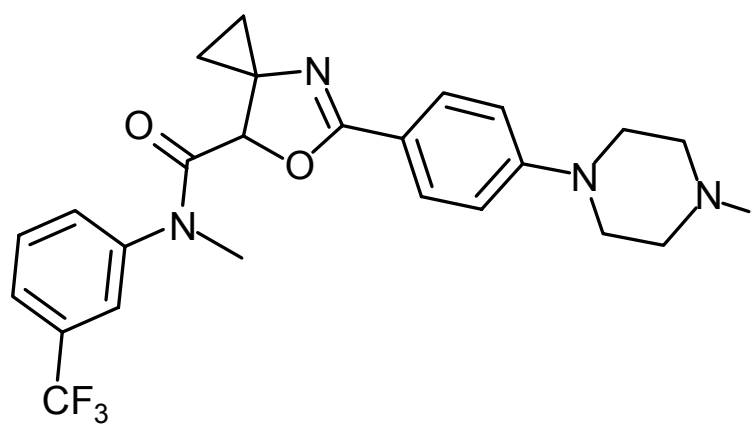
$1611,1520,1330,1233,1133,1068 .-{ }^{1} \mathrm{H}-\mathrm{NMR}\left(300 \mathrm{MHz}, \mathrm{CDCl}_{3}\right): \delta=0.69-0.81(\mathrm{~m}, 1 \mathrm{H}, \mathrm{cPr}-$ H), 1.05-1.14 (m, $1 \mathrm{H}, \mathrm{cPr}-\mathrm{H}), 1.18-1.35$ (m, $2 \mathrm{H}, \mathrm{cPr}-\mathrm{H}), 2.35$ (s, $\left.3 \mathrm{H}, \mathrm{CH}_{3}\right), 2.51-3.58(\mathrm{~m}, 4 \mathrm{H}$, $\mathrm{NCH}_{2}$ ), 3.20-3.28 (m, $4 \mathrm{H}, \mathrm{NCH}_{2}$ ), 3.30 (s, $\left.3 \mathrm{H}, \mathrm{CH}_{3}\right), 4.98$ (s, $\left.1 \mathrm{H}, \mathrm{CH}\right), 6.70$ (d, ${ }^{3} J=8.7 \mathrm{~Hz}, 2$ $\mathrm{H}$, aryl-H), 7.15-7.40 (m, $5 \mathrm{H}$, aryl-H), $7.44\left(\mathrm{~s}, 1 \mathrm{H}\right.$, aryl-H). ${ }^{13} \mathrm{C}-\mathrm{NMR}\left(75.5 \mathrm{MHz}, \mathrm{CDCl}_{3}\right.$, APT): $\delta=11.8$ (-, cPr-C), 14.4 (-, cPr-C), $39.0\left(+, \mathrm{CH}_{3}\right), 46.1\left(+, \mathrm{CH}_{3}\right), 47.7\left(-, 2 \mathrm{C}, \mathrm{CH}_{2}-\mathrm{C}\right)$, 53.4 (-, cPr-C), 54.7 (-, $\left.2 \mathrm{C}, \mathrm{CH}_{2}-\mathrm{C}\right), 80.8$ (+, CH-C), 113.9 (+, $2 \mathrm{C}$, aryl-C), 116.5 (-, aryl-C), $123.2\left(-, \mathrm{q},{ }^{1} J_{\mathrm{C}-\mathrm{F}}=273 \mathrm{~Hz}, \mathrm{CF}_{3}\right), 124.1\left(+, \mathrm{q},{ }^{3} J_{\mathrm{C}-\mathrm{F}}=3.4 \mathrm{~Hz}\right.$, aryl-C), $124.3\left(+, \mathrm{q},{ }^{3} J_{\mathrm{C}-\mathrm{F}}=3.4 \mathrm{~Hz}\right.$, aryl-C), 128.9 (+, 2 C, aryl-C), 130.1 (+, aryl-C), $131.8\left(-\right.$, q, ${ }^{2} J_{\mathrm{C}-\mathrm{F}}=32.1 \mathrm{~Hz}$, aryl-C), $143.1(-$, aryl-C), 153.0 (-, aryl-C), 162.4 (-, CN-C), 168.3 (-, CO-C). - MS (70 eV), m/z (\%): 472 (22) $\left[\mathrm{M}^{+}\right], 270$ (19), 242 (16), 203 (100).

5-[4-(4-Benzyl-piperazin-1-yl)-phenyl]-6-oxa-4-aza-spiro[2.4]hept-4-ene-7-carboxylic acid methyl-(3-trifluoromethyl-phenyl)-amide (71d): The crude product obtained from 70a (90.6 $\mathrm{mg}$, $0.20 \mathrm{mmol}), \mathrm{Pd}_{2}(\mathrm{dba})_{3}(3.65 \mathrm{mg}),( \pm)$-BINAP $(3.74 \mathrm{mg}), \mathrm{N}$-benzylpiperazine $(53 \mathrm{mg}, 0.30$ $\mathrm{mmol})$ and $\mathrm{NaOt} \mathrm{Bu}(28.8 \mathrm{mg}, 0.30 \mathrm{mmol})$ was purified by column chromatography $\left(R_{\mathrm{f}}=0.30\right.$, $\mathrm{DCM} / \mathrm{MeOH}=10: 1)$ to yield $68 \mathrm{mg}(62 \%)$ of 71d as white solid, m.p. $=144-146^{\circ} \mathrm{C} .-\mathrm{IR}$ (KBr): $v=2945 \mathrm{~cm}^{-1}, 2830,2814,1653,1611$,

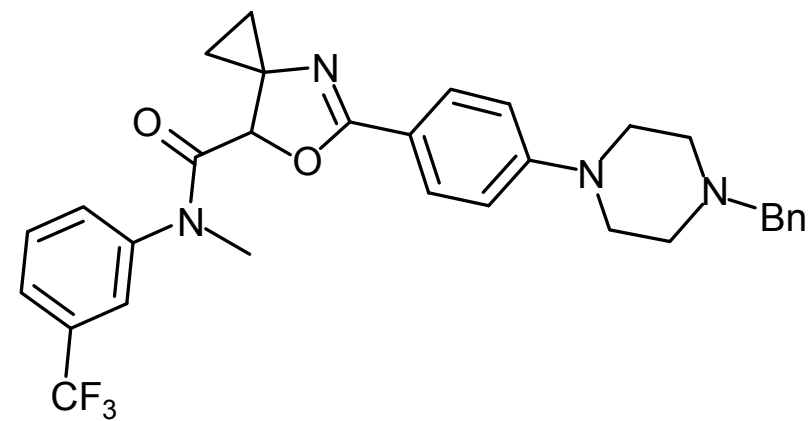
1520, 1333, 1134, 700. $-{ }^{1} \mathrm{H}-\mathrm{NMR}\left(300 \mathrm{MHz}, \mathrm{CDCl}_{3}\right): \delta=0.71-0.82(\mathrm{~m}, 1 \mathrm{H}, \mathrm{cPr}-\mathrm{H}), 1.04-1.14$ (m, $1 \mathrm{H}, \mathrm{cPr}-\mathrm{H}), 1.18-1.37$ (m, $2 \mathrm{H}, \mathrm{cPr}-\mathrm{H}), 2.54-2.62$ (m, $\left.4 \mathrm{H}, \mathrm{NCH}_{2}\right), 3.18-3.27$ (m, $4 \mathrm{H}$, $\mathrm{NCH}_{2}$ ), 3.29 (s, $3 \mathrm{H}, \mathrm{CH}_{3}$ ), 3.57 (s, $\left.2 \mathrm{H}, \mathrm{Bn}-\mathrm{H}\right), 5.00$ (s, $\left.1 \mathrm{H}, \mathrm{CH}\right), 6.67\left(\mathrm{~d},{ }^{3} J=8.7 \mathrm{~Hz}, 2 \mathrm{H}\right.$, aryl- 
H), 7.14-7.39 (m, $10 \mathrm{H}$, aryl-H), $7.41\left(\mathrm{~s}, 1 \mathrm{H}\right.$, aryl-H). $-{ }^{13} \mathrm{C}-\mathrm{NMR}\left(75.5 \mathrm{MHz}, \mathrm{CDCl}_{3}, \mathrm{APT}\right): \delta=$ 11.8 (-, cPr-C), 14.4 (-, cPr-C), $39.0\left(+, \mathrm{CH}_{3}\right), 47.8$ (-, $\left.2 \mathrm{C}, \mathrm{NCH}_{2}-\mathrm{C}\right), 52.7$ (-, $\left.2 \mathrm{C}, \mathrm{NCH}_{2}-\mathrm{C}\right)$, 53.4 (-, cPr-C), 62.9 (-, Bn-C), 80.8 (+, CH-C), 113.8 (+, 2 C, aryl-C), 116.4 (-, aryl-C), 123.2 (-, $\left.\mathrm{q},{ }^{1} J_{\mathrm{C}-\mathrm{F}}=273 \mathrm{~Hz}, \mathrm{CF}_{3}\right), 124.1\left(+, \mathrm{q},{ }^{3} J_{\mathrm{C}-\mathrm{F}}=3.4 \mathrm{~Hz}\right.$, aryl-C), $124.2\left(+, \mathrm{q},{ }^{3} J_{\mathrm{C}-\mathrm{F}}=3.4 \mathrm{~Hz}\right.$, aryl-C), $127.2(+$, aryl-C), 128.3 (+, 2 C, aryl-C), 128.9 (+, aryl-C), 129.2 (+, 2 C, aryl-C), 130.1 (+, arylC), 130.2 (+, aryl-C), 131.8 (-, q, ${ }^{2} J_{\mathrm{C}-\mathrm{F}}=32.5 \mathrm{~Hz}$, aryl-C), 137.6 (-, aryl-C), 143.2 (-, aryl-C),

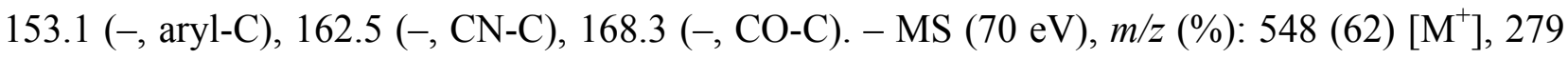
(58), 185 (18), $91(100)\left[\mathrm{C}_{7} \mathrm{H}_{7}^{+}\right] .-\mathrm{C}_{31} \mathrm{H}_{31} \mathrm{~F}_{3} \mathrm{~N}_{4} \mathrm{O}_{2}$ (541.6): calcd. C 67.87, H 5.71, N 10.21; found C 67.66, H 5.85, N 10.07.

5-[4-(6-Dibenzylamino-3-aza-bicyclo[3.1.0]hex-3-yl)-phenyl]-6-oxa-4-aza-spiro[2.4]hept-4-ene7-carboxylic acid methyl-(3-trifluoromethyl-phenyl)amide (71e): The crude product obtained from 70a $(90.6$ $\mathrm{mg}, 0.20 \mathrm{mmol}), \mathrm{Pd}_{2}(\mathrm{dba})_{3}(3.65 \mathrm{mg}),( \pm)$-BINAP $(3.74$ $\mathrm{mg}$, exo-6-( $N, N$-dibenzylamino)-3azabicyclo[3,1,0]hexane $(83 \mathrm{mg}, 0.30 \mathrm{mmol})$ and $\mathrm{NaO} t \mathrm{Bu}$ (28.8 $\mathrm{mg}, \quad 0.30 \mathrm{mmol}$ ) was purified in column chromatography $\left(R_{\mathrm{f}}=0.45, \mathrm{Et}_{2} \mathrm{O}\right)$ to yield $87 \mathrm{mg}(67 \%)$ of 71e as pale yellow solid, m.p. $=96{ }^{\circ} \mathrm{C}$. $-\mathrm{IR}(\mathrm{KBr})$ : $v=3061 \mathrm{~cm}^{-1}, 2841,1684,1611,1524,1388,1332,1186$, 1130, 699. $-{ }^{1} \mathrm{H}-\mathrm{NMR}\left(300 \mathrm{MHz}, \mathrm{CDCl}_{3}\right): \delta=0.70-0.80$ (m, $1 \mathrm{H}, \mathrm{cPr}-\mathrm{H}), 1.02-1.14$ (m, $1 \mathrm{H}, \mathrm{cPr}-\mathrm{H}), 1.16-1.34$ (m,

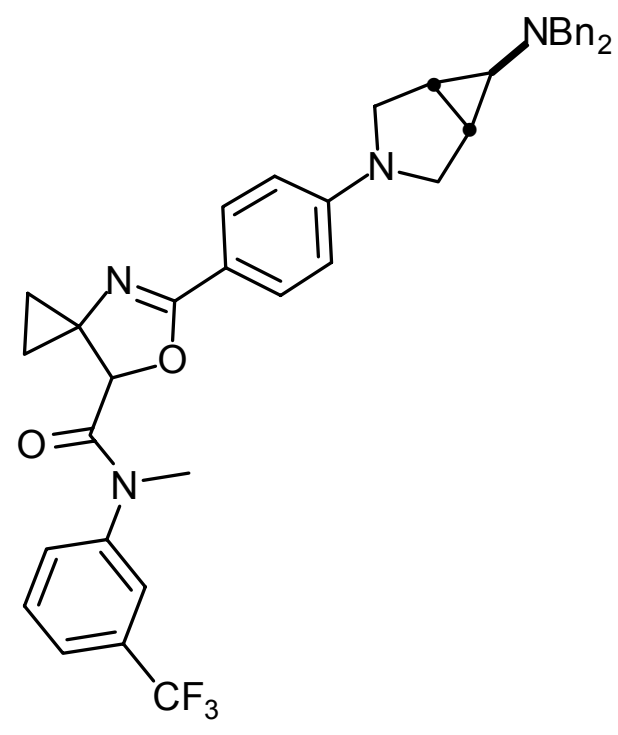
$2 \mathrm{H}, \mathrm{cPr}-\mathrm{H}), 1.44-1.58(\mathrm{~m}, 2 \mathrm{H}), 1.61-1.64(\mathrm{~m}, 1 \mathrm{H}), 3.12-3.20\left(\mathrm{~m}, 2 \mathrm{H}, \mathrm{NCH}_{2}-\mathrm{H}\right), 3.24-3.35(\mathrm{~m}$, $5 \mathrm{H}, \mathrm{CH}_{3}$ and $\left.\mathrm{NCH}_{2}-\mathrm{H}\right), 3.68(\mathrm{~s}, 4 \mathrm{H}, \mathrm{Bn}-\mathrm{H}), 5.00(\mathrm{~s}, 1 \mathrm{H}, \mathrm{CH}), 6.24\left(\mathrm{~d}, 2 \mathrm{H},{ }^{3} J=8.2 \mathrm{~Hz}\right.$, aryl-H), 7.18-7.38 (m, $15 \mathrm{H}$, aryl-H), 7.45 (s, $1 \mathrm{H}$, aryl-H). $-{ }^{13} \mathrm{C}-\mathrm{NMR}$ (75.5 MHz, CDCl 3 , APT): $\delta=11.8$ (-, cPr-C), 14.3 (-, cPr-C), 25.9 (+, CH-C), 26.0 (+, CH-C), 39.0 (+, $\left.\mathrm{CH}_{3}\right), 48.6$ (+, CH-C), 49.7 (-, $\left.2 \mathrm{C}, \mathrm{CH}_{2}-\mathrm{C}\right), 53.4$ (-, cPr-C), 59.0 (-, $\left.2 \mathrm{C}, \mathrm{Bn}-\mathrm{C}\right), 80.7$ (+, CH-C), 110.9 (+, $2 \mathrm{C}$, aryl-C), $113.7\left(-\right.$, aryl-C), $123.1\left(-, \mathrm{q},{ }^{1} J_{\mathrm{C}-\mathrm{F}}=273 \mathrm{~Hz}, \mathrm{CF}_{3}\right), 123.9\left(+, \mathrm{q},{ }^{3} J_{\mathrm{C}-\mathrm{F}}=3.4 \mathrm{~Hz}\right.$, aryl-C), $124.3(+, \mathrm{q}$, ${ }^{3} J_{\mathrm{C}-\mathrm{F}}=3.4 \mathrm{~Hz}$, aryl-C), $127.1(+$, aryl-C), $128.1(+, 2 \mathrm{C}$, aryl-C), $129.0(+$, aryl-C), $129.4(+$, arylC), 130.1 (+, aryl-C), 130.2 (+, aryl-C), 131.8 (-, q, ${ }^{2} J_{\mathrm{C}-\mathrm{F}}=32.5 \mathrm{~Hz}$, aryl-C), 138.6 (-, aryl-C), 143.2 (-, aryl-C), 149.6 (-, aryl-C), 162.9 (-, CN-C), 168.4 (-, CO-C). - MS (70 eV), m/z (\%): 
$650(19)\left[\mathrm{M}^{+}\right], 559(29)\left[\mathrm{M}^{+}-\mathrm{C}_{7} \mathrm{H}_{7}\right], 249$ (34), 158 (57), 91 (100) $\left[\mathrm{C}_{7} \mathrm{H}_{7}^{+}\right] .-\mathrm{C}_{39} \mathrm{H}_{37} \mathrm{~F}_{3} \mathrm{~N}_{4} \mathrm{O}_{2}$ (650.7): calcd. C 71.98, H 5.73, N 8.61; found C 71.75, H 5.57, N 8.79.

5-(3-Morpholino-phenyl)-6-oxa-4-aza-spiro[2.4]hept-4-ene-7-carboxylic acid methyl-(3trifluromethyl-phenyl)-amide (71f): The crude product obtained from $70 \mathrm{~b}$ (90.6 mg, $0.20 \mathrm{mmol}$ ), $\mathrm{Pd}_{2}(\mathrm{dba})_{3}(9.1 \mathrm{mg}, 5 \mathrm{~mol} \%),( \pm)$-BINAP (9.3 mg, $\left.7.5 \mathrm{~mol} \%\right)$, morpholine (26 mg, $0.30 \mathrm{mmol})$ and $\mathrm{NaO} t \mathrm{Bu}(28.8 \mathrm{mg}, 0.30$ mmol) was purified by column chromatography $\left(R_{\mathrm{f}}=0.25\right.$, $\left.\mathrm{Et}_{2} \mathrm{O}\right)$ to yield $75 \mathrm{mg}(82 \%)$ of $\mathbf{7 1 f}$ which crystallized from Pentane $/ \mathrm{Et}_{2} \mathrm{O}$ at $0{ }^{\circ} \mathrm{C}$ to get white solid, m.p. $=122{ }^{\circ} \mathrm{C}$. $-\mathrm{IR}$ $(\mathrm{KBr}): v=2959 \mathrm{~cm}^{-1}, 2924,2853,1684,1653,1599,1331$, 1265, 1220. $-{ }^{1} \mathrm{H}-\mathrm{NMR}\left(300 \mathrm{MHz}, \mathrm{CDCl}_{3}\right): \delta=0.72-0.80(\mathrm{~m}$, $1 \mathrm{H}, \mathrm{cPr}-\mathrm{H}), 1.04-1.14$ (m, $1 \mathrm{H}, \mathrm{cPr}-\mathrm{H}), 1.21-1.38$ (m, $2 \mathrm{H}$, cPr-H), 3.08 (d, $\left.{ }^{3} J=4.8 \mathrm{~Hz}, 4 \mathrm{H}\right), 3.30$ (s, $\left.3 \mathrm{H}, \mathrm{CH}_{3}\right), 3.81$ (d,

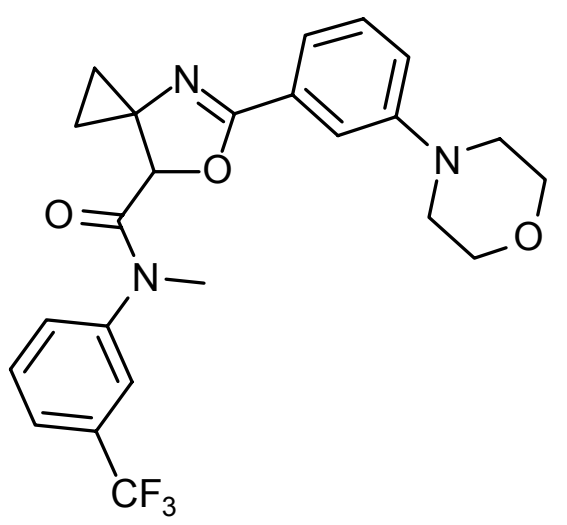
$\left.{ }^{3} J=4.8 \mathrm{~Hz}, 4 \mathrm{H}\right), 5.00$ (s, $\left.1 \mathrm{H}, \mathrm{CH}\right), 6.88-7.00$ (m, $2 \mathrm{H}$, aryl-H), 7.03-7.18 (m, $2 \mathrm{H}$, aryl-H), 7.20$7.40\left(\mathrm{~m}, 3 \mathrm{H}\right.$, aryl-H), 7.42 (s, $1 \mathrm{H}$, aryl-H). $-{ }^{13} \mathrm{C}-\mathrm{NMR}\left(75.5 \mathrm{MHz}, \mathrm{CDCl}_{3}, \mathrm{APT}\right): \delta=12.0(-$, cPr-C), 14.6 (-, cPr-C), 38.9 (+, $\mathrm{CH}_{3}$ ), 49.1 (-, $\left.2 \mathrm{C}, \mathrm{CH}_{2}-\mathrm{C}\right), 53.5$ (-, cPr-C), 66.8 (-, $2 \mathrm{C}, \mathrm{CH}_{2}-\mathrm{C}$ ), $80.6(+, \mathrm{CH}-\mathrm{C}), 114.5\left(+\right.$, aryl-C), $118.6\left(+\right.$, aryl-C), $123.3\left(-, \mathrm{q},{ }^{1} J_{\mathrm{C}-\mathrm{F}}=274 \mathrm{~Hz}, \mathrm{CF}_{3}\right), 123.9(+, \mathrm{q}$, ${ }^{3} J_{\mathrm{C}-\mathrm{F}}=3.4 \mathrm{~Hz}$, aryl-C), $124.4\left(+, \mathrm{q},{ }^{3} J_{\mathrm{C}-\mathrm{F}}=3.4 \mathrm{~Hz}\right.$, aryl-C), 127.2 (-, aryl-C), 128.7 (+, aryl-C), $130.2\left(+\right.$, aryl-C), $131.0\left(-\right.$, q, ${ }^{2} J_{\mathrm{C}-\mathrm{F}}=32.2 \mathrm{~Hz}$, aryl-C), 143.1 (-, aryl-C), 150.9 (-, aryl-C), 162.7 (-, CN-C), 168.1 (-, CO-C). - MS (DCI), m/z (\%): $919\left(2 \mathrm{M}+\mathrm{H}^{+}\right), 460(100)\left[\mathrm{M}+\mathrm{H}^{+}\right]$.

5-(3-Pyrrolidin-1-yl-phenyl)-6-oxa-4-aza-spiro[2.4] hept-4-ene-7-carboxylic acid methyl-(3trifluoro-methylphenyl)-amide (71g): The crude product obtained from 70b $(90.6 \mathrm{mg}, 0.20 \mathrm{mmol}), \mathrm{Pd}_{2}(\mathrm{dba})_{3}(9.1 \mathrm{mg}, 5$ mol\%), ( \pm )-BINAP (9.3 mg, $7.5 \mathrm{~mol} \%$ ), pyrrolidin (26 mg, $0.30 \mathrm{mmol})$ and $\mathrm{NaO} t \mathrm{Bu}(28.8 \mathrm{mg}, 0.30 \mathrm{mmol})$ was purified by column chromatography $\left(R_{\mathrm{f}}=0.40, \mathrm{Et}_{2} \mathrm{O}\right)$ to yield $65 \mathrm{mg}$ (73\%) of $71 \mathrm{~g}$ which crystallized from Pentane/Et $2 \mathrm{O}$ at $0{ }^{\circ} \mathrm{C}$ to give white solid, m.p. $=122{ }^{\circ} \mathrm{C} .-{ }^{1} \mathrm{H}-\mathrm{NMR}(300 \mathrm{MHz}$, $\left.\mathrm{CDCl}_{3}\right): \delta=0.71-0.80(\mathrm{~m}, 1 \mathrm{H}, \mathrm{cPr}-\mathrm{H}), 1.06-1.15(\mathrm{~m}, 1 \mathrm{H}$,

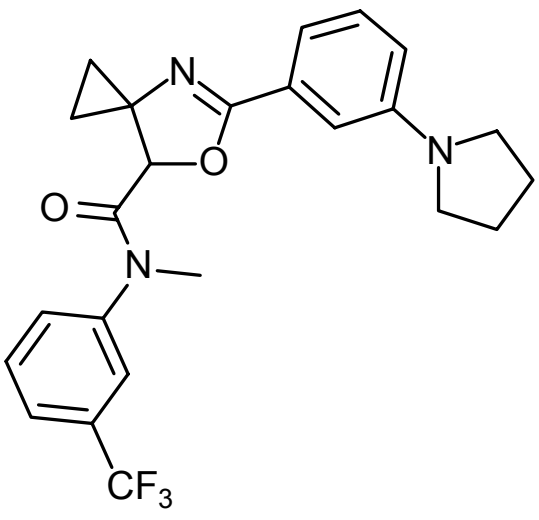
cPr-H), 1.21-1.39 (m, 2 H, cPr-H), 1.92-2.02 (m, 4 H), 3.18-3.30 (m, 4 H), 3.33 (s, 3 H, $\mathrm{CH}_{3}$ ), 
$5.03(\mathrm{~s}, 1 \mathrm{H}, \mathrm{CH}), 6.54-6.61(\mathrm{~m}, 1 \mathrm{H}$, aryl-H), 6.69-6.82 (m, $2 \mathrm{H}$, aryl-H), 7.03-7.16 (m, $1 \mathrm{H}$, aryl-H), 7.22-7.40 (m, $3 \mathrm{H}$, aryl-H), $7.46\left(\mathrm{~s}, 1 \mathrm{H}\right.$, aryl-H). $-{ }^{13} \mathrm{C}-\mathrm{NMR}\left(75.5 \mathrm{MHz}, \mathrm{CDCl}_{3}, \mathrm{APT}\right): \delta$ $=12.0$ (-, cPr-C), 14.5 (-, cPr-C), 25.4 (-, $\left.2 \mathrm{C}, \mathrm{CH}_{2}-\mathrm{C}\right), 38.9\left(+, \mathrm{CH}_{3}\right), 47.6\left(-, 2 \mathrm{C}_{2} \mathrm{CH}_{2}-\mathrm{C}\right), 53.5$ $(-, \mathrm{cPr}-\mathrm{C}), 80.6(+, \mathrm{CH}-\mathrm{C}), 110.5$ (+, aryl-C), $114.7\left(+\right.$, aryl-C), $123.3\left(-, \mathrm{q},{ }^{1} J_{\mathrm{C}-\mathrm{F}}=273 \mathrm{~Hz}, \mathrm{CF}_{3}\right)$, $123.9\left(+, \mathrm{q},{ }^{3} J_{\mathrm{C}-\mathrm{F}}=3.5 \mathrm{~Hz}\right.$, aryl-C), $124.4\left(+, \mathrm{q},{ }^{3} J_{\mathrm{C}-\mathrm{F}}=3.5 \mathrm{~Hz}\right.$, aryl-C), $127.0(-$, aryl-C), $128.5(+$, aryl-C), 130.2 (+, aryl-C), 131.9 (-, q, ${ }^{2} J_{\mathrm{C}-\mathrm{F}}=32.5 \mathrm{~Hz}$, aryl-C), 143.1 (-, aryl-C), 147.5 (-, aryl-C), 163.3 (-, CN-C), 168.2 (-, CO-C). - MS (70 eV), m/z (\%): 443 (23) [M+'], 241 (62), 174 (100), 84 (68).

5-[3-(4-Methyl-piperazin-1-yl)-phenyl]-6-oxa-4-aza-spiro[2.4]hept-4-ene-7-carboxylic

acid methyl-(3-trifluoromethyl-phenyl)-amide (71h): The crude product obtained from $\mathbf{7 0 b}(90.6 \mathrm{mg}, 0.20 \mathrm{mmol}), \mathrm{Pd}_{2}(\mathrm{dba})_{3}$ (9.1 mg, $5 \mathrm{~mol} \%)$, ( $( \pm)$-BINAP (9.3 mg, $7.5 \mathrm{~mol} \%)$, piperonyl (100 mg, $1.00 \mathrm{mmol})$ and $\mathrm{NaO} t \mathrm{Bu}(28.8 \mathrm{mg}, 0.30$ mmol) was purified by column chromatography $\left(R_{\mathrm{f}}=0.25\right.$, $\mathrm{DCM} / \mathrm{MeOH}=10: 1)$ to yield $12 \mathrm{mg}(13 \%)$ of $71 \mathbf{h} .-\mathrm{IR}$ $(\mathrm{KBr}): v=2925 \mathrm{~cm}^{-1}, 1684,1653,1600,1457,1331,1131$.

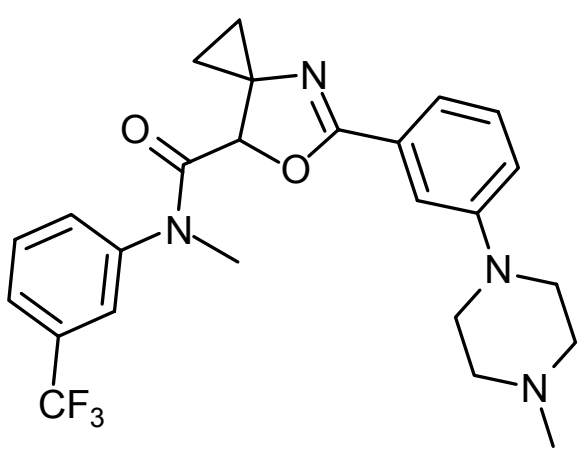
$-{ }^{1} \mathrm{H}-\mathrm{NMR}\left(200 \mathrm{MHz}, \mathrm{CDCl}_{3}\right): \delta=0.63-0.80(\mathrm{~m}, 1 \mathrm{H}, \mathrm{cPr}-$ H), 0.99-1.12 (m, 1 H, cPr-H), 1.18-1.31 (m, 2 H, cPr-H), 2.29 (s, $\left.3 \mathrm{H}, \mathrm{CH}_{3}\right), 2.49$ (t, ${ }^{3} \mathrm{~J}=4.9 \mathrm{~Hz}$, $4 \mathrm{H}), 3.14\left(\mathrm{t},{ }^{3} \mathrm{~J}=4.9 \mathrm{~Hz}, 4 \mathrm{H}\right), 3.24$ (s, $\left.3 \mathrm{H}, \mathrm{CH}_{3}\right), 4.98$ (s, $\left.1 \mathrm{H}, \mathrm{CH}\right), 6.83-6.96$ (m, $2 \mathrm{H}$, aryl-H), 7.01-7.17 (m, 2 H, aryl-H), 7.18-7.37 (m, 3 H, aryl-H), 7.39 (s, $1 \mathrm{H}$, aryl-H). $-{ }^{13} \mathrm{C}-\mathrm{NMR}(75.5$ $\left.\mathrm{MHz}, \mathrm{CDCl}_{3}, \mathrm{APT}\right): \delta=12.0(-, \mathrm{cPr}-\mathrm{C}), 14.0(-, \mathrm{cPr}-\mathrm{C}), 38.9\left(+, \mathrm{CH}_{3}\right), 46.1\left(+, \mathrm{CH}_{3}\right), 47.8(-, 2$ C, $\left.\mathrm{CH}_{2}-\mathrm{C}\right), 53.6$ (-, cPr-C), 55.0 (-, $\left.2 \mathrm{C}, \mathrm{CH}_{2}-\mathrm{C}\right), 80.7$ (+, CH-C), 114.8 (+, aryl-C), $118.9\left(^{+}\right.$, aryl-C), 119.0 (+, aryl-C), $123.0\left(-\right.$, q, $\left.{ }^{1} J_{\mathrm{C}-\mathrm{F}}=273 \mathrm{~Hz}, \mathrm{CF}_{3}\right), 124.0\left(+, \mathrm{q},{ }^{3} J_{\mathrm{C}-\mathrm{F}}=3.5 \mathrm{~Hz}\right.$, aryl-C), $124.4\left(+, \mathrm{q},{ }^{3} J_{\mathrm{C}-\mathrm{F}}=3.5 \mathrm{~Hz}\right.$, aryl-C), 127.1 (-, aryl-C), $128.6(+$, aryl-C), $130.2(+$, aryl-C), 130.3 (+, aryl-C), $132.0\left(-, \mathrm{q},{ }^{2} J_{\mathrm{C}-\mathrm{F}}=33.2 \mathrm{~Hz}\right.$, aryl-C), 143.1 (-, aryl-C), 150.8 (-, aryl-C), 162.8 (-, CN-

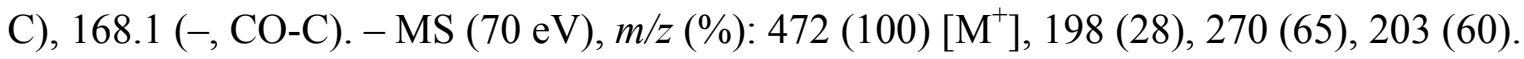


5-[3-(4-Benzyl-piperazin-1-yl)-phenyl]-6-oxa-4-aza-spiro[2.4]hept-4-ene-7-carboxylic

acid methyl-(3-trifluoromethyl-phenyl)-amide (71i): The crude product obtained from 70b (90.6 mg, $0.20 \mathrm{mmol}), \mathrm{Pd}_{2}(\mathrm{dba})_{3}(9.1 \mathrm{mg}, 5 \mathrm{~mol} \%),( \pm)$-BINAP (9.3 mg, 7.5 mol\%), N-benzylpiperazine (70.4 mg, $0.40 \mathrm{mmol}$ ) and $\mathrm{NaO} t \mathrm{Bu}(28.8 \mathrm{mg}, 0.30 \mathrm{mmol})$ was purified by column chromatography $\left(R_{\mathrm{f}}=0.25, \mathrm{DCM} / \mathrm{MeOH}=10: 1\right)$ to yield $13 \mathrm{mg}(12 \%)$ of $\mathbf{7 1 i}$ as a viscous oil. - IR ( $\mathrm{KBr})$ : $v=2962 \mathrm{~cm}^{-1}, 2925,2855,1675,1653,1457,1331,1167$, 1069, 701. - ${ }^{1} \mathrm{H}-\mathrm{NMR}\left(300 \mathrm{MHz}, \mathrm{CDCl}_{3}\right): \delta=0.70-0.85$ (m, $1 \mathrm{H}, \mathrm{cPr}-\mathrm{H}), 1.05-1.15$ (m, $1 \mathrm{H}, \mathrm{cPr}-\mathrm{H}), 1.20-1.35$ (m, $2 \mathrm{H}, \mathrm{cPr}-\mathrm{H}$ ), 2.63-2.78 (m, $4 \mathrm{H}, \mathrm{NCH}_{2}$ ), 3.18-3.24 (m, 4

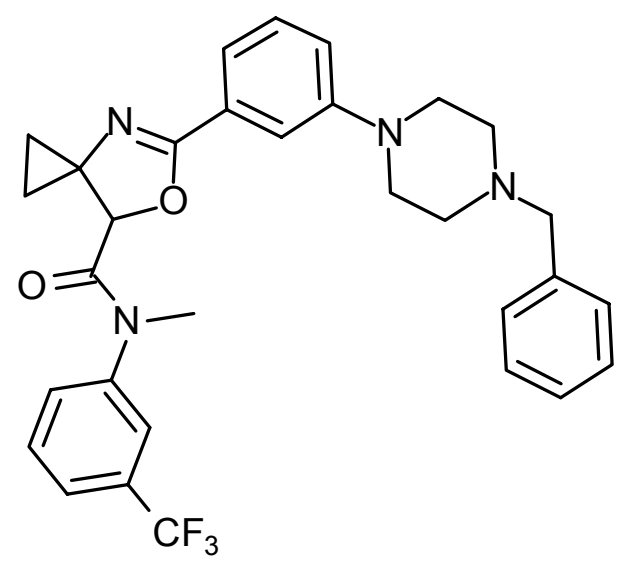
$\left.\mathrm{H}, \mathrm{NCH}_{2}\right), 3.25\left(\mathrm{~s}, 3 \mathrm{H}, \mathrm{CH}_{3}\right), 3.67$ (s, $\left.2 \mathrm{H}, \mathrm{Bn}-\mathrm{H}\right), 5.01(\mathrm{~s}, 1 \mathrm{H}, \mathrm{CH}), 6.87-7.00(\mathrm{~m}, 2 \mathrm{H}, \operatorname{aryl}-\mathrm{H})$, 7.08-7.17 (m, $2 \mathrm{H}$, aryl-H), 7.27-7.45 (m, $9 \mathrm{H}$, aryl-H). $\left.-{ }^{13} \mathrm{C} \mathrm{NMR} \mathrm{(75.5} \mathrm{MHz,} \mathrm{CDCl}_{3}, \mathrm{APT}\right): \delta=$ 12.0 (-, cPr-C), 14.6 (-, cPr-C), 38.9 (+, $\left.\mathrm{CH}_{3}\right), 48.4$ (-, $\left.2 \mathrm{C}, \mathrm{NCH}_{2}-\mathrm{C}\right), 52.6$ (-, $\left.2 \mathrm{C}, \mathrm{NCH}_{2}-\mathrm{C}\right)$, $53.6(-$, cPr-C), 62.5 (-, Bn-C), 80.7 (+, CH-C), 115.1 (+, aryl-C), 119.0 (+, aryl-C), $119.3(+$, aryl-C), $123.6\left(-\right.$, q, $\left.{ }^{1} J_{\mathrm{C}-\mathrm{F}}=272 \mathrm{~Hz}, \mathrm{CF}_{3}\right), 123.9$ (+, aryl-C), 124.4 (+, aryl-C), 128.9 (+, aryl-C), 127.0 (-, aryl-C), 127.8 (+, aryl-C), 128.5 (+, aryl-C), 128.7 (+, aryl-C), 129.7 (+, aryl-C), 130.3 (+, aryl-C), $132.0\left(-\right.$, q,${ }^{2} J_{\mathrm{C}-\mathrm{F}}=33.1 \mathrm{~Hz}$, aryl-C), 143.1 (-, aryl-C), 150.6 (-, aryl-C), 162.7 (-, CN-

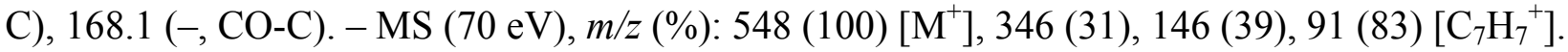

5-[3-(6-Dibenzylamino-3-aza-bicyclo[3.1.0]hex-3-yl)-phenyl]-6-oxa-4-aza-spiro[2.4]hept-4-ene7-carboxylic acid methyl-(3-trifluoromethyl-phenyl)amide (71j): The crude product obtained from $\mathbf{7 0 b}$ (90.6 mg, $0.20 \mathrm{mmol}), \mathrm{Pd}_{2}(\mathrm{dba})_{3}(9.1 \mathrm{mg}, 5 \mathrm{~mol} \%)$, $( \pm)$-BINAP $\quad(9.3 \quad \mathrm{mg}, \quad 7.5 \quad \mathrm{~mol} \%), \quad$ exo-6- $(N, N-$ dibenzylamino)-3-azabicyclo[3,1,0]hexane (139 $\mathrm{mg}$, $0.50 \mathrm{mmol})$ and $\mathrm{NaO} t \mathrm{Bu}(28.8 \mathrm{mg}, 0.30 \mathrm{mmol})$ was purified in column chromatography $\left(R_{\mathrm{f}}=0.40\right.$, Pentane $\left./ \mathrm{Et}_{2} \mathrm{O}=1: 2\right)$ to yield $105 \mathrm{mg}(81 \%)$ of $\mathbf{7 1} \mathbf{j}$ as

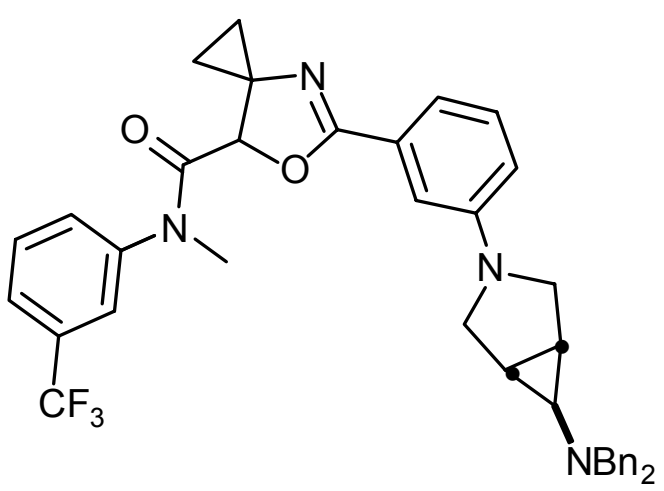
pale-yellow solid, m.p. $=85-88{ }^{\circ} \mathrm{C} .-\mathrm{IR}(\mathrm{KBr}): \mathrm{v}=3025 \mathrm{~cm}^{-1}, 2887,2812,1653,1599,1493$, 1332, 1129, 699. $-{ }^{1} \mathrm{H}-\mathrm{NMR}\left(300 \mathrm{MHz}, \mathrm{CDCl}_{3}\right): \delta=0.74-0.85(\mathrm{~m}, 1 \mathrm{H}, \mathrm{cPr}-\mathrm{H}), 1.12-1.20(\mathrm{~m}, 1$ H, cPr-H), 1.22-1.40 (m, 2 H, cPr-H), 1.50-1.61 (m, 2 H), 1.71-1.79 (m, 1 H), 3.08-3.20 (m, 2 H, 
$\left.\mathrm{NCH}_{2}-\mathrm{H}\right), 3.32-3.48\left(\mathrm{~m}, 5 \mathrm{H}, \mathrm{CH}_{3}\right.$ and $\left.\mathrm{NCH}_{2}-\mathrm{H}\right), 3.73(\mathrm{~s}, 4 \mathrm{H}, \mathrm{Bn}-\mathrm{H}), 5.09(\mathrm{~s}, 1 \mathrm{H}, \mathrm{CH}), 6.48-$ $6.80(\mathrm{~m}, 1 \mathrm{H}$, aryl-H), 6.72-6.93 (m, 2 H, aryl-H), 7.05-7.19 (m, $1 \mathrm{H}$, aryl-H), 7.24-7.47 (m, 13 $\mathrm{H}$, aryl-H), $7.52\left(\mathrm{~s}, 1 \mathrm{H}\right.$, aryl-H). $-{ }^{13} \mathrm{C}-\mathrm{NMR}\left(75.5 \mathrm{MHz}, \mathrm{CDCl}_{3}, \mathrm{APT}\right): \delta=12.0$ (-, cPr-C), 14.5 (-, cPr-C), 26.0 (+, CH-C), 38.9 (+, $\left.\mathrm{CH}_{3}\right), 48.3$ (+, CH-C), 49.8 (-, $\left.2 \mathrm{C}, \mathrm{CH}_{2}-\mathrm{C}\right), 53.5$ (-, cPr-C), 59.0 (-, 2 C, Bn-C), 80.5 (+, CH-C), 110.9 (+, aryl-C), 114.9 (+, aryl-C), 115.7 (+, aryl-C), 123.2 $\left(-, \mathrm{q},{ }^{1} J_{\mathrm{C}-\mathrm{F}}=272 \mathrm{~Hz}, \mathrm{CF}_{3}\right), 123.9\left(+, \mathrm{q},{ }^{3} J_{\mathrm{C}-\mathrm{F}}=3.8 \mathrm{~Hz}\right.$, aryl-C), $124.4\left(+, \mathrm{q},{ }^{3} J_{\mathrm{C}-\mathrm{F}}=3.8 \mathrm{~Hz}\right.$, aryl-C), $127.0(+, 2$ C, aryl-C), 128.1 (+, 4 C, aryl-C), 128.5 (+, aryl-C), 129.4 (+, 4 C, aryl-C), $130.2(+$, aryl-C), 131.9 (-, q, ${ }^{2} J_{\mathrm{C}-\mathrm{F}}=33.2 \mathrm{~Hz}$, aryl-C), 138.7 (-, 2 C, aryl-C), 143.3 (-, aryl-C), 147.4 (-, aryl-C), 163.1 (-, CN-C), 168.2 (-, CO-C). - MS (70 eV), m/z (\%): 650 (17) $\left[\mathrm{M}^{+}\right], 559(14)\left[\mathrm{M}^{+}-\right.$ $\left.\mathrm{C}_{7} \mathrm{H}_{7}\right], 249$ (19), 158 (18), 91 (100) $\left[\mathrm{C}_{7} \mathrm{H}_{7}^{+}\right]$.

General procedure for reaction of aryl boronic acid with aryl-bromide (70) (The Suzuki reaction) (GP 13): To a $25 \mathrm{~mL}$ Schlenk flask $\mathrm{Pd}(\mathrm{OAc})_{2}(4.5 \mathrm{mg}, 10 \mathrm{~mol} \%)$ and $\mathrm{P}(\mathrm{Ph})_{3}(21 \mathrm{mg}, 40$ mol\%) were taken in $5 \mathrm{~mL}$ of toluene. The mixture was degassed for $5 \mathrm{~min}$. by bubbling nitrogen through it. To this solution 70a $(90.6 \mathrm{mg}, 0.2 \mathrm{mmol})$, the corresponding boronic acid $(0.30 \mathrm{mmol})$ and $\mathrm{Na}_{2} \mathrm{CO}_{3}(0.40 \mathrm{mmol}$, aq. $2 \mathrm{~N})$ was added and the mixture was stirred at $80{ }^{\circ} \mathrm{C}$ for $16 \mathrm{~h}$. The reaction mixture was cooled to room temperature, taken in $\mathrm{Et}_{2} \mathrm{O}(25 \mathrm{ml})$, washed with water (10 $\mathrm{mL})$ and the aq. layer was extracted with $\mathrm{Et}_{2} \mathrm{O}(2 \times 10 \mathrm{~mL})$. The combined organic phase was dried over $\mathrm{MgSO}_{4}$, filtered, and concentrated in vacuo. The crude product was purified by column chromatography.

5-Biphenyl-4-yl-6-oxa-4-aza-spiro[2.4]hept-4-ene-7-carboxylic acid methyl-(3-trifluoromethylphenyl)-amide (72a): The crude product obtained from 70a, phenyl boronic acid (36.5 $\mathrm{mg}$ ) according to the GP 13 was purified by column chromatography $\left(R_{\mathrm{f}}=0.30\right.$, pentane $\left./ \mathrm{Et}_{2} \mathrm{O}=1: 2\right)$, to yield $81 \mathrm{mg}(90 \%)$ of $\mathbf{7 2 a}$ as a white solid, m.p. $=$ $136-137^{\circ} \mathrm{C}$. - IR (KBr): $v=3081 \mathrm{~cm}^{-1}, 1660,1643$, 1334, 1128, 1067, 705. - ${ }^{1} \mathrm{H}-\mathrm{NMR}(200 \mathrm{MHz}$, $\left.\mathrm{CDCl}_{3}\right): \delta=0.74-0.83(\mathrm{~m}, 1 \mathrm{H}, \mathrm{cPr}-\mathrm{H}), 1.03-1.38$ $(\mathrm{m}, 3 \mathrm{H}, \mathrm{cPr}-\mathrm{H}), 3.25\left(\mathrm{~s}, 3 \mathrm{H}, \mathrm{CH}_{3}\right), 5.02(\mathrm{~s}, 1 \mathrm{H}$,

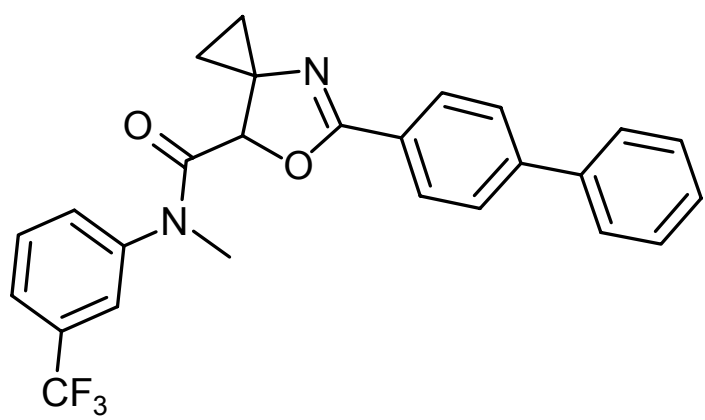
$\mathrm{CH}-\mathrm{H}), 7.09-7.59\left(\mathrm{~m}, 13 \mathrm{H}\right.$, aryl-H). $-{ }^{13} \mathrm{C}-\mathrm{NMR}\left(75.5 \mathrm{MHz}, \mathrm{CDCl}_{3}, \mathrm{APT}\right): \delta=12.0$ (-, cPr-C), 14.7 (-, cPr-C), $39.1\left(+, \mathrm{CH}_{3}\right), 53.7$ (-, cPr-C), $81.0(+, \mathrm{CH}-\mathrm{C}), 123.2\left(-, \mathrm{q},{ }^{1} J_{\mathrm{C}-\mathrm{F}}=262 \mathrm{~Hz}, \mathrm{CF}_{3}\right)$, 
$124.1\left(+, \mathrm{q},{ }^{3} J_{\mathrm{C}-\mathrm{F}}=3.8 \mathrm{~Hz}\right.$, aryl-C), $124.4\left(+, \mathrm{q},{ }^{3} J_{\mathrm{C}-\mathrm{F}}=3.8 \mathrm{~Hz}\right.$, aryl-C), $125.2(-$,aryl-C), $126.6(+$, 2 C, aryl-C), 127.1 (+, 2 C, aryl-C), 127.9 (+, aryl-C), 128.1 (+, 2 C, aryl-C), 128.9 (+, 2 C, arylC), $130.2\left(+\right.$, aryl-C), $132.0\left(-\right.$, q, ${ }^{2} J_{\mathrm{C}-\mathrm{F}}=33.2 \mathrm{~Hz}$, aryl-C), 140.1 (-, aryl-C), 143.2 (-, aryl-C), 143.9 (-, aryl-C), 162.2 (-, CN-C), 168.0 (-, CO-C). - MS (70 eV), m/z (\%): 450 (92) [M $\left.{ }^{+}\right], 276$ (91), 248 (61), 181 (100). - calcd. $\mathrm{C}_{26} \mathrm{H}_{21} \mathrm{~F}_{3} \mathrm{~N}_{2} \mathrm{O}_{2}$ (450.5) C 69.33, H 4.70, N 6.22; found C 69.26, H 4.48, N 6.18.

5-(4'-Trifluoromethoxy-biphenyl-4-yl)-6-oxa-4-aza-spiro[2.4] hept-4-ene-7-carboxylic

acid methyl-(3-trifluoromethyl-phenyl)-amide (72b): The crude product obtained from 70a, 4(trifluoromethoxy)phenyl boronic acid (66.5 $\mathrm{mg}$ ) according to the GP 13 was purified by column chromatography $\left(R_{\mathrm{f}}=\right.$ 0.26 , pentane $/ \mathrm{Et}_{2} \mathrm{O}=1: 2$ ), to yield $85 \mathrm{mg}$ $(80 \%)$ of $72 \mathbf{b}$ as colourless solid, m.p. $=$ $136-138{ }^{\circ} \mathrm{C}$. - IR (KBr): $v=2943 \mathrm{~cm}^{-1}$, 1654, 1646, 1494, 1331, 1270, 1178, 1068.

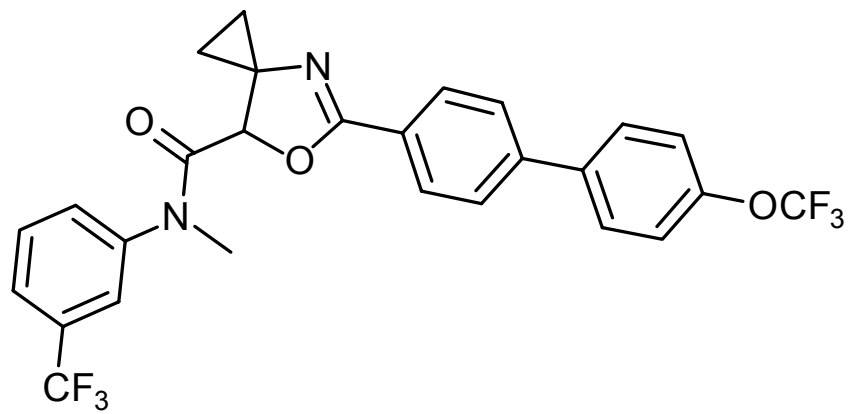

$-{ }^{1} \mathrm{H}-\mathrm{NMR}\left(200 \mathrm{MHz}, \mathrm{CDCl}_{3}\right): \delta=0.78-0.87(\mathrm{~m}, 1 \mathrm{H}, \mathrm{cPr}-\mathrm{H}), 1.12-1.40$ (m, $\left.3 \mathrm{H}, \mathrm{cPr}-\mathrm{H}\right), 3.34$ (s, $\left.3 \mathrm{H}, \mathrm{CH}_{3}\right), 5.07(\mathrm{~s}, 1 \mathrm{H}, \mathrm{CH}), 7.15-7.23(\mathrm{~m}, 1 \mathrm{H}$, aryl-H), 7.28-7.32 (m, 3 H, aryl-H), 7.35-7.40 (m, 1 H, aryl-H), 7.41-7.49 (m, 3 H, aryl-H), 7.55-7.62 (m, 4 H, aryl-H). $-{ }^{13} \mathrm{C}-\mathrm{NMR}(75.5 \mathrm{MHz}$, $\left.\mathrm{CDCl}_{3}, \mathrm{APT}\right): \delta=12.0$ (-, cPr-C), 14.7 (-, cPr-C), 39.1 (+, $\left.\mathrm{CH}_{3}\right), 53.7$ (-, cPr-C), 80.9 (+, CH-C), $120.4\left(-, \mathrm{q},{ }^{1} J_{\mathrm{C}-\mathrm{F}}=235 \mathrm{~Hz}, \mathrm{CF}_{3}\right), 121.3\left(+, 2 \mathrm{C}\right.$, aryl-C), $124.1\left(+, \mathrm{q},{ }^{3} J_{\mathrm{C}-\mathrm{F}}=3.8 \mathrm{~Hz}\right.$, aryl-C), 124.4 $\left(+, \mathrm{q},{ }^{3} J_{\mathrm{C}-\mathrm{F}}=3.8 \mathrm{~Hz}\right.$, aryl-C), $125.6(-$, aryl-C), $126.5(+, 2 \mathrm{C}$, aryl-C), $128.2(+, 2 \mathrm{C}$, aryl-C), 128.5 (+, 2 C, aryl-C), 130.2 (+, 2 C, aryl-C), $130.2\left(+\right.$, aryl-C), 132.1 (-, q, ${ }^{2} J_{\mathrm{C}-\mathrm{F}}=33.2 \mathrm{~Hz}$, arylC), 138.8 (-, aryl-C), 142.4 (-, aryl-C), 143.1 (-, aryl-C), 149.1 (-, aryl-C), 162.1 (-, CN-C), 168.0 (-, CO-C). - MS (70 eV), m/z (\%): 534 (27) $\left[\mathrm{M}^{+}\right], 360$ (43), 304 (32), 265 (100), 202 (76), 174 (86). - calcd. $\mathrm{C}_{27} \mathrm{H}_{20} \mathrm{~F}_{6} \mathrm{~N}_{2} \mathrm{O}_{2}$ (534.5) C 60.68, H 3.77, N 5.24; found $\mathrm{C}$ 60.41, H 3.58, N 5.06. 
5-(4-Naphthalen-2-yl-phenyl)-6-oxa-4-aza-spiro[2.4] hept-4-ene-7-carboxylic acid methyl-(3trifluoromethyl-phenyl)-amide (72c): The crude product obtained from 70a, 4-naphthalyl boronic acid $(51.5 \mathrm{mg})$ according to the GP 13 was purified by column chromatography $\left(R_{\mathrm{f}}=0.24\right.$, pentane/ $\left.\mathrm{Et}_{2} \mathrm{O}=1: 2\right)$, to yield $85 \mathrm{mg}(85 \%)$ of 72c as colourless solid, m.p. $=144^{\circ} \mathrm{C} .-\mathrm{IR}$ $(\mathrm{KBr}): v=3066 \mathrm{~cm}^{-1}, 1658,1492,1330,1170$, $1118, \quad 1053,818 . \quad-{ }^{1} \mathrm{H}-\mathrm{NMR} \quad(200 \mathrm{MHz}$, $\left.\mathrm{CDCl}_{3}\right): \delta=0.73-0.84(\mathrm{~m}, 1 \mathrm{H}, \mathrm{cPr}-\mathrm{H}), 1.03-$

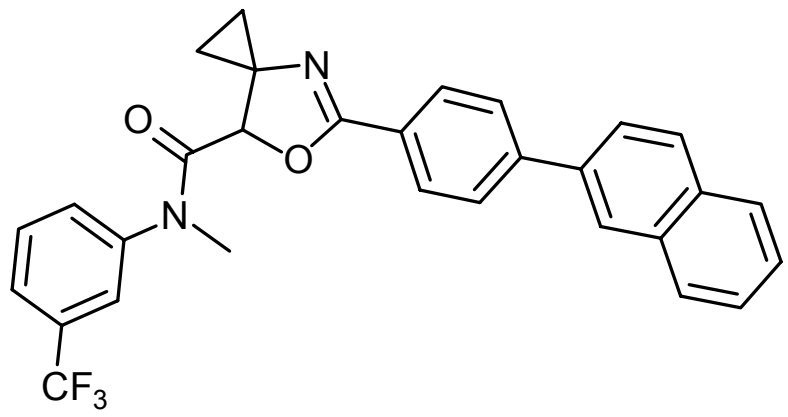
1.39 (m, $3 \mathrm{H}, \mathrm{cPr}-\mathrm{H}), 3.24$ (s, $\left.3 \mathrm{H}, \mathrm{CH}_{3}\right), 5.02$ (s, $\left.1 \mathrm{H}, \mathrm{CH}\right), 7.11-7.38$ (m, $3 \mathrm{H}$, aryl-H), 7.40-7.48 (m, $3 \mathrm{H}$, aryl-H), 7.52-7.60 (m, $4 \mathrm{H}$, aryl-H), 7.61-7.70 (m, $1 \mathrm{H}$, aryl-H), 7.74-7.90 (m, $3 \mathrm{H}$, arylH), 7.96-8.01 (m, $1 \mathrm{H}$, aryl-H). $-{ }^{13} \mathrm{C}-\mathrm{NMR}\left(75.5 \mathrm{MHz}, \mathrm{CDCl}_{3}, \mathrm{APT}\right): \delta=12.1$ (-, cPr-C), $14.7(-$ , cPr-C), $39.1\left(+, \mathrm{CH}_{3}\right), 53.7$ (-, cPr-C), $80.9(+, \mathrm{CH}-\mathrm{C}), 124.0\left(+, \mathrm{q},{ }^{3} \mathrm{~J}_{\mathrm{C}-\mathrm{F}}=3.8 \mathrm{~Hz}\right.$, aryl-C), 124.5 $\left(+, \mathrm{q},{ }^{3} J_{\mathrm{C}-\mathrm{F}}=3.8 \mathrm{~Hz}\right.$, aryl-C), 125.1 (+, aryl-C), 125.2 (-, aryl-C), 126.1 (+, aryl-C), 126.3 (+, arylC), 126.5 (+, aryl-C), 126.8 (+, aryl-C), 127.7 (+, aryl-C), 128.2 (+, 2 C, aryl-C), $128.3(+, 2$ C, aryl-C), $128.6\left(+\right.$, aryl-C), 130.3 (+, aryl-C), 132.1 (-, q, ${ }^{2} J_{\mathrm{C}-\mathrm{F}}=33.2 \mathrm{~Hz}$, aryl-C), 132.9 (-, arylC), 133.6 (-, aryl-C), 137.4 (-, aryl-C), 143.6 (-, aryl-C), 143.8 (-, aryl-C), 162.1 (-, CN-C),

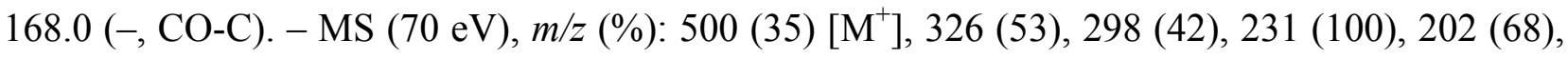
174 (28). $-\mathrm{C}_{30} \mathrm{H}_{23} \mathrm{~F}_{3} \mathrm{~N}_{2} \mathrm{O}_{2}$ (500.5): calcd. C 71.99, H 4.63, N 5.60; found C 72.25, H 4.46, N 5.35.

5-(4-Thiophen-3-yl-phenyl)-6-oxa-4-aza-spiro[2.4]hept-4-ene-7-carboxylic acid methyl-(3trifluoromethyl-phenyl)-amide (72d): The crude product obtained from 70a, 4-(thiophen-3-yl) boronic acid (26 mg) according to the GP 13 was purified by column chromatography $\left(R_{\mathrm{f}}=0.25\right.$, pentane $/ \mathrm{Et}_{2} \mathrm{O}=$ $1: 2)$ to yield $59 \mathrm{mg}(65 \%)$ of $\mathbf{7 2 d}$ as colourless solid, m.p. $=141-142{ }^{\circ} \mathrm{C} .-\mathrm{IR}(\mathrm{KBr}): v=2955 \mathrm{~cm}^{-1}, 1664$, 1644, 1340, 1165, 1127, 1070, 792. $-{ }^{1} \mathrm{H}-\mathrm{NMR}(300$

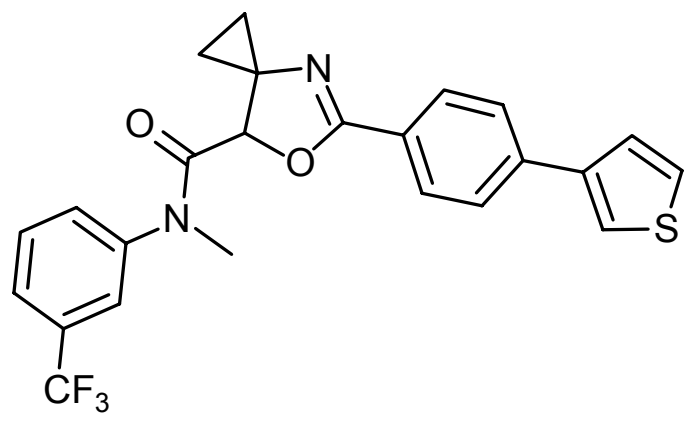
$\left.\mathrm{MHz}, \mathrm{CDCl}_{3}\right): \delta=0.78-0.88$. (m, $\left.1 \mathrm{H}, \mathrm{cPr}-\mathrm{H}\right), 1.11-1.40(\mathrm{~m}, 3 \mathrm{H}, \mathrm{cPr}-\mathrm{H}), 3.32\left(\mathrm{~s}, 3 \mathrm{H}, \mathrm{CH}_{3}\right), 5.06$ (s, $1 \mathrm{H}, \mathrm{CH}), 7.17-7.35$ (m, $2 \mathrm{H}$, aryl-H), 7.36-7.41 (m, $4 \mathrm{H}$, aryl-H), 7.43-7.57 (m, $5 \mathrm{H}$, aryl-H). $-{ }^{13} \mathrm{C}-\mathrm{NMR}\left(75.5 \mathrm{MHz}, \mathrm{CDCl}_{3}, \mathrm{APT}\right): \delta=12.0$ (-, cPr-C), 14.7 (-, cPr-C), $39.1\left(+, \mathrm{CH}_{3}\right), 53.7$ (-, cPr-C), $80.9(+, \mathrm{CH}-\mathrm{C}), 121.3\left(+\right.$, aryl-C), $124.0\left(+, \mathrm{d},{ }^{3} J_{\mathrm{C}-\mathrm{F}}=3.8 \mathrm{~Hz}\right.$, aryl-C), $124.4\left(+, \mathrm{d},{ }^{3} J_{\mathrm{C}-\mathrm{F}}=\right.$ 
$3.8 \mathrm{~Hz}$, aryl-C), 125.0 (-, aryl-C), 125.8 (+, 2 C, aryl-C), 126.1 (+, 2 C, aryl-C), 126.5 (+, aryl-C), $128.2\left(+, 2\right.$ C, aryl-C), 128.3 (+, 2 C, aryl-C), $130.2\left(+\right.$, aryl-C), $132.0\left(-\right.$, q, ${ }^{2} J_{\mathrm{C}-\mathrm{F}}=33.2 \mathrm{~Hz}$, arylC), 138.4 (-, aryl-C), 141.3 (-, aryl-C), 143.1 (-, aryl-C), 162.2 (-, CN-C), 168.0 (-, CO-C). - MS $(70 \mathrm{eV}), m / z(\%): 456(27)\left[\mathrm{M}^{+}\right], 282$ (53), 254 (38), 202 (42), 187 (100), 175 (42). $\mathrm{C}_{24} \mathrm{H}_{19} \mathrm{~F}_{3} \mathrm{~N}_{2} \mathrm{O}_{2} \mathrm{~S}$ (456.5): calcd. C 63.15, H 4.20, N 6.14; found C 63.18, H 4.14, N 6.6.02.

\subsubsection{Michael addition of dialky thiourea onto methyl 2-chloro-2-cyclopropyledeneacetate}

General Procedure: To a solution of meth (5) in $25 \mathrm{~mL}$ of dioxane in a $50 \mathrm{~mL}$ of flamed dried reaction flask, the corresponding dialkyl thiourea and $\mathrm{Et}_{3} \mathrm{~N}$ were added. The resulting slurry was stirred for $15 \mathrm{~h}$, solvent was removed in vacuum. The resulting crude product was dissolved in $\mathrm{DCM} / \mathrm{MeOH}$ (25:1) and purified in column chromatography to yield the product as white solid.

2-cyclopropyl-2,3,5,6,7,8-hexahydro-thiazolo[3,2-a] [1,3] diazepine-3-carboxylic acid methyl ester (74a): The crude product obtained from methyl 2-chloro-2-cyclopropylideneacetate (366 mg, 2.5 $\mathrm{mmol})$, tetramethylenethiourea $(650 \mathrm{mg}, 5 \mathrm{mmol})$ and $\mathrm{Et}_{3} \mathrm{~N}(1.01 \mathrm{~g}, 10$ mmol) was purified in column chromatography $\left(R_{\mathrm{f}}=0.22, \mathrm{DCM} / \mathrm{MeOH}\right.$ $=9: 1)$ to yield $568 \mathrm{mg}(82 \%)$ of product $74 \mathbf{a}$ as a white solid, m.p. $=$ $175-176{ }^{\circ} \mathrm{C}$. The product was crystallized from $\mathrm{Et}_{2} \mathrm{O} / \mathrm{MeOH}(50 / 1)$ at 0 ${ }^{\circ} \mathrm{C}$. - IR (KBr): $v=3171,3117,2929,2869,1741,1616,1559,1359$,

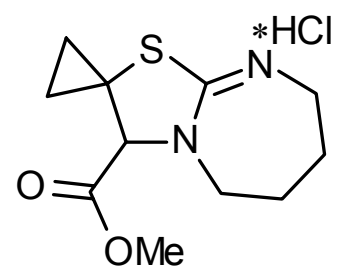
1039, 994, $856 \mathrm{~cm}^{-1} .-{ }^{1} \mathrm{H}-\mathrm{NMR}\left(250 \mathrm{MHz}, \mathrm{CDCl}_{3}\right): \delta=1.10-1.18$ (m, $\left.2 \mathrm{H}, \mathrm{cPr}-\mathrm{H}\right), 1.34-1.48$ (m, 2 H, cPr-H), 2.01-2.20 (m, 4 H, $\left.\mathrm{CH}_{2}\right), 3.68-3.80$ (m, $\left.4 \mathrm{H}, \mathrm{NCH}_{2}\right), 3.81$ (s, $\left.3 \mathrm{H}, \mathrm{OMe}\right), 4.38$ (s, $1 \mathrm{H}, \mathrm{CH}), 12.11$ (br s, $1 \mathrm{H}, \mathrm{NH}) .-{ }^{13} \mathrm{C}-\mathrm{NMR}\left(63.9 \mathrm{MHz}, \mathrm{CDCl}_{3}, \mathrm{DEPT}\right): \delta=6.4(-, \mathrm{cPr}), 18.4(-$, cPr), $25.0\left(-, \mathrm{CH}_{2}\right), 25.6\left(-, \mathrm{CH}_{2}\right), 29.3\left(\mathrm{C}_{\text {quat, }}, \mathrm{cPr}\right), 44.8\left(-, \mathrm{NCH}_{2}\right), 50.7\left(-, \mathrm{NCH}_{2}\right), 53.2(+$, $\mathrm{OMe}), 74.5(+, \mathrm{CH}), 167.5\left(\mathrm{C}_{\text {quat }}, \mathrm{C}=\mathrm{O}\right), 171.4\left(\mathrm{C}_{\text {quat }}, \mathrm{C}=\mathrm{N}\right) .-\mathrm{MS}(\mathrm{DCI}), m / z(\%): 241(100)\left[\mathrm{M}^{+}\right.$ $+\mathrm{H}-\mathrm{HCl}$. 
2-cyclopropyl-2,3,7,8-tetrahydro-5H-thiazolo[3,2-a]pyrimidine-3-carboxylic acid methyl ester (74b): The crude product obtained from methyl 2-chloro-2-cyclopropylidene acetate (366 mg, 2.5 $\mathrm{mmol})$, trimethylenethiourea $(580 \mathrm{mg}, 5 \mathrm{mmol})$ and $\mathrm{Et}_{3} \mathrm{~N}(1.01 \mathrm{~g}, 10$ mmol) was purified in column chromatography $\left(R_{\mathrm{f}}=0.20, \mathrm{DCM} / \mathrm{MeOH}\right.$ $=9: 1)$ to yield $235 \mathrm{mg}(41 \%)$ of product $\mathbf{7 4 \mathbf { b }}$ as a white solid, m.p. $=$ $180^{\circ} \mathrm{C}$. - IR (KBr): $v=3093,2955,1880,2767,2680,1734,1630$, $1548,1312,1225,1004 \mathrm{~cm}^{-1}$. ${ }^{1} \mathrm{H}-\mathrm{NMR}\left(250 \mathrm{MHz}, \mathrm{CDCl}_{3}\right): \delta=1.12-$

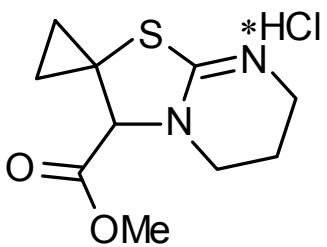
1.36 (m, $3 \mathrm{H}, \mathrm{cPr}-\mathrm{H}), 1.38-1.50$ (m, $1 \mathrm{H}, \mathrm{cPr}-\mathrm{H}), 2.12-2.21$ (m, $\left.2 \mathrm{H}, \mathrm{CH}_{2}\right), 3.48-3.67$ (m, $4 \mathrm{H}$, $\left.\mathrm{NCH}_{2}\right), 3.83(\mathrm{~s}, 3 \mathrm{H}, \mathrm{OMe}), 4.18(\mathrm{~s}, 1 \mathrm{H}, \mathrm{CH}) .-{ }^{13} \mathrm{C}-\mathrm{NMR}\left(75.6 \mathrm{MHz}, \mathrm{CDCl}_{3}, \mathrm{APT}\right): \delta=6.7(-$, cPr), 18.6 (-, cPr), 18.8 (-, $\left.\mathrm{CH}_{2}\right), 29.0$ (-, cPr), $39.7\left(-, \mathrm{CH}_{2}\right), 44.8$ (-, $\left.\mathrm{NCH}_{2}\right), 53.5$ (+, $\left.\mathrm{OMe}\right), 72.0$ $(+, \mathrm{CH}), 166.9\left(\mathrm{C}_{\text {quat }}, \mathrm{C}=\mathrm{O}\right), 167.1\left(\mathrm{C}_{\text {quat, }}, \mathrm{C}=\mathrm{N}\right) .-\mathrm{MS}(70 \mathrm{eV}), \mathrm{m} / z(\%): 226(23)\left[\mathrm{M}^{+}-\mathrm{CO}_{2} \mathrm{Me}-\right.$ $\mathrm{HCl}$, 140 (29). 
3.2.6. Sequential addition of Grignard reagent and aldehyde with methyl 2-chloro-2cylopropylideneacetate

General procedure for the one-pot reaction of methyl 2-chloro-2-cylopropylideneacetate (5) with Grignard reagents and benzaldehydes: To a solution of 5 (147 mg, $1.00 \mathrm{mmol})$ in THF $(10 \mathrm{~mL})$ was slowly (within $10 \mathrm{~min})$ added at $0{ }^{\circ} \mathrm{C}$ a solution of the Grignard reagent $(1.00 \mathrm{mmol})$. The mixture was stirred for $30 \mathrm{~min}$ under rewarming to $20^{\circ} \mathrm{C}$, and then cooled again, at $0{ }^{\circ} \mathrm{C}$ a solution of the respective aldehyde $(1.05 \mathrm{mmol})$ in THF $(2 \mathrm{~mL})$ was added, and the mixture was stirred for an additional $30 \mathrm{~min}$ under rewarming to $20^{\circ} \mathrm{C}$. The mixture was cooled again to $0{ }^{\circ} \mathrm{C}$, the reaction was quenched with sat. $\mathrm{NH}_{4} \mathrm{Cl}$ solution $(4 \mathrm{~mL})$, and the mixture was stirred at $20{ }^{\circ} \mathrm{C}$ for $15 \mathrm{~min}, \mathrm{Et}_{2} \mathrm{O}(20 \mathrm{~mL})$ was added, the phases were separated, the aq. phase was extracted with $20 \mathrm{~mL}$ of $\mathrm{Et}_{2} \mathrm{O}$, the combined organic phase was dried over $\mathrm{Na}_{2} \mathrm{SO}_{4}$, and the volatiles removed in vacuo. Chromatographic purification of the residue on silica gel yielded the products.

Methyl 2-chloro-3-hydoxy-2-(1-isopropylcylopropyl)-3-phenylpropionate (75a-iPr):From $\mathbf{5}$ $(147 \mathrm{mg}, 1.00 \mathrm{mmol}), \mathrm{iPrMgCl}\left(2 \mathrm{M}\right.$ in $\left.\mathrm{Et}_{2} \mathrm{O}, 0.50 \mathrm{~mL}, 1.00 \mathrm{mmol}\right)$, and benzaldehyde $(111 \mathrm{mg}$, $1.05 \mathrm{mmol})$, chromatographic purification on $20 \mathrm{~g}$ of silica gel $\left(R_{\mathrm{f}}=0.30\right.$, pentane $\left./ \mathrm{Et}_{2} \mathrm{O}=3: 1,1.5 \times 15 \mathrm{~cm}\right)$ yielded $273 \mathrm{mg}(92 \%)$ of $75 \mathbf{a}-1 \mathrm{Pr}$ as a colorless oil. - IR (film): $v=3516 \mathrm{~cm}^{-1}, 2960,2929,2873,1723,1454$, 1262, 1054, 702. $-{ }^{1} \mathrm{H}-\mathrm{NMR}\left(250 \mathrm{MHz}, \mathrm{CDCl}_{3}\right): \delta=-0.14$ to 0.08 (m, $1 \mathrm{H}, \mathrm{cPr}-\mathrm{H}), 0.16-0.26$ (m, $2 \mathrm{H}, \mathrm{cPr}-\mathrm{H}), 0.69\left(\mathrm{~d},{ }^{3} \mathrm{~J}=6.7 \mathrm{~Hz}, 3 \mathrm{H}, \mathrm{CH}_{3}\right)$, $0.78\left(\mathrm{~d},{ }^{3} J=6.7 \mathrm{~Hz}, 3 \mathrm{H}, \mathrm{CH}_{3}\right), 0.85-1.03(\mathrm{~m}, 1 \mathrm{H}, \mathrm{cPr}-\mathrm{H}), 2.42$ (sept, ${ }^{3} J$

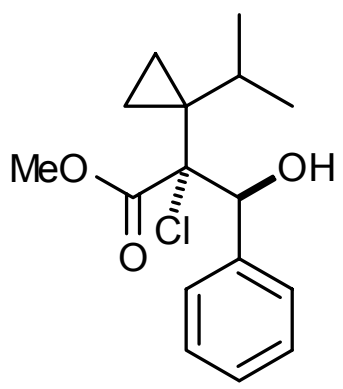
$=6.7,6.97 \mathrm{~Hz}, 1 \mathrm{H}, \mathrm{CH}), 3.82\left(\mathrm{~s}, 3 \mathrm{H}, \mathrm{OCH}_{3}\right), 5.64(\mathrm{~s}, 1 \mathrm{H}, \mathrm{CH}), 7.21-7.51(\mathrm{~m}, 5 \mathrm{H}, \operatorname{aryl}-\mathrm{H}) \mathrm{ppm}$. $-{ }^{13} \mathrm{C}-\mathrm{NMR}\left(62.9 \mathrm{MHz}, \mathrm{CDCl}_{3}, \mathrm{DEPT}\right): \delta=5.1(-, \mathrm{cPr}-\mathrm{C}), 8.5(-, \mathrm{cPr}-\mathrm{C}), 20.9\left(+, \mathrm{CH}_{3}\right), 21.2(+$, $\left.\mathrm{CH}_{3}\right), 26.1(+, \mathrm{CH}), 28.0\left(\mathrm{C}_{\text {quat }}, \mathrm{cPr}-\mathrm{C}\right), 53.1\left(+, \mathrm{OCH}_{3}\right), 75.1(+, \mathrm{CH}), 81.8\left(\mathrm{C}_{\text {quat }}, \mathrm{Cl}-\mathrm{C}\right), 127.5(+$, $2 \mathrm{C}$, aryl-C), 128.3 (+, aryl-C), 128.7 (+, $2 \mathrm{C}$, aryl-C), $138.3\left(\mathrm{C}_{\text {quat, }}, \mathrm{C}_{\text {ipso }}\right), 172.4\left(\mathrm{C}_{\text {quat }}, \mathrm{C}=\mathrm{O}\right) \mathrm{ppm}$. - MS (DCI), $m / z(\%): 610.6(<1)\left[2 \mathrm{M}^{+}+\mathrm{NH}_{4}\right], 314.3(100)\left[\mathrm{M}^{+}+\mathrm{NH}_{4}\right] .-\mathrm{C}_{16} \mathrm{H}_{21} \mathrm{ClO}_{3}(296.8):$ calcd. C 64.75, H 7.13; found C 64.97, H 6.86. 
Methyl 2-chloro-2-(1-cyclohexylcyclopropyl)-3-hydroxy-3-phenylpropionate (75a-cHex): From $\mathbf{5}$ $(147 \mathrm{mg}, 1.00 \mathrm{mmol}), \mathrm{cHexMgBr}\left(2 \mathrm{M}\right.$ in $\left.\mathrm{Et}_{2} \mathrm{O}, 0.5 \mathrm{~mL}, 1.00 \mathrm{mmol}\right)$, and benzaldehyde (111 mg, $1.05 \mathrm{mmol})$, chromatographic purification on $30 \mathrm{~g}$ of silica gel $\left(R_{\mathrm{f}}=0.13\right.$, pentane $\left./ \mathrm{Et}_{2} \mathrm{O}=3: 1,3 \times 10 \mathrm{~cm}\right)$ yielded $91 \mathrm{mg}(27 \%)$ of $75 \mathrm{a}-\mathrm{cHex}$ as a colorless oil. - IR (film): $v=3521 \mathrm{~cm}^{-1}, 2928,1718,1453,1263,701$. ${ }^{1} \mathrm{H}-\mathrm{NMR}\left(\mathrm{CDCl}_{3}, 250 \mathrm{MHz}\right): \delta=-0.02$ to $0.07(\mathrm{~m}, 1 \mathrm{H}, \mathrm{cPr}-\mathrm{H}), 0.18$ 0.32 (m, $2 \mathrm{H}, \mathrm{cPr}-\mathrm{H}), 0.45-0.60$ (m, $1 \mathrm{H}, \mathrm{cHex}-\mathrm{H}), 0.92-1.93$ (m, $10 \mathrm{H}$, cHex-H, $1 \mathrm{H}, \mathrm{cPr}-\mathrm{H}$ ), 3.14 (br s, $1 \mathrm{H}, \mathrm{OH}), 3.84$ (s, $3 \mathrm{H}, \mathrm{OCH}_{3}$ ), 5.62 (s, $1 \mathrm{H}, \mathrm{OCH}), 7.26-7.34$ (m, $3 \mathrm{H}$, aryl-H), 7.40-7.44 (m, $2 \mathrm{H}$, aryl-H) ppm.

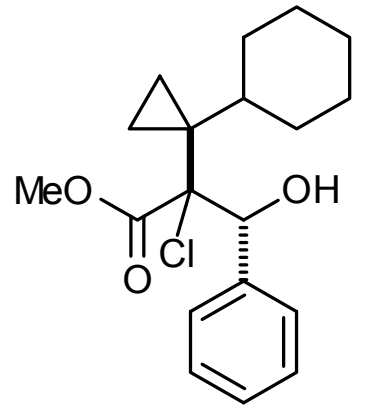
$-{ }^{13} \mathrm{C}-\mathrm{NMR}\left(62.9 \mathrm{MHz}, \mathrm{CDCl}_{3}, \mathrm{DEPT}\right): \delta=5.9$ (-, cPr-C), 7.1 (-, cPr-C), $26.1\left(-, \mathrm{CH}_{2}, \mathrm{cHex}\right)$, 26.8 (-, $\left.\mathrm{CH}_{2}, \mathrm{cHex}\right), 27.1$ (-, $\left.\mathrm{CH}_{2}, \mathrm{cHex}\right), 28.0$ ( $\mathrm{C}_{\text {quat }}$, cPr-C), 31.3 (-, $\left.\mathrm{CH}_{2}, \mathrm{cHex}\right), 32.1\left(-, \mathrm{CH}_{2}\right.$, cHex), 36.5 (+, CH, cHex), $53.0\left(+, \mathrm{OCH}_{3}\right), 75.3(+, \mathrm{OCH}), 82.4\left(\mathrm{C}_{\text {quat }}, \mathrm{Cl}-\mathrm{C}\right), 127.5(+, 2 \mathrm{C}$, arylC), 128.2 (+, aryl-C), 128.6 (+, 2 C, aryl-C), $138.4\left(\mathrm{C}_{\text {quat, }}, \mathrm{C}_{\text {ipso }}\right), 172.1\left(\mathrm{C}_{\text {quat, }}, \mathrm{C}=\mathrm{O}\right) \mathrm{ppm}$. - MS (DCI), $m / z$ (\%): 690.9 (20) $\left[2 \mathrm{M}^{+}+\mathrm{NH}_{4}\right], 354.5$ (100) $\left[\mathrm{M}^{+}+\mathrm{NH}_{4}\right] .-\mathrm{C}_{19} \mathrm{H}_{25} \mathrm{ClO}_{3}$ (336.9): calcd. C 67.75, H 7.48; found C 67.82, H 7.36.

Methyl 2-chloro-2-(1-ethylcyclopropyl)-3-hydroxy-3-phenylpropionate (75a-Et): From 5 (294 mg, $2.0 \mathrm{mmol}), \mathrm{EtMgBr}\left(2 \mathrm{M}\right.$ in $\left.\mathrm{Et}_{2} \mathrm{O}, 1.1 \mathrm{~mL}, 2.2 \mathrm{mmol}\right)$, and benzaldehyde (233 mg, $2.2 \mathrm{mmol}$ ), chromatographic purification on $30 \mathrm{~g}$ of silica gel $\left(R_{\mathrm{f}}=0.60\right.$, pentane $/ \mathrm{Et}_{2} \mathrm{O}$ $=5: 1,3 \times 10 \mathrm{~cm})$ yielded $180 \mathrm{mg}(32 \%)$ of $75 \mathrm{a}-$ Et as a colorless oil. $-\mathrm{IR}$ (film): $v=3530 \mathrm{~cm}^{-1}, 3030,2970,2945,1721,1453,1269 .-{ }^{1} \mathrm{H}-\mathrm{NMR}$ $\left(250 \mathrm{MHz}, \mathrm{CDCl}_{3}\right): \delta=0.01-0.07(\mathrm{~m}, 1 \mathrm{H}, \mathrm{cPr}-\mathrm{H}), 0.31-0.37(\mathrm{~m}, 1 \mathrm{H}$, cPr-H), 0.74 (t, $\left.{ }^{3} J=7.5 \mathrm{~Hz}, 3 \mathrm{H}, \mathrm{CH}_{3}\right), 0.86-0.93$ (m, $\left.2 \mathrm{H}, \mathrm{cPr}-\mathrm{H}\right), 1.63-$ $1.88\left(\mathrm{~m}, 2 \mathrm{H}, \mathrm{CH}_{2}\right), 2.90-3.40$ (br s, $\left.1 \mathrm{H}, \mathrm{OH}\right), 3.84$ (s, $\left.3 \mathrm{H}, \mathrm{OCH}_{3}\right), 5.59$

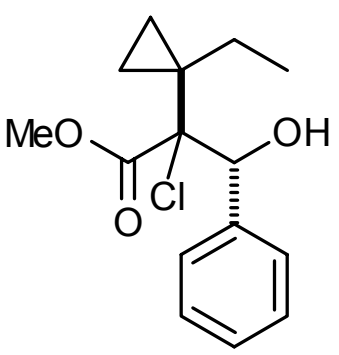
$(\mathrm{s}, 1 \mathrm{H}, \mathrm{OCH}), 7.26-7.33\left(\mathrm{~m}, 3 \mathrm{H}\right.$, aryl-H), 7.43-7.47 (m, $2 \mathrm{H}$, aryl-H) ppm. $-{ }^{13} \mathrm{C}-\mathrm{NMR}$ $\left(62.9 \mathrm{MHz}, \mathrm{CDCl}_{3}, \mathrm{DEPT}\right): \delta=15.4(-, \mathrm{cPr}-\mathrm{C}), 16.5(-, \mathrm{cPr}-\mathrm{C}), 18.2\left(+, \mathrm{CH}_{3}\right), 31.2\left(\mathrm{C}_{\text {quat }}, \mathrm{cPr}-\mathrm{C}\right)$, $33.4\left(-, \mathrm{CH}_{2}\right), 61.5\left(+, \mathrm{OCH}_{3}\right), 82.9(+, \mathrm{OCH}), 88.6\left(\mathrm{C}_{\text {quat }}, \mathrm{Cl}-\mathrm{C}\right), 135.7(+, 2 \mathrm{C}$, aryl-C), $136.5(+$, aryl-C), 136.9 (+, $2 \mathrm{C}$, aryl-C), $146.4\left(\mathrm{C}_{\text {quat, }}, \mathrm{C}_{\text {ipso }}\right), 180.8\left(\mathrm{C}_{\text {quat }}, \mathrm{C}=\mathrm{O}\right) \mathrm{ppm} .-\mathrm{MS}(\mathrm{DCI}), \mathrm{m} / z(\%)$ : $582.6(75)\left[2 \mathrm{M}^{+}+\mathrm{NH}_{4}\right], 300.3(100)\left[\mathrm{M}^{+}+\mathrm{NH}_{4}\right] .-\mathrm{C}_{15} \mathrm{H}_{19} \mathrm{ClO}_{3}$ (282.8): calcd. C 63.72, H 6.77; found C 63.76, H 6.44 . 
Methyl 2-chloro-3-hydroxy-3-phenyl-2-(1-phenyl-cyclopropyl)propionate (75a-Ph): From 5 (147 mg, $1.00 \mathrm{mmol}), \mathrm{PhMgCl}(2 \mathrm{M}$ in THF, $0.5 \mathrm{~mL}, 1.00 \mathrm{mmol})$, and benzaldehyde (111 $\mathrm{mg}$, $1.10 \mathrm{mmol})$, chromatographic purification on $20 \mathrm{~g}$ of silica gel $\left(R_{\mathrm{f}}=0.30\right.$, pentane $/ \mathrm{Et}_{2} \mathrm{O}=3: 1,1.5 \times 15 \mathrm{~cm}$ ) and subsequent crystallization from Pentane/Et $2 \mathrm{O}$ gave the pure product $75 \mathbf{a}-\mathrm{Ph}(139 \mathrm{mg}, 42 \%)$ as colorless crystals, m.p. $105-107^{\circ} \mathrm{C}$. - IR (KBr): $v=3549 \mathrm{~cm}^{-1}, 1729,1320,1182$, 700. - ${ }^{1} \mathrm{H}-\mathrm{NMR}\left(250 \mathrm{MHz}, \mathrm{CDCl}_{3}\right): \delta=0.88-0.94$ (m, $\left.2 \mathrm{H}, \mathrm{cPr}-\mathrm{H}\right), 1.08-$ $1.16(\mathrm{~m}, 1 \mathrm{H}, \mathrm{cPr}-\mathrm{H}), 1.48-1.54(\mathrm{~m}, 1 \mathrm{H}, \mathrm{cPr}-\mathrm{H}), 3.75\left(\mathrm{~s}, 3 \mathrm{H}, \mathrm{OCH}_{3}\right)$, 4.30 (br s, $1 \mathrm{H}, \mathrm{OH}), 4.79$ (br s, $1 \mathrm{H}, \mathrm{OCH}), 7.23-7.37$ (m, $8 \mathrm{H}$, aryl-H),

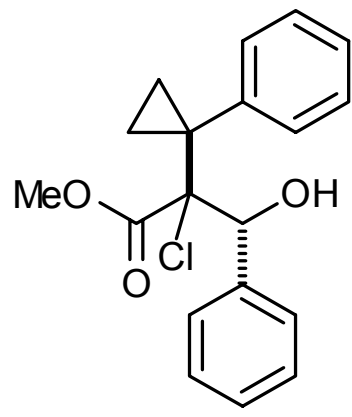
7.62-7.66 (m, $2 \mathrm{H}$, aryl-H) ppm. $-{ }^{13} \mathrm{C}-\mathrm{NMR}\left(62.9 \mathrm{MHz}, \mathrm{CDCl}_{3}, \mathrm{DEPT}\right): \delta=10.8$ (-, cPr-C), 14.3 (-, cPr-C), $31.8\left(\mathrm{C}_{\text {quat }}, \mathrm{cPr}-\mathrm{C}\right), 53.2\left(+, \mathrm{OCH}_{3}\right), 77.5(+, \mathrm{OCH}), 79.9\left(\mathrm{C}_{\text {quat }}, \mathrm{Cl}-\mathrm{C}\right), 127.5(+, 2 \mathrm{C}$, aryl-C), $127.6(+, 2$ C, aryl-C), 127.7 (+, aryl-C), 128.1 (+, aryl-C), 128.7 (+, 2 C, aryl-C), 132.4 $\left(+, 2 \mathrm{C}\right.$, aryl-C), $138.8\left(\mathrm{C}_{\text {quat }}, \mathrm{C}_{\mathrm{ipso}}\right), 140.4\left(\mathrm{C}_{\text {quat }}, \mathrm{C}_{\mathrm{ipso}}\right), 171.0\left(\mathrm{C}_{\text {quat }}, \mathrm{C}=\mathrm{O}\right) \mathrm{ppm} .-\mathrm{MS}(\mathrm{DCI}), m / z$ (\%): 678.6 (3) $\left[2 \mathrm{M}^{+}+\mathrm{NH}_{4}\right], 348.3(100)\left[\mathrm{M}^{+}+\mathrm{NH}_{4}\right] .-\mathrm{C}_{19} \mathrm{H}_{19} \mathrm{ClO}_{3}$ (330.8): calcd. C 68.98, $\mathrm{H}$ 5.79; found: C 69.00, H 5.51.

Methyl 2-chloro-2-[1-(4-fluorophenyl)cyclopropyl]-3-hydroxy-3-phenylpropionate

(75a-4-F-

$\left.\mathbf{C}_{6} \mathbf{H}_{4}\right)$ : From 5 (147 mg, $\left.1.0 \mathrm{mmol}\right)$, 4-F-PhMgCl (1 M in THF, $\left.0.5 \mathrm{~mL}, 1.0 \mathrm{mmol}\right)$, and benzaldehyde (111 mg, $1.05 \mathrm{mmol})$, and chromatographic purification on $25 \mathrm{~g}$ of silica gel $\left(R_{\mathrm{f}}=0.52\right.$, pentane $\left./ \mathrm{Et}_{2} \mathrm{O}=5: 11,5 \times 20 \mathrm{~cm}\right)$ and subsequent crystallization from Pentane/ $\mathrm{Et}_{2} \mathrm{O}$ gave $136 \mathrm{mg}$ (39\%) of 75a-4- $\mathrm{FC}_{6} \mathrm{H}_{4}$ as a colorless solid, m.p. $125-127^{\circ} \mathrm{C}$. - IR $(\mathrm{KBr})$ : $v=3548 \mathrm{~cm}^{-1}, 1731,1217,701,557 .-{ }^{1} \mathrm{H}-\mathrm{NMR}\left(\mathrm{CDCl}_{3}, 300 \mathrm{MHz}\right)$ : $\delta=0.86-0.96(\mathrm{~m}, 2 \mathrm{H}, \mathrm{cPr}-\mathrm{H}), 1.03-1.13$ (m, $1 \mathrm{H}, \mathrm{cPr}-\mathrm{H}), 1.45-1.54$ (m, $1 \mathrm{H}, \mathrm{cPr}-\mathrm{H}), 3.74$ (s, $\left.3 \mathrm{H}, \mathrm{OCH}_{3}\right), 4.40$ (br s, $\left.1 \mathrm{H}, \mathrm{OH}\right), 4.66$ (br s,

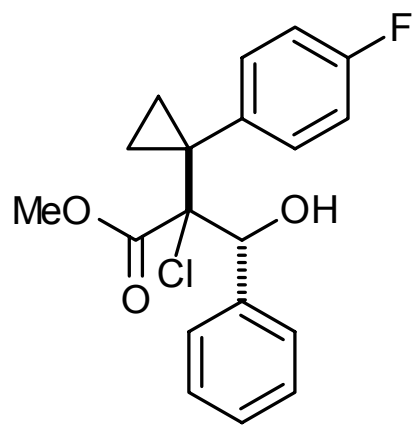
$1 \mathrm{H}, \mathrm{OCH}), 6.97-7.05(\mathrm{~m}, 2 \mathrm{H}$, aryl-H), 7.26-7.29 (m, $6 \mathrm{H}$, aryl-H), 7.61-7.67 (m, $1 \mathrm{H}$, aryl-H) ppm. $-{ }^{13} \mathrm{C}-\mathrm{NMR}\left(\mathrm{APT}, \mathrm{CDCl}_{3}, 75.5 \mathrm{MHz}\right): \delta=11.1$ (-, cPr-C), 14.8 (-, cPr-C), 31.0 (-, cPr-C), $53.4\left(+, \mathrm{OCH}_{3}\right), 77.9(+, \mathrm{OCH}), 79.8(-, \mathrm{Cl}-\mathrm{C}), 114.6\left(+, \mathrm{d},{ }^{2} J_{\mathrm{C}-\mathrm{F}}=21.1 \mathrm{~Hz}\right.$, aryl-C), $127.7(+, 2 \mathrm{C}$, aryl-C), 128.2 (+, aryl-C), 128.6 (+, 2 C, aryl-C), 134.2 (+, d, $J=8.3 \mathrm{~Hz}$, aryl-C), 136.23 (-, d, $J=$ $3.0 \mathrm{~Hz}$, aryl-C), 138.7 (-, aryl-C), 162.1 (-, d, ${ }^{1} J_{\mathrm{C}-\mathrm{F}}=247 \mathrm{~Hz}$, aryl-C-F ), 170.8 (-, C=O) ppm. $\mathrm{C}_{19} \mathrm{H}_{18} \mathrm{ClFO}_{3}$ (348.8): calcd. C 65.43, H 5.20; found: C 65.43, H 5.04. 
Methyl 2-chloro-3-hydroxy-2-(1-vinylcyclopropyl)-3-phenylpropionate (75a-Vin): To a solution of vinyl-magnesiumbromide (1.12 mL, $1.4 \mathrm{mmol}, 1.25 \mathrm{M})$ in THF $(10 \mathrm{~mL})$ was added $\mathrm{CuCN}(126 \mathrm{mg}, 1.4 \mathrm{mmol})$ at $-78^{\circ} \mathrm{C}$, the solution was stirred for $15 \mathrm{~min}$, then $\mathrm{BF}_{3} \cdot \mathrm{OEt}_{2}(100 \mathrm{mg}, 0.70 \mathrm{mmol})$ and 5 (147 mg, $1.00 \mathrm{mmol}$ ) was added and the solution was stirred at $-78^{\circ} \mathrm{C}$ for $1 \mathrm{~h}$ at which point benzaldehyde (148 $\mathrm{mg}, 1.4 \mathrm{mmol})$ was added and stirring was continued for $12 \mathrm{~h}$ at $20{ }^{\circ} \mathrm{C}$. Water $(10 \mathrm{~mL})$ and $\mathrm{Et}_{2} \mathrm{O}(20 \mathrm{~mL})$ was added,

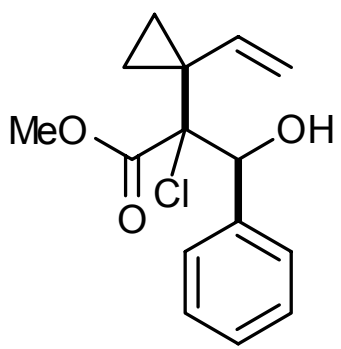
layers were separated and the aq. layer was extracted with $\mathrm{Et}_{2} \mathrm{O}(2 \times 10$ $\mathrm{mL}$ ) drying over $\mathrm{Na}_{2} \mathrm{SO}_{4}$, removal of the solvent in the vacuum and chromatografic purification of the residue on $20 \mathrm{~g}$ of silica $\left(1.5 \times 20 \mathrm{~cm}, R_{\mathrm{f}}=0.28\right.$ pentane $\left./ \mathrm{Et}_{2} \mathrm{O}=5: 1\right)$ to yield $55 \mathrm{mg}(20 \%)$ of syn isomer as a colorless oil and $122 \mathrm{mg}(44 \%)$ of anti isomer, $\left(R_{\mathrm{f}}=0.21\right.$ Pentane $\left./ \mathrm{Et}_{2} \mathrm{O}=5: 1\right)$ as a colorless oil. - Syn isomer: IR (film):= $v=3523,3086,3031,2951,1734,1453,1386,1264,1031,916,701 \mathrm{~cm}^{-1}$. $-{ }^{1} \mathrm{H}-\mathrm{NMR}\left(250 \mathrm{MHz}, \mathrm{CDCl}_{3}\right): \delta=0.51-0.60(\mathrm{~m}, 1 \mathrm{H}, \mathrm{cPr}-\mathrm{H}), 0.79-0.98$ (m, $2 \mathrm{H}, \mathrm{cPr}-\mathrm{H}), 1.13-1.23(\mathrm{~m}, 1 \mathrm{H}, \mathrm{cPr}-\mathrm{H}), 3.05\left(\mathrm{~d},{ }^{3} J=5.50 \mathrm{~Hz}, 1 \mathrm{H}\right.$, $\mathrm{OH}), 3.74\left(\mathrm{~s}, 3 \mathrm{H}, \mathrm{CH}_{3}\right), 5.04\left(\mathrm{~d},{ }^{3} \mathrm{~J}=10.0 \mathrm{~Hz}, 1 \mathrm{H}\right.$, vinyl-H), $5.10\left(\mathrm{~d},{ }^{3} \mathrm{~J}=\right.$

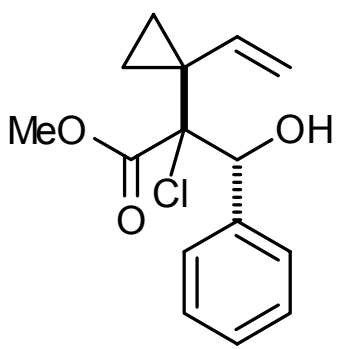
$3.7 \mathrm{~Hz}, 1 \mathrm{H}$, vinyl-H), 5.42 (d, $\left.{ }^{3} J=5.5 \mathrm{~Hz}, 1 \mathrm{H}, \mathrm{CH}\right), 6.48\left(\mathrm{dd},{ }^{3} J=17.0 \mathrm{~Hz},{ }^{2} J=10.1 \mathrm{~Hz}, 1 \mathrm{H}\right.$, vinyl-H), 7.27-7.37 (m, $3 \mathrm{H}$, aryl-H), 7.42-7.51 (m, $2 \mathrm{H}$, aryl-H). $-{ }^{13} \mathrm{C}-\mathrm{NMR}$ (DEPT, $62.9 \mathrm{MHz}$, $\left.\mathrm{CDCl}_{3}\right): \delta=11.0(-, \mathrm{cPr}-\mathrm{C}), 11.9(-, \mathrm{cPr}-\mathrm{C}), 27.3\left(\mathrm{C}_{\text {quat }}, \mathrm{cPr}-\mathrm{C}\right), 53.0\left(+, \mathrm{OCH}_{3}\right), 77.2(+, \mathrm{CH})$, $78.6\left(\mathrm{C}_{\text {quat }}, \mathrm{Cl}-\mathrm{C}\right), 114.4$ (-, vinyl-C), 127.5 (+, 2 C, aryl-C), 128.2 (+, aryl-C), 128.6 (+, 2 C, arylC ), $138.1\left(\mathrm{C}_{\text {quat }}, \mathrm{C}_{\mathrm{ipso}}\right), 138.7$ (+, vinyl-C), 170.2 (-, CO-C). - MS (DCI), m/z (\%): 578 (7) $\left[2 \mathrm{M}^{+}+\mathrm{NH}_{4}\right], 315(60)\left[\mathrm{M}^{+}+\mathrm{NH}_{3}+\mathrm{NH}_{4}\right], 298$ (100) $\left[\mathrm{M}^{+}+\mathrm{NH}_{4}\right]$. anti isomer: IR (film): $v=3523,3089,3032,2953,1718,1454,1266,1069,911,701 \mathrm{~cm}^{-1} .-{ }^{1} \mathrm{H}-\mathrm{NMR}(250 \mathrm{MHz}$, $\left.\mathrm{CDCl}_{3}\right): \delta=0.14-0.23(\mathrm{~m}, 1 \mathrm{H}, \mathrm{cPr}-\mathrm{H}), 0.38-0.56(\mathrm{~m}, 2 \mathrm{H}, \mathrm{cPr}-\mathrm{H}), 1.18-1.28(\mathrm{~m}, 1 \mathrm{H}, \mathrm{cPr}-\mathrm{H})$, 3.68 (br s, $1 \mathrm{H}, \mathrm{OH}), 3.87$ (s, $\left.3 \mathrm{H}, \mathrm{CH}_{3}\right), 4.98$ (d, ${ }^{3} \mathrm{~J}=17.0 \mathrm{~Hz}, 1 \mathrm{H}$, vinyl-H), 5.10 (d, ${ }^{3} \mathrm{~J}=6.5 \mathrm{~Hz}$, $1 \mathrm{H}$, vinyl-H), 5.52 (s, $1 \mathrm{H}, \mathrm{CH}-\mathrm{H}), 6.14$ (dd, ${ }^{3} J=16.3 \mathrm{~Hz},{ }^{2} J=10.5 \mathrm{~Hz}, 1 \mathrm{H}$, vinyl-H), 7.27-7.37 $\left(\mathrm{m}, 3 \mathrm{H}\right.$, aryl-H), 7.42-7.56 (m, $2 \mathrm{H}$, aryl-H). $-{ }^{13} \mathrm{C}-\mathrm{NMR}$ (DEPT, $\left.62.9 \mathrm{MHz}, \mathrm{CDCl}_{3}\right): \delta=9.84(-$, cPr-C), 13.86 (-, cPr-C), 26.5 ( $\left.\mathrm{C}_{\text {quat }}, \mathrm{cPr}-\mathrm{C}\right), 53.4$ (+, $\left.\mathrm{OCH}_{3}\right), 75.1$ (+, $\left.\mathrm{CH}-\mathrm{C}\right), 78.3$ ( $\left.\mathrm{C}_{\text {quat }}, \mathrm{Cl}-\mathrm{C}\right)$, 114.8 (-, vinyl-C), 127.3 (+, 2 C, aryl-C), 128.2 (+, aryl-C), 128.7 (+, 2 C, aryl-C ), $137.6(+$, 
vinyl-C), 137.7 ( $\mathrm{C}_{\text {quat }}, \mathrm{C}_{\text {ipso }}$ ), 172.1 (-, CO-C). - MS (DCI), $m / z(\%): 578$ (27) [2 $\left.\mathrm{M}^{+}+\mathrm{NH}_{4}\right], 298$ (100) $\left[\mathrm{M}^{+}+\mathrm{NH}_{4}\right] .-\mathrm{C}_{15} \mathrm{H}_{17} \mathrm{ClO}_{3}$ (280.8): calcd. C 64.17, H 6.17; found: C 64.27, H 5.88.

Methyl 2-chloro-3-hydroxy-3-phenyl-2-(1-phenyl-cyclo-propyl)propionate (75a-Ph): From phenylmagnesiumchloride $(0.55 \mathrm{~mL}, 1.1 \mathrm{mmol}, 2 \mathrm{M}), \mathrm{CuCN}(99 \mathrm{mg}, 1.1 \mathrm{mmol}), \mathrm{BF}_{3} \cdot \mathrm{OEt}_{2}$ (100 mg, $0.70 \mathrm{mmol}), 5$ (147 mg, $1.00 \mathrm{mmol})$ and benzaldehyde (148 mg, $1.4 \mathrm{mmol})$, chromatographic purification on $20 \mathrm{~g}$ of silica $(1.5 \times 20 \mathrm{~cm}$, $R_{\mathrm{f}}=0.45$ pentane $\left./ \mathrm{Et}_{2} \mathrm{O}=5: 1\right)$ yielded $39 \mathrm{mg}(12 \%)$ of $\operatorname{syn}$ isomer as a colorless oil and $77 \mathrm{mg}(23 \%)$ of anti isomer, $\left(R_{\mathrm{f}}=0.30\right.$ Pentane $/ \mathrm{Et}_{2} \mathrm{O}=$ 5:1) as a colorless oil. Anti isomer: (same as described before). - Syn isomer: IR (film): $v=3523,2961,1734,1456,1264,1069,1031,703 \mathrm{~cm}^{-}$ 1. - ${ }^{1} \mathrm{H}-\mathrm{NMR}\left(\mathrm{CDCl}_{3}, 250 \mathrm{MHz}\right): \delta=0.80-0.91(\mathrm{~m}, 1 \mathrm{H}, \mathrm{cPr}-\mathrm{H}), 1.05-$

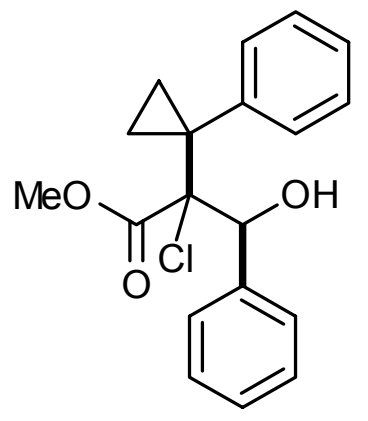
1.18 (m, $1 \mathrm{H}, \mathrm{cPr}-\mathrm{H}), 1.52-1.61$ (m, $1 \mathrm{H}, c \operatorname{Pr}-\mathrm{H}), 1.67-1.79$ (m, $1 \mathrm{H}, \mathrm{cPr}-\mathrm{H}), 2.56\left(\mathrm{~d},{ }^{3} J=5.7 \mathrm{~Hz}\right.$, $1 \mathrm{H}, \mathrm{OH}), 3.54\left(\mathrm{~s}, 3 \mathrm{H}, \mathrm{OCH}_{3}\right), 5.24\left(\mathrm{~d},{ }^{3} J=5.7 \mathrm{~Hz}, 1 \mathrm{H}, \mathrm{CH}\right), 7.24-7.36(\mathrm{~m}, 6 \mathrm{H}$, aryl-H), 7.39$7.43\left(\mathrm{~m}, 2 \mathrm{H}\right.$, aryl-H), 7.48-7.55 (m, $2 \mathrm{H}$, aryl-H) ppm. $-{ }^{13} \mathrm{C}-\mathrm{NMR}$ (DEPT, $\mathrm{CDCl}_{3}$, $62.9 \mathrm{MHz}): \delta=9.91$ (-, cPr-C), 12.7 (-, cPr-C), $32.4\left(\mathrm{C}_{\text {quat }}, \mathrm{cPr}-\mathrm{C}\right), 52.3\left(+, \mathrm{OCH}_{3}\right), 74.9(+$, $\left.\mathrm{OCH}_{3}\right), 82.2\left(\mathrm{C}_{\text {quat }}, \mathrm{Cl}-\mathrm{C}\right), 115.2(+$, aryl-C), $120.6(+$, aryl-C), $127.7(+, 2 \mathrm{C}$, aryl-C), $128.2(+$, $2 \mathrm{C}$, aryl-C), 128.2 (+, aryl-C), 128.7(+, 2 C, aryl-C), 129.6 (+, aryl-C), 131.9 (+, 2 C, aryl-C), $139.3\left(\mathrm{C}_{\text {quat, }}, \mathrm{C}_{\text {ipso }}\right), 141.4\left(\mathrm{C}_{\text {quat }}, \mathrm{C}_{\text {ipso }}\right), 168.6\left(\mathrm{C}_{\text {quat }}, \mathrm{C}=\mathrm{O}\right) \mathrm{ppm} .-\mathrm{MS}(\mathrm{DCI}), m / z(\%): 678.6(2)$ $\left[2 \mathrm{M}^{+}+\mathrm{NH}_{4}\right], 348.3(100)\left[\mathrm{M}^{+}+\mathrm{NH}_{4}\right]$.

Methyl 2-chloro-3-hydoxy-2-(1-isopropylcylopropyl)-3-(4-methoxyphenyl)propionate (75b-iPr): From 5 ( $800 \mathrm{mg}, 5.46 \mathrm{mmol}), \quad \mathrm{PrMgCl}^{2}\left(2 \mathrm{M}\right.$ in $\left.\mathrm{Et}_{2} \mathrm{O}, 3.00 \mathrm{~mL}, 6.00 \mathrm{mmol}\right)$, and $4-$ methoxybenzaldehyde $(818 \mathrm{mg}, 6.00 \mathrm{mmol})$, chromatographic purification on $30 \mathrm{~g}$ of silica gel $\left(3 \times 10 \mathrm{~cm}\right.$, pentane $/ \mathrm{Et}_{2} \mathrm{O}=3: 1, R_{\mathrm{f}}=0.13, p$ anisaldehyde) yielded $1.29 \mathrm{~g}$ (72\%) of $\mathbf{7 5 b}$-iPr as a colorless oil, which crystallized spontaneously, m. p. $95^{\circ} \mathrm{C}$. $-\mathrm{IR}(\mathrm{KBr}): v=3505,2968,2947$, $2841,1715,1613,1516,1252,1034,835 \mathrm{~cm}^{-1} .-{ }^{1} \mathrm{H}-\mathrm{NMR}\left(\mathrm{CDCl}_{3}\right.$, $250 \mathrm{MHz}$ ): $\delta=-0.12$ to 0.10 (m,1 H, cPr-H), $0.11-0.35$ (m, $2 \mathrm{H}, \mathrm{cPr}-\mathrm{H})$, $0.68\left(\mathrm{~d},{ }^{3} J=6.4 \mathrm{~Hz}, 3 \mathrm{H}, \mathrm{CH}_{3}\right), 0.77\left(\mathrm{~d},{ }^{3} J=6.4 \mathrm{~Hz}, 3 \mathrm{H}, \mathrm{CH}_{3}\right), 0.85-1.03$

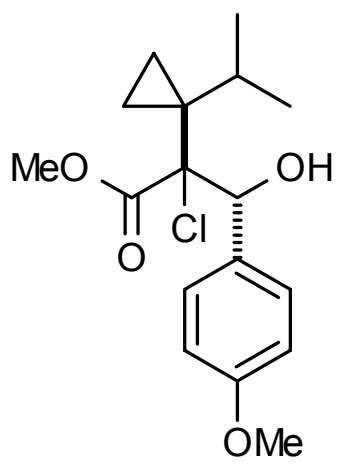
(m, $1 \mathrm{H}, \mathrm{cPr}-\mathrm{H}), 2.40\left(\mathrm{sept},{ }^{3} \mathrm{~J}=6.4 \mathrm{~Hz}, 1 \mathrm{H}, \mathrm{CH}\right), 3.81\left(\mathrm{~s}, 6 \mathrm{H}, 2 \times \mathrm{OCH}_{3}\right), 3.84(\mathrm{~s}, 1 \mathrm{H}, \mathrm{OH})$, 
$5.59(\mathrm{~s}, 1 \mathrm{H}, \mathrm{CH}), 6.85\left(\mathrm{~d},{ }^{3} \mathrm{~J}=8.8 \mathrm{~Hz}, 2 \mathrm{H}\right.$, aryl-H), $7.35\left(\mathrm{~d},{ }^{3} \mathrm{~J}=8.8 \mathrm{~Hz}, 2 \mathrm{H}\right.$, aryl-H) ppm. $-{ }^{13} \mathrm{C}-$ NMR (DEPT, $\left.\mathrm{CDCl}_{3}, 62.9 \mathrm{MHz}\right): \delta=5.1(-, \mathrm{cPr}-\mathrm{C}), 8.5(-, \mathrm{cPr}-\mathrm{C}), 20.9\left(+, \mathrm{CH}_{3}\right), 21.2\left(+, \mathrm{CH}_{3}\right)$, $26.1(+, \mathrm{CH}), 28.1\left(\mathrm{C}_{\text {quat }}, \mathrm{cPr}-\mathrm{C}\right), 53.0\left(+, \mathrm{OCH}_{3}\right), 55.2\left(+, \mathrm{OCH}_{3}\right), 74.7(+, \mathrm{CH}), 82.0\left(\mathrm{C}_{\text {quat }}, \mathrm{Cl}-\mathrm{C}\right)$, 112.8 (+, 2 C, aryl-C), 129.8 (+, 2 C, aryl-C), $130.5\left(\mathrm{C}_{\text {quat }}, \mathrm{C}_{\mathrm{ipso}}\right), 159.5\left(\mathrm{C}_{\text {quat }}, \mathrm{C}_{\text {ipso }}\right), 172.5\left(\mathrm{C}_{\text {quat }}\right.$, $\mathrm{C}=\mathrm{O}$ ) ppm. $-\mathrm{C}_{17} \mathrm{H}_{23} \mathrm{ClO}_{4}$ (326.8): calcd. C 62.48, H 7.09; found: C 62.53, H 6.80.

Methyl 2-chloro-3-hydroxy-2-(1-isopropylcyclopropyl)-3-(1,3-benzodioxole-5-yl)propionate (75c-

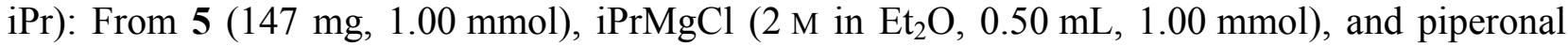
(158 mg, $1.05 \mathrm{mmol}$ ), chromatographic purification on $20 \mathrm{~g}$ of silica gel $\left(3 \times 10 \mathrm{~cm}\right.$, pentane $/ \mathrm{Et}_{2} \mathrm{O}=2: 1, R_{\mathrm{f}}=0.31, p$-anisaldehyde $)$ yielded $264 \mathrm{mg}(78 \%)$ of $75 \mathrm{c}-\mathrm{iPr}$ as a colorless solid, m. p. $113-115^{\circ} \mathrm{C}$. - IR $(\mathrm{KBr}): v=3544,2975,2872,1712,1440,1248,1041,929 \mathrm{~cm}^{-1} .-{ }^{1} \mathrm{H}-$ NMR (250 MHz, $\left.\mathrm{CDCl}_{3}\right): \delta=0.04-0.18(\mathrm{~m}, 1 \mathrm{H}, \mathrm{cPr}-\mathrm{H}), 0.22-0.36(\mathrm{~m}$, $2 \mathrm{H}, \mathrm{cPr}-\mathrm{H}), 0.68$ (d, $\left.{ }^{3} J=7.0 \mathrm{~Hz}, 3 \mathrm{H}, \mathrm{CH}_{3}\right), 0.80$ (d, ${ }^{3} J=7.0 \mathrm{~Hz}, 3 \mathrm{H}$, $\mathrm{CH}_{3}$ ), 0.95-1.02 (m, $\left.1 \mathrm{H}, \mathrm{cPr}-\mathrm{H}\right), 2.41$ (sept, $\left.{ }^{3} J=7.0 \mathrm{~Hz}, 1 \mathrm{H}, \mathrm{iPr}-\mathrm{H}\right)$,

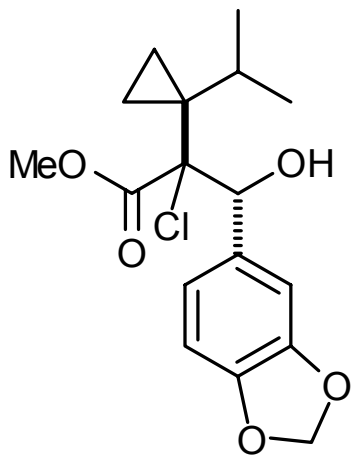
$3.38\left(\mathrm{~d},{ }^{3} J=5.7 \mathrm{~Hz}, 1 \mathrm{H}, \mathrm{OH}\right), 3.84\left(\mathrm{~s}, 3 \mathrm{H}, \mathrm{CH}_{3}\right), 5.59\left(\mathrm{~d},{ }^{3} J=5.7 \mathrm{~Hz}\right.$, $1 \mathrm{H}, \mathrm{CH}), 5.98\left(\mathrm{~s}, 2 \mathrm{H}, \mathrm{CH}_{2}\right), 6.72-6.94\left(\mathrm{~m}, 2 \mathrm{H}\right.$, aryl-H), 7.02 (s, $1 \mathrm{H}$, aryl-H) ppm. - ${ }^{13} \mathrm{C}-\mathrm{NMR}$ (DEPT, $\left.62.9 \mathrm{MHz}, \mathrm{CDCl}_{3}\right): \delta=5.25$ (-, cPr-C), 8.50 (-, cPr-C), 20.9 (+, iPr-C), 21.2 (+, iPr-C), $26.1(+, \mathrm{PPr}-\mathrm{C}), 28.0\left(\mathrm{C}_{\text {quat }}, \mathrm{cPr}-\mathrm{C}\right), 53.1\left(+, \mathrm{OCH}_{3}\right), 74.8(+, \mathrm{CH}), 81.8\left(\mathrm{C}_{\text {quat }}, \mathrm{Cl}-\mathrm{C}\right), 101.0(-$, $\left.\mathrm{CH}_{2}\right), 107.2\left(+\right.$, aryl-C), $109.0\left(+\right.$, aryl-C), $122.4\left(+\right.$, aryl-C ), $132.2\left(\mathrm{C}_{\text {quat }}\right.$, aryl-C), $147.0\left(\mathrm{C}_{\text {quat }}\right.$, aryl-C), $147.4\left(\mathrm{C}_{\text {quat}}\right.$, aryl-C), $172.4(-, \mathrm{C}=\mathrm{O})$ ppm. - MS (70 eV), m/z (\%): $340\left[\mathrm{M}^{+}\right](1), 151$ (100), 93 (64), 65 (66). $-\mathrm{C}_{17} \mathrm{H}_{21} \mathrm{ClO}_{5}$ (340.8): calcd. C 59.91, H 6.21; found: C 60.27, H 5.96.

Methyl 2-chloro-3-hydroxy-2-(1-isopropylcyclopropyl)-3-(4-dimethylaminophenyl)propionate (75d-iPr): From 5 (147 mg, $1.00 \mathrm{mmol})$, iPrMgCl $\left(2 \mathrm{M}\right.$ in $\left.\mathrm{Et}_{2} \mathrm{O}, 0.50 \mathrm{~mL}, 1.00 \mathrm{mmol}\right)$, and 4dimethylaminobenz-aldehyde $(156 \mathrm{mg}, 1.05 \mathrm{mmol})$, chromatographic purification on $20 \mathrm{~g}$ of silica gel $\left(3 \times 10 \mathrm{~cm}\right.$, pentane $/ \mathrm{Et}_{2} \mathrm{O}=1: 1, R_{\mathrm{f}}=0.40, p$-anisaldehyde $)$ yielded $270 \mathrm{mg}(80 \%)$ of $75 \mathbf{d}-\mathrm{iPr}$ as a colorless solid, m. p. $88^{\circ} \mathrm{C} .-\mathrm{IR}(\mathrm{KBr}): v=3529,2974,2953,2796,1716,1617,1525,1344$, 
1271, 945, $821 \mathrm{~cm}^{-1} .-{ }^{1} \mathrm{H}-\mathrm{NMR}\left(250 \mathrm{MHz}, \mathrm{CDCl}_{3}\right): \delta=0.03-0.14(\mathrm{~m}, 1 \mathrm{H}, \mathrm{cPr}-\mathrm{H}), 0.20-0.31$ (m, $2 \mathrm{H}, \mathrm{cPr}-\mathrm{H}), 0.67\left(\mathrm{~d},{ }^{3} J=6.9 \mathrm{~Hz}, 3 \mathrm{H}, \mathrm{CH}_{3}\right), 0.78\left(\mathrm{~d},{ }^{3} J=6.9 \mathrm{~Hz}, 3 \mathrm{H}, \mathrm{CH}_{3}\right), 0.90-0.98(\mathrm{~m}$, $1 \mathrm{H}, \mathrm{cPr}-\mathrm{H}$ ), 2.38 (sept, $\left.{ }^{3} J=6.9 \mathrm{~Hz}, 1 \mathrm{H}, \mathrm{iPr}-\mathrm{H}\right), 2.98$ (s, $6 \mathrm{H}, \mathrm{NMe}_{2}$ ), 3.19 (d, $\left.{ }^{3} J=5.8 \mathrm{~Hz}, 1 \mathrm{H}, \mathrm{OH}\right), 3.82\left(\mathrm{~s}, 3 \mathrm{H}, \mathrm{CH}_{3}\right), 5.55\left(\mathrm{~d},{ }^{3} J=5.8 \mathrm{~Hz}, 1 \mathrm{H}\right.$, $\mathrm{CH}), 6.63-6.72\left(\mathrm{~m}, 2 \mathrm{H}\right.$, aryl-H), 7.24-7.32 (m, $2 \mathrm{H}$, aryl-H) ppm. $-{ }^{13} \mathrm{C}-$ NMR (APT, 75.5 MHz, $\mathrm{CDCl}_{3}$ ): $\delta=5.36$ (-, cPr-C), 8.61 (-, cPr-C), 21.0 (+, iPr-C), 21.3 (+, iPr-C), 26.1 (+, iPr-C), 28.4 (-, cPr-C), $40.5(+$, $\left.\mathrm{NCH}_{3}\right), 53.0\left(+, \mathrm{OCH}_{3}\right), 75.1(+, \mathrm{CH}), 82.7(-, \mathrm{Cl}-\mathrm{C}), 111.5(+, 2 \mathrm{C}$, arylC), 126.2 (-, aryl-C), 129.3 (+, 2 C, aryl-C), 150.5 (-, aryl-C), 172.4 (-,

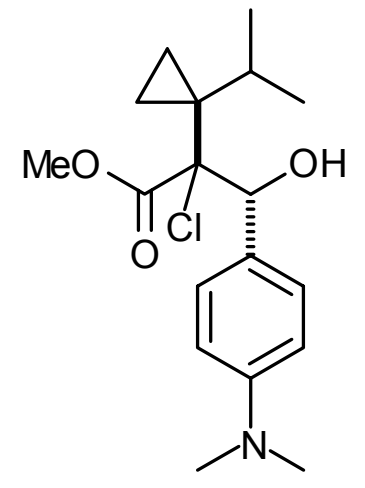
$\mathrm{C}=\mathrm{O})$ ppm. - MS (DCI), $m / z(\%): 679$ (2) $\left[2 \mathrm{M}^{+}+\mathrm{H}\right], 340(38)\left[\mathrm{M}^{+}+\mathrm{H}\right]$, 150 (100). $-\mathrm{C}_{18} \mathrm{H}_{26} \mathrm{ClNO}_{3}$ (339.9): calcd. C 63.61, H 7.71, N 4.12; found: $\mathrm{C}$ 63.91, H 7.46, N 4.21 .

Methyl 2-chloro-3-hydroxy-2-(1-isopropylcyclopro-pyl)-3-(3,4,5-trimethoxyphenyl)propionate (75e-iPr): From 5 (147 mg, $1.00 \mathrm{mmol})$, $\mathrm{iPrMgCl}\left(2 \mathrm{M}\right.$ in $\mathrm{Et}_{2} \mathrm{O}, 0.50 \mathrm{~mL}, 1.00 \mathrm{mmol}$ ), and 3,4,5trimethoxybenzalde-hyde $(206 \mathrm{mg}, 1.05 \mathrm{mmol})$, chromatographic purification on $20 \mathrm{~g}$ of silica gel $\left(3 \times 10 \mathrm{~cm}\right.$, pentane $/ \mathrm{Et}_{2} \mathrm{O}=1: 2, R_{\mathrm{f}}=$ $0.50, p$-anisaldehyde) yielded $278 \mathrm{mg}(72 \%)$ of $75 \mathrm{e}$-iPr as a colorless solid, m. p. $105^{\circ} \mathrm{C}$. - IR (KBr): $v=3533$, 3026, 2951, 2916, 2882, 1722, 1593, 1506, 1462, 1266, 1122, $846 \mathrm{~cm}^{-1}$. - ${ }^{1} \mathrm{H}-\mathrm{NMR}(300 \mathrm{MHz}$, $\left.\mathrm{CDCl}_{3}\right): \delta=0.05-0.13(\mathrm{~m}, 1 \mathrm{H}, \mathrm{cPr}-\mathrm{H}), 0.18-0.28$ (m, $\left.2 \mathrm{H}, \mathrm{cPr}-\mathrm{H}\right)$, $0.67\left(\mathrm{~d},{ }^{3} J=7.0 \mathrm{~Hz}, 3 \mathrm{H}, \mathrm{CH}_{3}\right), 0.77\left(\mathrm{~d},{ }^{3} J=7.0 \mathrm{~Hz}, 3 \mathrm{H}, \mathrm{CH}_{3}\right), 0.89-$

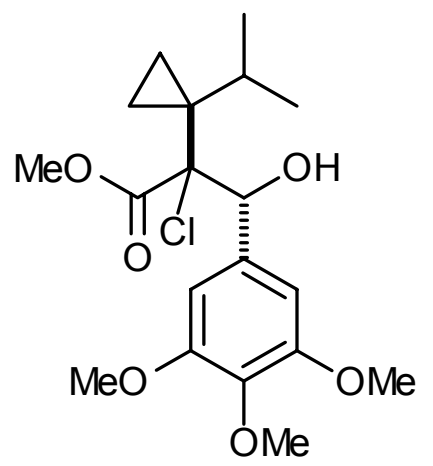
0.97 (m, $1 \mathrm{H}, \mathrm{cPr}-\mathrm{H}), 2.38$ (sept, ${ }^{3} J=7.0 \mathrm{~Hz}, 1 \mathrm{H}, \mathrm{iPr}-\mathrm{H}$ ), 3.32-3.42 (br s, $\left.1 \mathrm{H}, \mathrm{OH}\right), 3.80$ (s, $3 \mathrm{H}$, $\mathrm{CH}_{3}$ ), $3.82\left(\mathrm{~s}, 9 \mathrm{H}, \mathrm{CH}_{3}\right.$ ), 5.54 (s, $\left.1 \mathrm{H}, \mathrm{CH}\right), 6.67$ (s, $2 \mathrm{H}$, aryl-H) ppm. $-{ }^{13} \mathrm{C}-\mathrm{NMR}$ (APT, 75.5 $\left.\mathrm{MHz}, \mathrm{CDCl}_{3}\right): \delta=5.38$ (-, cPr-C), 8.49 (-, cPr-C), 21.0 (+, iPr-C), 21.2 (+, iPr-C), $26.2(+$, iPr-C), $28.1(-, \mathrm{cPr}-\mathrm{C}), 53.1\left(+, \mathrm{OCH}_{3}\right), 56.1\left(+, 2 \mathrm{C}, \mathrm{OCH}_{3}\right), 60.9\left(+, \mathrm{OCH}_{3}\right), 75.2(+, \mathrm{CH}), 81.7(-, \mathrm{Cl}-$ C), 106.2 (+, 2 C, aryl-C), 133.9 (-, aryl-C), 138.1 (-, aryl-C), 152.4 (-, aryl-C), 172.5 (-, C=O) ppm. - MS (DCI), m/z (\%): 790 (45) $\left[2 \mathrm{M}^{+}+\mathrm{NH}_{4}\right], 404$ (100) $\left[\mathrm{M}^{+}+\mathrm{NH}_{4}\right], 369$ (90). $\mathrm{C}_{19} \mathrm{H}_{27} \mathrm{ClO}_{6}$ (386.9): calcd. C 58.99, H 7.03; found: C 59.21, H 6.88. 
Methyl 2-chloro-3-hydroxy-2-(1-isopropylcyclopropyl)-3-(pentafluorophenyl)propionate (75f-iPr):

From 5 (147 mg, $1.00 \mathrm{mmol}), \quad i \operatorname{PrMgCl}\left(2 \mathrm{M}\right.$ in $\left.\mathrm{Et}_{2} \mathrm{O}, \quad 0.50 \mathrm{~mL}, 1.00 \mathrm{mmol}\right)$, and pentafluorobenzaldehyde (206 $\mathrm{mg}, 1.05 \mathrm{mmol}$ ), chromatographic purification on $20 \mathrm{~g}$ of silica gel $\left(3 \times 10 \mathrm{~cm}\right.$, pentane $/ \mathrm{Et}_{2} \mathrm{O}=3: 1, R_{\mathrm{f}}=0.50, p$-anisaldehyde $)$ yielded $302 \mathrm{mg}$ (78\%) of $\mathbf{7 5 f}$-iPr as a colorless solid, m. p. $74^{\circ} \mathrm{C}$. - IR (KBr): $v=3389,3032,2992,2962,2880,1753,1658,1528,1500,1237,999,946 \mathrm{~cm}^{-}$ 1. - ${ }^{1} \mathrm{H}-\mathrm{NMR}\left(300 \mathrm{MHz}, \mathrm{CDCl}_{3}\right): \delta=0.40-0.54(\mathrm{~m}, 3 \mathrm{H}, \mathrm{cPr}-\mathrm{H}), 0.67\left(\mathrm{~d},{ }^{3} \mathrm{~J}=\right.$ $\left.7.0 \mathrm{~Hz}, 3 \mathrm{H}, \mathrm{CH}_{3}\right), 0.78\left(\mathrm{~d},{ }^{3} J=7.0 \mathrm{~Hz}, 3 \mathrm{H}, \mathrm{CH}_{3}\right), 1.00-1.08$ (m, $\left.1 \mathrm{H}, \mathrm{cPr}-\mathrm{H}\right)$, 2.28 (sept, $\left.{ }^{3} J=7.0 \mathrm{~Hz}, 1 \mathrm{H}, \mathrm{iPr}-\mathrm{H}\right), 3.83\left(\mathrm{~s}, 3 \mathrm{H}, \mathrm{CH}_{3}\right), 4.07$ (d, $J=5.6 \mathrm{~Hz}, 1 \mathrm{H}$, $\mathrm{OH}$ ), $5.80\left(\mathrm{~d},{ }^{3} J=5.6 \mathrm{~Hz}, 1 \mathrm{H}, \mathrm{CH}\right) \mathrm{ppm} .-{ }^{13} \mathrm{C}-\mathrm{NMR}\left(\mathrm{APT}, 75.5 \mathrm{MHz}, \quad \mathrm{E}=\mathrm{CO}_{2} \mathrm{Me}\right.$ $\left.\mathrm{CDCl}_{3}\right): \delta=6.94$ (-, cPr-C), 8.71 (-, cPr-C), 21.1 (+, iPr-C), 21.5 (+, iPr-C), $26.6(+$, iPr-C), 29.5 $\left(-\right.$, cPr-C), $53.6\left(+, \mathrm{OCH}_{3}\right), 70.1(+, \mathrm{CH}), 80.7(-, \mathrm{Cl}-\mathrm{C}), 113.5$ (-, m, aryl-C), 135.8 (-, m, aryl-C), 139.1 (-, m, aryl-C), 142.8 (-, m, aryl-C), 143.6 (-, m, aryl-C), 146.9 (-, m, aryl-C), 170.6 (-, $\mathrm{C}=\mathrm{O}) \mathrm{ppm} .-\mathrm{MS}(\mathrm{DCI}), \mathrm{m} / z(\%): 790(10)\left[2 \mathrm{M}^{+}+\mathrm{NH}_{4}\right], 404(100)\left[\mathrm{M}^{+}+\mathrm{NH}_{4}\right] .-\mathrm{C}_{16} \mathrm{H}_{16} \mathrm{ClF}_{5} \mathrm{O}_{3}$ (386.7): calcd. C 49.69, H 4.17; found: C 49.81, H 3.96.

Methyl 2-chloro-3-hydroxy-2-(1-isopropylcyclopropyl)-3-(2-fluoro-4-nitrophenyl)propio-nate (75g-iPr): From 5 (147 mg, $1.00 \mathrm{mmol})$, iPrMgCl $\left(2 \mathrm{M}\right.$ in $\left.\mathrm{Et}_{2} \mathrm{O}, 0.50 \mathrm{~mL}, 1.00 \mathrm{mmol}\right)$, and 2fluoro-4-nitrobenzaldehyde $\quad(177 \mathrm{mg}, 1.05 \mathrm{mmol})$, chromatographic purification on $20 \mathrm{~g}$ of silica gel $\left(3 \times 10 \mathrm{~cm}\right.$, pentane $/ \mathrm{Et}_{2} \mathrm{O}=3: 1, R_{\mathrm{f}}=$ $0.45, p$-anisaldehyde) yielded $299 \mathrm{mg}(83 \%)$ of $\mathbf{7 5 g}$-iPr as a colorless solid, m. p. $98^{\circ}$ C. - IR (KBr): $v=3530,3199,2999,2978,2878,1717$, 1616, 1539, 1357, 1242, 876, $801 \mathrm{~cm}^{-1} .{ }^{1} \mathrm{H}-\mathrm{NMR}\left(300 \mathrm{MHz}, \mathrm{CDCl}_{3}\right): \delta$ $=0.11-0.20(\mathrm{~m}, 1 \mathrm{H}, \mathrm{cPr}-\mathrm{H}), 0.42-0.54(\mathrm{~m}, 2 \mathrm{H}, \mathrm{cPr}-\mathrm{H}), 0.62\left(\mathrm{~d},{ }^{3} J=\right.$ $\left.7.0 \mathrm{~Hz}, 3 \mathrm{H}, \mathrm{CH}_{3}\right), 0.83\left(\mathrm{~d},{ }^{3} J=7.0 \mathrm{~Hz}, 3 \mathrm{H}, \mathrm{CH}_{3}\right), 0.95-1.02(\mathrm{~m}, 1 \mathrm{H}$,

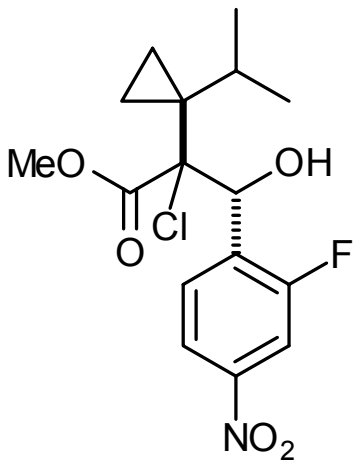
cPr-H), 2.25 (sept, $\left.{ }^{3} J=7.0 \mathrm{~Hz}, 1 \mathrm{H}, \mathrm{iPr}-\mathrm{H}\right), 3.74$ (s, $\left.3 \mathrm{H}, \mathrm{CH}_{3}\right), 4.35$ (br s, $\left.1 \mathrm{H}, \mathrm{OH}\right), 6.42$ (s, $1 \mathrm{H}$, $\mathrm{CH}), 7.26-7.31$ (m, $1 \mathrm{H}$, aryl-H), 7.58 (m, $1 \mathrm{H}$, aryl-H), 7.80 (m, $1 \mathrm{H}$, aryl-H) ppm. - ${ }^{13} \mathrm{C}-\mathrm{NMR}$ (APT, $\left.75.5 \mathrm{MHz}, \mathrm{CDCl}_{3}\right): \delta=7.88(-, \mathrm{cPr}-\mathrm{C}), 8.35$ (-, cPr-C), $21.2(+, \mathrm{iPr}-\mathrm{C}), 21.3(+, \mathrm{Pr}-\mathrm{C})$, $26.5(+, \mathrm{iPr}-\mathrm{C}), 29.4(-, \mathrm{cPr}-\mathrm{C}), 53.4\left(+, \mathrm{OCH}_{3}\right), 68.9(+, \mathrm{CH}), 81.5(-, \mathrm{Cl}-\mathrm{C}), 111.9\left(+, \mathrm{d},{ }^{2} J_{\mathrm{C}-\mathrm{F}}=\right.$ $26.4 \mathrm{~Hz}$, aryl-C), $119.4\left(+, \mathrm{d},{ }^{3} J_{\mathrm{C}-\mathrm{F}}=20.4 \mathrm{~Hz}\right.$, aryl-C), $129.9\left(-, \mathrm{d},{ }^{3} J_{\mathrm{C}-\mathrm{F}}=3.8 \mathrm{~Hz}\right.$, aryl-C), $132.0(+$, $\mathrm{d},{ }^{4} J_{\mathrm{C}-\mathrm{F}}=7.5 \mathrm{~Hz}$, aryl-C), $150.3\left(-, \mathrm{d},{ }^{2} J_{\mathrm{C}-\mathrm{F}}=15.1 \mathrm{~Hz}\right.$, aryl-C), $161.5\left(-, \mathrm{d},{ }^{1} J_{\mathrm{C}-\mathrm{F}}=252 \mathrm{~Hz}\right.$, aryl-C), 
$171.6(-, \mathrm{C}=\mathrm{O})$ ppm. $-\mathrm{MS}(\mathrm{DCI}), m / z(\%): 736(56)\left[2 \mathrm{M}^{+}+\mathrm{NH}_{4}\right], 377(100)\left[\mathrm{M}^{+}+\mathrm{NH}_{4}\right] .-$ $\mathrm{C}_{16} \mathrm{H}_{19} \mathrm{ClFNO}_{5}$ (359.8): calcd. C 53.41, H 5.32, N 3.89; found: C 53.58, H 5.11, N 3.97.

Methyl 2-chloro-3-hydroxy-2-(1-isopropylcyclopropyl)-3-(4-fluorophenyl)propionate (75h-iPr):

From $5(147 \mathrm{mg}, 1.00 \mathrm{mmol}),{ }_{i P r M g C l}\left(2 \mathrm{M}\right.$ in $\left.\mathrm{Et}_{2} \mathrm{O}, 0.50 \mathrm{~mL}, 1.00 \mathrm{mmol}\right)$, and $4-$ fluorobenzaldehyde (129 $\mathrm{mg}, 1.05 \mathrm{mmol})$, chromatographic purification on $20 \mathrm{~g}$ of silica gel $\left(3 \times 10 \mathrm{~cm}\right.$, pentane $/ \mathrm{Et}_{2} \mathrm{O}=3: 1, R_{\mathrm{f}}=0.35, p$ anisaldehyde) yielded $252 \mathrm{mg}$ ( $80 \%)$ of $\mathbf{7 5 h}$-iPr as a colorless solid, m. p. $78^{\circ} \mathrm{C}$. - IR (KBr): $v=3556,3002,2981,2955,2877,1716,1604,1511$, $1267,1218,840 \mathrm{~cm}^{-1} .-{ }^{1} \mathrm{H}-\mathrm{NMR}\left(250 \mathrm{MHz}, \mathrm{CDCl}_{3}\right): \delta=-0.13$ to 0.04 (m, $1 \mathrm{H}, \mathrm{cPr}-\mathrm{H}), 0.12-0.33$ (m, $2 \mathrm{H}, \mathrm{cPr}-\mathrm{H}), 0.67\left(\mathrm{~d},{ }^{3} J=7.0 \mathrm{~Hz}, 3 \mathrm{H}\right.$, $\left.\mathrm{CH}_{3}\right), 0.79\left(\mathrm{~d},{ }^{3} J=7.0 \mathrm{~Hz}, 3 \mathrm{H}, \mathrm{CH}_{3}\right), 0.86-1.00(\mathrm{~m}, 1 \mathrm{H}, \mathrm{cPr}-\mathrm{H}), 2.38$ (sept, ${ }^{3} J=7.0 \mathrm{~Hz}, 1 \mathrm{H}, \mathrm{iPr}-\mathrm{H}$ ), $3.43\left(\mathrm{~d},{ }^{3} J=5.6 \mathrm{~Hz}, 1 \mathrm{H}, \mathrm{OH}\right.$ ), 3.83 (s,

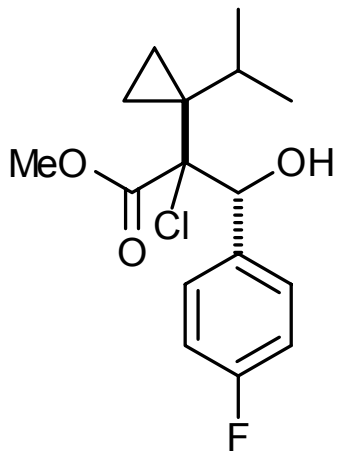
$\left.3 \mathrm{H}, \mathrm{CH}_{3}\right), 5.63\left(\mathrm{~d},{ }^{3} \mathrm{~J}=5.6 \mathrm{~Hz}, 1 \mathrm{H}, \mathrm{CH}\right), 6.97-7.08$ (m, $2 \mathrm{H}$, aryl-H), 7.37-7.46 (m, $2 \mathrm{H}$, aryl-H) ppm. $-{ }^{13} \mathrm{C}-\mathrm{NMR}\left(\mathrm{APT}, 50.3 \mathrm{MHz}, \mathrm{CDCl}_{3}\right.$ ): $\delta=5.09$ (-, cPr-C), 8.51 (-, cPr-C), 20.9 (+, iPr-C), 21.2 (+, iPr-C), 26.1 (+, iPr-C), $28.0\left(-\right.$, cPr-C), $53.1\left(+, \mathrm{OCH}_{3}\right), 74.4(+, \mathrm{CH}), 81.5\left(\mathrm{C}_{\text {quat }}, \mathrm{Cl}-\mathrm{C}\right)$, $114.4\left(+, \mathrm{d},{ }^{2} J_{\mathrm{C}-\mathrm{F}}=21.6 \mathrm{~Hz}, 2 \mathrm{C}\right.$, aryl-C), $130.4\left(+, \mathrm{d},{ }^{3} J_{\mathrm{C}-\mathrm{F}}=8.0 \mathrm{~Hz}, 2 \mathrm{C}\right.$, aryl-C), $134.1\left(-, \mathrm{d},{ }^{4} J_{\mathrm{C}-\mathrm{F}}\right.$ $=3.0 \mathrm{~Hz}$, aryl-C), $162.7\left(-, \mathrm{d},{ }^{1} J_{\mathrm{C}-\mathrm{F}}=247 \mathrm{~Hz}\right.$, aryl-C), $172.6(-, \mathrm{C}=\mathrm{O}) \mathrm{ppm} .-\mathrm{MS}(\mathrm{DCI}), \mathrm{m} / z(\%)$ : $296(100)\left[\mathrm{M}^{+}-\mathrm{F}+\mathrm{H}\right], 332(62)\left[\mathrm{M}^{+}+\mathrm{NH}_{4}\right] .-\mathrm{C}_{16} \mathrm{H}_{20} \mathrm{ClFO}_{3}$ (314.8): calcd. C 61.05, H 6.40; found C 61.30, H 6.21 .

Methyl 2-chloro-3-hydroxy-2-(1-isopropylcyclopropyl)-3-(4-nitrophenyl)propionate (75i-iPr): From $5(147 \mathrm{mg}, 1.00 \mathrm{mmol}), \quad{ }^{\mathrm{PrMgCl}}\left(2 \mathrm{M}\right.$ in $\left.\mathrm{Et}_{2} \mathrm{O}, 0.50 \mathrm{~mL}, 1.00 \mathrm{mmol}\right)$, and $4-$ nitrobenzaldehyde (159 mg, $1.05 \mathrm{mmol}$ ), chromatographic purification on $20 \mathrm{~g}$ of silica gel $\left(3 \times 10 \mathrm{~cm}\right.$, pentane $/ \mathrm{Et}_{2} \mathrm{O}=2: 1, R_{\mathrm{f}}=0.35, p$ anisaldehyde) yielded $250 \mathrm{mg}$ (73\%) of $75 \mathrm{i}-\mathrm{iPr}$ as a colorless solid, m. p. $138^{\circ} \mathrm{C}$. - IR (KBr): $v=3580,2972,2950,2877,1729,1515,1346,1250$, $1078,855 \mathrm{~cm}^{-1}$. $-{ }^{1} \mathrm{H}-\mathrm{NMR}\left(250 \mathrm{MHz}, \mathrm{CDCl}_{3}\right): \delta=-0.18$ to $0.02(\mathrm{~m}, 1 \mathrm{H}$, cPr-H), 0.16-0.39 (m, $2 \mathrm{H}, \mathrm{cPr}-\mathrm{H}), 0.67$ (d, $\left.{ }^{3} J=7.0 \mathrm{~Hz}, 3 \mathrm{H}, \mathrm{CH}_{3}\right), 0.82$ (d, $\left.{ }^{3} J=7.0 \mathrm{~Hz}, 3 \mathrm{H}, \mathrm{CH}_{3}\right), 0.95-1.00$ (m, $1 \mathrm{H}, \mathrm{cPr}-\mathrm{H}$ ), 2.46 (sept, ${ }^{3} J=$

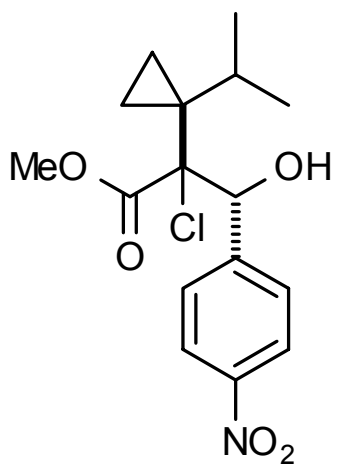
$7.0 \mathrm{~Hz}, \mathrm{iPr}-\mathrm{H}), 3.67\left(\mathrm{~d},{ }^{3} \mathrm{~J}=5.7 \mathrm{~Hz}, 1 \mathrm{H}, \mathrm{OH}\right), 3.85\left(\mathrm{~s}, 3 \mathrm{H}, \mathrm{CH}_{3}\right), 5.78\left(\mathrm{~d},{ }^{3} J=5.7 \mathrm{~Hz}, 1 \mathrm{H}, \mathrm{CH}\right)$, 
$7.61\left(\mathrm{~m}, 2 \mathrm{H}\right.$, aryl-H), $8.19\left(\mathrm{~m}, 2 \mathrm{H}\right.$, aryl-H) ppm. $-{ }^{13} \mathrm{C}-\mathrm{NMR}$ (DEPT, 62.9 MHz, $\left.\mathrm{CDCl}_{3}\right): \delta=$ 5.08 (-, cPr-C), 8.66 (-, cPr-C), 20.8 (+, iPr-C), $21.2(+, \mathrm{iPr}-\mathrm{C}), 26.2(+, \mathrm{iPr}-\mathrm{C}), 27.8\left(\mathrm{C}_{\text {quat }}, \mathrm{cPr}-\right.$ C), $53.3\left(+, \mathrm{OCH}_{3}\right), 74.1(+, \mathrm{CH}), 80.5\left(\mathrm{C}_{\text {quat }}, \mathrm{Cl}-\mathrm{C}\right), 122.5(+, 2 \mathrm{C}$, aryl-C), $129.8(+, 2 \mathrm{C}$, aryl-C), 145.4 ( $\mathrm{C}_{\text {quat }}$, aryl-C), 147.7 ( $\mathrm{C}_{\text {quat }}$, aryl-C), $172.4(-, \mathrm{C}=\mathrm{O})$ ppm. - MS (DCI), m/z (\%): 359 (5) $\left[\mathrm{M}^{+}+\mathrm{NH}_{4}\right], 210 / 208$ (32/100), 139 (93). $-\mathrm{C}_{16} \mathrm{H}_{20} \mathrm{ClNO}_{5}$ (341.8): calcd. C 56.23, H 5.90, N 4.10; found: C 56.38, H 5.80, N 4.00.

Methyl 2-chloro-3-hydroxy-2-(1-isopropylcyclopropyl)propionate (75j-iPr): From 5 (147 mg, $1.00 \mathrm{mmol}), \mathrm{iPrMgCl}\left(2 \mathrm{M}\right.$ in $\left.\mathrm{Et}_{2} \mathrm{O}, 0.5 \mathrm{~mL}, 1.0 \mathrm{mmol}\right)$, paraformaldehyde $(150 \mathrm{mg}, 5 \mathrm{mmol}$ of monomer) after $15 \mathrm{~h}$, Chromatografic purification on $20 \mathrm{~g}$ of silica $\left(1.5 \times 15 \mathrm{~cm}\right.$, pentane $\left./ \mathrm{Et}_{2} \mathrm{O}=3: 1, R_{\mathrm{f}}=0.35\right)$ yielded $135 \mathrm{mg}(61 \%)$ of 75j-iPr as colorless oil. - IR (film): $v=3464,2961,2875,1733,1436$, 1266, 1077, 1031, $733 \mathrm{~cm}^{-1} .-{ }^{1} \mathrm{H}-\mathrm{NMR}\left(250 \mathrm{MHz}, \mathrm{CDCl}_{3}\right): \delta=0.48-0.61$ (m, $2 \mathrm{H}, \mathrm{cPr}-\mathrm{H}), 0.78\left(\mathrm{~d},{ }^{3} J=2.1 \mathrm{~Hz}, 3 \mathrm{H}, \mathrm{CH}_{3}\right), 0.81\left(\mathrm{~d},{ }^{3} J=2.1 \mathrm{~Hz}, 3 \mathrm{H}\right.$,

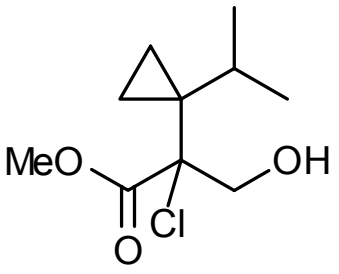
$\mathrm{CH}_{3}$ ), 0.85-0.93 (m, $\left.2 \mathrm{H}, \mathrm{cPr}-\mathrm{H}\right), 2.08$ (sept, $\left.{ }^{3} \mathrm{~J}=6.8 \mathrm{~Hz}, \mathrm{PPr}-\mathrm{H}\right), 2.37$ (br s, $\left.1 \mathrm{H}, \mathrm{OH}\right), 3.71$ (d, ${ }^{3} J=$ $12.3 \mathrm{~Hz}, \mathrm{CH}_{2}$ ), 3.81 (s, $3 \mathrm{H}, \mathrm{CH}_{3}$ ), 4.19 (d, ${ }^{3} J=12.3 \mathrm{~Hz}, \mathrm{CH}_{2}$ ). $-{ }^{13} \mathrm{C}-\mathrm{NMR}$ (DEPT, $62.9 \mathrm{MHz}$, $\left.\mathrm{CDCl}_{3}\right): \delta=7.76(-, \mathrm{cPr}-\mathrm{C}), 8.33$ (-, cPr-C), 21.2 (+, iPr-C), 27.8 (+, iPr-C), 29.1 ( $\left.\mathrm{C}_{\text {quat }}, \mathrm{cPr}-\mathrm{C}\right)$, $53.0\left(+, \mathrm{OCH}_{3}\right), 67.3\left(-, \mathrm{CH}_{2}\right), 80.6\left(\mathrm{C}_{\text {quat }}, \mathrm{Cl}-\mathrm{C}\right), 170.8$ (-, CO-C). - MS (DCI), m/z (\%): 458 (1) $\left[2 \mathrm{M}^{+}+\mathrm{NH}_{4}\right], 255(13)\left[\mathrm{M}^{+}+\mathrm{NH}_{3}+\mathrm{NH}_{4}\right], 238(100)\left[\mathrm{M}^{+}+\mathrm{NH}_{4}\right] .-\mathrm{C}_{10} \mathrm{H}_{17} \mathrm{ClO}_{3}$ (220.7): calcd. C 54.42, H 7.76; found: C 54.67, H 5.51.

2-Chloro-3-hydroxy-2-(1-isopropyl-cyclopropyl)-succinic acid 4-ethyl ester 1-methyl ester (75kiPr): From $5(147.0 \mathrm{mg}, 1.00 \mathrm{mmol})$, $i \operatorname{PrMgCl}\left(2 \mathrm{M}\right.$ in $\left.\mathrm{Et}_{2} \mathrm{O}, 0.50 \mathrm{~mL}, 1.00 \mathrm{mmol}\right)$, and ethylglyoxylate $(224 \mathrm{mg}, 50 \%$ solution in toluene, $1.1 \mathrm{mmol})$, chromatographic purification on $20 \mathrm{~g}$ of silica $\left(1.5 \times 15 \mathrm{~cm}\right.$, pentane $/ \mathrm{Et}_{2} \mathrm{O}$ $\left.=5: 1, R_{\mathrm{f}}=0.35\right)$ yielded $211 \mathrm{mg}(72 \%)$ of $75 \mathrm{i}-\mathrm{iPr}$ as colorless oil. $-\mathrm{IR}$ (film): $v=3496,2968,1734,1436,1268,1121,1029,763 \mathrm{~cm}^{-1} .-{ }^{1} \mathrm{H}-$ $\operatorname{NMR}\left(\mathrm{CDCl}_{3}, 250 \mathrm{MHz}\right): \delta=0.39-0.46(\mathrm{~m}, 1 \mathrm{H}, \mathrm{cPr}-\mathrm{H}), 0.65-0.74(\mathrm{~m}$, $1 \mathrm{H}, \mathrm{cPr}-\mathrm{H}), 0.76\left(\mathrm{~d},{ }^{3} \mathrm{~J}=6.8 \mathrm{~Hz}, 3 \mathrm{H}, \mathrm{CH}_{3}\right), 0.85\left(\mathrm{~d},{ }^{3} \mathrm{~J}=6.8 \mathrm{~Hz}, 3 \mathrm{H}\right.$,

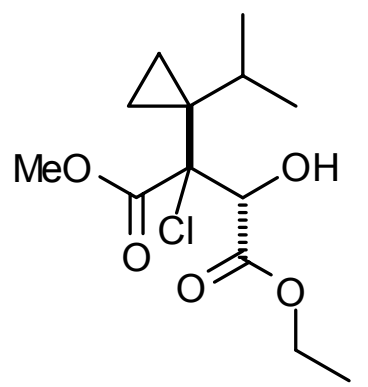
$\mathrm{CH}_{3}$ ), 1.01-1.09 (m, $2 \mathrm{H}, \mathrm{CH}_{2}$ ), 1.27 (t, ${ }^{3} J=7.0 \mathrm{~Hz}, 3 \mathrm{H}, \mathrm{CH}_{3}$ ), 2.39 (sept, ${ }^{3} J=6.8 \mathrm{~Hz}, \mathrm{Pr}-\mathrm{H}$ ), $3.20\left(\mathrm{~d},{ }^{3} \mathrm{~J}=6.8 \mathrm{~Hz}, 1 \mathrm{H}, \mathrm{OH}\right), 3.81\left(\mathrm{~s}, 3 \mathrm{H}, \mathrm{OCH}_{3}\right), 4.15-4.32\left(\mathrm{~m}, 2 \mathrm{H}, \mathrm{CH}_{2}\right), 5.03\left(\mathrm{~d},{ }^{3} \mathrm{~J}=6.8 \mathrm{~Hz}\right.$, 
$1 \mathrm{H}, \mathrm{CH}) .-{ }^{13} \mathrm{C}-\mathrm{NMR}\left(\mathrm{DEPT}, \mathrm{CDCl}_{3}, 62.9 \mathrm{MHz}\right): \delta=6.31(-, \mathrm{cPr}-\mathrm{C}), 9.73(-, \mathrm{cPr}-\mathrm{C}), 13.9(+$, $\left.\mathrm{CH}_{3}\right), 21.1(+, \mathrm{CH}, \mathrm{iPr}-\mathrm{C}), 21.7\left(\mathrm{C}_{\text {quat, }}, \mathrm{Pr}-\mathrm{C}\right), 26.6(+, \mathrm{CH}), 30.2\left(\mathrm{C}_{\text {quat }}, \mathrm{cPr}-\mathrm{C}\right), 53.0\left(+, \mathrm{OCH}_{3}\right)$, $62.7\left(-, \mathrm{CH}_{2}\right), 74.4(+, \mathrm{CH}), 80.3\left(\mathrm{C}_{\text {quat }}, \mathrm{Cl}-\mathrm{C}\right), 169.2\left(\mathrm{C}_{\text {quat }}, \mathrm{C}=\mathrm{O}\right), 171.2\left(\mathrm{C}_{\text {quat }}, \mathrm{C}=\mathrm{O}\right) \mathrm{ppm} .-\mathrm{MS}$ (DCI), $m / z$ (\%): $602.6(1)\left[2 \mathrm{M}^{+}+\mathrm{NH}_{4}\right], 310.3(100)\left[\mathrm{M}^{+}+\mathrm{NH}_{4}\right] .-\mathrm{C}_{13} \mathrm{H}_{21} \mathrm{ClO}_{5}$ (292.8): calcd. C 53.34, H 7.23; found: C 53.54, H 7.11.

Methyl 2-chloro-3-hydroxy-2-(1-isopropylcyclopropyl)-3-butyl propionate (751-iPr): From 5 $(147.0 \mathrm{mg}, 1.00 \mathrm{mmol}), i \operatorname{PrMgCl}\left(2 \mathrm{M}\right.$ in $\left.\mathrm{Et}_{2} \mathrm{O}, 0.5 \mathrm{~mL}, 1.0 \mathrm{mmol}\right)$, butyraldehyde (260 $\mathrm{mg}$, $5 \mathrm{mmol})$, chromatografic purification on $20 \mathrm{~g}$ of silica $(1.5 \times 15 \mathrm{~cm}$, pentane/ $\left.\mathrm{Et}_{2} \mathrm{O}=5: 1, R_{\mathrm{f}}=0.35\right)$ yielded $85 \mathrm{mg}(32 \%)$ of $75 \mathrm{l}-\mathrm{iPr}$ as colorless oil. - IR (film): $v=3530,2962,2873,1717,1467,1260,1123$, $1079,1033,1013 \mathrm{~cm}^{-1} .-{ }^{1} \mathrm{H}-\mathrm{NMR}\left(\mathrm{CDCl}_{3}, 250 \mathrm{MHz}\right): \delta=0.42-0.66(\mathrm{~m}$, $2 \mathrm{H}, \mathrm{cPr}-\mathrm{H}), 0.75\left(\mathrm{~d},{ }^{3} \mathrm{~J}=3.6 \mathrm{~Hz}, 3 \mathrm{H}, \mathrm{CH}_{3}\right), 0.79\left(\mathrm{~d},{ }^{3} \mathrm{~J}=3.6 \mathrm{~Hz}, 3 \mathrm{H}\right.$,

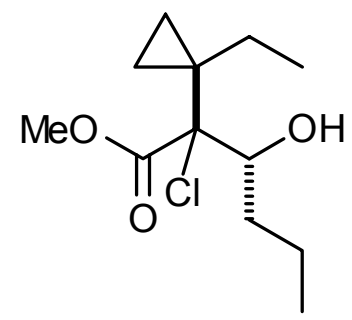
$\left.\mathrm{CH}_{3}\right), 0.87-1.11$ (m, $\left.5 \mathrm{H}\right), 1.38-1.56\left(\mathrm{~m}, 2 \mathrm{H}, \mathrm{CH}_{2}\right), 1.61-1.77$ (m, $2 \mathrm{H}$, $\mathrm{CH}_{2}$ ), 2.12 (sept, ${ }^{3} \mathrm{~J}=6.8 \mathrm{~Hz}, \mathrm{PPr}-\mathrm{C}$ ), $3.80\left(\mathrm{~s}, 3 \mathrm{H}, \mathrm{OCH}_{3}\right), 4.38-4.46(\mathrm{~m}, 1 \mathrm{H}, \mathrm{CH}) \cdot-{ }^{13} \mathrm{C}-\mathrm{NMR}$ (DEPT, $\left.\mathrm{CDCl}_{3}, 62.9 \mathrm{MHz}\right): \delta=7.16$ (-, cPr-C), 8.13 (-, cPr-C), $14.1\left(+, \mathrm{CH}_{3}\right), 19.0\left(-, \mathrm{CH}_{2}\right), 21.2$ $\left(+, \mathrm{CH}_{3}\right), 21.4\left(+, \mathrm{CH}_{3}\right), 26.6(+, \mathrm{CH}, \mathrm{iPr}), 28.0\left(\mathrm{C}_{\text {quat }}, \mathrm{cPr}-\mathrm{C}\right), 33.3\left(-, \mathrm{CH}_{2}\right), 52.9\left(+, \mathrm{OCH}_{3}\right), 73.0$ $(+, \mathrm{CH}), 81.8\left(\mathrm{C}_{\text {quat }}, \mathrm{Cl}-\mathrm{C}\right), 172.5\left(\mathrm{C}_{\text {quat }}, \mathrm{C}=\mathrm{O}\right) \mathrm{ppm}$. - MS (DCI), m/z (\%): 280.3 (100) $\left[\mathrm{M}^{+}+\mathrm{NH}_{4}\right] .-\mathrm{C}_{13} \mathrm{H}_{23} \mathrm{ClO}_{3}$ (262.8): calcd. C 59.42, $\mathrm{H}$ 8.82; found: C 59.34, H 8.54.

Methyl chloro(1-isopropylcyclopropyl)acetate (78-iPr): From 5 (294 mg, $2.0 \mathrm{mmol}), \mathrm{iPrMgCl}(2 \mathrm{M}$ in $\mathrm{Et}_{2} \mathrm{O}, 1.1 \mathrm{~mL}, 2.2 \mathrm{mmol}$ ) and aq. sat. $\mathrm{NH}_{4} \mathrm{Cl}$, chromatographic purification on $20 \mathrm{~g}$ of silica gel $\left(1.5 \times 10 \mathrm{~cm}\right.$, pentane $\left./ \mathrm{Et}_{2} \mathrm{O}=5: 1, R_{\mathrm{f}}=0.80\right)$ yielded $373 \mathrm{mg}(98 \%)$ of 78-iPr as a colorless oil. - IR (film): $v=3085,2960,2875$, $1758,1434,1168,1015,772 \mathrm{~cm}^{-1} .{ }^{1} \mathrm{H}-\mathrm{NMR}\left(\mathrm{CDCl}_{3}, 250 \mathrm{MHz}\right): \delta=0.61-$ 0.56 (m, $4 \mathrm{H}, \mathrm{cPr}-\mathrm{H}), 0.94-0.85$ (m, $\left.6 \mathrm{H}, 2 \mathrm{CH}_{3}\right), 1.64-1.72(\mathrm{~m}, 1 \mathrm{H}, \mathrm{CH})$,

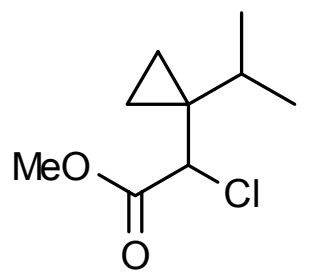
$3.73\left(\mathrm{~s}, 3 \mathrm{H}, \mathrm{OCH}_{3}\right), 4.38(\mathrm{~s}, 1 \mathrm{H}, \mathrm{CH}) \mathrm{ppm} .-{ }^{13} \mathrm{C}-\mathrm{NMR}\left(\mathrm{DEPT}, \mathrm{CDCl}_{3}, 62.9 \mathrm{MHz}\right): \delta=10.4(-$, cPr-C), 11.9 (-, cPr-C), 19.5 (+, iPr-C), 20.0 (+, iPr-C), 28.1 ( $\left.\mathrm{C}_{\text {quat }}, \mathrm{cPr}-\mathrm{C}\right), 31.3$ (+, iPr-C), 52.6 $(+, \mathrm{C}-\mathrm{Cl}), 62.7\left(+, \mathrm{OCH}_{3}\right), 169.3\left(\mathrm{C}_{\text {quat }}, \mathrm{C}=\mathrm{O}\right) \mathrm{ppm} . \quad-\mathrm{MS}(\mathrm{DCI}), \mathrm{m} / z(\%): 225.3$ (50) $\left[\mathrm{M}^{+}+\mathrm{NH}_{3}+\mathrm{NH}_{4}\right], 208.2(100)\left[\mathrm{M}^{+}+\mathrm{NH}_{4}\right]$. 
Methyl chloro-(1-vinylcyclopropyl)acetate (78-Vin): To a solution of vinylmagnesium bromide (4.78 $\mathrm{mL}, 9.56 \mathrm{mmol}, 2 \mathrm{M})$ in THF $(40 \mathrm{~mL})$ was added at $-78^{\circ} \mathrm{C} \mathrm{CuCN}(855 \mathrm{mg}, 9.55 \mathrm{mmol})$, the mixture was stirred for $15 \mathrm{~min}$, then $\mathrm{BF}_{3} \cdot \mathrm{OEt}_{2}(600 \mu \mathrm{l}, 4.78 \mathrm{mmol})$ and $\mathbf{5}$ $(1.00 \mathrm{~g}, 6.82 \mathrm{mmol})$ were added, and the mixture was stirred at $-78^{\circ} \mathrm{C}$ for $3 \mathrm{~h}$. Water $(20 \mathrm{~mL})$ was added at this temperature and the mixture was allowed to warm to $20{ }^{\circ} \mathrm{C}$. Extraction of the aq. phase with $\mathrm{Et}_{2} \mathrm{O}$

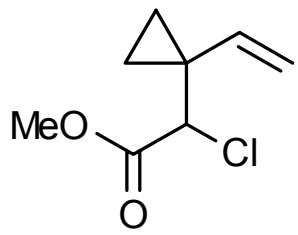
$(3 \times 30 \mathrm{~mL})$, drying over $\mathrm{Na}_{2} \mathrm{SO}_{4}$, removal of the solvent in vacuo and chromatographic purification of the residue on $20 \mathrm{~g}$ of silica gel $\left(1.5 \times 20 \mathrm{~cm}\right.$, pentane $/ \mathrm{Et}_{2} \mathrm{O}=100: 1, R_{\mathrm{f}}=0.42$ [pentane $/ \mathrm{Et}_{2} \mathrm{O}=10: 1$ ]) yielded $957 \mathrm{mg}(80 \%)$ of 78 -Vin as a colorless oil. - IR (film): $v=3087$, 3010, 2955, $1756(\mathrm{C}=\mathrm{O}), 1699,1653,1641,1436,1289,1195,1168,1014,908,808,668 \mathrm{~cm}^{-1}$. ${ }^{1} \mathrm{H}-\mathrm{NMR}\left(250 \mathrm{MHz}, \mathrm{CDCl}_{3}\right): \delta=0.85-1.13(\mathrm{~m}, 4 \mathrm{H}, \mathrm{cPr}-\mathrm{H}), 3.78\left(\mathrm{~s}, 3 \mathrm{H}, \mathrm{OCH}_{3}\right), 4.01(\mathrm{~s}, 1 \mathrm{H}$, $\mathrm{CH}$ ), $5.02\left(\mathrm{dd},{ }^{3} J=17.3,{ }^{2} J=0.7 \mathrm{~Hz}, 1 \mathrm{H}\right.$, vinyl-H), $5.06\left(\mathrm{dd},{ }^{3} J=10.6,{ }^{2} J=0.7 \mathrm{~Hz}, 1 \mathrm{H}\right.$, vinylH), $6.05\left(\mathrm{dd},{ }^{3} J=17.3 \mathrm{~Hz},{ }^{3} J=10.6 \mathrm{~Hz}, 1 \mathrm{H}\right.$, vinyl-H) ppm. $-{ }^{13} \mathrm{C}-\mathrm{NMR}$ (DEPT, $62.9 \mathrm{MHz}$, $\left.\mathrm{CDCl}_{3}\right): \delta=14.8(-, \mathrm{cPr}-\mathrm{C}), 15.1(-, \mathrm{cPr}-\mathrm{C}), 26.3\left(\mathrm{C}_{\text {quat }}, \mathrm{cPr}-\mathrm{C}\right), 52.6(+, \mathrm{CHCl}), 64.3\left(+, \mathrm{OCH}_{3}\right)$, 114.0 (-, vinyl-C), 136.8 (+, vinyl-C), $168.5\left(\mathrm{C}_{\text {quat, }} \mathrm{C}=\mathrm{O}\right) \mathrm{ppm}$. $-\mathrm{MS}(70 \mathrm{eV}), m / z(\%): 174(<1)$ $\left[\mathrm{M}^{+}\right], 173(1)\left[\mathrm{M}^{+}-\mathrm{H}\right], 139(100)\left[\mathrm{M}^{+}-\mathrm{Cl}\right], 107$ (12), 79 (35), 67 (10), 59 (8). - $\mathrm{C}_{8} \mathrm{H}_{11} \mathrm{ClO}_{2}$ (174.6): calcd. C 55.02, H 6.35, Cl 20.30; found C 55.09, H 6.31, Cl 20.24.

Methyl 2-bromo-3-hydoxy-2-(1-isopropylcylopropyl)-3-phenylpropionate (79a-iPr): From 1-Br (191.0 mg, $1.00 \mathrm{mmol}), i \operatorname{PrMgCl}\left(2 \mathrm{M}\right.$ in $\left.\mathrm{Et}_{2} \mathrm{O}, 0.50 \mathrm{~mL}, 1.00 \mathrm{mmol}\right)$, and benzaldehyde (111 mg, $1.05 \mathrm{mmol}$ ), chromatographic purification on $20 \mathrm{~g}$ of silica gel $\left(1.5 \times 15 \mathrm{~cm}\right.$, pentane $/ \mathrm{Et}_{2} \mathrm{O}=10: 1, R_{\mathrm{f}}=0.50$, MOPS $)$ yielded $249 \mathrm{mg}(73 \%)$ of 75a-iPr as a colorless oil. - IR (film): $v=2959 \mathrm{~cm}^{-1}, 2875$, $1738,1453,1268,1038,699 .-{ }^{1} \mathrm{H}-\mathrm{NMR}\left(300 \mathrm{MHz}, \mathrm{CDCl}_{3}\right): \delta=0.24-0.41$ (m, $3 \mathrm{H}, \mathrm{cPr}-\mathrm{H}), 0.47\left(\mathrm{~d},{ }^{3} \mathrm{~J}=6.9 \mathrm{~Hz}, 3 \mathrm{H}, \mathrm{CH}_{3}\right), 0.78\left(\mathrm{~d},{ }^{3} \mathrm{~J}=6.9 \mathrm{~Hz}, 3 \mathrm{H}\right.$,

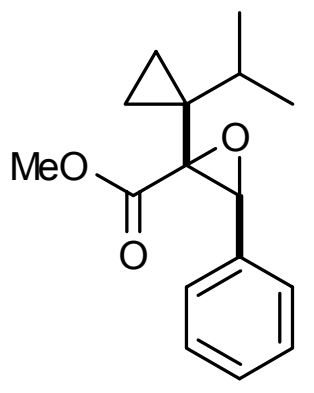
$\mathrm{CH}_{3}$ ), 0.84-0.94 (m, $\left.1 \mathrm{H}, \mathrm{cPr}-\mathrm{H}\right), 2.42$ (sept, ${ }^{3} J=6.9 \mathrm{~Hz}, 1 \mathrm{H}, \mathrm{CH}$ ), 3.78 (s, $3 \mathrm{H}, \mathrm{OCH}_{3}$ ), 4.31 (s, $1 \mathrm{H}), 7.28-7.40$ (m, $5 \mathrm{H}$, aryl-H) ppm. $-{ }^{13} \mathrm{C}-\mathrm{NMR}\left(75.5 \mathrm{MHz}, \mathrm{CDCl}_{3}, \mathrm{APT}\right): \delta=7.7$ (-, cPr-C), 9.3 (-, cPr-C), 19.9 (+, $\left.\mathrm{CH}_{3}\right), 20.0\left(+, \mathrm{CH}_{3}\right), 22.7$ (-, cPr-C), $30.1(+, \mathrm{CH}), 52.5\left(+, \mathrm{OCH}_{3}\right), 63.6$ (+, CH), 66.6 (-, Br-C), 127.2 (+, 2 C, aryl-C), 127.9 (+, 2 C, aryl-C), 128.0 (+, aryl-C), 134.3 (-, 
$\left.\mathrm{C}_{\text {ipso }}\right), 171.1(-, \mathrm{C}=\mathrm{O})$ ppm. $-\mathrm{MS}(\mathrm{DCI}), m / z(\%): 538.5(<1)\left[2 \mathrm{M}^{+}+\mathrm{NH}_{4}-2 \mathrm{HBr}\right], 278.2(100)$ $\left[\mathrm{M}^{+}+\mathrm{NH}_{4}-\mathrm{HBr}\right] .-\mathrm{C}_{16} \mathrm{H}_{20} \mathrm{O}_{3}(260.3)$ : calcd. C 73.82, H 7.74; found C 73.69, H 7.39.

Methyl 2-(1-isopropylcyclopropyl)-3-(4-methoxyphenyl)oxirane-2-carboxylate (79b-iPr): To a solution of 75b-iPr $(222 \mathrm{mg}, 679 \mu \mathrm{mol})$ in THF $(20 \mathrm{~mL})$ was added at $20^{\circ} \mathrm{C} \mathrm{KO} t \mathrm{Bu}(80 \mathrm{mg}$, $713 \mu \mathrm{mol})$, and the suspension was stirred at this temperature for $3 \mathrm{~h}$. Removal of all volatiles in vacuo and chromatographic purification of the residue on $20 \mathrm{~g}$ of silica gel $\left(3 \times 20 \mathrm{~cm}\right.$, pentane $/ \mathrm{Et}_{2} \mathrm{O}=3: 1, R_{\mathrm{f}}=0.75$, MOPS) yielded $122 \mathrm{mg}$ (62\%) of $\mathbf{7 9 b}$-iPr as a colorless oil. - IR (film): $v=2957,2874,2837,1734,1614,1516,1436,1251,1038,831 \mathrm{~cm}^{-1} .{ }^{1} \mathrm{H}-$ NMR $\left(\mathrm{CDCl}_{3}, 250 \mathrm{MHz}\right): \delta=0.21-0.50(\mathrm{~m}, 3 \mathrm{H}, \mathrm{cPr}-\mathrm{H}), 0.58\left(\mathrm{~d},{ }^{3} J=\right.$ $\left.7.0 \mathrm{~Hz}, 3 \mathrm{H}, \mathrm{CH}_{3}\right), 0.81\left(\mathrm{~d},{ }^{3} J=7.0 \mathrm{~Hz}, 3 \mathrm{H}, \mathrm{CH}_{3}\right), 0.85-1.03$ (m, $1 \mathrm{H}, \mathrm{cPr}-$

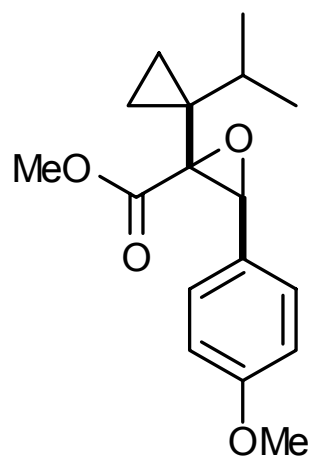
H), 2.65 (sept, $\left.{ }^{3} J=7.0, \mathrm{~Hz}, 1 \mathrm{H}, \mathrm{iPr}\right), 3.79\left(\mathrm{~s}, 3 \mathrm{H}, \mathrm{OCH}_{3}\right), 3.81\left(\mathrm{~s}, 3 \mathrm{H}, \mathrm{OCH}_{3}\right), 4.24(\mathrm{~s}, 1 \mathrm{H}, \mathrm{CH})$, $6.84\left(\mathrm{~d},{ }^{3} J=8.8 \mathrm{~Hz}, 2 \mathrm{H}\right.$, aryl-H), $7.28\left(\mathrm{~d},{ }^{3} J=8.8 \mathrm{~Hz}, 2 \mathrm{H}\right.$, aryl-H) ppm. $-{ }^{13} \mathrm{C}-\mathrm{NMR}$ (DEPT, $\left.\mathrm{CDCl}_{3}, 62.9 \mathrm{MHz}\right): \delta=7.7$ (-, cPr-C), 9.3 (-, cPr-C), 19.9 (+, $\left.\mathrm{CH}_{3}, 2 \mathrm{C}\right), 22.5\left(\mathrm{C}_{\text {quat }}, \mathrm{cPr}-\mathrm{C}\right), 30.1$ $(+, \mathrm{CH}), 52.4\left(+, \mathrm{OCH}_{3}\right), 55.2\left(+, \mathrm{OCH}_{3}\right), 63.4(+, \mathrm{CH}), 66.4\left(\mathrm{C}_{\text {quat }}, \mathrm{C}-\mathrm{O}\right), 113.4(+, 2 \mathrm{C}$, aryl-C), $126.1\left(\mathrm{C}_{\text {quat }}, \mathrm{C}_{\mathrm{ipso}}\right), 128.3\left(+, 2 \mathrm{C}\right.$, aryl-C), $159.3\left(\mathrm{C}_{\text {quat, }}, \mathrm{C}_{\mathrm{ipso}}\right), 171.1\left(\mathrm{C}_{\text {quat }}, \mathrm{C}=\mathrm{O}\right) \mathrm{ppm} .-\mathrm{MS}$ (DCI), $m / z$ (\%): 598.7 (1) $\left[2 \mathrm{M}^{+}+\mathrm{NH}_{4}\right], 308.3(100)\left[\mathrm{M}^{+}+\mathrm{NH}_{4}\right], 291.3(46)\left[\mathrm{M}^{+}+\mathrm{H}\right]$. $\mathrm{C}_{17} \mathrm{H}_{22} \mathrm{O}_{4}$ (290.4): calcd. C 70.32, H 7.64; found C 70.23, H 7.35. 


\subsubsection{Synthesis of 6-amino-3-azabicyclo[3,1,0]hexanes}

$N$-tert-Butoxycarbonyl-3-pyrroline (81): $N$-tert-butoxycarbonyl diallylamine (216 g, $1.1 \mathrm{~mol}$ ) was added over $1.5 \mathrm{~h}$ at $20{ }^{\circ} \mathrm{C}$ into a two litre round bottomed flask equipped with stirrer, dropping funnel and thermometer containing bis(tricyclohexylphosphine)benzylidene ruthenium(IV)dichloride (Grubbs catalyst, $905 \mathrm{mg}, 1.1 \mathrm{mmol}, 0.1 \mathrm{~mol} \%$ ) in dichloromethane $(1.2 \mathrm{~L})$. After $15 \mathrm{~h}$ of stirring at $20{ }^{\circ} \mathrm{C}$ the solvent was removed by<smiles>O=C(O)N1CC=CC1</smiles>
reduced pressure and the remaining dark oil was distilled ( $\mathrm{bp}=60{ }^{\circ} \mathrm{C}, 1$ torr) to get the pure product ( $184 \mathrm{~g}, 99 \%$ ) as a colourless oil which crystallized on standing at $0{ }^{\circ} \mathrm{C}$ to give the pure $\mathrm{N}$ tert-butoxycarbonyl-3-pyrroline. $-{ }^{1} \mathrm{H}-\mathrm{NMR}\left(\mathrm{CDCl}_{3}, 250 \mathrm{MHz}\right): \delta=1.43\left(\mathrm{~s}, 9 \mathrm{H}, \mathrm{CH}_{3}\right), 3.99-4.13$ $\left(\mathrm{m}, 4 \mathrm{H}, \mathrm{CH}_{2}\right), 5.60-5.78(\mathrm{~m}, 2 \mathrm{H}, \mathrm{CH}) .{ }^{13} \mathrm{C}-\mathrm{NMR}\left(\mathrm{CDCl}_{3}, 62.9 \mathrm{MHz}, \mathrm{DEPT}\right): \delta=28.3(+$, $\left.\mathrm{CH}_{3}\right), 48.6\left(-, \mathrm{CH}_{2}\right), 79.5\left(\mathrm{C}_{\text {quat }}\right.$, Boc-C), $133.9(+, \mathrm{CH}), 155.3\left(\mathrm{C}_{\text {quat, }}, \mathrm{CO}\right)$.

exo-6-(N,N-dibenzylamino)-3-aza-bicyclo[3,1,0] hexane-3-carboxylic acid tert-butyl ester (82):In to a four litre 3 necked flask equipped with a mechanical stirrer, reflux condenser and dropping funnel and thermometer, $N$-tert-butoxycarbonyl-3-pyrroline (169 g, $1.00 \mathrm{~mol})$ was taken in 1.5 litre of dry THF. To this solution $\mathrm{Ti}(\mathrm{O} i \mathrm{Pr})_{4}(313 \mathrm{~g}, 1.10 \mathrm{~mol})$ was added in one portion followed by addition of $\mathrm{CH}_{3} \mathrm{MgCl}(3 \mathrm{M}$ in THF, $367 \mathrm{~mL}, 1.1 \mathrm{~mol})$ at $10{ }^{\circ} \mathrm{C}$ within a period of $30 \mathrm{~min}$. The ice bath was removed and $\mathrm{N}, \mathrm{N}$-dibenzyl formamide (148 $\mathrm{g}, 0.65 \mathrm{~mol})$ was added at a time to the above solution. After stirring

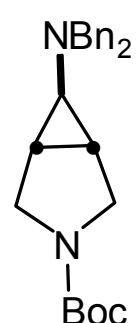

Boc for 15 min cyclohexyl magnesium bromide $\left(2 \mathrm{M}^{\text {in }} \mathrm{Et}_{2} \mathrm{O}, 1.10 \mathrm{~L}, 2.2 \mathrm{~mol}\right)$ was added slowly within a period of 4 hours. Then the resulting black solution was refluxed for $1 \mathrm{~h}$, cooled down to $0{ }^{\circ} \mathrm{C}$ and quenched with $200 \mathrm{ml}$ of $\mathrm{H}_{2} \mathrm{O}$ and stirred for $10 \mathrm{~h}$ in the presence of air. The reaction mixture was filtered and the solid material was washed with $\mathrm{Et}_{2} \mathrm{O}(3 \times 500 \mathrm{~mL})$, combined organic phahe dried over $\mathrm{MgSO}_{4}$, concentrated to $\sim 700 \mathrm{~mL}$ and the crude product was filtered through a small column $\left(\mathrm{Al}_{2} \mathrm{O}_{3}\right.$ with $2 \%$ water, $600 \mathrm{~g}, 10 \mathrm{~cm}$ height). The product was crystallized from pentane/ether $(10: 1,800 \mathrm{~mL})$ at $0{ }^{\circ} \mathrm{C}$ to get $(270 \mathrm{~g}, 71 \%)$ as a colourless crystals m.p. xx ${ }^{\circ} \mathrm{C} .-$ ${ }^{1} \mathrm{H}-\mathrm{NMR}\left(\mathrm{CDCl}_{3}, 250 \mathrm{MHz}\right): \delta=1.30-1.37,(\mathrm{~m}, 2 \mathrm{H}, \mathrm{CH}), 1.45\left(\mathrm{~s}, 9 \mathrm{H}, \mathrm{CH}_{3}\right), 1.58-1.60(\mathrm{~m}, 1 \mathrm{H}$, $N \mathrm{CH}$ ), 3.17-3.27 (m, 2 H, CH$)_{2}$, 3.29-3.47 (m, 2 H, $\mathrm{CH}_{2}$ ), 3.58-3.76 (m, $\left.4 \mathrm{H}, \mathrm{Bn}-\mathrm{H}\right), 7.20-7.39$ (m, $10 \mathrm{H}$, aryl-H). $-{ }^{13} \mathrm{C}-\mathrm{NMR}\left(\mathrm{CDCl}_{3}, 62.9 \mathrm{MHz}, \mathrm{DEPT}\right): \delta=25.2(+, \mathrm{CH}), 25.8(+, \mathrm{CH}), 28.4$ $\left(+, \mathrm{CH}_{3}\right), 47.5(+, \mathrm{CH}), 47.6\left(-, \mathrm{CH}_{2}\right), 47.8\left(-, \mathrm{CH}_{2}\right), 58.7$ (-, Bn-CH$), 79.1\left(\mathrm{C}_{\text {quat }}, \mathrm{Boc}-\mathrm{C}\right), 126.9$ 
(+, 2 C, aryl-C), $128.0\left(+, 4 \mathrm{C}\right.$, aryl-C), $129.3\left(+, 4 \mathrm{C}\right.$, aryl-C), $138.4\left(\mathrm{C}_{\text {quat }}, \mathrm{C}_{\text {ipso }}\right), 154.4\left(\mathrm{C}_{\text {quat }}\right.$, $\mathrm{CO})$.

exo-6-Amino-3-azabicyclo[3,1,0]hexane-3-carboxylic acid tert-butyl ester (84): To a $500 \mathrm{~mL}$ flask equipped with stirrer, flushed with nitrogen, $\mathrm{Pd} / \mathrm{C}(10 \% \mathrm{Pd}$, Merck, $2.3 \mathrm{~g})$ was taken followed by careful addition of methanol $(100 \mathrm{~mL})$. The resulting mixture was stirred in the presence of $\mathrm{H}_{2}$ (1 atm). After $30 \mathrm{~min}$ exo-6-(N,N-dibenzylamino)-3-tertbutoxycarbonyl-3-azabicyclo[3,1,0]hexane (37.8 g, 0.1 mol) dissolved in methanol $(150 \mathrm{~mL})$ was added to it. The resulting mixture was stirred under $\mathrm{H}_{2}$ until the $\mathrm{H}_{2}$ uptake became equal to the theoretical value $(4.48 \mathrm{~L})$. Then the crude product was filtered through a pad of Celite ${ }^{\circledR}$, the solvent was removed under reduced pressure. The product crystallised on standing at $5{ }^{\circ} \mathrm{C}(18.9 \mathrm{~g}, 96 \%)$ as a white solid, m.p. $=$

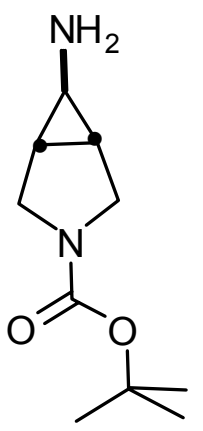
$51{ }^{\circ} \mathrm{C} .-{ }^{1} \mathrm{H}-\mathrm{NMR}\left(\mathrm{CDCl}_{3}, 250 \mathrm{MHz}\right): \delta=1.37\left(\mathrm{~s}, 9 \mathrm{H}, \mathrm{CH}_{3}\right), 1.39-1.42$, (m, $\left.2 \mathrm{H}, \mathrm{CH}\right), 1.65$ (br s, $2 \mathrm{H}, \mathrm{NH}), 2.03$ (br s, $1 \mathrm{H}, \mathrm{NCH}), 3.22-3.36\left(\mathrm{~m}, 2 \mathrm{H}, \mathrm{CH}_{2}\right), 3.38-3.52\left(\mathrm{~m}, 2 \mathrm{H}, \mathrm{CH}_{2}\right) .-{ }^{13} \mathrm{C}-\mathrm{NMR}$ $\left(\mathrm{CDCl}_{3}, 62.9 \mathrm{MHz}, \mathrm{DEPT}\right): \delta=25.2(+, \mathrm{CH}), 26.0(+, \mathrm{CH}), 28.4\left(+, \mathrm{CH}_{3}\right), 35.2(+, \mathrm{CH}), 47.5(-$, $\left.\mathrm{CH}_{2}\right), 47.8\left(-, \mathrm{CH}_{2}\right), 154.4\left(\mathrm{C}_{\text {quat }}, \mathrm{CO}\right)$.

exo-6-(N,N-dibenzylamino)-3-aza-bicyclo[3,1,0]hexane (83): To a suspension of exo-6-(N,Ndibenzylamino)-3-aza-bicyclo[3,1,0]hexane-3-carboxylic acid tert-butyl ester (18.9 g, $50 \mathrm{mmol})$ in $20 \mathrm{~mL}$ of EtOAc was added $\mathrm{HCl}(2 \mathrm{~N}$ in EtOAc, $250 \mathrm{~mL})$ over $15 \mathrm{~min}$. The resulting white slurry was stirred at $20^{\circ} \mathrm{C}$ for $15 \mathrm{~h}$. To this was added $10 \mathrm{~N} \mathrm{NaOH}(100 \mathrm{~mL})$, the organic layer was separated, aq. layer extracted with EtOAc $(3 \times 100 \mathrm{~mL})$, dried over $\mathrm{Na}_{2} \mathrm{SO}_{4}$, concentrated and the product crystallized on standing at room temperature to give $13.5 \mathrm{~g}(97 \%)$ as white solid, m.p $68^{\circ} \mathrm{C}$. - IR (KBr): $v=3283,3026,2919,2850$,

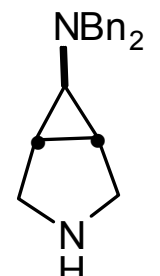
1495, 1453, 1093, 826, 746, $698 \mathrm{~cm}^{-1} .-{ }^{1} \mathrm{H}-\mathrm{NMR}\left(250 \mathrm{MHz}, \mathrm{CDCl}_{3}\right): \delta=1.25(\mathrm{~m}, 2 \mathrm{H}, \mathrm{CH})$, 1.48-1.55 (m, 1 H, NCH), 1.57 (br s, $1 \mathrm{H}, \mathrm{NH}), 2.72-2.91$ (m, $\left.4 \mathrm{H}, \mathrm{CH}_{2}\right), 3.63\left(\mathrm{~s}, 4 \mathrm{H}, \mathrm{Bn}_{-} \mathrm{CH}_{2}\right)$, 7.21-7.37 (m, $10 \mathrm{H}$, aryl-H). $-{ }^{13} \mathrm{C}-\mathrm{NMR}\left(62.9 \mathrm{MHz}, \mathrm{CDCl}_{3}\right): \delta=27.3(\mathrm{CH}), 44.1(\mathrm{NCH}), 48.7$ $\left(\mathrm{CH}_{2}\right), 58.7$ (Bn-CH$), 126.9$ (aryl-C), 128.0 (aryl-C), 129.4 (aryl-C), 138.5 (aryl-C).

exo-6-Benzyloxycarbonylaminoexo-3-aza-bicyclo[3,1,0] hexane-3-carboxylic acid tert-butyl-ester (85): To a solution of exo-6-Amino-3-azabicyclo[3,1,0]hexane-3-carboxylic acid tert-butyl ester 
(12.9 g, $65 \mathrm{mmol})$ in $200 \mathrm{~mL}$ of acetone was added $\mathrm{N}$-(benzyloxy-carbonyloxy)-succinamide (17.8 g, $71.5 \mathrm{mmol}$ ) and $\mathrm{NaHCO}_{3}$ (aq. $\left.1 \mathrm{~N}, 130 \mathrm{~mL}\right)$. This mixture was stirred for $15 \mathrm{~h}$ at $20^{\circ} \mathrm{C}$. Acetone was removed, aq. layer extracted with $\mathrm{Et}_{2} \mathrm{O}(3 \times 200$ $\mathrm{mL}$ ), dried over MgSO4. After removing solvent the crude product was crystallized from $\mathrm{Et}_{2} \mathrm{O}$ to yield the title compound (18.3 g, 85\%) as white solid, m.p. $=111-112^{\circ} \mathrm{C} .-\mathrm{IR}(\mathrm{KBr}): v=3289,3068,2974,2941,2872,1676,1540$, 1390, 1164, 1105, $745 \mathrm{~cm}^{-1} .-{ }^{1} \mathrm{H}-\mathrm{NMR}\left(300 \mathrm{MHz}, \mathrm{CDCl}_{3}\right): \delta=1.40(\mathrm{~s}, 9 \mathrm{H}$, Boc- $\left.\mathrm{CH}_{3}\right), 1.58-1.70$ (m, $\left.2 \mathrm{H}, \mathrm{CH}\right), 2.31-2.36$ (m, $\left.1 \mathrm{H}, \mathrm{NCH}\right), 3.27-2.39$ (m, 2

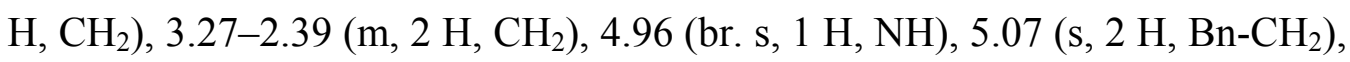
7.30-7.37 (m, $5 \mathrm{H}$, aryl-C). $-{ }^{13} \mathrm{C}-\mathrm{NMR}\left(75.5 \mathrm{MHz}, \mathrm{CDCl}_{3}, \mathrm{APT}\right): \delta=24.1(+$, $\mathrm{CH}), 25.3(+, \mathrm{CH}), 28.4\left(+, \mathrm{Boc}_{-} \mathrm{CH}_{3}\right), 33.3(+, \mathrm{CH}), 47.4\left(-, \mathrm{CH}_{2}\right), 47.7(-$,

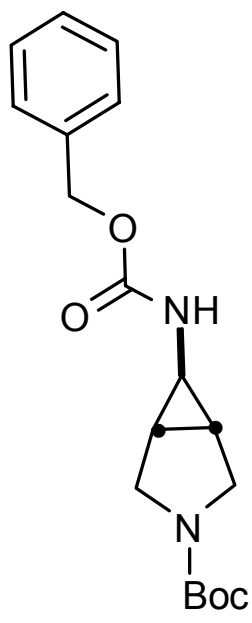
$\left.\mathrm{CH}_{2}\right), 66.8$ (-, Bn-CH 2$), 79.5$ (-, Boc-C), 128.2 (+, aryl-C), 128.5 (+, 4 C, aryl-C), 136.3 (-, $\mathrm{C}_{\mathrm{ipso}}$ ),

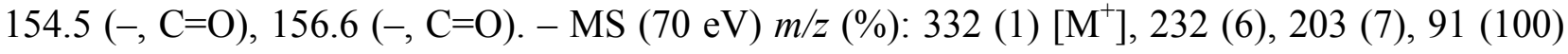
$\left[\mathrm{C}_{7} \mathrm{H}_{7}\right] \cdot-\mathrm{C}_{18} \mathrm{H}_{24} \mathrm{~N}_{2} \mathrm{O}_{4}(332.4)$ : calcd. C

exo-6-Benzyloxycarbonylamin-3-aza-bicyclo[3,1,0]hexane (86): To a suspension of exo-6Benzyloxycarbonylamino-3-aza-bicyclo[3,1,0]hexane-3-carboxylic acid tert-butyl-ester (17 g, 51 $\mathrm{mmol})$ in $25 \mathrm{~mL}$ of EtOAc was added $\mathrm{HCl}(2 \mathrm{~N}$ in EtOAc, $255 \mathrm{~mL})$ over $15 \mathrm{~min}$. The resulting white slurry was stirred at $20^{\circ} \mathrm{C}$ for $15 \mathrm{~h}$. To this was added $10 \mathrm{~N}$ $\mathrm{NaOH}(100 \mathrm{~mL})$, organic layer was separated, aq. layer extracted with EtOAc $(2 \times 100 \mathrm{~mL})$, combined organic layer was dried over $\mathrm{Na}_{2} \mathrm{SO}_{4}$, concentrated and the product crystallized from Pentane/ $\mathrm{Et}_{2} \mathrm{O}(1: 1)$ to give $11.6 \mathrm{~g}(98 \%)$ of product as a white solid, m.p. $=141{ }^{\circ} \mathrm{C} .-\mathrm{IR}(\mathrm{KBr}): v=3325,3166,2950,2874,1703$, 1567, 1297, 1179, 1037, $895 \mathrm{~cm}^{-1} .{ }^{1} \mathrm{H}-\mathrm{NMR}\left(300 \mathrm{MHz}, \mathrm{CDCl}_{3}\right): \delta=1.46(\mathrm{~s}, 1$ $\mathrm{H}, \mathrm{NH}), 1.52-1.58(\mathrm{~m}, 2 \mathrm{H}, \mathrm{CH}), 2.32-2.37$ (m, $1 \mathrm{H}, \mathrm{NCH}), 2.86\left(\mathrm{~m}, 2 \mathrm{H}, \mathrm{CH}_{2}\right)$, 3.10 (m, $2 \mathrm{H}, \mathrm{CH}_{2}$ ), 5.00 (br. s, $\left.1 \mathrm{H}, \mathrm{NH}\right), 5.05$ (s, $\left.2 \mathrm{H}, \mathrm{Bn}-\mathrm{CH}_{2}\right), 7.30-7.38$ (m,

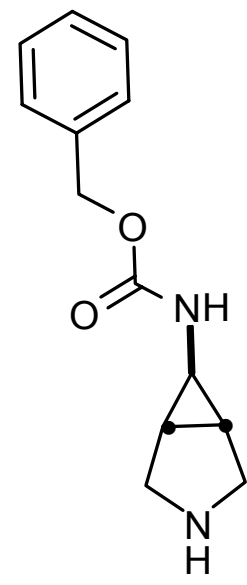
$5 \mathrm{H}$, aryl-H). $-{ }^{13} \mathrm{C}-\mathrm{NMR}\left(75.5 \mathrm{MHz}, \mathrm{CDCl}_{3}, \mathrm{APT}\right): \delta=26.1(+, 2 \mathrm{C}, \mathrm{CH}), 30.3(+, \mathrm{CH}), 48.5(-$, $\left.\mathrm{CH}_{2}\right), 66.7$ (-, Bn-CH$), 128.1$ (+, 2 C, aryl-C), 128.2 (+, aryl-C), 128.5 (+, 2 C, aryl-C), 136.4 (-,

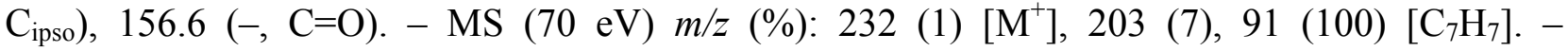
$\mathrm{C}_{13} \mathrm{H}_{16} \mathrm{~N}_{2} \mathrm{O}_{2}$ (232.3): calcd. C 67.22, H 6.94, N 12.06; found C 67.57, H 6.97, N 11.89. 
exo-6-(N,N-dibenzylamino)-3-pheny-3-aza-bicyclo[3,1,0]hexane (87): To an oven-dried Schlenk flask purged with nitrogen exo-6-( $N, N$-dibenzylamino)-3-aza-bicyclo[3,1,0]hexane (274 mg, $1.00 \mathrm{mmol})$ was taken with $\mathrm{CuI}(19 \mathrm{mg}, 10.0 \mathrm{~mol} \%)$ ethylene glycol (124 $\mathrm{mg}, 2 \mathrm{mmol}) \mathrm{K}_{3} \mathrm{PO}_{4}(425 \mathrm{mg}, 2.2 \mathrm{mmol})$ and iodobenzene (449 $\left.\mathrm{mg}, 2.2 \mathrm{mmol}\right)$ in 2 $\mathrm{mL}$ of isopropanol. The mixture was heated to $80{ }^{\circ} \mathrm{C}$ for $16 \mathrm{~h}$. The mixture was cooled to room temperature, diluted with $\mathrm{Et}_{2} \mathrm{O}(25 \mathrm{~mL})$, filtered over a pad of $\mathrm{Al}_{2} \mathrm{O}_{3}$, and concentrated in vacuo. The crude product was then purified by crystallization from pentane $/ \mathrm{Et}_{2} \mathrm{O}$ m.p. $=79^{\circ} \mathrm{C} .-\mathrm{IR}(\mathrm{KBr}): v=3022,2829,1602,1508,1508,1360$,

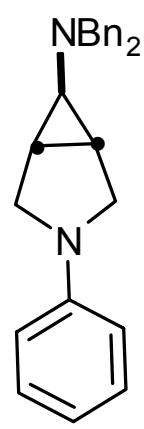
1162, 753, $702 \mathrm{~cm}^{-1} .{ }^{1} \mathrm{H}$ NMR $\left(300 \mathrm{MHz} \mathrm{CDCl}_{3}\right): \delta=1.50-1.56(\mathrm{~m}, 2 \mathrm{H}, \mathrm{CH})$, 2.70-2.75 (m, $1 \mathrm{H}, \mathrm{CH}), 3.08-3.16\left(\mathrm{~m}, 2 \mathrm{H}, \mathrm{CH}_{2}\right), 3.37$ (m, $\left.2 \mathrm{H}, \mathrm{CH}_{2}\right), 3.67$ (s, $\left.4 \mathrm{H}, \mathrm{Bn}-\mathrm{H}\right), 6.48$ $\left(\mathrm{d}, J=8.2 \mathrm{~Hz}, 2 \mathrm{H}\right.$, aryl-H), 6.66 (t, $J=\mathrm{xx} \mathrm{Hz}, 1 \mathrm{H}$, aryl-H), 7.16-7.38 (m, $12 \mathrm{H}$, aryl-H). $-{ }^{13} \mathrm{C}$ NMR (75.5 MHz, $\left.\mathrm{CDCl}_{3}\right): \delta=26.1(+, 2 \mathrm{C}, \mathrm{CH}), 48.2(+, \mathrm{CH}), 49.7\left(-, \mathrm{CH}_{2}\right), 59.0\left(-, \mathrm{Bn}^{-} \mathrm{CH}_{2}\right)$, 112.0 (+, 2 C, aryl-C), 116.0 (+, aryl-C), 127.0 (+, 2 C, aryl-C), 128.1 (+, 4 C, aryl-C), $129.1(+, 2$ C, aryl-C), 129.4 (+, 4 C, aryl-C), 138.7 (-, 2 C, $\mathrm{C}_{\mathrm{ipso}}$ ), 147.8 (-, $\mathrm{C}_{\mathrm{ipso}}$ ). - MS (DCI), m/z (\%): 709 $[2 \mathrm{M}+\mathrm{H}]^{+}(1), 372\left[\mathrm{M}+\mathrm{NH}_{4}\right]^{+}(8), 355\left[\mathrm{M}+\mathrm{H}^{+}\right](100) .-\mathrm{C}_{25} \mathrm{H}_{26} \mathrm{~N}_{2}$ (354): calcd. C 84.71, H 7.39, N 7.97, found; C 84.99, 7.16, 8.08.

3-pheny-3-aza-bicyclo[3,1,0]hexylamine (88): Into a $100 \mathrm{~mL}$ flame-dried flask flushed with nitrogen, $\mathrm{Pd} / \mathrm{C}(540 \mathrm{mg}, 10 \% \mathrm{Pd}$ w/w) was added, followed by $20 \mathrm{~mL}$ of DMA. This mixture was stirred under hydrogen atmosphere and after $30 \mathrm{~min}$ a solution of $\mathbf{8 7}(1.77 \mathrm{~g}, 5 \mathrm{mmol})$ in $25 \mathrm{~mL}$ of DMA was added with a syringe and stirring was continued at room temperature for $2 \mathrm{~d}$. Reaction mixture was filtered through a pad of Celite ${ }^{\circledR}$, and the solvent was removed in vacuo, the product was crystallized by standing at $4{ }^{\circ} \mathrm{C}$ to yield $793 \mathrm{mg}(91 \%)$ of 88 as a white solid, m.p. $=121^{\circ} \mathrm{c},-\mathrm{IR}(\mathrm{KBr}): v=3340,2935,2841$, 1629, 1600, 1505, 1448, 1355, 1160, $745 \mathrm{~cm}^{-1} .-{ }^{1} \mathrm{H}$ NMR $\left(300 \mathrm{MHz}, \mathrm{CDCl}_{3}\right): \delta=$

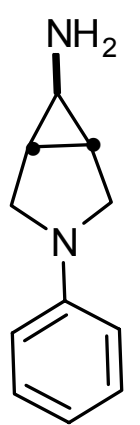
1.61-1.66 (m, 2 H, CH), 1.71 (br. s, 2 H NH), 2.20-2.25 (m, 1 H, CH), 3.19-3.27 (m, 2 $\left.\mathrm{H}, \mathrm{CH}_{2}\right), 3.44-3.57\left(\mathrm{~m}, 2 \mathrm{H}, \mathrm{CH}_{2}\right), 6.48(\mathrm{~d}, J=8.2 \mathrm{~Hz}, 2 \mathrm{H}$, aryl-H), 6.64 (t, $J=7.8 \mathrm{~Hz}, 1 \mathrm{H}$, aryl$\mathrm{H}), 7.16-7.24\left(\mathrm{~m}, 2 \mathrm{H}\right.$, aryl-H). $-{ }^{13} \mathrm{C} \mathrm{NMR}\left(75.5 \mathrm{MHz}, \mathrm{CDCl}_{3}\right): \delta=26.2(+, 2 \mathrm{C}, \mathrm{CH}), 35.8(+$, $\mathrm{CH}), 49.6\left(-, \mathrm{CH}_{2}\right), 112.0$ (+, $2 \mathrm{C}$, aryl-C), 116.0 (+, aryl-C), 129.0 (+, $2 \mathrm{C}$, aryl-C), 147.8 (-, $\left.\mathrm{C}_{\mathrm{ipso}}\right)$. - MS (70 eV) m/z (\%): $174(20)\left[\mathrm{M}^{+}\right], 106(12), 69$ (100). 


\section{Summary}

The work in this thesis starts with the advanced synthesis of methyl 2-chloro- and 2-bromo-2cyclopropyledeneacetate (4-Me and 5). The cyclopropanol derivative (31) obtained by Kulinkovich reductive cyclopropanation of 3,3-diethoxy propionate, was easily converted to 2-(1'mesyloxycyclopropyl)-acetic acid (28) by mesylating the alcohol group followed by oxidation. $\alpha$-Chlorination of the in situ formed acid chloride of the acid 28 with $\mathrm{N}$-chlorosuccinamide in presence of catalytic amount of $\mathrm{HCl}$ generated the chloride 32a which under basic elimination furnished the methyl 2-chloro-2-cyclopropyledeneacetate (5). In the similar way the bromo analogue 4-Me was prepared by using $N$-bromosuccinamide.

A series of different amidines (34b-f) was easily prepared from the corresponding nitriles which underwent Michael addition followed by domino transformation in presence of triethyl amine to afford cyclobutene annelated pyrimidinones $(\mathbf{3 5 a}-\mathbf{j})$. These pyrimidinones were reacted with phenyl vinyl sulfone to produce 6-sulfone substituted tetrahydroquinazolinones (37a-h) under thermal or microwave assisted conditions. Basic elimination of $\mathbf{3 7} \mathbf{a}-\mathbf{h}$ followed by hydrogenation led to 2-substituted 5,6,7,8-tetrahydroquinazolinones (39a-f). Alkylation of the tetrahydro- ring was done by use of base such as $n$-BuLi to get the alkylated product $(\mathbf{4 0}, \mathbf{4 2 a}, \mathbf{b})$. Nucleophilic substitution of $S \mathrm{Me}$ group of the compound $\mathbf{3 7} \mathbf{h}$ with different secondary amines produced the 2 amino derivative of 5,6,7,8-tetrahydroquinazolinones $46 \mathbf{a}-\mathbf{c}$.

Michael addition of indole onto methyl 2-chloro-2-cyclopropylideneacetate (5) in presence of a Lewis acid furnished the methyl 2-chloro-2-[1-(1H-indol-3-yl)cyclopropyl]acetate (55). The cyclopropanated tryptophane methyl ester 56 was prepared by nucleophilic substitution of the chloride 55 with sodium azide under phase transfer catalysis followed by subsequent reduction of the azido ester 57. The Pictet-Spengler reaction of the ester $\mathbf{5 6}$ with $p$-anisaldehde or piperonal produced the tetrahydro- $\beta$-carbolines $(\mathbf{5 9 a}, \mathbf{b})$ with greater efficiency than the corresponding noncyclopropanated precursor. In both cases products were obtained as a mixture of two diasteriomers (cis/trans $=1: 1.7$ for 59a and $1: 2.5$ for 59b) from which the trans-isomers were easily separated by crystallization from ethyl acetate. Treatment of the tetrahydro- $\beta$-carboline 59a with $n$-butyl isocyanate gave the cyclopropanated hydantoin lead structure to Tadalafil (25). The tetrahydro- $\beta$-carboline $\mathbf{5 9 b}$ was efficiently converted to the spirocyclopropanated analogue of Tadalafil (26) in two steps with a very good yield. 
Michael addition of carboxamides with compound 5 gave the spirocyclopropanated oxazoline carboxylate methyl esters (13a-e), from which free carboxylic acids $(\mathbf{6 4 a}-\mathbf{e})$ were obtained under basic hydrolysis. Coupling reaction of $\mathbf{6 4 a}-\mathbf{e}$ with different anilines in presence of HOAt/EDC and colidine gave the amide $\mathbf{6 8 a}-\mathbf{j}$ with very good yields. The coupling products with $\alpha$-hydroxy anilines under Mitsunobu reaction conditions $\left(\mathrm{Ph}_{3} \mathrm{P} / \mathrm{DEAD}\right)$ gave the benzoxazole derivatives of oxazolines $(69 \mathbf{a}-\mathbf{e})$. The bromide group present in the $N$-methylated products of 70a,b were reacted with varieties of amines under Buchwald reaction conditions to give the amino-aryl derivatives 71a-j. Similarly, Suzuki reaction conditions were successfully applied to synthesize the biary-derivatives of oxazolines $(\mathbf{7 2 a}-\mathbf{d})$.

Sequential Michael reaction of Grignard reagent and aldol reaction with the compound $\mathbf{5}$ produced the $\beta$-chloro alcohols 75a-l and in most cases single diasteriomers $\left(>97 \%\right.$, anti $\left.2 \mathrm{~S}^{*}, 2 \mathrm{R}^{*}\right)$ were obtained. The structure was deduced from a single crystal X-ray structure analysis. These $\beta$-chloro alcohols 75a-l are good precursors for the synthesis of $\alpha, \beta$-epoxyesters. The $\beta$-chloro alcohol 75biPr underwent clean cyclization under basic condition to produce diasteriomerically pure $\alpha, \beta-$ epoxyester 79b-iPr. The analogous $\alpha, \beta$-epoxyester 79a-iPr was obtained directly from methyl 2bromo-2-cyclopropylideneacetate (4-Me) upon treatment with isopropylmagnesium chloride and benzaldehyde. Apparently, the bromohydrine analogous to 75a-iPr is not stable under the work-up conditions and undergoes cyclization by intramolecular nucleophilic displacement.

The orthogonally protected diamine exo-6-( $N, N$-dibenzylamino)-3-aza-bicyclo[3,1,0]hexane-3carboxylic acid tert-butyl ester (82) was also prepared from $N$-tert-butoxycarbonyl-3-pyrroline (81). Selective deprotection of either the tert-butoxycarbonyl group or dibenzyl group provided monoprotected derivatives $\mathbf{8 3}$ and $\mathbf{8 4}$ with good yields. The phenylation reaction of $\mathbf{8 3}$ catalyzed by $\mathrm{CuI}$ was found to be more efficient than the corresponding $\operatorname{Pd}_{2}(\mathrm{dba})_{3}$ catalyzed reaction to produce $\mathbf{8 7}$ Unlike in the case of $\mathbf{8 4}$ the debenzylation reaction was successful in dimethy acetamide to give the product $\mathbf{8 8}$. 


\section{Zusammenfassung}

In der vorliegenden Arbeit wurde zunächst eine verbesserte Synthese von 2-Chlor- und 2-Brom-2cyclopropylidenessigsäuremethylester (4-Me und 5) vorgestellt. Hierzu wurde das CyclopropanolDerivat 31, das durch eine Kulinkovich-Reaktion von 3,3-Diethoxypropionsäuerester dargestellt wurde, durch Mesitylierung und nachfolgender Oxidation in die 1'-Mesityloxycyclopropansäure (28) überführt. $\alpha$-Chlorierung des in situ gebildeten Säurechlorids mit N-Chlorsuccinimid in Gegenwart katalytischer Mengen Chlorwasserstoff ergab das Chlorid 32a, welches durch basische Eliminierung 2-Chlor-2-cyclopropylidenessigsäuremethylester (5) ergibt. Durch die analoge Reaktion mit $N$-Bromosuccinimid wurde 4-Me dargestellt.

Eine Reihe verschiedener Amidine (34b-f) wurde aus den Nitrilen durch eine Michael-Addition mit nachfolgender Domino-Reaktion in Gegenwart von Triethylamin zu den Cyclobutenannelierten Pyrimidinonen (35a-j) synthetisiert. Diese wurden dann thermisch oder durch Mikrowellen mit Vinylsulfon zu den substituierten Tetrahydrochinolidinen (37a-h) umgesetzt. Basische Eliminierung gefolgt von Hydrogenolyse fuehrte $\mathrm{zu}$ den 2-substituierten 5,6,7,8Tetrahydrochinolidinen 39a-f. Alkylierung nach der Deprotonierung mit einer Base wie $n$-BuLi ergab die Produkte 40, 42a,b. Durch nucleophile Substitution der SMe-Gruppe in der Verbindung 37h mit verschiedenen sekundären Aminen konnten die 2-Amino-Derivate 46a-c erhalten werden. Mittels Michael-Addition von Indol an 2-Chlor-2-cyclopropylidenessigsäuremethylester (5) in Gegenwart einer Lewis-Säure wurden 2-Chlor-2-[1-(1H-indol-3-yl)-cyclopropyl)-essigester (55) dargestellt. Der cyclopropanierte Tryptophanester 56 wurde durch die nucleophile Substitution von 55 mit Natriumazid unter Phasentransferbedingungen und nachfolgender Reduktion erhalten. Die Pictet-Spengler-Reaktion mit $p$-Anisaldehyd oder Piperonal ergab die Tetrahydro- $\beta$-carboline 59a,b effizienter als der entsprechende nicht-cyclopropanierte Vorläufer. In beiden Fällen wurde eine Mischung aus zwei Diastereomeren (cis/trans $=1: 1.7$ fuer 59a bzw. $1: 2.5$ fuer 59b) isoliert. Das trans-Isomer konnte durch Kristallisation aus Essigester erhalten werden. Die Reaktion von 59a mit $n$-Butylisocyanate ergab die cyclopropanierte Hydantoin Leitstruktur für Tadalafil (25). Das Tetrahydro- $\beta$-carbolin 59b wurde in zwei Stufen mit guter Ausbeute in das spirocyclopropanierte Analogon von Tadalafil (26) ueberfuehrt. 
Eine weitere Michael-Addition von Carboxamiden mit Verbindung $\mathbf{5}$ ergab die spirocyclopropanierten Oxazolinecarbonsaeuremethylester 13a-e, aus denen die freie Carbonsaeure (64a-e) durch Hydrolyse erhalten wurde. Kupplung mit verschiedenen Anilinen in Gegenwart von HOAt/EDC und Colidin ergab die Amide 68a-j in guten Ausbeuten. Die Reaktion mit $\alpha$-Hydroxyanilinen unter Mitsunobu-Bedingungen $\left(\mathrm{Ph}_{3} \mathrm{P} / \mathrm{DEAD}\right)$ erbrachte die BenzoxazolDerivate 69a-e. Eine Buchwald-Reaktion der $N$-methylierten Produkte 70a,b mit einer Vielzahl von Aminen fuehrte zu den Aminoaryl-Derivaten 71a-j. Analog fuehrte die Suzuki-Reaktion erfolgreich zu den Biaryl-Oxazolinen 72a-d.

Die Addition von Grignard-Reagenzien an Verbindung 5 und nachfolgende Aldol-Reaktion fuehrte zu den $\beta$-Chloralkoholen 75a-I meist in Form eines einzigen Diastereomers ( $>$ 97\%, anti $\left.2 \mathrm{~S}^{*}, 2 \mathrm{R}^{*}\right)$. Die Struktur wird durch die Röntgenstruktur belegt. Die Verbindungen 75a-I sind hervorragende Ausgangsverbindungen zur Synthese von $\alpha, \beta$-Epoxyestern. Der $\beta$-Chloralkohol 75b-iPr konnte zu dem $\alpha, \beta$-Epoxyester 79b-iPr in Form eines einzigen Diastereomers cyclisiert werden. Der analoge $\alpha, \beta$-Epoxyester 79a-iPr wurde direkt aus 2-Brom-2cyclopropylidenessigsäuremethylester (4-Me) durch Reaktion mit Isobutylmagnesiumchlorid und Benzaldehyd erhalten. Offensichtlich ist das Bromhydrin nicht stabil und cyclisiert durch eine intramolekulare nucleophile Substitution.

Der orthogonal geschuetzte exo-6-( $N, N$-Dibenzylamino)-3-aza-bicyclo[3.1.0]hexan-3carbonsaeure-tert-butylester (82) wurde ebenfalls aus $N$-tert-Butoxycarbonyl-3-pyrrolin (81) hergestellt. Selektive Entfernung der tert-Butoxycarbonylgruppe bzw. der Dibenzylgruppe ergab die Derivate 83 und 84. Die Kupfer-katalysierte Phenylierung von 83 zu 87 erwies sich als effizienter als die entsprechende Palladium-katalysierte Reaktion. Im Gegensatz dazu war die Dibenzylierung von 84 zu 88 mit Dimethylacetamid erfolreich. 


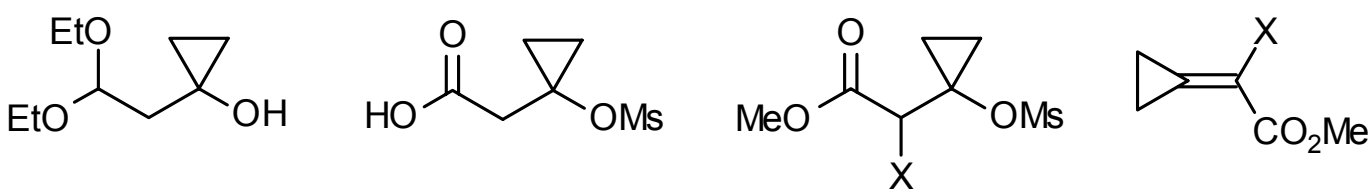

28

32a: $X=\mathrm{Cl}$

5: $\mathrm{X}=\mathrm{Cl}$

32b: $X=\mathrm{Br}$

4-Me: $\mathrm{X}=\mathrm{Br}$<smiles>[R]c1nc2c(c(=O)[nH]1)CC2</smiles>

$35 a-i$<smiles>[R]c1nc2c(c(=O)[nH]1)CC(S(=O)(=O)c1ccccc1)CC2</smiles>

$37 a-h$<smiles>[R]c1nc2c(c(=O)[nH]1)CCCC2</smiles>

$39 a-f$<smiles>[R]C1CCc2nc(-c3ccccc3)[nH]c(=O)c2C1</smiles>

42a,b<smiles>CC1Cc2nc(-c3ccccc3)[nH]c(=O)c2C[C@H]1S(=O)(=O)[GeH2]</smiles>

44<smiles>COC(=O)C(Cl)C1(c2c[nH]c3ccccc23)CC1</smiles>

55<smiles>CC1CC(S(=O)(=O)c2ccccc2)Cc2c1nc(-c1ccccc1)n(C(C)(C)C)c2=O</smiles>

40<smiles>COC(=O)C(N)C1(c2c[nH]c3ccccc23)CC1</smiles>

56<smiles></smiles>

46a-c<smiles></smiles>

$59 a, b$<smiles>CCCCN1C(=O)C2N(C1=O)C(c1ccc(OC)cc1)c1[nH]c3ccccc3c1C21CC1</smiles><smiles>CN1CC(=O)N2C(c3ccc4c(c3)OCO4)c3[nH]c4ccccc4c3C3(CC3)C2C1=O</smiles> 

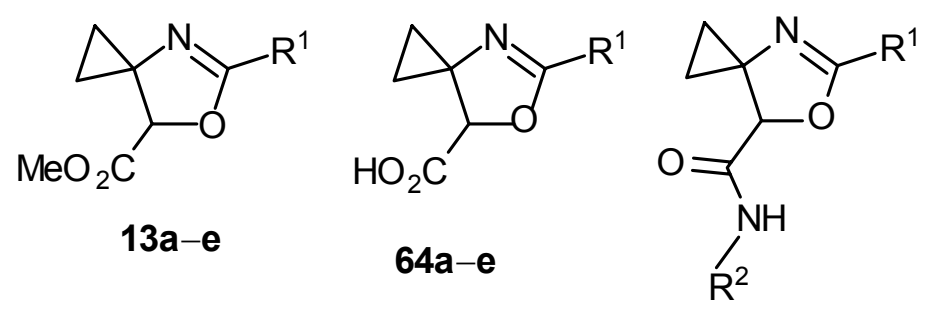

$68 a-j$

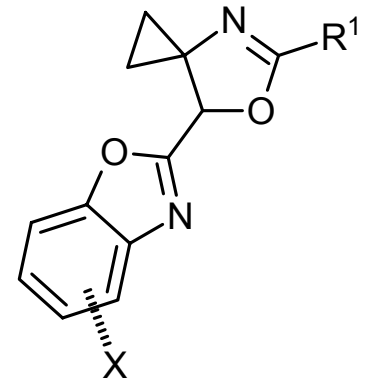<smiles>[R]N(C)C(=O)C1OC(c2ccc(Br)c(Br)c2)=NC12CC2</smiles>

$70 a-b$<smiles>[R]N(C)C(=O)[C@H]1OC(c2ccc(N)c([N+]#N)c2)=NC12CC2</smiles>

71a-j<smiles>[R]N(C)C(=O)[C@H]1OC(c2ccc([Al])cc2)=NC12CC2</smiles>

$72 a-d$<smiles>[R]C(O)C(Cl)(C(=O)OC)C1([R])CC1</smiles>

$75 a-I-R^{1}$<smiles>COC(=O)C1(C2(C(C)C)CC2)OC1c1ccc(OC)cc1</smiles>

79b-iPr<smiles>CC(C)(C)OC(=O)N1CC2C3C(C(=O)OCc4ccccc4)C3C21</smiles>

82<smiles>CCCCC1C2CNCC12</smiles>

83

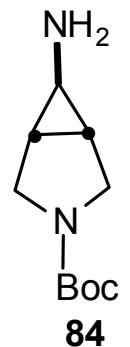<smiles>COC(=O)C1(C2(C(C)C)CC2)OC1c1ccccc1</smiles>

79a-iPr 


\section{Spectral Data}
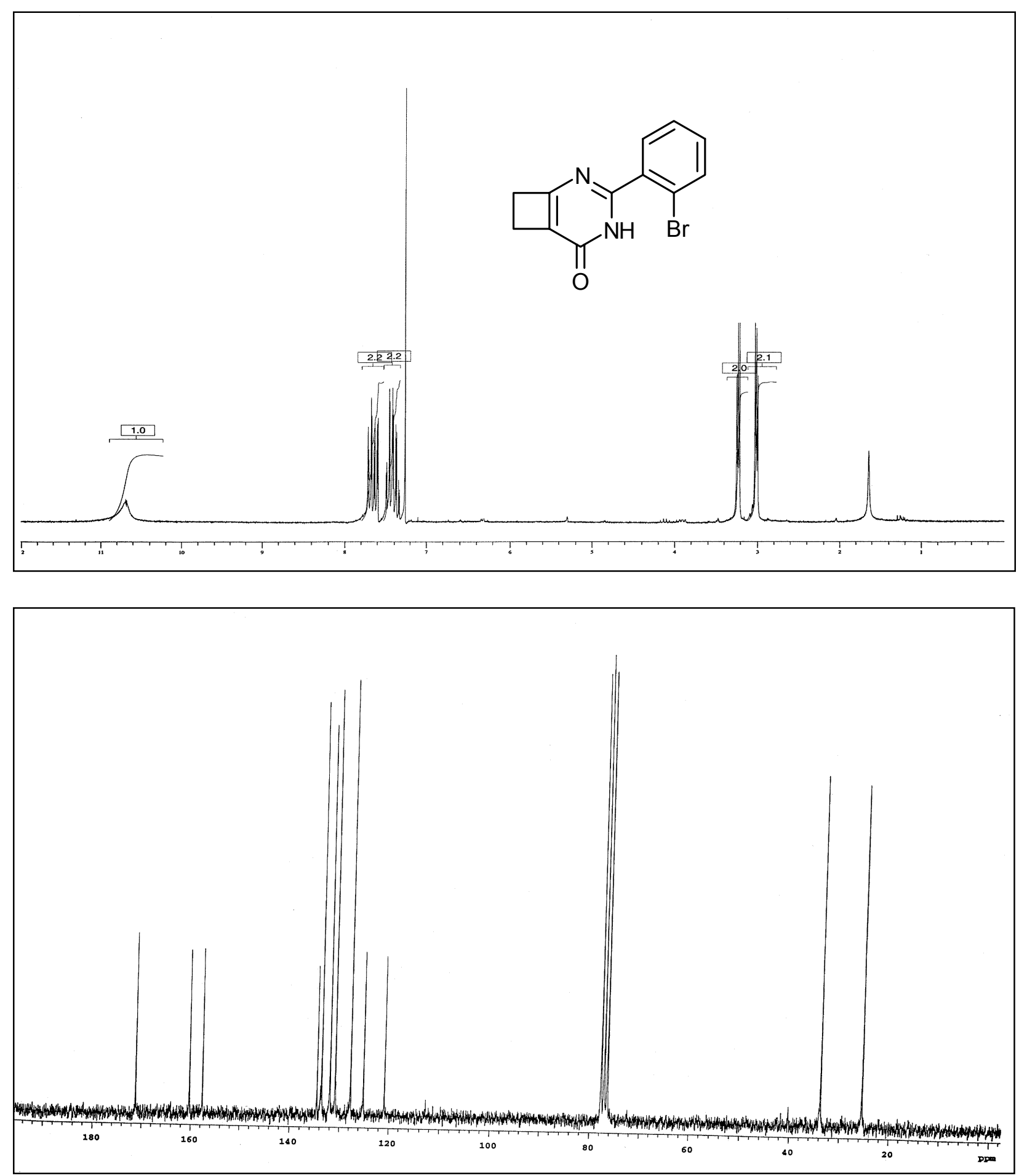

3-(o-Bromophenyl)-2,4-diazabicyclo[4.2.0]octa-1(6),2-dien-5-one (35c) 

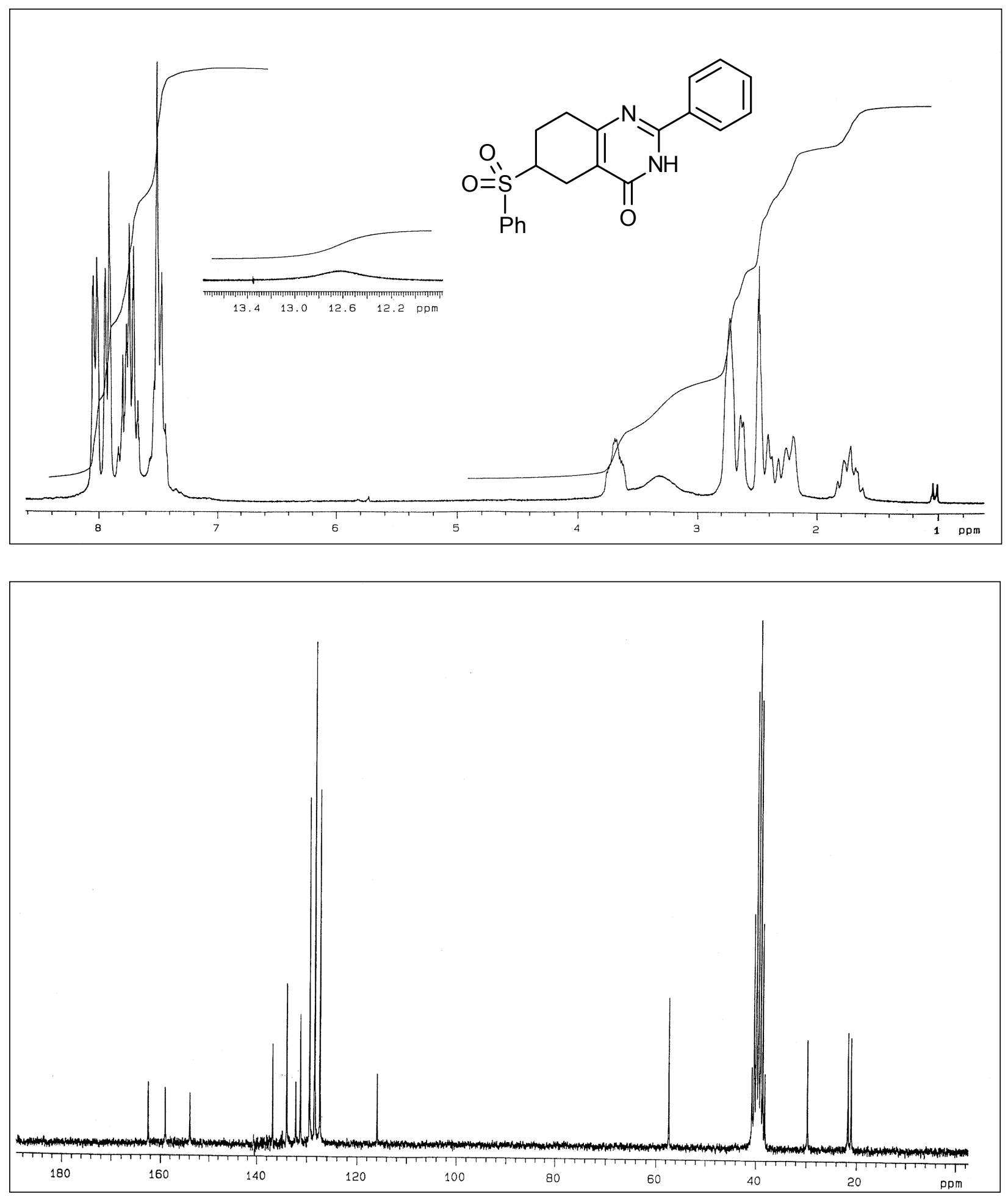

6-Benzenesulfonyl-2-phenyl-5,6, 7,8-tetrahydroquinazolin-4(3H)-one (37a) 

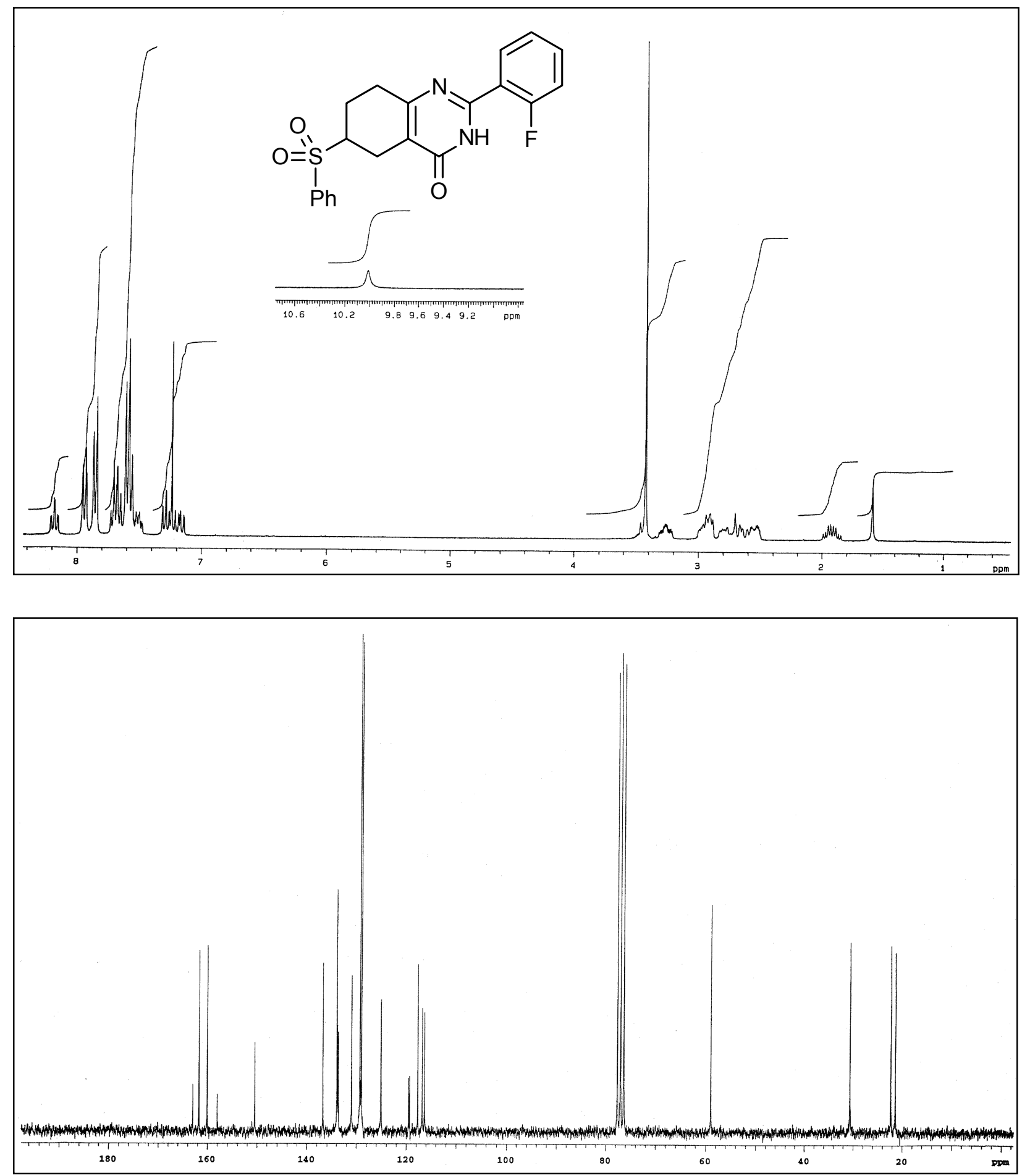

6-Benzenesulfonyl-2-(o-fluorophenyl)-5,6,7,8-tetrahydroquinazolin-4(3H)-one (37d) 

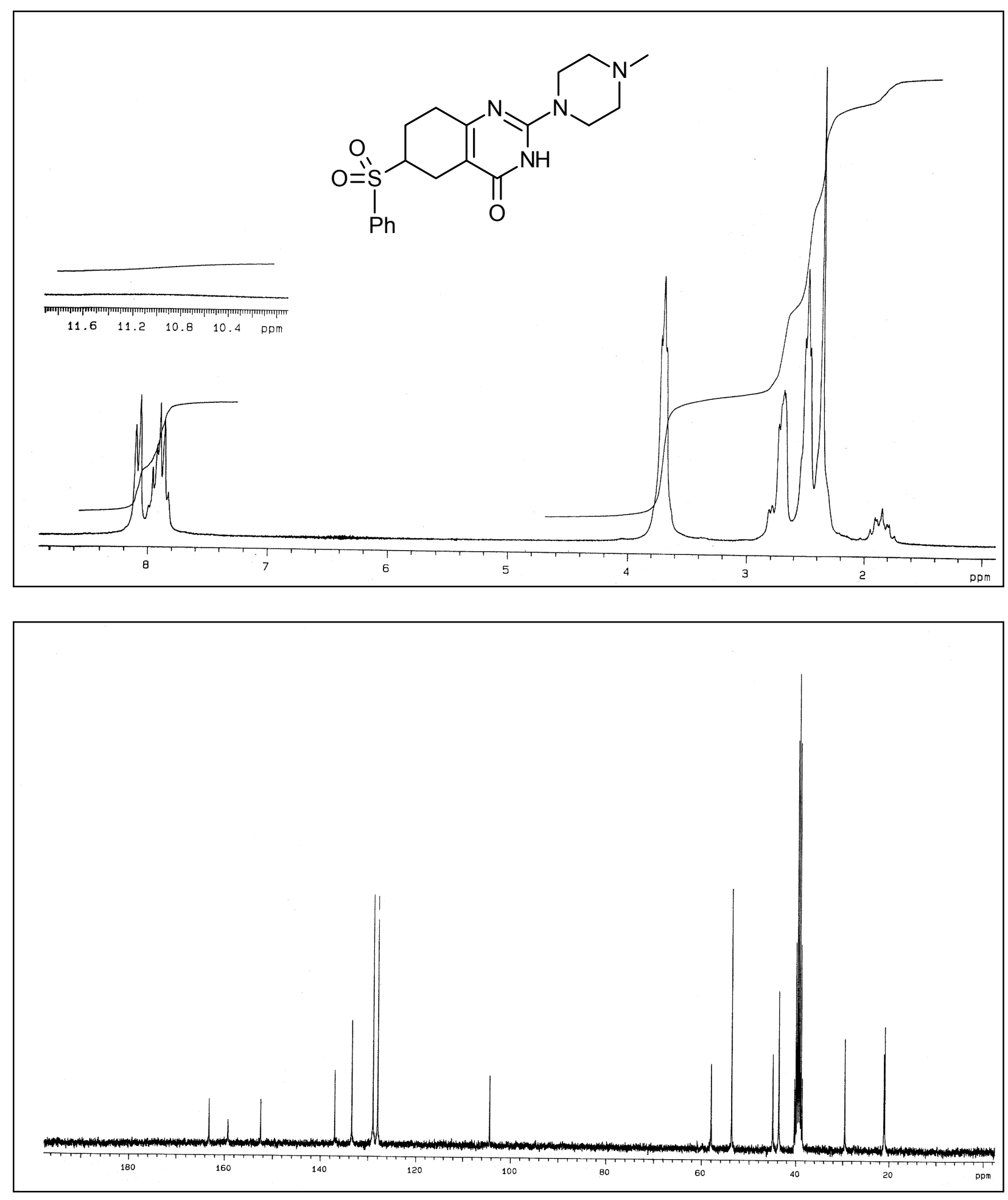

6-Benzenesulfonyl-2-(4-methylpiperazin-1-yl)-5,6,7,8-tetrahydroquinazolin-4(3H)-one (45c) 

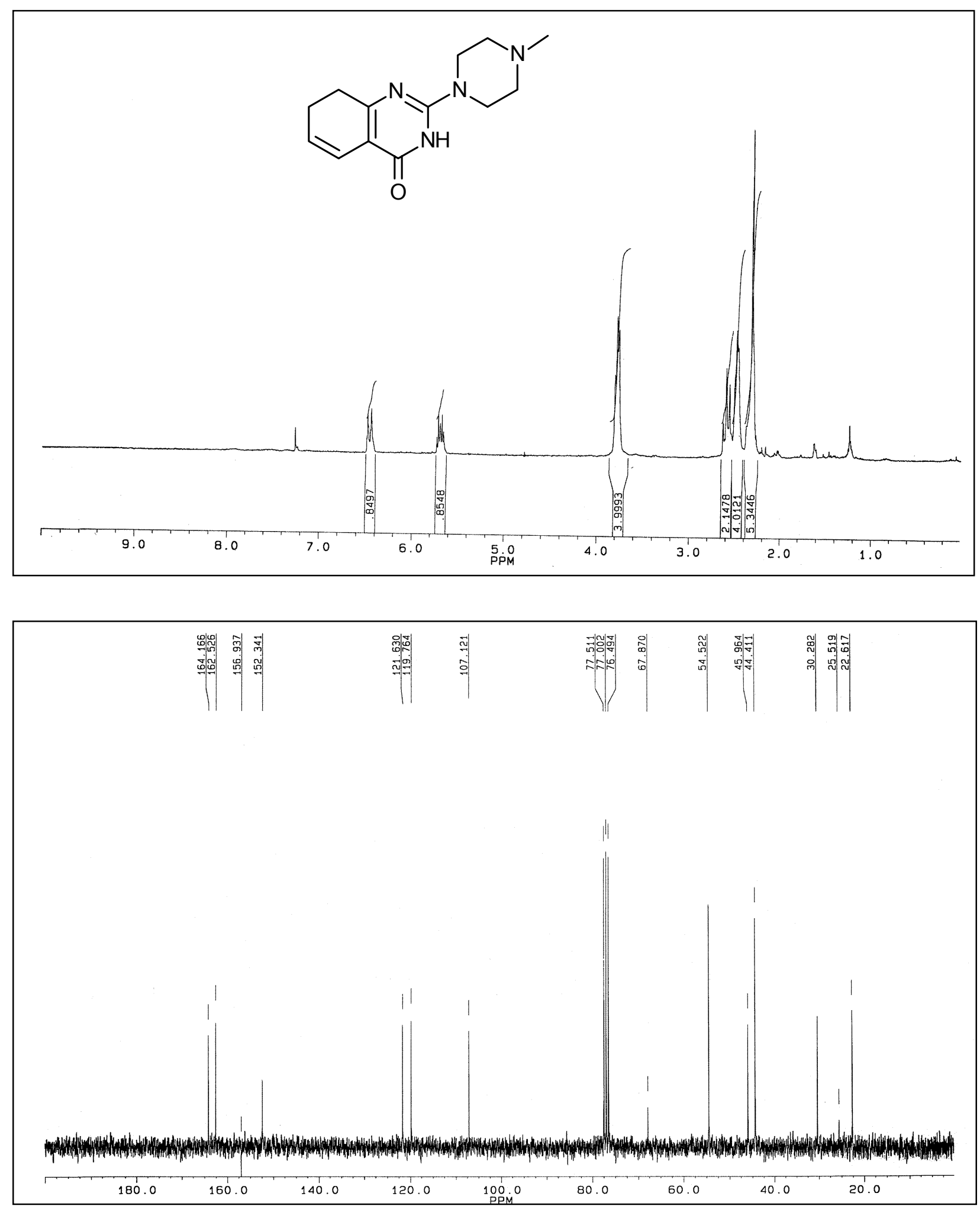

2-(4-Methylpiperazin-1-yl)-7,8-dihydroquinazolin-4(3H)-one (47c) 

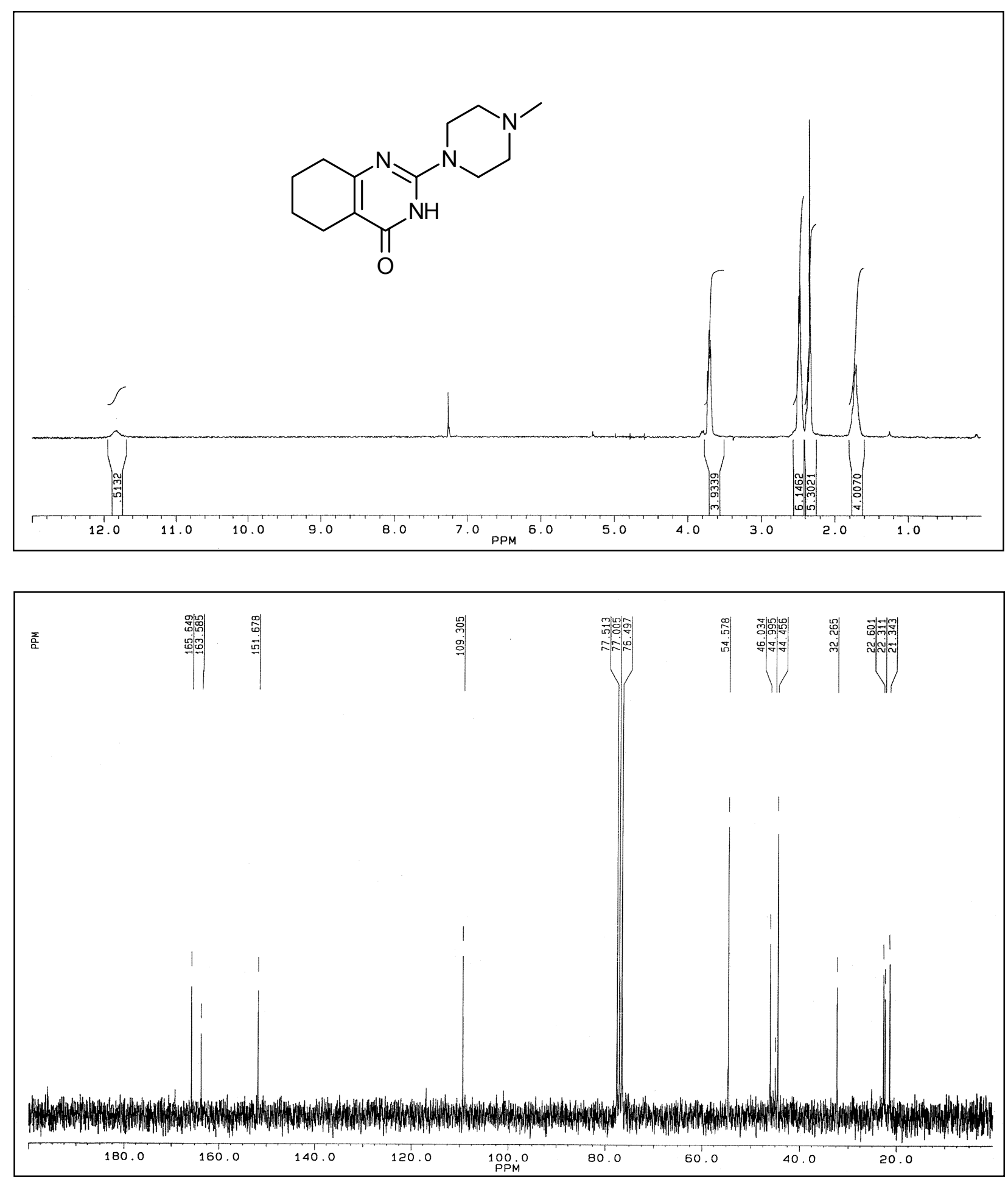

2-(4-Methylpiperazin-1-yl)-5,6,7,8-tetrahydroquinazolin-4(3H)-one (46c) 

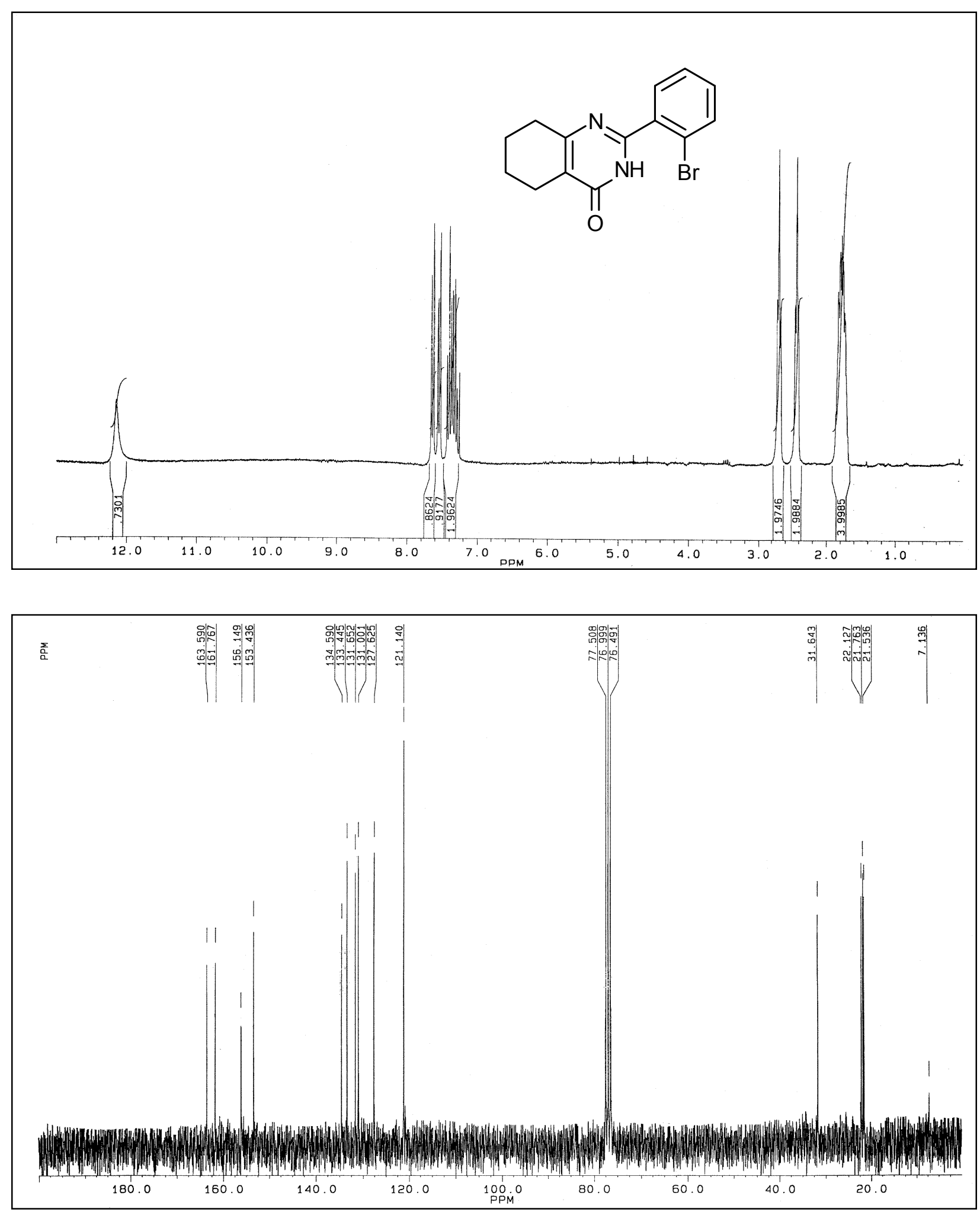

2-(o-Bromophenyl)-5, 6, 7,8-tetrahydroquinazolin-4(3H)-one (39c) 

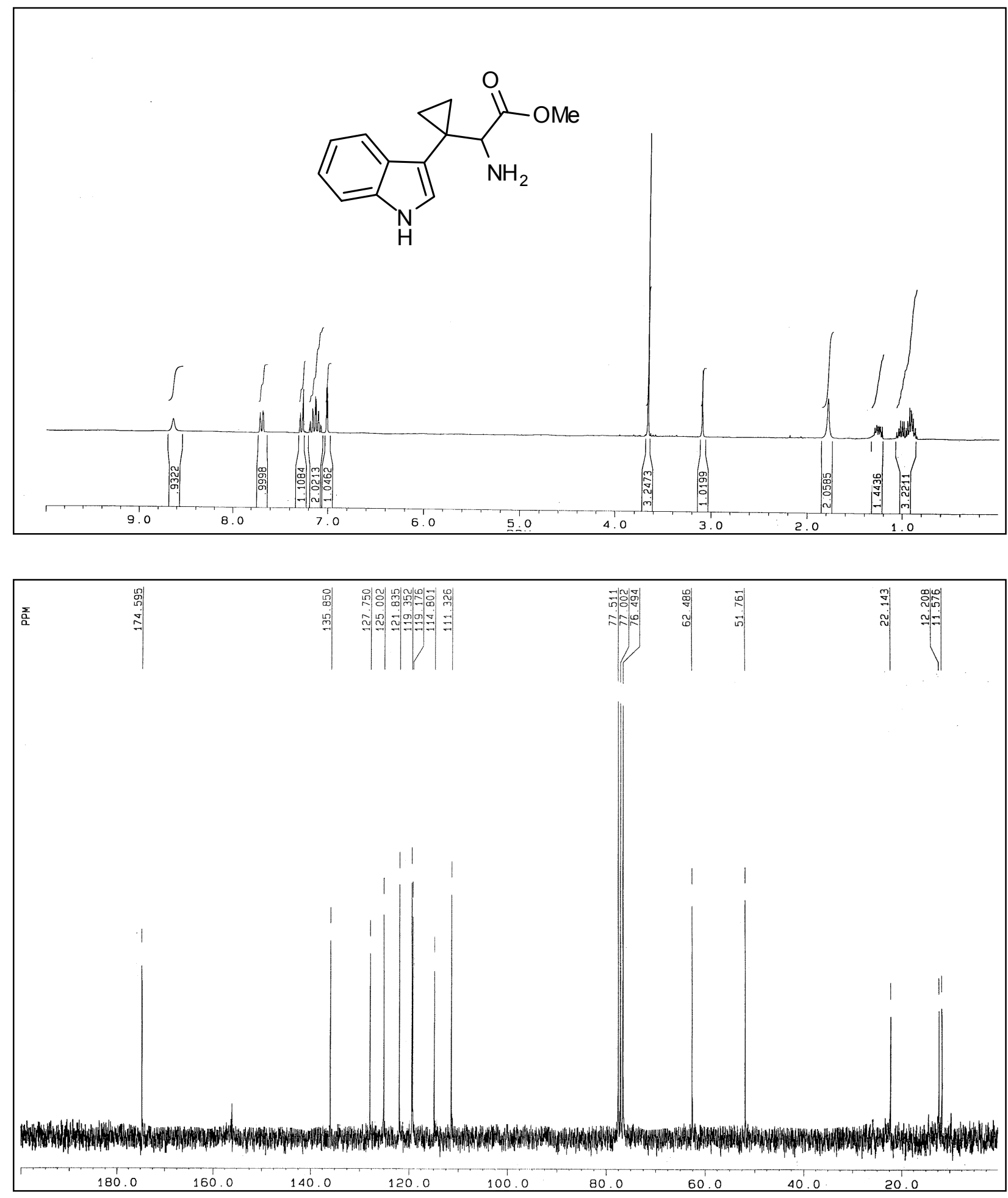

Methyl 2-amino-2-[1-(1H-indol-3-yl)cyclopropyl]acetate (56) 

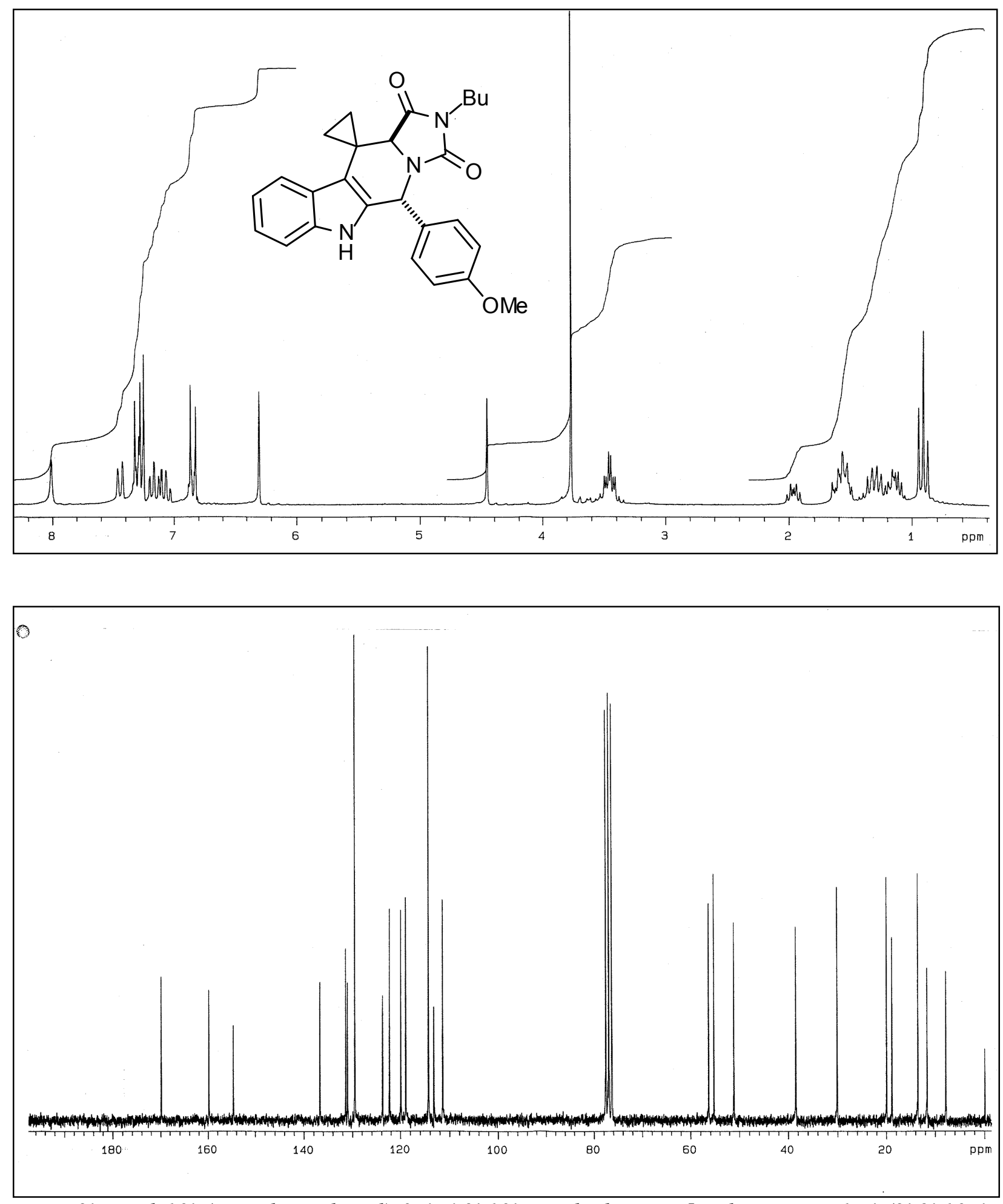

trans-2'-Butyl-10'-(4-methoxyphenyl)-3a',4',9',10'-tetrahydrospiro[cyclopropane-1,4'-(2',9',10a'triazacyclopenta[b]fluorene)]-1',3'-dione (trans-25) 

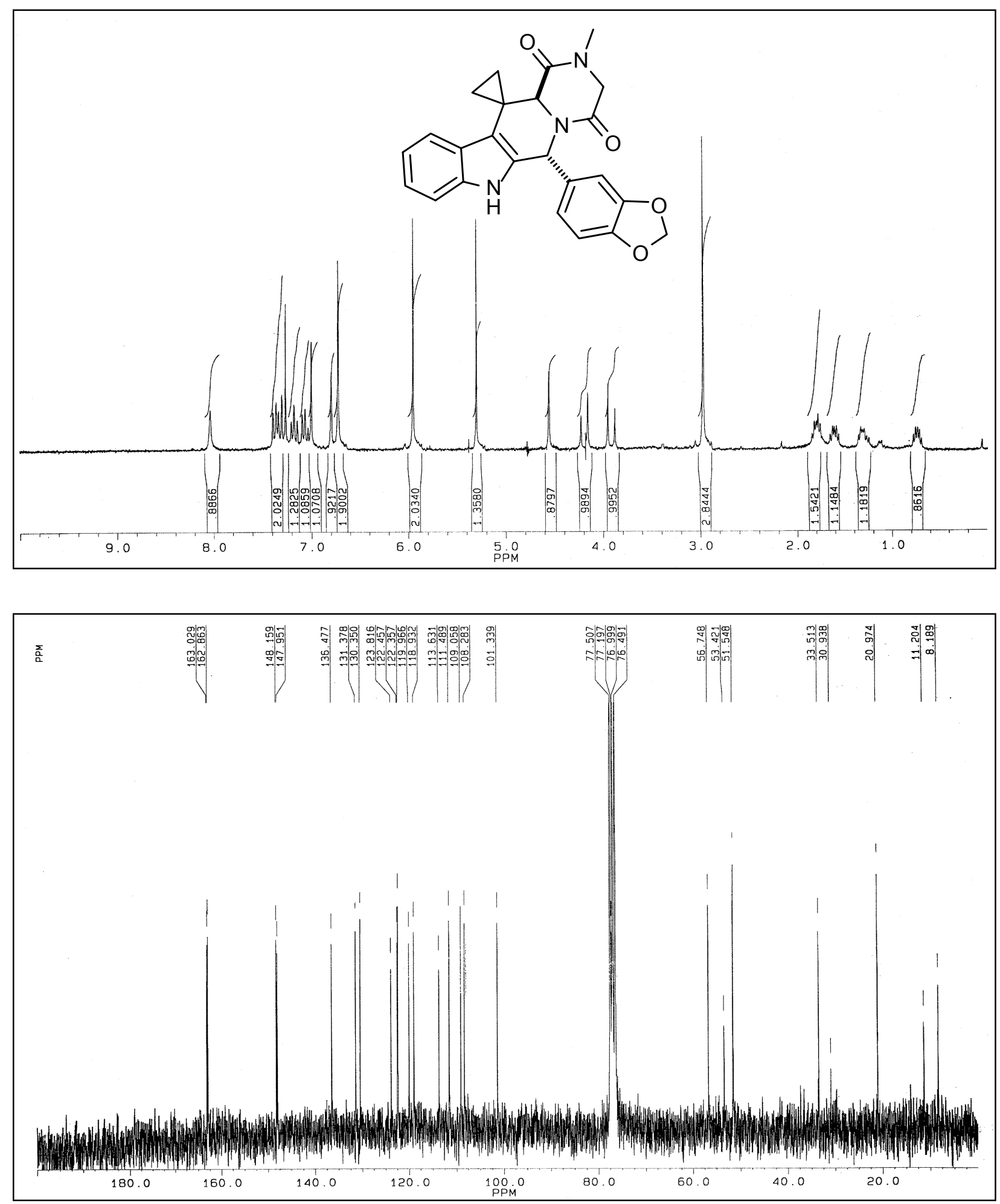

trans-6'-(1,3-Benzodioxole-5-yl)-2'-methyl-12'-spirocyclopropyl-2', 3',6',7',12',12a'-hexa-hydropyrazino $\left[1^{\prime}, 2^{\prime}: 1,6\right]$ pyrido[3,4-b]indole-1',4'-dione (trans-26) 

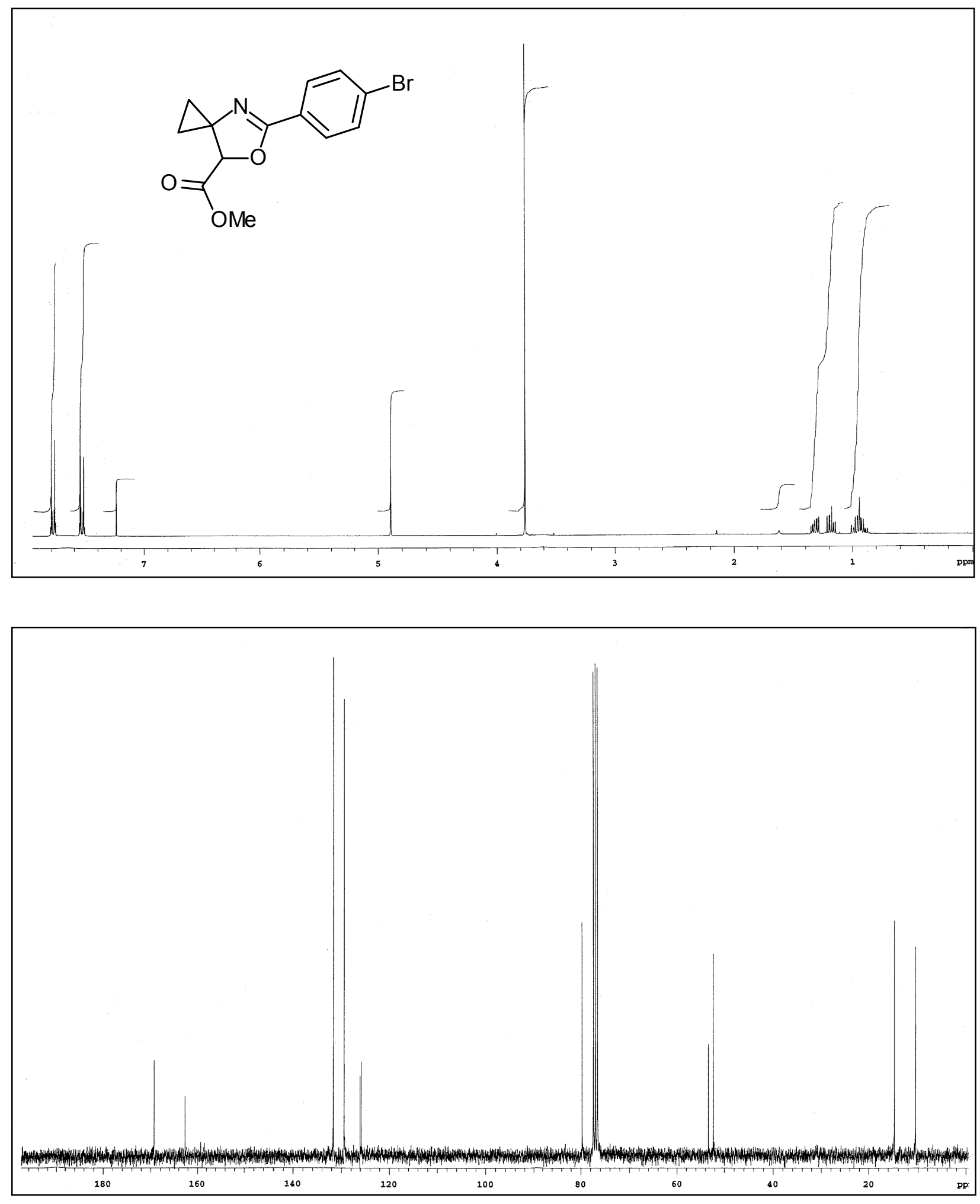

5-(4-Bromophenyl)-6-oxa-4-aza-spiro[2.4]hept-4-ene-7-carboxylic acid methyl ester (13a) 

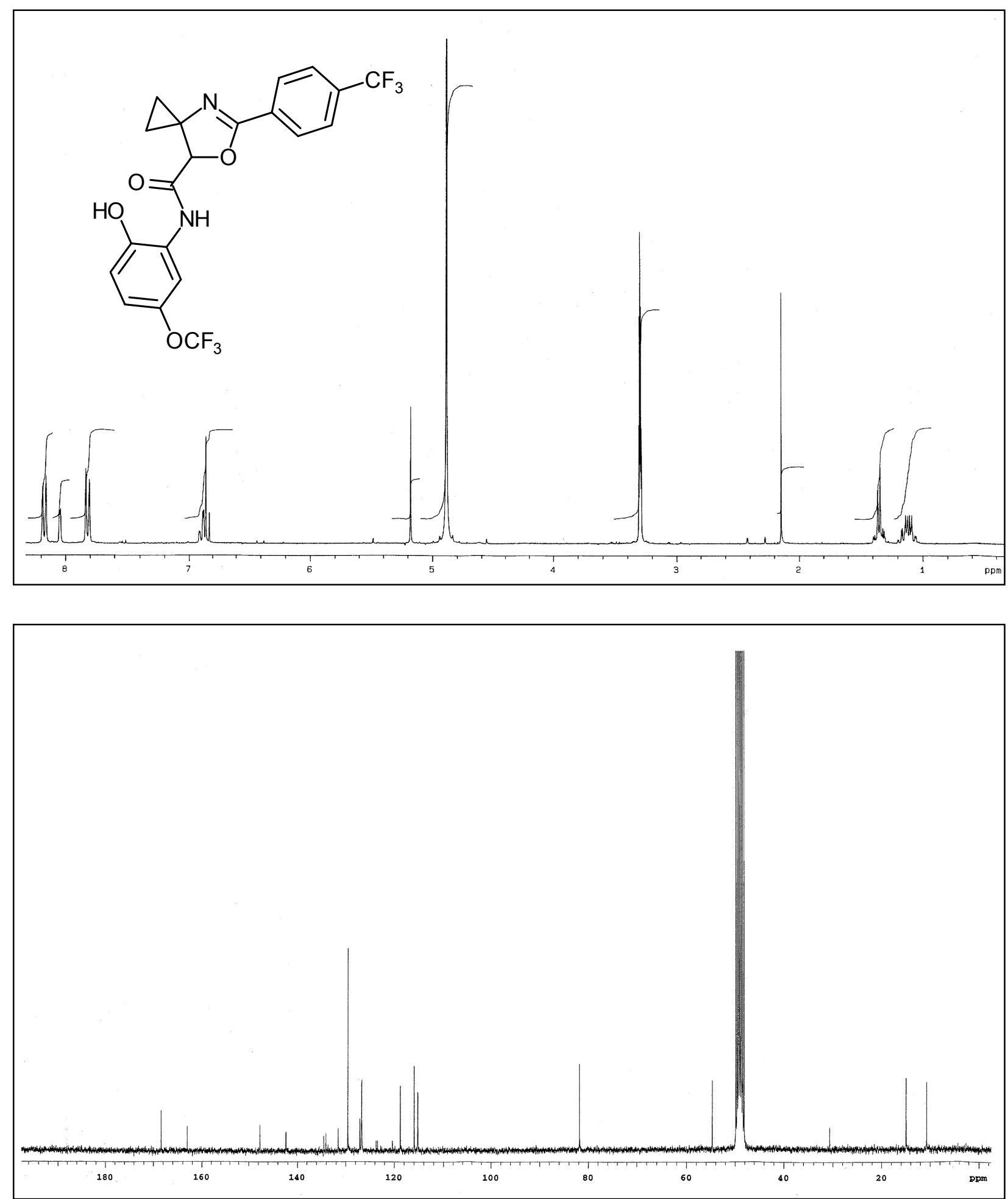

5-(4-Trifluoromethyl-phenyl)-6-oxa-4-aza-spiro[2.4]hept-4-ene-7-carboxylic acid (5-trifluoromethoxy-2-hydroxy-phenyl)-amide (68f) 

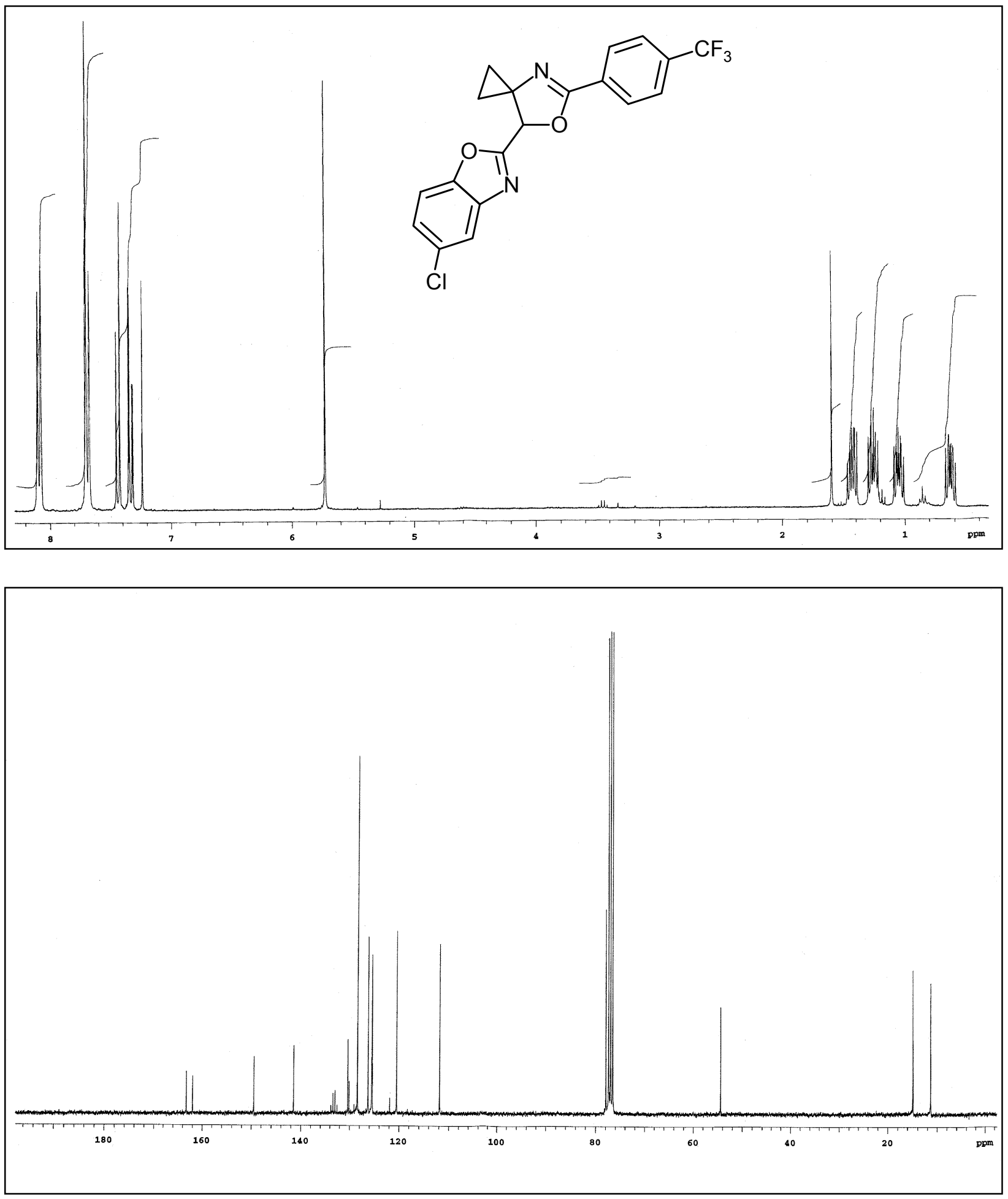

5-Chloro-2-[5-(4-trifluoromethyl-phenyl)-6-oxa-4-aza-spiro[2.4]hept-4-en-7-yl]-benzooxazole (69a) 

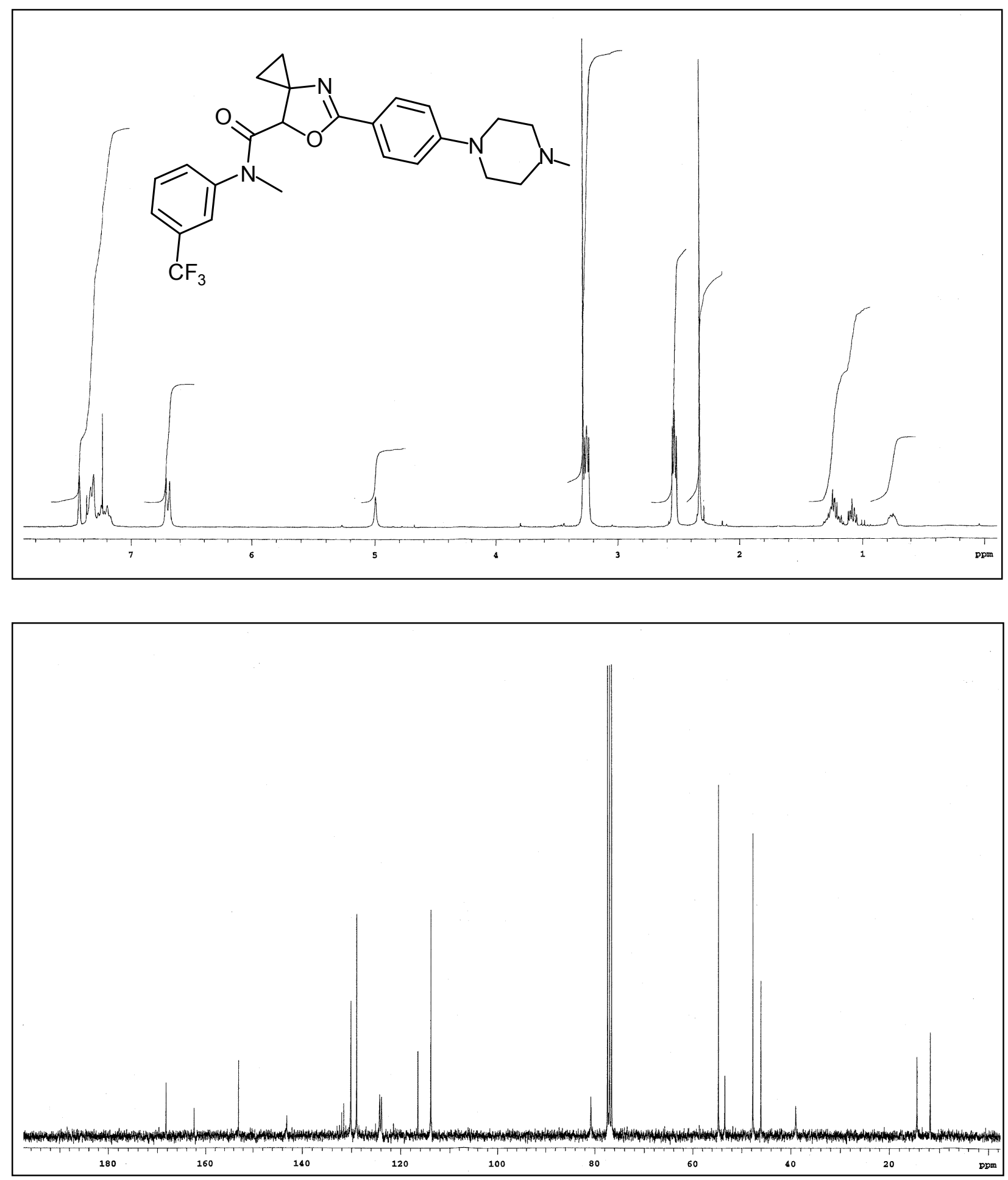

5-[4-(4-Methyl-piperazin-1-yl)-phenyl]-6-oxa-4-aza-spiro[2.4] hept-4-ene-7-carboxylic acid methyl-(3-trifluoromethyl-phenyl)-amide (71c) 

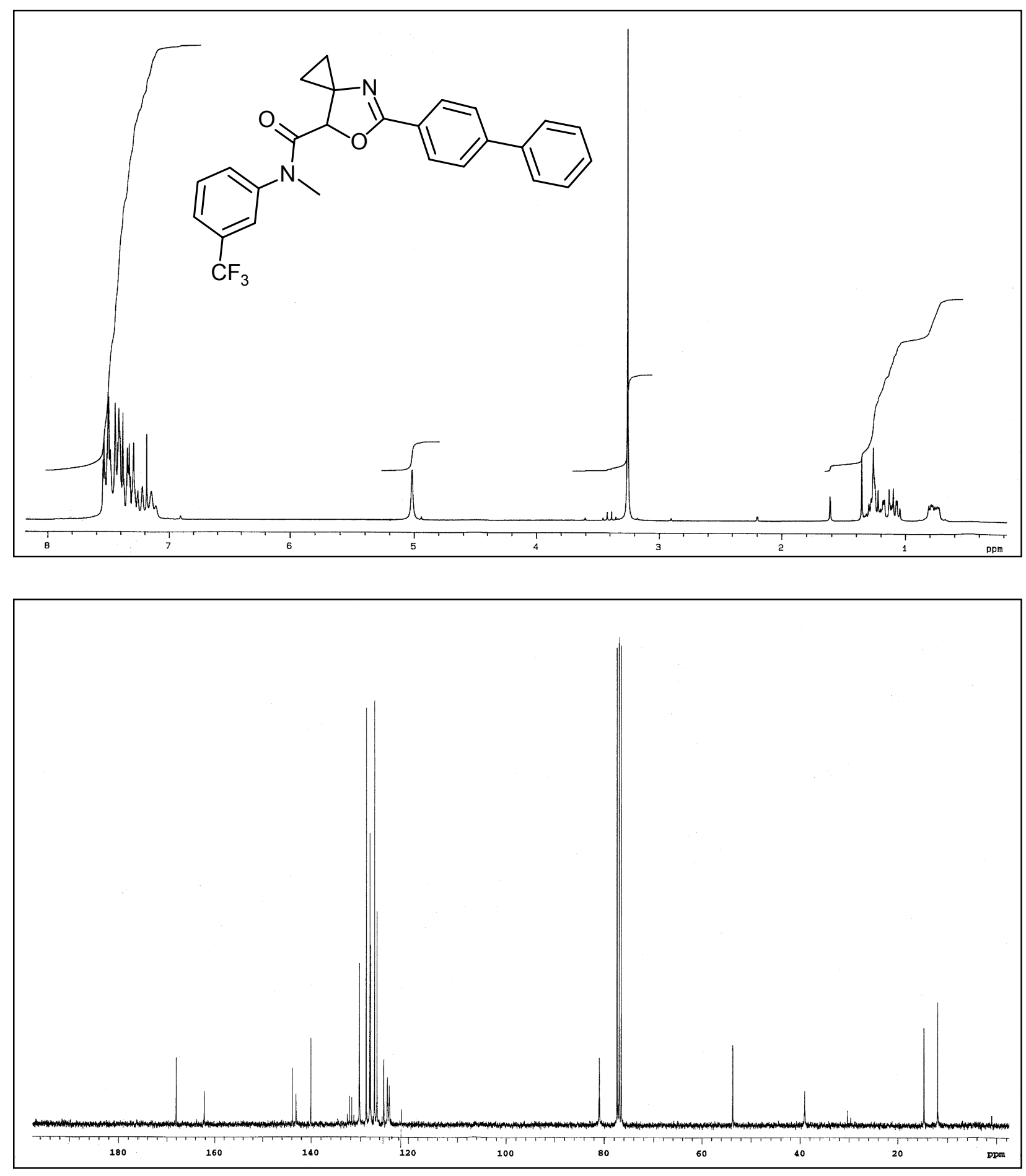

5-Biphenyl-4-yl-6-oxa-4-aza-spiro[2.4] hept-4-ene-7-carboxylic acid methyl-(3-trifluoromethylphenyl)-amide (72a) 


\section{Crystal Data}

1. 6-Benzenesulfonyl-6-ethyl-2-phenyl-5,6,7,8-tetrahydroquinazolin-4(3H)-one (41b)

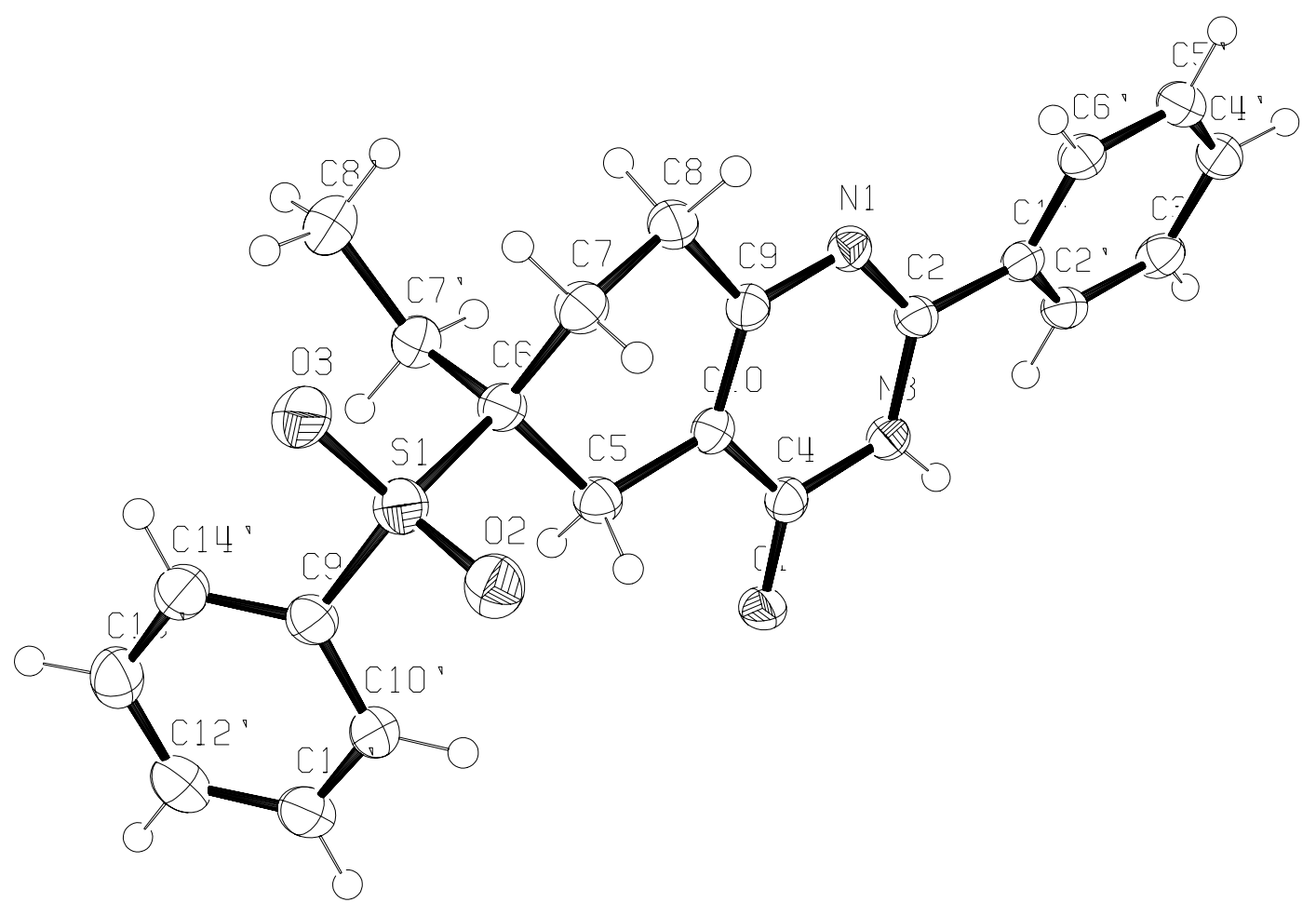


Table 18.. Crystal data and structure refinement for $\mathbf{4 1 b}$

Empirical formula

Formula weight

Temperature

Wavelength

Crystal system

Space group

Unit cell dimensions

Volume

Z

Density (calculated)

Absorption coefficient

$\mathrm{F}(000)$

Crystal size

Theta range for data collection

Index ranges

Reflections collected

Independent reflections

Completeness to theta $=56.74^{\circ}$

Refinement method

Data / restraints / parameters

Goodness-of-fit on $\mathrm{F}^{2}$

Final R indices [I $>2 \operatorname{sigma}(\mathrm{I})]$

$\mathrm{R}$ indices (all data)

Largest diff. peak and hole
$\mathrm{C}_{22} \mathrm{H}_{22} \mathrm{~N}_{2} \mathrm{O}_{3} \mathrm{~S}$

394.48

100(2) K

$1.54178 \AA$

Monoclinic

$\mathrm{P} 2(1) / \mathrm{c}$

$$
\begin{array}{ll}
\mathrm{a}=13.17(2) \AA & \alpha=90 \mathrm{deg} . \\
\mathrm{b}=10.98(4) \AA & \beta=94.05(6) \mathrm{deg} . \\
\mathrm{c}=13.03(3) \AA & \gamma=90 \mathrm{deg} .
\end{array}
$$

1881(8) $\AA^{3}$

4

$1.393 \mathrm{Mg} / \mathrm{m}^{3}$

$1.748 \mathrm{~mm}^{-1}$

832

$0.2 \times 0.2 \times 0.2 \mathrm{~mm}^{3}$

3.36 to $56.74^{\circ}$.

$-14<=\mathrm{h}<=12,-11<=\mathrm{k}<=11,-14<=\mathrm{l}<=14$

12555

$2461[\mathrm{R}(\mathrm{int})=0.0525]$

$98.0 \%$

Full-matrix least-squares on $\mathrm{F}^{2}$

$2461 / 0 / 253$

1.035

$\mathrm{R} 1=0.0366, \mathrm{wR} 2=0.0843$

$\mathrm{R} 1=0.0493, \mathrm{wR} 2=0.0900$

0.152 and -0.329 e. $\AA^{-3}$ 
Table 19. Atomic coordinates $\left(\times 10^{4}\right)$ equivalent isotropic displacement parameters $\left(\AA^{2} \times 10^{3}\right)$ for 41b. $U(\mathrm{eq})$ is defined as one third of the trace of the orthogonalized $\mathrm{U}^{\mathrm{ij}}$ tensor.

\begin{tabular}{|c|c|c|c|c|}
\hline- & $\mathrm{x}$ & $\mathrm{y}$ & z & $\mathrm{U}(\mathrm{eq})$ \\
\hline - & & & & \\
\hline $\mathrm{S}(1)$ & $8017(1)$ & 2131(1) & $1182(1)$ & $32(1)$ \\
\hline $\mathrm{O}(3)$ & $8944(1)$ & $2122(2)$ & $657(1)$ & $39(1)$ \\
\hline $\mathrm{N}(1)$ & $8180(1)$ & $-425(2)$ & $5217(1)$ & $24(1)$ \\
\hline$C\left(5^{\prime}\right)$ & $8347(2)$ & $-1523(2)$ & $8313(2)$ & $30(1)$ \\
\hline $\mathrm{O}(2)$ & $7734(1)$ & $3232(2)$ & $1692(1)$ & $37(1)$ \\
\hline $\mathrm{N}(3)$ & $6404(1)$ & $-352(2)$ & $5200(1)$ & $24(1)$ \\
\hline$C\left(4^{\prime}\right)$ & $7521(2)$ & $-2115(2)$ & $8687(2)$ & $30(1)$ \\
\hline $\mathrm{O}(1)$ & $5397(1)$ & $299(2)$ & $3809(1)$ & $29(1)$ \\
\hline$C\left(3^{\prime}\right)$ & $6628(2)$ & $-2247(2)$ & $8066(2)$ & $29(1)$ \\
\hline $\mathrm{C}\left(2^{\prime}\right)$ & $6556(2)$ & $-1776(2)$ & $7080(2)$ & $26(1)$ \\
\hline$C\left(1^{\prime}\right)$ & $7379(2)$ & $-1164(2)$ & $6703(2)$ & $24(1)$ \\
\hline$C\left(6^{\prime}\right)$ & $8277(2)$ & $-1046(2)$ & $7330(2)$ & $28(1)$ \\
\hline $\mathrm{C}(2)$ & $7337(2)$ & $-625(2)$ & $5664(2)$ & $23(1)$ \\
\hline $\mathrm{C}(4)$ & $6276(2)$ & $117(2)$ & $4218(2)$ & $24(1)$ \\
\hline$C(10)$ & $7192(2)$ & $368(2)$ & $3740(2)$ & $23(1)$ \\
\hline $\mathrm{C}(9)$ & $8103(2)$ & $109(2)$ & $4261(2)$ & $24(1)$ \\
\hline $\mathrm{C}(8)$ & $9089(2)$ & $382(2)$ & $3807(2)$ & $29(1)$ \\
\hline $\mathrm{C}(7)$ & $8963(2)$ & $1290(2)$ & $2916(2)$ & $30(1)$ \\
\hline$C(6)$ & $8080(2)$ & $918(2)$ & $2143(2)$ & $28(1)$ \\
\hline$C\left(7^{\prime}\right)$ & $8217(2)$ & $-346(2)$ & $1646(2)$ & $32(1)$ \\
\hline$C(5)$ & $7084(2)$ & $935(2)$ & $2684(2)$ & $26(1)$ \\
\hline$C\left(8^{\prime}\right)$ & $9249(2)$ & $-660(3)$ & $1239(2)$ & $41(1)$ \\
\hline $\mathrm{C}\left(9^{\prime}\right)$ & $7001(2)$ & $1744(2)$ & $280(2)$ & $31(1)$ \\
\hline$C\left(10^{\prime}\right)$ & $6002(2)$ & $1998(2)$ & $498(2)$ & $35(1)$ \\
\hline $\mathrm{C}\left(11^{\prime}\right)$ & $5214(2)$ & $1663(3)$ & $-211(2)$ & $41(1)$ \\
\hline$C\left(12^{\prime}\right)$ & $5426(2)$ & $1118(2)$ & $-1128(2)$ & $40(1)$ \\
\hline$C\left(13^{\prime}\right)$ & $6419(2)$ & $903(2)$ & $-1353(2)$ & $39(1)$ \\
\hline $\mathrm{C}\left(14^{\prime}\right)$ & $7212(2)$ & $1199(2)$ & $-641(2)$ & $35(1)$ \\
\hline
\end{tabular}


Table 20. Bond lengths $[\AA]$ and angles $\left[{ }^{\circ}\right]$ for $\mathbf{4 1 b}$.

\begin{tabular}{ll}
\hline $\mathrm{S}(1)-\mathrm{O}(3)$ & $1.441(3)$ \\
$\mathrm{S}(1)-\mathrm{O}(2)$ & $1.442(4)$ \\
$\mathrm{S}(1)-\mathrm{C}\left(9^{\prime}\right)$ & $1.769(3)$ \\
$\mathrm{S}(1)-\mathrm{C}(6)$ & $1.827(4)$ \\
$\mathrm{N}(1)-\mathrm{C}(2)$ & $1.308(3)$ \\
$\mathrm{N}(1)-\mathrm{C}(9)$ & $1.375(4)$ \\
$\mathrm{C}\left(5^{\prime}\right)-\mathrm{C}\left(6^{\prime}\right)$ & $1.382(4)$ \\
$\mathrm{C}\left(5^{\prime}\right)-\mathrm{C}\left(4^{\prime}\right)$ & $1.384(4)$ \\
$\mathrm{N}(3)-\mathrm{C}(2)$ & $1.364(3)$ \\
$\mathrm{N}(3)-\mathrm{C}(4)$ & $1.379(4)$ \\
$\mathrm{C}\left(4^{\prime}\right)-\mathrm{C}\left(3^{\prime}\right)$ & $1.387(4)$ \\
$\mathrm{O}(1)-\mathrm{C}(4)$ & $1.257(3)$ \\
$\mathrm{C}\left(3^{\prime}\right)-\mathrm{C}\left(2^{\prime}\right)$ & $1.383(4)$ \\
$\mathrm{C}\left(2^{\prime}\right)-\mathrm{C}\left(1^{\prime}\right)$ & $1.394(4)$ \\
$\mathrm{C}\left(1^{\prime}\right)-\mathrm{C}\left(6^{\prime}\right)$ & $1.395(4)$ \\
$\mathrm{C}\left(1^{\prime}\right)-\mathrm{C}(2)$ & $1.476(4)$ \\
$\mathrm{C}(4)-\mathrm{C}(10)$ & $1.422(4)$ \\
$\mathrm{C}(10)-\mathrm{C}(9)$ & $1.367(4)$ \\
$\mathrm{C}(10)-\mathrm{C}(5)$ & $1.508(4)$ \\
$\mathrm{C}(9)-\mathrm{C}(8)$ & $1.496(4)$ \\
$\mathrm{C}(8)-\mathrm{C}(7)$ & $1.531(4)$ \\
$\mathrm{C}(7)-\mathrm{C}(6)$ & $1.540(4)$ \\
$\mathrm{C}(6)-\mathrm{C}(5)$ & $1.534(4)$ \\
$\mathrm{C}(6)-\mathrm{C}\left(7^{\prime}\right)$ & $1.547(5)$ \\
$\mathrm{C}\left(7^{\prime}\right)-\mathrm{C}\left(8^{\prime}\right)$ & $1.533(4)$ \\
$\mathrm{C}\left(9^{\prime}\right)-\mathrm{C}\left(14^{\prime}\right)$ & $1.387(4)$ \\
$\mathrm{C}\left(9^{\prime}\right)-\mathrm{C}\left(10^{\prime}\right)$ & $1.394(4)$ \\
$\mathrm{C}\left(10^{\prime}\right)-\mathrm{C}\left(11^{\prime}\right)$ & $1.390(4)$ \\
$\mathrm{C}\left(11^{\prime}\right)-\mathrm{C}\left(12^{\prime}\right)$ & $1.382(4)$ \\
$\mathrm{C}\left(12^{\prime}\right)-\mathrm{C}\left(13^{\prime}\right)$ & $1.381(4)$ \\
$\mathrm{C}\left(13^{\prime}\right)-\mathrm{C}\left(14^{\prime}\right)$ & $1.387(4)$ \\
& \\
$\mathrm{O}(3)-\mathrm{S}(1)-\mathrm{O}(2)$ & $118.69(13)$ \\
$\mathrm{O}(3)-\mathrm{S}(1)-\mathrm{C}\left(9^{\prime}\right)$ & $108.02(18)$ \\
&
\end{tabular}




\begin{tabular}{|c|c|c|c|}
\hline $\mathrm{O}(2)-\mathrm{S}(1)-\mathrm{C}\left(9^{\prime}\right)$ & $107.46(14)$ & & \\
\hline $\mathrm{O}(3)-\mathrm{S}(1)-\mathrm{C}(6)$ & 108.74(13) & & \\
\hline $\mathrm{O}(2)-\mathrm{S}(1)-\mathrm{C}(6)$ & $107.2(2)$ & & \\
\hline $\mathrm{C}\left(9^{\prime}\right)-\mathrm{S}(1)-\mathrm{C}(6)$ & 106.01(17) & & \\
\hline $\mathrm{C}(2)-\mathrm{N}(1)-\mathrm{C}(9)$ & $117.6(2)$ & & \\
\hline$C\left(6^{\prime}\right)-C\left(5^{\prime}\right)-C\left(4^{\prime}\right)$ & $120.1(2)$ & & \\
\hline $\mathrm{C}(2)-\mathrm{N}(3)-\mathrm{C}(4)$ & $122.8(2)$ & & \\
\hline $\mathrm{C}\left(5^{\prime}\right)-\mathrm{C}\left(4^{\prime}\right)-\mathrm{C}\left(3^{\prime}\right)$ & $119.9(2)$ & & \\
\hline $\mathrm{C}\left(2^{\prime}\right)-\mathrm{C}\left(3^{\prime}\right)-\mathrm{C}\left(4^{\prime}\right)$ & $120.2(2)$ & & \\
\hline $\mathrm{C}\left(3^{\prime}\right)-\mathrm{C}\left(2^{\prime}\right)-\mathrm{C}\left(1^{\prime}\right)$ & $120.3(2)$ & & \\
\hline $\mathrm{C}\left(2^{\prime}\right)-\mathrm{C}\left(1^{\prime}\right)-\mathrm{C}\left(6^{\prime}\right)$ & $119.1(2)$ & & \\
\hline $\mathrm{C}\left(2^{\prime}\right)-\mathrm{C}\left(1^{\prime}\right)-\mathrm{C}(2)$ & $122.5(2)$ & & \\
\hline$C\left(6^{\prime}\right)-C\left(1^{\prime}\right)-C(2)$ & $118.4(2)$ & & \\
\hline $\mathrm{C}\left(5^{\prime}\right)-\mathrm{C}\left(6^{\prime}\right)-\mathrm{C}\left(1^{\prime}\right)$ & $120.4(2)$ & & \\
\hline $\mathrm{N}(1)-\mathrm{C}(2)-\mathrm{N}(3)$ & $122.2(2)$ & & \\
\hline $\mathrm{N}(1)-\mathrm{C}(2)-\mathrm{C}\left(1^{\prime}\right)$ & $119.8(2)$ & & \\
\hline $\mathrm{N}(3)-\mathrm{C}(2)-\mathrm{C}\left(1^{\prime}\right)$ & $118.0(2)$ & & \\
\hline $\mathrm{O}(1)-\mathrm{C}(4)-\mathrm{N}(3)$ & $120.1(2)$ & & \\
\hline $\mathrm{O}(1)-\mathrm{C}(4)-\mathrm{C}(10)$ & $124.6(2)$ & & \\
\hline $\mathrm{N}(3)-\mathrm{C}(4)-\mathrm{C}(10)$ & $115.2(2)$ & & \\
\hline$C(9)-C(10)-C(4)$ & $119.0(2)$ & & \\
\hline$C(9)-C(10)-C(5)$ & $124.2(2)$ & & \\
\hline$C(4)-C(10)-C(5)$ & $116.8(2)$ & & \\
\hline $\mathrm{C}(10)-\mathrm{C}(9)-\mathrm{N}(1)$ & $123.0(2)$ & & \\
\hline $\mathrm{C}(10)-\mathrm{C}(9)-\mathrm{C}(8)$ & $121.2(2)$ & $\mathrm{C}\left(8^{\prime}\right)-\mathrm{C}\left(7^{\prime}\right)-\mathrm{C}(6)$ & $118.6(2)$ \\
\hline $\mathrm{N}(1)-\mathrm{C}(9)-\mathrm{C}(8)$ & $115.7(2)$ & $C(10)-C(5)-C(6)$ & $112.7(2)$ \\
\hline $\mathrm{C}(9)-\mathrm{C}(8)-\mathrm{C}(7)$ & $112.4(2)$ & $\mathrm{C}\left(14^{\prime}\right)-\mathrm{C}\left(9^{\prime}\right)-\mathrm{C}\left(10^{\prime}\right)$ & $120.8(2)$ \\
\hline$C(8)-C(7)-C(6)$ & $111.1(2)$ & $\mathrm{C}\left(14^{\prime}\right)-\mathrm{C}\left(9^{\prime}\right)-\mathrm{S}(1)$ & $119.4(2$ \\
\hline$C(5)-C(6)-C(7)$ & 109.1(2) & $\mathrm{C}\left(10^{\prime}\right)-\mathrm{C}\left(9^{\prime}\right)-\mathrm{S}(1)$ & $119.8(2)$ \\
\hline$C(5)-C(6)-C\left(7^{\prime}\right)$ & $109.5(2)$ & $\mathrm{C}\left(11^{\prime}\right)-\mathrm{C}\left(10^{\prime}\right)-\mathrm{C}\left(9^{\prime}\right)$ & $118.9(3)$ \\
\hline $\mathrm{C}(7)-\mathrm{C}(6)-\mathrm{C}\left(7^{\prime}\right)$ & $114.0(2)$ & $\mathrm{C}\left(12^{\prime}\right)-\mathrm{C}\left(11^{\prime}\right)-\mathrm{C}\left(10^{\prime}\right)$ & $120.2(3)$ \\
\hline$C(5)-C(6)-S(1)$ & 107.91(17) & $\mathrm{C}\left(13^{\prime}\right)-\mathrm{C}\left(12^{\prime}\right)-\mathrm{C}\left(11^{\prime}\right)$ & $120.7(3)$ \\
\hline$C(7)-C(6)-S(1)$ & $104.5(2)$ & $\mathrm{C}\left(12^{\prime}\right)-\mathrm{C}\left(13^{\prime}\right)-\mathrm{C}\left(14^{\prime}\right)$ & $119.8(3)$ \\
\hline$C\left(7^{\prime}\right)-C(6)-S(1)$ & $111.6(3)$ & $\mathrm{C}\left(9^{\prime}\right)-\mathrm{C}\left(14^{\prime}\right)-\mathrm{C}\left(13^{\prime}\right)$ & $119.6(3)$ \\
\hline
\end{tabular}

Symmetry transformations used to generate equivalent atoms: 
Table 21. Anisotropic displacement parameters $\left(\AA^{2} \times 10^{3}\right)$ for $\mathbf{4 1 b}$. The anisotropic displacement factor exponent takes the form: $-2 \quad 2\left[h^{2} a^{* 2} U^{11}+\ldots+2 h_{k} * b^{*} U^{12}\right]$

\begin{tabular}{|c|c|c|c|c|c|c|}
\hline & $\mathrm{U}^{11}$ & $\mathrm{U}^{22}$ & $\mathrm{U}^{33}$ & $\mathrm{U}^{23}$ & $\mathrm{U}^{13}$ & $\mathrm{U}^{12}$ \\
\hline $\mathrm{S}(1)$ & $32(1)$ & $34(1)$ & $31(1)$ & $7(1)$ & $7(1)$ & $1(1)$ \\
\hline $\mathrm{O}(3)$ & $32(1)$ & $48(1)$ & $38(1)$ & $13(1)$ & $14(1)$ & $0(1)$ \\
\hline $\mathrm{N}(1)$ & $22(1)$ & $28(1)$ & $23(1)$ & $-1(1)$ & $3(1)$ & $-1(1)$ \\
\hline$C\left(5^{\prime}\right)$ & $27(1)$ & $35(2)$ & $28(1)$ & $1(1)$ & $-2(1)$ & $2(1)$ \\
\hline $\mathrm{O}(2)$ & $45(1)$ & $32(1)$ & $36(1)$ & $4(1)$ & $7(1)$ & $0(1)$ \\
\hline $\mathrm{N}(3)$ & 19(1) & $30(1)$ & $24(1)$ & 1(1) & $5(1)$ & $-1(1)$ \\
\hline$C\left(4^{\prime}\right)$ & $34(2)$ & $30(1)$ & $26(1)$ & $5(1)$ & 2(1) & $5(1)$ \\
\hline $\mathrm{O}(1)$ & $20(1)$ & $41(1)$ & $26(1)$ & $5(1)$ & 2(1) & $3(1)$ \\
\hline$C\left(3^{\prime}\right)$ & $28(1)$ & $28(1)$ & $33(1)$ & $4(1)$ & $8(1)$ & $3(1)$ \\
\hline$C\left(2^{\prime}\right)$ & $25(1)$ & $25(1)$ & $29(1)$ & $0(1)$ & 1(1) & $2(1)$ \\
\hline$C\left(1^{\prime}\right)$ & $23(1)$ & $24(1)$ & $25(1)$ & $0(1)$ & $3(1)$ & $3(1)$ \\
\hline$C\left(6^{\prime}\right)$ & $23(1)$ & $32(1)$ & $28(1)$ & $1(1)$ & $4(1)$ & $0(1)$ \\
\hline$C(2)$ & 21(1) & $23(1)$ & $24(1)$ & $-3(1)$ & $0(1)$ & $0(1)$ \\
\hline $\mathrm{C}(4)$ & $26(1)$ & $23(1)$ & $23(1)$ & $-1(1)$ & 2(1) & $1(1)$ \\
\hline$C(10)$ & $23(1)$ & $24(1)$ & $23(1)$ & $-1(1)$ & $5(1)$ & $-1(1)$ \\
\hline $\mathrm{C}(9)$ & $24(1)$ & $25(1)$ & $23(1)$ & $-2(1)$ & 2(1) & $-1(1)$ \\
\hline $\mathrm{C}(8)$ & $22(1)$ & $39(2)$ & $27(1)$ & $1(1)$ & 2(1) & $-1(1)$ \\
\hline $\mathrm{C}(7)$ & $24(1)$ & $36(2)$ & $30(1)$ & $3(1)$ & $5(1)$ & $-2(1)$ \\
\hline$C(6)$ & $23(1)$ & $35(2)$ & $26(1)$ & $6(1)$ & $5(1)$ & $1(1)$ \\
\hline$C\left(7^{\prime}\right)$ & $30(1)$ & $36(2)$ & $29(1)$ & $0(1)$ & 4(1) & $1(1)$ \\
\hline$C(5)$ & $23(1)$ & $29(1)$ & $26(1)$ & $3(1)$ & 2(1) & $0(1)$ \\
\hline$C\left(8^{\prime}\right)$ & $40(2)$ & $47(2)$ & $37(1)$ & $-3(1)$ & 11(1) & $6(1)$ \\
\hline$C\left(9^{\prime}\right)$ & $33(2)$ & $30(1)$ & $29(1)$ & $9(1)$ & $5(1)$ & $5(1)$ \\
\hline $\mathrm{C}\left(10^{\prime}\right)$ & $38(2)$ & $40(2)$ & $27(1)$ & $6(1)$ & $6(1)$ & $10(1)$ \\
\hline$C\left(11^{\prime}\right)$ & $36(2)$ & $51(2)$ & $36(2)$ & 11(1) & 2(1) & $10(1)$ \\
\hline $\mathrm{C}\left(12^{\prime}\right)$ & $46(2)$ & $39(2)$ & $35(2)$ & $7(1)$ & $-5(1)$ & $1(1)$ \\
\hline $\mathrm{C}\left(13^{\prime}\right)$ & $54(2)$ & $31(2)$ & $32(1)$ & $-1(1)$ & $5(1)$ & $5(1)$ \\
\hline $\mathrm{C}\left(14^{\prime}\right)$ & $40(2)$ & $32(2)$ & $35(2)$ & $6(1)$ & $10(1)$ & $6(1)$ \\
\hline
\end{tabular}


Table 22. Hydrogen coordinates ( $\left.\mathrm{x} 10^{4}\right)$ and isotropic displacement parameters $\left(\AA^{2} \times 10^{3}\right)$ for 41b.

\begin{tabular}{|c|c|c|c|c|}
\hline & $\mathrm{x}$ & $\mathrm{y}$ & $\mathrm{z}$ & $\mathrm{U}(\mathrm{eq})$ \\
\hline \multicolumn{5}{|l|}{-} \\
\hline $\mathrm{H}(1 \mathrm{C})$ & 8962 & -1446 & 8733 & 36 \\
\hline $\mathrm{H}(2 \mathrm{~B})$ & 5862 & -480 & 5541 & 29 \\
\hline $\mathrm{H}(2 \mathrm{C})$ & 7566 & -2429 & 9367 & 36 \\
\hline $\mathrm{H}(3 \mathrm{~A})$ & 6065 & -2662 & 8320 & 35 \\
\hline $\mathrm{H}(4 \mathrm{~A})$ & 5944 & -1870 & 6657 & 32 \\
\hline $\mathrm{H}(6 \mathrm{~A})$ & 8843 & -634 & 7079 & 33 \\
\hline $\mathrm{H}(11 \mathrm{~A})$ & 9380 & -385 & 3556 & 35 \\
\hline $\mathrm{H}(11 \mathrm{~B})$ & 9575 & 718 & 4348 & 35 \\
\hline $\mathrm{H}(12 \mathrm{~A})$ & 9602 & 1328 & 2560 & 36 \\
\hline $\mathrm{H}(12 \mathrm{~B})$ & 8830 & 2111 & 3189 & 36 \\
\hline $\mathrm{H}(14 \mathrm{~A})$ & 8067 & -971 & 2162 & 38 \\
\hline $\mathrm{H}(14 \mathrm{~B})$ & 7693 & -426 & 1067 & 38 \\
\hline $\mathrm{H}(01 \mathrm{~A})$ & 6854 & 1789 & 2747 & 31 \\
\hline $\mathrm{H}(01 \mathrm{~B})$ & 6554 & 490 & 2257 & 31 \\
\hline $\mathrm{H}(15 \mathrm{~A})$ & 9223 & -1484 & 950 & 61 \\
\hline $\mathrm{H}(15 \mathrm{~B})$ & 9780 & -620 & 1804 & 61 \\
\hline $\mathrm{H}(15 \mathrm{C})$ & 9403 & -76 & 703 & 61 \\
\hline $\mathrm{H}(17 \mathrm{~A})$ & 5862 & 2393 & 1121 & 42 \\
\hline $\mathrm{H}(18 \mathrm{~A})$ & 4528 & 1808 & -65 & 49 \\
\hline $\mathrm{H}(19 \mathrm{~A})$ & 4883 & 890 & -1608 & 48 \\
\hline $\mathrm{H}(20 \mathrm{~A})$ & 6558 & 552 & -1995 & 46 \\
\hline $\mathrm{H}(21 \mathrm{~A})$ & 7895 & 1030 & -783 & 42 \\
\hline
\end{tabular}


2. 2-cyclopropyl-2,3,7,8-tetrahydro-5H-thiazolo[3,2-a]pyrimidine-3-carboxylic acid methyl ester (74b)

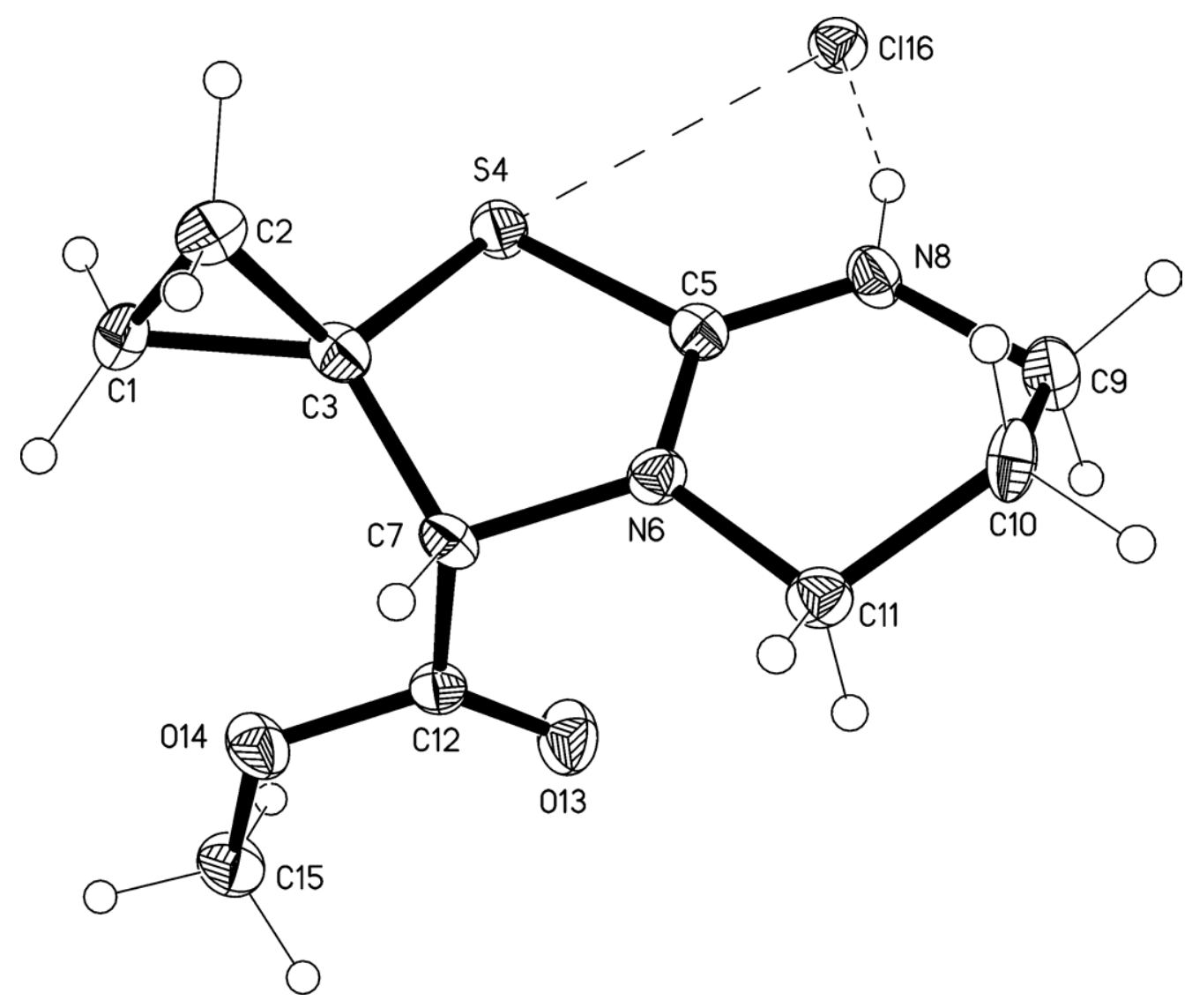


Table 23. Crystal data and structure refinement for $\mathbf{7 4 b}$.

Identification code

Empirical formula

Formula weight

Temperature

Wavelength

Crystal system

Space group

Unit cell dimensions

Volume

Z

Density (calculated)

Absorption coefficient

$\mathrm{F}(000)$

Theta range for data collection

Index ranges

Reflections collected

Independent reflections

Completeness to theta $=58.66^{\circ}$

Refinement method

Data / restraints / parameters

Goodness-of-fit on $\mathrm{F}^{2}$

Final $\mathrm{R}$ indices [I $>2 \operatorname{sigma}(\mathrm{I})]$

$\mathrm{R}$ indices (all data)

Absolute structure parameter

Extinction coefficient

Largest diff. peak and hole

\section{$74 b$}

$\mathrm{C}_{10} \mathrm{H}_{15} \mathrm{Cl} \mathrm{N} \mathrm{O}_{2} \mathrm{~S}$

262.75

100(2) K

$1.54178 \AA$

Orthorhombic

P2(1)2(1)2(1)

$\mathrm{a}=8.9542(2) \AA \quad \alpha=90^{\circ}$.

$\mathrm{b}=10.3036(3) \AA \quad \beta=90^{\circ}$.

$\mathrm{c}=12.8711(3) \AA \quad \gamma=90^{\circ}$.

$1187.49(5) \AA^{3}$

4

$1.470 \mathrm{Mg} / \mathrm{m}^{3}$

$4.404 \mathrm{~mm}^{-1}$

552

5.50 to $58.66^{\circ}$.

$-9<=\mathrm{h}<=9,-10<=\mathrm{k}<=11,-14<=\mathrm{l}<=13$

7764

$1667[\mathrm{R}(\mathrm{int})=0.0325]$

$99.7 \%$

Full-matrix least-squares on $\mathrm{F}^{2}$

1667 / 0 / 146

0.722

$\mathrm{R} 1=0.0246, \mathrm{wR} 2=0.0761$

$\mathrm{R} 1=0.0252, \mathrm{wR} 2=0.0771$

$0.054(15)$

$0.0046(6)$

0.272 and -0.271 e. $\AA^{-3}$ 
Table 24. Atomic coordinates ( $\left.\mathrm{x} 10^{4}\right)$ and equivalent isotropic displacement parameters $\left(\AA^{2} \mathrm{x}\right.$ $10^{3}$ )

for $\mathbf{7 4 b}$. $\mathrm{U}(\mathrm{eq})$ is defined as one third of the trace of the orthogonalized $\mathrm{Uij}^{\mathrm{ij}}$ tensor.

\begin{tabular}{lrrrr}
\hline- & & & \\
& $\mathrm{x}$ & $\mathrm{y}$ & $\mathrm{z}$ & $\mathrm{U}(\mathrm{eq})$ \\
\hline & & & & \\
\hline $\mathrm{O}(13)$ & & & & \\
$\mathrm{N}(6)$ & $-1158(2)$ & $7253(2)$ & $975(1)$ & $26(1)$ \\
$\mathrm{C}(12)$ & $194(2)$ & $5115(2)$ & $1795(1)$ & $16(1)$ \\
$\mathrm{Cl}(16)$ & $-1908(2)$ & $6564(2)$ & $1514(1)$ & $16(1)$ \\
$\mathrm{S}(4)$ & $3838(1)$ & $7829(1)$ & $3796(1)$ & $20(1)$ \\
$\mathrm{N}(8)$ & $296(1)$ & $6339(1)$ & $3547(1)$ & $19(1)$ \\
$\mathrm{O}(14)$ & $2576(2)$ & $5860(2)$ & $2241(1)$ & $18(1)$ \\
$\mathrm{C}(7)$ & $-3350(2)$ & $6766(1)$ & $1741(1)$ & $23(1)$ \\
$\mathrm{C}(3)$ & $-1372(2)$ & $5320(2)$ & $2050(2)$ & $16(1)$ \\
$\mathrm{C}(1)$ & $-1369(2)$ & $5437(2)$ & $3225(2)$ & $18(1)$ \\
$\mathrm{C}(2)$ & $-2737(2)$ & $5556(2)$ & $3883(2)$ & $20(1)$ \\
$\mathrm{C}(5)$ & $-1843(3)$ & $4315(2)$ & $3896(2)$ & $24(1)$ \\
$\mathrm{C}(9)$ & $1140(2)$ & $5724(2)$ & $2427(2)$ & $16(1)$ \\
$\mathrm{C}(10)$ & $3241(3)$ & $5356(2)$ & $1272(2)$ & $22(1)$ \\
$\mathrm{C}(11)$ & $2330(2)$ & $4201(2)$ & $897(2)$ & $21(1)$ \\
$\mathrm{C}(15)$ & $690(2)$ & $4581(2)$ & $796(2)$ & $18(1)$ \\
& $-4007(3)$ & $7956(2)$ & $1335(2)$ & $24(1)$ \\
\hline
\end{tabular}


Table 25. Bond lengths $[\AA]$ and angles $\left[^{\circ}\right]$ for $\mathbf{7 4 b}$.

\begin{tabular}{|c|c|c|c|}
\hline $\mathrm{O}(13)-\mathrm{C}(12)$ & $1.198(3)$ & & \\
\hline $\mathrm{N}(6)-\mathrm{C}(5)$ & $1.332(3)$ & & \\
\hline $\mathrm{N}(6)-\mathrm{C}(7)$ & $1.456(3)$ & & \\
\hline $\mathrm{N}(6)-\mathrm{C}(11)$ & $1.467(2)$ & & \\
\hline $\mathrm{C}(12)-\mathrm{O}(14)$ & $1.340(3)$ & & \\
\hline$C(12)-C(7)$ & $1.533(3)$ & & \\
\hline $\mathrm{S}(4)-\mathrm{C}(5)$ & $1.747(2)$ & & \\
\hline $\mathrm{S}(4)-\mathrm{C}(3)$ & $1.806(2)$ & & \\
\hline $\mathrm{N}(8)-\mathrm{C}(5)$ & $1.316(3)$ & & \\
\hline $\mathrm{N}(8)-\mathrm{C}(9)$ & $1.477(3)$ & & \\
\hline $\mathrm{O}(14)-\mathrm{C}(15)$ & $1.457(3)$ & & \\
\hline$C(7)-C(3)$ & $1.517(3)$ & & \\
\hline $\mathrm{C}(3)-\mathrm{C}(1)$ & $1.494(3)$ & & \\
\hline $\mathrm{C}(3)-\mathrm{C}(2)$ & $1.504(3)$ & & \\
\hline $\mathrm{C}(1)-\mathrm{C}(2)$ & $1.510(3)$ & & \\
\hline $\mathrm{C}(9)-\mathrm{C}(10)$ & $1.521(3)$ & & \\
\hline $\mathrm{C}(10)-\mathrm{C}(11)$ & $1.525(3)$ & & \\
\hline $\mathrm{C}(5)-\mathrm{N}(6)-\mathrm{C}(7)$ & 114.01(17) & & \\
\hline$C(5)-N(6)-C(11)$ & $121.26(18)$ & & \\
\hline $\mathrm{C}(7)-\mathrm{N}(6)-\mathrm{C}(11)$ & $122.93(17)$ & & \\
\hline $\mathrm{O}(13)-\mathrm{C}(12)-\mathrm{O}(14)$ & $125.0(2)$ & & \\
\hline $\mathrm{O}(13)-\mathrm{C}(12)-\mathrm{C}(7)$ & $125.50(19)$ & $C(1)-C(3)-S(4)$ & $120.32(15)$ \\
\hline $\mathrm{O}(14)-\mathrm{C}(12)-\mathrm{C}(7)$ & $109.48(17)$ & $C(2)-C(3)-S(4)$ & $119.80(15)$ \\
\hline$C(5)-S(4)-C(3)$ & $88.91(10)$ & $C(7)-C(3)-S(4)$ & $105.74(14)$ \\
\hline $\mathrm{C}(5)-\mathrm{N}(8)-\mathrm{C}(9)$ & $120.67(19)$ & $C(3)-C(1)-C(2)$ & $60.10(14)$ \\
\hline $\mathrm{C}(12)-\mathrm{O}(14)-\mathrm{C}(15)$ & $116.19(17)$ & $C(3)-C(2)-C(1)$ & $59.44(14)$ \\
\hline $\mathrm{N}(6)-\mathrm{C}(7)-\mathrm{C}(3)$ & $103.56(16)$ & $\mathrm{N}(8)-\mathrm{C}(5)-\mathrm{N}(6)$ & $124.1(2)$ \\
\hline$N(6)-C(7)-C(12)$ & $108.76(17)$ & $\mathrm{N}(8)-\mathrm{C}(5)-\mathrm{S}(4)$ & $122.30(17)$ \\
\hline$C(3)-C(7)-C(12)$ & $112.48(17)$ & $\mathrm{N}(6)-\mathrm{C}(5)-\mathrm{S}(4)$ & $113.56(16)$ \\
\hline $\mathrm{C}(1)-\mathrm{C}(3)-\mathrm{C}(2)$ & $60.46(15)$ & $\mathrm{N}(8)-\mathrm{C}(9)-\mathrm{C}(10)$ & 109.07(18) \\
\hline $\mathrm{C}(1)-\mathrm{C}(3)-\mathrm{C}(7)$ & $124.73(18)$ & $\mathrm{C}(9)-\mathrm{C}(10)-\mathrm{C}(11)$ & $110.01(18)$ \\
\hline$C(2)-C(3)-C(7)$ & $120.70(19)$ & $\mathrm{N}(6)-\mathrm{C}(11)-\mathrm{C}(10)$ & $108.27(16)$ \\
\hline
\end{tabular}

Symmetry transformations used to generate equivalent atoms: 
Table 26. Anisotropic displacement parameters $\left(\AA^{2} \times 10^{3}\right)$ for $\mathbf{7 4 b}$. The anisotropic displacement factor exponent takes the form: $-2 \quad 2\left[h^{2} a^{* 2} U^{11}+\ldots+2 h k a^{*} b^{*} U^{12}\right]$

\begin{tabular}{lcccccc}
\hline & $\mathrm{U}^{11}$ & $\mathrm{U}^{22}$ & $\mathrm{U}^{33}$ & $\mathrm{U}^{23}$ & $\mathrm{U}^{13}$ & $\mathrm{U}$ \\
\hline $\mathrm{O}(13)$ & $23(1)$ & $25(1)$ & $29(1)$ & $7(1)$ & $6(1)$ & $0(1)$ \\
$\mathrm{N}(6)$ & $17(1)$ & $16(1)$ & $14(1)$ & $-2(1)$ & $0(1)$ & $-1(1)$ \\
$\mathrm{C}(12)$ & $16(1)$ & $19(1)$ & $14(1)$ & $-3(1)$ & $-2(1)$ & $-1(1)$ \\
$\mathrm{Cl}(16)$ & $20(1)$ & $21(1)$ & $19(1)$ & $-1(1)$ & $-2(1)$ & $-1(1)$ \\
$\mathrm{S}(4)$ & $16(1)$ & $23(1)$ & $17(1)$ & $-4(1)$ & $-1(1)$ & $0(1)$ \\
$\mathrm{N}(8)$ & $13(1)$ & $21(1)$ & $21(1)$ & $-4(1)$ & $-1(1)$ & $0(1)$ \\
$\mathrm{O}(14)$ & $18(1)$ & $22(1)$ & $28(1)$ & $8(1)$ & $-1(1)$ & $3(1)$ \\
$\mathrm{C}(7)$ & $12(1)$ & $18(1)$ & $18(1)$ & $-1(1)$ & $-3(1)$ & $-1(1)$ \\
$\mathrm{C}(3)$ & $16(1)$ & $17(1)$ & $21(1)$ & $1(1)$ & $-2(1)$ & $-1(1)$ \\
$\mathrm{C}(1)$ & $19(1)$ & $24(1)$ & $18(1)$ & $3(1)$ & $5(1)$ & $-1(1)$ \\
$\mathrm{C}(2)$ & $24(1)$ & $29(1)$ & $19(1)$ & $7(1)$ & $0(1)$ & $0(1)$ \\
$\mathrm{C}(5)$ & $16(1)$ & $13(1)$ & $18(1)$ & $1(1)$ & $-1(1)$ & $1(1)$ \\
$\mathrm{C}(9)$ & $17(1)$ & $25(1)$ & $25(1)$ & $-2(1)$ & $3(1)$ & $1(1)$ \\
$\mathrm{C}(10)$ & $20(1)$ & $20(1)$ & $23(1)$ & $-5(1)$ & $9(1)$ & $4(1)$ \\
$\mathrm{C}(11)$ & $18(1)$ & $18(1)$ & $17(1)$ & $-2(1)$ & $-2(1)$ & $-2(1)$ \\
$\mathrm{C}(15)$ & $22(1)$ & $23(1)$ & $27(1)$ & $4(1)$ & $-2(1)$ & $6(1)$ \\
& & & & & & \\
\hline
\end{tabular}


Table 27. Hydrogen coordinates ( x 104) and isotropic displacement parameters $\left(\AA^{2} \times 10^{3}\right)$ for $\mathbf{7 4 b}$.

\begin{tabular}{lrrrl}
\hline- & & & \\
& & & & \\
& & & & \\
& & & & \\
- & & & & \\
& & & & \\
$\mathrm{H}(8 \mathrm{~A})$ & 3147 & 6255 & 2699 & 22 \\
$\mathrm{H}(7 \mathrm{~A})$ & -1987 & 4560 & 1825 & 19 \\
$\mathrm{H}(1 \mathrm{~A})$ & -3718 & 5544 & 3528 & 25 \\
$\mathrm{H}(1 \mathrm{~B})$ & -2684 & 6124 & 4502 & 25 \\
$\mathrm{H}(2 \mathrm{~A})$ & -1240 & 4124 & 4522 & 29 \\
$\mathrm{H}(2 \mathrm{~B})$ & -2275 & 3544 & 3548 & 29 \\
$\mathrm{H}(9 \mathrm{~A})$ & 4287 & 5087 & 1399 & 27 \\
$\mathrm{H}(9 \mathrm{~B})$ & 3244 & 6044 & 734 & 27 \\
$\mathrm{H}(10 \mathrm{~A})$ & 2431 & 3475 & 1396 & 25 \\
$\mathrm{H}(10 \mathrm{~B})$ & 2712 & 3906 & 215 & 25 \\
$\mathrm{H}(11 \mathrm{~A})$ & 569 & 5238 & 242 & 21 \\
$\mathrm{H}(11 \mathrm{~B})$ & 84 & 3811 & 613 & 21 \\
$\mathrm{H}(15 \mathrm{~A})$ & -5057 & 8007 & 1545 & 36 \\
$\mathrm{H}(15 B)$ & -3941 & 7955 & 575 & 36 \\
$\mathrm{H}(15 \mathrm{C})$ & -3464 & 8707 & 1611 & 36 \\
& & & & \\
\hline
\end{tabular}


3. Methyl 2-chloro-3-hydoxy-2-(1-isopropylcylopropyl)-3-(4-methoxyphenyl)propionate (75b-iPr).

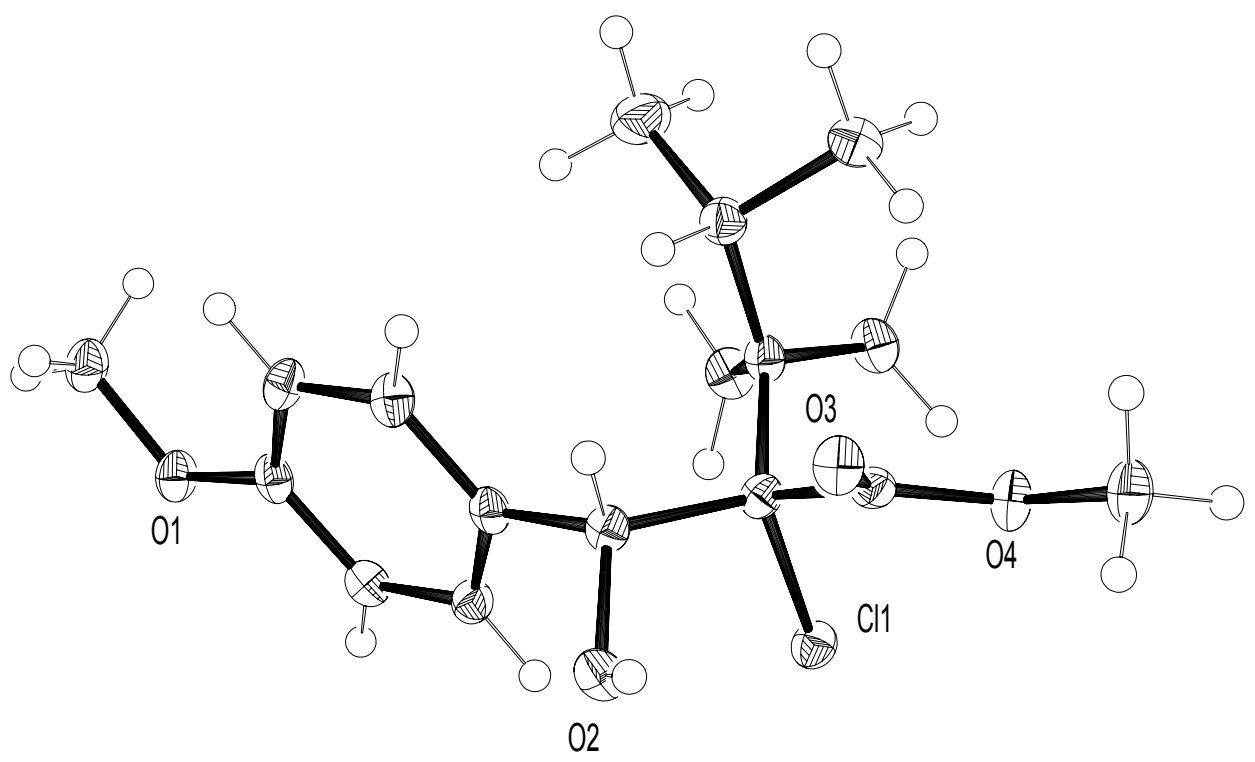


Table 28. Crystal data and structure refinement for $\mathbf{7 5 b} \mathbf{b}-\mathrm{PPr}$.

Empirical formula

Formula weight

Temperature

Wavelength

Crystal system

Space group

Unit cell dimensions

Volume

Z

Density (calculated)

Absorption coefficient

$\mathrm{F}(000)$

Crystal size

Theta range for data collection

Index ranges

Reflections collected

Independent reflections

Completeness to theta $=56.97^{\circ}$

Refinement method

Data / restraints / parameters

Goodness-of-fit on $\mathrm{F}^{2}$

Final R indices [I $>2 \operatorname{sigma}(\mathrm{I})]$

$\mathrm{R}$ indices (all data)

Extinction coefficient

Largest diff. peak and hole
$\mathrm{C}_{17} \mathrm{H}_{23} \mathrm{Cl} \mathrm{O}_{4}$

326.80

100(2) K

$1.54178 \AA$

Monoclinic

$\mathrm{P} 2(1) / \mathrm{n}$

$\mathrm{a}=13.008 \AA$

$\alpha=90^{\circ}$.

$\mathrm{b}=8.581 \AA$

$\beta=93.42^{\circ}$.

$\mathrm{c}=14.591 \AA$

$\gamma=90^{\circ}$.
4

$1.335 \mathrm{Mg} / \mathrm{m}^{3}$

$2.214 \mathrm{~mm}^{-1}$

696

$0.5 \times 0.5 \times 0.5 \mathrm{~mm}^{3}$

4.42 to $56.97^{\circ}$.

$-14<=\mathrm{h}<=14,-9<=\mathrm{k}<=9,-15<=\mathrm{l}<=15$

12541

$2186[\mathrm{R}(\mathrm{int})=0.0272]$

$99.5 \%$

Full-matrix least-squares on $\mathrm{F}^{2}$

2186 / 0 / 200

1.137

$\mathrm{R} 1=0.0343, \mathrm{wR} 2=0.0848$

$\mathrm{R} 1=0.0343, \mathrm{wR} 2=0.0849$

$0.0242(9)$

0.242 and -0.334 e. $\AA^{-3}$ 
Table 29. Atomic coordinates $\left(\mathrm{x} 10^{4}\right)$ and equivalent isotropic displacement parameters $\left(\AA^{2} \mathrm{x}\right.$ $\left.10^{3}\right)$

for $\mathbf{7 5 b}$-iPr. $U($ eq $)$ is defined as one third of the trace of the orthogonalized $\mathrm{Uij}^{\mathrm{ij}}$ tensor.

\begin{tabular}{|c|c|c|c|c|}
\hline & $\mathrm{x}$ & $\mathrm{y}$ & z & $\mathrm{U}(\mathrm{eq})$ \\
\hline \multicolumn{5}{|l|}{-} \\
\hline $\mathrm{Cl}(1)$ & $3720(1)$ & 1859(1) & $2574(1)$ & $20(1)$ \\
\hline $\mathrm{O}(2)$ & $5619(1)$ & $1849(1)$ & $3752(1)$ & $21(1)$ \\
\hline $\mathrm{O}(4)$ & $2944(1)$ & $-632(1)$ & $3634(1)$ & $22(1)$ \\
\hline $\mathrm{O}(3)$ & $4486(1)$ & $-1085(1)$ & $4352(1)$ & $21(1)$ \\
\hline $\mathrm{O}(1)$ & $8119(1)$ & 2181(1) & $232(1)$ & $22(1)$ \\
\hline $\mathrm{C}(1)$ & $7514(1)$ & $1687(2)$ & $915(1)$ & $19(1)$ \\
\hline$C(4)$ & $6222(1)$ & $968(2)$ & $2328(1)$ & $17(1)$ \\
\hline$C(8)$ & $4444(1)$ & $102(2)$ & $2853(1)$ & $17(1)$ \\
\hline$C(3)$ & $7020(1)$ & $-16(2)$ & 2111(1) & $21(1)$ \\
\hline$C(16)$ & $4253(1)$ & $-3863(2)$ & $2675(1)$ & $24(1)$ \\
\hline$C(13)$ & $4937(1)$ & $-2625(2)$ & $2257(1)$ & $21(1)$ \\
\hline$C(10)$ & $4356(1)$ & $-1079(2)$ & 2051(1) & $18(1)$ \\
\hline$C(2)$ & $7670(1)$ & $322(2)$ & $1412(1)$ & $21(1)$ \\
\hline$C(6)$ & $6708(1)$ & $2678(2)$ & 1111(1) & $20(1)$ \\
\hline$C(15)$ & $2463(1)$ & $-1285(2)$ & $4415(1)$ & $25(1)$ \\
\hline$C(9)$ & $3970(1)$ & $-586(2)$ & $3700(1)$ & $17(1)$ \\
\hline$C(5)$ & $6079(1)$ & $2329(2)$ & 1813(1) & $19(1)$ \\
\hline$C(11)$ & $4269(1)$ & $-425(2)$ & 1086(1) & $23(1)$ \\
\hline$C(7)$ & $5583(1)$ & $569(2)$ & $3125(1)$ & $18(1)$ \\
\hline$C(12)$ & $3350(1)$ & $-1175(2)$ & 1480(1) & $23(1)$ \\
\hline$C(14)$ & $5433(2)$ & $-3309(2)$ & $1420(1)$ & $33(1)$ \\
\hline$C(17)$ & $8899(1)$ & $1131(2)$ & $-32(1)$ & $25(1)$ \\
\hline
\end{tabular}


Table 30. Bond lengths $[\AA]$ and angles $\left[{ }^{\circ}\right]$ for $\mathbf{7 5 b}$-iPr.

\begin{tabular}{lc}
\hline $\mathrm{Cl}(1)-\mathrm{C}(8)$ & $1.8121(16)$ \\
$\mathrm{O}(2)-\mathrm{C}(7)$ & $1.429(2)$ \\
$\mathrm{O}(4)-\mathrm{C}(9)$ & $1.333(2)$ \\
$\mathrm{O}(4)-\mathrm{C}(15)$ & $1.445(2)$ \\
$\mathrm{O}(3)-\mathrm{C}(9)$ & $1.209(2)$ \\
$\mathrm{O}(1)-\mathrm{C}(1)$ & $1.373(2)$ \\
$\mathrm{O}(1)-\mathrm{C}(17)$ & $1.426(2)$ \\
$\mathrm{C}(1)-\mathrm{C}(2)$ & $1.386(3)$ \\
$\mathrm{C}(1)-\mathrm{C}(6)$ & $1.392(3)$ \\
$\mathrm{C}(4)-\mathrm{C}(3)$ & $1.390(3)$ \\
$\mathrm{C}(4)-\mathrm{C}(5)$ & $1.395(3)$ \\
$\mathrm{C}(4)-\mathrm{C}(7)$ & $1.508(2)$ \\
$\mathrm{C}(8)-\mathrm{C}(9)$ & $1.533(2)$ \\
$\mathrm{C}(8)-\mathrm{C}(10)$ & $1.547(2)$ \\
$\mathrm{C}(8)-\mathrm{C}(7)$ & $1.563(2)$ \\
$\mathrm{C}(3)-\mathrm{C}(2)$ & $1.393(3)$ \\
$\mathrm{C}(16)-\mathrm{C}(13)$ & $1.535(3)$ \\
$\mathrm{C}(13)-\mathrm{C}(14)$ & $1.530(3)$ \\
$\mathrm{C}(13)-\mathrm{C}(10)$ & $1.548(2)$ \\
$\mathrm{C}(10)-\mathrm{C}(12)$ & $1.511(2)$ \\
$\mathrm{C}(10)-\mathrm{C}(11)$ & $1.515(2)$ \\
$\mathrm{C}(6)-\mathrm{C}(5)$ & $1.381(3)$ \\
$\mathrm{C}(11)-\mathrm{C}(12)$ & $1.502(3)$ \\
$\mathrm{C}(9)-\mathrm{O}(4)-\mathrm{C}(15)$ & $115.69(13)$ \\
$\mathrm{C}(1)-\mathrm{O}(1)-\mathrm{C}(17)$ & $116.63(13)$ \\
$\mathrm{O}(1)-\mathrm{C}(1)-\mathrm{C}(2)$ & $124.60(16)$ \\
$\mathrm{O}(1)-\mathrm{C}(1)-\mathrm{C}(6)$ & $115.57(15)$ \\
$\mathrm{C}(2)-\mathrm{C}(1)-\mathrm{C}(6)$ & $119.81(16)$ \\
$\mathrm{C}(3)-\mathrm{C}(4)-\mathrm{C}(5)$ & $117.57(15)$ \\
$\mathrm{C}(3)-\mathrm{C}(4)-\mathrm{C}(7)$ & $119.44(15)$ \\
$\mathrm{C}(5)-\mathrm{C}(4)-\mathrm{C}(7)$ & $122.93(15)$ \\
$\mathrm{C}(9)-\mathrm{C}(8)-\mathrm{C}(10)$ & $110.03(13)$ \\
$\mathrm{C}(9)-\mathrm{C}(8)-\mathrm{C}(7)$ & $108.37(13)$ \\
&
\end{tabular}




$\begin{array}{lr}\mathrm{C}(10)-\mathrm{C}(8)-\mathrm{C}(7) & 112.79(13) \\ \mathrm{C}(9)-\mathrm{C}(8)-\mathrm{Cl}(1) & 105.79(11) \\ \mathrm{C}(10)-\mathrm{C}(8)-\mathrm{Cl}(1) & 111.17(11) \\ \mathrm{C}(7)-\mathrm{C}(8)-\mathrm{Cl}(1) & 108.40(11) \\ \mathrm{C}(4)-\mathrm{C}(3)-\mathrm{C}(2) & 122.32(16) \\ \mathrm{C}(14)-\mathrm{C}(13)-\mathrm{C}(16) & 109.48(15) \\ \mathrm{C}(14)-\mathrm{C}(13)-\mathrm{C}(10) & 113.47(15) \\ \mathrm{C}(16)-\mathrm{C}(13)-\mathrm{C}(10) & 112.44(14) \\ \mathrm{C}(12)-\mathrm{C}(10)-\mathrm{C}(11) & 59.54(11) \\ \mathrm{C}(12)-\mathrm{C}(10)-\mathrm{C}(8) & 118.42(14) \\ \mathrm{C}(11)-\mathrm{C}(10)-\mathrm{C}(8) & 117.29(14) \\ \mathrm{C}(12)-\mathrm{C}(10)-\mathrm{C}(13) & 117.23(14) \\ \mathrm{C}(11)-\mathrm{C}(10)-\mathrm{C}(13) & 120.43(14) \\ \mathrm{C}(8)-\mathrm{C}(10)-\mathrm{C}(13) & 113.67(13) \\ \mathrm{C}(1)-\mathrm{C}(2)-\mathrm{C}(3) & 118.85(16) \\ \mathrm{C}(5)-\mathrm{C}(6)-\mathrm{C}(1) & 120.44(16) \\ \mathrm{O}(3)-\mathrm{C}(9)-\mathrm{O}(4) & 123.58(16) \\ \mathrm{O}(3)-\mathrm{C}(9)-\mathrm{C}(8) & 122.60(15) \\ \mathrm{O}(4)-\mathrm{C}(9)-\mathrm{C}(8) & 113.77(14) \\ \mathrm{C}(6)-\mathrm{C}(5)-\mathrm{C}(4) & 121.00(16) \\ \mathrm{C}(12)-\mathrm{C}(11)-\mathrm{C}(10) & 60.11(11) \\ \mathrm{O}(2)-\mathrm{C}(7)-\mathrm{C}(4) & 108.74(13) \\ \mathrm{O}(2)-\mathrm{C}(7)-\mathrm{C}(8) & 110.74(13) \\ \mathrm{C}(4)-\mathrm{C}(7)-\mathrm{C}(8) & 114.82(13) \\ \mathrm{C}(11)-\mathrm{C}(12)-\mathrm{C}(10) & 60.35(11) \\ & \\ & \\ \end{array}$

Symmetry transformations used to generate equivalent atoms: 
Table 31. Anisotropic displacement parameters $\left(\AA^{2} \times 10^{3}\right)$ for $\mathbf{7 5 b}$-iPr. The anisotropic displacement factor exponent takes the form: $-2 \quad 2\left[h^{2} a^{* 2} U^{11}+\ldots+2 h \mathrm{k}^{*} b^{*} U^{12}\right]$

\begin{tabular}{lcccccc}
\hline & $\mathrm{U}^{11}$ & $\mathrm{U}^{22}$ & $\mathrm{U}^{33}$ & $\mathrm{U}^{23}$ & $\mathrm{U}^{13}$ & $\mathrm{U}^{12}$ \\
\hline $\mathrm{Cl}(1)$ & $19(1)$ & $21(1)$ & $21(1)$ & $3(1)$ & $0(1)$ & $3(1)$ \\
$\mathrm{O}(2)$ & $26(1)$ & $25(1)$ & $12(1)$ & $-2(1)$ & $0(1)$ & $-5(1)$ \\
$\mathrm{O}(4)$ & $15(1)$ & $32(1)$ & $19(1)$ & $4(1)$ & $2(1)$ & $-1(1)$ \\
$\mathrm{O}(3)$ & $20(1)$ & $29(1)$ & $15(1)$ & $2(1)$ & $-1(1)$ & $-1(1)$ \\
$\mathrm{O}(1)$ & $20(1)$ & $29(1)$ & $17(1)$ & $2(1)$ & $5(1)$ & $-2(1)$ \\
$\mathrm{C}(1)$ & $16(1)$ & $27(1)$ & $12(1)$ & $-2(1)$ & $-2(1)$ & $-5(1)$ \\
$\mathrm{C}(4)$ & $15(1)$ & $22(1)$ & $14(1)$ & $-2(1)$ & $-4(1)$ & $-3(1)$ \\
$\mathrm{C}(8)$ & $15(1)$ & $19(1)$ & $16(1)$ & $1(1)$ & $-1(1)$ & $2(1)$ \\
$\mathrm{C}(3)$ & $18(1)$ & $25(1)$ & $18(1)$ & $5(1)$ & $-2(1)$ & $0(1)$ \\
$\mathrm{C}(16)$ & $27(1)$ & $22(1)$ & $24(1)$ & $-1(1)$ & $0(1)$ & $-1(1)$ \\
$\mathrm{C}(13)$ & $21(1)$ & $22(1)$ & $20(1)$ & $-1(1)$ & $1(1)$ & $-1(1)$ \\
$\mathrm{C}(10)$ & $16(1)$ & $23(1)$ & $15(1)$ & $0(1)$ & $0(1)$ & $-2(1)$ \\
$\mathrm{C}(2)$ & $15(1)$ & $29(1)$ & $19(1)$ & $0(1)$ & $0(1)$ & $3(1)$ \\
$\mathrm{C}(6)$ & $22(1)$ & $21(1)$ & $16(1)$ & $2(1)$ & $-2(1)$ & $-2(1)$ \\
$\mathrm{C}(15)$ & $21(1)$ & $35(1)$ & $21(1)$ & $3(1)$ & $5(1)$ & $-2(1)$ \\
$\mathrm{C}(9)$ & $18(1)$ & $17(1)$ & $17(1)$ & $-4(1)$ & $-1(1)$ & $0(1)$ \\
$\mathrm{C}(5)$ & $17(1)$ & $22(1)$ & $20(1)$ & $-3(1)$ & $0(1)$ & $0(1)$ \\
$\mathrm{C}(11)$ & $26(1)$ & $28(1)$ & $14(1)$ & $0(1)$ & $1(1)$ & $-3(1)$ \\
$\mathrm{C}(7)$ & $17(1)$ & $21(1)$ & $15(1)$ & $0(1)$ & $-2(1)$ & $0(1)$ \\
$\mathrm{C}(12)$ & $21(1)$ & $30(1)$ & $18(1)$ & $0(1)$ & $-5(1)$ & $-3(1)$ \\
$\mathrm{C}(14)$ & $39(1)$ & $27(1)$ & $35(1)$ & $-4(1)$ & $14(1)$ & $2(1)$ \\
$\mathrm{C}(17)$ & $20(1)$ & $33(1)$ & $21(1)$ & $-2(1)$ & $5(1)$ & $-1(1)$ \\
& & & & & & \\
\hline & & & & & & \\
\end{tabular}


Table 32. Hydrogen coordinates ( $\left.\mathrm{x} 10^{4}\right)$ and isotropic displacement parameters $\left(\AA^{2} \times 10^{3}\right)$ for $75 \mathbf{b}-\mathrm{iPr}$.

\begin{tabular}{|c|c|c|c|c|}
\hline & $\mathrm{x}$ & $\mathrm{y}$ & $\mathrm{z}$ & $\mathrm{U}(\mathrm{eq})$ \\
\hline - & & & & \\
\hline $\mathrm{H}(2 \mathrm{~A})$ & 5585 & 1513 & 4290 & 32 \\
\hline $\mathrm{H}(3 \mathrm{~A})$ & 7127 & -953 & 2451 & 25 \\
\hline $\mathrm{H}(16 \mathrm{~A})$ & 4654 & -4817 & 2793 & 37 \\
\hline $\mathrm{H}(16 \mathrm{~B})$ & 3664 & -4090 & 2246 & 37 \\
\hline $\mathrm{H}(16 \mathrm{C})$ & 4006 & -3471 & 3253 & 37 \\
\hline $\mathrm{H}(13 \mathrm{~A})$ & 5508 & -2389 & 2726 & 25 \\
\hline $\mathrm{H}(2 \mathrm{~B})$ & 8211 & -371 & 1278 & 25 \\
\hline $\mathrm{H}(6 \mathrm{~A})$ & 6590 & 3599 & 760 & 23 \\
\hline $\mathrm{H}(15 \mathrm{~A})$ & 1713 & -1268 & 4300 & 38 \\
\hline $\mathrm{H}(15 \mathrm{~B})$ & 2654 & -668 & 4963 & 38 \\
\hline $\mathrm{H}(15 \mathrm{C})$ & 2695 & -2363 & 4510 & 38 \\
\hline $\mathrm{H}(5 \mathrm{~A})$ & 5541 & 3027 & 1947 & 23 \\
\hline $\mathrm{H}(11 \mathrm{~A})$ & 4264 & 723 & 1015 & 27 \\
\hline $\mathrm{H}(11 \mathrm{~B})$ & 4616 & -999 & 603 & 27 \\
\hline $\mathrm{H}(7 \mathrm{~A})$ & 5918 & -340 & 3451 & 21 \\
\hline $\mathrm{H}(12 \mathrm{~A})$ & 3132 & -2208 & 1238 & 28 \\
\hline $\mathrm{H}(12 \mathrm{~B})$ & 2780 & -487 & 1650 & 28 \\
\hline $\mathrm{H}(14 \mathrm{~A})$ & 5787 & -4282 & 1595 & 50 \\
\hline $\mathrm{H}(14 \mathrm{C})$ & 5930 & -2563 & 1196 & 50 \\
\hline $\mathrm{H}(14 \mathrm{D})$ & 4898 & -3520 & 935 & 50 \\
\hline $\mathrm{H}(17 \mathrm{C})$ & 9279 & 1603 & -521 & 37 \\
\hline $\mathrm{H}(17 \mathrm{D})$ & 8579 & 157 & -254 & 37 \\
\hline $\mathrm{H}(17 \mathrm{~A})$ & 9374 & 911 & 499 & 37 \\
\hline
\end{tabular}




\section{References}

[1] K. C. Nicolaou, D Vourloumis, N. Winssinger, P. S. Baran, Angew. Chem. 2000, 112, 46-126; Angew. Chem. Int. Ed. 2000, 39, 44-122.

[2] [a] H-W. Lio and C. T. Walsh: The Chemistry of cyclopropyl group, Ed.: Z. Rappoport, Wiley 1987, 959-1021. [b] J. Salaün, Top. Curr. Chem. 2000, 207, 1-67. [c] J. Salaün, M. S. Baird, Curr. Med. Chem. 1995, 2, 511.

[3] A. de Meijere, Angew. Chem. 1979, 91, 867-884; Angew. Chem. Int. Ed. 1979, 18, 809826.

[4] [a] G. Moss, Topics in current chemistry, 1987, 137, 75-253. [b] J. M. fraile, J. I. Garcia, J. A. Mayoral, Chem. Commun. 1996, 1319-1320. [c] M. M. C. Lo, G. C. Fu, J. Am. Chem. Soc. 1998, 120, 10270-10271.

[5] A. J. Anciaux, A. J. Hubert, A. F. Noels, N. Petniot, P. Teyssie, J. Org. Chem. 1980, 45, 695-701. [b] H. J. Callot, C. Piechochi, Tetrahedron Lett. 1980, 21, 3489-3492.

[6] [a] O. G. Kulinkovich, A. de Meijere, Chem. Rev. 2000, 100, 2789-2834. [b] F. Sato, H. Urabe, S. Okamoto, Chem. Rev. 2000, 100, 2835-2886.

[7] D. H. Gibson, C. H. DePuy, Chem. Rev. 1974, 74, 605-623.

[8] [a] O. G. Kulinkovich, S. V. Sviridov, D. A. Vasilevskii, A. I. Savchenko, T. S. Pritytskaya, J. Org. Chem. USSR (Engl. Transl.) 1991, 27, 250-253; Zh. Org. Khim. 1991, 27, 294-298. [b] O. G. Kulinkovich, S. V. Sviridov, D. A. Vasilevski, Synthesis 1991, 234. [c] O. G. Kulinkovich, D. A. Vasilevskii, A. I. Savchenko, S. V. Sviridov, J. Org. Chem. USSR (Engl. Transl.) 1991, 27, 1249-1251; Zh. Org. Khim. 1991, 27, 14281430. [d] S. V. Sviridov, D. A. Vasilevskii, O. G. Kulinkovich, J. Org. Chem. USSR (Engl. Transl.) 1991, 27, 1251-1253; Zh. Org. Khim. 1991, 27, 1431-1433.

[9] [a] V. Chaplinski, A. de Meijere, Angew. Chem. 1996, 108, 491-492; Angew. Chem. Int. Ed. 1996, 35, 413-414. [b] V. Chaplinski, H. Winsel, M. Kordes, A. de Meijere, Synlett, 1997, 111-114. [c] For a recent reveiw see: A. de Meijere, S. I. Kozhushkov, A. I. Savchenko, J. Orgmet. Chem. 2004, 2033-2055.

[10] [a] D. M. Campoli-Richards, J. P. Monk, A. Price, P. Benfield, P. A. Todd, A. Ward, Drugs 1988, 35, 373-447. [b] R. Wise, J. M. Andrews, Antimicrob. Chemother. 1983, 23, 559-564. 
[11] [a] K. E. Brighty, WO Patent 91/02526, 1991; EU Patent 413455, 1991; (Chem. Abstr. 1991, 115, 232216); U. S. Patent 5.165.402, 1993: (Chem. Arstr. 1993, 119, 117227); [b] T. Norrish, T. F. Braish, M. Butters, K. M. DeVries, J. M. Hawkins, S. S. Massett, P. R. Rose, D. Santafianos, C. Sklavounos, J. Chem. Soc., Perkin Trans. 1, 2000, 16151622 .

[12] [a] P. M. Dunnill, L. Fowde, Phytochemistry 1965, 4, 935. [b] H. Shinozaki, S. Konishi, Neuropharmacology 1974, 13, 665.

[13] [a] Y. Han, S. Liao, W. Qiu, C. Cai, V. J. Hruby, Tetrahedron Lett. 1997, 38, 51355138. [b] S. E. Gibson, N. Guillo, R. J. Middleton, A. Thuillie, M. J. Tozer, J. Chem. Soc., Perkin Trans. 1, 1997, 447-455. [c] C. Haskell-Luevano, L. W. Boteju, H. Miwa, C. Dickinson, I. Gantz, T. Yamada, M. E. Hadley, V. J. Hruby, J. Med. Chem. 1995, 38, $4720-4729$.

[14] V. J. Hruby, Biopolymers 1993, 33, 1073-1082. [b] V. J. Hruby, F. Al-qebidi, W. Kazmierski, Biochem. J. 1990, 268, 249-262.

[15] [a] A. Brandi, S. Cicchi, F. M. Cardero, A. Goti, Chem. Rev. 2003, 103, 1213-1269. [b] I. Nakamura, Y. Yamamoto, Adv. Synth. Catal. 2002, 344, 111-129. [c] A. de Meijere, S. I. Kozhushkov, L. P. Hadjiarapoglou, Topics Curr. Chem. 2000, 207, 149-227.

[16] [a] Methyl: F. Seyed-Mahdavi, S. Teichmann, A. de Meijere, Tetrahedron Lett. 1986, 27, 6185-6188. [b] Ethyl: J. Salaün, F. Bennani, J.-C. Compain, A. Fadel, J. Ollivier, J. Org. Chem. 1980, 45, 4129-4135. [c] D. Spitzner, H. Swoboda, Tetrahedron Lett. 1986, 27, 1281-1284. [d] T. Thiemann, D. Ohira, Y. Li, T. Sawada, S. Mataka, K. Rauch, M. Noltemeyer, A. de Meijere, J. Chem. Soc. Perkin Trans. 1 2000, 17, 2968-2976. [e] Benzyl: L. Wessjohann, N. Krass, D. Yu, A. de Meijere, Chem. Ber. 1992, 125, 867-882.

[17] [a] Ethyl: A. de Meijere, S. Teichmann, F. Seyed-Mahdavi, S. Kohlstruk, Liebigs Ann. Org. Bioorg. Chem. 1996, 1989-2000. [b] Benzyl: N. F. Osborne, J. Chem. Soc. Perkin Trans. 1 1982, 1435-1439.

[18] [a] T. Liese, G. Splettstösser, A. de Meijere, Angew. Chem. 1982, 94, 799. Angew. Chem. Int. Ed. Engl. 1982, 21, 790. [b] T. Liese, F. Sayed-Mahdavi, A. de Meijere, Org. Synth. 1990, 69, 148-152.

[19] C. Glück, V. Poignee, H. Schwager, Synthesis 1987, 260 [b] J. Sepiol, R. L. Saulen, J. Org. Chem. 1975, 40, 3791. 
[20] [a] A. de Meijere, L. Wessjohan, Synlett, 1990, 20-32. [b] L. Wessjohan, L. Skattebol, A. de Meijere, J. Chem. Soc.,Chem. Commun. 1990, 574-576.

[21] [a] L. Hadjiarapoglou, I. Klein, D. Spitzner, A. de Meijere, Synthesis 1996, 4, 525528. [b] L. J. van Boxtel, S. Körbe, M. Noltemeyer, A. de Meijere, Eur. J. Org. Chem. 2001, 2283-2292. [c] K. H. Ang, S. Bräse, A. G. Steinig, F. E. Meyer, A. Llebaria, K. Voigt, A. de Meijere, Tetrahedron 1996, 52, 11503-11528. [d] G.-A. Cao, K.-F. Cheng, Synth. Commun. 1996, 26, 1525-1538. [e] K. Tokuzaki, Y. Kanemitu, T. Yoshimitsu, H. Nagaoka, Tetrahedron Lett. 2000, 41, 5923-5926.

[22] [a] A. de Meijere, I. D. Kuchuk, V. V. Sokolov, T. Labahn, K. Rauch, M. Es-Sayed, T. Krämer, Eur. J. Org. Chem. 2003, 985-997. [b] C. Zorn, A. Goti, A. Brandi, K. Johnsen, M. Noltemeyer, S. I. Kozhushkov, A. de Meijere, J. Org. Chem. 1999, 64, 755-763. [c] F. M. Cordero, B. Anichini, A. Goti, A. Brandi, Tetrahedron 1993, 49, 9867-9876. [d] X. Huang, H. Zhou, W. Chen, J. Org. Chem. 2004, 839-842.

[23] [a] A. Rulev, J. Maddaluno, Eur. J. Org. Chem. 2001, 2569-2576. [b] M. Tamm, M. Thutewohl, C. B. Ricker, N. T. Bes, A. de Meijere, Eur. J. Org. Chem. 1999, 2017-2024. [c] L. Wessjohann, K. Giller, B. Zuck, L. Skattebøl, A. de Meijere, J. Org. Chem. 1993, 58, 6442-6450. [d] L. Wessjohann, G. McGaffin, A. de Meijere, Synthesis 1989, 359363.

[24] [a] V. N. Belov, C. Funke, T. Labahn, M. Es-Sayed, A. de Meijere, Eur. J. Org. Chem. 1999, 1345-1356. [b] C. Funke, M. Es-Sayed, A. de Meijere, Org. Lett. 2000, 2, 42494251 .

[25] L. Wessjohann, N. Krass, D. Yu, A. de Meijere, Chem. Ber. 1992, 125, 867.

[26] M. Nötzel, K. Rauch, T. Labahn, A. de Meijere, Org. Lett. 2002, 4, 839-841.

[27] M. W. Nötzel, M. Tamm, T. Labahn, M. Noltemeyer, M. Es-Sayed, A. de Meijere, J. Org. Chem. 2000, 65, 3850-3852.

[28] M. Nötzel, T. Labahn, M. Es-Sayed, A. de Meijere, Eur. J. Org. Chem. 2001, 3025-3030.

[29] [a] M. R. Prinsep, R. E. Moore, I. A. Levine, G. M. Patterson, J. Nat. Prod. 1992, 55, 140-142. [b] M. North, G. Pattenden, Tetrahedron 1990, 46, 8267-8290. [c] T. Ishida, M. Tanaka, M. Nabae, M. Inoue, S. Kato, Y. Hamada, T. Shioiri, J. Org. Chem. 1988, 53, $107-112$. 
[30] [a] S. Kato, Y. Hamada, T. Shioiri, Tetrahedron Lett. 1986, 27, 2653-2656. [b] T. Shioiri, Y. Hamada, Studies in Natural Products Chemistry 1989, 44, 83-110.

[31] T. Hosokawa, T. Ohta, S. Murahashi, J. Chem. Soc. Chem. Commun. 1983, 848-849.

[32] L. J. Reddy, P. Saravanan, E. J. Corey, J. Am. Chem. Soc. 2004, 126, 6230-6231.

[33] [a] A. J. Robichaud, A. I. Meyers, J. Org. Chem. 1991, 56, 2607-2609. [b] D. A. Evans, K. A. Woerpel, M. J. Scott, Angew. Chem. 1992, 104, 439-441; Angew. Chem. Int. Ed. 1992, 31, 430-432.

[34] D. G. I. Kingston, A. G. Chaudhary, A. A. Gunatilaka, M. L. Middleton, Tetrahedron Lett. 1994, 35, 4483-4484.

[35] M. C. Wani, H. L. Taylor, M. E. Wall, P. Coggon, A. T. McPhail, J. Am. Chem. Soc. 1971, 93, 2325-2327.

[36] [a] F. A. Holmes, R. S. walters, R. L. Theriault, A. D. Forman, L. K. Newton, M. N. Raber, A. U. Buzdar, D. K. Frye, G. N. Hortobagye, J. Nat. Cancer Inst. 1991, 83, 17971805. [b] M. Markman, Yale Biol. Med. 1991, 64, 583-590. [c] S. M. Swain, S. F. Honig, M. C. Tefft, L. Walton, Invest. New drugs 1995, 13, 217-222.

[37] [a] K. C. Nicolaou, Z. Yang, J. J. Liu, H. Ueno, P. G. Nantermet, R. K. Guy, C. F. Claiborne, J. Reneud, E. A. Couladouros, K. Paulvannan, E. J. Sorensen, Nature (London) 1994, 367, 630. [b] K. C. Nicolaou, R. K. Guy, Angew. Chem. 1995, 107, 2247-2259; Angew. Chem. Int. Ed. Engl. 1995, 34, 2079-2090.

[38] For a review on the synthesis of cyclopropane-containing natural products, see: [a] W. A. Donaldson, Tetrahedron, 2001, 57, 8589-8627. [b] J. Pietruszka, Chem. Rev. 2003, 103, 1051-1070.

[39] [a] I. Ojima, S. Lin, J. Org. Chem. 1998, 63, 224-225. [b] S. -H. Chen, S. Huang, J. Wei, V. Farina, J. Org, Chem. 1993, 58, 4520-4521.

[40] C. Liu, M. Tamm, M. W. Nötzel, A. de Meijere, J. K. Schilling, D. G. I. Kingston, Tetrahedron Lett. 2003, 44, 2049-2052.

[41] K. Minn, P. Braun, B. Sachse, H. Wicke, WO 92/05159. [b] U. Schindler, K. Schönafinger, H. Hartmut, WO 00/46214.

[42] R. Koehler, L. Goodman, J. DeGraw, B. R. Baker, J. Amer. Chem. Soc. 1958, 80, 57795786. 
[43] [a] A. Gangjee, A. Vasudevan, R. L. Kisliuk, J. Heterocycl. Chem. 1997, 34, 1669-1676.

[b] G. Bernáth, J. Kóbor, J. Lázár, F. Fülöp, J. Heterocycl. Chem. 1996, 33, 1983-1987. [c]

G. Bernáth, T. Janáky, G. Göndös, J. Lázár, Z. Ecsery, Pharmazie 1983, 38, 270-271. [d]

T. Nishio, M. Fujisawa, Y. Omote, J. Chem. Soc., Perkin Trans. 1, 1987, 2523-2529.

[44] [a] T. Sekiya, H. Hiranuma, M. Uchide, S. Hata, S. Yamada, Chem. Pharm. Bull. 1981, 29, 948-954. [b] F. Claudi, G. Giorgioni, L. Scoccia, R. Ciccocioppo, I. Panocka, M. Massi, Eur. J. Med. Chem. 1997, 32, 651-660..

[45] M. G. Nair, R. Dhawan, M. Ghazala, T. I. Kalman, R. Ferone, Y. Gaumont, R. L. Kisliuk, J. Med. Chem. 1987, 30, 1256-1261.

[46] A: Rosowsky, R. A. Forsch, R. G. Moran, J. Med. Chem. 1989, 32, 709-715.

[47] [a] S. Kawamura, Y. Sanemitsu, J. Org. Chem. 1993, 58, 414-418; b) F. Fülöp, G. Bernáth, Synthesis, 1985, 1148-1149.

[48] [a] P. López-Alvarado, S. García-Granda, C. Álvarez-Rúa, C. Avendaño, Eur. J. Org. Chem. 2002, 1702-1707. [b] P. M. T. Ferreira, H. L. S. Maia, L. S. Monteiro, J. Sacramento, J. Sebastião, J. Chem. Soc., Perkin Trans. 1 2000, 3317-3324. [c] J. H. Poupaert, J. Bukuru, A. Gozzo, Monatsh. Chem. 1999, 130, 929-932. [d] M.-F. Hsieh, P. D. Rao, C.-C. Liao, Chem. Comm. 1999, 1441-1442. [e] X.-L. Li, Y.-M. Wang, T. Matsuura, J.-B. Meng, J. Het. Chem. 1999, 36, 697-701.

[49] [a] C. Balsamini, G. Diamantini, A. Duranti, G. Spadoni, A. Tontini, Synthesis 1995, 370-372. [b] G. Spadoni, C. Balsamini, A. Bedini, E. Duranti, A. Tontini, J. Het. Chem. 1992, 29, 305-309.

[50] [a] M.-Y. Chang, S.-T. Chen, N.-C. Chang, Heterocycles 2002, 57, 2321-2334. [b] K. Yamada, F. Yamada, M. Somei, Heterocycles 2002, 57, 1231-1234. [c] G. Galambos, P. Csókási, C. Szántay Jr., C. Szantay, Liebigs Ann. Recl. 1997, 1969-1978. [d] G. Galambos, P. Csókási, C. Szántay Jr., G. Czira, C. Szántay, Heterocycles 1994, 38, 1727-1732. [e] M. Toyota, K. Fukumoto, J. Chem. Soc., Perkin Trans. 1 1992, 547-552. [f] M. Somei, F. Yamada, H. Ohnishi, Y. Makita, M. Kuriki, Heterocycles 1987, 26, 2823-2828.

[51] A. Dougan, P. Grondin, C. Ruault, A.-C. Le Monnier de Gouville, H. Coste, J. Kirilovsky, F. Hyafil, R. Labaudinière, J. Med. Chem. 2003, 46, 4525-4532. 
[52] [a] S. J. Whitaker, I. S. de Tejada, K. M. Ferguson, WO 01/80860 A2. [b] A. Dougan, P. Grondin, C. Ruault, A-C. Le M. de Gouville, H. Coste, J. M. Linget, J. Kirilovsky, F. Hyafil, R. Labaudinière, J. Med. Chem. 2003, 46, 4533-4542.

[53] [a] Posner, G. H. Chem. Rev. 1986, 86, 831-844. [b] Tietze, L. F.; Beifuss, U. Angew. Chem. 1993, 105, 137-170; Angew. Chem. Int. Ed. Engl. 1993, 32, 131-164. [c] Tietze, L. F.; Chem. Rev. 1996, 96, 115-136.

[54] [a] A. G. M. Barrett, A. Kamimura, J. Chem. Soc. Chem. Commun. 1995, 17, 1755-1756. [b] N. Tsukada, T. K. Nishimura, H. Tsubouchi, M. Ono, T. Hayama, Y. Nagaoka, K. Tomioka, Tetrahedron Lett. 2003, 44, 2323-2326. [c] N. Tsukada, T. Shimida, Gyoung, Y. S.; Asao, N.; Yamamoto, Y. J. Org. Chem. 1995, 60, 143-148.

[55] Reviews see: [a] R. E. Gawley, Synthesis 1976, 777-794. [b] W. Notz, F. Tanaka, C. F. Barbas, Acc. Chem. Res. 2004, 37, 580-591.

[56] [a] O. Hara, K. Sugimoto, K. Makino, Y. Hamada, Synlett 2004, 1625-1627. [b] H. S. P Rao, S. P. Senthilkumar, J. Org. Chem. 2004, 69, 2591-2594. [c] K. Makino, O. Hara, Y. Takiguchi, T. Katano, Y. Asakawa, K. Hatano, Y. Hamada, Tetrahedron Lett. 2003, 44, 8925-8929. [d] X. Huang, Xiong, Chem. Commun. 2003, 14, 1714-1715. [e] Yamamoto, Y.; Momiyama, N.; Yamamoto, H. J. Am. Chem. Soc. 2004, 126, 59625963.

[57] M. Limbach, Dissertation, University of Goettingen, 2004.

[58] [a] A. Kishi, S. Sakaguchi, Y. Ishii, Org. Lett. 2000, 2, 523-526. [b] T. Hosokawa, S. Aoki, S.-I. Murahashi, Synthesis 1992, 558-561. [c] L. Q. Jia, H. F. Jiang, J. H. Li, Chem. Commun. 1999, 985-986. [d] T. Hosokawa, T. Ohta, S. Kanayama, S. Murahashi, J. Org. Chem. 1987, 52; 1758-1764.

[59] A. Holy, Collect. Czech. Chem. Commun. 1974, 39, 3177-3186.

[60] Ethyl 3,3-diethylpropionate $\mathbf{1 6}$ has previously been employed in a titanium-mediated reductive cyclopropanation with butylmagnesium bromide to yield, in a diastereoselective fashion, 2-ethyl-1-(2',2'-diethoxyethyl)cyclopropanol: [a] Y. Y. Kozyrkov, A. Pukin, O. G. Kulinkovich, J. Ollivier, J. Salaün, Tetrahedron Lett. 2000, 41, 6399-6402. [b] S. Racouchot, I. Sylvestre, J. Ollivier, Y. Y. Kozyrkov, A. Pukin, O. G. Kulinkovich, J. Salaün, Eur. J. Org. Chem. 2002, 2160-2176.

[61] M. Limbach, S. Dalai, A. de Meijere, Adv. Synth. Catal. 2004, 346, 760-766. 
[62] For syntheses of amidines from nitriles see: [a] R. A. Moss, J. Terpinski, D. P. Cox, D. Z. Denney, K. Krogh-Jespersen, J. Am. Chem. Soc. 1985, 107, 2743-2748. [b] R. T. Boeré, R. T. Oakley, R. W. Reed, J. Organomet. Chem. 1987, 331, 161-167. [c] F. C. Shaefer, A. P. Krapcho, J. Org. Chem. 1962, 27, 1255-1258. [d] A. Thurkauf, A. Hutchison, J. Peterson, L. Cornfield, R. Meade, K. Huston, K. Harris, P. C. Ross, K. Gerber, T. V. Ramabhadran, J. Med. Chem. 1995, 38, 2251-2255. [e] W. von der Saal, R. A. Engh, A. Eichinger, B. Gabriel, R. Kucznierz, J. Sauer, Arch. Pharm. 1996, 329, 73-82.

[63] M. W. Nötzel, Dissertation, University of Goettingen, 2001

[64] K. J. M. Andrews, N. Anand, A. R. Todd, A. Topham, J. Chem. Soc. 1949, 2490-2497.

[65] For a book see: F. Fringuelli, A. Taticchi, The Diels-Alder Reaction, Selected Practical Methods, John Willy \& Sons, 2002.

[66] K. C. Nicolaou, S. A. Snyder, T. Montagnon, G. Vassilikogiannakis, Angew. Chem. 2002, 114, 1742-1773. Angew. Chem. Int. Ed. Engl. 2002, 41, 1668-1698.

[67] [a] R. V. Wheeler, W. L. Wood, J. Chem. Soc. 1930, 1819. [b] P. D. Bartlett, K. E. Schueller, J. Am. Chem. Soc. 1968, 90, 6071-6077.

[68] [a] R. V. C. Carr, L. Paquette, J. Amer. Chem. Soc. 1980, 102, 853-855. [b] R. V. C. Carr, R. V. Williams, L. Paquette, J. Org. Chem. 1983, 48, 4976-4986.

[69] [a] E. J. Bush, D. W. Jones, J. Chem. Soc., Perkin Trans. 1, 1997, 3531-3536. [b] E. J. Bush, D. W. Jones, T. P. Mark, Tetrahedron Lett. 1994, 35, 9755-9758. [c] T. Kametani, Y. Suzuki, T. Honda, Chem. Pharma. Bull. 1986, 34, 4971-4977.

[70] [a] L. Perreux, A. Loupy, Tetrahedron 2001, 57, 9199; [b] R. S. Berma, Green Chem. 1999, $1,43-54$.

[71] For recent books on microwaves: [a] B. L. Hayes, Microwave synthesis, Chemistry at the speed of light, CEM publishing, 2002. [b] A. Loupy, Microwaves in organic synthesis, Wiley VCH, Weinheim, 2003.

[72] [a] R. K. Bansal, A. Dandia, N. Gupta, D. Jain, Heteroatom Chem. 2003, 14, 560-563. [b] N. Kaval, J. Van der Eychen, J. Caroen, W. Dehaen, G. A. Strohmeier, C. O. Kappe, E. Van der Eychen, J. Comb. Chem. 2003, 5, 560-568. [c] E. Van der Eychen, P. Appukkuttan, W. de Borggraeve, W. Dehaen, D. Dallinger, C. O. Kappe, J. Org. Chem. 2002, 67, 7904-7907. 
[73] B. M. Trost, H. C. Arndt, P. E. Strege, T. R. Verhoeven, Tetrahedron. Lett. 1976, $3477-$ 3478.

[74] [a] Y. Masaki, Y. Serizawa, K. Nagata, K. Kaji, Chem. Lett. 1984, 2105-2108. [b] D. F. Taber, Q. Jiang, B. Chen, W. Zhang, C. L. Campbell, J. Org. Chem. 2002, 67, 4821-4827.

[75] [a] J. A. Beavo, Physiol. Rev. 1995, 75, 725-748. [b] S. H. Soderling, J. A. Biavo, Curr. Opin. Cell Biol. 2000, 12, 174-179. [c] J. D. Corbin, S. H. Francis, J. Biol. Chem. 1999, 274, 13729-13732.

[76] [a] M. Czarniecki, H. S. Ahn, E. J. Sybertz, Annu. Rep. Med. Chem. 1996, 31, 61-70. [b] J. C. Stoclet, T. Keravis, N. Komas, C. Lugnier, Exp. Opin. Invest. Drugs. 1995, 4, 1081-1100.

[77] [a] J. Rajfer,W. J. Aronson, P. A. Bush, F. J. Dorey, L. J. Ignarro, N. Engl. J. Med. 1992, 326, 90-94. [b] D. P. Rotella, Drugs Future 2001, 26, 153-164. [c] A. W. Stanford, Annu. Rep. Med. Chem. 1996, 31, 61-70.

[78] I. H. Osterloh, M. Collins, P. Wicker, G. Wagner, Int. J. Clin. Pract., Suppl. 1999, 102, $3-5$.

[79] J. Carledge, I. Eardley, Exp. Opin. Pharmacother. 1999, 1, 137-147.

[80] A. Dougan, P. Grondin, C. Ruault, A-C. Le M. de Gouville, H. Coste, J. M. Linget, J. Kirilovsky, F. Hyafil, R. Labaudinière, J. Med. Chem. 2003, 46, 4533-4542.

[81] E. D. Cox, J. M. Cook. Chem. Rev. 1995, 95, 1797-1842.

[82] J. Li, T. Wang, P. Yu, A. Peterson, R. Weber, D. Soerens, D. Grubisha, D. Bennett, J. M. Cook, J. Am. Chem. Soc. 1999, 121, 6998-7010.

[83] [a] C. H. stammer, Tetrahedron 1990, 46, 2231-2254. [b] R. M. Williams, J. G. Fegley, J. Am. Chem. Soc. 1991, 113, 8796-8806. [c] S. Ahmed, R. S. Phillips, C. H. Stammer, J. Med. Chem. 1992, 35, 1410-1417. [d] G. Flouret, W. Brieher, T. Majewsky, K. Mahan, L. Wilson, J. Med. Chem. 1991, 34, 2089-2094. [e] A. Doherty, J. Med. Chem. 1992, 35, 1493-1508.

[84] This so called Thorpe-Ingold effect had originally been attributed to an angle enlargement. $C f$. [a] R. M. Beesley, C. K. Ingold, J. F. Thorpe, J. Chem. Soc. 1915, 107, 1080-1106. [b] C. K. Ingold, J. Chem. Soc. 1921, 119, 305. It was later shown that the major influence stems from the conformational preference of such a system: $C f$. [c] M. E. 
Jung, M. Kiankarimi, J. Org. Chem. 1998, 63, 2968-2974. [d] M. E. Jung, J. Gervay, J. Am. Chem. Soc. 1991, 113, 224-232.

[85] [a] J. Sandrin, D. Soerens, J. M. Cook, Heterocycles 1976, 4, 1249-1255. [b] F. Ungemach, D. Soerens, R. Weber, M. Dipierro, O. Campos, P. Mokry, J. M. Cook, J. V. Silverton, J. Am. Chem. Soc. 1980, 102, 6976-6984.

[86] M. Limbach, S. Dalai, A. Janssen, M. Es-Sayed, J. Magull, A. de Meijere, Eur. J. Org. Chem. 2005, 610-617.

[87] J. C. Powers, P. E. Wilcox, J. Am. Chem. Soc. 1970, 92, 1782-1783.

[88] P. Chen, P. T. W. Cheng, S. H. Spergel, R. Zahler, X. Wang, J. Thottathil, J. C. Barrish, R. P. Polniaszek, Tetrahedron Lett. 1997, 38, 3175-3178.

[89] L. Luc, A. Jocelyne, D. Philippe, D. Patrice, J. Med. Chem. 1999, 42, 277-290.

[90] [a] D. M. Raush, M. M. Patel, Synth. Commun. 1985, 15, 675-679. [b] F. J. Wang, J. R. Hauske, Tetrahedron Lett. 1992, 33, 6267-6270. [c] P. Jiao, J. Xu, Q. Zhang, M. C. K. Choi, A. S. C. Chan, Tetrahedron Assym. 2001, 22, 3081-3088.

[91] [a] A. S. Guram, R. A. Rennels, S. L. Buchwald, Angew. Chem. 1995, 107, 1456-1459; Angew. Chem. Int. Ed. 1995, 34, 1348-1350. [b] J. Louie, J. F. Hartwig, Tetrahedron Lett. 1995, 36, 21, 3609-3612.

[92] L. Jiang, S. L. Buchwald, Metel Caralyzed-Cross coupling reactions, WILEY-VHC, Ed: A. de Meijere, F. Diederich, 2004, 699-760.

[93] J. Hassan, M. Sevignon, C. Gozzi, E. Schulz, L. Lamaire, Chem. Rev. 2002, 102, 1359.

[94] [a] J. Thibonnet, P. Knochel, Tetrahedron Lett. 2000, 41, 3319-3322. - [b] J. Thibonnet, V. A. Vu, L. Berillon, P. Knochel, Tetrahedron 2002, 58, 4787-4800.

[95] S. Chandrasekhar, C. Narsihmulu, M. R. Reddy, M. S. Reddy, Tetrahedron Lett. 2003, $44,2583-2585$.

[96] [a] A. Meheta, A. Silamkoti, B. Miriyala, S. K. Arora, B. Srinivasulu, B. Mukherjee, J. B. Gupta, WO Patent 04/052857 A1, 2004. [b] A. Meheta, S. K. Srivastava, J. B. Gupta, WO Patent 04/056810 A1, 2004; WO Patent 04/056811 A1, 2004. [c] A. Meheta, B. Miriyala, N. Kumar, J. B. Gupta, WO Patent 04/069835 A1, 2004.

[97] R. Stuermer, B. Schaefer, V. Wolfart, H. Stahr, U. Kazmaier, G. Halmchen, Synthesis, 2001, 46-48.

[98] P. Schwab, R. H. Grubbs, J. W. Ziller, J. Am. Chem. Soc. 1996, 118, 100-118. 
[99] T. W. Greene, P. G. M. Wuts, Protective in Organic Synthesis, Wiley-Interscience.

[100] J. P. Wolf, S. Wagaw, J. -F. Marcoux, S. L. Buchwald, Acc. Chem. Res. 1998, 31, 805818.

[101] F. Y. Kwong, A. Klopars, S. L. Buchwald, Org. Lett. 2002, 4, 581-584.

[102] E. Rogana, D. L. Nelson, M. Mares-Guia, J. Am. Chem. Soc. 1975, 97, 6844-6848.

[103] P. Reynaud, J.-D. Brion, E. Nguyen-Tri-Xuong, C. Davrinche, F. Pieri, M.-L. ArnouldGuerin, Eur. J. Med. Chem. 1989, 24, 427-434. 


\section{Acknowledgements}

First of all, I owe my sincere thanks and deepest sense of gratitude to Professor A. de Meijere for his guidance and giving me the opportunity to carry out my doctoral dissertation in his group.

I wish to thank the Professors J. Magull, C. Schnieder, A. Zeeck and A. de Meijere at the University of Göttingen, M. Periasamy, D. Basavaiah, M. Durgaprasad, and K. C. Kumaraswamy at University of Hyderabad, Dr. S. Mishra and Dr. A. K. Panigrahi at Ravenshaw College Cuttack for their scientific lectures.

I am very thankful to Mr. Machinek for the measurement of the NMR spectra. I thank Dr. Frauendorf and Mrs. Udvarnoki for the measurement of the mass spectra and Mr. Hambloch for carrying out the elemental analyses. I am also very much grateful to M. Sevvana for the determination of the X-ray structures.

I express my sincere thanks to Dr. V. Belov and Dr. S. Nizamov for their help in the lab and for their interesting and fruitful discussions.

I would like to thank Dr. Mazen Es Sayed (Bayer AG) and Dr. D. Finsinger (Merck, Darmstadt) for their financial support and fruitful discussions.

I thank Alessandra, Michael, Marco, Mario, Ligang and Baris for the careful correction of this work. I would also like to thank all my lab mates in lab 308 for their help and encouragement at the time of working in the lab.

I am very grateful to Mrs. G. Keil Knepel, for her helping hand at any time.

I thank all my colleagues in our research group of Prof. de Meijere, especially Stefan, Daniel, Anna, Farina, Heiko, Hans, and Takuya for the good and motivating work atmosphere.

I would like to thank Pradip and Rekha for their friendship and helping hands in Göttingen without whom it would have been very difficult to stay away from home for such a long time.

I thank Manmohan, Devasmita for their friendliness during my stay in Göttingen.

I would like to warmly thank Pratap for always being there at the time of writing my thesis.

Finally, I thank my parents and other family members for their love, constant encouragement and support. 



\section{Lebenslauf}

Ich wurde am 20. September 1979 als 2. Kind von Anjanabala und Brahmananda Dalai in Jagatsingpur, Orissa, Indien geboren.

Von Juni 1984 bis Mai 1989 besuchte ich die "Kolar Jaypur Upper Primary School" in Kolar, wechselte dann im Juli 1989 zur "Jagannath High School Kolar Jaypur" in Kolar, in der ich bis Juli 1994 verblieb.

Von August 1994 bis Juni 1996 besuchte ich das "Ispat College Rourkela" in Orissa, in der ich mein I. Sc. (Intermediate in Science) ablegte.

Von 1996 bis 1999 studierte ich am "Ravenshaw College Cuttack, Utkal University Vanivihar” in Orissa im Fachgebiet Chemie (als Honours student).

Am 08. Juli 1999 wurde mir der akademische Grad "First Class B. Sc. with distinction" zugesprochen.

Zum Sommersemester 1999/2000 nahm ich das Studium der Chemie an der "University of Hyderabad, Hyderabad" auf. Anschließend bis Mai 2001 absolvierte ich unter der wissenschaftlichen Anleitung von Prof. Meriapan Periasamy meine Diplomarbeit zum Thema "Efforts Towards the Synthesis of Chromium Carbonyl Species for Application in Organic Synthesis". Am 31. Juli 2001 wurde mir der akademische Grad "M. Sc. (Master of Science with Distinction)" zuerkannt.

Seit September 2001 arbeite ich im Arbeitskreis von Prof. Dr. Armin de Meijere an meiner Doktorarbiet mit dem Titel "New Applications of Methyl 2-Chloro-2-cyclopropylideneacetate Towards the Synthesis of Biologically Important Heterocycles".

Im Wintersemester 2002/2003 nahm ich erfolgreich an einem Kurs zur Heterocyclen-chemie teil. Seit Januar 2005 bin ich als wissenschaftlicher Mitarbeiter angestellt.

Ich errang im Jahr 2001 im "GATE (Graduate Aptitude Test for Engineering)" den 39. Platz von allen Chemikeren in Indien mit 98,01 percentile. Zusätzlich bekam ich den "Junior Research Fellowship Award" von CSIR, Indien.

Suryakanta Dalai 